\title{
Data Recovery Investigations at the Tank Destroyer Site (41CV1378) at Fort Hood, Coryell County, Texas
}

\author{
Douglas K. Boyd
}

Prewitt and Associates, Inc.

John E. Dockall

Karl W. Kibler

Cross Timbers Geoarcheological Services

Gemma Mehalchick

Laura M. Short

Follow this and additional works at: https://scholarworks.sfasu.edu/ita

Part of the American Material Culture Commons, Archaeological Anthropology Commons, Environmental Studies Commons, Other American Studies Commons, Other Arts and Humanities Commons, Other History of Art, Architecture, and Archaeology Commons, and the United States History Commons

Tell us how this article helped you.

This Article is brought to you for free and open access by the Center for Regional Heritage Research at SFA ScholarWorks. It has been accepted for inclusion in Index of Texas Archaeology: Open Access Gray Literature from the Lone Star State by an authorized editor of SFA ScholarWorks. For more information, please contact cdsscholarworks@sfasu.edu. 


\section{Data Recovery Investigations at the Tank Destroyer Site (41CV1378) at Fort Hood, Coryell County, Texas}

\section{Licensing Statement}

This is a work for hire produced for the Texas Department of Transportation (TxDOT), which owns all rights, title, and interest in and to all data and other information developed for this project under its contract with the report producer. The report may be cited and brief passages from this publication may be reproduced without permission provided that credit is given to TxDOT and the firm that produced it. Permission to reprint an entire chapter, section, figures or tables must be obtained in advance from the Supervisor of the Archeological Studies Branch, Environmental Affairs Division, Texas Department of Transportation, 125 East 11th Street, Austin, Texas, 78701. 


\title{
DATA RECOVERY INVESTIGATIONS AT THE TANK DESTROYER SITE (41CV1378) AT FORT HOOD, CORYELL COUNTY, TEXAS
}

\author{
by \\ Douglas K. Boyd \\ John E. Dockall \\ Karl W. Kibler \\ Gemma Mehalchick \\ and \\ Laura M. Short \\ with contributions by \\ Chester P. Walker \\ Co-Principal Investigators: Douglas K. Boyd and Karl W. Kibler \\ REPORTS OF INVESTIGATIONS, NUMBER 172 \\ Prewitt and Associates, Inc. \\ Cultural Resources Services \\ Austin, Texas \\ PAI Nos. 207011, 207024, 209002, 210023, and 214014 \\ ARCHEOLOGICAL STUDIES PROGRAM, REPORT NO. 149 \\ Texas Department of Transportation \\ Environmental Affairs Division \\ Austin, Texas \\ CSJ No. 3623-01-001
}

July 2014 


\title{
DATA RECOVERY INVESTIGATIONS AT THE TANK DESTROYER SITE (41CV1378) AT FORT HOOD, CORYELL COUNTY, TEXAS
}

\author{
COPYRIGHT @ 2014
}

\section{Texas Department of Transportation (TxDOT)}

This is a work for hire produced by the Texas Department of Transportation (TxDOT), which owns all rights, title, and interest in and to all data and other information developed for this project under Contract No. 579XXSA002 (Work Authorizations 57902SA002 and 57911SA002), and Contract No. 577XXSA001 (Work Authorizations 57713SA001, 57702SA001, and 57305SA003). Brief passages from this publication may be reproduced without permission provided that credit is given to TxDOT and Prewitt and Associates, Inc. Permission to reprint an entire chapter, section, figures or tables must be obtained in advance from the Supervisor of the Archeological Studies Program, Environmental Affairs Division, Texas Department of Transportation, 125 East 11th Street, Austin, Texas, 78701. A copy of this final report will be submitted to the Texas State Library and Archives Commission, State Publications Depository Program in compliance with 13 TAC §26.24(a).

\author{
jointly published by \\ Texas Department of Transportation \\ Environmental Affairs Division \\ Archeological Studies Program \\ Scott Pletka, Ph.D., Supervisor \\ Archeological Studies Program, Report No. 149
}

and

Prewitt and Associates, Inc.

Cultural Resources Services

Austin, Texas

PAI Project Nos. 207001, 207024, 209002, 210023, and 214014

Printed by OneTouchPoint/Ginny's in Austin, Texas

ISBN 978-1-935545-16-3 


\section{TABLE OF CONTENTS}

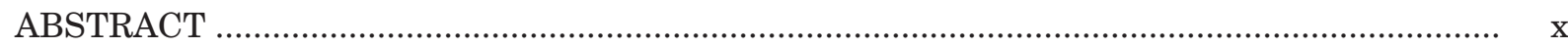

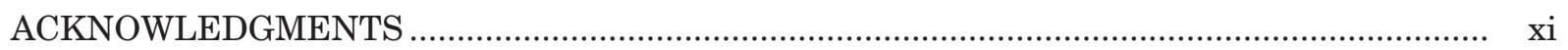

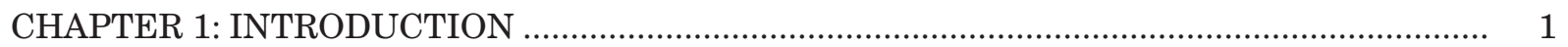

CHAPTER 2: ENVIRONMENTAL BACKGROUND AND SITE SETTING ............................. 3

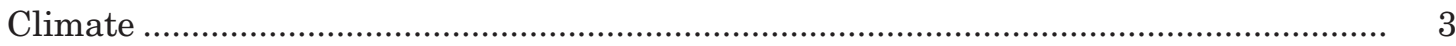

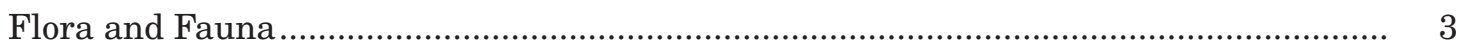

Geology, Geomorphology, and Late Quaternary Stratigraphy ........................................ 4

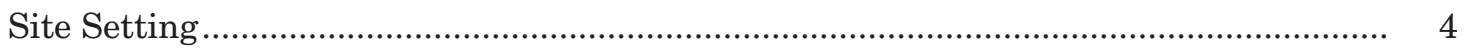

Burned Rock Mounds in Upland Settings .............................................................. 5

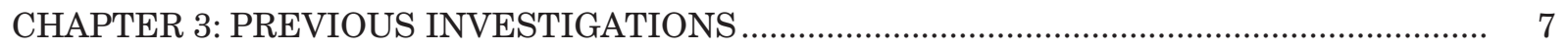

CHAPTER 4: METHODS OF INVESTIGATIONS AND WORK ACCOMPLISHED.................. 11

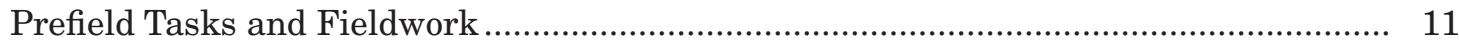

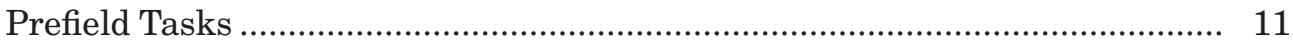

Remote Sensing Survey ........................................................................ 11

Excavations, Site Mapping, and Field Documentation .................................. 12

Laboratory Processing and Initial Reporting ......................................................... 15

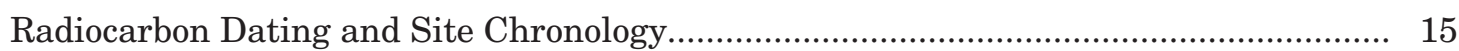

Comparing Absolute and Relative Chronologies .......................................... 19

Research Design for the Data Recovery Analysis ...................................................... 20

Research Issues and Goals ........................................................................ 21

CHAPTER 5: IMPLEMENTING AND EVALUATING THE TxDOT CHIPPED STONE

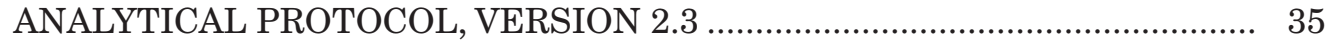

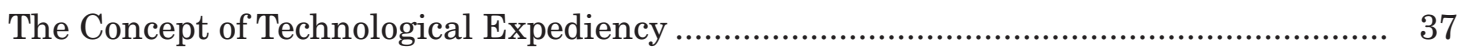

Comments on the Chipped Stone Tool Analytical Protocol........................................... 38

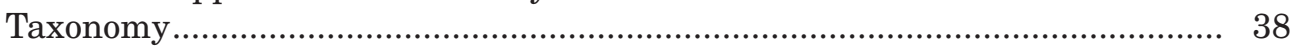

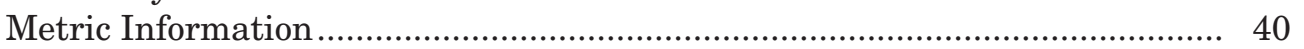

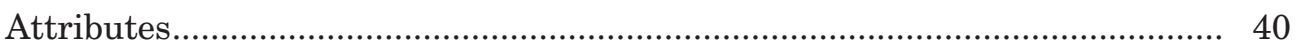

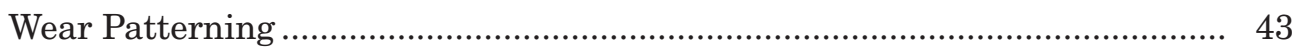

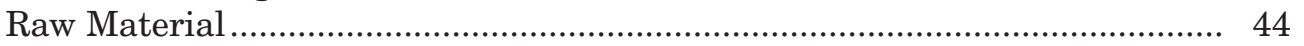

Comments on the Chipped Stone Unmodified Debitage Analytical Protocol .................. 44

CHAPTER 6: DESCRIPTION OF MATERIAL CULTURE ....................................................... 47

Chipped Stone Tools, Cores, and Debitage................................................................. 47

Arrow Point Preform .............................................................................. 47

Dart Points ................................................................................................... 47

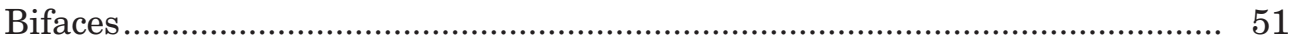

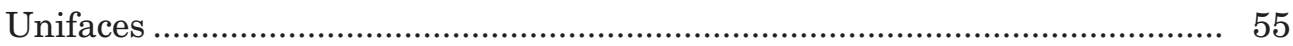

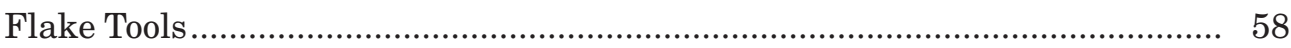

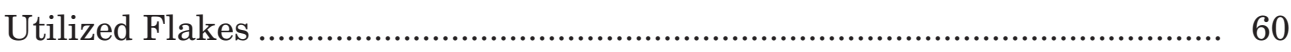




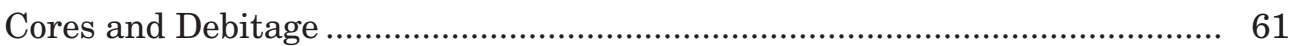

Spatial Patterning of Chipped Stone Artifacts ............................................ 66

Summary and Conclusions ......................................................................... 66

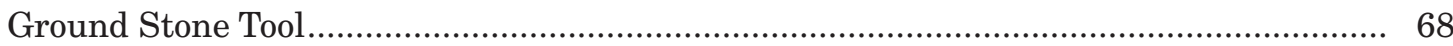

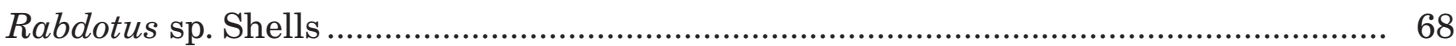

Rabdotus sp. Shell Distributions................................................................. 69

Age Structure of the Rabdotus sp. Shell Assemblage ..................................... 69

CHAPTER 7: DESCRIPTION OF FEATURES AND SPATIAL ANALYSIS OF

BURNED ROCKS AND ARTIFACTS .......................................................... 71

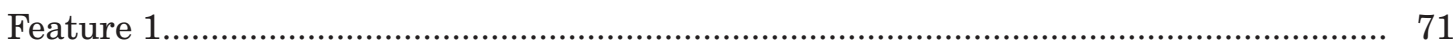

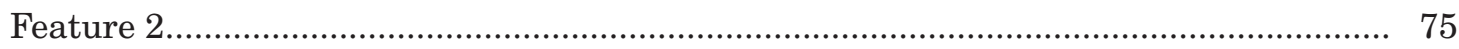

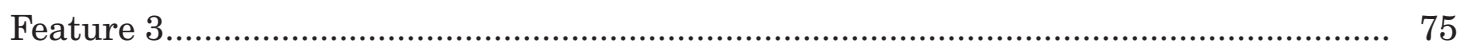

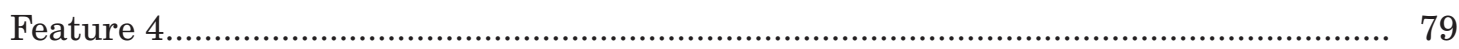

Analysis of Burned Rock and Artifact Data: Defining the Structure

of the Burned Rock Mound .................................................................................... 79

Burned Rock Data ...................................................................................... 80

Matrix Column Data ................................................................................... 86

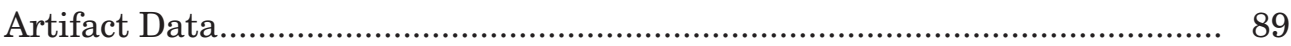

Summary of the Tank Destroyer Burned Rock Mound ................................... 92

The Status of Burned Rock Midden Studies in Central Texas ........................................ 93

The Tank Destroyer Site as a Central Texas Burned Rock Mound................................. 94

Size and Shape of the Burned Rock Mound at the Tank Destroyer Site ........ 94

Burned Rock Weights and Use-Episode Estimates ......................................... 95

CHAPTER 8: A LANDSCAPE ANALYSIS OF FORT HOOD ARCHEOLOGICAL AND ENVIRONMENTAL DATA.................................................................. 97

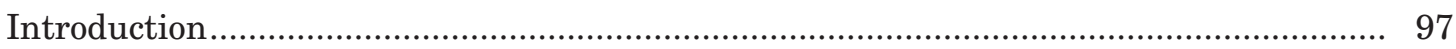

Landscape Study 1: Examining the Distribution of Burned Rock Mounds

The Fort Hood Study Area and GIS Database................................................ 98

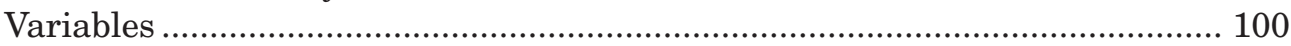

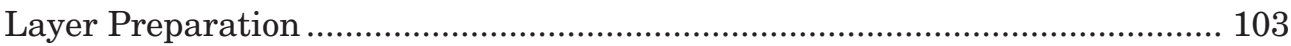

Analytical Methods and Results................................................................. 103

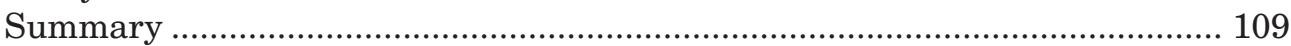

Landscape Study 2: Post Oaks, Paluxy Sands, and Burned Rock Features

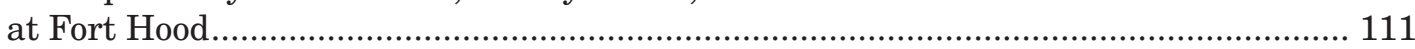

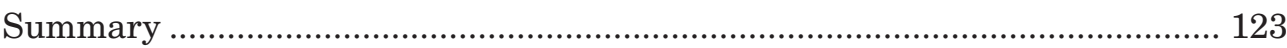

CHAPTER 9: EXAMINING SOCIAL IDENTITY IN THE CENTRAL TEXAS

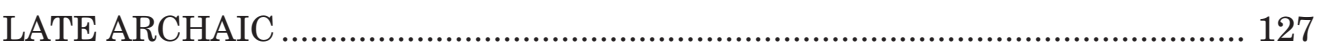

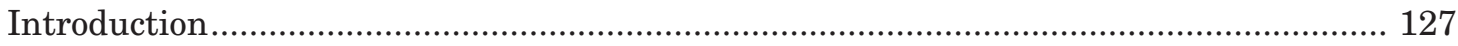

Archeological Patterns of the Central Texas Late Archaic ............................................ 127

Social Identity, Social Fields, and Social Boundaries.................................................... 129

Social Groups and Fields in Prehistoric Texas .............................................................. 132

Future Research on Social Identity in the Late Archaic of Central Texas ....................... 134 
CHAPTER 10: SUMMARY OF FINDINGS AT THE TANK DESTROYER SITE ...................... 143

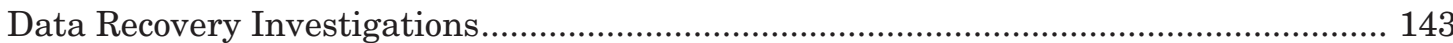

The Chipped Stone Assemblage and the TxDOT Lithic Analysis Protocol...... 143

The Rabdotus sp. Shell Assemblage as a Food Source ...................................... 146

Burned Rocks and Cooking Facilities............................................................... 146

Landscape Analysis of Burned Rock Mounds and Middens on Fort Hood....... 146

Social Identity in the Late Archaic of Central Texas ....................................... 148

Social Aspects of Burned Rock Middens and Mounds in Central Texas ........................... 149

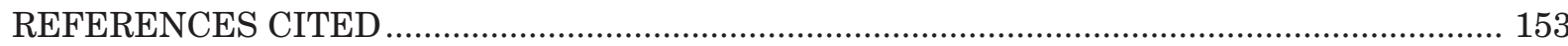

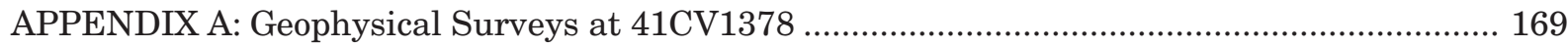

APPENDIX B: Inventory of Cultural Materials from 41CV1378 ............................................ 179

APPENDIX C: TxDOT Chipped Stone Analytical Protocol ....................................................... 185

APPENDIX D: Lithic Analysis Protocol Blank Data Entry Form for Chipped Stone Tools ......... 221

APPENDIX E: Lithic Analysis Protocol Blank Data Entry Form for Unmodified Debitage

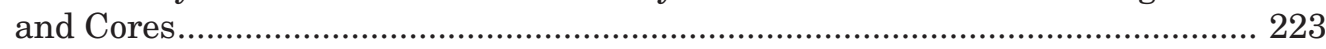

APPENDIX F: Lithic Analysis Protocol Form for Chipped Stone Tools from 41CV1378............. 225

APPENDIX G: Lithic Analysis Protocol Form for Unmodified Debitage and

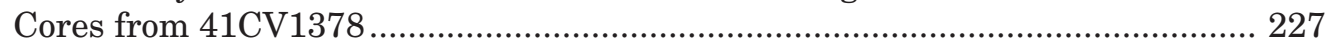

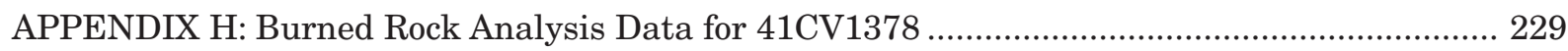




\section{LIST OF FIGURES}

1.1. Location of 41CV1378 on Fort Hood, Texas......................................................................... 2

3.1. TRC Mariah map of 41CV1378 showing the 1994 investigations .................................... 8

4.1. Map of data recovery excavations at 41CV1378 ....................................................... 13

4.2. Map of the features and locations of the 16 radiocarbon-dated samples .......................... 17

4.3. Comparison of calibrated radiocarbon dates.................................................................... 18

4.4. Comparison of calibrated and corrected radiocarbon dates ............................................ 19

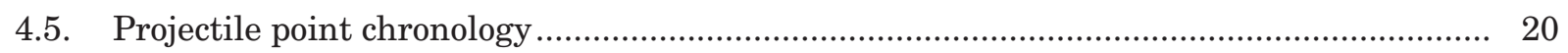

4.6. Comparison of radiocarbon dates and projectile point chronology …............................... 21

5.1. TxDOT's Chipped Stone Analytical Protocol, Version 2.3, classification scheme

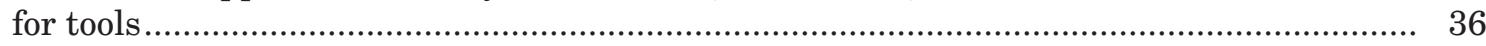

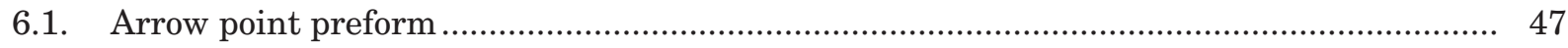

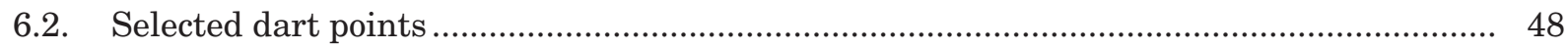

6.3. Selected untyped dart points and untypeable fragments.............................................. 51

6.4. Selected Stage 1-4 bifaces and biface fragments............................................................. 52

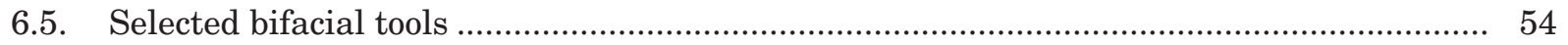

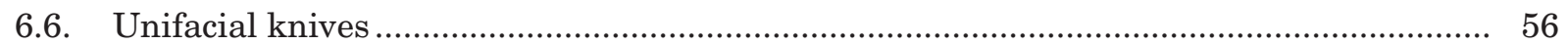

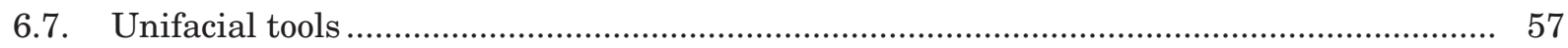

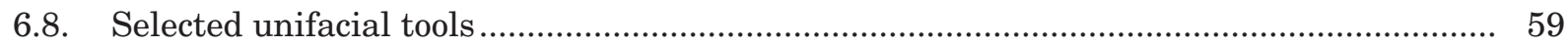

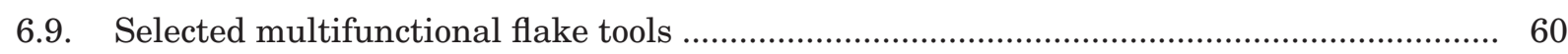

7.1. Topographic map showing excavation units and cultural features ................................... 72

7.2. Overviews of the burned rock mound at the Tank Destroyer site,

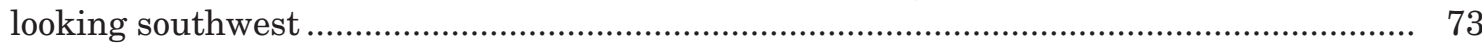

7.3. Views of data recovery excavations of the burned rock mound Feature 1 at the Tank Destroyer site.

7.4. Plan drawing of the burned and unburned limestone rocks comprising

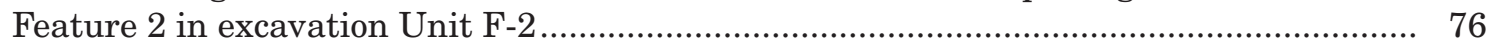

7.5. Views of Feature 3, a rock-lined pit within Feature 1 ….............................................. 77

7.6. Schematic plan and profile drawings of Feature 3 ....................................................... 78

7.7. Map showing the thickness of the burned rock deposits across the Feature 1 mound.

7.8. Maps showing the distribution of burned rocks by weight and density in and around the burned rock mound.....

7.9. Schematic profiles of the five matrix column samples comparing burned rocks, sediments, and Rabdotus sp. shells

7.10. Schematic profiles of the five matrix column samples comparing ratios of burned rocks to sediment, burned rocks to Rabdotus sp. shells, and sediment to Rabdotus sp. shells.... 
7.11. Bar graph comparing the total weight of burned rocks to sediment in all of the matrix column samples

7.12. Distribution map of chipped stone tools, cores, and unmodified debitage 91

8.1. The Fort Hood landscape analysis study area 99

8.2. Maximum likelihood classification comparing the predicted locations of burned rock mounds, middens, and scatters for the unmasked and masked data.... 105

8.3. Graph of maximum likelihood classification confidence levels for the masked and unmasked data sets. 107

8.4. Local $\mathrm{R}^{2}$ values for Geographically Weighted Regression of sites vs. nonsites ................. 112

8.5. Local $\mathrm{R}^{2}$ values for Geographically Weighted Regression of mounds vs. middens.............. 113

8.6. Generalized geologic cross section of the Lampasas Cut Plain in central Texas .....

8.7. Distribution of three post oak communities on Fort Hood using data provided by the Nature Conservancy...

8.8. Distribution of Paluxy outcrops in west-central Fort Hood based on geologic and soils data

8.9. Distribution of expanded Paluxy outcrops in west-central Fort Hood based on geologic and soils data in conjunction with the distribution of post oak communities

8.10. Distribution of known Paluxy sites $(n=37)$ and expanded Paluxy outcrops from Figure 8.9

8.11. Distribution of all burned rock mounds and middens across Fort Hood

9.1. Map of sites containing significant components of the Marcos-Montell-Castroville projectile point interval, or Uvalde phase

9.2. Map of sites containing significant components of the Ensor-Frio-Fairland projectile point interval, or Twin Sisters phase

9.3. Map of sites containing significant components of the Darl projectile point interval, or Driftwood phase

A.1. Magnetometer data 173

A.2. Magnetometer data with interpretations. 174

A.3. Positive magnetic anomalies.... 175

A.4. GPR amplitude slice maps 176

A.5. GPR amplitude slice maps with interpretations 176 


\section{LIST OF TABLES}

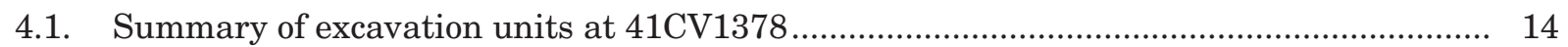

4.2. Radiocarbon dates from 41CV1378 .......................................................................... 16

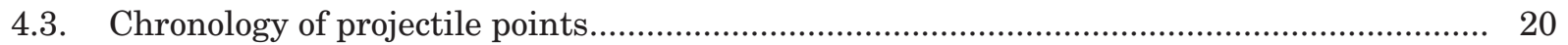

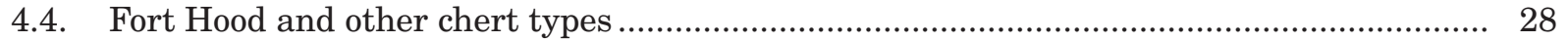

4.5. Technical definitions of various flake types .................................................................... 30

6.1. Dimensions and weights of identified projectile points ................................................ 49

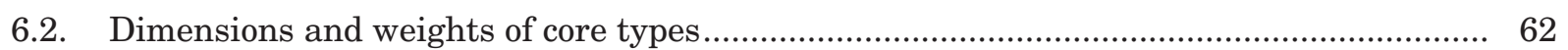

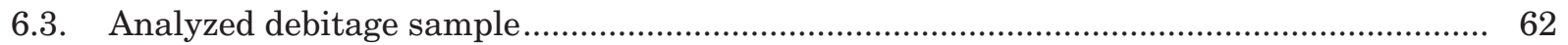

6.4. Identified raw materials of the tools and analyzed debitage sample ................................ 63

6.5. Flake type and probable technological origins of analyzed debitage.................................. 64

6.6. Flake and platform types of analyzed debitage ........................................................... 65

6.7. Proportions of cortex classes, size grade variability, and degree of fragmentation

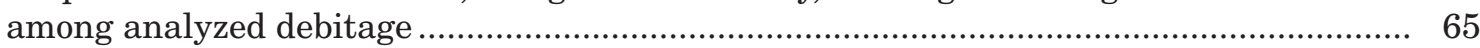

6.8. Correlation of selected flake types, total flake weight, and average flake weight

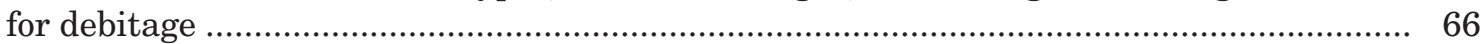

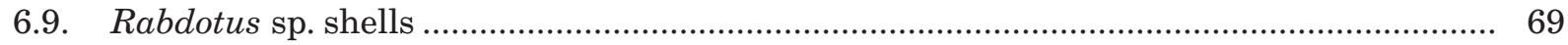

6.10. Rabdotus sp. shells recovered from flotation and soil column samples ............................ 70

7.1. Burned rocks and cultural sediments at 41CV1378 ..................................................... 79

7.2. Burned rock weights by feature and size class ........................................................... 80

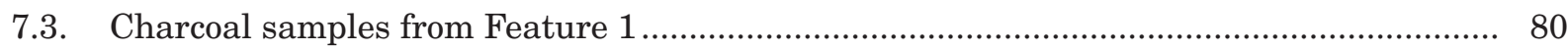

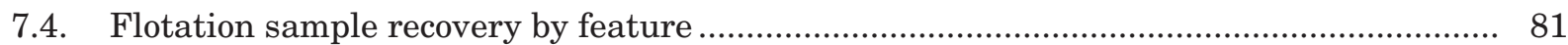

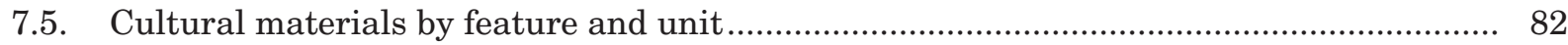

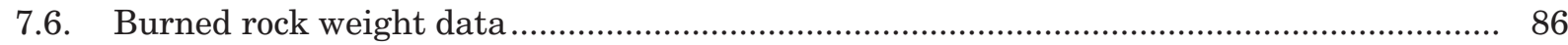

7.7. Percentage of size classes of burned rocks by feature …................................................ 86

7.8. Density and frequency of lithic artifacts recovered from the burned rock mound,

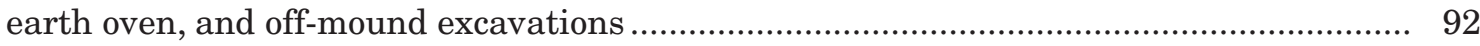

7.9. Estimated number of cooking episodes represented by the burned rock mound

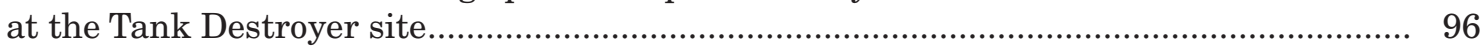

8.1. Feature types in the Fort Hood CRM database and nonsite locations ............................. 104

8.2. Ordinary least squares analysis diagnostics for sites and nonsites ................................ 108

8.3. Statistics for ordinary least squares analysis comparing environmental variables

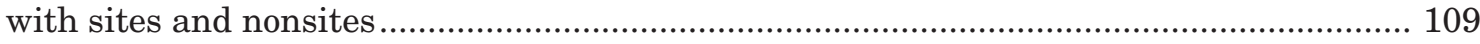

8.4. Ordinary least squares analysis diagnostics for burned rock mounds and middens.......... 110

8.5. Statistics for ordinary least squares analysis comparing environmental variables

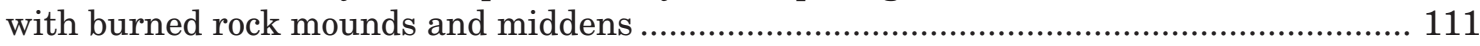

8.6. Summary of Paluxy sites on Fort Hood ............................................................................... 120 
9.1. Central Texas sites with significant components attributed to selected Late Archaic projectile point intervals and cultural phases .............................................................. 137

B.1. Inventory of cultural materals recovered from $41 \mathrm{CV} 1378$ by unit and provenience.......... 181

H.1. Summary of burned rock and sediment sample data for 41CV1378 ................................. 231

H.2. Summary of burned rock data for the 41CV1378 general excavations by size classes,

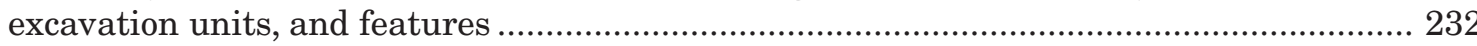

H.3. Summary of burned rock and sediment data for 41CV1378 samples.............................. 233

H.4. Summary of burned rock data for $41 \mathrm{CV} 1378$ samples by size classes, excavation

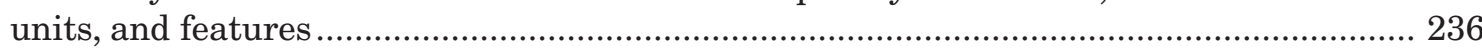

H.5. Adjusted burned rock data for Mariah's 1994 Test Pit 1............................................... 238

H.6. Summary of all 41CV1378 burned rock data by excavation units and features................ 239

H.7. Summary of all burned rock data for 41CV1378 by size classes, excavation units, and features

H.8. Summary of volumetric calculations for excavation units and feature

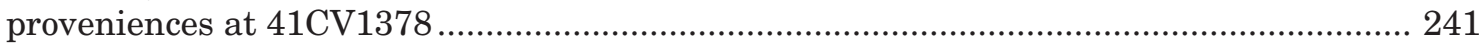

H.9. Summary of all burned rocks from 41CV1378 by weight and density ............................ 243

H.10. Summary of matrix column sample data for 41CV1378 ................................................ 245

H.11. Raw data, volume adjusted data, and calculated ratios for matrix sample columns at $41 \mathrm{CV} 1378$. 


\begin{abstract}
Data recovery investigations at the Tank Destroyer site (41CV1378) were conducted in August 2007 for the Texas Department of Transportation (TxDOT). This work was required because of potential impacts to the site from TxDOT's planned improvements of Tank Destroyer Boulevard and State Highway 9. The investigations focused on a burned rock mound (Feature 1), one-half of which has been destroyed by an adjacent tank trail. The mound contained two internal features: an off-centered earth oven and a small cluster of Rabdotus sp. shells. With the exception of the location of its earth oven, the mound at the Tank Destroyer is typical of a classic central Texas domed mound, though slightly flattened by postdepositional processes.

In all, an area of $30.5 \mathrm{~m}^{2}$ and volume of $11.8 \mathrm{~m}^{3}$ of cultural deposits were hand excavated, and an additional ca. $17.3 \mathrm{~m}^{2}$ was mechanically stripped. The mound excavations yielded 5,570.5 kg of burned rocks. Artifacts recovered from mound and nonmound contexts consist of 129 chipped stone tools, 9 cores and core fragments, 4,466 pieces of unmodified debitage, 1 ground stone tool, 2 unmodified bone fragments, 1,415 Rabdotus sp. shells, and 40 historic artifacts. In addition, 413 pieces of microdebitage and 251 Rabdotus sp. shells were recovered from flotation and soil column samples taken from the mound. There was virtually no preservation of vertebrate faunal remains and poor preservation of botanical remains. No economic plants (i.e., food resources) were recovered despite the collection and processing of flotation samples. Sixteen radiocarbon assays on charred wood and Rabdotus sp. shells date the site occupation to 1500 B.C. through A.D. 1650. The date range for the diagnostic projectile points recovered from the site (200 B.C. to A.D. 1200) fits nicely within the range of radiocarbon dates. As a group, the radiocarbon dates and the projectile points suggest that the most intensive period of site use occurred intermittently between 1000 B.C. and A.D. 1200 .

Like most burned rock mounds, the mound at the Tank Destroyer site consisted of a jumbled mass of burned rocks that episodically accreted around an earth oven. These processes and repeated use over centuries limit our ability to recognize distinct components for analysis. Given these limitations, our analysis took a different approach. While it includes traditional analyses of the lithic, burned rock, and snail assemblages, it also examines social identity during the Late Archaic period in central Texas and the relationships between burned rock mounds and middens and environmental variables through a landscape analysis.
\end{abstract}

\title{
CURATION
}

All project records and artifacts generated by this project will be submitted for permanent curation to the laboratory facility of the Cultural Resources Management Program, Environmental Management Office, Directorate of Public Works, Fort Hood, Texas. 


\section{ACKNOWLEDGMENTS}

The archeological investigations at the Tank Destroyer site were sponsored by the Texas Department of Transportation (TxDOT). Project manager Jim Abbott of TxDOT's Archeological Studies Program provided his usual sage advice, from the prefield planning through the final analysis and report. Jason Barrett served as the contact for our work pertaining to the TxDOT Chipped Stone Analytical Protocol. All of our fieldwork at Fort Hood was coordinated by Fort Hood's cultural resources program director, Karl Kleinbach, and archeologist Sunny Wood, and their assistance is greatly appreciated. During the analysis phase, Richard Jones took over as the Fort Hood cultural resources director.

For Prewitt and Associates, Doug Boyd served as the principal investigator, Gemma Mehalchick was project archeologist, and the field crew consisted of Roman Clem, Mark Holderby, Christopher Kugler, and Matt Taylor. Karl Kibler (co-principal investigator) joined the project during the analysis phase, and laboratory supervisor Rob Thrift processed all artifacts and special samples. John Dockall conducted the lithic analysis and reviewed the TxDOT Chipped Stone Analytical Protocol, and Sandy Hannum and Brian Wootan prepared the figures. Karl Kibler and Elaine Robbins edited the report.

Other contributors conducted special studies. Chet Walker of Archaeo-Geophysical Associates, LLC, conducted the magnetometer and ground-penetrating radar surveys, and Laura Short of the Department of Anthropology at Texas A\&M University conducted the GIS landscape analysis. The Center for Applied Isotope Studies at the University of Georgia performed the radiocarbon dating. 



\section{INTRODUCTION}

Gemma Mehalchick

In August 2007, Prewitt and Associates, Inc. (PAI), completed data recovery excavations of a portion of a burned rock mound at the Tank Destroyer site (41CV1378) at Fort Hood, a military reservation in Bell and Coryell Counties, Texas. The work was conducted for the Environmental Affairs Division of the Texas Department of Transportation (TxDOT) under Contract No. 577XXSA001, Work Authorization No. 57713SA001, and Excavation Permit Control No. 07-375 from Fort Hood. As per an agreement between TxDOT and the Texas Historical Commission (THC), no Texas Antiquities Permit was required for these investigations since the site is on the federal lands of Fort Hood. The burned rock mound at $41 \mathrm{CV} 1378$ was previously recommended as eligible for listing in the National Register of Historic Places. Therefore, archeological investigation of the site was required because of potential impacts from TxDOT's planned extension of Tank Destroyer Boulevard in conjunction with the construction of Northeast Copperas Cove Bypass. The results of the data recovery excavations are presented in this report under Contract No. 579XXSA002, Work Authorization Nos. 57911SA002 and 57305SA003.

Before starting the data recovery effort, PAI developed a work plan for investigating the site. Once the work plan was approved by TxDOT, the Fort Hood Cultural Resource Management Program, and THC, it became the scope of work for the data recovery effort. The plan specified the completion of three tasks. Task 1, which consisted of the prefield research, geophysical investigation, and field preparation, was completed in stages in May through July 2007. Task 2, the data recovery excavations, was conducted in August 2007. Task 3 consisted of the preliminary laboratory processing of the recovered prehistoric artifacts, radiocarbon dating of charcoal and snail shell samples, and the preparation of an interim report.

Site $41 \mathrm{CV} 1378$ is an upland surface site bordered by Turkey Run Creek and Tank Destroyer Boulevard on the Fort Hood military installation (Figure 1.1). The data recovery investigations focused on a burned rock mound, which is designated Feature 1, and the offmound area immediately surrounding it. A portion of the mound had been destroyed by a tank trail. The goal was to conduct a thorough archeological examination of the remaining portion of the mound to mitigate the adverse effects associated with the road enhancement project. Hand and machine excavations revealed that the feature was composed of a dense central core of burned rocks surrounded by thinner cultural deposits, which included one large intact pit feature or earth oven (Feature 3). The off-mound deposits produced sparse burned rocks, greater amounts of lithic artifacts, and one possible expedient hearth (Feature 2). The work revealed that less than half of the original burned rock mound remained intact. The PAI excavations effectively removed the remaining portion of the feature. 
Maps with site locations are not shown in report copies for public distribution.

Figure 1.1. Location of $41 \mathrm{CV} 1378$ on Fort Hood, Texas. Base map is the 1:50,000 scale "Fort Hood Military Installation Map, V782S, Edition 6-DMA," prepared and published by the Defense Mapping Agency, 1996. 


\section{ENVIRONMENTAL BACKGROUND AND SITE SETTING}

Karl W. Kibler, Gemma Mehalchick, and Douglas K. Boyd

The following is a brief summary of Fort Hood's natural environment, since many reports present this information in greater detail (Mehalchick et al. 1999; Nordt 1992; Trierweiler 1994, 1996; U.S. Army 1979). Much of this section is taken from the environmental background chapter of Mehalchick et al. (2004:9-13).

Fort Hood is in the Lampasas Cut Plain, a subprovince of the Grand Prairie (Hayward et al. 1996) and dissected northeastern edge of the Edwards Plateau (Hill 1901). The Lampasas Cut Plain exhibits a diverse floral community and contains important microhabitats due to its great topographic variability and deeply incised streams (Diggs et al. 1999). Nordt's (1992) study provides the groundwork specific to the archeological geology of Fort Hood. The flora and fauna are typical of the Balconian and Texan biotic provinces (Blair 1950). The biotic assemblage represents a mix of species typical of the Blackland Prairie to the east and the Edwards Plateau to the west.

\section{CLIMATE}

The modern climate of the Fort Hood area is subtropical, characterized by hot, humid summers and relatively short, dry winters (Natural Fibers Information Center 1987:6). The prevailing wind blows from the south, reaching its peak during the spring. Summer temperatures are high, with an overall average of $83^{\circ} \mathrm{F}\left(28.3^{\circ} \mathrm{C}\right)$ and an average daily maximum of $96^{\circ} \mathrm{F}\left(35.5^{\circ} \mathrm{C}\right)$ in Coryell County. The average temperature in winter is $49^{\circ} \mathrm{F}\left(9.4^{\circ} \mathrm{C}\right)$ but tends to vary considerably with the periodic passage of northern cold fronts, resulting in a pattern of alternating cold and mild days (McCaleb 1985:3).
Annual precipitation is approximately 32.5 inches $(826 \mathrm{~mm})$ for Coryell County (Natural Fibers Information Center 1987:121). Although rainfall occurs year-round, the overall distribution pattern is bimodal, with peak rainfall in the late spring and early fall.

\section{FLORA AND FAUNA}

The flora and fauna of Fort Hood are typical of the Balconian and Texan biotic provinces (Blair 1950). The biotic assemblage represents a mix of species from the Blackland Prairie to the east and the Edwards Plateau to the west. Many specific ecological niches also exist across the base, depending on the local topography, slope aspect, soil, and geology. Dense juniper and oak forest and scrub now characterize the eastern side of the military reservation, but upland areas to the west and south are generally more open. Grasslands are most prevalent on the intermediate upland surfaces. The high upland surface is typically covered by juniper and oak scrub. Riparian zones, exhibiting a variety of hardwood species, are common along drainages.

The Balconian faunal assemblage includes 57 species of mammals, but none of these are solely restricted to the Balconian province (Blair 1950:113). Eight of these species also inhabit the Texan province to the east and the interconnecting riparian zones (Blair 1950:101). Other native fauna include 36 species of snakes, 15 anuran species, and 16 species of lizards. In historic times, several prehistorically significant economic species, such as bison and pronghorn antelope, have been removed from the area.

The flora and fauna of Fort Hood have been significantly altered by Anglo American farming 
and ranching beginning in the mid-nineteenth century, followed by intensive military use of the landscape since the 1940s. Consequently, the modern data must be viewed in light of these extensive historic and modern changes. A case in point is the fact that many species of geophytes were probably abundant on the Fort Hood landscape but are now quite rare (Boyd, Mehalchick, and Kibler 2004:211-218).

\section{GEOLOGY, GEOMORPHOLOGY, AND LATE QUATERNARY STRATIGRAPHY}

The Fort Hood landscape consists of the dissected northeastern margin of the uplifted Edwards Plateau and reflects the variable resistance of the various underlying geologic formations to erosion. Structurally, the area is underlain by a deeply buried extension of the Paleozoic Ouachita Mountains, which divide the stable continental interior to the west from the subsiding Gulf basin to the southeast. During the Cretaceous Period, this region consisted of a very broad shelf covered by a shallow sea. Limestones and marls were deposited on the shelf as the shoreline fluctuated for more than 80 million years. Occasionally, relatively thin deposits of sand derived from terrestrial sources also accumulated on the shelf, resulting in interbedded formations like the Paluxy Formation and Trinity Sands. The Gulf Basin subsided during the Miocene, and the Balcones Fault Zone developed along the old Ouachita line and the uplift of the Edwards Plateau (Woodruff and Abbott 1986). West of the Balcones Fault, the Cretaceous limestones and marls remain relatively horizontal and structurally unmodified, but to the east the Cretaceous rocks dip sharply gulfward and are buried deeply by Gulfian and later lithological units.

Because Fort Hood is west of the fault zone, relatively flat-lying lower Cretaceous rocks showing a two-tiered topography locally termed the Lampasas Cut Plain underlie it (Hayward et al. 1990). This landscape developed between the Brazos and Colorado Rivers and consists of large, mesa-like remnants of an early Tertiary planation surface surrounded by a broad, rolling pediplain formed during the late Tertiary and early Quaternary. These two surfaces differ by 25 to $40 \mathrm{~m}$ in elevation and form what Hayward et al. (1990) called the "high" and "intermediate" uplands and Nordt (1992) referred to as the "Manning" and "Killeen" surfaces. Modern stream valleys are incised approximately 40 to $70 \mathrm{~m}$ into the pediplain surface.

The oldest rocks exposed at Fort Hood belong to the lower Cretaceous Trinity Group, which includes the Glen Rose Formation. This formation is surficially exposed on the western side of Fort Hood, where relatively deep incision of the landscape by Cowhouse Creek and its tributaries has removed the overlying rocks (Proctor et al. 1970; Sellards et al. 1932).

Resting on the Trinity Group are rocks of the lower Cretaceous Fredericksburg Group. The lowest unit is the Paluxy Formation, a terrigenous siliclastic unit of strandplain, fluvial, and deltaic deposits. The Walnut Clay, which is widely exposed at Fort Hood and forms the principle substrate of the Killeen surface, overlies the Paluxy Formation. Above the Walnut Clay lies the Comanche Peak Limestone, which forms the intermediate slopes of the higher Manning surface. The highest extensive lithological unit is the Edwards Limestone, which forms the resistant cap of the high upland mesas or Manning surface. The Edwards Limestone also is a very important source of high-quality chert (see Frederick and Ringstaff 1994; Frederick et al. 1994).

Nordt $(1992,1993,1995)$ identifies six principal alluvial units in the study area based on his extensive examinations of the stratigraphy and soil geomorphology of several larger Fort Hood streams. From oldest to youngest, these units are termed the Reserve alluvium, Jackson alluvium, Georgetown alluvium, Fort Hood alluvium, West Range alluvium, and Ford alluvium (Nordt 1992).

\section{SITE SETTING}

The burned rock mound (Feature 1) at $41 \mathrm{CV} 1378$ is on an intermediate upland that Nordt (1992) defines as the Killeen surface, a pediplain formed on lower Cretaceous carbonate rocks. The feature is composed of anthropogenic deposits, and the surrounding upland Killeen surface is relatively stable, though thin eroded residual soils overlie the bedrock. The Waco Sheet of the Geologic Atlas of Texas (Bureau of Economic Geology 1979) maps the site area as Walnut Clay, a Lower Cretaceous formation that 
occurs stratigraphically below the chert-bearing Edwards group formations. The county soil survey (U.S. Department of Agriculture 1985: Sheet 55, 16-17, 58-59) shows the site area mapped as Cho clay loam. The Cho series soils are very shallow to shallow, well-drained loamy soils on uplands. Typically, the Cho clay loam has an A horizon that is less than 11 inches (ca. $28 \mathrm{~cm}$ ) thick, and it grades quickly into an indurated white caliche formed on weathered limestone.

The Tank Destroyer site covers an area of $300 \mathrm{~m}$ (northwest to southeast) by $250 \mathrm{~m}$ (northeast to southwest), extending from the east bank of Turkey Run Creek upslope to the Killeen surface, where the burned rock mound is located. Feature 1 is ca. $250 \mathrm{~m}$ due east of Turkey Run Creek at an elevation of $1,020 \mathrm{ft}(311 \mathrm{~m})$ above mean sea level, which is about $18 \mathrm{~m}$ higher than the creek. In the spring and summer of 2007, the feature was covered with grasses, while the surrounding upland flora included juniper, oak, gum bumelia, and poison ivy.

\section{BURNED ROCK MOUNDS IN UPLAND SETTINGS}

On Fort Hood, large burned rock middens tend to be situated in alluvial deposits along major streams and in the colluvial toeslopes along the valley walls of major streams. In con- trast, many of the burned rock features found in upland settings on the Killeen and Manning surfaces are isolated burned rock mounds that are generally circular to oval in plan view and domed in cross section. The dichotomy between burned rock middens and mounds on Fort Hood was first observed by Abbott (1996:577-585) and Kleinbach et al. (1995:767-775), who argue that this typological distinction is of considerable importance. Kleinbach et al. (1995:773) believe that: "burned rock middens and burned rock mounds are indeed discrete, separable classes of features representing distinctly different types of behavior." The current authors agree that investigating the differences between middens and mounds should be a critical archeological research focus for understanding prehistoric hunter-gatherer behaviors and land-use patterns in central Texas. The Tank Destroyer site as an excellent example of an upland site with a classic large burned rock mound generated by repeated earth oven cooking activities. As discussed in Chapters 8 and 10 , the locations of burned rock mounds on the landscape are intimately tied to the distribution and proximity of particular resources. In central Texas, we found that three key resources determined when and where earth ovens would be used: root foods of certain geophytic plants, limestone rocks, and hardwood trees needed to provide firewood. 



\section{PREVIOUS INVESTIGATIONS}

When 41CV1378 was first recorded by Texas A\&M University archeologists in 1987, the northern half of the mound feature had been destroyed by a tank trail (MuellerWille and Carlson 1990:147-148; Trierweiler 1996:473-478). The burned rock mound was noted as one of the largest on Fort Hood, with maximum dimensions were estimated to be $40 \times 10 \times 1.25 \mathrm{~m}$, although this estimate now appears inaccurate.

The site was revisited and reevaluated by Mariah Associates archeologists in 1992 (Trierweiler 1994:A-1508-A-1510), and it was divided into two subareas based on different geomorphic contexts for management purposes. Subarea B was a narrow alluvial terrace along Turkey Run Creek. Subarea A encompassed the entire Killeen surface and accounted for more than 90 percent of the site. This area included the burned rock mound, which was designated Feature 1. Bisected by a tank trail, the remaining portion of the feature measured $9.0 \times 2.5 \times 0.4 \mathrm{~m}$. One shovel test was placed in an area investigators believed to be on the mound. It was excavated to bedrock at $10 \mathrm{~cm}$ and contained burned rocks and glass fragments. Because of the shallow depth of the deposits and apparent disturbance, no further work was recommended.

TRC Mariah archeologists returned to 41CV1378 in 1994 and reexamined the site and the burned rock mound (Trierweiler 1996:473-478). They observed the feature to measure approximately $10 \times 5 \mathrm{~m}$. A profile dug along the edge of the tank trail revealed that the burned rock mound cultural deposit was $60 \mathrm{~cm}$ thick. Furthermore, it appeared that the previously excavated shovel test had been dug outside the mound area. Consequently, a 1x1-m test pit was excavated on the highest portion of the mound (Figure 3.1). Feature matrix was present from the surface to $55 \mathrm{~cm}$, and the unit was terminated on sloping bedrock encountered between 60 and $78 \mathrm{~cm}$. The excavation yielded $472 \mathrm{~kg}$ of burned rocks and recovered 14 flakes and a Scallorn arrow point (the latter was found $10-20 \mathrm{~cm}$ below surface). Amino acid epimerization analysis on Rabdotus sp. shells from 40 to $50 \mathrm{~cm}$ yielded an estimated age of between 3425 and 4275 B.P. (based on two different age calculation equations) for the initial mound construction. An AMS radiocarbon assay on one of the Rabdotus sp. shells yielded a radiocarbon age of 3110-2910 B.P. (uncalibrated). Based on these findings, TRC Mariah investigators recommended that the burned rock mound was eligible for listing in the National Register.

Prewitt and Associates, Inc. (PAI) began work on 41CV1378 in March 2006 in conjunction with TxDOT's planned road improvements for Tank Destroyer Boulevard. Investigators conducted a literature review of previous investigations of burned rock middens and mounds on Fort Hood, examined prehistoric research issues relating to middens and mounds, and developed a field investigation strategy for the mound at 41CV1378. PAI (Mehalchick and Boyd) and Fort Hood Cultural Resource Management Program personnel (Kleinbach) then conducted an onsite meeting on March 6,2007 . The remaining portion of Feature 1 appeared to have a maximum length of about $8 \mathrm{~m}$ east-west and $4 \mathrm{~m}$ north-south. Based on the cultural remains exposed all along the tank trail, it appeared that Feature 1 was about one-half of an isolated burned rock mound, 


\section{Maps with site locations are not shown} in report copies for public distribution.

Figure 3.1. TRC Mariah map of 41CV1378 showing the 1994 investigations. "FEA. 1" is the burned rock mound, and "TP 1" is the 1994 test pit. Map is reproduced from Trierweiler (1996:Figure 5.208). The "5/8 Iron Rod" was found and used as the primary site datum for the 2007 data recovery excavations. It was assigned an arbitrary elevation of $100.00 \mathrm{~m}$. 
and that it might be as much as $80 \mathrm{~cm}$ thick. In most places, the burned rock zone appeared to be lying directly on limestone bedrock. Based on the site visit and subsequent consultation with the Texas Department of Transportation (TxDOT) and the Fort Hood Cultural Resource Management Program, PAI developed a formal
Data Recovery Field Investigation Plan (Prewitt and Associates, Inc. 2007) that was submitted to TxDOT in May 2007. Once approved by TxDOT, the Texas Historical Commission (THC), and the Fort Hood Cultural Resource Management Program, the plan became the scope of work for the data recovery effort. 



\title{
METHODS OF INVESTIGATIONS AND WORK ACCOMPLISHED
}

\author{
Douglas K. Boyd, John E. Dockall, Karl W. Kibler, and Gemma Mehalchick
}

\section{PREFIELD TASKS AND FIELDWORK}

\section{Prefield Tasks}

The initial prefield tasks consisted of research and planning in preparation for the data recovery excavations. Site records and previously collected materials from $41 \mathrm{CV} 1378$ were obtained on loan from Fort Hood. The Fort Hood Cultural Resource Management Program also provided Prewitt and Associates, Inc. (PAI) with digital GIS data for all recorded burned rock mound and midden sites on Fort Hood to aid in an examination and synthesis of these types of features. The prefield tasks also included a variety of logistical matters, such as acquiring a Fort Hood excavation permit, obtaining necessary personnel passes, and arranging site access and crew housing.

\section{Remote Sensing Survey}

The first phase of the fieldwork consisted of a remote sensing survey of the mound area using a proton magnetometer and groundpenetrating radar (GPR). The primary goal of these surveys was to identify the location of a main internal pit feature within the mound, if one existed. Previous attempts have shown that magnetometer surveys can be successful in locating internal features within burned rock middens (Abbott and Frederick 1990), and this site was considered a good candidate for both magnetometer and GPR surveys. Chet Walker (Archaeo-Geophysical Associates, LLC) conducted the geophysical investigations of Feature 1 on June 8, 2007. Archeologists from
PAI and Fort Hood were present to prepare the survey area and to help develop an appropriate survey strategy for the burned rock mound. After clearing vegetation, metal detectors were used to sweep the surface of the survey area. All audible metal hits were examined, and numerous metal objects were removed. A 7-x-7-m grid was then established to cover the main mound area and some adjacent off-mound areas. To facilitate the survey and subsequent excavations, the grid was oriented perpendicular to the east-west tank trail along the north edge of the mound (and the resulting grid was skewed 18 degrees off of magnetic north).

Magnetic data were collected using a 0.5$\mathrm{m}$ traverse interval and a $0.125-\mathrm{m}$ ( 8 readings per meter) sample interval. Radar data were collected at a $0.5-\mathrm{m}$ traverse interval, and 32 samples per meter were recorded. Both instruments were passed over the grid in a bidirectional pattern. GPS points were taken on wooden stakes that marked the corners of the survey grid. Once the geophysical surveys were completed, iron rebar was put in at key grid points for later reference and to serve as elevation control datum points for the subsequent excavations.

The geophysical survey data were presented in a draft report by Walker that was submitted to TxDOT in July 2007. The survey results hinted at one possible internal feature within the mound, though subsequent excavations revealed there was no feature causing the anomaly. Because the survey results were not conclusive, the field investigation strategy was not altered. The geophysical survey data and interpretations are presented in Appendix A of this report. 


\section{Excavations, Site Mapping, and Field Documentation}

The next phase of fieldwork, site mapping and hand excavation, was completed from August 3 to 31,2007. The site was mapped with a Sokkia electronic total station, and absolute elevations were tied to the previously established datum, which had been assigned an arbitrary elevation of $100 \mathrm{~m}$ during the 1994 testing. The surface of the burned rock mound and adjacent area was mapped in detail, with shots taken at the same $0.5-\mathrm{m}$ traverse interval used during the geophysical surveys. Additional mapping included the surrounding upland area and survey transect lines to provide data for a topographic cross section of the landform on which the mound is situated.

Because the mound at the Tank Destroyer site was relatively small and discrete, only hand excavations were proposed in the data recovery field investigation plan. However, the work was eventually expanded to include some mechanical excavations. The work ultimately included hand excavation of 31 units and 5 matrix sample columns, two mechanically excavated block areas, and one shovel test (Figure 4.1). As summarized in Table 4.1, the 31 units consist of $301 \times 1-m$ and one $1.0 \times 0.5-\mathrm{m}$ excavation units. All aspects of the mound feature and the archeological excavations were documented with digital photographs and videotape (an 8-mm camcorder).

The excavation units were initially laid out to correspond with the $7 \times 7-\mathrm{m}$ grid block established for the geophysical survey, and the grid was expanded as needed. To designate units with something other than a sequential number, designations were used for east-west and north-south rows. East-west rows were assigned a letter designation in alphabetical order from north to south, beginning with Row A on the north. North-south rows were assigned a number, with Rows 1 through 9 from west to east covering what was believed to be the main portion of the mound. When it became necessary to excavate other units to the east and west of the block to find the edges of the burned rock mound, Rows 10 to 14 were assigned numbers in the order excavated.

Row A units were excavated first to expose a clean east-west cross section through the mound. The stratigraphy along this cross section was closely examined for any signs that might indicate the presence of an internal pit feature or other possible evidence of discrete episodes of mound formation. Profile observations were made of characteristics such as changes in burned rock size, layering of rocks, changes in the quantity or type of fine-grained matrix, lenses or layering of fine-grained matrix, and unusual concentrations of certain materials (e.g., snail shells). The cross-section profile was documented with measured sketches and photographs. Special samples were taken as warranted due to observations of stratigraphic layers, anomalies, and features.

Notably, the size and volume of fill removed from each Row A unit was not equivalent to the amount of fill removed from other units. This discrepancy occurs because of the slope along the edge of the tank trail. While most of the $1 \mathrm{x} 1-\mathrm{m}$ excavation units covered a full square meter, the units in Row A did not because they were laid out along the artificial cut adjacent to the tank trail. Because the north edge sloped down into the tank trail, bedrock was already exposed on the northern edge of most Row A units. Thus, the north-south dimension of each Row A units was generally less than $100 \mathrm{~cm}$ but more than $70 \mathrm{~cm}$. The volume of fill removed from the Row A units was about 50 percent of the fill removed from other units. Consequently, a 50 percent adjustment was used to derive the estimated volume of fill excavated from the Row A units (see Table 4.1).

When the excavation of Row A was completed, the north-south line of $1 \times 1-m$ units designated as Row 4 was excavated next. This was done to provide a north-south cross section that slices through the approximate center of the burned rock mound. The Row 4 profiles (east and west sides) were carefully inspected, and the exposed stratigraphy was documented with measured sketches and photographs. TRC Mariah's test pit falls in Row 4 and corresponds with Unit C-4 on the PAI grid system. This 1994 test unit was reexcavated to maintain an uninterrupted northsouth profile of the mound. For the data recovery investigations, the test pit was redesignated as Unit C-4, and the 1994 testing data was integrated into the current analyses.

To ensure that the east edge of the mound feature had been identified, a shovel test $(40 \times 30 \mathrm{~cm})$ was dug about $1.5 \mathrm{~m}$ east of the southeast corner of Unit A-13. Although some small burned rocks were present in the fill from 


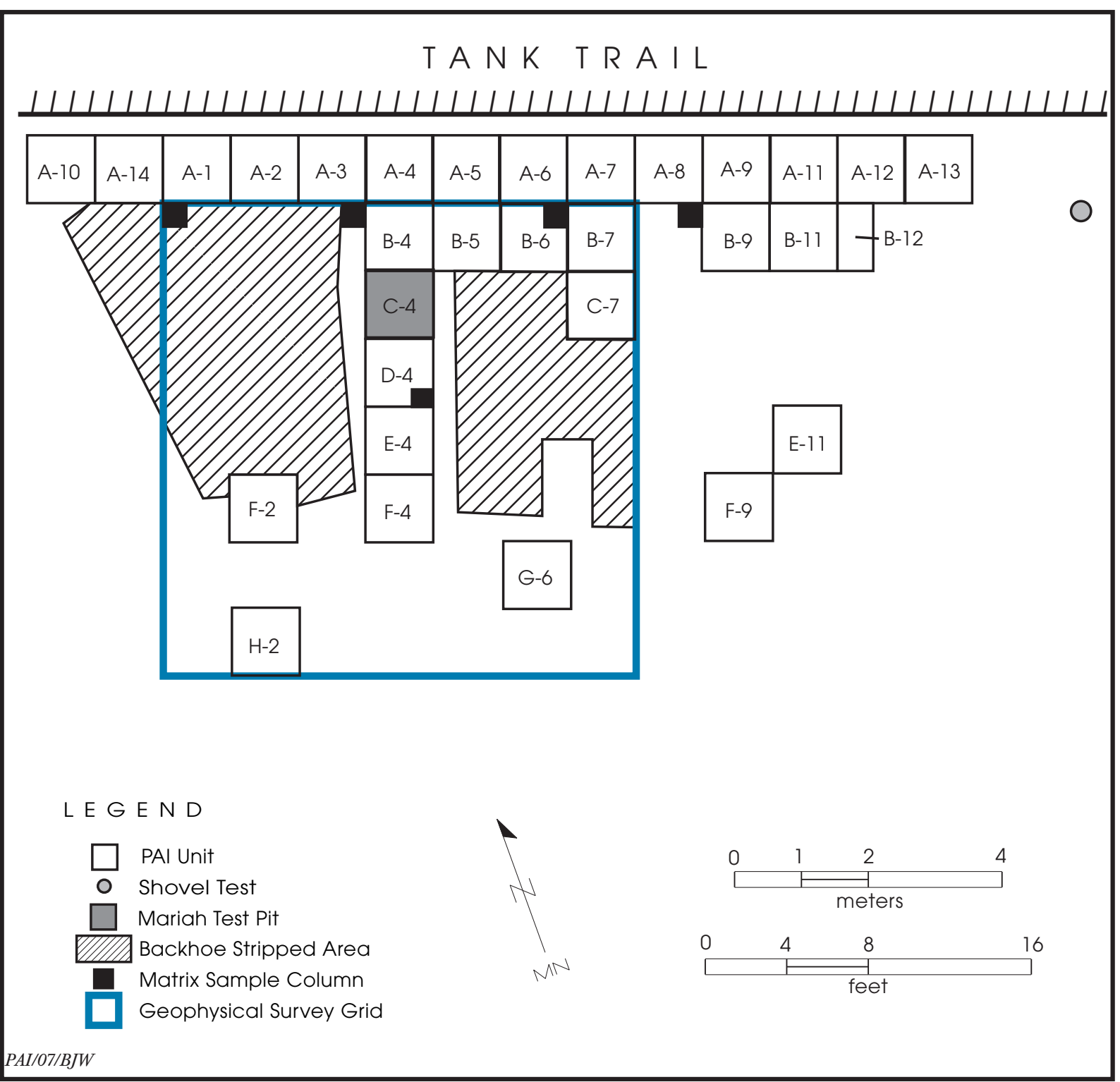

Figure 4.1. Map of data recovery excavations at 41CV1378.

the surface to the bedrock at $29 \mathrm{~cm}$, there was no significant concentration to indicate the presence of Feature 1 in the shovel test.

Archeological research has shown that the general matrix comprising burned rock mounds and middens, while structured in some ways, is often extensively mixed and disturbed through cultural processes (Black et al. 1997:284-285, 295-296). Such deposits generally lack recognizable stratification, which makes excavation by stratigraphic levels difficult if not impossible, and the use of arbitrary levels does not provide meaningful data due to the mixed and jumbled nature of the deposits. For these reasons, the deposits of the burned rock mound were excavated as a single unit or level starting from the surface to the base of the mound. Excavations consisted of horizontal stripping of each unit using picks, shovels, and trowels without digging in any arbitrary levels. Any definable stratigraphic zones, anomalies, or features encountered were excavated, recorded, and sampled as separate analysis units. Vertical provenience was noted solely with absolute beginning (surface of the mound) 
Data Recovery Investigations at the Tank Destroyer Site, Fort Hood

Table 4.1. Summary of excavation units at $41 \mathrm{CV} 1378$

\begin{tabular}{|c|c|c|c|c|c|c|}
\hline Unit No. & Size & $\begin{array}{c}\text { Starting } \\
\text { Elevation (m) }\end{array}$ & $\begin{array}{c}\text { Ending } \\
\text { Elevation }(\mathrm{m})\end{array}$ & $\begin{array}{c}\text { Maximum } \\
\text { Depth of } \\
\text { Excavation }(\mathrm{m}) \\
\end{array}$ & $\begin{array}{c}\text { Excavation } \\
\text { Volume } \\
\left(\mathrm{m}^{3}\right) \\
\end{array}$ & $\begin{array}{c}\text { Volume Adjusted } \\
\text { for Slope or Unit } \\
\text { Size* }\end{array}$ \\
\hline A-1 & $1 \mathrm{x} 1 \mathrm{~m}$ & 99.63 & 99.23 & 0.40 & 0.20 & slope \\
\hline A-2 & $1 \mathrm{x} 1 \mathrm{~m}$ & 99.70 & 99.18 & 0.52 & 0.26 & slope \\
\hline A-3 & $1 \mathrm{x} 1 \mathrm{~m}$ & 99.68 & 99.25 & 0.43 & 0.22 & slope \\
\hline A-4 & $1 \mathrm{x} 1 \mathrm{~m}$ & 99.69 & 99.30 & 0.39 & 0.20 & slope \\
\hline A-5 & $1 \mathrm{x} 1 \mathrm{~m}$ & 99.77 & 99.15 & 0.62 & 0.31 & slope \\
\hline A-6 & $1 \mathrm{x} 1 \mathrm{~m}$ & 99.79 & 99.19 & 0.60 & 0.30 & slope \\
\hline A-7 & $1 \mathrm{x} 1 \mathrm{~m}$ & 99.79 & 99.15 & 0.64 & 0.32 & slope \\
\hline A-8 & $1 \mathrm{x} 1 \mathrm{~m}$ & 99.73 & 99.12 & 0.61 & 0.31 & slope \\
\hline A-9 & $1 \mathrm{x} 1 \mathrm{~m}$ & 99.75 & 99.10 & 0.65 & 0.33 & slope \\
\hline A-10 & $1 \mathrm{x} 1 \mathrm{~m}$ & 99.91 & 99.20 & 0.71 & 0.35 & slope \\
\hline A-11 & $1 \mathrm{x} 1 \mathrm{~m}$ & 99.74 & 99.10 & 0.64 & 0.32 & slope \\
\hline A-12 & $1 \mathrm{x} 1 \mathrm{~m}$ & 99.64 & 99.12 & 0.52 & 0.26 & slope \\
\hline A-13 & $1 \mathrm{x} 1 \mathrm{~m}$ & 99.66 & 99.28 & 0.38 & 0.19 & slope \\
\hline A-14 & $1 \mathrm{x} 1 \mathrm{~m}$ & 99.62 & 99.18 & 0.44 & 0.22 & slope \\
\hline B-4 & $1 \mathrm{x} 1 \mathrm{~m}$ & 99.84 & 99.29 & 0.55 & 0.55 & - \\
\hline B-5 & $1 \mathrm{x} 1 \mathrm{~m}$ & 99.91 & 99.36 & 0.55 & 0.55 & - \\
\hline B-6 & $1 \mathrm{x} 1 \mathrm{~m}$ & 99.91 & 99.32 & 0.59 & 0.59 & - \\
\hline B-7 & $1 \mathrm{x} 1 \mathrm{~m}$ & 99.89 & 99.27 & 0.62 & 0.62 & - \\
\hline B-9 & $1 \mathrm{x} 1 \mathrm{~m}$ & 99.85 & 99.12 & 0.73 & 0.73 & - \\
\hline B-11 & $1 \mathrm{x} 1 \mathrm{~m}$ & 99.75 & 99.16 & 0.59 & 0.59 & - \\
\hline B-12 & $1.0 \times 0.5 \mathrm{~m}$ & 99.70 & 99.23 & 0.47 & 0.23 & size \\
\hline C-4 ** & $1 \mathrm{x} 1 \mathrm{~m}$ & 99.90 & 99.18 & 0.72 & 0.72 & - \\
\hline $\mathrm{C}-7$ & $1 \mathrm{x} 1 \mathrm{~m}$ & 99.87 & 99.35 & 0.52 & 0.52 & - \\
\hline D-4 & $1 \mathrm{x} 1 \mathrm{~m}$ & 99.80 & 99.42 & 0.38 & 0.38 & - \\
\hline E-4 & $1 \mathrm{x} 1 \mathrm{~m}$ & 99.81 & 99.37 & 0.44 & 0.44 & - \\
\hline E-11 & $1 \mathrm{x} 1 \mathrm{~m}$ & 99.73 & 99.36 & 0.37 & 0.37 & - \\
\hline F-2 & $1 \mathrm{x} 1 \mathrm{~m}$ & 99.73 & 99.45 & 0.28 & 0.28 & - \\
\hline F-4 & $1 \mathrm{x} 1 \mathrm{~m}$ & 99.76 & 99.32 & 0.44 & 0.44 & - \\
\hline F-9 & $1 \mathrm{x} 1 \mathrm{~m}$ & 99.72 & 99.42 & 0.30 & 0.30 & - \\
\hline G-6 & $1 \mathrm{x} 1 \mathrm{~m}$ & 99.73 & 99.33 & 0.40 & 0.40 & - \\
\hline $\mathrm{H}-2$ & $1 \mathrm{x} 1 \mathrm{~m}$ & 99.72 & 99.40 & 0.32 & 0.32 & - \\
\hline Total & & & & & 11.81 & \\
\hline
\end{tabular}

and ending elevations (base of the mound) for each excavation unit, as well as ending and beginning elevations for any stratigraphic zones, anomalies, or features encountered. Each day, a laser level was set up near the excavations and backsighted to a rebar grid point with a known elevation. The level was then used to obtain relative elevations throughout the day. All elevations were based on the arbitrary 100-m datum established by TRC Mariah archeologists in 1994.

Excavation of each unit was terminated when the weathered bedrock was encountered. Hand-excavated matrix was dry-screened through 1/4-inch-mesh hardware cloth. Rabdotus sp. shells and all cultural materials except burned rocks were collected, bagged, and labeled with appropriate provenience information. 
The data recovery excavations generally followed the recommendations by Black and Ellis (1997) on how to document burned rock middens and other burned rock features that may occur within middens. Burned rocks were size graded (in centimeters), and each size category was weighed (in kilograms) using a Chatillon heavy-duty 15-kg hanging scale. Burned rock attributes documented included shape (e.g., angular or rounded), lithology, and number of complete clasts. Other general observations pertaining to burned rocks were also noted on the excavation record and feature forms. These observations included the relative density of burned rocks (e.g., tightly packed or loose), the thickness and number of rock layers, and the orientation of the rocks (e.g., vertical, sloping, or flat). Other observations were made regarding the fine-grained matrix and nonmatrix constituents of the mound.

The following types of samples were collected systematically during the data recovery excavations: burned rock (BR), charcoal (C), flotation (F), thermoluminescence (TL), and bulk matrix columns (MC). Within each group of samples, individual samples were given a unique number. Charcoal samples, for example, were numbered $\mathrm{C}-1$ through $\mathrm{C}-12$, indicating that 12 charcoal samples were collected.

The data recovery field investigation plan estimated that the complete excavation of Feature 1 could be accomplished with about 20 units, which was true for the core area of the mound. However, the feathered edges of the feature enlarged its overall maximum dimensions from $8 \times 4 \mathrm{~m}$ to almost $14 \times 5 \mathrm{~m}$. Because of this increased size, the Texas Department of Transportation (TxDOT) authorized PAI to excavate additional units and strip the rest of the mound deposits with a backhoe to search for internal features. In the end, 24 new units and one that was previously excavated sampled various portions of the mound and accounted for nearly half of it being hand dug. Off-mound deposits immediately around Feature 1 were tested with 6 units. Excluding the reexcavated unit, the excavation volume totaled $11.8 \mathrm{~m}^{3}$ compared with the approximately $18 \mathrm{~m}^{3}$ estimated in the data recovery field plan. Lastly, the remaining core area of Feature 1 and most of its thinner outer margins were mechanically stripped. No internal features were identified, on which the data recovery excavations ended.

\section{LABORATORY PROCESSING AND INITIAL REPORTING}

After the data recovery excavations were finished, cultural materials were processed in the laboratory, and an interim report was produced (Mehalchick and Boyd 2007). The laboratory processing consisted of washing and cataloging the artifacts. In addition, broad categories of artifact types were identified and tabulated. General descriptions of the chipped stone tools were made for the interim report, which also provided feature descriptions and described the effort and size of the data recovery excavations. After TxDOT's review of the interim report, it issued a work authorization to process 22 flotation samples from feature and nonfeature contexts and 26 bulk matrix samples collected from five matrix sample columns and wash and catalog cultural materials from these samples. Artifacts and other materials recovered from these samples are presented in Appendix B. The work authorization also called for the submittal of samples for radiocarbon dating and the development of a final research design.

\section{RADIOCARBON DATING AND SITE CHRONOLOGY}

Seven charcoal and eight snail shell samples were selected and submitted for radiocarbon dating. Of the resulting 15 radiocarbon ages, 4 (2 charcoal and 2 snail shell dates) were from Feature 3, a large burned rock-filled pit, while the other 11 are associated with Feature 1, the burned rock mound. No charcoal was recovered from Feature 2, the small off-mound hearth, and no dated samples are associated with the off-mound hearth.

Table 4.2 presents the radiocarbon dates along with all pertinent provenience data for the 15 samples dated by PAI and 1 sample dated by TRC Mariah Associates in 1996. The PAI samples are numbered sequentially 1 through 15 and are designated with a "C" for charcoal or an "S" for snail. Figure 4.2 is a map of the burned rock mound (Feature 1) and Features $2-4$ at the Tank Destroyer site showing the locations of the 16 dated samples.

Radiocarbon dating of Rabdotus sp. shells from prehistoric archeological sites on Fort Hood and the application of a correction factor are discussed by Abbott and Trierweiler (1995). In 


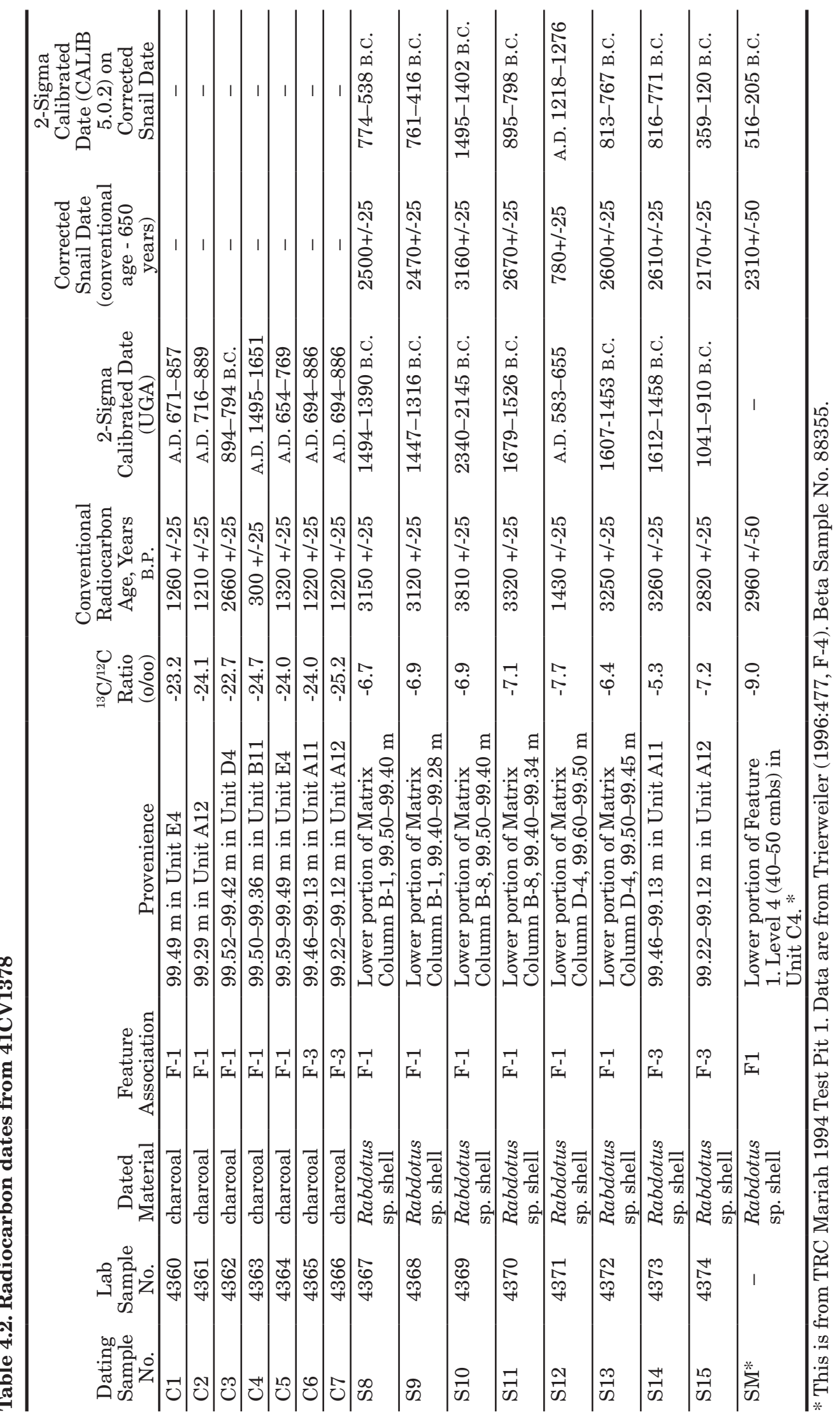




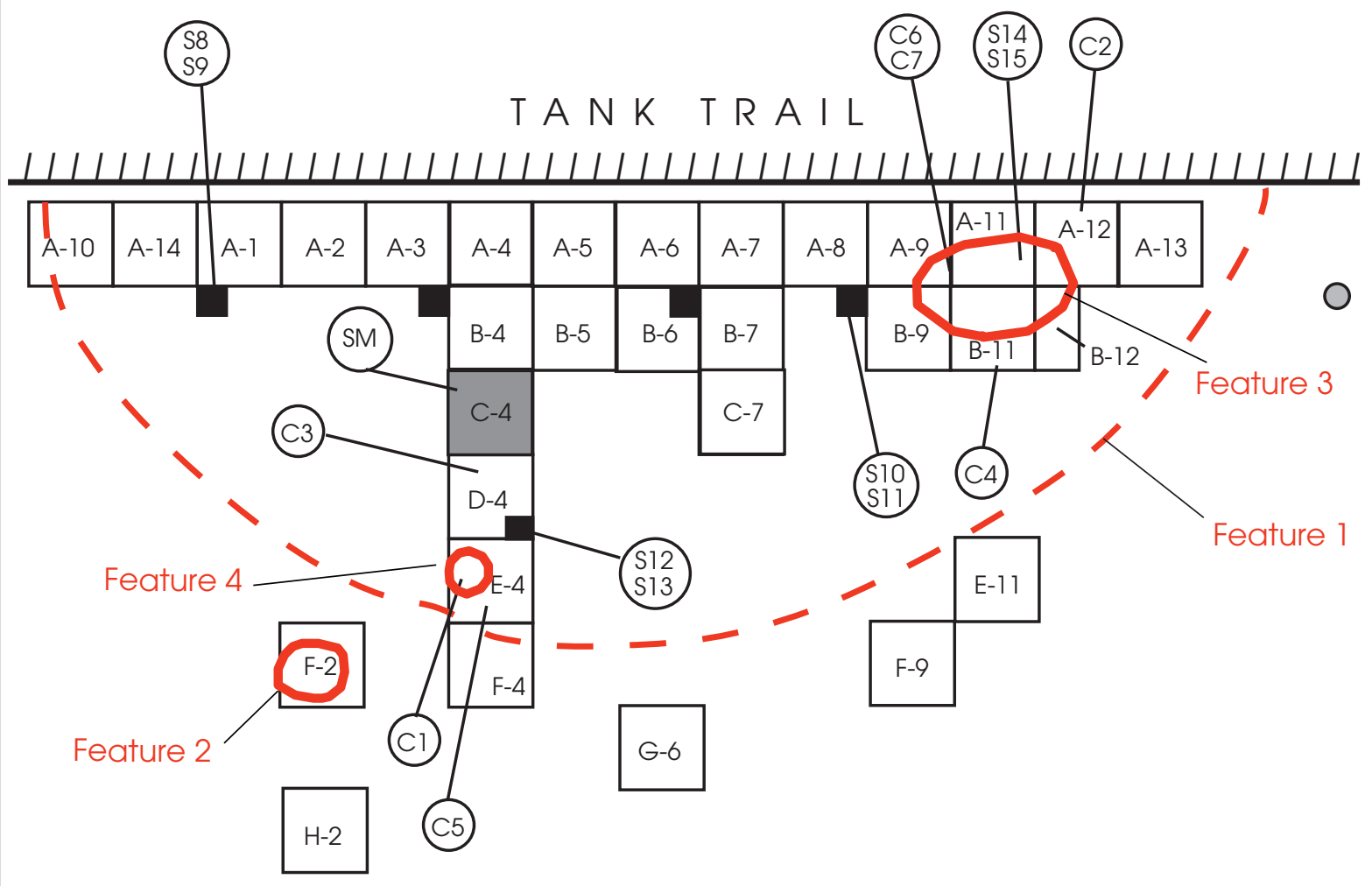

$L E G E N D$

$\square$ PAI Unit

O Shovel Test

$\square$ Mariah Test Pit

- Matrix Sample Column

(S) Snail Date

(C) Charcoal Date
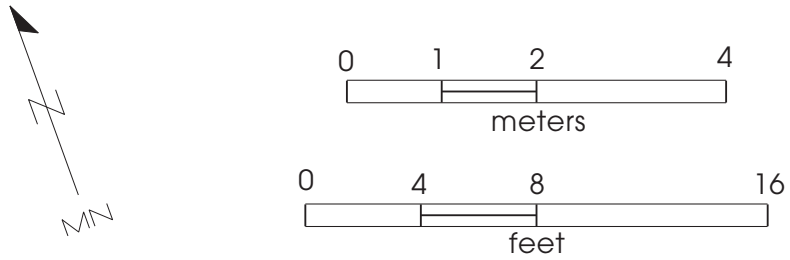

Figure 4.2. Map of the features and locations of the 16 radiocarbon-dated samples.

simplest terms, previous researchers radiocarbon-dated paired samples of Rabdotus sp. shells and charcoal and concluded that the snail radiocarbon ages were consistently too old by about 650 years. They attributed this to the fact that snails ingest old carbonates during their lifetimes and suggested that Rabdotus sp. shell dates may be corrected by subtracting 650 years from the conventional radiocarbon age B.P. (Abbott and Trierweiler 1995:803, 806-808). Subsequent research suggests that that this 650 -year correction factor is fairly accurate when snail dates are paired with charcoal dates (Mehalchick et al. 1999:270). However, this perception is admittedly based on a limited number of paired samples, and the 650-year correction factor is far from certain. Because of this, the snail data presented in Table 4.2 include both the original calibrated dates and the old carbon-corrected dates that are calibrated using CALIB 5.0 (Stuiver and Reimer 1993).

Figures 4.3 and 4.4 are graphic comparisons of the radiocarbon dating results for the Tank Destroyer site using two-sigma calibrated dates. The two graphs are almost identical except that Figure 4.3 uses only the original snail dates 
from the radiocarbon laboratory while Figure 4.4 uses snail dates that have been corrected (by subtracting 650 years) and then calibrated using the computerized CALIB 5.0 calibration program (Stuiver and Reimer 1993). The correction factor is based on the assumption that snails ingest and incorporate only a specified amount of old carbon (i.e., depleted of ${ }^{14} \mathrm{C}$ ) into their shell during their lifetimes. It is our opinion that there is validity to the correction factor of 650 years; however, more study is needed to confirm this, and to verify if it is really a constant number that does not fluctuate through time or with the lifespans of individual snails. Although most of the Rabdotus sp. shells that have been radiocarbon dated are adults, one must wonder whether the amount of old carbon added to shell varies in relation to the age of the snail. However, for the sake of argument, the remainder of this discussion assumes that the correction factor is valid to some degree.

At first glance, it appears that all of the snail dates are older than all of the charcoal dates, but a closer look reveals that this is not the case. While six of the seven charcoal dates cluster between A.D. 650 and 1650, one snail date also falls within this range at A.D. 1218-1276.
In contrast, seven of the eight snail shell dates cluster between 1495 B.C. and 120 B.C., while one charcoal date falls within this range at 894-794 B.C. Thus, if the charcoal and corrected snail dates are valid-and there is no reason to believe they are not-the age span for the use of the burned rock mound at 41CV1378 could be from as early 1495 B.c. to as late as A.D. 1650 . This represents a maximum age span of 3,145 years.

One conclusion that may be reached based on the dates and the projectile point data presented below is that differential preservation has affected the recovery of charcoal samples and limited the materials available for radiocarbon dating. The paucity of piece-plotted charcoal samples and charcoal recovered from flotation highlights the poor preservation. Furthermore, it is likely that the recovered charcoal is biased toward younger specimens, as they are more likely to be preserved than the older specimens. This suggests that the seven charcoal dates may not truly represent the temporal span of activities that generated the burned rock mound and are heavily biased toward later activities and occupational episodes. It also means that the single charcoal

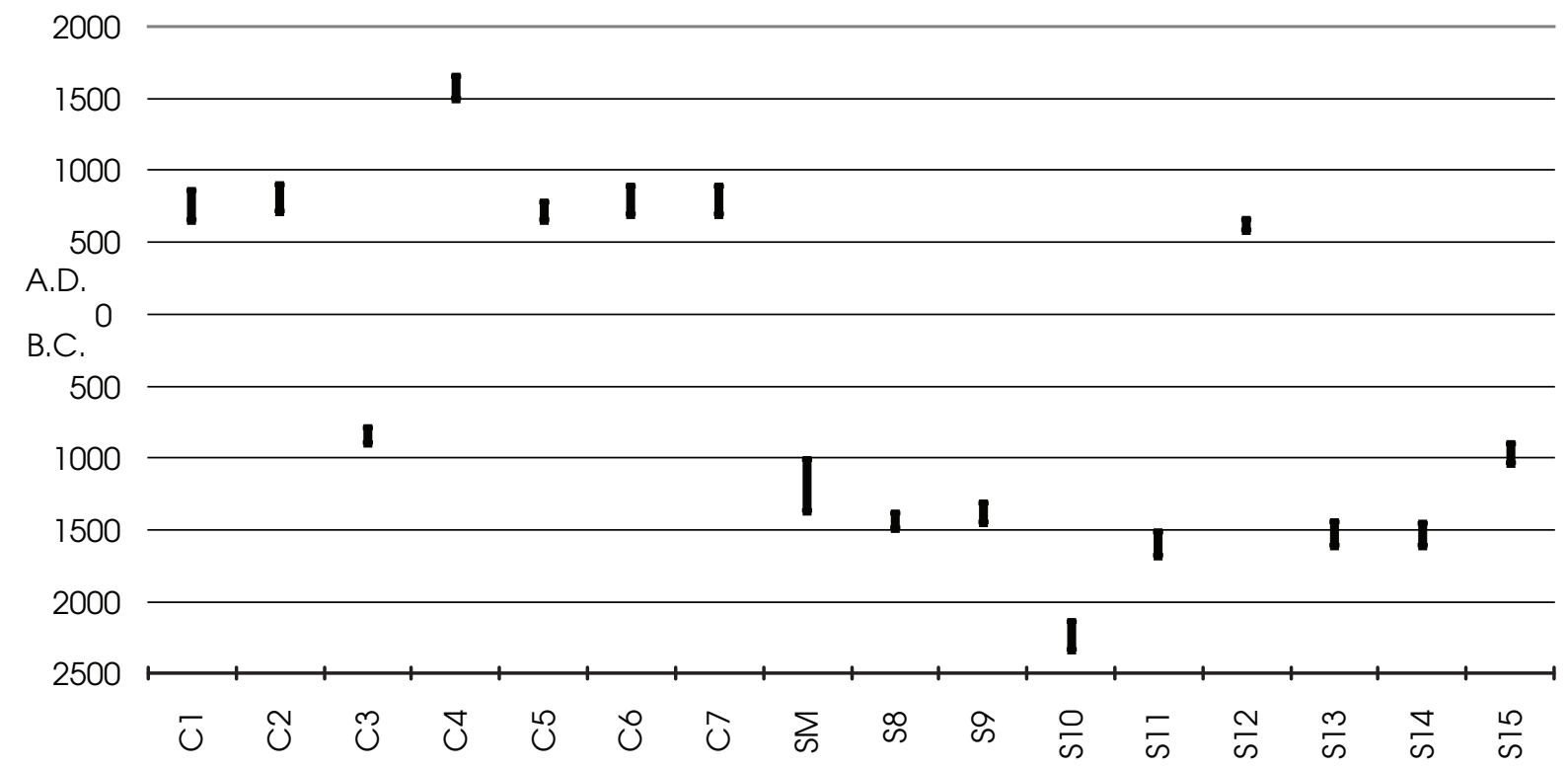

Figure 4.3. Comparison of calibrated radiocarbon dates. Graph uses two-sigma calibrated dates for all charcoal and snail assays from the University of Georgia's Center for Applied Isotope Studies and Beta Analytic, Inc. 


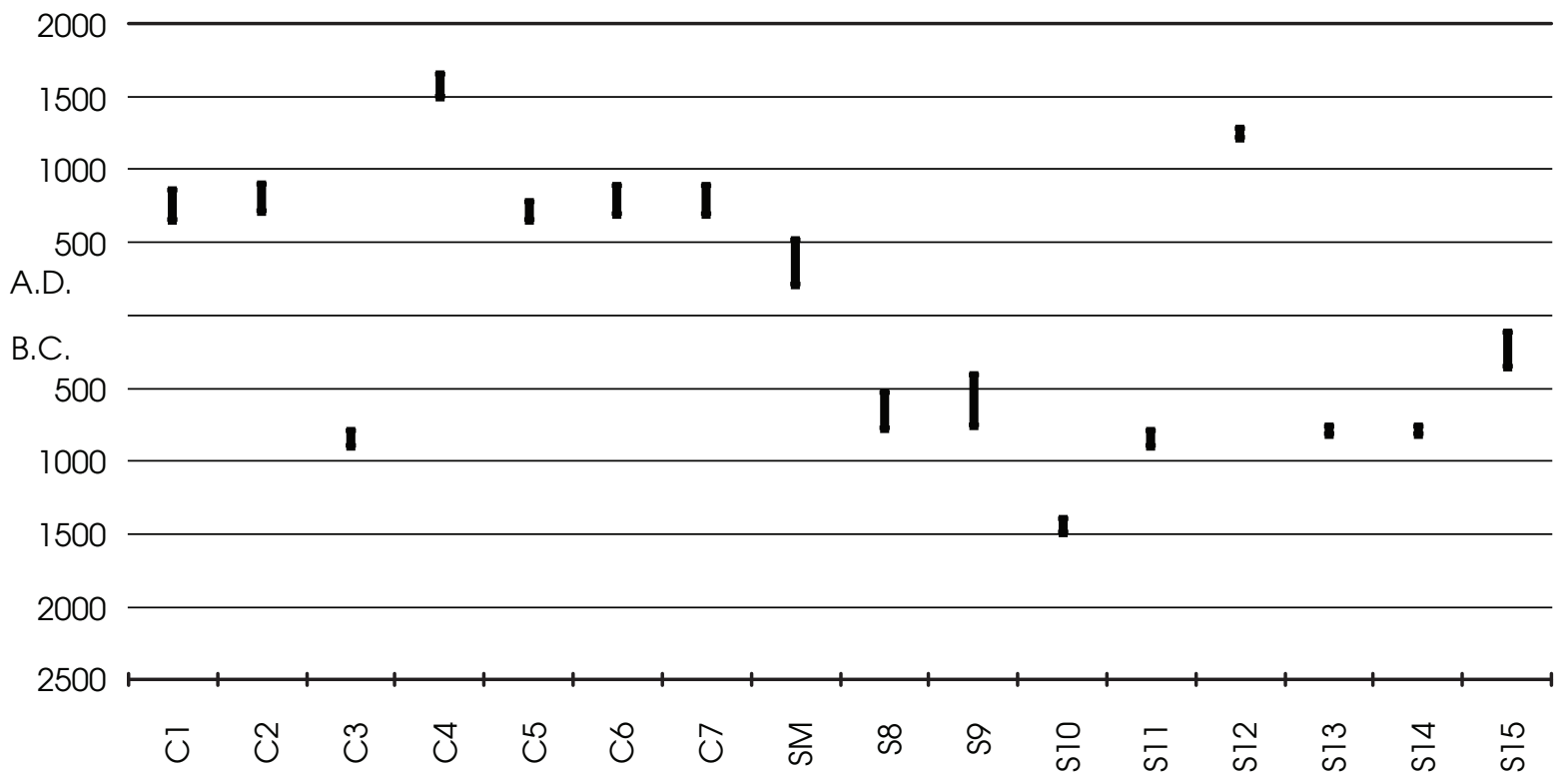

Figure 4.4. Comparison of calibrated and corrected radiocarbon dates. Graph uses two-sigma calibrated dates. The charcoal dates are calibrated by the Center for Applied Isotope Studies at the University of Georgia. Rabdotus sp. shell dates are corrected with 650-year correction factor (following Abbott and Trierweiler 1995) and then calibrated using CALIB 5.0 (Stuiver and Reimer 1993).

specimen (Sample C3) that dates to 894-794 B. C. may not be a true reflection of the intensity of activities that might have occurred at this time.

An understanding of the site's chronology is also provided by projectile points. Table 4.3 summarizes the 27 projectile points recovered from the data recovery excavations, along with 1 projectile point previously recovered by TRC Mariah Associates in 1994. Notably, all of the specimens recovered by PAI are dart points, and 16 of them can be confidently assigned to Late Archaic period. The only arrow point was a single Scallorn point recovered in 1994.

Figure 4.5 plots the chronology of the 17 temporally diagnostic projectile points from 41CV1378 (excluding the 11 untyped or untypeable specimens). Several things are notable about the assemblage. First, the points would suggest a more or less continuous occupation or site use from about 200 B.C. to A.D. 1200. Second, the most intensive occupation and activities seems to have occurred during three Late Archaic projectile point style intervals defined by Collins (1995:384-385, Table 2): Marcos-Montell-Castroville (200 B.C.-A.D. 150), Ensor-Frio-Fairland (A.D. 200-550), and Darl (A.D. 550-750).

\section{Comparing Absolute and Relative Chronologies}

Figure 4.6 compares the radiocarbon dates and relative projectile point chronologies, and the results are revealing. While the early and late groups of radiocarbon dates suggest that a gap in occupations or activities occurred between $416 \mathrm{~B}$. C. and A.D. 651 , the projectile points completely fill in this gap. Collectively, the radiocarbon dates and projectile point chronology suggest that the maximum use life of the burned rock mound is from 1500 B.C. through A.D. 1650, but that the period of most intensive use occurred from around 1000 B.C. to A.D. 1200 . However, it is not suggested that the site occupations or use of the burned rock mound was continuous during this time. In fact, the overall paucity of cultural materials and the relatively small size of the 
Table 4.3. Chronology of projectile points

\begin{tabular}{l|c|l|l|l|l}
\hline \multicolumn{1}{|c|}{ Point Type } & $\begin{array}{c}\text { No. of } \\
\text { Specimens } \\
\text { Recovered }\end{array}$ & \multicolumn{1}{|c|}{ Unit No. } & $\begin{array}{c}\text { Beginning } \\
\text { Date }\end{array}$ & $\begin{array}{c}\text { Ending } \\
\text { Date }\end{array}$ & \multicolumn{1}{c}{ Reference } \\
\hline Castroville & 3 & B-11, E-4, E-11 & 200 B.C. & A.D. 150 & Collins (1995:Table 2) \\
\hline Marcos & 5 & A-8, A-13, B-5, B-7, F-9 & 200 B.C. & A.D. 150 & Collins (1995:Table 2) \\
\hline Montell & 2 & A-9, C-7 & 200 B.C. & A.D. 150 & Collins (1995:Table 2) \\
\hline Ensor & 4 & B-9 $(\mathrm{n}=2)$, B-11, E-11 & A.D. 200 & A.D. 550 & Collins (1995:Table 2) \\
\hline Fairland & 1 & G-6 & A.D. 200 & A.D. 550 & Collins (1995:Table 2) \\
\hline Darl & 1 & B-9 & A.D. 550 & A.D. 750 & Collins (1995:Table 2) \\
\hline Scallorn* & 1 & C-4 (Mariah Test Pit 1) & A.D. 700 & A.D. 1200 & $\begin{array}{l}\text { Turner and Hester } \\
(1993: 230)\end{array}$ \\
\hline $\begin{array}{l}\text { Untyped or } \\
\text { Untypeable }\end{array}$ & 11 & $\begin{array}{l}\text { A-12, A-13, B-9, B-11 (n }=4), \\
\text { D-4 (n = 2), E-4, G-6 }\end{array}$ & - & - & \\
\hline
\end{tabular}

* Recovered by TRC Mariah Associates archeologists in 1994. It is from Level 5 (40-50 cm below surface) in Test Pit No. 1 (redesignated as Unit C-4).

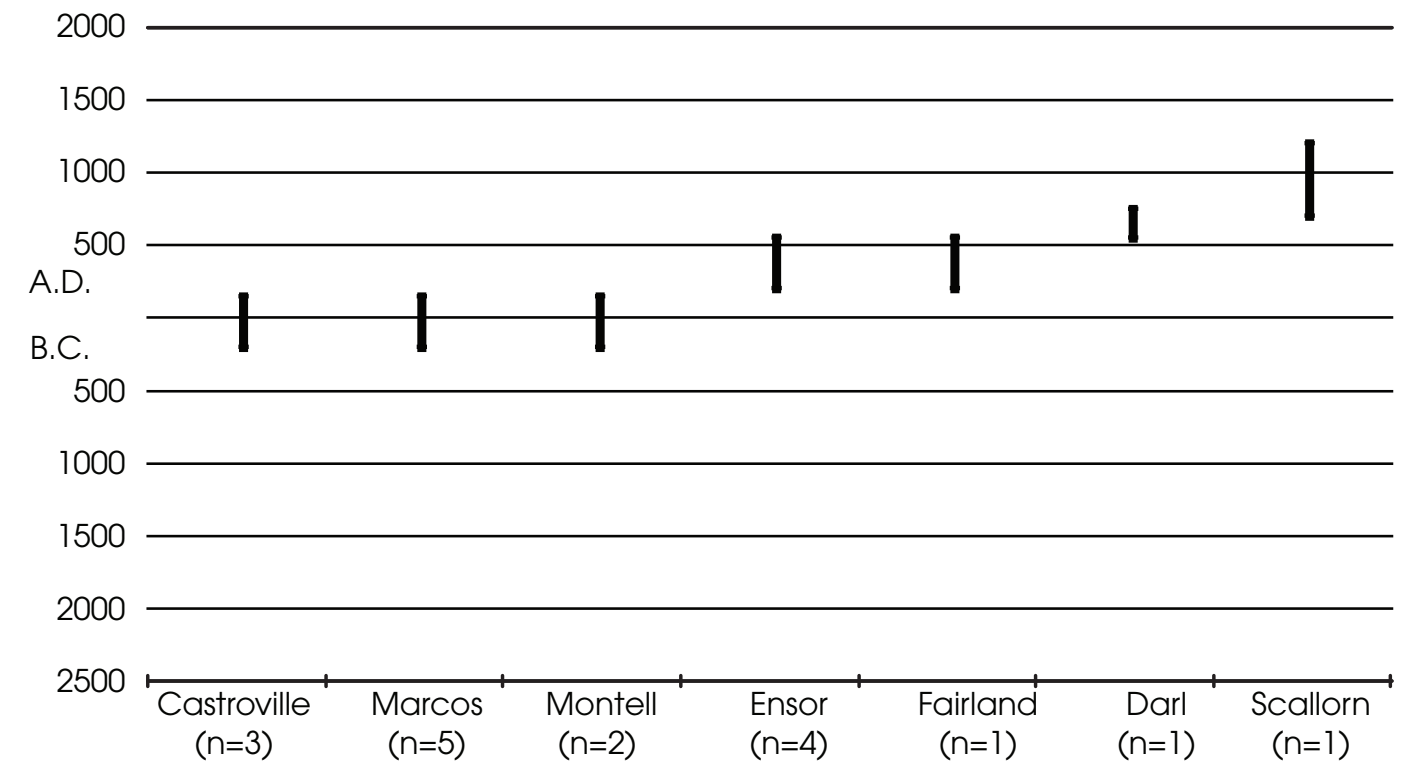

Figure 4.5. Projectile point chronology. Date ranges for dart points are from Collins (1995:Table 2).

remaining portion of the burned rock mound would suggest intermittent and sporadic use over a period of 2,200 years or more.

\section{RESEARCH DESIGN FOR THE DATA RECOVERY ANALYSIS}

The artifacts, features, and other materials recovered from the Tank Destroyer site represent repeated use of the same locale for more than 3,000 years, with most of the activities oc- curring during the latter part of the Late Archaic period. Despite the chronological evidence of multiple episodes of occupation and use, temporally and stratigraphically discrete components cannot be isolated. Burned rock mound deposits are notorious for being jumbled by cultural activities, so it is not surprising that there are no meaningful patterns to the horizontal and vertical distributions of radiocarbon dates and diagnostic projectile points at 41CV1378. It is very difficult to tease out meaningful or inter- 


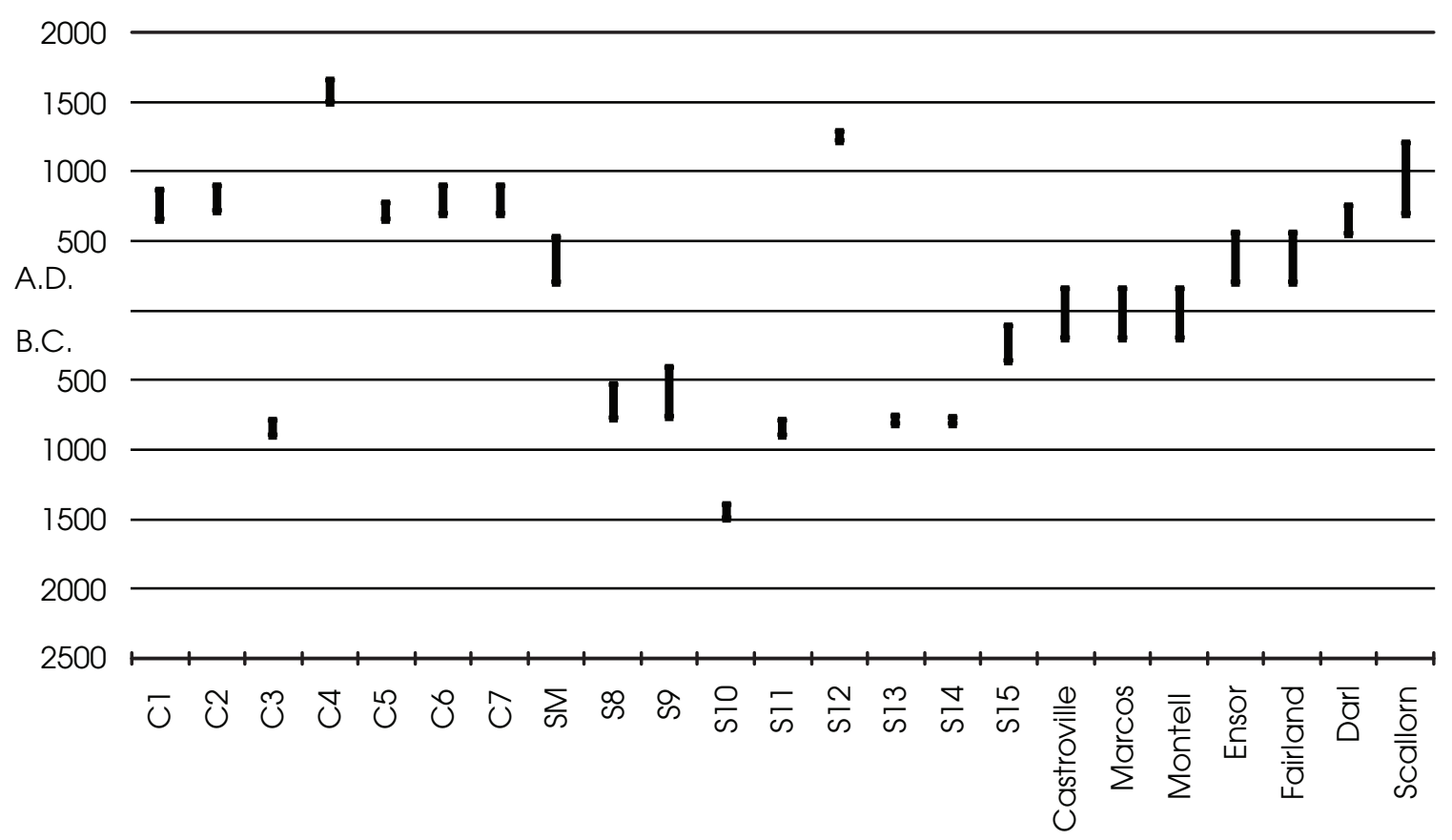

Figure 4.6. Comparison of radiocarbon dates and projectile point chronology. Graph uses two-sigma calibrated dates.

pretable groups of material culture that belong to specific times within the Late Archaic. The site data, however, can be analyzed as representative of a broad time period, though the treatment of the assemblage as a single analysis unit or component places limitations on how the artifacts and features can be interpreted and what can be said about the site overall.

The lack of isolable components is not the only limiting factor to interpreting the Tank Destroyer site. The relatively small assemblage poses another limitation. Despite the excavation of an area of more than $30 \mathrm{~m}^{2}$, the cultural materials recovered, including those from TRC Mariah's 1994 test excavations, consist of 1 arrow point perform, 1 arrow point, 27 dart points, 102 other chipped stone tools, 9 cores and core fragments, 4,466 pieces of unmodified debitage, 413 pieces of microdebitage (recovered from flotation), 1 ground stone tool (mano), 2 bone fragments, 1,666 Rabdotus sp. shells, various amounts of other species of snail shells and freshwater mussel shell fragments, $5,729.6 \mathrm{~kg}$ of burned rocks, and 40 historic/modern artifacts (see Appendix B for provenience data).
The 40 historic/modern artifacts are notable in that they indicate there is a fair amount of recent bioturbation and disturbance at the site. A higher proportion of these specimens were found in the off-mound excavation units, and excavation records indicate that most of the historic/modern artifacts found in Feature 1 were in the upper portions of the mound deposits. The scarcity of ground stone tools and vertebrate faunal remains also is notable.

\section{Research Issues and Goals}

The goal of the data recovery investigations was to ascertain the structure, use life, and function of the burned rock mound at the Tank Destroyer site by analyzing specific sets of archeological data. These data would be compiled, interpreted, and compared to data from other mounds and middens, particularly those at Fort Hood, for a more comprehensive regional analysis and synthesis, which could address broader hunter-gatherer issues, such as residential mobility, settlement systems, foraging strategies, social identity, and regional 
cultural interactions (e.g., Binford 1978, 1980; Kelly 1983, 1992, 1995; Shott 1986).

Unfortunately, the small site assemblage and the inability to recognize and define multiple components, particularly the scarcity of certain artifact and material types and categories, limit what can be said about the burned rock mound at $41 \mathrm{CV} 1378$. Even basic issues associated with chronology, subsistence, technology, and paleoenvironments can not be fully addressed due to these factors.

Given these limiting factors, a different analytical approach was taken. This approach was comprised of four tasks, part of a work authorization issued by TxDOT in July 2010: 1) analyses of the chipped stone and Rabdotus sp. shell assemblages; 2) analysis of the burned rock data; 3) landscape analysis of burned rock mounds and middens at Fort Hood; and 4) examination of social identity in the Late Archaic period in central Texas.

\section{Analysis of the Chipped Stone Assemblage}

The methodology for analysis and interpretation of the chipped stone artifacts recovered from the Tank Destroyer site is guided by the TxDOT Chipped Stone Analytical Protocol, Version 2.3, and the research design developed for this project and therefore consists of two parts. For the first part, observations about tools, cores, and unmodified debitage were recorded and entered into a spreadsheet. The TxDOT Chipped Stone Analytical Protocol, Version 2.3 (Appendix C), "specifies the observations to be made with respect to chipped stone artifacts during fieldwork and analysis" and is an integral part of data collection associated with "problemoriented" research. The analytical procedures rely on standardizing taxonomy and distinguishing between tools and non-tools and core-derived versus core-based tools. This type of dichotomous framework is intended to provide a relatively stable and standardized method of sorting the assemblage into meaningful categories of artifacts (e.g., cores, tools, non-tool debris).

Once the observations on the tools, cores, and unmodified debitage were made and the data were entered into the appropriate spreadsheets, the second part of the analysis involved classifying the assemblage into the following categories: projectile points, bifaces, unifaces, expedient flake tools, utilized flakes, cores, and unmodified debitage. The analysis methods used are briefly discussed below. The analysis is predicated on the understanding of lithic technology as a continuum from the procurement of raw material through manufacture, rejuvenation, and eventual tool discard (Bradley 1975; Collins 1975; Holmes 1894; Muto 1971).

\section{TOOLS}

The chipped stone tool assemblage was sorted into projectile points, bifaces, unifaces, expedient flake tools, and utilized flakes. Following the TxDOT Chipped Stone Analytical Protocol, Version 2.3, metric data was collected for each tool. Measurements taken were maximum length, maximum width, maximum thickness, weight (grams), and edge angle. Dimensions were not projected or estimated for broken tools. Edge angle for tools was recorded as an averaged measure along the used portion of the tool and was recorded to the nearest 5-degree increment; edge angle measurements were made with a goniometer. More specific metric data was taken for projectile points and other bifacial artifacts and is discussed below in the appropriate sections.

During analysis, an assessment of the stage that a tool had reached in its use life was determined from technological analysis, use wear, and fracture patterns. Stage of manufacture was recorded for all tool groups with the assumption that all tools proceed along a relatively linear trajectory from manufacture to discard. This theoretical construct provides the analyst with the means necessary to place the lithic assemblage in a behavioral and functional perspective. The theory behind the linear reduction process is based on previous archeological and experimental studies (Callahan 1979; Collins 1975; Crabtree 1966; Muto 1971; Shafer 1973; Young and Bonnichsen 1984). The protocol also follows closely the manufacture stage scheme discussed by Black et al. (1997:455-457).

The stages used in this analysis are expanded slightly from the original five stages in Version 2.3 of the analytical protocol but conform to the intent. Seven stages of reduction were defined: initial reduction, earlystage forming, late-stage preform, finished product, recycled, rejuvenated/repaired, and indeterminate. The "rejuvenated/repaired" and "indeterminate" stages were added to the ana- 
lytical protocol to make it easier to categorize non-bifacial tools.

The first stage, initial reduction, represents the beginning of the manufacturing process and can include the production of flakes or blades for tools or the initial thinning and shaping process for bifaces. For bifaces, the tool form is usually irregular in shape and is equivalent to Stage 1 of other studies (e.g., Dial and Collins 1998:539-543). Bifaces and flake/blade tools in this stage of manufacture can retain large areas of cortex, and size can vary according to the tool blank. In this analysis, the majority of non-bifacial tools are attributed to this stage of the manufacturing process, unless there were other indications of later-stage reduction (such as evidence of recycling or rejuvenation/repair). If non-bifacial tools were deemed to have been recycled or otherwise repaired/rejuvenated, then it was possible for them to have transitioned from the initial reduction stage to one of the final two stages in the use history of the artifact. The same rationale also holds true for some bifaces. For example, a middle- or early-stage biface fragment that had been subjected to a radial break and then subsequently used as a scraping implement or burin would be classified as recycled or rejuvenated/repaired and not as initial reduction or early-stage forming. At 41CV1378, examples of these types of artifacts were identified, which underscores the need for careful technological analysis and understanding of manufacture versus use-related breakage.

Early-stage forming or blank preparation applies to middle-stage bifaces that are equivalent to Stage 2-3 bifaces, which are characterized by continued thinning and shaping so that it is difficult to determine the original flake or blank attributes. Little cortex may remain, and the artifact morphology is more refined and regularized in outline. A mix of hard-hammer and soft-hammer percussion techniques may be apparent on artifact surfaces. At this stage, hafting elements may also be apparent.

Late-stage preforms have more refined artifact outlines and advanced shaping and thinning and typically have no cortex. Preforms have a significant reduction in thickness over their earlier stages. Stems or other haft elements may be essentially complete. All that is often lacking is the final shaping of the lateral edges of the biface blade or haft element attributes. Technology may still include use of both hard- and soft-hammer percussion techniques to achieve refined artifact outline. Previous studies that have included multiple biface manufacture stages would assign these artifacts to Stage 3 or 4 , depending on the number of stages employed by the analyst (Black et al. 1997; Dial and Collins 1998:545-548). Young and Bonnichsen (1984:76-82) suggest that during this stage of manufacture in bifaces, the focus is on the shaping and thinning of the form, whereas earlier manufacture efforts are on edge or platform preparation and shaping (Young and Bonnichsen 1984:72-76). At this stage of manufacture, such techniques as pressure flaking and notching are also conducted.

The finished product stage was used in lieu of final edge trimming and shaping as suggested in Version 2.3 of the TxDOT Chipped Stone Analytical Protocol. Generally, this stage will include finished artifacts or artifacts very close to completion in terms of manufacture prior to use. At this level of completion, bifacial and other artifacts have been refined in outline shape and overall morphology except for terminal shaping by such techniques as pressure flaking or indirect (punch) flaking. Notching and other haft element aspects are complete. There may be some expected overlap in assigning artifacts to the last two stages described here.

The final two stages, recycled and rejuvenated/repaired, are best discussed together even though they involve very different technological choices on the part of the tool maker/user. Version 2.3 of the protocol makes no real distinction between recycling and rejuvenation and considers them roughly equivalent in meaning. For this analysis, however, a distinction is made between these strategies.

"Rejuvenation" implies a restoration of function to an otherwise broken or worn implement. In this case, the restored function is the same as the original function of the tool. Technological indicators of rejuvenation or repair would include beveled edges on bifacial knives or projectile points, reworked blade edges on projectile points, or unifaces displaying evidence of resharpening episodes. Indirectly this would be indicated by the presence of uniface or biface resharpening flakes as part of the unmodified debitage assemblage.

"Recycling" implies a refurbishment of a tool for a different function. In the assemblage from $41 \mathrm{CV} 1378$, several instances of recycling are 
identified. These include but are not limited to bifaces or unifaces that display deliberate radial or transverse breaks, the presence of use wear on radial or transverse break fracture edges, implements repurposed as drills or perforators, cores reused as hammerstones, and broken bifacial or unifacial tools with burin retouch facets. Similar distinctions have been made by other researchers (Amick 2007). Recycling and rejuvenation do not necessarily occur only when raw material is scarce or of unknown supply. According to Amick (2007:228), such lithic strategies can be the result of opportunistic behavior, mobility constraints, restrictions to raw material access, or how the lithic technology is organized. Both can be a regular component to "expedient" or "curated" technology.

\section{Projectile Points}

Dart and arrow points are a functional group that is inclusive of all artifacts used to tip projectiles or other similar weapons. Typically they are characterized as bifacial (but sometimes unifacial) flaked tools with triangular to leaf-shaped blade sections, pointed distal ends, and uniform lateral blade edges. Distinctions between dart and arrow points are size-based. Where possible, projectile points were assigned to known and established formal type names (e.g., Castroville). Specimens that could not be assigned to a named type were classified as untyped. Fragments that could not be classified because they were too incomplete were classified as untypeable. Completeness, breakage type, and raw material were noted for each specimen. In addition to maximum length, maximum width, maximum thickness, and weight, stem length, stem width, and neck thickness were recorded for projectile points.

\section{Bifaces}

Bifaces and bifacial artifacts from 41CV1378 were classified generally according to technological assessments of manufacture stage, breakage type, and tool type. Completeness and raw material were also noted. Unfinished bifaces were classified as Stage 1,2,3, or 4, and finished functional bifacial tools were classified by tool type. Tool type categories consist of knife, indeterminate fragments, fragments with burin retouch, adze fragments, and thick battered bifaces. Fragmentary bifacial tools and complete unfinished specimens dominated the biface types.

\section{Unifacial Tools}

Unifaces were classified according to technological aspects and were classified with names generally indicative of their function and morphology. These tools encompass knives, drills, denticulates, notched flakes, side scrapers, end scrapers, end/side scrapers, and generalized unifacial tools of unknown function. Tools identified as scrapers had at least one edge altered by direct percussion retouch. These tools had edge retouch that was regular, somewhat invasive, and could be continuous or localized to a portion of the edge or edges. Completeness, breakage type, and raw material types were noted for these tools.

\section{Expedient Flake Tools}

Expedient flake tools are flakes that have been further modified by varying degrees and types of intentional retouch into a variety of tools. Attributes recorded include metric dimensions, weight, stage (of manufacture or use), degree of completeness, type of secondary alteration (e.g., patination, thermal), shape, retouch pattern, tool edge/element construction, edge grinding, use-wear characteristics, and evidence of hafting, and lithology.

\section{Utilized Flakes}

Utilized flakes are flakes that display edge modification through use such as cutting or scraping but no deliberate edge retouch or modification. These tools were identified based on the presence of consistent unifacial, bifacial, or other microwear. Implements in this group were classified according to function as determined from microscopic and macroscopic use-wear analysis. Utilized flake tool types that were identified include radial break tools and unretouched flakes that were used to cut, scrape, or both.

Radial break tools are flakes that were broken by deliberate transverse snapping or radial compression fractures, with one or more of the resulting abrupt edges or tip being used as a tool. In some cases, radial and snap breaks 
can be identified as deliberate based on the presence of specific features that indicate it was done on an anvil of some sort. These have been identified in Texas in several contexts associated with Archaic-age and other occupations (Dockall and Boyd 2006a:55, 2006b:100-102; Dockall and Pevney 2007: 195-197). Similar broken implements have been observed in pre-Clovis, Paleoindian, and later archeological contexts (Amick 2007:223-249; Dellar and Ellis 2001; Frison and Bradley 1980; Goodyear 2005; Root et al. 1999, 1978) and have occasionally been associated with probable ritual activities in Paleoindian to Caddo occupations (Dellar and Ellis 2001; Shafer 1973:224-228). Dockall and Pevney (2007:197) illustrated some of the distinctive fracture features associated with these types of deliberate breaks, as have other authors mentioned above. Unfortunately, there is little information in the literature to compare these implements in central Texas, and it is difficult to place these technological features into a broader behavioral framework. However, where these occur in the assemblage, they are discussed within the context of the lithic technology represented at the site.

\section{CORES}

Cores are angular lithic chunks with evidence of single or multiple flake removal. Analysis of these artifacts is not addressed in the protocol, but the attributes and variables used in the analysis of the $41 \mathrm{CV} 1378$ assemblage are presented below. Cores were assigned to a specific group and type. For each core identified, presence/absence of thermal alteration, the flake removal pattern, and type of platform preparation apparent were also recorded. Maximum length, width, thickness, and weight were recorded.

\section{Core Group}

Core groups were categorized as flake, blade, bipolar, other, and indeterminate, based on the end product(s) removed. Generally speaking, it is possible to determine the end product removed from a core by examining the flake scars, platform type, and overall core morphology. There are two principal blank production trajectories that can be identified: flake and blade (Odell 2004:91-103).
Flake cores are parent pieces from which percussion flakes were removed. Following the TxDOT analytical protocol for stone tools, these types of cores would easily be classified as a source of simple detachment-based products. Unless the analyst is dealing with specialized types of flake cores, then it is relatively easy to identify pieces related to this mode of flake production. Cores and flakes of this trajectory often will have no consistent set of attributes (J. Johnson 1986, 1989; Teltser 1991). Typically, cores of this type have been referred to as generalized cores, free hand cores, or hard-hammer percussion cores, but the mode of detachment could also have included a soft-hammer technique as well. An often-held assumption regarding the flake trajectory is that there is a distinct relationship between the production of flakes and an expedient technology in which flakes are selected and used as tools with minimal or no modification. Following Teltser (1991:365), it is best to consider such generalized production strategies not as unstructured or expedient in nature, but as organized around and in response to a different set of factors distinct from either blade or biface production. Such factors might include raw material provisioning and other types of activities (Kuhn 1990, 1991, 1995).

Blade cores are also rather obvious and are identified primarily based on the presence of remnant scars that indicate the systematic removal of flakes that fit the descriptive attributes of blades-laminar in form and a length dimension at least twice the width dimension. General shape and thickness of blades removed would be variable according to the degree of platform preparation and core surface preparation. Blade production is accomplished by a distinct set of production and core preparation techniques, most notably the construction of a straight ridge running the length of the core from the platform to the base (Odell 2004:95-96). Variations of this basic technique exist, but this mode of blank production differs from flake production because of its emphasis on creation of a blank with standardized attributes and method(s) of knapping.

Bipolar cores are more difficult to identify in an assemblage and can often resemble small battered pieces that are often called pieces esquillees in assemblages recovered from Paleoindian and a host of other sites (LeBlanc 
1992; Shott 1999). Although not a special-purpose technique, bipolar cores and flakes are more often present in areas where raw material occurred in small pebble or small cobble form-too small to efficiently remove flakes by holding the core in the hand or where raw materials were in short supply, necessitating extreme forms of curation (Goodyear 1993; Jeske and Lurie 1993; Shott 1989). Bipolar cores/flakes can often be mistakenly classified as nondescriptive and nondiagnostic shatter associated with other forms of flake production. Common visible signatures of this mode of flake production are opposed smashed surfaces or ends on flakes and cores, sheared bulbs of percussion, small or absent striking platforms, and features indicating removal of flakes from both ends of the core/flake as indicated by ripple marks and other direction indicators on flake scars.

Core Type

Cores from each core group were assigned to one of several core types. A core is classified as a cobble/pebble core if it retains enough of the material to determine the general size and shape of the initial mass. A tested cobble/pebble core displays multiple flake scars and retains enough material to determine the general size and shape of the initial mass, whereas a partial cobble/pebble core denotes a core that only has enough of the material to determine if it was either a cobble or a pebble but not enough to observe the general size and shape. Cortical cores exhibit exterior cortex, while decorticated cores are lacking of any exterior cortex. Other possibilities include decorticated and cortical core fragments or core trimming pieces. Such pieces are too small to allow for a distinction between cobble- and pebble-sized masses. Large flake cores or macroflake cores are percussion cores in which the mass of material used for flake production is a large flake blank. These are usually identified only if they retain evidence of a former ventral or dorsal surface and/or a remnant of the original striking platform. Other core types considered include bipolar cores, blade cores, and bifacial or discoid cores. Each of these are identified based on flake scar morphology of removed products or blanks, core preparation, and patterns of surface flake scars.

\section{Flake Removal Pattern}

Flakes may be removed from a core unidirectionally (one direction), bi-directionally (two directions), and multi-directionally (three or more directions). The number of directions of flake removal generally can correspond to the number and arrangement of striking platforms and the intensity or extensiveness of core reduction.

\section{Core Platform Preparation}

The pattern(s) of core platform preparation that were selected in this analysis correspond closely to the types of striking platforms that are included in the unmodified debitage analytical protocol. The following types of preparation or platform types are included in the analysis: natural cortex (no abrasion or abraded), single facet (no abrasion or abraded), multi-facet (no abrasion or abraded), multiple (more than one type present), other (special cases), and indeterminate (for core fragments or core trimming pieces). Preparation includes the deliberate modification for flake removal of natural cortex, single facet, and multi-faceted platforms. Natural cortex preparation is a platform consisting of the unmodified exterior cortex of the raw material mass. Single-faceted platforms can be abraded or nonabraded and refer to a core platform that has only one facet or plane from which flakes are struck. Multifaceted core platforms suggest more systematic platform preparation and maintenance of the flake initiation surface such that the flakes or blades removed will have multiple intersecting flake scars on their corresponding striking platforms. Multi-faceted platforms are often associated with specialized techniques of tool blank production-bifaces, prismatic blades, or sequent flakes, for example, in which the core is prepared to control and standardize the products removed. Platform abrasion can be performed at any stage of the core reduction process and serves to remove weak areas along the platform/core face intersection and further ensure successful flake removal. It is also a common aspect of biface manufacture and frequently observed on flake debris produced during the process. Occasionally, a core can have more than one platform of different types. In these cases, an "other" option is in- 
cluded and observations on the nature of the platforms present are made in the comments field during data entry.

\section{UNMODIFIED DEBITAGE}

Version 2.3 of the analytical protocol specifies a limited number of observations to be recorded for unmodified debitage. These consist of raw material, form (completeness), size grade, cortex percent, striking platform type, and the presence or absence of thermal alteration. An additional variable, flake type, was also included as part of the analysis.

\section{Raw Materials and Chert Types}

Chert was virtually the only material identified in the chipped stone lithic assemblage from 41CV1378. Cherts consist of opaque to partially translucent cryptocrystalline quartz. Fine-grained cherts lack visible crystalline structure, have weak to moderate luster, and are partially translucent. Coarse-grained cherts have a visible crystalline structure, opaque appearance, and a grainy feel.

All chert specimens regardless of artifact class, were compared with the established Fort Hood chert taxonomy. Because central Texas is so important as a chert resource area for local and extraregional use, much attention has been devoted to developing a typology of the chert resources present on Fort Hood lands (Abbott and Trierweiler 1995; Dickens 1993a 1993b, Frederick and Ringstaff 1994). The Fort Hood chert typology that is used in this study is summarized in Table 4.4.

\section{Size Grade}

The general process followed was to take each analysis grouping and size grade the lot through the four nested size-grade screens: Size Grade $1=$ inch, Size Grade $2=3 / 4$ inch, Size Grade $3=1 / 2$ inch, Size Grade $4=1 / 4$ inch. Material that fell through the 1/4 inch sieve was recorded as Size Grade 5.

\section{Completeness}

Following size grading, material within each size grade was sorted according to com- pleteness. Flake completeness is recorded as one of four states: complete, broken, fragment, and debris. Complete flakes exhibit striking platforms and distal ends. Broken includes flake fragments with an intact striking platform. Fragment includes medial, distal or other portions. Debris is reserved for all other fragments and pieces that represent angular shatter. This scheme for recording flake or piece completeness is almost identical to that method employed by Dibble and Lenoir (1995).

\section{Cortex Percent}

Cortex was recorded as 0 percent or none, 1-25 percent, $26-50$ percent, $51-75$ percent, and 76-100 percent. Often, cortex is recorded as a presence/absence variable or flakes are assigned to primary, secondary, or tertiary (interior) based on cortex presence. The problems with using any cortex recording technique stem from varying definitions of what constitutes primary, secondary or tertiary flakes and the application of ambiguous estimates of each type. Using more or fewer categories appears to make little difference.

The amount of cortex has been used often to determine the stage(s) of reduction represented in a lithic assemblage or the intensity of reduction present. Researchers have usually found cortex useful only for determining the ends of a core reduction or tool manufacturing sequence (Odell 2004:127). Cortex is probably one of those variables that covaries with the size and shape of the initial raw material (Andrefsky 2001:12).

The importance of recording cortex varies with the occurrence of available raw materials. Cortex presence is significant for cobble and pebble forms of material, but raw material obtained from bedrock sources as ledge material naturally has little cortex, or becomes essentially cortex free early in the reduction process. The patterns that can be observed in cortex will also vary with how the technology is organized. For instance, cortex could be a significant variable at lithic procurement sites where material testing, core shaping, and stages of biface manufacture are occurring, but it will be of little importance at sites dominated by late-stage biface manufacture or repair of finished tools. 
Table 4.4. Fort Hood and other chert types

\begin{tabular}{|c|c|c|c|}
\hline Type No. & Type Name & Abbreviation & Fort Hood Chert Province \\
\hline 1 & Heiner Lake Blue-Light & HLB-LT & Southeast Range \\
\hline 2 & Cowhouse White & $\mathrm{CW}$ & Southeast Range \\
\hline 3 & Anderson Mountain Gray & AMG & West Fort \\
\hline 4 & Seven Mile Mountain Novaculite & SMN & West Fort \\
\hline 5 & Texas Novaculite & TN & North Fort \\
\hline 6 & Heiner Lake Tan & HLT & Southeast Range \\
\hline 7 & Fossiliferous Pale Brown & FPB & Southeast Range \\
\hline 8 & Fort Hood Yellow & FHY & North Fort \\
\hline 9 & Heiner Lake Translucent Brown & HLTB & Southeast Range \\
\hline 10 & Heiner Lake Blue & HLB & Southeast Range \\
\hline 11 & East Range Flat & ERF & North Fort \\
\hline 13 & East Range Flecked & ER Flecked & Southeast Range \\
\hline 14 & Fort Hood Gray & FHG & North Fort \\
\hline 15 & Gray-Brown-Green & GBG & North Fort \\
\hline 16 & Leona Park & LP & North Fort \\
\hline 17 & Owl Creek Black & OCB & Cowhouse Alluvial \\
\hline 18 & Cowhouse Two Tone & CTT & Cowhouse Alluvial \\
\hline 19 & Cowhouse Dark Gray & CDG & Cowhouse Alluvial \\
\hline 20 & Cowhouse Shell Hash & $\mathrm{CSH}$ & Cowhouse Alluvial \\
\hline 21 & Cowhouse Light Gray & CLG & Cowhouse Alluvial \\
\hline 22 & Cowhouse Mottled with Flecks & $\mathrm{CMF}$ & Cowhouse Alluvial \\
\hline 23 & Cowhouse Banded and Mottled & CBM & Cowhouse Alluvial \\
\hline 24 & Cowhouse Fossiliferous Light Brown & CFLB & Cowhouse Alluvial \\
\hline 25 & Cowhouse Brown Flecked & $\mathrm{CBF}$ & Cowhouse Alluvial \\
\hline 26 & Cowhouse Streaked & $\mathrm{CS}$ & Cowhouse Alluvial \\
\hline 27 & Cowhouse Novaculite & $\mathrm{CN}$ & Cowhouse Alluvial \\
\hline 28 & Table Rock Flat & TRF & West Fort \\
\hline 29 & Indeterminate white & & None \\
\hline 30 & Indeterminate yellow & & None \\
\hline 31 & Indeterminate mottled & & None \\
\hline 32 & Indeterminate light gray & & None \\
\hline 33 & Indeterminate dark gray & & None \\
\hline 34 & Indeterminate light brown & & None \\
\hline 35 & Indeterminate dark brown & & None \\
\hline 36 & Indeterminate black & & None \\
\hline 37 & Indeterminate blue & & None \\
\hline 38 & Indeterminate red & & None \\
\hline 39 & Indeterminate nonlocal & & None \\
\hline
\end{tabular}

\section{Striking Platform Type}

Flake striking platforms were recorded as 10 distinct technological states: indeterminate, cortical, flat, complex, abraded, faceted, multifaceted, rejuvenated, crushed, and missing.
Striking platform characteristics can be used to determine the mode of flake detachment and the type of hammer used during the flaking process and has also been used to assess the stage(s) of core reduction or tool production present within an assemblage (Andrefsky 
1998:87-88). These specific striking platforms are described in the TxDOT Chipped Stone Analytical Protocol, Version 2.3 (see Appendix C). Characteristics of certain platform states can be diagnostic to varying degrees of certain types of flakes or types of flake initiation and hence can be informative of core reduction or tool manufacture techniques (Andrefsky 1998:92-96; Cotterell and Kamminga 1987; Morrow 1984; Odell 1989; Whittaker 1994:98105, 140-147, 194-199).

\section{Thermal Alteration}

Thermal alteration of chipped stone debris was recorded as indeterminate, observed, or not observed with no attempts to classify the degree or type of burning or the presence or absence of deliberately heat-treated materials due to the difficulty in assessing the presence and type of burning represented on a piece.

Flake Type

The identification of flake types is not part of the TxDOT analytical protocol for unmodified debitage, but it was added to the analysis for this project. Complete flakes and fragments retaining enough of the striking platform were sorted into several distinct flake type categories. Where possible, fragments of special flake types such as burin spalls and uniface resharpening flakes were also identified. Quantification of size-graded material by flake type was initiated because of the behavioral information contained within an array of specialized flake types that would normally be missed during conventional size-grade analysis. There has been considerable discussion regarding the validity of using flake types as a sorting criterion (e.g., Andrefsky 1998:23-29; Odell 2004:121-124; Sullivan and Rozen 1985). The array of flake types employed in this study is based on observations and experience distilled from an understanding of fracture mechanics of brittle solids (Cotterell and Kamminga 1987), experimental flintknapping with a variety of techniques, a type collection of flake types produced by a variety of flaking techniques, and the diagnostic value of specific attribute features of various types of fracture initiation; conchoidal, bending, and wedging (after Cotterell and Kamminga 1979, 1987; Tsirk 1979).
As Odell (2004:121-124) has noted, various flake types are indicative of particular technological strategies of tool production, while others are more indicative of certain behaviors. For this study, flake type identification was used along with the standard procedure outlined in the analytical protocol to address the following twopart question: What stage(s) of tool manufacture occurred on the site, and in what form was lithic material imported to this location? Artifact morphology and technology observations are also used to address these questions. Flake types that were identified during this lithic analysis are defined in Table 4.5.

\section{Analysis of Rabdotus sp. Shells}

Interpreting snail shells from archeological contexts as food sources often relies on ethnographic accounts of aboriginal consumption and the amount of shells. Alternatively, large amounts of shells are often interpreted as a post-site abandonment phenomenon, the remains of snail populations naturally attracted to the organic-rich deposits left behind (e.g., Fullington 1978; Gadus et al. 1999:80). Whether they represent a food source or simply a natural phenomenon is the question we asked about the shell assemblage from 41CV1378.

The many hundreds of Rabdotus sp. shells recovered from the site were quantified, and their distributions were examined for possible patterns that might illuminate whether they are a cultural or natural phenomenon. If the snail shells were evenly distributed throughout the mound and nonmound deposits, it would argue that the snails represent a natural accumulation. In contrast, significant accumulations of snail shells in some areas more likely indicate a cultural phenomenon, like the dumping of food remains. If any accumulations were recognized, the snails were examined to search for cultural modifications. For example, a high frequency of specimens with pinholes in the shells might be evidence of extraction of the snails by humans.

A second level of analysis was to examine the snail shells specifically from flotation samples and the soil columns to look at age structure. The age structure is based on shell size, with larger shells representing adults and smaller shells representing juveniles. The numbers of 
Table 4.5. Technical definitions of various flakes types

\begin{tabular}{|c|c|}
\hline Flake Type & Key Attributes \\
\hline $\begin{array}{l}\text { Bending initiation } \\
\text { (soft hammer or } \\
\text { biface thinning) }\end{array}$ & $\begin{array}{l}\text { No or diffuse bulb of percussion. Distinct lip or ridge on the ventral aspect or edge } \\
\text { of the striking platform. Some may have a waisted or constricted appearance just } \\
\text { below the striking platform. Profile shape is typically arched, and plan view is often } \\
\text { expanded distally. Crushed or collapsed platforms may be present. Abraded, faceted, } \\
\text { or multifaceted platforms are also common. }\end{array}$ \\
\hline $\begin{array}{l}\text { Conchoidal initiation } \\
\text { (hard hammer) }\end{array}$ & $\begin{array}{l}\text { Typically has an exuberant or pronounced bulb of percussion and thickened cross } \\
\text { section. Flake shape is variable and often dependent on core surface morphology. }\end{array}$ \\
\hline $\begin{array}{l}\text { Wedging initiation } \\
\text { (bipolar) }\end{array}$ & $\begin{array}{l}\text { No bulb of percussion or only a sheared bulb present. Ripple marks and crushed } \\
\text { and sheared faces on opposed ends of fracture surface indicate force from opposing } \\
\text { directions. Can be associated with abundant nondiagnostic shatter. }\end{array}$ \\
\hline Notching & $\begin{array}{l}\text { Can be produced by pressure or punch techniques. Flakes are typically } \\
\text { C- or S-shaped with previous similarly shaped dorsal flake scars where removed } \\
\text { in sequence. Platforms are typically single-faceted (Titmus 1985; Weber 1994:635). } \\
\text { Notching flakes expand laterally and ventrally like the Hertzian cone. }\end{array}$ \\
\hline Punch & $\begin{array}{l}\text { Similar striking platform morphology as notching flakes but variable flake } \\
\text { morphology. When viewed from above onto the striking platform, has pronounced } \\
\text { gull-wing appearance. Typically a noticeable lip is below the striking platform } \\
\text { ventral edge and the top of the bulb of percussion. Exuberant bulb of percussion or } \\
\text { corresponding deep negative bulbar scar on biface. }\end{array}$ \\
\hline Pressure & $\begin{array}{l}\text { Typically displays laminar or elongate tonguelike shapes with a small contact } \\
\text { platform area. Some are constricted below the striking platform because the platform } \\
\text { was isolated by pressure flaking before flake removal (Whittaker 1994:147). Common } \\
\text { very small bulbs of percussion produced during static loading. Crushed platforms } \\
\text { and broken flakes are common due to thinness. }\end{array}$ \\
\hline $\begin{array}{l}\text { Outrepassé or } \\
\text { overshot }\end{array}$ & $\begin{array}{l}\text { These flakes can be either bending or conchoidal initiated but preserve a remnant } \\
\text { of opposing lateral edge(s) of the biface. The distal end terminates in removal or a } \\
\text { portion of the opposing biface edge or in a feather or hinge termination well onto } \\
\text { the surface of the biface. In cross section the flake will often have an arched profile } \\
\text { following the contour of the biface surface. Usually created by use of excessive force } \\
\text { in flaking and generally associated with biface manufacture. }\end{array}$ \\
\hline Biface edge collapse & $\begin{array}{l}\text { Both faces of the biface lateral edge are preserved on the proximal end as the } \\
\text { striking platform. This would create a corresponding open C shape along the edge of } \\
\text { the biface. Produced as a result of manufacturing error. Termination morphology is } \\
\text { variable (Masson 1998:686). }\end{array}$ \\
\hline $\begin{array}{l}\text { Uniface or tool edge } \\
\text { retouch }\end{array}$ & $\begin{array}{l}\text { Flakes variable depending on detachment technique. On some, striking platform is } \\
\text { rounded, stepped, or crushed from use wear. Common use wear on dorsal surface, } \\
\text { trailing distally from the striking platform. Retouch technique dictates presence or } \\
\text { absence of bulb of percussion. Previous dorsal flake scars representing previous edge } \\
\text { retouch removals are common. Retouch flakes have an arched profile. Can expand } \\
\text { distally or have mostly parallel lateral edges. In profile, the distal termination is } \\
\text { curved (see Andrefsky 1998:120). Other flakes resembles a small microblade or burin } \\
\text { spall in form and size and may have been produced by a burin retouch technique. On } \\
\text { these flakes, one edge of the retouch flake will retain a portion or much of the uniface } \\
\text { edge and have a triangular cross section. Other various uniface retouch flakes have } \\
\text { been described by Shafer (1970). }\end{array}$ \\
\hline Burin spall & $\begin{array}{l}\text { Flakes variable depending on the parent piece from which they were detached. } \\
\text { Generally appear like small blades or microblades with trihedral, trapezoidal, or } \\
\text { rectangular cross sections. Can display some overlap with certain morphologies of } \\
\text { uniface or tool edge retouch techniques discussed above. Depending on the parent } \\
\text { piece, an edge of the burin spall can retain an unmodified lateral edge of the flake, a } \\
\text { bifacial edge of either projectile point or small biface, or previous burin spall removal } \\
\text { scars. Striking platforms vary from flat to faceted and occasionally are ground or } \\
\text { abraded with small contact areas. }\end{array}$ \\
\hline
\end{tabular}


Table 4.5, continued

\begin{tabular}{l|l}
\hline \multicolumn{1}{c|}{ Flake Type } & \multicolumn{1}{c}{ Key Attributes } \\
\hline $\begin{array}{l}\text { Core platform } \\
\text { rejuvenation }\end{array}$ & $\begin{array}{l}\text { Sections or flakes removed from core platforms or surfaces to rejuvenate or repair the } \\
\text { core for continued flaking. Commonly removed from unidirectional flake and blade } \\
\text { and flake blade cores. Category includes core tablets that are disc-shaped flakes with } \\
\text { remnant flake removal scars around the lateral edge (portion or all). These flakes } \\
\text { were removed to renew the striking platform. }\end{array}$ \\
\hline Hammerstone spall & $\begin{array}{l}\text { Dorsal surfaces consist of heavily battered surfaces and flake scar ridges. Typically of } \\
\text { limestone or quartz and quartzite, with no evidence of burning to suggest a thermal } \\
\text { spall. In absence of hammerstones, can be used to identify hammerstone use. }\end{array}$ \\
\hline $\begin{array}{l}\text { Shatter/angular and } \\
\text { thermal }\end{array}$ & $\begin{array}{l}\text { This category includes all fragments, chunks, chips, and pieces that could not be } \\
\text { assigned to a particular flake class or identified as a flake fragment. These pieces } \\
\text { retain no identifying technological features. Specimens with evidence of burning or } \\
\text { heat alteration are distinguished from those without. }\end{array}$ \\
\hline Flake fragments & $\begin{array}{l}\text { Includes all portions identified as fragments. Usually does not include proximal } \\
\text { fragments because these can be assigned to other known flake types. Pieces include } \\
\text { lateral edge remnants and medial, distal, and wedge-shaped fragments. }\end{array}$ \\
\hline
\end{tabular}

adults vs. juveniles were compared to determine if the assemblage represents a natural population (all ages represented) or a separate subset of the population (such as adults only) that may have been harvested as food.

\section{Analysis of Burned Rock Data}

Burned rocks were the most abundant cultural materials recovered from the Tank Destroyer site. Their ubiquitous nature makes them a valuable data set for addressing the types of features used at the site and the number of estimated cooking episodes or use life of the burned rock features.

The burned rocks associated with Features 1,2 , and 3 were systematically size-sorted, counted, weighed, and then discarded in the field. Burned rocks recovered from samples (i.e., soil column and flotation samples) also were size-sorted, counted, and weighed. In total, ca. $5,729 \mathrm{~kg}$ of burned rocks were recorded.

The burned rock data was used to describe the mound (Feature 1) and its internal basin (Feature 3 ) as accurately as possible using the protocol described by Black and Ellis (1997:781783). Each of the following attributes for the Tank Destroyer mound were addressed to the extent possible: landscape position, site context, mound integrity, dimensions, morphology, stratigraphic contacts (top, perimeter, bottom), internal structure, coarse matrix, fine matrix, and nonmatrix constituents.

The horizontal and vertical distributions of rocks were examined and the total rock weights by size category were quantified for the mound and the other two burned rock features. The data were used to help define the original sizes of limestone slabs and determine how much fracturing due to heating occurred before being discarded into the mound. From this, a gross estimate of the number of cooking episodes represented by the total amount of burned rocks was calculated. The estimate, or more precisely a range of estimates, were based on at least two main assumptions. The first was that approximately one-half of the burned rock mound at 41CV1378 was destroyed, so the estimate based on archeological burned rock data would be doubled. The second concerned how many heating episodes a large limestone slab can go through before it fractures and is no longer useable as a heating element. This assumption was based on a literature review of published experimental data on the use of limestone rocks in earth ovens (e.g., Lucas and Frederick 1998:187).

\section{Landscape Analysis of Burned Rock Mounds and Middens}

Mehalchick and Boyd (2007:10-17) suggested that the burned rock mound at 41CV1378 
could contribute to an understanding of the activities of the prehistoric hunter-gatherers across the Edwards Plateau. This suggestion was based on the idea that burned rock middens and mounds are different types of features or sites found on different parts of the landscape and characterized by different sets of activities (Abbott et al. 1996; Kleinbach et al. 1995:767-775). What these distinctions might mean in terms of prehistoric human behaviors is most clearly defined by Kleinbach et al. (1995:767-775), Abbott et al. (1996), Black et al. (1997:287-289), Boyd et al. (2000), Boyd and Mehalchick (2002), and Kleinbach et al. (1999:411-417).

To better understand the differences between mounds and middens, the activities and behaviors that generated them, and how these things might have varied across the landscape, the spatial distribution of mound and midden sites and various environmental variables were examined for patterns and relationships. The archeological and environmental GIS databases at Fort Hood were used to do this analysis. This analysis was exploratory in nature and conducted in two parts. The first study examined the environmental factors influencing the distribution of burned rock mounds and middens across the landscape of Fort Hood. The second study took an independent look at the relationship between the locations of large burned rock features at Fort Hood and the distribution of post oaks (Q. stellata) and Paluxy sands.

\section{An Examination of Social Identity in the Late Archaic of Central Texas}

Social identity is a "person's knowledge that he or she belongs to a social category or group" (Stets and Burke 2000:225). A social group is a "set of individuals who hold a common social identification or view themselves as members of the same social category" (Stets and Burke 2000:225). Social identity theory (following Tajfel [1972] and Tajfel and Turner [1986]) is an explanatory framework that attempts to explain that an individual's actions are a reflection of his or her group. In other words, an individual's actions represent a range of accepted practices and behaviors appropriate to an individual's status, role, or membership within a social group. In the archeological record, artifacts and artifact as- semblages can represent this range of accepted practices and behaviors. Cultural materials should reflect different group identities in the way that the group did things and what was considered acceptable within a particular group. Differences in artifacts and assemblages across a geographically wide area can be described as a social field, a space created by the actions and interactions of people and groups.

The radiocarbon dates and diagnostic projectile points indicate that the human occupations and activities at $41 \mathrm{CV} 1378$ primarily occurred during the Late Archaic, and more specifically during three periods defined by Prewitt (1981) as the Uvalde, Twin Sisters, and Driftwood phases. When initially defined by Prewitt, there appeared to be some meaningful differences among the three phases, based on artifact and feature assemblages, that might reflect real cultural differences representing various groups of people and changes through time. More recent research, however, suggests that each phase most likely represents a large social field composed of many different and distinctive groups.

For the period of European contact in the Edwards Plateau region, Wade (2003:224-232) notes that a large social field existed in the midto late 1600 s that was composed of as many as 21 individual groups. These distinctive social groups shared a broadly similar lifestyle and material culture and participated in various exchange networks and alliances. Although Wade presents her analysis in the context of native groups' relationships with Europeans, she clearly believes that these social mechanisms did not rise in response to the presence of Europeans. The complexity of these networks and alliances indicates that they existed in prehistory as well (Wade 2003:228, 231). Arnn (2007) proposed a similar scenario for the Late Prehistoric Toyah phase and suggested that the presence of large social fields has roots in the Late Archaic period. Based on work at Lake Waco, Mehalchick and Kibler (2008:368-371) proposed that the Late Archaic in central Texas may be characterized at any given time as a giant social field. They suggest that it was a "network of common socioeconomic interests" that would have been maintained through a complex and constantly changing system of alliances. They conclude that "the key to unraveling this is identifying local or geographically 
restricted variants of artifact types that signal social identity."

It was not suggested that the data from 41CV1378 would provide solid answers to the question of social identity in the Late Archaic of central Texas, or that a rigorous intersite comparative analysis of assemblages would be completed. Rather, based on a limited literature review, we present in Chapters 9 and
10 a brief discussion of how 41CV1378 might fit into developing ideas of Late Archaic social fields. This examination focuses on Prewitt's Uvalde, Twin Sisters, and Driftwood phrases, or more specifically the key markers of these phases (Marcos, Montell, Castroville, Ensor, Frio, Fairland, and Darl points), all of which (save for Frio points) were recovered from the Tank Destroyer site. 



\section{IMPLEMENTING AND EVALUATING THE TxDOT CHIPPED STONE ANALYTICAL PROTOCOL, VERSION 2.3}

John E. Dockall

Version 2.3 of the TxDOT Chipped Stone Analytical Protocol calls for developing spreadsheets with dropdown menus for recording observational and metric data on tools, cores, and unmodified debitage (Figure 5.1). The analytical procedure is designed to help researchers standardize taxonomy, identify distinctions between tools and non-tools, and distinguish core-derived tool and core-based tool portions of the chipped stone assemblage (see Appendix C). This dichotomous framework is intended to provide a standard method of sorting the assemblage or various components into meaningful subsets (e.g., cores, tools, non-tool debris).

At the highest level of the taxonomy, technological distinctions are made between chipped stone and ground stone portions of the lithic assemblage. At the next taxonomic level, the chipped stone assemblage is divided into tool and non-tool groups. Artifacts assigned to the tool group include those that were intended for use as tools or are inferred to have a specific function. The non-tool group includes chipped stone artifacts such as cores that have "indirect functionality" or that are "objects of an instructional, symbolic or artistic nature." This taxonomic framework does not include unmodified debitage. A separate protocol addresses the analysis of unmodified debitage and is discussed and evaluated later in this chapter.

The tool group is divided into three "subgroups": simple detachment-based tools, complex detachment-based tools, and core-based tools. ${ }^{1}$

\footnotetext{
1 Though Figure 5.1, which was produced by TxDOT for the analytical protocol, depicts two subgroupscore-derived tools and core-based tools-the protocol text (see Appendix C) references three subgroupssimple detachment-based tools, complex detachmentbased tools, and core-based tools.
}

The simple and complex detachment-based tools are considered core-derived tools, as opposed to core-based tools. The core-derived tools, both simple and complex detachment tools, consist of two "classes": flakes and blades. Core-based tools are sorted into biface or non-biface classes. Simple detachment flake or blade tools include only those with very little to no deliberate retouch following detachment from the core. Complex detachment flake and blade tools exhibit considerable modification of their original shape, although the final shape of the discarded artifact could have been achieved prior to use or as a result of extended use and maintenance. In this regard, some complex detachment tools may have started their use lives as simple detachment implements but transitioned to forms that may be considered complex detachment through use and maintenance. The intervening history of use and maintenance will not be particularly obvious but may be broadly interpretable from comparisons with the debitage and the overall character of the tool assemblage.

Flake, blade, biface, and non-biface classes each consist of two "subclasses." The flake and blade tool subclasses are modified and unmodified. Biface and non-biface tool subclasses are formal and informal. Subclass data provide the analyst with impressions of the degree that the tool producer followed an established manufacture or final-form template for the appearance of that tool. The TxDOT protocol depicts prehistoric lithic technology as a continuum, with formal tools at one end and informal tools at the other. Informal tools are understood to be unstandardized in form compared to formal tools. The informal category typically includes expedient tools, meaning tools made, used, and 
Data Recovery Investigations at the Tank Destroyer Site, Fort Hood

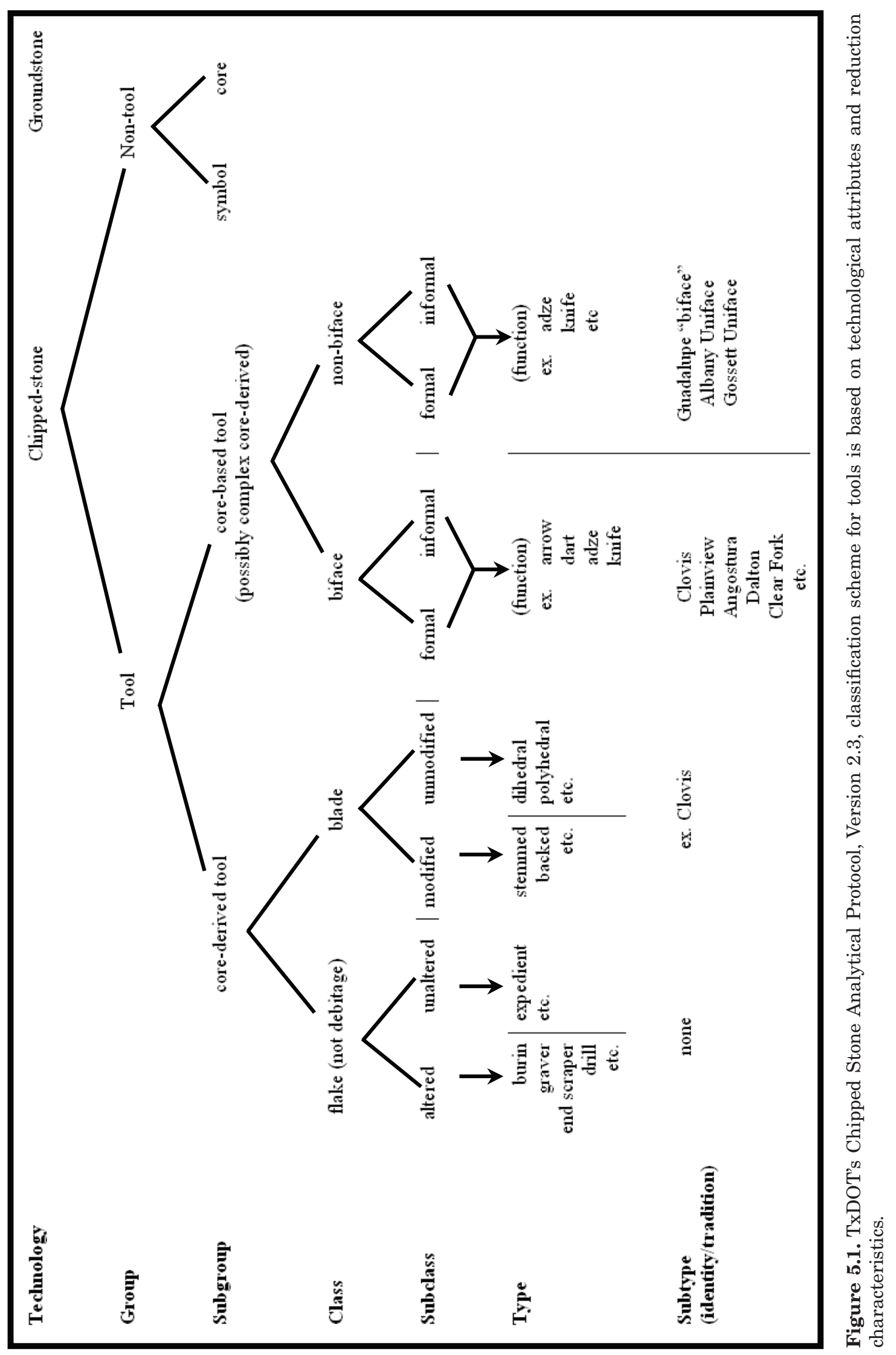


abandoned over a short time period (Andrefsky 1998:113-114). This information also can be used to ascertain the presence or absence and character of expediency associated with a tool or a chipped stone assemblage. Assessment of tool or assemblage expediency has been discussed in the literature and is still considered to be open to interpretation. Its use in any analysis should be defined as explicitly as possible; therefore, the concept of expediency is discussed in greater detail later in this chapter.

In accordance with Version 2.3 of the protocol, flake and blade tools are classified as modified when stages of manufacture were involved following their removal from the core and prior to initial use. Examples include sequent flake unifaces, various end scrapers, drills, and backed blades. For bifaces, formal tools conform to a "standardized, pervasive, recognizable morphology," while informal bifacial artifacts do not. An example of a formal biface would be a Gahagan or Friday biface or a known projectile point type, whereas an informal biface would be a thin bifacial preform or drill.

The final categories of artifact classification are "type" and "subtype." Tools are typed according to their function as determined by technology, form, and microscopic use-wear traces. Modified flake tools should be typed according to their function and include burins, gravers, scrapers, and drills. Modified blades considered tools should be typed in accordance with their type of modification (e.g., backed, stemmed, truncated, notched). Simple detachment implements subclassified as unmodified flakes with use wear are typed as "expedient," while tools subclassified as unmodified blades are typed according to morphology (e.g., dihedral, polyhedral, flake, prismatic). Examples of bifacial tool types include arrow points, dart points, drills, knives, and adzes. Non-bifacial tool types include various scrapers, some unifacial adzes and knives, denticulates, and drills. In most instances, there can be a non-bifacial functional counterpart to bifacial artifacts.

Tool "subtype" is employed in Version 2.3 to identify artifacts that correspond to well-established types or artifact traditions. Projectile points may be classified according to their type names-e.g., Ensor, Darl, Perdiz, Scallorn, Clovis, and Bassett. Some other bifacial and unifacial implements can be linked with specific type names-e.g., Guadalupe adze, Clear Fork gouge, Gahagan or Friday biface. Typically tools that have been subclassified as modified flakes or blades will not be associated with specific type names.

\section{THE CONCEPT OF TECHNOLOGICAL EXPEDIENCY}

Because the TxDOT Chipped Stone Analytical Protocol Version 2.3 relies in part on determinations of "expediency," it is necessary to provide a working definition of the concept for this report. This chapter follows Nelson's (1991:64) concept of expediency as a strategy of chipped stone tool manufacture. Expediency depends on two conditions. First, raw material must be in ample supply at a specific location, either naturally or through stockpiling. Second, there must be sufficient time to manufacture tools and use them at a specific location. In addition, expediency may involve long-term occupation or regular reuse of a specific location to take advantage of available raw materials, a nonlithic resource, or both.

When considering formal and expedient technologies, it is important to consider the way in which lithic raw materials are supplied. What are the strategies of raw material procurement that are represented at a given site? Kuhn (1990, 1995) has developed and discussed a multilevel model for raw material provisioning. Essentially, there are three modes of material supply:

1. Provisioning of activities occurs at the time when the need arises and tools are made without prior planning. The tools are then abandoned when the need ceases. Kuhn (1990) has referred to this as responsive technology. Artifact manufacture is controlled by material availability, and types of activities reflect a low level of technological investment.

2. Individual provisioning often occurs in response to low lithic resource availability or if a group knows it will be in an area of uncertain resource abundance. Often, this strategy is associated with personal gear and the curation of formal tool types (Binford 1980, 1982). Costs of this strategy are related to the limited amount of gear that an individual can carry and the high 
degree of attrition coming from use and maintenance.

3. Provisioning of places allows an individual or group to meet anticipated tool and raw material needs when future activities are predictable. Raw materials are supplied by caching, stockpiling or by locating activities near suitable supplies of lithic raw materials.

The lithic assemblage from any given site may contain the archeological residue of one or more of each of these modes, constituting a technological and temporally mixed assemblage (Gramly 1980; Torrence 1983:13-14). Any lithic assemblage will be composed of, to varying degrees, tools and debris produced for resource acquisition (extractive) tasks and repair/replacement (maintenance) tasks. The concepts of expedient and formal may apply differently to each of these, and the distinction between the two is not always clear (Torrence 1989:64). Often, the terms "curated," "formal," and "maintainable" are used interchangeably, as are "expedient," "informal," and "reliable," which has had a tendency to oversimplify our understanding of prehistoric technology (Torrence 1989) and can mask important behavioral observations. The dichotomy usually follows that curated-formal-maintainable tools are implements such as hunting weapons or similar gear (used for resource procurement) and expedientinformal-reliable tools are implements used to manufacture or repair other tools or are used in resource procurement where the risk of failure to procure a specific resource is low. Analytical decisions between expedient and formal technologies are heuristic. Often the dichotomy is based on subsistence mode or mobility (Parry and Kelly 1987). The dichotomies presented by reliable (expedient)/maintainable (formal) tool design and collector/forager subsistence modes are not necessarily mutually exclusive (Binford 1980; Bleed 1986; Bousman 1993; Nelson 1991; Torrence 1983, 1989a, 1989b). They reflect an array of strategies or technological options for given situations. Consequently they can be used exclusively or in varying combinations as needs arise.

For example, previous researchers have on occasion contrasted the abundance of formal tools, primarily bifaces, with expedient forms of core reduction (bipolar and general percussion) as if they were equivalent units of comparison (Parry and Kelly 1987). The comparison is questionable. The more appropriate question is what the use history of those artifacts can indicate regarding site activities and behavior. As Nelson (1991) states, expedient technologies are planned responses to scheduled tasks that require minimal preparation of tools; these tools have short use lives and are usually discarded at the site of use.

\section{COMMENTS ON THE CHIPPED STONE TOOL ANALYTICAL PROTOCOL}

The chipped stone tool analytical protocol consists of five parts: taxonomy, metric information, attributes, raw material, and wear patterning. These aspects of the protocol and their various categories and variables are evaluated below.

\section{Taxonomy}

The protocol employs seven taxonomic levels to classify chipped stone artifacts (excluding unmodified debitage). Below are comments on each regarding their appropriateness and degree of usefulness.

\section{Technology}

This field is straightforward and is subdivided into two broad categories: chipped stone and ground stone artifacts. Currently, the only protocol available is for chipped stone artifacts.

\section{Group}

Materials in the chipped and ground stone categories are further subdivided into "tool" and "non-tool" items. The definitions of these are quite clear and self-evident in the protocol; however, there is room for deviation in how different analysts assign tools and non-tools in some artifact categories. For example, cores are not typically considered tools, but analysts disagree about the subsequent use of cores as tools or the incidental use of larger cobble or core-based tools such as choppers as cores. Resolution is largely dependent on how artifacts 
of this nature are initially categorized - as cores first and tools second, or vice versa. How this is done could impact the recording of additional data later or instigate the removal of some artifacts from one database to another. Therefore, such decisions should be made at the start of the analysis process.

\section{Subgroup}

Three subgroups are recognized based on the item's primary technique of manufacture: "simple detachment-based tools," "complex detachment-based tools," and "core-based tools." Simple detachment tools include flakes and blades that are employed as tools with little to no prior modification. Complex detachment tools have undergone substantial modification prior to use according to the protocol. But substantial modification can also occur to a simple detachment tool that transforms it, for example, though repeated sharpening or modification, into a complex detachment tool. Tool fragment or implement recycling may lead to discrepancies in assignment to simple, complex, or core-based detachment categories. One can easily envision bifaces, projectile points, and various types of more formal unifaces as fitting neatly into a complex detachment category. However, there may be some confusion where to place some more "expedient" tool forms, such as minimally modified flakes that have a portion of an edge modified by deliberate retouch. It is also possible that some very early stage bifaces would be classified as simple detachment tools. In many cases modification is simply a continuum with no clear separation between "simple" and "complex" modification. There would also seem to be some overlap between complex and core-based detachment categories as well. These comments aside, the categories appear sufficient to catch the bulk of the variability within a given assemblage.

\section{Class and Subclass}

At the class level, the analyst classifies simple detachment tools into "flakes" and "blades," and complex and core-based detachment tools are segregated into "bifaces" and "non-bifaces." Flakes and blades are further sorted into "modified" and "unmodified" subclasses, and bifaces and non-bifaces are sorted into "formal" and "informal" subclasses. Behaviors such as recycling and rejuvenation of formal tools or fragments of formal tools should be recorded as secondary observations of each tool. For example, one burin manufactured on a simple percussion flake or blade that has been truncated by anvil percussion and then a burin spall removed along one edge may be classified differently from a burin manufactured on a broken dart point proximal medial fragment in which the transverse bending break has been used as a platform to remove burin spalls.

By this point in the analysis, however, the analyst is considering the simple and complex detachment dichotomy of an artifact that reflects both and could essentially be coded as one or the other and still be correct. The protocol manual indicates that modified simple detachment tools have undergone "additional stages of manufacture...following their initial detachment prior to their use" (see Subclass section of protocol in Appendix C). Herein there may be some confusion among analysts between modified simple detachment tools and complex detachment tools (which can be "reduced through bifacial or unifacial percussion"). There is a notion of distinct reduction or manufacture stages associated with complex detachment tools, but simple side or end scrapers that would initially be classified as simple detachment-based implements can go through an extended life history of resharpening episodes that can, in theory, transform it into something that may be considered a complex detachment tool. One may also consider whether certain types of convex end scrapers may be formal tools even though an accepted type name is not used for them. As another example, some Perdiz points display minimal modifications, either modified by pressure flaking or largely unifacially flaked. The analyst here is asked to decide between simple detachment and complex detachment. Can some Perdiz points be simple detachment tools yet be formal, depending on the degree or amount of unifacial or overall facial retouch? The distinction between simple and complex detachment has utility for most assemblages, but similar in formation is captured in the class and subclass categories.

\section{Type}

The type lists for tools are based on form and function, with function determined through use-wear analysis. The type list provided can be 
quite expansive, or has the potential to be, as analysts have a tendency to be either splitters or lumpers. Given this, tool type lists will vary among analysts.

Typing simple detachment-based unmodified flake tools as expedient allows the analyst to segregate them from the debitage sample. If the analyst is asked to collect use-wear information on tools, then such unmodified flakes employed as tools should be included, particularly since flakes that have been modified (by deliberate retouch) are included in this category and are supposed to be typed according to function (or morphology).

The concept of tool type is particularly difficult to implement for complex, multifunctional tools, which are often highly variable in form and function. Although the protocol provides independent fields for recording each attribute, there are not enough attribute variables to deal with variable multifunctional tools. The existing protocol only allows one to record certain attributes (e.g., edge angle, edge morphology, edge construction type, use wear type, and retouch type) only once per tool. The existing protocol format does not allow an analyst to record data for multiple working edges on a single multifunctional tool. As is discussed later, however, it is relatively easy to expand the existing protocol format to capture data for multiple working edges to more accurately represent functional variability and complex tool types in the database.

\section{Subtype}

This field is appropriate where formal tools conform to the definitions of well-established type names. For other tools, though, its utility is limited.

\section{Metric Information}

The collection of metric information is fairly straightforward and in most cases only involves recording the maximum length, width, thickness, and weight. For most tools this information is clear, but there is no standard for modified flake or blade tools unless the observable maximum dimension is recognizable. The illustration in the protocol (see Metric Information section in Appendix C) only shows how to orient a projectile point, not other tool types, to derive measurements. Some clarity on how to orient other implements would be helpful, or else it should be stated in the protocol that measurements for amorphous flake tools should be oriented toward maximum dimension. If the maximum size of the flake blank is desired, then systematic orientation of the tool during analysis will be in order.

The protocol calls for measurements of edge angle, though the discussion is rather brief and would be more useful if expanded. How many edges and what edges in particular should be measured? For instance, for projectile blades, does one just take the edge angle of both lateral edges, or should lateral stem edge angles also be included? If a modified flake has three distinct elements of use, do each of the three elements get an edge angle column in the database? If the functional element in question is a tip or point, does the analyst take the edge angles of both edges of the point, the planview angle of the tip, or the tip face? Should edge angles on burins or burin spalls that have been used as tools be measured? If the goal of the protocol is uniformity and standardization in data collection, then more direction for the analyst is in order.

Regarding edge angle, presumably there should be an edge angle for each edge of a modified flake or blade tool that displays wear or has been retouched. In this case, edge angle column data should correspond to edge wear data that is collected later in the database spreadsheet. This would provide some way to correlate certain wear patterns with an edge angle or type of edge/element modification. For our analysis, we have included four columns for edge angle, four columns for edge construction, and a separate column for each type of edge wear (flaking attrition, crushing, smoothing, etching/pitting). In this way, we have the potential to record up to four distinct attributes for edges that have been modified or utilized in some way. This allows some discussion of wear type, edge angle, and functional elements for tool classes if needed.

\section{Attributes}

The protocol lists a series of attributes to be recorded for each tool. These attributes are discussed below and their usefulness is evaluated. 


\section{Stage}

Five stages of artifact manufacture, use, and discard are recognized in the protocol: initial package reduction, blank preparation, preform shaping and thinning, final edge trimming, and rejuvenated forms. These stages generally work well for projectile points and other bifacial tools, as well as some unifacial artifact types. They are nebulous, however, when applied to utilized flakes, flake fragments used as tools, recycled tools, tool fragments, or minimally retouched implements. Attributing a stage of manufacture for these types of implements is not as straightforward as it is for formal tools. Although stages are listed for bifacial tools, it may not always be possible to assign many expedient tools to later manufacture stages.

According to the protocol, most expedient tool forms should be recorded as initial package reduction, or Stage 1. This assertion is based largely on the assumption that these tool types retain much of the original shape of the flake or blade blank and are minimally modified except through use or retouch. Technically, however, it is hard to assign these types of tools to the initial package reduction stage because by the fact of use, resharpening, and discard, they have already progressed into later stages; however, the transition between stages does not involve a significant change in morphology. It should be recognized that these implement types can transition between the initial and later stages without much change in form. Most analysts do not appear to regularly assign expedient tools to particular stages of manufacture and may not be entirely clear how to assess this information from these tool types. More clarification and visual examples of such tools assigned to particular stages would be in order. The stage attribute, however, is useful because it allows the analyst to record such esoteric behaviors as recycling or tool fragmentation/reuse. As noted in Chapter 4, for this analysis two more stages were added: recycled and resharpening/repair. In addition, the rejuvenated stage was changed to rejuvenated/repaired to more clearly capture these aspects of the tool manufacture-use-discard trajectory.

\section{Portion}

The portion attribute is clear and applicable to most assemblages. Other variables can be added if they are identified. The figures in the protocol (see Attributes section in Appendix C) only include examples of dart point and projectile point fragments. A few examples of fragments of other tool types may be helpful.

\section{Failure/Discard}

Failure/discard is an important aspect of tool history to consider, since it conveys useful information about site use and type. More thorough definitions of each of the variables in the protocol would be helpful. To capture more variability that might be present in an assemblage, for the current analysis, the following failure/ discard variables were added to the dropdown menu: multiple fractures, edge collapse, radial break, snap break, and radial/snap break. These variables are defined as follows.

\section{MULTIPLE FRACTURES}

This variable allows the analyst to code implement pieces that exhibit multiple fractures that may be the cause of failure. An example would be a biface medial fragment with a bending fracture on one end and a perverse fracture on the other.

\section{EDGE COLLAPSE}

This variable is reserved for bifaces or bifacial fragments, or perhaps other formal tools, that exhibit a C-shaped segment missing along one or more lateral edges that is not due to specific types of edge retouch. The variable can also be used to record the missing piece of the edge, and rather than recording it as a lateral edge fragment, it can be recorded as a tool manufacturing failure. This variable is similar in some respects to outrepassé or overshot, though there is no indication that the fracture traveled across the surface of the biface any significant distance. Edge collapse is recognized to be distinctly different from the variable of platform loss.

Edge collapse flakes are short and wide, with wide platforms representing the upper and lower biface surfaces. When they are removed, edge collapse flakes produce a crescent or Cshaped fracture along a biface edge. It is most likely due to a manufacturing error in which the flaking tool contacted the biface too far in from 
the edge (Collins 1974; Masson 1998; Waters et al. 2011).

\section{RADIAL BREAK, SNAP BREAK, AND RADIAL/SNAP BREAK}

Examples of these breaks are occasionally present in assemblages. These categories are modified from Frison and Bradley's (1980:90-98) discussion of hinge, radial, and bend break tools from the Hanson site in Wyoming. In some cases, radial and snap breaks can be identified as deliberate based on the presence of specific flake and fracture face features that indicate it was done on an anvil of some sort. Such breaks have been identified in several contexts in Texas associated with Archaic and other occupations (Dockall and Boyd 2006a:55, 2006b:100-102; Dockall and Pevney 2007:195-197), and they appear in the assemblage from 41CV1378. Similar broken implements have typically been observed and identified in various pre-Clovis, Paleoindian, and other later archeological contexts (Amick 2007:223-249; Dellar and Ellis 2001; Frison and Bradley 1980; Goodyear 2005; Root et al. 1999) and have occasionally been associated with various probable ritual activities (Dellar and Ellis 2001; Shafer 1973:224-228) in Paleoindian to Caddo occupations. Dockall and Pevney (2007:197) have illustrated some of the distinctive fracture features associated with these types of tool breaks, as have the other previously mentioned authors.

\section{Alteration}

This attribute, consisting of several variables (e.g., thermal, white patina), is straightforward and useful as defined in the protocol.

\section{Edge Morphology}

The application of edge morphology—distal, left lateral, and right lateral-appears straightforward until the analyst is required to record the edge morphology for tools with multiple edges or functional elements. Then the morphology for each edge must be integrated into the database. Here the number of edge morphology columns should correlate to the total number of identifiable edges with distinct types of edge wear, edge angle, edge construction, and the like. Otherwise, none of these data categories can be adequately summarized as a coherent unit for each tool.

\section{Distal}

The application of distal edge morphology criteria appears to be limited to unifacial or other tools that have a distal functional element that is linear or curvilinear in form. It seems to exclude tool types such as burins, drills, gravers, some denticulates, or beaked tools that have specific functional elements composed of points or edges of variable morphology. The selections offered in the variable list do not consider working points or protrusions unless the analyst is expected to code the lateral edge shape of the point in question. If so, this is not clear and should be more specific.

\section{Left and Right Lateral}

Recording these edge morphology attributes is straightforward and works well. For the current analysis, a column was added for "proximal edge morphology" for implements such as scrapers or utilized flake tools that have use wear or retouch on the proximal end.

\section{Flake Scar Pattern}

The flake scar pattern variables that are included in the protocol are appropriate for most formal bifacial and some of the more formalized unifacial artifact types. For the current analysis, a few additional types were added to accommodate retouch often found on simpler unifacial and other flake tools. These tools frequently have retouch-either for hafting or prehension modification or as a result of resharpening unifacial edges-that is as distinctive and functionally relevant as that often associated with formal implements. Although these other types may not reflect an aesthetic element that might inform social identity or cultural preferences, they are consistent enough to be related to certain tool functions or tool element morphologies. Types of retouch added are "invasive percussion," "marginal percussion," "invasive pressure," "marginal pressure," "marginal edge nibbling," "steep abrupt retouch," "burination or burin retouch," and "no retouch." Pictures or schematic drawings of each type of retouch should be included in the user's manual. 


\section{Edge Construction Type}

According to the protocol, edge construction refers to the location and form of preparatory edge chipping, and distinctions are made at a basic level between unifacial and bifacial. The one problem with this variable is that the choices are useful only for the tool overall and are not easily applicable to discrete retouched edges or tool elements. If one is to record use wear for specific elements, it only makes sense to try to correlate edge angle, use-wear types, and edge construction types for coherence in the tool database. We found it useful to add a few categories to accommodate other kinds of edge modifications: "no retouch," "deliberate radial break," "deliberate snap break," "unpatterned retouch," "burin spall retouch," "radial break with superimposed retouch," and "snap break with superimposed retouch." The final version of the protocol should include descriptions and/or illustrations to clearly specify what is meant by each edge construction type. It is also worth considering whether this variable should be applied to haft element edges/basal edges.

\section{Proximal Edge Grinding}

This attribute, recorded as present or absent, is self-explanatory.

\section{Wear Patterning}

Recording some of the wear patterning data in the spreadsheet requires more detailed knowledge of use-wear techniques and theory than the brief discussion in the protocol implies. While an analyst can identify presence or absence of particular kinds of use wear with an 18-20x jeweler's loupe, this information only supports very generalized interpretations. Only through higher-power magnification can an analyst make more meaningful interpretations of tool function. A tool edge may exhibit polish under low magnification, for example, but only at a higher magnification does it reveal the type of material that caused the polish and the striations that indicate direction of tool use. For most tools examined, flaking attrition, crushing, and smoothing are the most commonly observed wear types. However, the interpretation of the various types of wear patterns from the selection lists is confusing and difficult to visual- ize for some of the types. These wear pattern choices are also difficult to apply to discrete tool edges since some of them at least consider the location of two or more edges or parts of a tool rather than a discrete edge. Either additional types should be added or the list should be reworked to accommodate individual edges or tool elements. Where possible, we have adhered to the list of use-wear patterns as much as possible, but we have found it necessary to add some of our own types.

Crushing and smoothing are considered in Version 2.3 to be wear types associated with battering, grinding, and polishing. Flaking attrition wear can transition to have a crushed appearance which, with continued tool use or certain types of abrasive worked materials, can further transition or co-occur with smoothing, rounding, polish, or striations. The nature of use-wear formation is a continuum that runs from minor flaking attrition traces to very heavily rounded, abraded edges that may or may not include polish and striations. Use wear is dependent on the worked material (e.g., how abrasive is it, how wet or dry is it, and/or how hard is it) and the tool motion (e.g., scraping, cutting, drilling, engraving, chopping). The resulting wear is a combination of these factors and the length of time a tool is used.

The categories for location of use wear, again, reflect the location of wear for multiple portions of the tool, which makes it difficult to record wear traces for specific edges or particular portions of the tool. The categories for flaking attrition, crushing, smoothing, polish, and etching/pitting should be accompanied by better descriptions or graphic representations of some of them so they can be more easily visualized by the analyst. While an analyst can identify presence or absence of particular kinds of use wear with an 18-20x jeweler's loupe, this information only supports very generalized interpretations. Only through higher power magnification can an analyst make more meaningful interpretations of tool function. A tool edge may exhibit polish under low magnification, for example, but only at a higher magnification can the analyst infer the type of material that caused the polish and see the striations that indicate direction of tool use. This analyst has successfully used several use-wear recording protocols that allow the analyst to record observed wear traces for very specific portions of tools. Those developed by 
George Odell and John Shea (Odell 1979; Shea 1991), for example, employ an 8-segment polar coordinate graph concept, and wear is recorded as an 11-digit use-wear profile.

According to the protocol, hafting evidence is to be recorded as observed or not observed; however, some of the wear types recorded above may be associated with hafting and not tool use, depending on location. Recording presence or absence doesn't reveal the type of hafting evidence observed. Also, hafting evidence present only as microscopic usewear traces may occur without any indicative retouch to suggest that hafting may have been part of the tool design. An example, albeit an uncommon one, is utilized or edge-modified flakes that may have been used in a hafted state.

Tool prehension, whether hafting or handheld, is often quite visible and interpretable along the appropriate segments of a tool edge (Odell 1980; Odell and Odell-Vereecken 1980; Rots $2005,2008)$. The experimental and archeological literature is replete with examples of wear traces recorded as prehension and hafting. This information is easily recorded as part of the use-wear analysis of a tool. This analyst has observed very clear indications of hafting traces in the form of polishes, abrasion, residues, and patterned microscarring that are also routinely interpreted as hafting by other analysts. Location and type of edge wear in these cases can be used to develop ideas about how certain tools were hafted, which is pertinent to interpretations of tool use. Also, hafting observed in ethnographic contexts can be used to interpret microwear traces as evidence of a particular mode of hafting or prehension (Beyries and Rots 2008; Kehoe 2005).

\section{Raw Material}

The raw material list provided in the protocol can easily be modified, adding or deleting certain materials for specific projects. We have added a substantial list that incorporates all of the known types of Fort Hood lithic materials. Regionally specific raw materials can be added for projects in other parts of the state.

\section{COMMENTS ON THE CHIPPED STONE UNMODIFIED DEBITAGE ANALYTICAL PROTOCOL}

The TxDOT protocol for debitage analysis is specific with regard to the analytical process and data recorded for the analysis of unmodified debitage. The protocol combines aspects of flake attribute analysis and mass analysis (size grading) to maximize the amount of data collected for large assemblages and still provide sufficient data for a wide array of research questions. The types of data collected from unmodified debitage are primarily observations and quantities based on sorting variables.

Only total counts and weights of flake groupings or assemblages created during the analytical process are collected for analysis. These groupings can be based on raw material and technological similarities, shared (spatial) provenience, or combinations of other observations and data that suggest that the materials (tools, cores, unmodified debitage or subsets of these) are contextually related in some manner. Examples of analytical assemblages could include but are not limited to the following: discrete concentrations of unmodified debris and cores excavated in the vicinity of hearth features (Gadus et al. 2006:79-90), isolated surface scatters of a few flakes and/or cores, and manufacturing debris (cores, flakes, tool fragments) associated with quarry locations and other raw material procurement locales.

\section{Metric Attributes}

It seems appropriate to limit metric data to counts and weights in size-grade categories.

\section{Minimum Number of Nodules (MNN)}

Presumably this is the same as the Minimum Analytical Nodule (MAN) technique discussed by Larson and Kornfeld (1997). MNN and MAN were designed to allow the analyst to record the minimum number of "individual packages" or raw material nodules contributing to a specific analytical assemblage. The use of these techniques implies a certain degree of cohesion and context control over the assemblage, which may not be possible in many cases. The very limited discussion in the protocol of applying this technique to an archeological example is not very instructive for analysts that have not done refitting or MNN/MAN. One of the goals is to achieve some understanding of the minimum number of raw material packages that contributed to a specific assemblage. This 
can provide some additional information on such activities as raw material acquisition and selection, reduction or manufacture trajectories, and curation-all of which are worthy goals of data collection and research. Although the basic rationale behind MNN/MAN is clear, the limitations of its application are not. Obvious limitations to this technique would appear to include patination, raw material types that are not amenable to sorting on the basis of visual criteria, and the degree of horizontal and vertical mixing (i.e., context). Presumably, sites that have been repeatedly used for a significant period of time such as burned rock middens would not be as appropriate for such activities as refitting and MNN/MAN. These types of contexts limit what can be said about raw material selection over time or specific changes in knapping strategies.

The protocol should give more consideration to the utility of this technique in certain contexts and when its use is most appropriate. Use of MNN/MAN can be limited to debitage alone, but studies where this technique has been applied have included formal and informal tools. This suggests that the technique is something that could be done as part of the technical analysis of both tools and debris and not something done only with size grading and core analysis. Furthermore, this protocol includes no expressed instruction on how to collect this information within the context of the lithic analysis program. This should be elucidated more clearly. Analytical nodules are defined based on similarities in raw material type, color, texture, inclusions, and cortex characteristics, and nodule members can include debris, tools, cores, or other artifacts (Larson 1994).

Although the MNN variable is considered part of the analytical protocol for debitage, it is uncertain how it is to be recorded and integrated into the database. Due to a number of post-occupation disturbances and the site type, this approach was not used for this project analysis. There are a variety of ways in which this information can be recorded, but the most simple method would be to include a column in the spreadsheet in which pieces that can be assigned to specific raw material packages all share a unique number or other type of designation that can be used as a sorting variable. This could be included in both the tools database and the cores/debitage database. Both could be linked in
Access, and all artifacts considered part of the same analytical nodule could be retrieved by a search query for discussion. This level of analysis is beyond the scope of a basic lithic protocol.

\section{Form (Completeness)}

The categories for flake completeness are appropriate and should be easily replicable among analysts to varying degrees.

\section{Size-Grade Analysis}

The size grades selected in the protocol are commonly used, although the number of size grades can vary between analysts. Interestingly, there is no specific notation in the protocol to include raw material type as a sorting criterion prior to size grading. This criteria could easily be included, especially since MNN is a part of the analytical protocol.

\section{Cortex Percent}

The cortex proportion categories presented in the protocol seem to follow those used by other analysts. The protocol cites Ahler (1989:90) as noting that the presence and abundance of cortex will vary according to a number of factors, but Ahler (1989:90) also indicates such classification schemes do not need to be elaborate. The basic principle of cortex analysis suggests that the more dorsal cortex present on a piece (and the more dorsal cortex present in a lithic assemblage), the earlier the reduction stage. In some cases, and in dealing with assemblages that involve largely one or two reduction techniques only, this may be the case. However, an experimental study by Bradbury and Carr (1995:112) noted that the dorsal cortex can be more directly related to the initial size of the raw material being transformed (Andrefsky 1998:109, 112-114). They and other researchers suggest that cortex is most useful in determining the earliest and perhaps the latest stages of lithic reduction.

The amount of cortex cover varies considerably through the reduction sequence and is seldom a reliable indicator of where in the sequence a flake belongs (Andrefsky 1998:114; Bradbury and Carr 1995; Odell 2004:127). Odell (1989) determined cortex to be only moderately useful to define the extremes of a reduction 
sequence. Mauldin and Amick (1989:70) noted that cortex proportions were useful for defining only the initial phases of a reduction sequence. Patterns of dorsal cortex within an assemblage may be telling us more about the nature of the raw material (i.e., size of the nodules being reduced) than about the technology used to reduce them into tools. This has certainly been this analyst's experience when dealing with lithic assemblages that are derived from secondary gravel sources of large cobble to small pebble size or a mix of secondary gravel and primary ledge source materials. The smaller the parent material used for stone tool manufacture, the more cortex that remains present throughout the reduction process, even into the smaller debitage size grades.

In terms of replicability and accuracy between analysts, Bradbury and Carr (1995:102) indicate that a good degree of accuracy can be obtained using a five-category cortex classification scheme. Coupled with other technological information, this data can certainly be useful in determining reduction patterns and technological differences between local and nonlocal raw materials. However, just how meaningful this information is for different types of flake production or core reduction techniques remains to be seen. Cortex pattern data for biface production from cobble to finished form signifies something completely different than it would for bifaces manufactured from ledge material (essentially cortex-free at the start) or bifaces manufactured from primary or secondary flakes removed from cobbles. Couple this with attempting to interpret cortex patterns for assemblages that also include varying amounts of bipolar percussion of small cortex-bearing chert, petrified wood, and quartzite nodules, and the difficulties in interpreting a multicategory cortex scheme become readily apparent. Certainly such data are more interpretable if the analyst knows something beforehand of the types of raw materials that comprise the assemblage. Within that framework, the collected data can be more easily placed within a technological perspective.

\section{Platform Type}

The striking platform types selected for use in the protocol are appropriate and should not pose a problem for most analysts to recognize and code. Other types or combinations can be easily added by the analyst if a special need arises.

\section{Thermal Alteration}

By recording this variable as indeterminate or present/absent, the analyst is allowed to avoid certain technological inferences such as trying to determine whether a piece is deliberately heat treated. This scheme should work efficiently for large masses of size-graded material. 


\section{DESCRIPTION OF MATERIAL CULTURE}

6

John E. Dockall and Karl W. Kibler

\section{CHIPPED STONE TOOLS, CORES, AND DEBITAGE}

The chipped stone assemblage recovered from the data recovery excavations at 41CV1378 consists of 1 arrow point preform, 27 dart points, 48 bifaces, 27 unifaces, 7 flake tools, 19 utilized flakes, 9 cores and core fragments, 4,466 pieces of unmodified debitage, and 413 pieces of microdebitage recovered through flotation.

\section{Arrow Point Preform}

The single arrow point preform was recovered from Unit D-4 (99.80-99.59) in Feature 1. It is a proximal fragment of a small oval pressure flake biface with a convex proximal or basal edge. The piece has been broken in a transverse fracture oriented obliquely across the middle portion of the blade. It is manufactured from an indeterminate white chert (Figure 6.1).

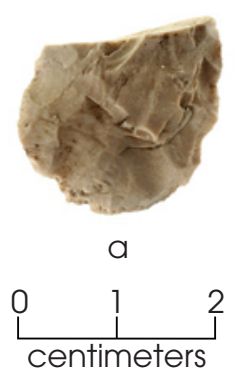

Figure 6.1. Arrow point preform.

\section{Dart Points}

Twenty-seven dart points were recovered from various excavation contexts at 41CV1378. The sample consists of 11 untyped and frag- mentary examples, 3 Castroville, 5 Marcos, 2 Montell, 4 Ensor, 1 Fairland, and 1 Darl dart point (Figure 6.2 and Table 6.1). These dart point styles all date to the Late Archaic period of central Texas and can be assigned to corresponding projectile point intervals (following Collins 1995: Table 2) or cultural phases (following Prewitt 1981:81-82; 1985).

\begin{tabular}{|c|c|c|}
\hline Time Span & $\begin{array}{l}\text { Collins's Projectile } \\
\underline{\text { Point Intervals }}\end{array}$ & $\begin{array}{l}\text { Prewitt's } \\
\text { Phases }\end{array}$ \\
\hline 200 B.C.-A.D. 150 & $\begin{array}{l}\text { Marcos, Montell, } \\
\text { Castroville }\end{array}$ & Uvalde \\
\hline A.D. $200-550$ & Ensor, Frio, Fairland & Twin Sisters \\
\hline D. $550-780$ & Darl & Driftwood \\
\hline
\end{tabular}

\section{Castroville}

Three Castroville points were identified from 41CV1378 (see Figure 6.2a-c). Raw materials for each of these points are East Range Flecked, Anderson Mountain Gray, and indeterminate heavily patinated chert. One completed point made of East Range Flecked chert is from Unit E-4 in Feature 1 at an elevation of $99.40 \mathrm{~m}$. It has been resharpened along each lateral blade edge, producing a mildly scalloped appearance to each edge. The stem is well made, with straight to slightly convex lateral edges and a convex basal edge. Shoulders are prominent to somewhat barbed. The haft element has been exposed to heat and has a reddish color on one side. Overall the piece is patinated. The two others are from beyond the edge of Feature 1 at 99.56 $\mathrm{m}$ and 99.49-99.36 $\mathrm{m}$, respectively. The specimen of Anderson Mountain Gray material is complete except for the tip, which was broken in a transverse bending fracture with a small 


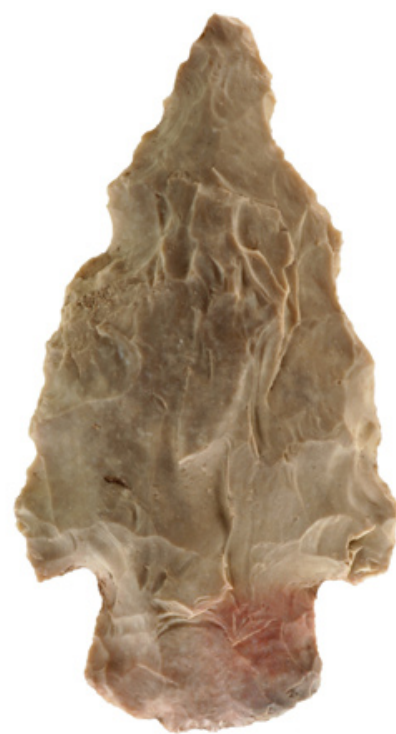

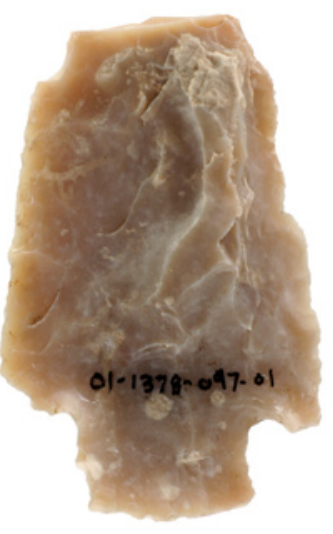

b

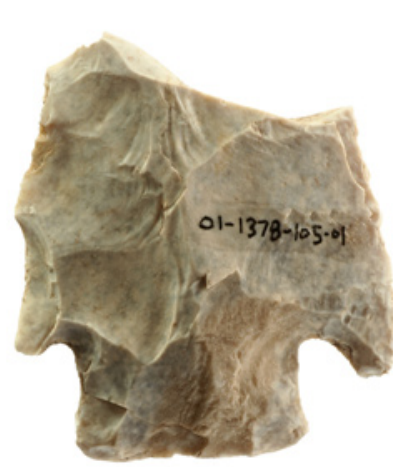

C

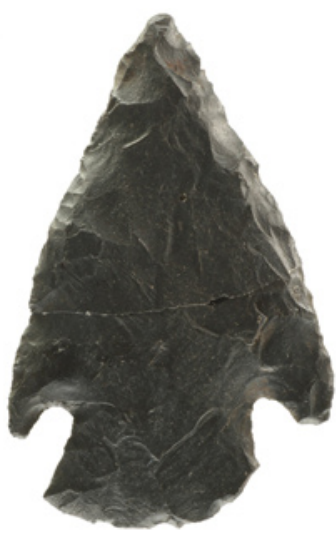

d a

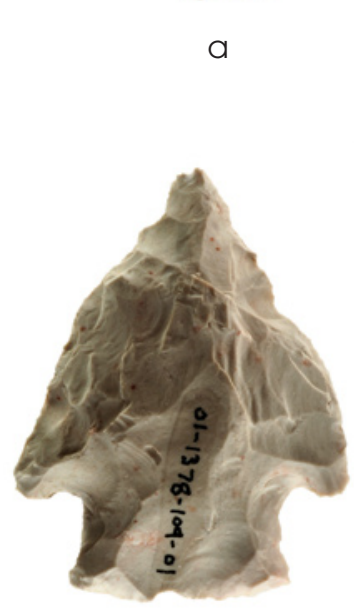

e

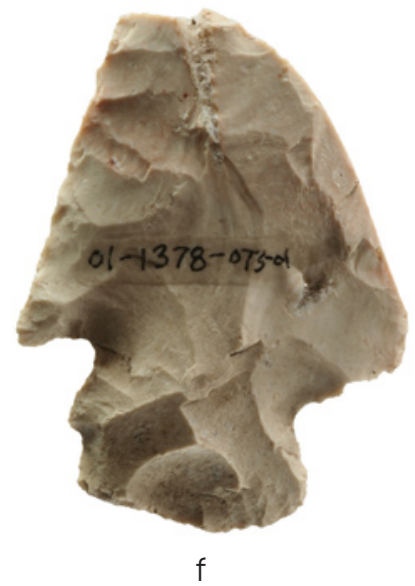

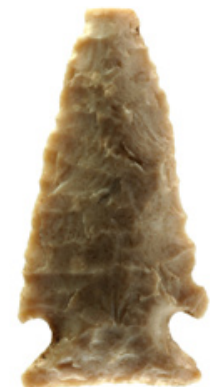

j

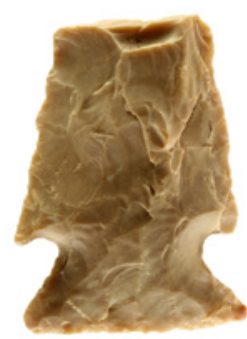

k

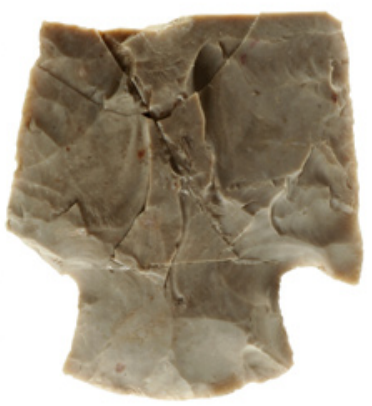

g

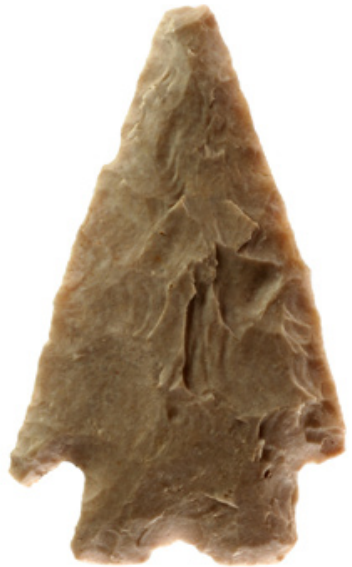

h

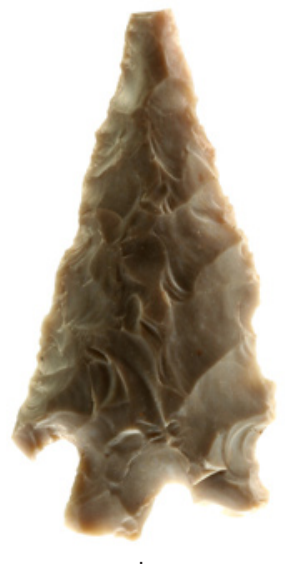

i

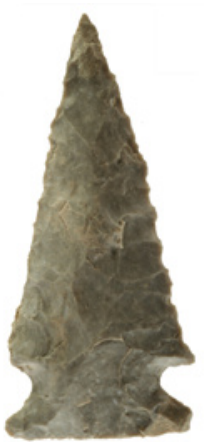

।

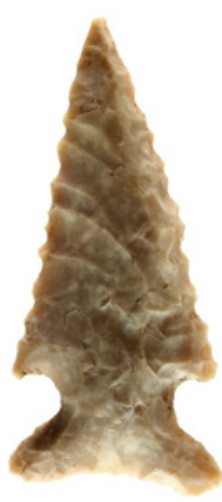

$\mathrm{m}$

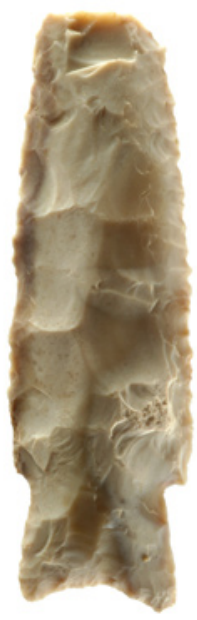

$\mathrm{n}$

Figure 6.2. Selected dart points. (a-c) Castroville; (d-g) Marcos; (h-i) Montell; (j-l) Ensor; (m) Fairland; and (n) Darl. 
Table 6.1. Dimensions and weights of identified projectile points

\begin{tabular}{l|l|c|c|c|c|c|c|c|c}
\hline \multicolumn{1}{c|}{ Type } & $\begin{array}{c}\text { Unit } \\
\text { No. }\end{array}$ & $\begin{array}{c}\text { Elevation } \\
(\mathrm{m})\end{array}$ & $\begin{array}{c}\text { Max } \\
\text { Length } \\
(\mathrm{mm})\end{array}$ & $\begin{array}{c}\text { Max } \\
\text { Width } \\
(\mathrm{mm})\end{array}$ & $\begin{array}{c}\text { Max } \\
\text { Thickness } \\
(\mathrm{mm})\end{array}$ & $\begin{array}{c}\text { Stem } \\
\text { Length } \\
(\mathrm{mm})\end{array}$ & $\begin{array}{c}\text { Stem } \\
\text { Width } \\
(\mathrm{mm})\end{array}$ & $\begin{array}{c}\text { Neck } \\
\text { Thickness } \\
(\mathrm{mm})\end{array}$ & $\begin{array}{c}\text { Weight } \\
(\mathrm{g})\end{array}$ \\
\hline Castroville & E-4 & 99.40 & 72.5 & 38.8 & 9.4 & 16.68 & 23.77 & 8.2 & 22.6 \\
\hline Castroville & B-11 & 99.56 & $*$ & 32.2 & 7.6 & 9.35 & 16.72 & 5.36 & 12.3 \\
\hline Castroville & E-11 & $99.49-99.36$ & $*$ & 38.6 & 8.1 & 11.46 & 23.71 & 7.36 & 13.1 \\
\hline Darl & B-9 & 99.43 & $*$ & 19.0 & 6.9 & 12.92 & 16.96 & 5.53 & 8.4 \\
\hline Ensor & B-9 & $99.85-99.41$ & $*$ & 23.3 & 6.5 & 10.03 & 22.82 & 5.43 & 5.4 \\
\hline Ensor & B-11 & 99.49 & 43.2 & 19.7 & 5.8 & 7.6 & 19.09 & 5.03 & 3.1 \\
\hline Ensor & E-11 & $99.49-99.36$ & 31.8 & 19.4 & 5.6 & 7.96 & 18.35 & 5.12 & 3.0 \\
\hline Ensor & B-9 & $99.85-99.41$ & 38.1 & 19.2 & 5.0 & 7.67 & 18.23 & 4.41 & 3.5 \\
\hline Fairland & G-6 & $99.64-99.47$ & 47.2 & 21.7 & 6.8 & 10.89 & 21.75 & 6.01 & 4.8 \\
\hline Marcos & F-9 & 99.45 & 43.4 & 33.5 & 7.6 & 11.17 & 22.21 & 5.03 & 8.4 \\
\hline Marcos & A-8 & $99.73-99.12$ & $*$ & 36.6 & 5.0 & 13.55 & 23.4 & 4.06 & 8.0 \\
\hline Marcos & A-13 & $99.48-99.28$ & $*$ & $*$ & $*$ & $*$ & 26.75 & $*$ & 2.9 \\
\hline Marcos & B-7 & 99.68 & 51.7 & 32.5 & 6.7 & 12.12 & 21.34 & 5.08 & 9.5 \\
\hline Marcos & B-5 & 99.74 & $*$ & $*$ & 1.3 & 17.51 & 23.28 & 7.8 & 14.7 \\
\hline Montell & C-7 & 99.57 & 53.7 & 26.7 & 5.9 & 11.13 & 20.81 & 7.8 & 6.4 \\
\hline Montell & A-9 & 99.24 & 56.5 & 34 & 5.0 & 11.03 & 22.14 & 4.53 & 9.2 \\
\hline
\end{tabular}

* Incomplete dimension

fracture remnant along one lateral edge. This point may have broken during impact. On one surface there was some difficulty in thinning and shaping the blade along the lateral edge. Lateral blade edges are straight, and the basal edge is slightly convex. Stem edges are expanding and straight. One barb is broken. The third Castroville point is also a proximo-medial fragment with the tip broken in a bending fracture related to projectile impact. One barb/shoulder portion is also broken and probably occurred during impact. Lateral edge remnants are slightly convex, and the basal edge is straight. Stem lateral edges are slightly convex and expanding. The specimen is heavily patinated.

\section{Marcos}

Marcos points represent the most abundant dart point type recovered at the site. Five excavation units yielded these points; three were from Feature 1, and two were from outside Feature 1 (see Figure 6.2d-g). Four were found between 99.74 and $99.45 \mathrm{~m}$. All of the Marcos are of chert: one Anderson Mountain Gray, one Owl Creek Black, two indeterminate light gray, and one indeterminate white. Two are complete and three are fragmentary. Specimens of Owl Creek Black and indeterminate white chert are complete and both have been reworked or re- sharpened at the tip and along the lateral blade edges. Stem basal edges are convex and stem lateral edges are straight, expanding with wellshaped corner notches. The blade edges of the white chert example are slightly recurved from resharpening, and those of the Owl Creek Black example are uniformly convex. The remaining fragmentary specimens are represented by two proximo-medial portions and a stem portion that compares favorably to a Marcos stem. One proximo-medial fragment of Anderson Mountain Gray chert exhibits a well-defined impact fracture that produced an oblique break across the mid portion of the blade. A broken barb may also be associated with the impact fracture. The second proximo-medial fragment was broken in an apparent snap or bending fracture but does not appear related to manufacture. Both have convex basal stem edges and straight to slightly convex expanding lateral stem edges. The single complete stem is similar in morphology and also has a transverse snap or bending fracture. Only the Anderson Mountain Gray specimen exhibits any sign of thermal alteration, with a slight pinkish or reddish hue.

\section{Montell}

Two examples of this point style were recovered (see Figure 6.2h-i). Both are complete. Raw 
materials are a nonlocal gray fine-grained chert (very similar to Georgetown chert) and an indeterminate fine-grained light gray chert. These points were recovered from 99.24 and $99.57 \mathrm{~m}$ respectively. The specimen of fine-grained gray chert has been considerably reworked along one lateral edge and a portion of the stem and may reflect repair of an impact-fractured point. Lateral blade edges on both specimens are straight, and blades are well shaped and thinned. The basal edges are characteristic and have the basal notch identifying them as Montell points.

\section{Ensor}

Raw materials used for Ensor points consist of East Range Flecked, Cowhouse Banded and Mottled, indeterminate light gray, and an unknown patinated chert. Three of the four specimens are complete but show evidence of distal reworking, and one has a transverse fracture across the middle of the blade (see Figure $6.2 \mathrm{j}-1)$. Two of these were recovered from Unit B9 between 99.85 and 99.41 and another was from $99.49 \mathrm{~m}$ in Unit B-11 in association with Feature 1. The East Range Flecked and indeterminate light gray specimens have very straight lateral blade edges and corner-notched expanding stems with straight to slightly convex basal edges. Both also exhibit well-controlled pressure flaking over the blade faces but do not appear beveled. The third complete example of patinated chert was reworked along both edges of the blade and has a remnant impact fracture scar still visible on one blade face. One barb or shoulder is also missing. Stem morphology is identical to those described above, although the blade is slightly asymmetrical. The final broken specimen of Cowhouse Banded and Mottled chert is broken transversely across the midsection of the blade in a bending/impact fracture combination. Previous to this breakage, the blade edges had been reworked and are alternately beveled in appearance.

\section{Fairland}

A single Fairland dart point of East Range Flecked chert was found in Unit G-6 between 99.64 and $99.47 \mathrm{~m}$ (see Figure 6.2m). This specimen is only lightly patinated on both surfaces and is complete. Lateral blade edges are well made, straight, and finished with fine pressure flaking and have a slightly serrated appearance. The basal edge is concave, and the stem expands to just beyond the shoulder barbs. Stem lateral edges are convex, and basal ears flare away from the stem center line.

\section{Darl}

The single Darl point was found in Unit B-9 as part of the Feature 1 fill at a depth of $99.43 \mathrm{~m}$ (see Figure 6.2n). Raw material is chert but does not appear to be local Fort Hood chert. The chert is brownish purple and white and is slightly translucent at the very edges of the point. The tip is broken in a transverse bending fracture due to projectile impact. On one blade face the distal portion of the fracture ended in a hinge or step termination. Flaking on the blade is well executed with controlled pressure flaking along each lateral edge, giving each edge a serrated appearance. Lateral blade edges are straight to slightly convex. The stem edges are convex, slightly expanding, and flaring. The basal edge is concave. Some white patina is present on both point surfaces.

\section{Untyped Dart Points and Untypeable Fragments}

A single complete dart point and 10 dart point fragments could not be assigned to any particular established type (Figure 6.3). Fragments consist of 1 stem portion, 1 medial, 1 proximo-medial, 1 barb/shoulder, and 6 distal fragments. The complete point is from Unit G6 at an elevation of $99.54 \mathrm{~m}$ (see Figure 6.3a). The raw material is Anderson Mountain Gray. Lateral blade edges are recurved and resharpened, and one edge is quite blunted from step fractures. The stem edges are parallel, and the basal edge is also straight. Barbs are absent, although the point is shouldered. Morphologically, this point resembles Bulverde, Morrill, or other parallel-stemmed shouldered points but could not be definitively classified as any of these.

Raw materials represented among dart point fragments include five indeterminate light gray cherts, one indeterminate dark gray, one indeterminate white, one Cowhouse White chert, and two unknown cherts. Of the six distal fragments, four exhibit breakage patterns associated with impact fractures and two have transverse snap or end 


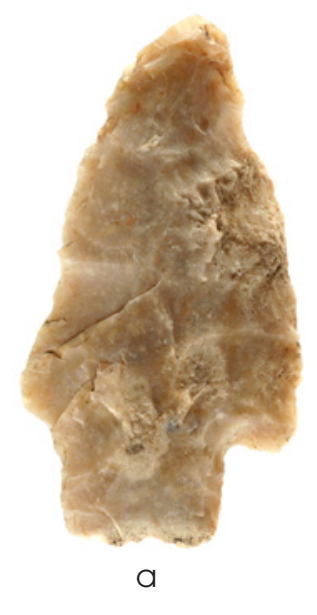

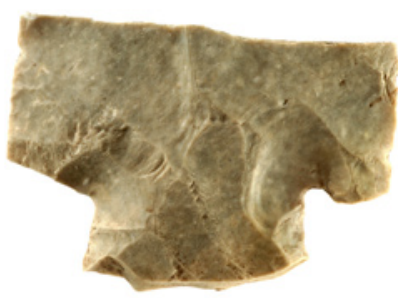

b

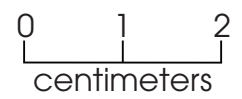

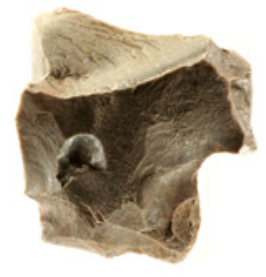

C

Figure 6.3. Selected untyped dart points and untypeable fragments.

shock breaks, perhaps related to breakage during manufacture or repair. The stem portion and the barb/shoulder segment were broken in end shock or a transverse snap, the proximo-medial portion was broken in a snap break, and the medial fragment had been exposed to excessive heating and thermal damage. The stem portion was possibly a portion of a corner-notched point style with an expanding stem.

As a group, the sample of complete and fragmentary dart points and dart point fragments exhibit a fairly consistent array of failure or discard types, suggesting that most points at the site were arriving in a well-used or broken state. The following failure or discard types are represented: nine impact/bending fractures, four exhausted or worn out, six transverse snap or end shock, one snap break, one excessive heating, and six indeterminate.

\section{Bifaces}

Forty-eight artifacts are classified as complete or fragments of non-projectile point bifaces. The unfinished bifaces $(n=36)$, in terms of manufacture or reduction stages, consist of 6 Stage 1 bifaces, 15 Stage 2 bifaces, 7 Stage 3, and 8 Stage 4 bifaces. Bifaces and fragments from various stages of manufacture are depicted in Figure 6.4. Representative complete specimens and fragments that exhibit the typical suite of technological traits associated with each stage of manufacture are included to indicate the range of biface technology represented at 41CV1378.
The six Stage 1 bifaces consist of one proximal piece, two distal fragments, two pieces that may be proximal or distal, and one indeterminate fragment (see Figure 6.4a-b). Most breakage and discard was due to apparent manufacture-related failures: four exhibit snap or end shock breaks, one has a perverse fracture across the blade portion, and one is an indeterminate fracture. Two fragments exhibit thermal damage, and one has postdepositional patina. Remnant ventral surface flake features are visible on four fragments, indicating that some of the bifaces being manufactured at 41CV1378 were made on flake blanks.

The biface assemblage includes 15 Stage 2 bifaces and fragments (see Figure 6.4c-e). Only one specimen is complete. Represented fragments are 2 proximal, 1 proximal-medial, 3 medial, 2 distal, 2 indeterminate end portions, 3 indeterminate fragments, and 1 fragment with missing lateral edges. Manufacturing errors are the most common cause of breakage and discard. Seven snap or end shock, 2 perverse fractures, 2 overshot (outrepasse), 1 material flaw, 1 biface edge collapse, and 2 thermal fractures account for the breakage patterns. There are 3 fragments that exhibit a white patina on one or more faces and 7 that show secondary thermal alteration of some type, including the two fragments with thermal fractures. The one complete specimen was recovered from Unit B-11 at $99.42 \mathrm{~m}$ (see Figure 6.4c). The lateral edges and base are convex for the ovate biface. This biface has coarse percussion flaking over both faces and 

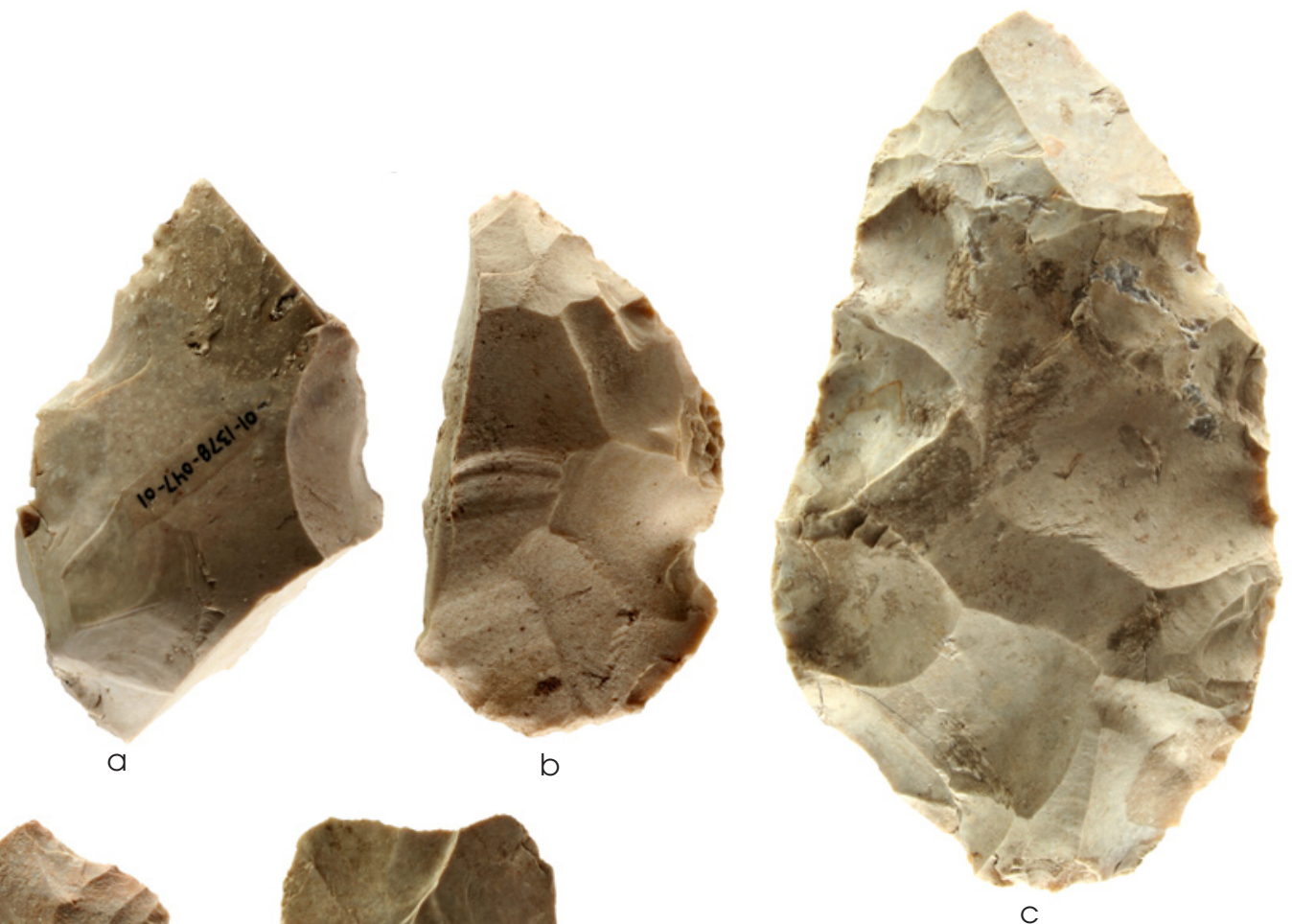

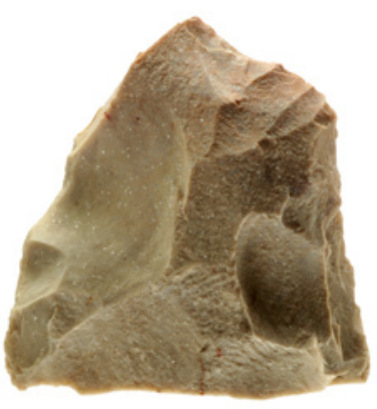

d
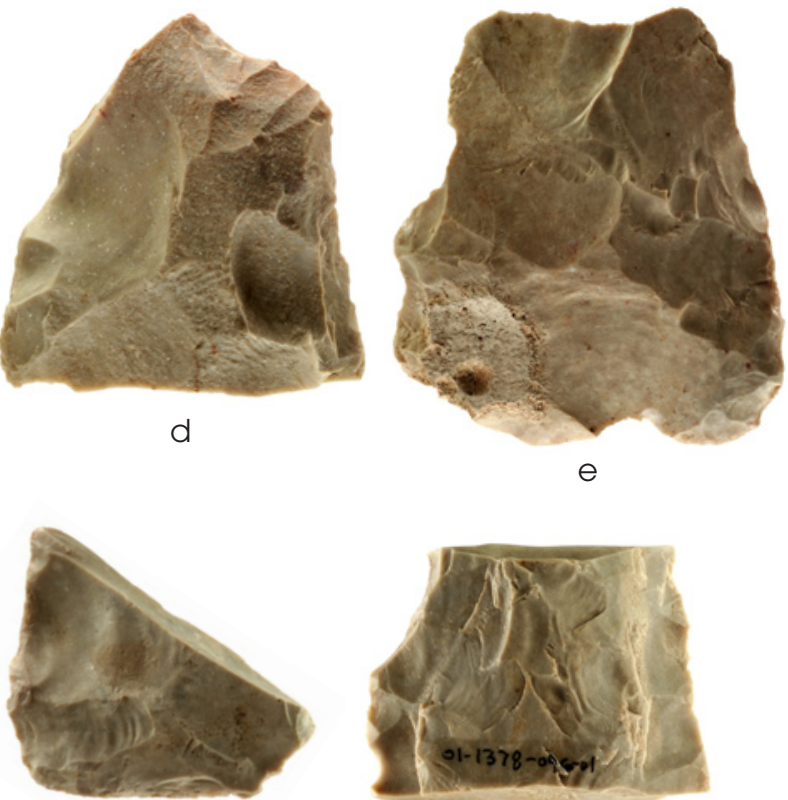

h
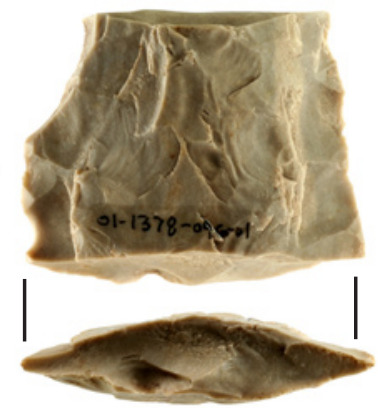

i

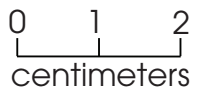

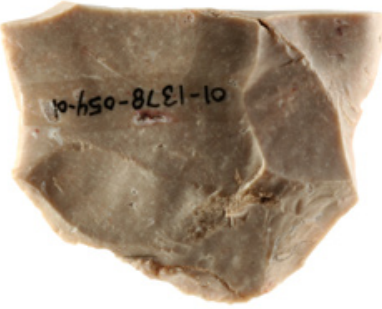

f

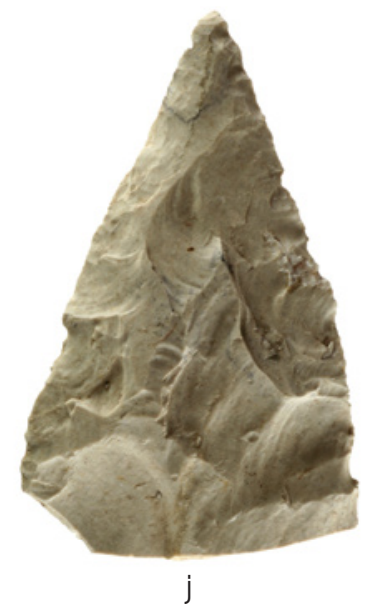

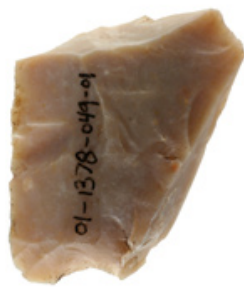

g

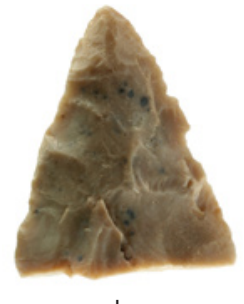

k

Figure 6.4. Selected Stage 1-4 bifaces and biface fragments. (a-b) Stage 1; (c-e) Stage 2; (f-i) Stage 3; (j-k) Stage 4. 
has no visible remnants of a ventral flake surface to indicate initial blank type; in fact, all of the fragments have been reduced and refined to the point where they lack any indication of the initial flake blank. Proximal and proximomedial fragment shapes indicate an ovate to subtriangular biface or preform outline, and other fragments indicate that lateral blade edges were typically convex. Surfaces of complete and fragmentary Stage 2 bifaces exhibit a combination of apparent hard-hammer and soft-hammer percussion for thinning and shaping.

Seven fragments are attributed to Stage 3 manufacture (see Figure 6.4f-i). Stage 3 fragments consist of one stem, two proximal, one medial, one distal, one distal-medial, and one undetermined end fragment. The majority of these were broken due to manufacture-related knapping problems. Four were broken in snap or end shock, one in a perverse fracture, and one due to excessive thermal damage. Two fragments exhibit evidence of probable recycling in the form of deliberate transverse snap breaks. One of these is a stem or basal fragment, and the other is a medial fragment. The stem fragment and the medial fragment have transverse breaks with indications of bipolar percussion used to snap the biface (see Figure 6.4i), indicating that some biface fragments were exploited for tool material. The medial fragment also exhibits an end shock or snap failure. Proximal and other Stage 3 fragments indicate that biface shapes were ovate, triangular, or subtriangular. Fragments exhibit a combination of hard- and soft-hammer percussion over the faces.

Eight fragments are attributed to Stage 4 biface manufacture: one proximal, two medial, two distal, one medial-distal, one undetermined end fragment, and one indeterminate fragment (see Figure 6.4j-k). The proximal fragment is a small basal portion of a pressure-flaked biface that may have been heat treated. It was recovered in Unit F-4 between 99.71 and $99.42 \mathrm{~m}$. Of these artifacts, six are associated with snap or end shock failure, and two are broken due to excessive thermal damage. Three exhibit thermal alteration, and one has white surface patina. It is difficult to gain an impression of Stage 4 biface shapes, but lateral edge remnants on most fragments appear convex or slightly convex, suggesting oval or ovate preform outlines similar to those observed among earlier stage bifaces and fragments. The presence of resharpening and use wear suggests a cutting function for these artifacts. All specimens but one distal fragment exhibit bifacial microscars with hinge and step terminations distributed along the lateral edges or edge remnants. Such wear traces are often indicative and characteristic of tool use in a cutting motion. In addition, two of the distal fragments have light polish along the lateral edges.

An absence of cortex on all of the bifaces suggests that at least a majority arrived onsite in a virtually cortex-free state. This indicates that the procurement and initial trimming of biface blanks may have occurred elsewhere, either nearby or close to the geological source of the raw material.

Ten of the bifacial artifacts were identified as finished or functional tools or fragments of functional tools and consist of one adze fragment, six bifacial knives or knife fragments, one thick battered biface, and two biface fragments with burin retouch (Figure 6.5). The single example of an adze is a bit or cutting edge fragment recovered from Unit F-4 from 99.76-99.52 m (see Figure 6.5a). The raw material is an indeterminate dark gray chert, and breakage appears to have been during use and is classified as a transverse bending fracture. The bit edge has significant use-wear damage consisting of crushing and macroflake scars with hinge and step terminations. The edge is unifacially beveled by direct percussion.

Bifacial knives are represented by two complete specimens and four distal fragments. Complete knives were recovered in Units B-9 (99.85-99.41 m) and B-11 (99.75-99.42 m) and are ovate in shape but considerably resharpened and reworked along both lateral edges (see Figure 6.5b-c). Both exhibit thermal alteration and may have been heat treated prior to manufacture. No hafting wear was observed on either of these bifaces. These bifaces were discarded because they had been retouched to the end of their use life, whereas the knife fragments were discarded due to snap or end shock breakage. Breakage may have occurred during use or during maintenance and resharpening. Two of the distal fragments were from knives that had been alternately beveled during resharpening and have distinctive diamond shaped or parallelogram cross sections (see Figure 6.5d).

A single example of a thick battered biface was recovered from Unit B-9 in Feature 1 at 99.70-99.34 m (see Figure 6.5e). This artifact 


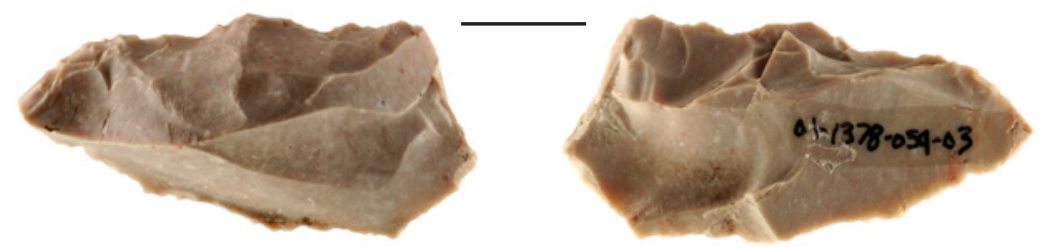

a

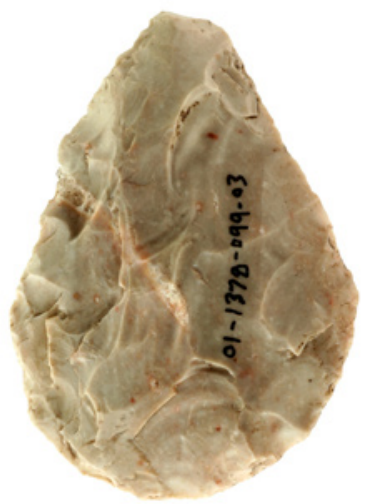

b

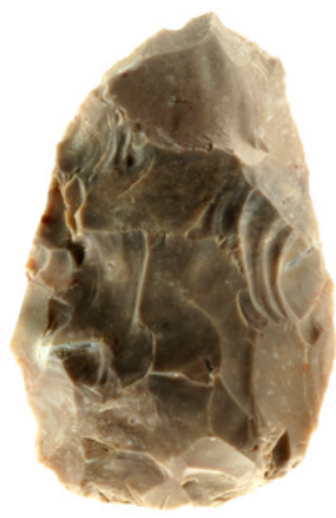

C
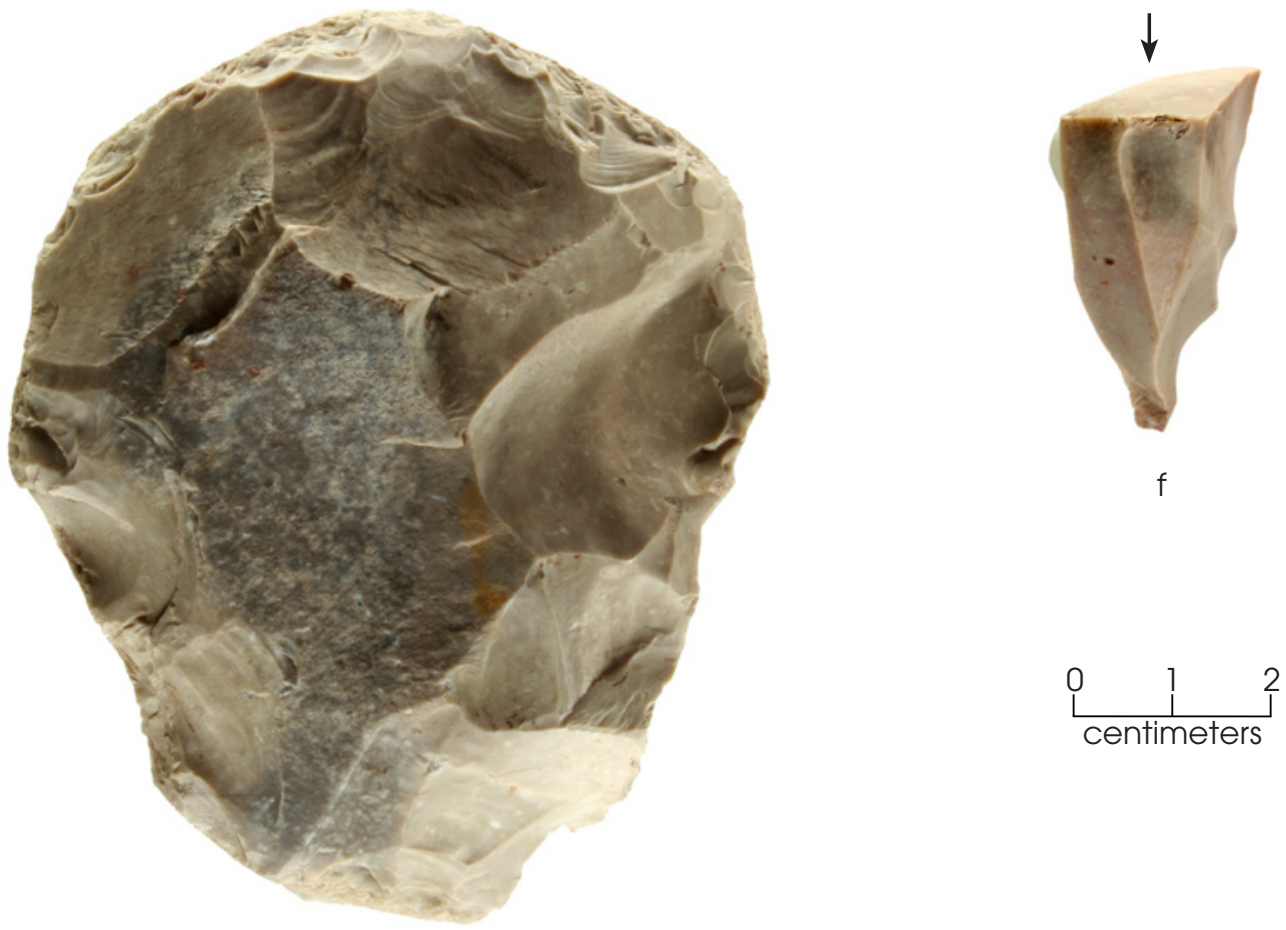

e

Figure 6.5. Selected bifacial tools. a) Adze; (b-d) knives; (e) thick battered biface; and (f) biface fragment with burin spall.

may have initially served as a large percussion core but much of the lateral edge circumference has been bifacially flaked by invasive percussion retouch and exhibits considerable wear along all of the retouched edge. Visible use wear is characterized by considerable edge crushing and overlapping areas of step and hinge terminated flake scars from contact with a hard material. 
The wear is not characteristic of hammerstones that have been used in stone tool manufacture or core reduction since it is confined to what appears to have been a functional cutting edge. Dense but yielding materials such as wood or bone may have been the worked material, but not stone.

Two biface fragments were observed to have burin spall or burin retouch scars along one or more lateral edges. They represent the reuse or recycling of biface fragments. One example is a distal fragment from Unit F-2 between 99.73 and $99.40 \mathrm{~m}$ (see Figure 6.5f), and the second is an indeterminate fragment from Unit B-9 at 99.85$99.41 \mathrm{~m}$. The distal fragment from Unit F-2 has a transverse snap or end shock break that served as a striking platform for removal of one burin spall flake along one lateral edge. Technically, this artifact is sometimes referred to as a burin on a break. The biface fragment from Unit B-9 has two burin spall scars along one lateral edge that were removed from a percussion retouched striking platform, often referred to as a burin on a truncation (Barton et al. 1996; Dial and Collins 1998:576-586; Knecht 1988; Tomaskova 2005). Dial and Collins (1998:576-586) identified several burin groups based on technology and platform types at the Wilson-Leonard site in central Texas. Two of their groups, Group 3 and Group 5, correspond to the burin on a break and burin on a truncation forms discussed above. An absence of observed microscopic use wear along either of the specimens from 41CV1378 makes it difficult to determine if these were produced as tools or if the burin spall was the desired product. In keeping with other burin retouched pieces from this site discussed further below, it is most probable that they were burin retouched for use as tools.

Two biface fragments were identified that could not be assigned to a particular manufacture stage or other biface type. Both specimens were recovered from Unit G-6 between 99.64 and $99.33 \mathrm{~m}$ but were not associated with any feature. Each retains a portion of a lateral edge and one may be a haft element fragment based on the presence of edge rounding or smoothing.

The raw materials represented among the biface group consist of Anderson Mountain Gray $(\mathrm{n}=7)$, Fort Hood Gray $(\mathrm{n}=4)$, Cowhouse Shell Hash ( $\mathrm{n}=1)$, Cowhouse Novaculite $(\mathrm{n}=1)$, East Range Flecked $(\mathrm{n}=1)$, Owl Creek Black $(\mathrm{n}=1)$, indeterminate light gray $(\mathrm{n}=21)$, indeterminate white chert $(n=2)$, indeterminate dark gray $(n=1)$, and 9 cherts that could not be identified as materials from Fort Hood.

The discard of non-projectile point bifaces appears largely to have been related to onsite manufacture of bifaces and the repair of finished bifacial knives with minor representation of discard of use-broken and recycled pieces. Manufacture-related discard or breakage accounts for 77.1 percent $(n=37)$ of all complete and fragmentary non-projectile bifaces. Discard of use-broken or worn-out complete bifaces represents 10.4 percent $(n=5)$.

\section{Unifaces}

Included in this group of 27 unifaces are several different tool types, including knives, drills, denticulates, and various scrapers. Each of these tool types is discussed separately below.

Four unifacial tools were identified as knives based on tool morphology and the type and location of microscopic use wear (Figure 6.6). Two were recovered from Unit C-7 in Feature 1 between 99.87 and $99.50 \mathrm{~m}$ and Unit F-4 at 99.54-99.32 m (see Figure 6.6a-b). Both of these implements were manufactured on large ovate percussion flakes and retain cortex on the dorsal surface or platform area. The cortex is weathered and indicates that the flakes were removed from large chert cobbles. Marginal soft-hammer percussion has produced broad convex edges along much of the edge circumference of these tools. The bulbs of percussion have also been thinned and removed by percussion retouch, which has created a partial bifacial edge in the area of the striking platform. This retouch may have been to facilitate hafting or to increase efficiency as an unhafted handheld tool. Flake scars and ventral/dorsal surfaces of both tools exhibit a white patina, but the specimen from Unit C-7 displays later secondary soft-hammer retouch on the ventral surface that has removed the earlier patination and created a partial bifacial edge along a portion of the tool margin. The morphology and use wear on these tools compares well to similar implements identified and described by Shafer and Holloway $(1977,1979)$ from sites in the Lower Pecos and Trans-Pecos areas of Texas that were used as knives to cut the leaves of succulent plants like sotol and agave. It is possible that these implements were produced from specially prepared or removed tool blanks. 
Data Recovery Investigations at the Tank Destroyer Site, Fort Hood

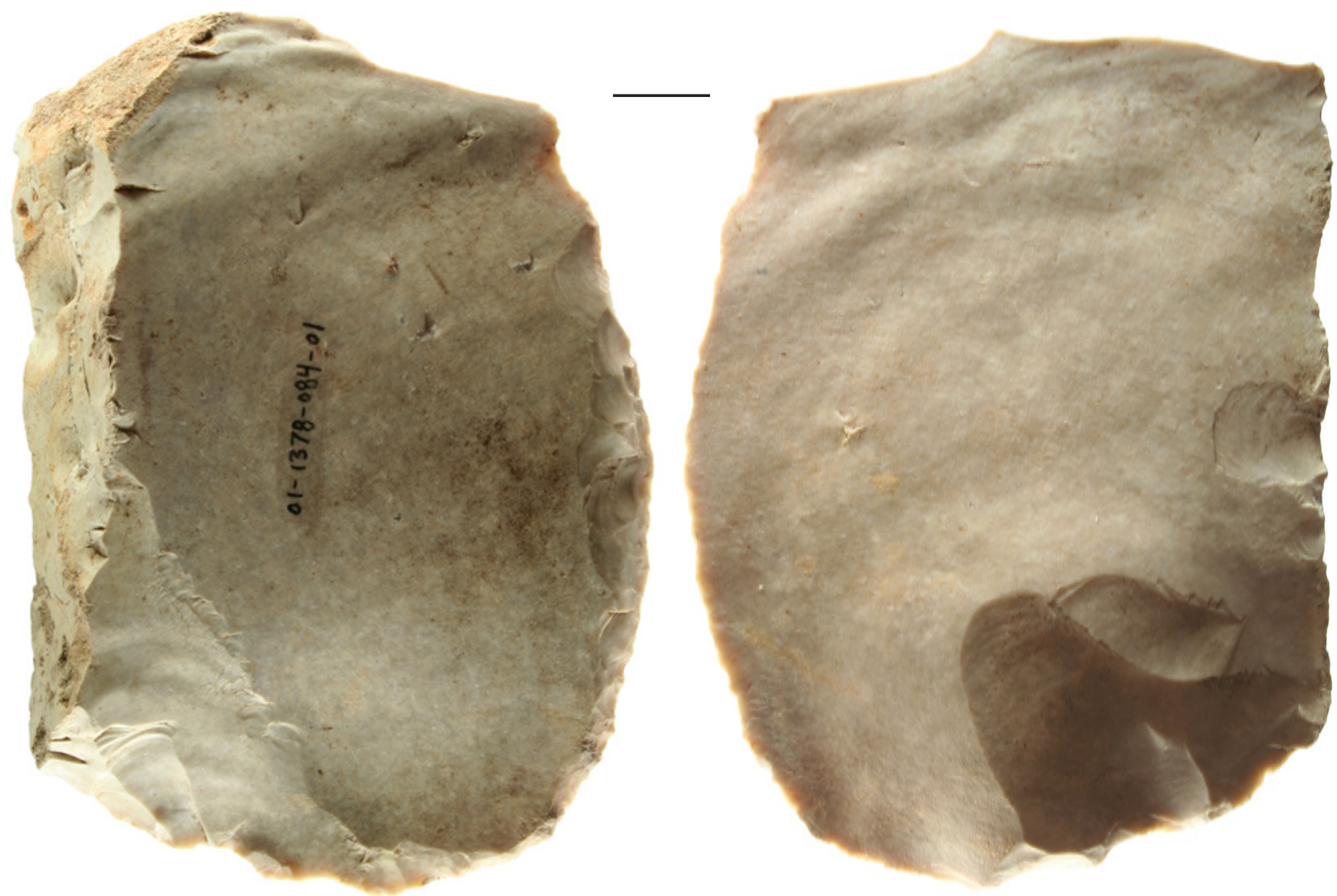

a

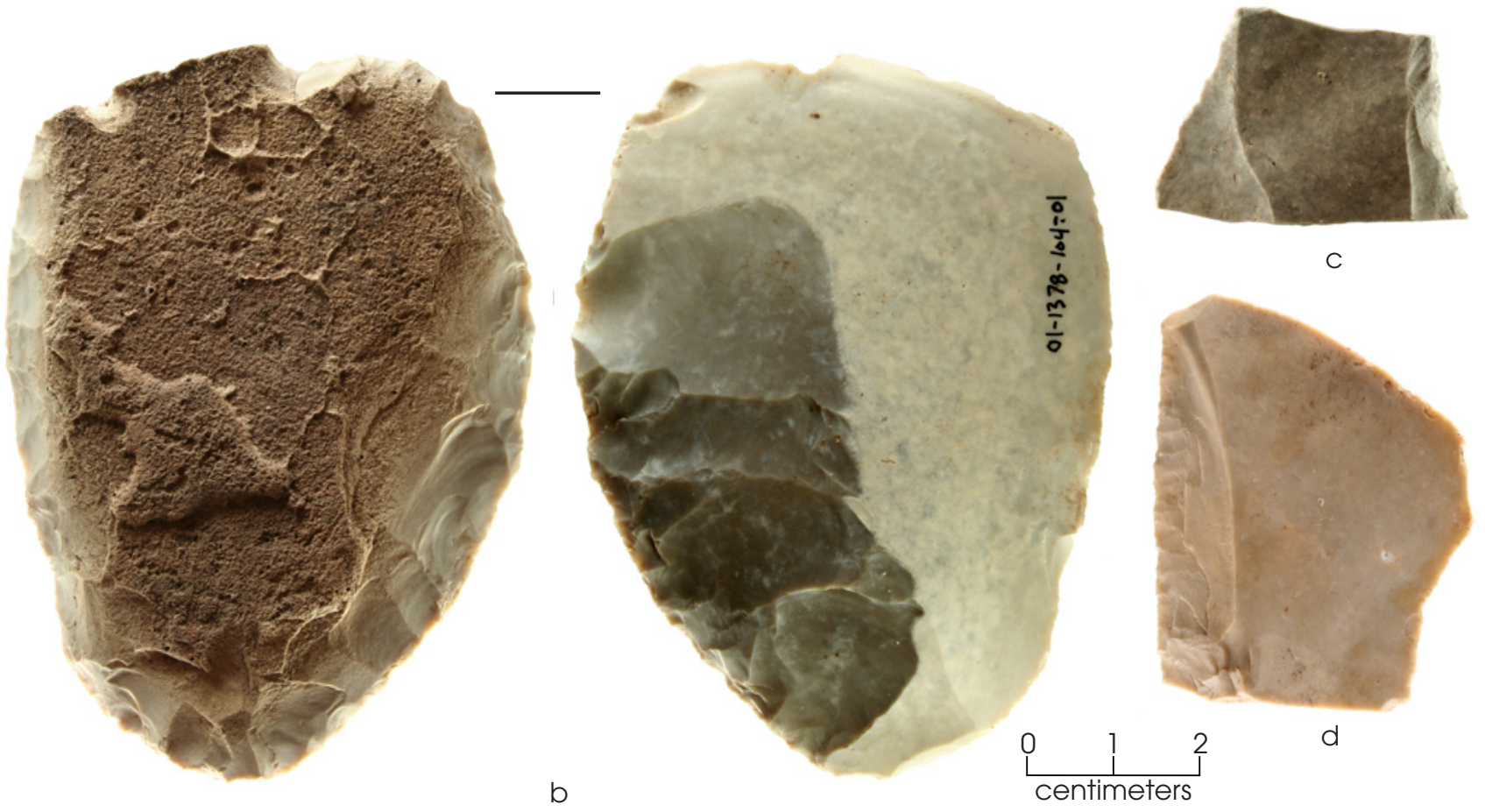

Figure 6.6. Unifacial knives. 
The other unifacial knives are a medial fragment and a distal fragment of thin percussion flakes (see Figure 6.6c-d). The medial fragment has fine unifacial edge retouch along one edge, perhaps to regularize the edge for use. The distal fragment displays very uniform unifacial soft-hammer retouch with superimposed pressure retouch. None of these unifacial knives specimens show evidence burning or other thermal alteration.

A single drill, made on a proximal flake fragment with a transverse bending fracture, was recovered from Unit F-2 at 99.73-99.46 m (Figure 6.7a). Unifacial retouch along one remnant lateral edge has created a projecting tip element at the intersection of the transverse fracture and the retouched edge. The specimen shows no evidence of burning or thermal alteration, but a white patina is present.

Six unifacial implements are identified as denticulates (see Figure 6.7b-c). These tools exhibit one or more peripheral areas of retouch, creating a coarsely serrated or multiple-notched effect on the tool edge. Retouch varies from rather fine pressure flaking to coarser percussion flake removal along the tool periphery. Denticulate implements have been identified in assemblages from central Texas, particularly at the Wilson-Leonard site (Prilliman and Bousman 1998:611-612) and elsewhere in west central Texas (Black et al. 1997:467-475) in association with burned rock middens and mounds. The denticulates from 41CV1378 are made on percussion flakes, although one is quite blade-like in form. Despite such edge modifications, microscopic use wear was only observed on three specimens and consists of micro or fine unifacial flake scars with hinge and step terminations. The morphology and technology of these tools suggests functions comparable to similar tools from other central Texas sites that exhibit wear attributable to various cutting, scraping, shaving, and planing tasks that may be primarily indicative of woodworking (Black et al. 1997:467). Two exhibit a white patina, and one displays thermal alteration from burning. Two have traces of weathered surface cobble cortex. One tool has a transverse snap or radial fracture produced by deliberate bipolar percussion. No particular cause for discard is apparent for any of the specimens other than they probably were function or task specific and served out their use life.

A single notched flake was found in Unit E-11 but is not associated with any feature (see Figure 6.7d). The tool is manufactured from a thick percussion flake of Anderson Mountain Gray chert and has had one or two smaller percussion flakes removed along one lateral edge to produce a single large concavity or notch. Although no use wear was observed on this specimen, a similar function to denticulates described above is inferred based on morphological and technological similarities.
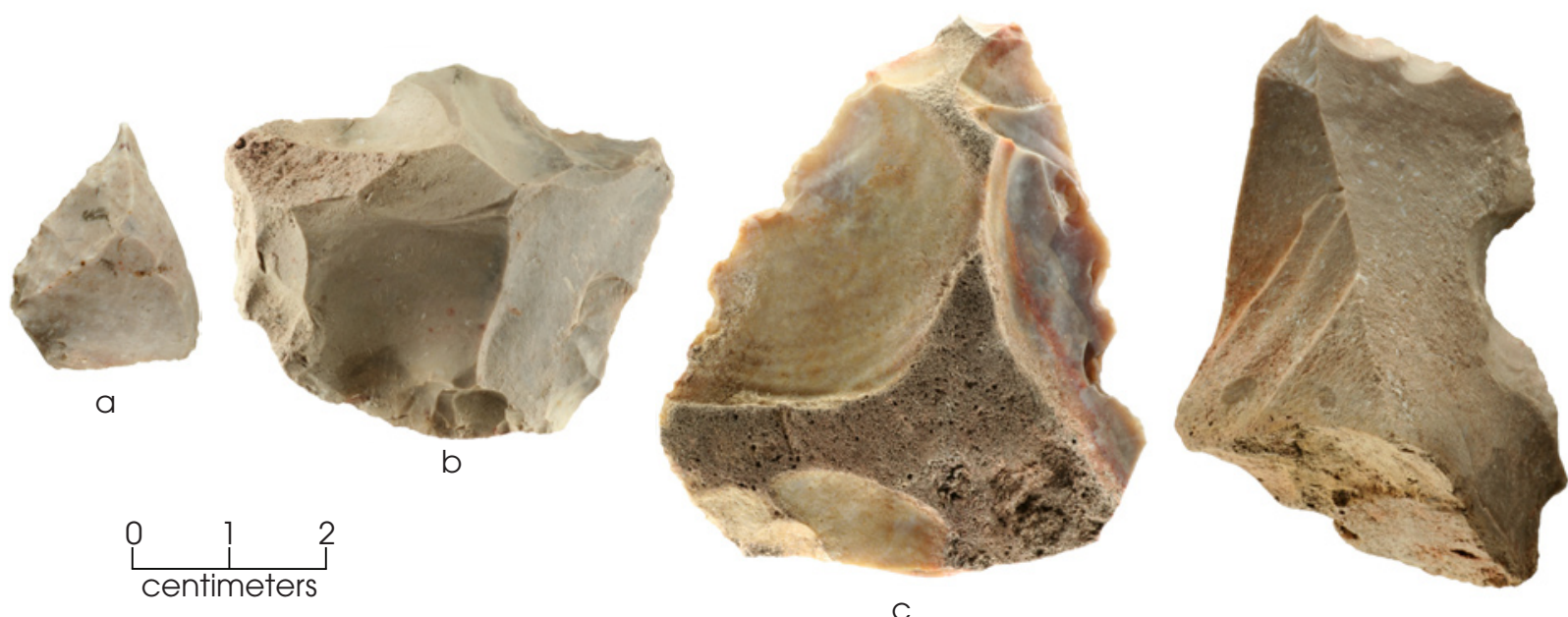

d

Figure 6.7. Unifacial tools. (a) Drill; (b-c) select denticulates; (d) notched flake. 
The remaining 15 unifacial implements in the assemblage consist of 10 scrapers and 5 indeterminate uniface fragments (Figure 6.8). Although this group of tools shows considerable variation in retouch location and intensity, they are discussed as a group because they represent a nice cross section of variability, from minimally retouched pieces to some that appear to have been resharpened multiple times. These implements display deliberate snap or radial breaks associated with their manufacture or discard. Eight of the tools have one or more fractures associated with the use of bipolar percussion or a transverse/radial snapping technique to either produce a suitable fragment that can be retouched or to recycle worn-out unifacial implements. Transverse snap breaks are present on five specimens, and radial or radiating breaks are apparent on three others. Fracture attributes include opposed rebound stress indicators from bipolar percussion, bulb(s) of percussion on some specimens, ring cracks on the central portions of dorsal and ventral surfaces, opposed cones of percussion, and fracture faces radiating from a central point of impact.

The scrapers consist of five side scrapers, two end scrapers, two end/side scrapers, and one side scraper/graver. The five indeterminate unifaces are identified as generalized modified flakes of an unknown function (no use wear was observed on these specimens). Retouch types along lateral or distal edges consist of marginal percussion $(n=9)$, fine edge flaking $(n=1)$, and steep abrupt retouch $(\mathrm{n}=4)$. Microscopic use wear, observed on five of the tools or tool fragments, is consistent with a scraping motion, but the scraped materials could not be distinguished. Wear consists of unifacial micro flake scars with hinge and step terminations, but polish was only observed on two of these implements. The scarcity of identifiable wear and the very light nature of the wear that was identified indicates that the majority of these tools had either been resharpened prior to discard and recycling or the last episode of tool use had been very light. It may also suggest that tasks involving the use of retouched hafted and unhafted unifacial tools were not common.

\section{Flake Tools}

Flake tools with burin spall facets account for seven specimens (Figure 6.9). The burin spall technique was employed as a form of retouch to produce a specific type of functional edge with certain technological characteristics. Based on the location of this type of retouch and the location of use wear, it is apparent that the desired end product of this type of modification was not the production of burin spall flakes but the edge created by their removal. A similar or identical technique of flake removal was used to resharpen other artifacts in the assemblage based on the presence of these types of flakes or spalls. The small sample from $41 \mathrm{CV} 1378$ represents a rather opportunistic use of flake blanks to create functional edges. Five of these tools are considered to be multifunctional, exhibiting wear diagnostic of cutting, scraping, and/or graving tasks. Two specimens are considered dihedral burins, three are burins on breaks, one is a burin on retouched truncation, and one has had spalls removed from the flat striking platform of a percussion flake blank. Each dihedral burin is formed by the intersection of two burin facets. One of these is made on a percussion flake fragment or shatter fragment, and another may be a biface fragment. Burins on breaks are fairly explanatory, with at least one dihedral tip being created by the intersection of a transverse break and one burin spall facet. In the case of those in this assemblage, one is manufactured on a percussion flake broken in a bending fracture and two have had spalls removed from deliberate radial break or snap break fractures. The single example of a burin on a truncation has a single spall facet removed from a small concave retouched platform on the distal end of a flake. Lateral edges of this specimen have use wear indicative of cutting tasks in addition to the small burin spall at the distal end. The last burin has had two, possibly three, small burin spall removals along one edge, with the striking platform of the flake serving as the platform for burin spall removal. These burins are comparable in morphology and technology to the much larger assemblage analyzed by Dial and Collins (1998:576-593) from the Wilson-Leonard site. Most of the burin retouched artifacts from 41CV1378 appear to have been manufactured, used, and discarded soon after task completion. Only one appears to have been discarded because it was exhausted. Microscopic use wear was observed on six specimens. Of these, three exhibited wear traces along the burin spall facet edge(s) and dihedral tip diagnostic with scraping 

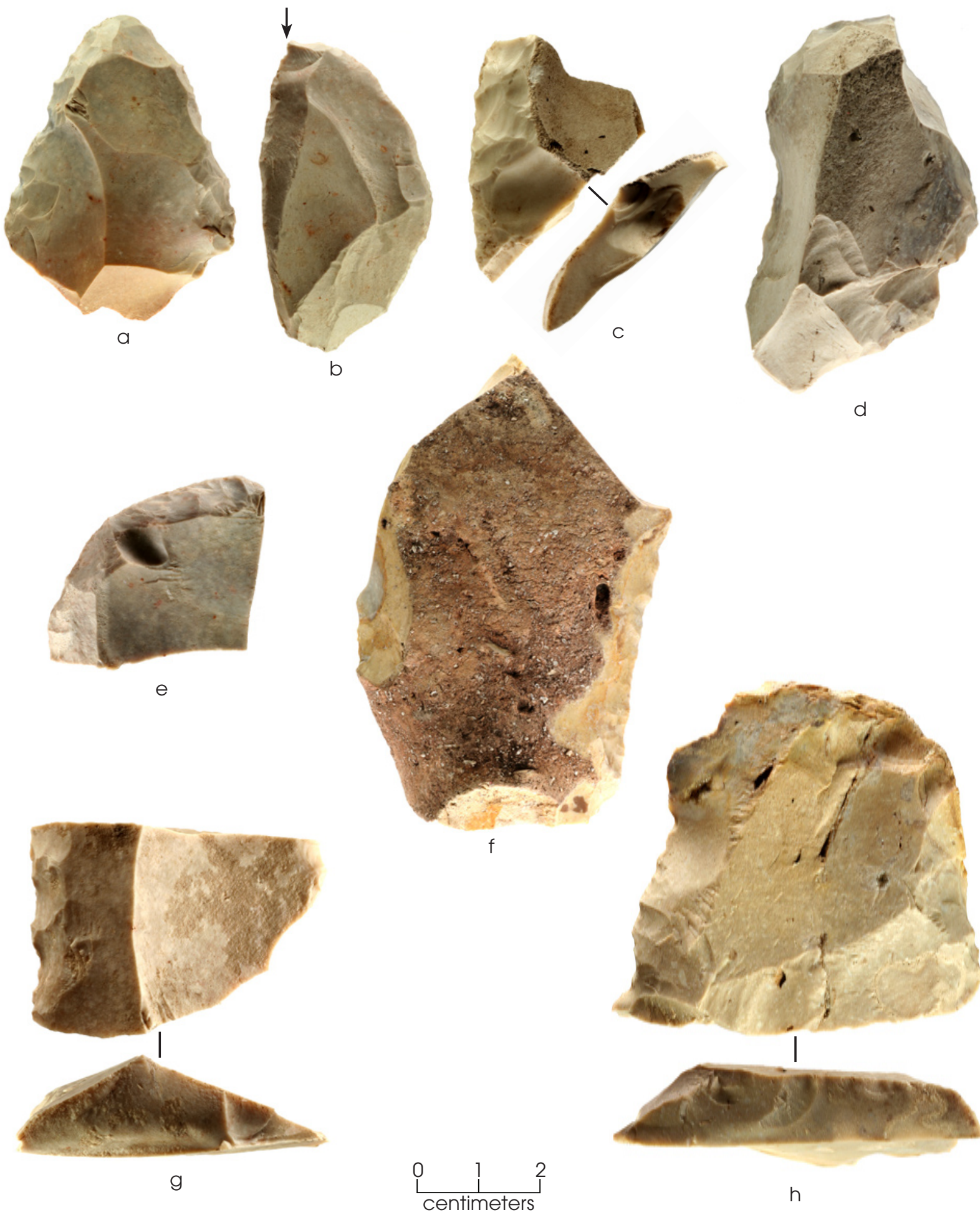

d
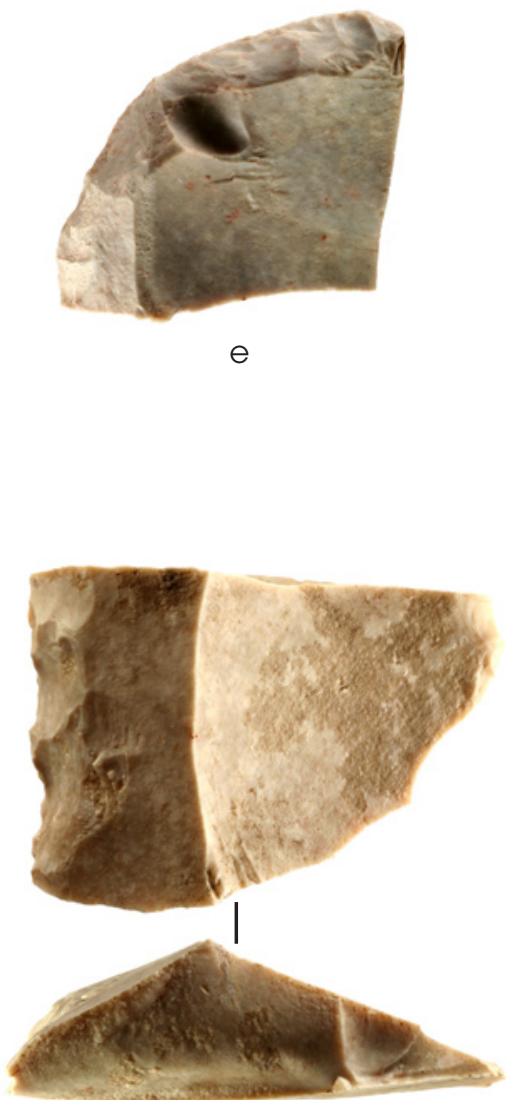

9

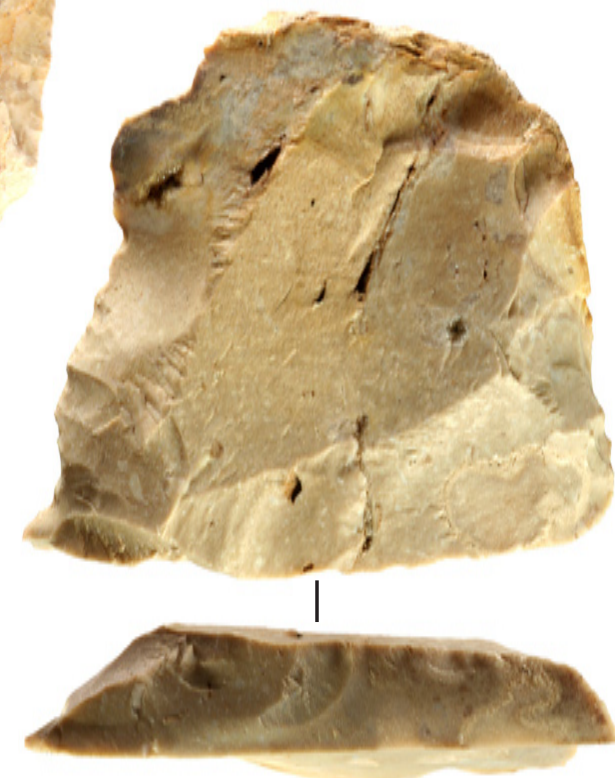

h

Figure 6.8. Selected unifacial tools. (a-d) side scrapers, (e) end scraper, (f) end/side scraper, and (g-h) indeterminate uniface fragments. 
and graving tasks, one had graving wear at the dihedral tip, and one had cutting wear along a lateral edge. Another tool had pronounced scraping wear along each lateral flake edge but no observed wear along the burin spall facet. A white patina is present on two specimens. None exhibit thermal damage of any type.

\section{Utilized Flakes}

The final category of tools to be discussed are those that essentially are unretouched flakes or fragments of chipping debris that were selected for use as tools for a variety of tasks. Visible edge modification is attributed entirely to the process of tool use and does not appear to represent any type of deliberate flaking, retouch, or post-use cultural modification. Nineteen tools fall within this category. Use wear on each of these tools was identified microscopically be- tween 10 and 50x. Functional interpretations of wear only address the basic tool motion as revealed through microflake scar distribution, orientation, and the presence/absence of edge crushing, smoothing, and polish. No attempts were made to interpret the possible worked materials.

The blanks selected for tool use consist of 3 blades or blade flakes and 16 flakes. Of these, 7 are complete, 2 proximal, 3 proximal-medial, 3 medial, 1 distal-medial, 2 distal, and 1 is an indeterminate fragment. Evidence of deliberate bipolar segmenting is apparent on six flakes and consists of 4 with snap breaks, 1 with an end shock/ snap break, and 1 with a radial/snap break. This indicates that such tools were subjected to similar recycling or reuse as other tools in this assemblage. It also indicates that blades or similar tool blanks were not a major part of the tool kit employed at the site—at least not as
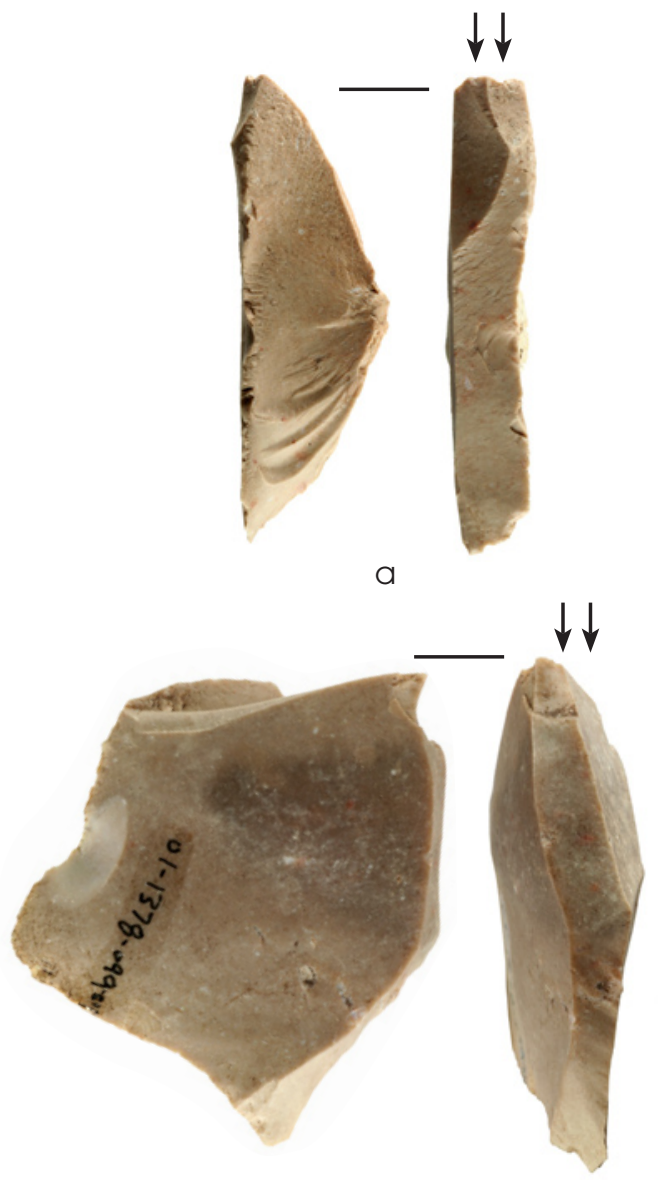

C

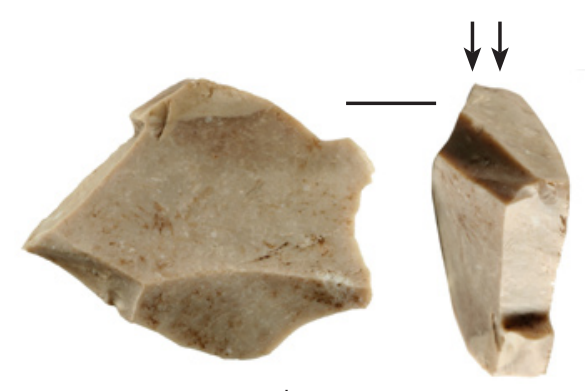

b

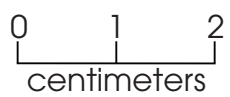

Figure 6.9. Selected multifunctional flake tools. 
represented within the site areas investigated. There are six specimens with a white surface patina and only one with thermal damage. The raw materials represented consist of 7 pieces of Anderson Mountain Gray, 1 Cowhouse Dark Gray, 1 Cowhouse White, 7 indeterminate light gray, 2 indeterminate dark gray, and 1 of undetermined chert.

Within this small group of tools, the following general tool motions or functions were interpreted based on the location and characteristics of macro and microscopic use wear. Of this grouping, there are 10 that exhibit wear associated with cutting tasks, 3 that have both cutting and scraping wear, 4 with only scraping attrition, and 1 each that can be classified as a snap break tool and a combination radial break burin and cutting implement. The functional specificity of this assemblage of unmodified flake tools is apparent and is presumed, in large part, to reflect an array of specific tasks that were conducted onsite and related to the site's function as a processing locale or related to downtime of site inhabitants while at the site. Certainly, it reflects the selection of suitable flakes and other pieces of debitage that could be used without further modification, despite the presence of an abundant supply of available raw material. It can be argued that raw material scarcity is not a logical argument for the presence of these expedient implements as part of the assemblage.

Apparent reasons for tool discard could only be ascertained for six implements, three of which have snap breaks, one with a snap/end shock, one with a radial/snap break combination, and one discarded for multiple fractures. These observations suggest that even these impromptu types of tools were occasionally considered fair game for recycling into other types of implements, even when raw material availability was not an issue.

\section{Cores and Debitage}

Nine cores or core fragments and 4,466 pieces of unmodified debitage were recovered from the $41 \mathrm{CV} 1378$ excavations. An additional 413 pieces of microdebitage were recovered from flotation. Due to the obvious difficulty of assigning microdebitage to flake categories and raw material types, these flakes were not included as part of the technological analysis. All 9 cores or core fragments in the assemblage were analyzed; however, only 469 flakes (1025 g), representing 10.5 percent of the total debitage, were analyzed.

\section{Cores}

Nine percussion cores and core fragments were identified. Raw materials represented include Anderson Mountain Gray $(\mathrm{n}=4)$, indeterminate light gray chert $(\mathrm{n}=2)$, indeterminate dark gray chert $(n=1)$, indeterminate white chert $(n=1)$, and indeterminate chert $(n=1)$. These materials correspond in general to the raw materials identified as part of the analyzed subassemblage of 469 flakes and flake fragments. Core types identified include 1 bifacial or discoid core, 4 noncortical cores, 1 tested cobble/pebble, 1 cortical core fragment, and 2 noncortical core fragments. Of these cores and fragments, 6 exhibit a multidirectional flake removal pattern, 2 are unidirectional, and 1 is bidirectional. None of the cores and fragments have any type of specialized striking platform preparation such as abrasion, but 4 are single-faceted, 2 are multiple-faceted, 1 is cortical, 1 has multiple platform types, and 1 is indeterminate. None of the cores appear to have been used as a source to produce blades, and all exhibit hard-hammer percussion flake scars. Core types in the lithic assemblage from 41CV1378 indicate that flake production was not intensive or extensive and primarily appears to have been geared toward the production of tool flake blanks that could be minimally modified into other tool forms or used with little or no modification. These tools would probably have served multiple functions. Exceptions would be the modified macroflake tools that resemble agave or sotol knives. These have been manufactured from specialized larger flakes, but no cores associated with the production of these tools were found in the tested portion of the site. Metric dimensions of the cores are provided in Table 6.2.

\section{Unmodified Debitage}

The analyzed sample of debitage was selected from several excavation units both on and off the burned rock mound, including a unit next to Feature 3, a pit feature within the mound (Table 6.3). The sample was drawn from Units A-10, A12 , B-7, F-2, and G-6, though not all flakes from these units were analyzed. Six flakes from four 
Data Recovery Investigations at the Tank Destroyer Site, Fort Hood

Table 6.2. Dimensions and weights of core types

\begin{tabular}{l|c|c|c|c|c}
\hline \multicolumn{1}{c|}{ Core Type } & Unit No. & $\begin{array}{c}\text { Maximum } \\
\text { Length }(\mathrm{mm})\end{array}$ & $\begin{array}{c}\text { Maximum } \\
\text { Width (mm) }\end{array}$ & $\begin{array}{c}\text { Thickness } \\
(\mathrm{mm})\end{array}$ & Weight (g) \\
\hline Bifacial or discoid & B-7 & 60.1 & 50.3 & 35.9 & 98.1 \\
\hline Noncortical & D-4 & 75.3 & 43.1 & 42.7 & 145.0 \\
\hline Noncortical & B-11 & 43.4 & 34.0 & 26.2 & 34.6 \\
\hline Noncortical & C-7 & 62.2 & 30.5 & 26.5 & 44.6 \\
\hline Noncortical & B-11 & 54.1 & 43.6 & 22.6 & 56.2 \\
\hline Noncortical fragment & C-7 & 127.1 & 72.7 & 43.3 & 230.9 \\
\hline Noncortical fragment & B-12 & 42.0 & 48.5 & 17.1 & 35.2 \\
\hline Cortical core fragment & E-4 & 42.3 & 39.3 & 19.9 & 28.3 \\
\hline Tested cobble/pebble & B-9 & 47.0 & 39.4 & 26.1 & 60.8 \\
\hline
\end{tabular}

Table 6.3. Analyzed debitage sample

\begin{tabular}{|c|c|c|c|}
\hline Unit No. & $\begin{array}{c}\text { Total } \\
\text { Flakes } \\
\end{array}$ & $\begin{array}{c}\text { Flakes } \\
\text { Analyzed }\end{array}$ & $\begin{array}{c}\text { Percent } \\
\text { Analyzed } \\
\end{array}$ \\
\hline $\mathrm{A}-1$ & 5 & 0 & \\
\hline A-2 & 16 & 0 & \\
\hline A-3 & 6 & 0 & \\
\hline $\mathrm{A}-4$ & 2 & 0 & \\
\hline $\mathrm{A}-5$ & 6 & 0 & \\
\hline A-6 & 1 & 0 & \\
\hline $\mathrm{A}-7$ & 14 & 0 & \\
\hline A-8 & 55 & $\begin{array}{l}1 \text { scraper re- } \\
\text { touch flake* }\end{array}$ & $<2$ \\
\hline A-9 & 126 & 0 & \\
\hline A-10 & 78 & 50 & 64 \\
\hline A-11 & 180 & 0 & \\
\hline A-12 & 154 & 91 & 59 \\
\hline A-13 & 138 & 0 & \\
\hline A-14 & 15 & 0 & \\
\hline B-1 & 0 & 0 & \\
\hline B-3 & 0 & 0 & \\
\hline B-4 & 7 & 0 & \\
\hline B-5 & 16 & 0 & \\
\hline B-6 & 24 & 0 & \\
\hline B-7 & 183 & 132 & 72 \\
\hline B-8 & 0 & 0 & \\
\hline B-9 & 449 & 2 burin spalls* & $<1$ \\
\hline B-11 & 474 & 2 burin spalls* & $<1$ \\
\hline B-12 & 146 & 0 & \\
\hline C-4 & 17 & 0 & \\
\hline $\mathrm{C}-7$ & 253 & 0 & \\
\hline D-4 & 143 & 0 & \\
\hline E-4 & 167 & 0 & \\
\hline E-11 & 446 & 0 & \\
\hline F-2 & 218 & 94 & 43 \\
\hline F-4 & 265 & 0 & \\
\hline F-9 & 307 & 1 burin spall & $<1$ \\
\hline
\end{tabular}

Table 6.3, continued

\begin{tabular}{l|c|c|c}
\hline Unit No. & $\begin{array}{c}\text { Total } \\
\text { Flakes }\end{array}$ & $\begin{array}{c}\text { Flakes } \\
\text { Analyzed }\end{array}$ & $\begin{array}{c}\text { Percent } \\
\text { Analyzed }\end{array}$ \\
\hline G-6 & 362 & 96 & 27 \\
\hline H-2 & 193 & 0 & \\
\hline All Units & 4,466 & 469 & 10.5 \\
\hline
\end{tabular}

* pulled during sorting

additional excavation units, A-8, B-9, B-11, and F-9, were also pulled as specialized flakes and are included in the analyzed sample. In total, 469 flakes were analyzed.

The raw materials represented among the analyzed sample are listed in Table 6.4. These coincide closely to the materials identified for the tools, particularly the unifacial and other flake tools. There is a greater variability of Fort Hood material types represented among the laterstage and finished bifaces, suggesting that most were being brought to the site as finished or relatively complete tools, although the assemblage is too small to state this with certainty. Obviously, the raw material data is biased toward various indeterminate light and dark gray and brown cherts among the analyzed flake assemblage, which in larger artifacts like bifaces could be identified with more certainty to known Fort Hood chert types. The trend of more diversity in raw material types among bifacial tools is generally supported, although this is not apparent in the total numbers of bifaces, flake/blade tools, or total flakes for each raw material type. Table 6.4 compares overall material diversity represented within the analyzed lithic assemblage. When discussing the use of raw materials, it is also necessary to consider both tools and debitage 
to obtain a more complete picture of where raw materials are coming from geographically, as some of the raw materials identified are only represented among bifaces and other tools and could not be distinguished among the analyzed debitage assemblage.

The geographic distribution of the Fort Hood raw materials in Table 6.4 indicates the general direction of movement of raw materials into 41CV1378. Site 41CV1378 lies along Turkey Creek within the West Fort chert province (Frederick and Ringstaff 1994:155-156). The cherts of this province occur on a southern Manning surface remnant, primarily on Seven Mile Mountain and Anderson Mountain. These localities are known for two chert types: Seven Mile Mountain Novaculite and Anderson Mountain Gray. Anderson Mountain Gray and indeterminate light and dark gray cherts comprise a significant portion of the tool and debitage assemblages recovered from 41CV1378. It is probable that some of the unidentified light and dark gray cherts are also Anderson Mountain Gray. The variety of other Fort Hood chert types indicate the use of sources located in the South East Range and North Fort chert provinces. However, these chert types were coming to the site in finished tool form and contributed very little to the overall analyzed debitage sample. A cursory examination of the unanalyzed portion of the debitage assemblage noted the predominance of other Anderson Mountain Gray material and unidentified gray cherts. Interestingly, several bifaces were manufactured of Anderson Mountain Gray chert, which is somewhat surprising based on an earlier study (Frederick and Ringstaff 1994:168-169) that demonstrated the difficulty of working this material in non heattreated form.

Perhaps the best way to present technological data for the analyzed assemblage of flakes and fragments is to treat the group as a whole. Too few identified cherts and specific Fort Hood chert types were found to address any potential

Table 6.4. Identified raw materials of the tools and analyzed debitage sample

\begin{tabular}{|c|c|c|c|c|}
\hline Raw Material & $\begin{array}{c}\text { Total Bifacial } \\
\text { Tools } \\
\end{array}$ & $\begin{array}{l}\text { Total Flake/ } \\
\text { Blade Tools }\end{array}$ & $\begin{array}{c}\text { Total } \\
\text { Flakes }\end{array}$ & $\begin{array}{l}\text { Total Weight } \\
\text { of Flakes }(\mathrm{g})\end{array}$ \\
\hline Chalcedony & 0 & 0 & 1 & 0.7 \\
\hline Chert, indeterminate & 13 & 2 & 8 & 23.6 \\
\hline Chert, indeterminate white & 5 & 0 & 30 & 64.1 \\
\hline Chert, indeterminate yellow & 0 & 0 & 12 & 32.6 \\
\hline Chert, indeterminate mottled & 0 & 0 & 17 & 151.8 \\
\hline Chert, indeterminate light gray & 30 & 24 & 263 & 484.7 \\
\hline Chert, indeterminate dark gray & 2 & 4 & 106 & 166.9 \\
\hline Chert, indeterminate light brown & 0 & 0 & 15 & 16.7 \\
\hline Chert, indeterminate dark brown & 0 & 0 & 2 & 7.1 \\
\hline Chert, indeterminate nonlocal & 1 & 0 & 0 & 0 \\
\hline Silex, unidentified & 1 & 0 & 0 & 0 \\
\hline Anderson Mountain Gray & 10 & 13 & 11 & 149 \\
\hline Heiner Lake Tan & 0 & 1 & 1 & 66.6 \\
\hline Orthoquartzite & 0 & 0 & 3 & 9.4 \\
\hline East Range Flecked & 4 & 0 & 0 & 0 \\
\hline Fort Hood Gray & 4 & 0 & 0 & 0 \\
\hline Owl Creek Black & 2 & 0 & 0 & 0 \\
\hline Cowhouse White & 1 & 0 & 0 & 0 \\
\hline Cowhouse Shell Hash & 1 & 0 & 0 & 0 \\
\hline Cowhouse Banded/Mottled & 1 & 1 & 0 & 0 \\
\hline Cowhouse Dark Gray & 0 & 1 & 0 & 0 \\
\hline Cowhouse Novaculite & 1 & 0 & 0 & 0 \\
\hline Heiner Lake Translucent Brown & 0 & 1 & 0 & 0 \\
\hline Fort Hood Yellow & 0 & 2 & 0 & 0 \\
\hline Total & 76 & 49 & 469 & $1,173.2$ \\
\hline
\end{tabular}


technological differences. In addition, vertical and spatial mixing and other site disturbances preclude any useful discussion of spatial differences within this portion of the lithic assemblage. Where feasible, specific raw materials will be discussed.

Flake types can indicate the types of core reduction or tool manufacture techniques that were used at a site. Certain flake types may be more related to the early-stage manufacture of tools and tool blank production, while others might suggest the presence of tool repair and later-stage manufacture. Table 6.5 presents the total number of various flake types in the analyzed debitage sample. It is apparent that biface manufacture, maintenance, and use dominate the onsite lithic technology. These activities may not have been directly related to the presence and function of the burned rock mound at 41CV1378. Most likely, the core reduction related flake types and at least a portion of the utilized flakes and unifaces are closely related to tasks or behaviors associated with the function of the burned rock mound, but spatial data and vertical mixing of the deposits preclude definite statements.

Technological information derived from differences in striking platforms is informative in two particular areas. First, platform types can be broadly associated with different techniques of core reduction or tool manufac- ture. Second, they can indicate the presence and nature of biface manufacture or platform core reduction/tool blank production. Various platform types can also be directly related to differences in core platform preparation and the application of standardized forms of preparation to produce certain types of tool blanks or flake types. Platform types that were identified consist of abraded, complex, cortical, crushed, faceted, flat, multifaceted, missing, and indeterminate. Table 6.6 shows the number of flakes or platform-bearing fragments associated with various identified striking platform types in the analyzed sample.

With the exception of flakes with missing striking platforms, the most abundant platform types are multifaceted (26.7 percent), faceted (24.1 percent), and flat 21.9 percent. From Table 6.6 , it is possible to suggest that certain flake types are more likely to be associated with particular types of striking platform preparation. For instance, flake types typically attributed to biface manufacture have multifaceted platforms or faceted platforms as a common attribute, while hard-hammer flakes suggesting core reduction have more flakes with flat or faceted platforms. This suggests that the generalized core reduction that was conducted at the site was not accompanied by special techniques of core platform shaping or preparation to produce specialized tool blanks. The few abraded

Table 6.5. Flake type and probable technological origins of analyzed debitage

\begin{tabular}{l|c|l}
\hline \multicolumn{1}{c|}{ Flake Type } & $\begin{array}{c}\text { No. of } \\
\text { Flakes }\end{array}$ & \multicolumn{1}{c}{ Technological Origin } \\
\hline Biface edge collapse segment & 2 & Biface manufacture, bifacial tool repair, and finishing \\
\hline Biface/tool edge resharpening & 29 & Biface manufacture, bifacial tool repair, and finishing \\
\hline Soft hammer & 85 & $\begin{array}{l}\text { Biface manufacture, bifacial tool repair, and finishing; } \\
\text { core trimming and shaping }\end{array}$ \\
\hline Notching & 1 & Biface manufacture, bifacial tool repair, and finishing \\
\hline Pressure & 5 & $\begin{array}{l}\text { Biface manufacture, bifacial tool repair, and finishing; } \\
\text { other tool maintenance and repair }\end{array}$ \\
\hline Punch & 9 & Biface manufacture, bifacial tool repair, and finishing \\
\hline Blade & 1 & Core reduction, trimming and shaping \\
\hline Burin spall & 7 & Tool manufacture and maintenance \\
\hline Hard hammer & 31 & $\begin{array}{l}\text { Core reduction, trimming, and shaping; tool manufacture } \\
\text { and maintenance }\end{array}$ \\
\hline Scraper retouch & 3 & Tool manufacture and maintenance \\
\hline Debitage shatter & 14 & Multiple \\
\hline Thermal shatter & 47 & Indeterminate \\
\hline Flake fragments (medial and distal) & 235 & Multiple \\
\hline
\end{tabular}


striking platforms that were observed are only present among soft-hammer and biface/tool edge resharpening flake types. Although this is certainly not unexpected, it does confirm the use of edge abrasion as part of the manufacture process for the middle and late-stage bifaces being produced at 41CV1378.

Proportions of flake size grades and cortex percentage classes should reveal aspects of the

Table 6.6. Flake and platform types of analyzed debitage

\begin{tabular}{|c|c|c|}
\hline Flake Type & Platform Type & Total \\
\hline $\begin{array}{l}\text { Biface edge collapse } \\
\text { segment }\end{array}$ & Multifaceted & 2 \\
\hline \multirow{4}{*}{$\begin{array}{l}\text { Biface/tool edge } \\
\text { resharpening }\end{array}$} & Flat & 1 \\
\hline & Abraded & 3 \\
\hline & Multifaceted & 23 \\
\hline & Faceted & 2 \\
\hline Blade & Cortical & 1 \\
\hline \multirow[t]{3}{*}{ Burin spall } & Faceted & 2 \\
\hline & Flat & 3 \\
\hline & Missing & 2 \\
\hline \multirow[t]{5}{*}{ Hard hammer } & Multifaceted & 5 \\
\hline & Cortical & 1 \\
\hline & Crushed & 1 \\
\hline & Faceted & 6 \\
\hline & Flat & 18 \\
\hline $\begin{array}{l}\text { Indeterminate flake } \\
\text { fragment }\end{array}$ & Missing & 235 \\
\hline Notching & Faceted & 1 \\
\hline \multirow{3}{*}{ Pressure } & Multifaceted & 2 \\
\hline & Faceted & 2 \\
\hline & Flat & 1 \\
\hline \multirow[t]{2}{*}{ Punch } & Flat & 7 \\
\hline & Faceted & 2 \\
\hline \multirow[t]{3}{*}{ Scraper retouch } & Flat & 1 \\
\hline & Faceted & 1 \\
\hline & Missing & 1 \\
\hline \multirow{2}{*}{$\begin{array}{l}\text { Shatter associated with } \\
\text { debitage }\end{array}$} & Missing & 9 \\
\hline & Indeterminate & 5 \\
\hline \multirow[t]{7}{*}{ Soft hammer } & Abraded & 15 \\
\hline & Complex & 2 \\
\hline & Flat & 14 \\
\hline & Cortical & 4 \\
\hline & Crushed & 7 \\
\hline & Faceted & 25 \\
\hline & Multifaceted & 18 \\
\hline \multirow[t]{2}{*}{ Thermal shatter } & Indeterminate & 12 \\
\hline & Missing & 35 \\
\hline Total & & 469 \\
\hline
\end{tabular}

technology such as core preparation, reduction intensity of cores, and stages of manufacture of such formal implements as bifaces. The degree of fragmentation (broken versus whole flakes and shatter) suggests possible taphonomic impacts on the assemblage. Some flaking techniques are also more prone to produce broken flakes. Table 6.7 provides data on cortex and flake size groupings for the analyzed sample of debitage. The proportions of cortex recorded indicate that 95.5 percent $(n=448)$ of the debitage is cortexfree and only 4.5 percent $(n=21)$ has any dorsal cortex at all. The lack of cortex suggests the predominance of later-stage lithic production such as biface manufacture or finishing and tool maintenance rather than core reduction. As with the distribution of cortex, the distribution of flakes and fragments among the various size grades is skewed, with an abundance of pieces in the smallest three size grades. The two smallest size grades contain 67.2 percent $(n=315)$ of all size-graded material.

Flake weight can also be useful in sorting out flake types associated with different types of flake production or tool manufacture techniques (Ahler 1989; Dockall 1991). Ahler (1989:90-91) and other researchers have demonstrated a relationship between flake type and flake weight.

Table 6.7. Proportions of cortex class, size grade variability, and degree of fragmentation among analyzed debitage

\begin{tabular}{l|c|c}
\hline & $\begin{array}{c}\text { Total } \\
\text { Flakes }\end{array}$ & $\begin{array}{c}\text { Percent of } \\
\text { Sample }\end{array}$ \\
\hline Cortex Class & 448 & 95.5 \\
\hline $0 \%$ & 13 & 2.7 \\
\hline $1-25 \%$ & 6 & 1.3 \\
\hline $26-50 \%$ & 1 & 0.2 \\
\hline $51-75 \%$ & 1 & 0.2 \\
\hline $76-100 \%$ & 8 & 1.7 \\
\hline Size Grade Class & 18 & 3.8 \\
\hline $1(1$ inch) & 127 & 27.0 \\
\hline $2(3 / 4$ inch) & 201 & 42.9 \\
\hline $3(1 / 2$ inch) & 114 & 24.3 \\
\hline $4(1 / 4$ inch) & 111 & 23.6 \\
\hline $5(<1 / 4$ inch) & 64 & 13.6 \\
\hline Degree of Fragmentation & 224 & 47.8 \\
\hline Complete & 70 & 14.9 \\
\hline Broken (retains platform)
\end{tabular}


The average weight of flakes removed from a core by hard hammer is greater than the average flake weight of pieces removed from the edges of a biface or other tool. That is simply because hard-hammer or hertzian fracture is more effective for removing mass, while soft-hammer or bending fracture is more effective for shaping, since it allows better control of the amount of mass removed. Therefore, average flake weights for hard-hammer flakes and bipolar flakes will be greater than for soft-hammer flakes or bifacial/ tool edge retouch flakes because of their greater mass. This is demonstrated within the small sample of 469 flakes here. Table 6.8 presents total flake counts, total flake weights, and average weight for selected flake types. Flake types with greater mass such as hard hammer, burin spall, and punch flakes have greater average and total flake weights, and less massive flake types like soft-hammer and biface edging flakes have smaller average and total flake weights. In some instances, certain patterns may be specific to particular raw materials. Although the analyst may not be able to associate technologies with different time periods, the technological nature of the assemblage can be determined. This provides some useful interpretive information for identifying the site type-information that is often overlooked by a more cursory analysis of lithic debitage.

Table 6.8. Correlation of selected flake types, total flake weight, and average flake weight for debitage

\begin{tabular}{l|c|c|c}
\hline \multicolumn{1}{c|}{ Type } & $\begin{array}{c}\text { Total } \\
\text { (No.) }\end{array}$ & $\begin{array}{c}\text { Weight } \\
(\mathrm{g})\end{array}$ & $\begin{array}{c}\text { Average } \\
\text { Weight (g) }\end{array}$ \\
\hline $\begin{array}{l}\text { Biface/tool edge } \\
\text { resharpening }\end{array}$ & 29 & 5.9 & 0.20 \\
\hline Hard hammer & 31 & 226.4 & 7.30 \\
\hline Soft hammer & 85 & 176.2 & 2.07 \\
\hline Burin spall & 7 & 11.6 & 1.66 \\
\hline Punch & 9 & 13.1 & 1.45 \\
\hline
\end{tabular}

\section{Spatial Patterning of Chipped Stone Artifacts}

A brief analysis of the horizontal and vertical provenience of lithic artifacts across 41CV1378 was conducted to determine if any patterns (spatial or otherwise) could be detected. Plots of the total numbers of unmodified deb- itage from units across the site revealed that the majority of debitage was recovered from units in the eastern portion of the site. There were high frequencies of lithic debitage in units both on and off mound, including in the vicinity of Feature 3. Unfortunately, little more can be said about why some units have significantly higher numbers of flakes than others-only that the distribution of these materials across the site is relatively uneven. Spatial distributions of debitage, dart points, bifaces, and other tools were examined for possible patterns that would suggest the presence of activity areas, but none were apparent. No concentrations of tool types were found, and tool presence or absence coincided with the relative abundance of debitage overall across the site. There was also little distinction between artifact types or abundance on or off mound.

This finding supports the initial assessment that the Tank Destroyer site must be treated as a single component for analytical and interpretive purposes, despite the temporal range of the radiocarbon dates and dart points. Spatial interpretations are limited by the shallow and overprinted character of the site deposits (Black 1997:145), uneven sampling across the site, and post-occupation disturbances.

\section{Summary and Conclusions}

Central Texas burned rock midden and mound sites oftentimes contain voluminous amounts of chipped stone artifacts and debris. The dynamic processes of midden and mound formation, however, regularly leave these assemblages in mixed contexts and limit our ability to group the materials into discrete analytical units. Scavenging and reuse of tools from earlier occupations by subsequent site occupiers also contribute to the contextual problems that characterize assemblages from midden and mound sites (Black and Creel 1997:280). These factors impact the types of useful information that can be gleaned from these assemblages.

The lithic assemblage from $41 \mathrm{CV} 1378$ is similar to assemblages recovered from similar sites elsewhere on Fort Hood. These assemblages can be characterized as a low-density, generalized lithic technology with low tool diversity reflecting a mix of residential types of debris (broken, resharpened, and discarded worn tools such as bifaces and dart points) and limited 
types of multifunctional tools (scrapers, denticulates, and utilized flakes). Such assemblages can be predicted in situations where tools must be used in many different tasks and are characteristic of mobile hunter-gatherer groups who can only transport a limited amount of personal gear between residences (Torrence 1983:13).

Evidence of retooling and replacement of worn tools is present in the form of resharpened and refurbished projectile points and points with impact damage. Fully a third of the dart points are represented by various portions of the proximal end, suggesting that broken dart points were brought to $41 \mathrm{CV} 1378$ for replacement or repair. A similar pattern of point fragment representation was documented for the Firebreak site (41CV595) (Boyd et al. 2004). It is probable that a majority of the biface manufacturing that occurred at 41CV1378 was conducted with the express purpose of replacing worn implements or to augment personal tool kits with bifacial tool blanks rather than to produce bifacial tools for use in activities directly related to the burned rock features at the site.

The vast majority of the analyzed debitage is cortex-free and 1/2 inch or less in maximum dimension, which is strongly indicative of lithic activities centered around the manufacture and maintenance of bifacial artifacts. Flake types indicative of this biface production represent 28.5 percent of the debitage and 58.4 percent of identified flake types. Core reduction, primarily represented by hard-hammer percussion flakes, represents a much smaller portion of the overall lithic debitage and was associated with generalized core reduction and the production of flake blanks for a variety of edge-modified flake tools. Overall, these characteristics indicate that 41CV1378 was not primarily associated with the procurement of raw material. Most of the raw material procurement that did take place undoubtedly occurred within a few kilometers of the site based on the types of identified cherts in the assemblage, which makes the relative lack of cortex-bearing debris and cores surprising.

The presence of recycling-or transformation of tools and tool fragments into other more informal implements-is represented by bifaces, flakes, and other implements such as unifaces broken deliberately by smashing (radial fractures) and truncating (breakage into one or more pieces on an anvil of some sort). These types of implements have often been used to argue that raw material was in scarce supply, that raw materials were being conserved, or that the occupants of the site were stressed for time and were achieving serviceable implements by impromptu means rather than making tools for a specific function. In the case of the Tank Destroyer site, it can be argued that these types of implements are no more unexpected than any other type of tool-and that their creation and use lends itself well to situations in which tasks can be accomplished without the need of highly specialized implements. Burin spall retouch and deliberately broken artifacts yield edges that have durable facets that can be used for a variety of heavy scraping or planing tasks on hard materials. It is quite possible that these types of recycling activities could have made use of previously discarded tools and tool fragments from earlier occupations (Amick 2007:230-231). At $41 \mathrm{CV} 1378$ it could be argued that this behavior was opportunistic rather than a specific aspect of the lithic production strategies on a regional scale. In this case, lithic material (e.g., cores, tools, and flakes) discarded within the mound represents usable sources of raw material. Scavenging and recycling of lithic materials, and deliberate breakage of tools and tool fragments, places a different perspective on our understanding of lithic procurement and raw material reduction patterns that goes beyond the typical scenario for raw material procurement in resource-rich areas such as Fort Hood-all which add to the complexity of interpreting these types of lithic assemblages.

The presence of denticulates, notched flakes, and burins/burin spall flakes indicate the need for a variety of multifunctional implements and perhaps certain function-specific implements at the site during its intermittent occupation. Use wear on these and utilized pieces of debitage demonstrates that a variety of tasks, including scraping and cutting, took place. Burins were primarily used as specialized graving or scraping tools, probably for woodworking or working other similarly dense, hard materials.

Overall the lithic assemblage from the Tank Destroyer site is indicative of behaviors oriented toward the production and use of tools for maintenance and extractive tasks. At the risk of oversimplifying this notion, extractive tasks at the site were probably primarily associated with the procurement and processing of plant resources such as bulbs, roots, and tubers. 
Presently, the only chipped stone artifacts that can arguably be inferred to represent specialized procurement tools for such resources are the unifacial flake knives associated with Feature 1. Tools associated with the procurement of more risky extractive tasks, such as deer hunting, are the various dart points. Generalized flake tools can be inferred to represent implements used for primarily maintenance and repair of other gear and the processing of predictable resources. In general, these tasks were conducted in support of, and incidental to, the activities that were associated with the use and formation of the burned rock mound.

The tasks and activities that produced the assemblage suggest that the Tank Destroyer site might have functioned in two ways. First, the site could represent a residential base camp at which a range of activities occurred, with the associated tools and debris accumulating over an unknown number of short-term intermittent occupations. Or it may have functioned as a special-purpose site associated with the procurement and processing of specific resources, where the location was reoccupied intermittently for a period of time, and during which other activities not directly related to the primary function of the site were conducted. Each scenario can be viewed as opposite ends of Binford's (1980) forager-collector model of subsistence and resource acquisition. Forager and collector strategies, however, are not mutually exclusive, and foraging groups may employ collector strategies for certain seasons. Given this, the lithic assemblage from 41CV1378 probably represents a congruence of strategies that represent behaviors commonly associated with both forager and collector patterns and hence cannot be attributed exclusively to one strategy. This strategy produced lithic assemblages that, with the exception of diagnostic projectile points, remained largely unchanged from the Archaic into the Late Prehistoric period. As Boyd et al. (2004:219) have concluded for similar sites at Fort Hood, such locales have produced evidence supporting the idea of long-term continuity in repeated site use and patterns of resource exploitation.

\section{GROUND STONE TOOL}

A single coarse-grained limestone mano was recovered. It is oblate or disk-shaped and is $101 \mathrm{~mm}$ long, $76 \mathrm{~mm}$ wide, and $38 \mathrm{~mm}$ thick. The implement displays one grinding facet and weighs $417 \mathrm{~g}$. The scarcity of ground stone artifacts at the Tank Destroyer site indicates that activities associated with such tools were rare or were not conducted in the immediate vicinity of the burned rock mound.

\section{RABDOTUS SP. SHELLS}

Ethnographic accounts indicate that aboriginal populations in parts of Texas consumed snails (Campbell and Campbell 1981:17). The shipwrecked Spanish explorer Cabeza de Vaca witnessed the consumption of snails by Native Americans in south Texas (Clark 1969:43, 1976; Hester and Hill 1975). Often found numbering in the thousands at archeological sites throughout central and south Texas, the shells of Rabdotus $\mathrm{sp}$. are sometimes interpreted as the remains of an aboriginal food source (e.g., Gadus et al. 2006:170; Highley 1986:87-89; Johnson et al. 1962:47; Kibler and Scott 2000:67, 72; Neck 1994:496). Interpreting snail shells from archeological contexts as food sources often tenuously relies solely on early ethnographic accounts of aboriginal consumption and the abundance of shells. Alternatively, copious shells are often interpreted as the remains of snail populations naturally attracted to the organic-rich deposits left behind when a site is abandoned (e.g., Fullington 1978; Gadus et al. 1999:80). Brown (2002) provides an excellent examination of this issue and the methods that may be used to address it.

Does the Rabdotus sp. shell assemblage from the Tank Destroyer site represent a natural phenomenon or the remains of a food source? To address this issue, Rabdotus sp. shells were sorted into size categories with the assumption that larger shells represent more mature snails and that smaller shells represent adolescents and juveniles. Examining the age structure of a Rabdotus sp. assemblage can provide insight into whether the snails were a food source for prehistoric peoples (Neck 1994:496). An adultdominated population with few young snails might indicate that they were purposefully collected. In contrast, an even age distribution might indicate that the snails were simply a natural occurrence, perhaps representing snails that moved in to occupy and feed on debris after a site was abandoned. 
In addition, the distribution of snail shells was examined to see if the snails were randomly distributed across the site or if they were concentrated only in features. The latter might suggest that people used the snails as food.

\section{Rabdotus sp. Shell Distributions}

A total of 1,666 snail shells identified as Rabdotus sp. were recovered from feature and nonfeature contexts. Most of these snail shells were recovered through hand excavations and on 1/4-inch-mesh screens, although 251 specimens were recovered through flotation and fine-screening of soil column samples collected from Features 1, 2, and 3. The cluster of seven shells designated as Feature 4 are included in the count for Feature 1. As summarized in Table 6.9 , recovery varied by provenience. Feature 1 yielded 1,247 snail shells from an excavated volume of $8.57 \mathrm{~m}^{3}$. Feature 2 yielded 1 snail shell from an excavated volume of $0.1 \mathrm{~m}^{3}$, and Feature 3 yielded 139 snails from an excavated volume of $1.3 \mathrm{~m}^{3}$. The excavation of nonfeature contexts totaled $1.93 \mathrm{~m}^{3}$ and yielded 279 snail shells. All of these contexts generated similar densities of shells, with 145.5 snails per $\mathrm{m}^{3}$ in Feature 1 and 106.9 snails per $\mathrm{m}^{3}$ in Feature 3, while the nonfeature contexts produced 144.5 snails per $\mathrm{m}^{3}$. Only Feature 2 yielded a vastly different density of shells-at 9.1 snails per $\mathrm{m}^{3}$. But this is probably due to the small size of the feature and the limited volume of excavated sediment. Qualitatively, Feature 2 is quite different from Features 1 and 3. Regardless of the shell density differences between Feature 2 and the other contexts, a scatter plot of feature and nonfeature excavated volume versus the number of snail shells recovered returns a Pearson's $r$ of 0.99 . This suggests that the number of snails recovered is related to the size (volume) of the feature or excavated context, and that there are no differences in terms of the number of snails between feature and nonfeature contexts. It appears that the density of snail shells is relatively consistent throughout most of the site, with an average recovery of 140 snails per $\mathrm{m}^{3}$.

\section{Age Structure of the Rabdotus sp. Shell Assemblage}

Of the 1,666 snail shells recovered, 251 were from heavy fraction flotation and soil column samples collected from burned rock features. Fine screening of these samples ensured that all snails would be recovered from these feature-associated sediments, in contrast to the rest of the assemblage, which is only composed of snail shells that would not pass through 1/4-inch mesh screen. The recovery of all sizes of shells made it possible to look at the age structure of the snail population by sorting the shells into size groups.

Of the 251 snail shells recovered from the flotation and soil column samples, 25 were too incomplete or fragmentary to be assigned to a size group. The shells were sorted into the following size groups: $<5 \mathrm{~mm}, 5-10 \mathrm{~mm}, 10-15$ $\mathrm{mm}, 15-20 \mathrm{~mm}, 20-25 \mathrm{~mm}$, and $>25 \mathrm{~mm}$. Table 6.10 presents the results of the size sorting by feature.

Only Feature 3 yielded a large majority of adult Rabdotus sp. shells, suggesting that those snails may represent a food source, albeit a very limited one. Feature 3, which appears to be the remains of an earth oven within the burned rock mound, is distinguished from the surrounding burned rock matrix of Feature 1 by vertical to inclined tabular limestone rocks and complete cobbles that line a depression in some areas. Other parts of the feature display

Table 6.9. Rabdotus sp. shells

\begin{tabular}{l|c|c|c}
\hline \multicolumn{1}{c|}{ Feature Number and Type } & $\begin{array}{c}\text { Volume Excavated } \\
\left(\mathrm{m}^{3}\right)\end{array}$ & $\begin{array}{c}\text { Number of Snail } \\
\text { Shells Recovered }\end{array}$ & $\begin{array}{c}\text { Number of Snail } \\
\text { Shells }\left(\mathrm{m}^{3}\right)\end{array}$ \\
\hline $\begin{array}{l}\text { Feature 1 } \\
\text { Burned rock mound }\end{array}$ & 8.57 & 1,247 & 145.5 \\
\hline $\begin{array}{l}\text { Feature 2 } \\
\text { Burned rock cluster or expedient hearth }\end{array}$ & 0.10 & 1 & 9.1 \\
\hline $\begin{array}{l}\text { Feature 3 } \\
\text { Earth oven }\end{array}$ & 1.30 & 139 & 106.9 \\
\hline Nonfeature areas & 1.93 & 279 & 144.5 \\
\hline Total & 11.90 & 1,666 & 140.0 \\
\hline
\end{tabular}


Data Recovery Investigations at the Tank Destroyer Site, Fort Hood

Table 6.10. Rabdotus sp. shells recovered from flotation and soil column samples

\begin{tabular}{l|c|c|c|c|c|c|c|c}
\hline \multicolumn{1}{c|}{$\begin{array}{c}\text { Feature } \\
\text { Number and Type }\end{array}$} & $<5 \mathrm{~mm}$ & $\begin{array}{c}5-10 \\
\mathrm{~mm}\end{array}$ & $\begin{array}{c}10-15 \\
\mathrm{~mm}\end{array}$ & $\begin{array}{c}15-20 \\
\mathrm{~mm}\end{array}$ & $\begin{array}{c}20-25 \\
\mathrm{~mm}\end{array}$ & $\begin{array}{c}>25 \\
\mathrm{~mm}\end{array}$ & Fragments & Total \\
\hline $\begin{array}{l}\text { Feature 1 } \\
\text { Burned rock mound }\end{array}$ & 6 & 4 & 6 & 32 & 70 & 28 & 20 & 166 \\
\hline $\begin{array}{l}\text { Feature 2 } \\
\text { Burned rock cluster or } \\
\text { expedient hearth }\end{array}$ & 0 & 0 & 0 & 0 & 0 & 0 & 1 & 1 \\
\hline $\begin{array}{l}\text { Feature 3 } \\
\text { Earth oven }\end{array}$ & 3 & 0 & 0 & 20 & 48 & 9 & 4 & 84 \\
\hline Total & 9 & 4 & 6 & 52 & 118 & 37 & 25 & 251 \\
\hline
\end{tabular}

a more haphazard jumble of burned rocks-a characteristic that, along with the absence of charcoal, suggests that the feature might have been cleaned out following the last cooking episode and later filled with burned rock rubble. If Feature 3 was filled in, were snail shells part of or included in the backfill materials? If so, where did the snail shells originate? Are they the remains of a meal not processed in Feature 3 but consumed nearby? The flotation sample from Feature 3 also yielded many small broken shell fragments, but the presence of so many complete shells makes it difficult to imagine that so many fragile adult shells survived intact if they were part of the backfill materials. This scenario, if accurate, suggests that the snails recovered from Feature 3 are more likely a postsite abandonment phenomena than an aboriginal food source. In all likelihood, most of the Rabdotus sp. shells recovered at 41CV1378 represent the introduction of snails to the site after it was abandoned. The distribution of the shells by age groups supports this inference in most contexts. Although the adult-dominated population recovered from Feature 3 seems to represent discarded food remains, this interpretation is unlikely. 


\title{
DESCRIPTION OF FEATURES AND SPATIAL ANALYSIS OF BURNED ROCKS AND ARTIFACTS
}

\author{
Douglas K. Boyd and Karl W. Kibler
}

Four features were recorded during the data recovery excavations at the Tank Destroyer site. The features and their associated cultural materials are described below. Feature 1 is the large burned rock mound that was the focus of the archeological investigations at the site. Feature 2 is a smaller off-mound burned rock feature, and Feature 3 is a rock-lined pit within the larger burned rock mound. Feature 4 is a cluster of terrestrial snail shells found within Feature 1. The locations of these four features are shown in Figures 7.1 and 7.2.

\section{FEATURE 1}

Feature 1 is the number assigned to the entire burned rock mound by Mariah archeologists during their 1994 testing investigations, which consisted of a single 1x1-m test pit in the mound (see Figure 7.1) (Trierweiler 1996:473478). In the 2007 data recovery investigations, PAI sampled the mound feature with 23.5 additional 1x1-m units, bringing the total number of units in the mound to 24.5. The total area of the hand excavations in Feature 1 may be summarized as:

Mariah test unit

$1.0 \mathrm{~m}^{2}$

PAI excavation units $\quad 23.5 \mathrm{~m}^{2}$

PAI matrix columns outside units $\quad 0.75 \mathrm{~m}^{2}$

Total area of Feature 1 hand excavations $\quad 25.25 \mathrm{~m}^{2}$

A portion of these units, however, accounting for about $2 \mathrm{~m}^{2}$, sampled the subfeature designated as Feature 3. If the Feature 3 area is subtracted from the total, the Feature 1 handexcavation sample is $23.25 \mathrm{~m}^{2}$.

The mound area investigated by PAI measured approximately $14 \mathrm{~m}$ (east-west) by $5 \mathrm{~m}$ (north-south)_about half of the original mound. The missing portion was destroyed over several decades by the east-west tank trail (see Figure 3.1). The estimate that half of the circular mound was destroyed is based on observations made when archeologists first recorded the site in 1987 (as reported by Trierweiler 1996:473). After PAI hand-excavated a substantial portion of the remaining half of the mound, a backhoe was used to carefully excavate much of the remaining area to search for internal subfeatures (Figure 7.3; see Figure 4.1). No additional subfeatures were encountered, and it is notable that Feature 3 was the only large baking pit or earth oven found within the surviving portion of the mound. The area of the burned rock mound investigations is calculated as follows:

\begin{tabular}{lr} 
Hand-excavations in Feature 1 mound & $23.25 \mathrm{~m}^{2}$ \\
Hand-excavations in Feature 3 & $2.00 \mathrm{~m}^{2}$ \\
Machine-scraped area in mound & $17.30 \mathrm{~m}^{2}$ \\
Unexcavated area in mound & $14.20 \mathrm{~m}^{2}$ \\
Previously destroyed portion of mound & $56.75 \mathrm{~m}^{2}$ \\
\hline Total estimated area of Feature 1 mound & $113.50 \mathrm{~m}^{2}$
\end{tabular}

If the burned rock mound were perfectly circular, its size would be about $12 \mathrm{~m}$ in diameter, covering an area of $113 \mathrm{~m}^{3}$.

The core area of the mound was originally defined horizontally based on field observations of where burned rocks were most concentrated and where the density began to decline. The initial field tally of burned rocks (weight per unit) from the general excavations supported this inference. As discussed later in this chapter, the burned rock sample confirms the concept of a burned rock mound with a dense core area trailing off to a dispersed feather edge holds true. From the edge of the core area, the mound 


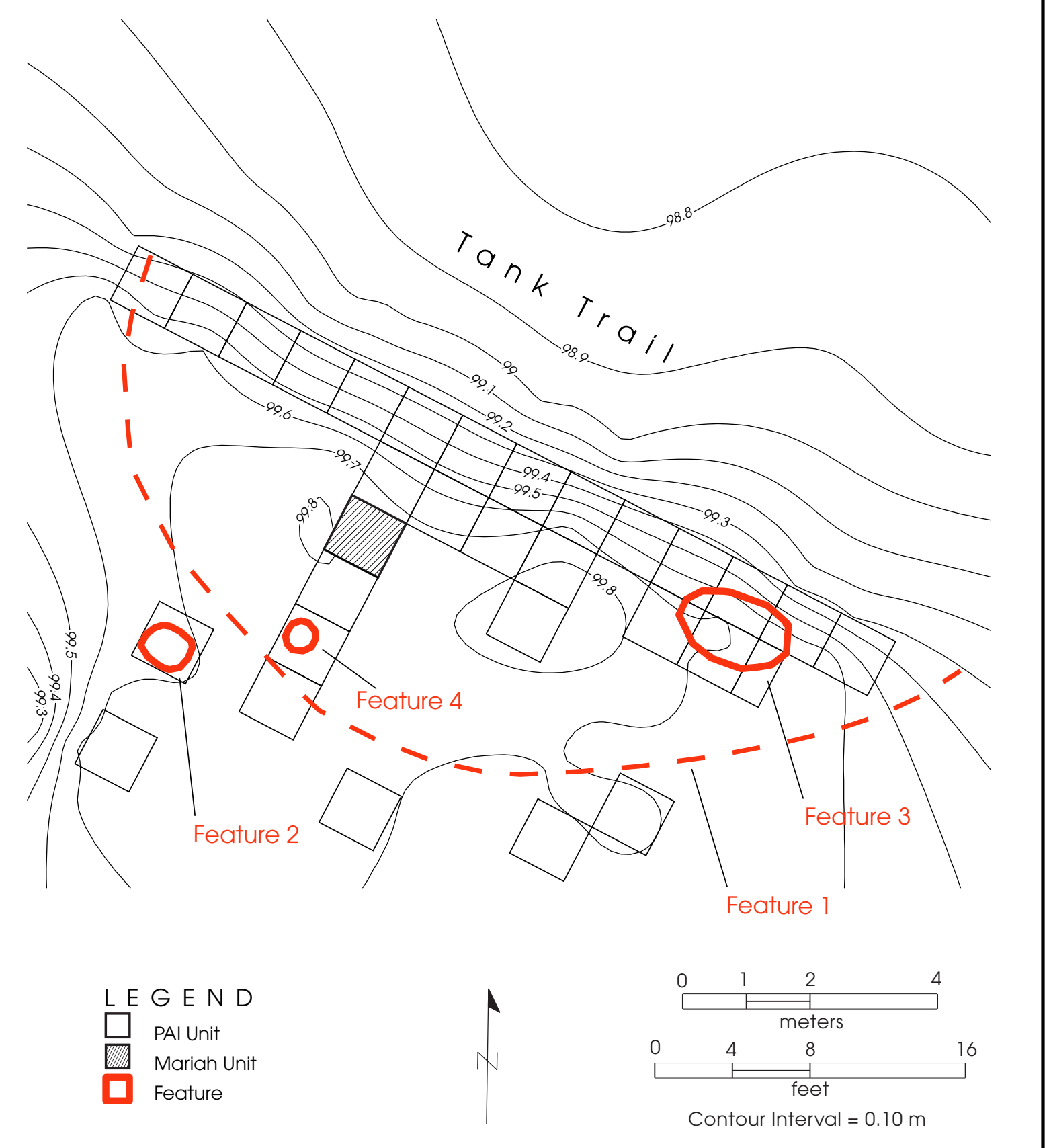

Figure 7.1. Topographic map showing excavation units and cultural features.

deposits became less dense and thinner in all directions, with the outer edge of the feature being only $1-2 \mathrm{~m}$ beyond the core.

As described in the previous chapter, cultural materials recovered from the general Feature 1 mound matrix consist of 21 dart points, 1 arrow point (from testing) and 1 preform, 31 bifaces, 17 unifaces, 15 utilized flakes, 6 flake tools, 9 cores and core fragments, 2,635 unmodified flakes, and 1 mano (recycled as a heating stone), and 5,165.5 kg of burned rocks. The typeable dart points are classified as Castroville, Darl, Ensor, Marcos, and Montell with several of the specimens having been resharpened. Late-stage and finished bifaces are well represented in the assemblage, and 


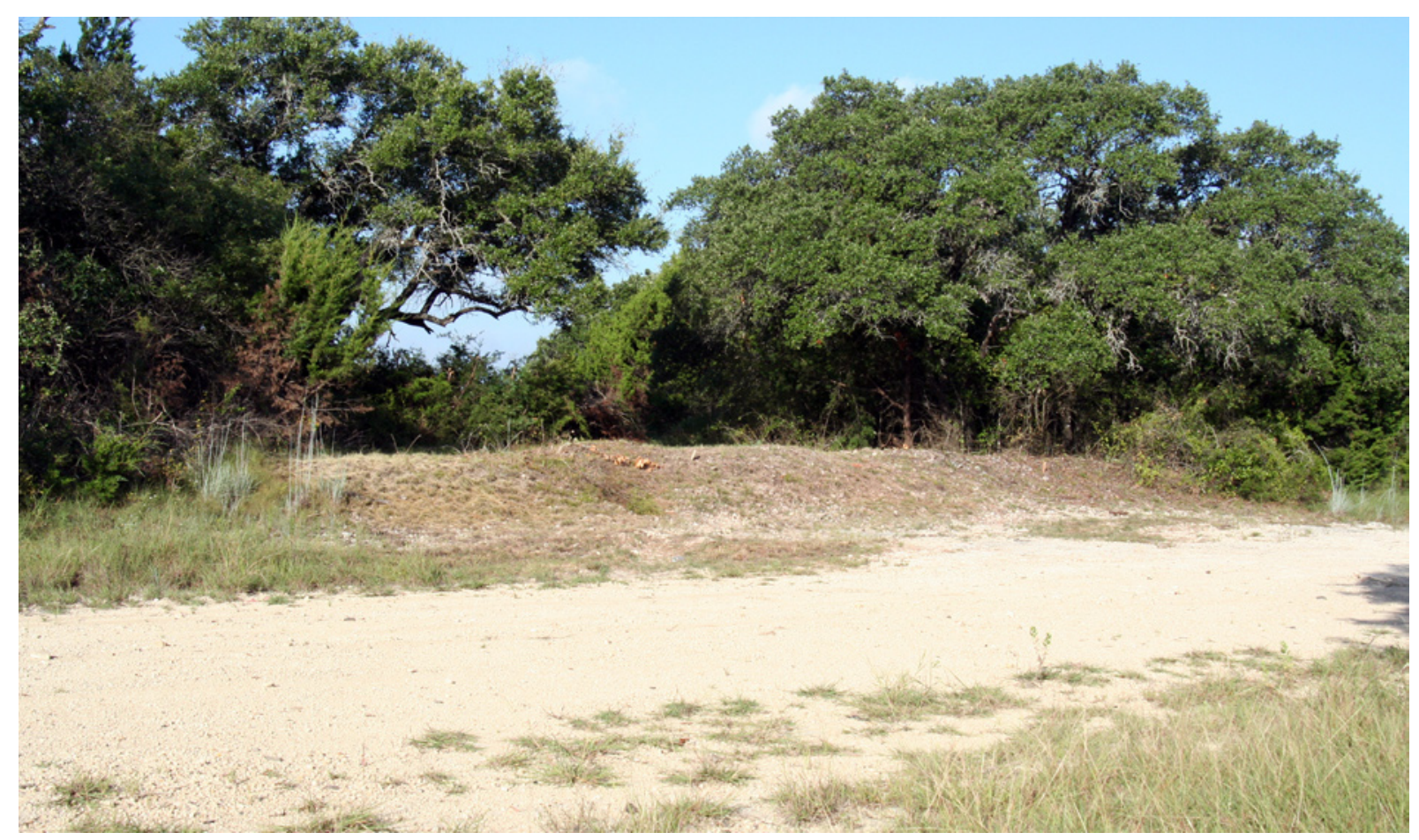

a

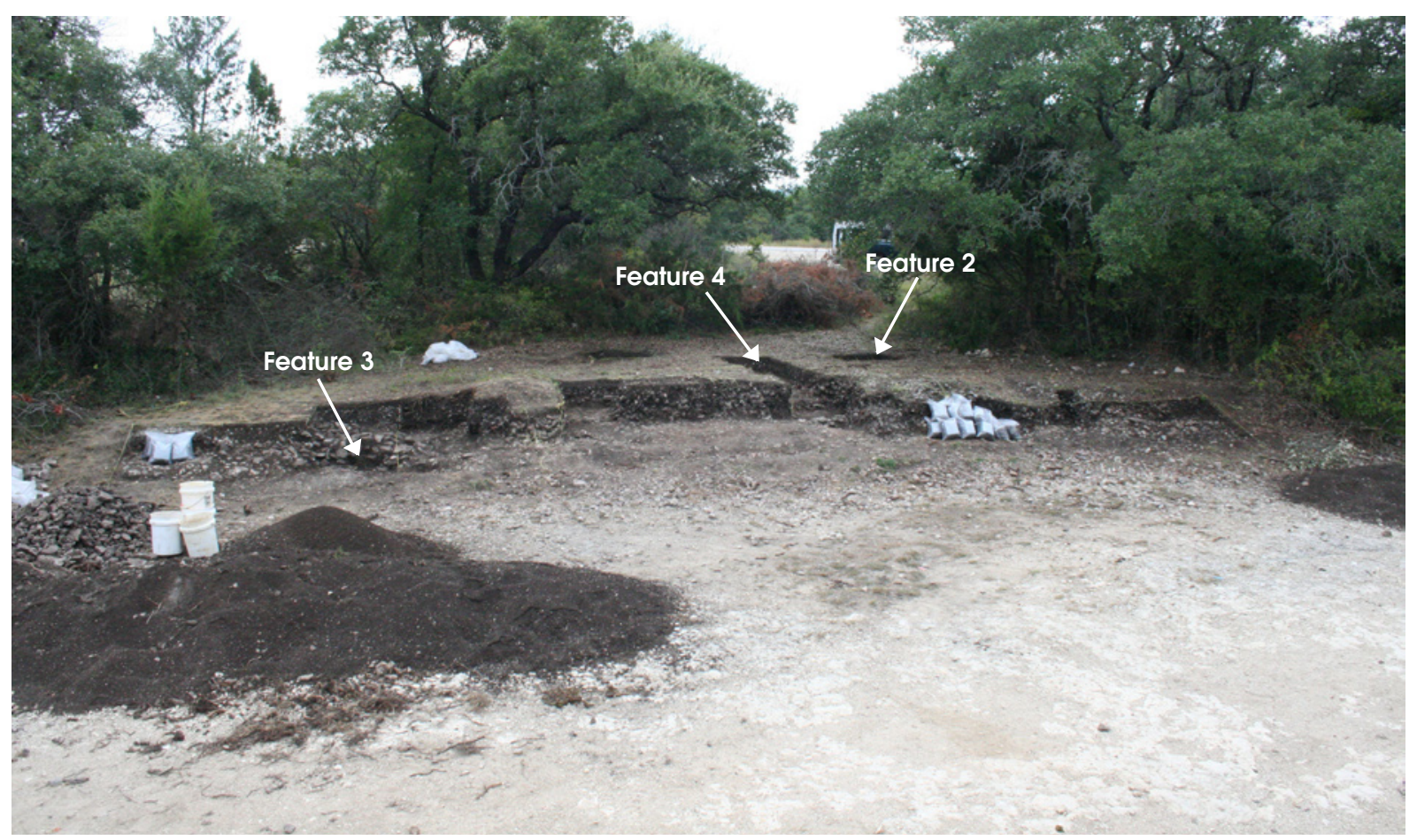

$b$

Figure 7.2. Overviews of the burned rock mound at the Tank Destroyer site, looking southwest. (a) Photograph from ground level looking across the tank trail at the burned rock mound before any excavations occurred. (b) Low-angle photograph showing the excavations in progress. Feature 1 is the entire mound, and arrows mark the locations of Features 2 through 4. 


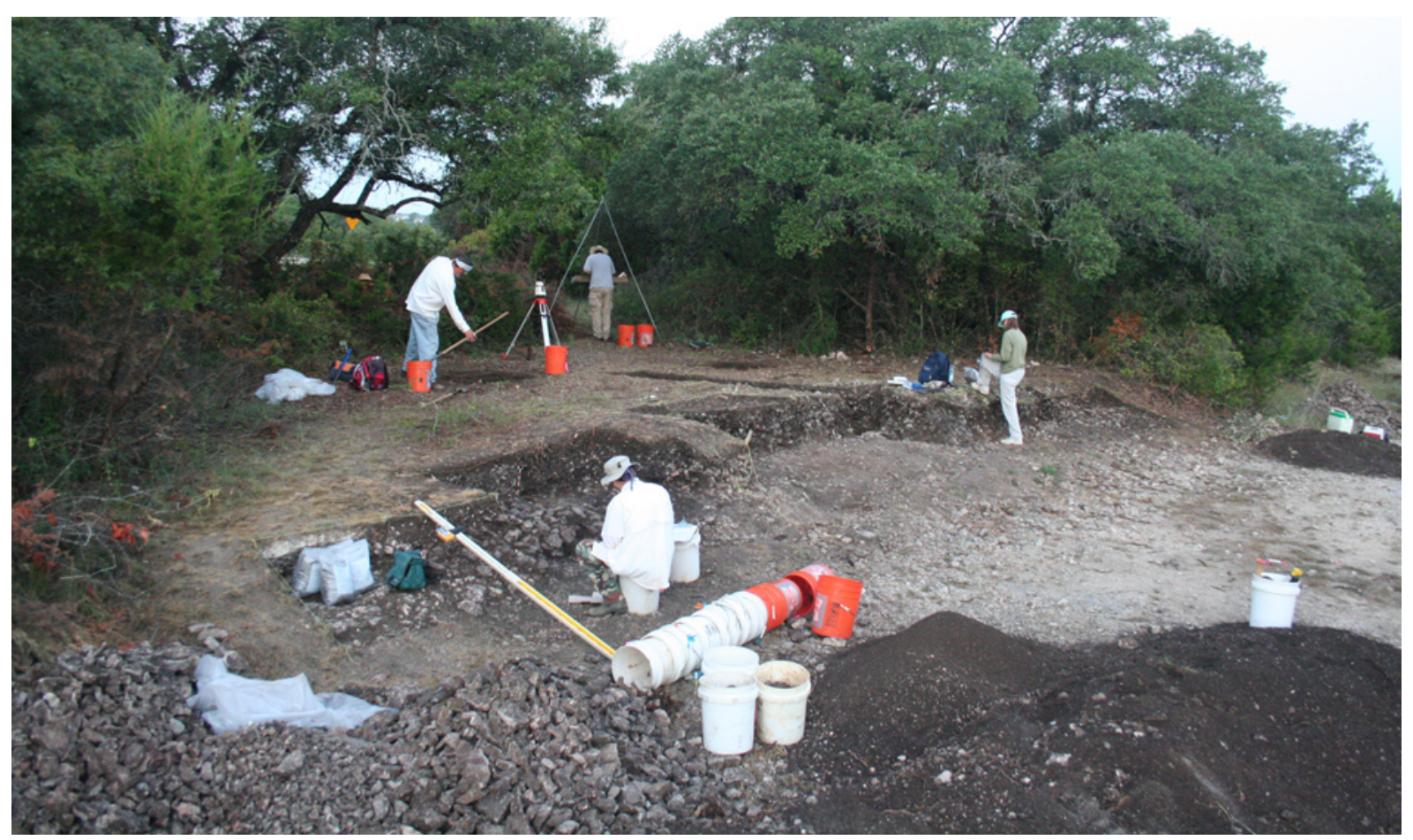

a

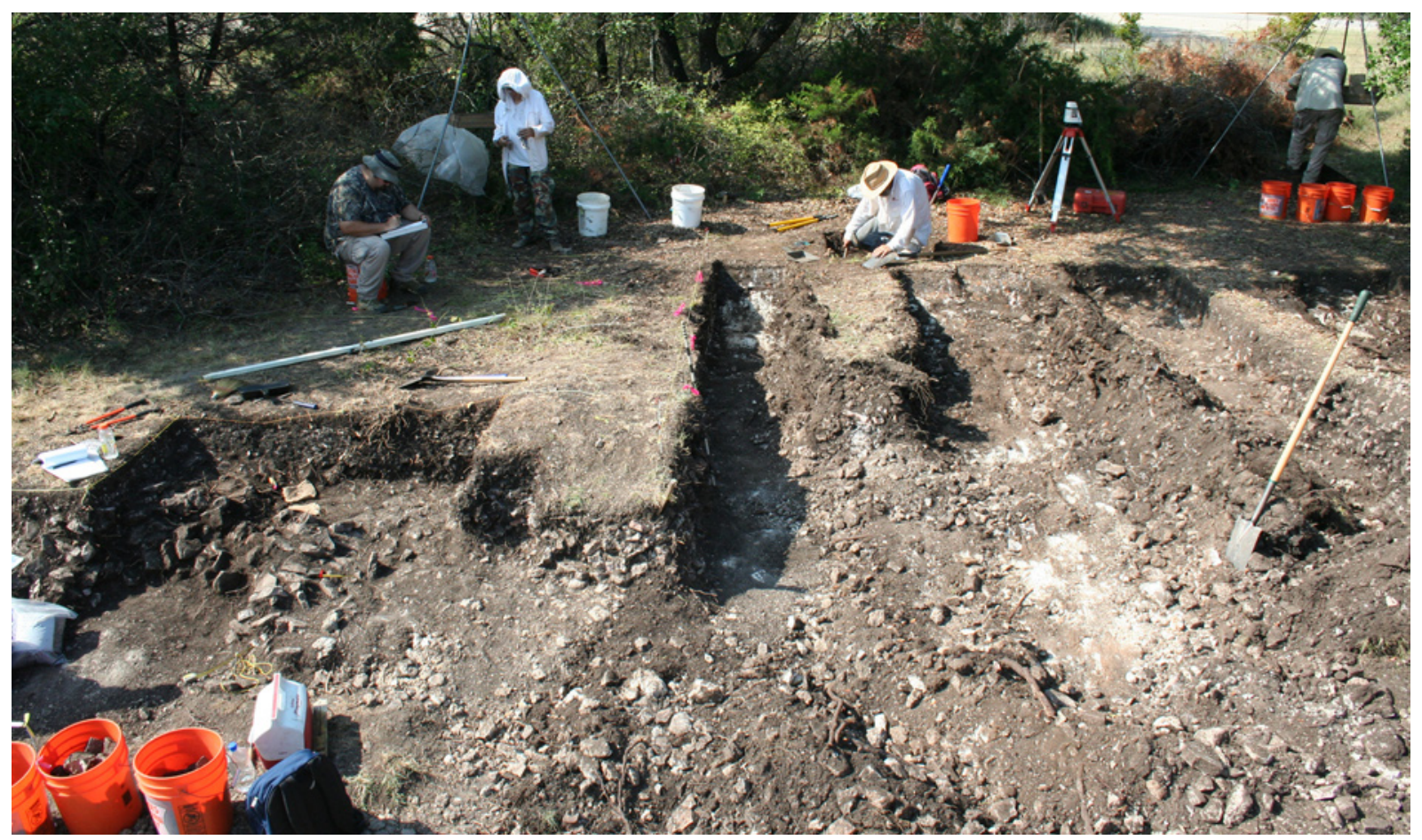

b

Figure 7.3. Views of data recovery excavations of the burned rock mound Feature 1 at the Tank Destroyer site. (a) Photograph of the hand excavations in progress, looking west; (b) photograph of the final backhoe scraping to search for subfeatures in the burned rock mound. 
the lithic debitage consists mainly of small- to medium-sized flakes.

Several different types of samples were collected from various parts of the mound, including charcoal, individual burned rocks, flotation samples, and five matrix sample columns. The total fill processed from 17 flotation samples associated with Feature 1 was 197.5 liters; they yielded burned rocks, sparse charred plant remains, microdebitage, and snail shells.

\section{FEATURE 2}

Just beyond the southwest edge of the mound, Feature 2 was encountered in Unit F-2 from 99.55 to $99.45 \mathrm{~m}$ (see Figure 7.1). It was centrally located in the unit and had maximum dimensions of $83 \mathrm{~cm}$ east-west by $72 \mathrm{~cm}$ northsouth (Figure 7.4). The feature was a single layer of burned limestone rocks, with only a few rocks slightly imbricated. The rocks, which have a total weight of $11.5 \mathrm{~kg}$, are primarily large tabular pieces and slabs ranging from 15 to $25 \mathrm{~cm}$ in maximum length and 3 to $7 \mathrm{~cm}$ in thickness. Most were lying flat; those that were sloping may have been moved by many smalland medium-sized roots dissecting the feature. The sediment among the rocks was a dark gray brown to brown clay loam-a residual soil-with patches of limestone bedrock exposed around the edges of the feature. Notably, several unburned limestone rocks were observed around the burned rocks.

Being relatively small, Feature 2 is interpreted as an expedient surface hearth based on its configuration-a single course of large rocks resting just above bedrock. This small rock-lined cooking or warming hearth was probably placed on an unprepared surface at the edge of the burned rock mound. The spatial relationship between Features 1 and 2 is reminiscent of a pattern of similar small burned rock hearths found surrounding an incipient burned rock midden at the Higginbotham site (41ML195) in the North Bosque River valley (Mehalchick and Kibler 2008). It is possible that the hearth could have been constructed inside a shallow basinshaped pit, but that erosion and deflation of the thin soils have obliterated all traces of the pit. All of the feature sediment was collected for flotation, and burned rock and thermoluminescence samples were taken. The one flotation sample contained 5.0 liters of fill that yielded 13 pieces of microdebitage and 1 snail shell. No charcoal was recovered from the sample.

\section{FEATURE 3}

Feature 3 is a large rock-filled pit discovered less than $1 \mathrm{~m}$ east of the mound's core area (Figure 7.5). It was spread across portions of six units: A-9, A-11, A-12, B-9, B-11, and B-12 (see Figure 7.1). The burned rocks, which have a total weight of $405.0 \mathrm{~kg}$, occurred from 99.51 to $99.12 \mathrm{~m}$. Feature 3 is $184 \mathrm{~cm}$ east-west by $132 \mathrm{~cm}$ north-south and has a maximum thickness of about $39 \mathrm{~cm}$. The pit was constructed in a bedrock depression that appears natural but could have been modified slightly, perhaps to enlarge it somewhat. The depression was approximately $20 \mathrm{~cm}$ deeper than the surrounding regolith, and much of it was lined with slabs, large tabular clasts, and whole cobbles. Many of the burned rocks were oriented vertically or at a steep angle, particularly around the perimeter of the pit, while the central portion of the feature consisted of up to five layers of rocks that were haphazardly oriented. No charcoal or dark organic-stained soils were detected while excavating Feature 3, but many burned rock, thermoluminescence, and flotation samples were taken. Screening of the feature matrix produced 1 dart point (Montell), 1 unifacial tool, 60 flakes, and 139 Rabdotus sp. shells. Flotation of 97 liters of feature fill, taken as four samples from different parts of the feature, yielded burned rocks, abundant microdebitage, a single animal bone, sparse charcoal, and many snails.

Feature 3 is interpreted as the remains of a substantial earth oven cooking facility that was constructed in a prepared pit (Figure 7.6). Some of the angled perimeter rocks were probably in situ since the last cooking episode, and they probably represent portions of the original heating element composed of slabs used to line the edges of the pit. In contrast, the central portion of the feature exhibited a thick jumble of rocks that appear haphazard in their arrangement. Consequently, it is likely that these rocks were dumped in this location, perhaps having been thrown back into the rock-lined pit sometime after the last cooking and pit-cleanout episode. However, the relative paucity of charcoal could indicate that the pit might have been left open for some time before being backfilled. 


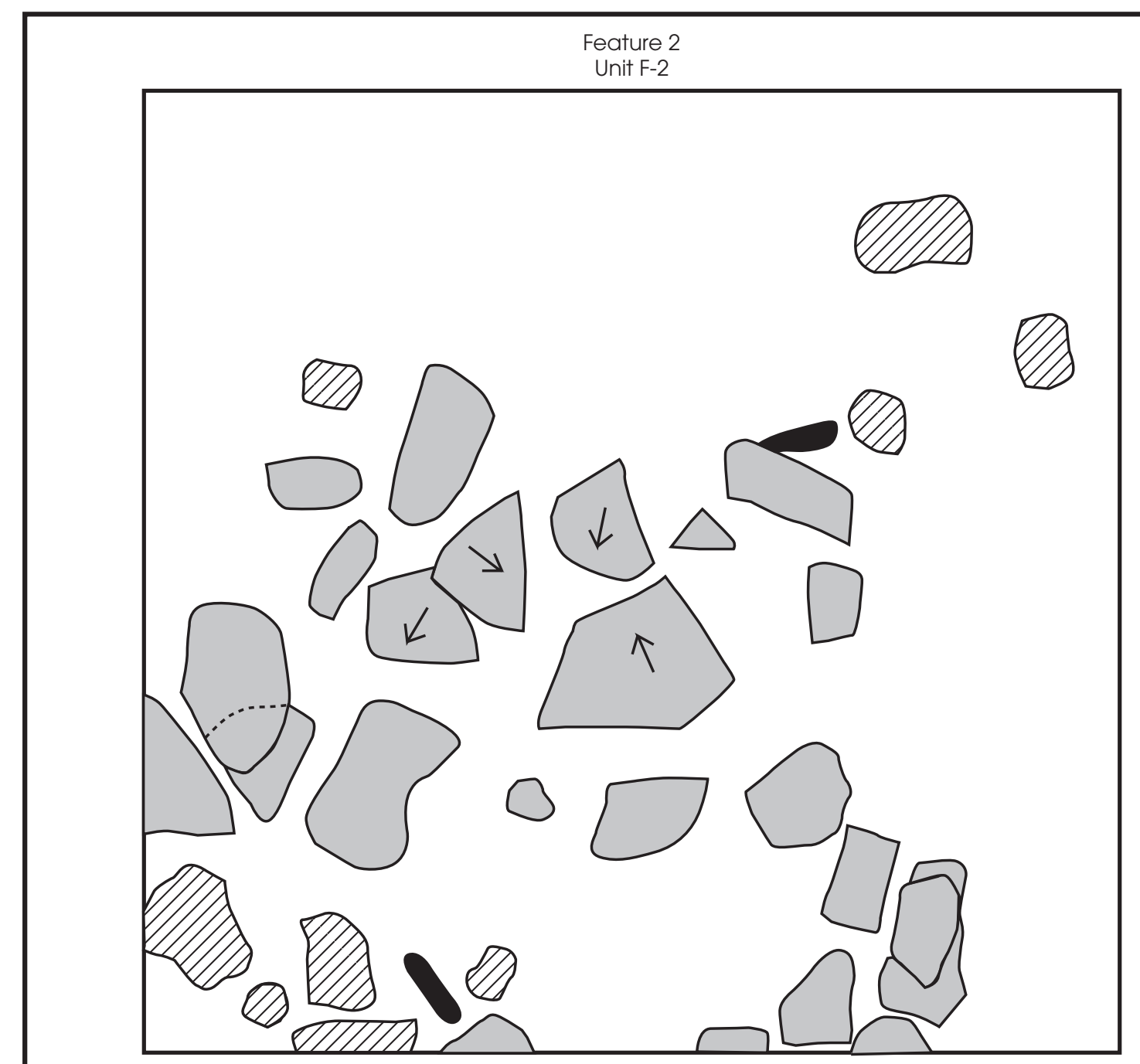

\footnotetext{
$L E G E N D$

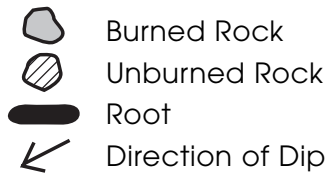

PAI/12/BW

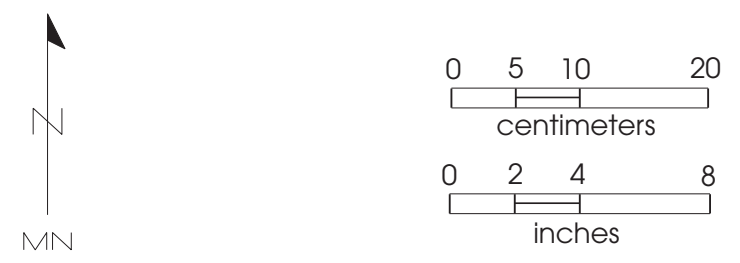

Figure 7.4. Plan drawing of the burned and unburned limestone rocks comprising Feature 2 in excavation Unit F-2. The bottoms of the exposed rocks ranged in elevation from $99.56 \mathrm{~m}$ to $99.48 \mathrm{~m}$, and most of the rocks lay flat. 


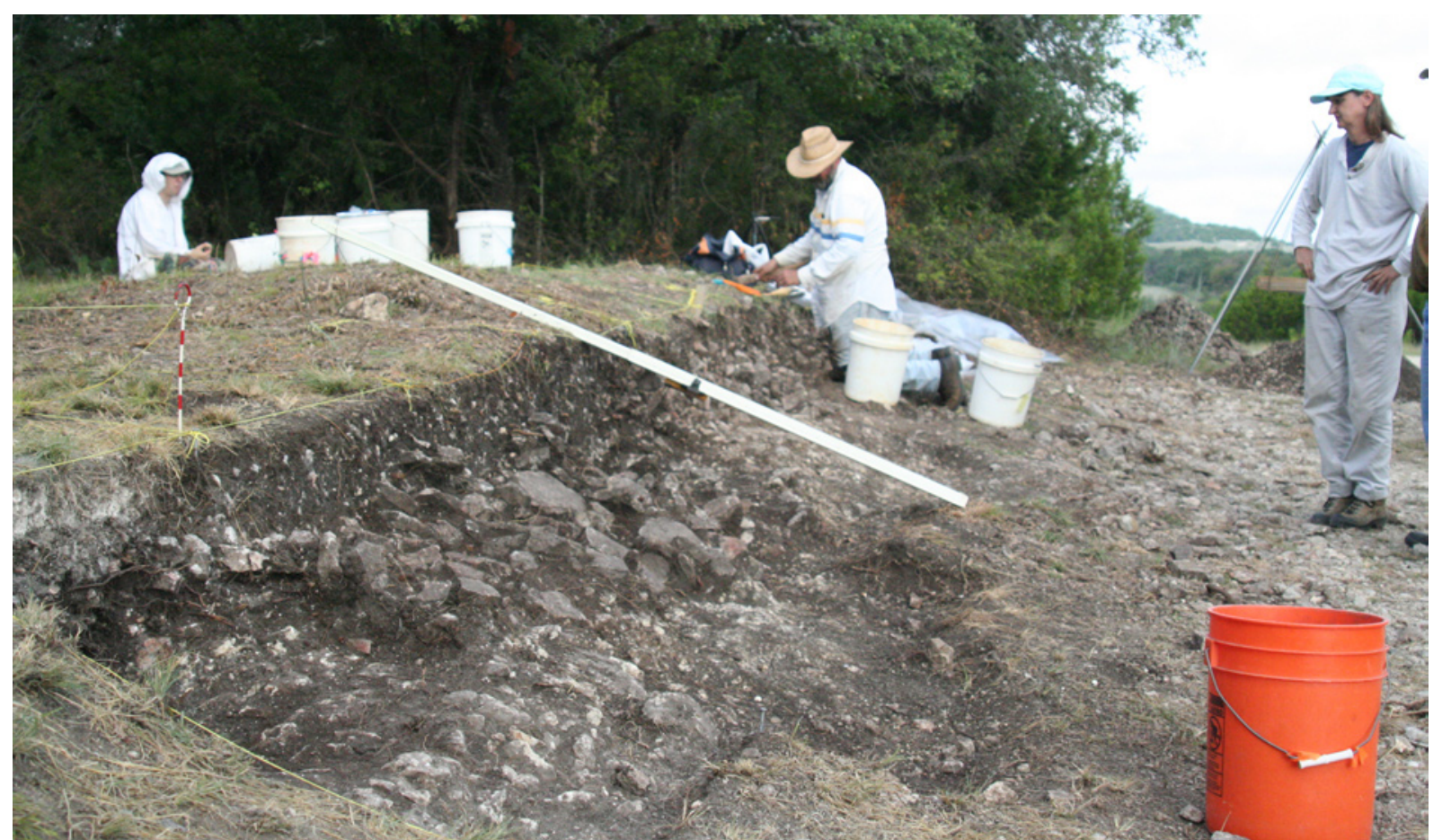

a

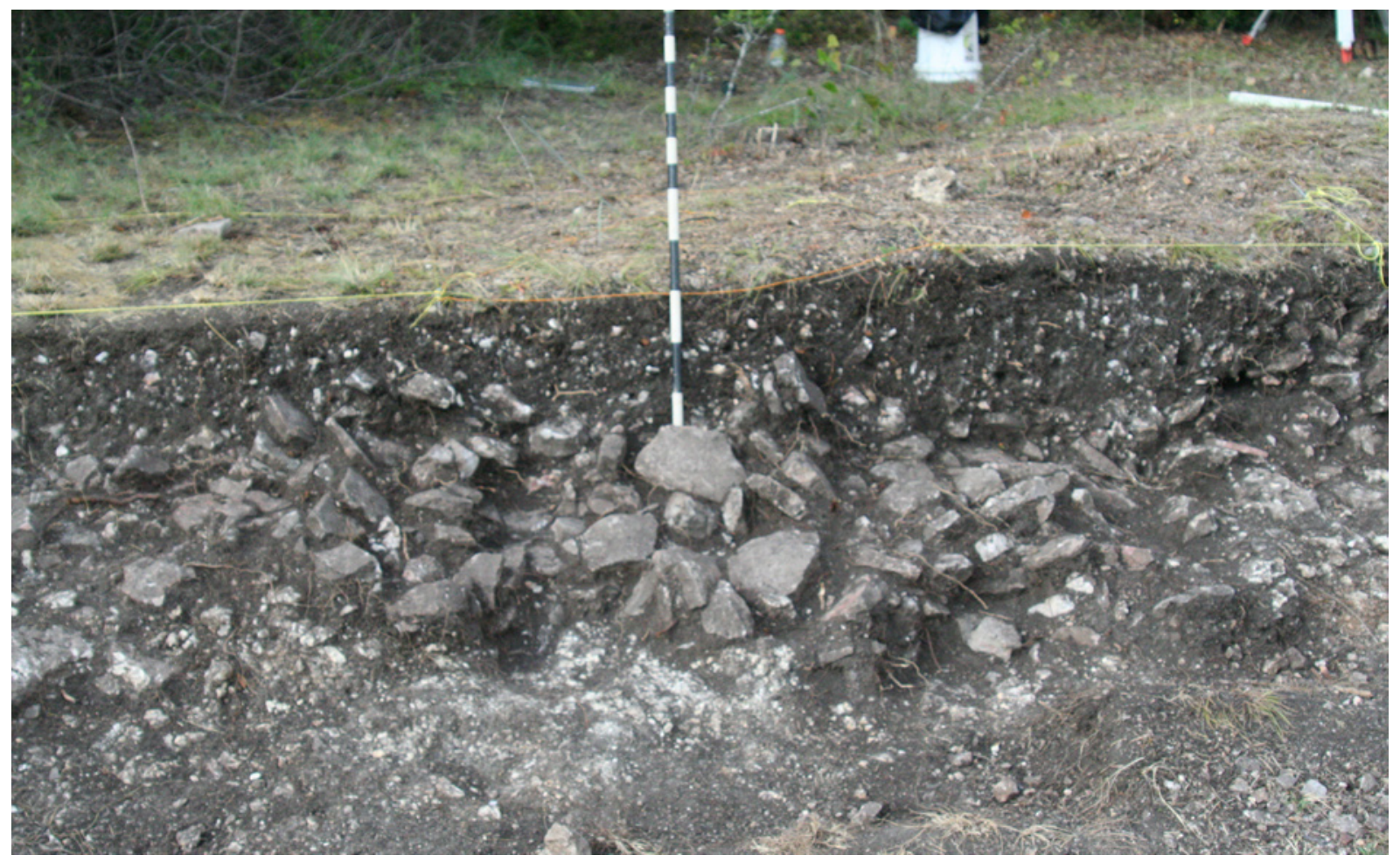

b

Figure 7.5. Views of Feature 3, a rock-lined pit within Feature 1. (a) Feature 3, looking west-northwest; (b) the rocks associated with Feature 3 (center) are very large compared with the rocks in the surrounding mound matrix, looking south-southwest. 


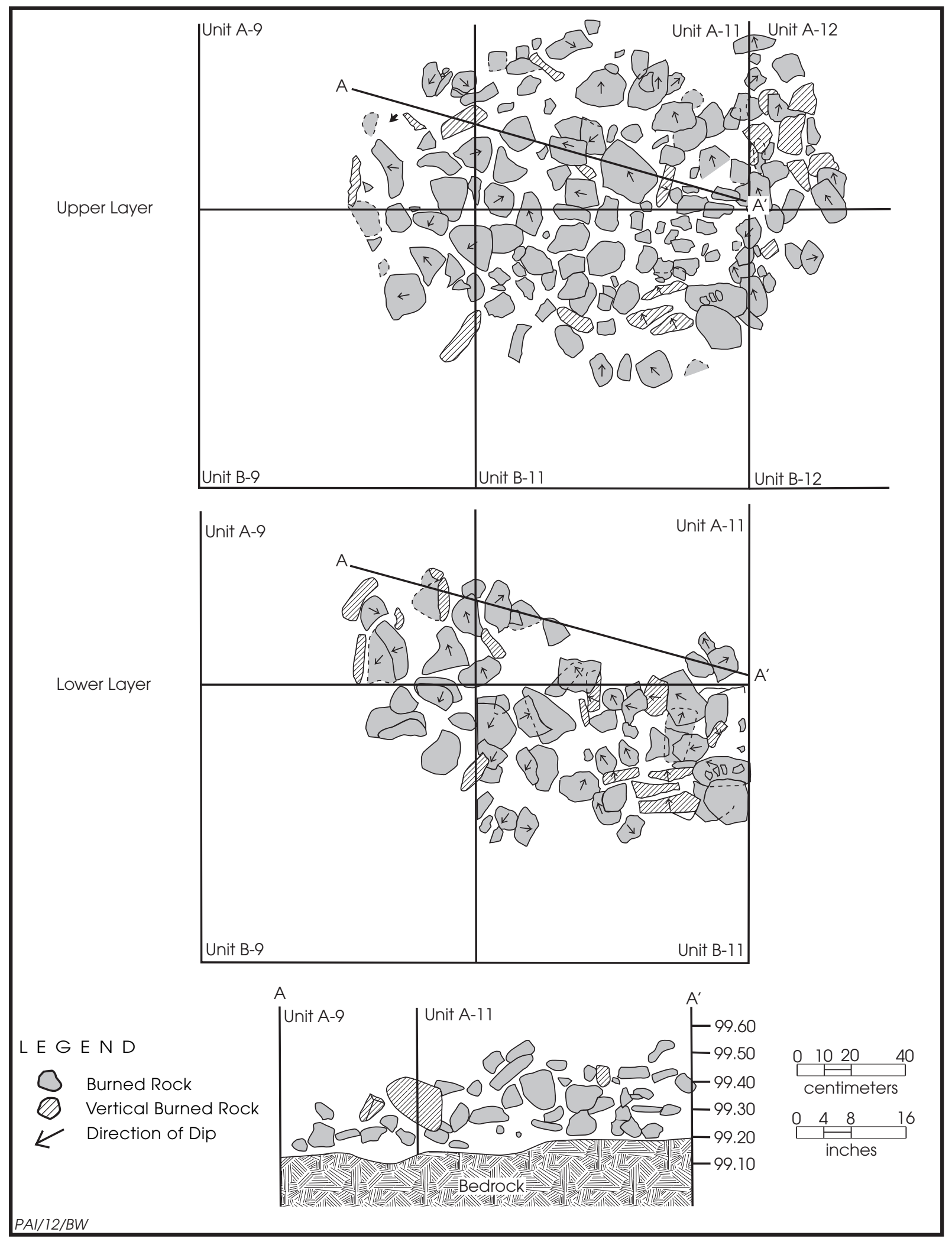

Figure 7.6. Schematic plan and profile drawings of Feature 3. 


\section{FEATURE 4}

Feature 4 was a small concentration of Rabdotus sp. shells found in the northwest corner of Unit E-4 (see Figure 7.1). The cluster consisted of 7 complete shells within a $20 \times 15-\mathrm{cm}$ area; it was partially removed before it was recognized as a feature. All of the shells were found within a 3-cm layer from 99.47 to $99.44 \mathrm{~m}$. The cluster is within Feature 1 very near the southern edge of the mound. When observed in the field, the shells were tightly grouped, but there was no evidence of a pit or disturbance of any kind. The shells do not appear to be modified.

Notably, a complete corner-notched dart point (Lot 61-1; see Chapter 5) was found about $5 \mathrm{~cm}$ west of and $4 \mathrm{~cm}$ below (at $99.40 \mathrm{~m}$ ) the snail shell cluster. The point is typed as a Castroville; it has heavily reworked blade edges and extensive surface patination. Although it was mapped in place and found in close proximity to the snail shell cluster, the dart point does not appear to have any functional association with this feature.

There are at least two alternative explanations to account for Feature 4. It is possible that the snail shell cluster is a prehistoric cultural feature, perhaps a discard pile of shells that were boiled as food or even a dump of snails collected by a child. Alternatively, the snails could be a natural accumulation, perhaps of snails that congregated in a particular spot or that were gathered by a burrowing animal. ${ }^{2}$ However, given the nature of the shallow upland sediments at this location, evidence of an ancient animal burrow or prehistoric cultural pit should not be expected to have survived. Consequently, no definitive interpretation of this feature can be offered. Since there is no way to determine if the shells were boiled or heated, the interpretation of these snails as food remains conjectural at best.

\section{ANALYSIS OF BURNED ROCK AND ARTIFACT DATA: DEFINING THE STRUCTURE OF THE BURNED ROCK MOUND}

This final section presents a comparative analysis of quantified burned rock, sediment, and artifact data from the cultural features to interpret the burned rock mound cooking facility at the Tank Destroyer site. Table 7.1 summarizes the burned rocks and sediments from general excavations and samples, and Table 7.2 summarizes the burned rock weights by feature and size class. Tables 7.3 and 7.4 summarize the charcoal and flotation samples associated with the cultural features. Detailed information on the burned rocks and samples associated with these features is presented in Appendix H. Table 7.5 lists all the cultural materials recovered by feature and unit. Detailed data tables and analytical steps are presented in Appendix H.

The investigated portion of Feature 1 had a distinctive shape, with the surface topography

Table 7.1. Burned rocks and cultural sediments at 41CV1378

\begin{tabular}{l|c|c|c|c}
\hline \multicolumn{1}{c|}{ Provenience } & $\begin{array}{c}\text { No. of Samples } \\
\text { Collected }\end{array}$ & $\begin{array}{c}\text { Total Burned } \\
\text { Rock Weight } \\
(\mathrm{kg})\end{array}$ & $\begin{array}{c}\text { Total Sediment } \\
\text { Volume } \\
\text { (liters) }\end{array}$ & $\begin{array}{c}\text { Total Sediment } \\
\text { Weight } \\
(\mathrm{kg})\end{array}$ \\
\hline General excavation* & - & $5,353.8$ & - & - \\
\hline Matrix column samples & 26 & 226.6 & 121.0 & 107.4 \\
\hline Flotation samples & 22 & 103.6 & 296.5 & - \\
\hline Burned rock samples & 36 & 26.7 & - & - \\
\hline Thermoluminescence samples & 14 & 18.9 & - & - \\
\hline Total & 98 & $5,729.6$ & 417.5 & 107.4 \\
\hline
\end{tabular}

*Burned rocks from general excavations were recorded in the field and discarded.

\begin{abstract}
2 The senior author has seen natural snail shell clusters inside animal burrows within burned rock middens. An example was observed within Feature 3-2, Area 3, at $41 \mathrm{ME} 147$ during the 2011 Texas Archeological Society Field School. Given sufficient time, all traces of an animal burrow could disappear, leaving only a cluster of snail shells within the general midden matrix.
\end{abstract}

defining a flattened mound that was about $50 \mathrm{~cm}$ tall (see Figure 7.1). Figure 7.7 shows the measured thickness of the burned rock deposits across the Feature 1 mound. The thickness varies considerably due to several factors, 
Table 7.2. Burned rock weights by feature and size class

\begin{tabular}{|c|c|c|c|c|c|}
\hline \multicolumn{6}{|c|}{ Burned Rock Weight (kg) by Size Class } \\
\hline Provenience & $<5 \mathrm{~cm}$ & $5-15 \mathrm{~cm}$ & $15-25 \mathrm{~cm}$ & $>25 \mathrm{~cm}$ & Total \\
\hline \multicolumn{6}{|c|}{ General excavation burned rocks (discarded in the field) } \\
\hline Feature 1 & 881.5 & $3,581.7$ & 386.8 & 0.0 & $4,850.0$ \\
\hline Feature 2 & 0.0 & 4.0 & 7.5 & 0.0 & 11.5 \\
\hline Feature 3 & 5.0 & 189.7 & 149.2 & 8.7 & 352.6 \\
\hline Nonfeature & 43.0 & 82.3 & 14.4 & 0.0 & 139.7 \\
\hline Total & 929.5 & $3,857.7$ & 557.9 & 8.7 & $5,353.8$ \\
\hline \multicolumn{6}{|c|}{ Sample burned rocks (BR, F, SC, and TL samples) } \\
\hline Feature 1 & 103.9 & 181.5 & 28.0 & 2.1 & 315.5 \\
\hline Feature 2 & 1.2 & 0.5 & 6.2 & 0.0 & 7.9 \\
\hline Feature 3 & 30.0 & 3.8 & 18.6 & 0.0 & 52.4 \\
\hline Nonfeature & 0.0 & 0.0 & 0.0 & 0.0 & 0.0 \\
\hline Total & 135.1 & 185.8 & 52.8 & 2.1 & 375.8 \\
\hline \multicolumn{6}{|c|}{ All burned rocks (from general excavations and samples) } \\
\hline Feature 1 & 985.4 & $3,763.2$ & 414.8 & 2.1 & $5,165.5$ \\
\hline Feature 2 & 1.2 & 4.5 & 13.7 & 0.0 & 19.4 \\
\hline Feature 3 & 35.0 & 193.5 & 167.8 & 8.7 & 405.0 \\
\hline Nonfeature & 43.0 & 82.3 & 14.4 & 0.0 & 139.7 \\
\hline Total & $1,064.6$ & $4,043.5$ & 610.7 & 10.8 & $5,729.6$ \\
\hline
\end{tabular}

Table 7.3. Charcoal samples from Feature 1

\begin{tabular}{l|c|l|c|l|c}
\hline Sample No. & Lot No. & \multicolumn{1}{|c|}{ Unit No. } & Elevation $(\mathrm{m})$ & \multicolumn{1}{|c}{ Association } & $\begin{array}{c}\text { Charred Plant } \\
\text { Remains }(\mathrm{g})\end{array}$ \\
\hline C1 & 162 & E-4 & 99.49 & Feature 1 & 0.1 \\
\hline C2 & 163 & B-11 & $99.52-99.44$ & above Feature 1 & 0.2 \\
\hline C3 & 164 & B-11 & $99.49-99.36$ & Feature 1 & 0.1 \\
\hline C4 & 165 & A-12 & 99.29 & Feature 1 & 0.4 \\
\hline
\end{tabular}

including undulations in the underlying substrate, variations in the original human deposition (i.e., discard of the burned rocks), and postdepositional disturbances and hummocks in the surface topography. But the general pattern is that the burned rock layer is thickest (50-64 cm thick) in the central part of the mound, although the burned rock layer in some core units is only $38-39 \mathrm{~cm}$ thick. The rapid drop in the thickness of the deposits is one of the characteristics defining the edge of the mound.

\section{Burned Rock Data}

Using data from Tables H-6 through H-9, Figure 7.8 compares the distributions of burned rocks in and around the mound by weight (total $\mathrm{kg}$ per $\left.\mathrm{m}^{2}\right)$ and density $\left(\mathrm{kg}\right.$ per $\left.\mathrm{m}^{3}\right)$. The total amount of burned rocks is highest (more than $100 \mathrm{~kg}$ per $\mathrm{m}^{2}$ ) in the 19 or 20 units considered to be the core area of the mound. This core area, which is ca. 10x4 m, generally corresponds with the surface topography of the mound (see Figure 7.1). The total weight of burned rocks drops dramatically outside the mound area (i.e., to less than $30 \mathrm{~kg}$ per $\mathrm{m}^{2}$ ). The burned rock density data show a similar pattern, with the density per cubic meter being generally highest ( 300 to more than $1,000 \mathrm{~kg}$ per $\mathrm{m}^{3}$ ) in the central core and very low beyond the mound (50-136 kg per $\left.\mathrm{m}^{3}\right)$.

When looking at Figure 7.8, one must consider that the burned rock weights in the Row $\mathrm{A}$ units are somewhat misleading when compared with data in the other 1x1-m units. As discussed earlier, vehicle traffic and erosion on the south 
4

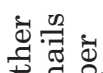
O

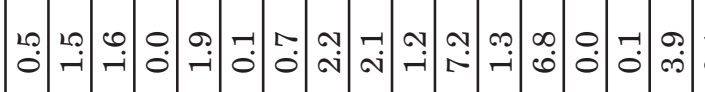

空这.

8 .

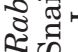

ת ת

(1)

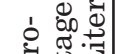

只

这要

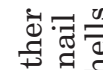

ठथี

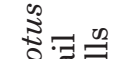

จ बiा

कृ

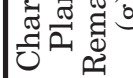

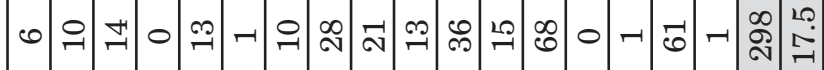

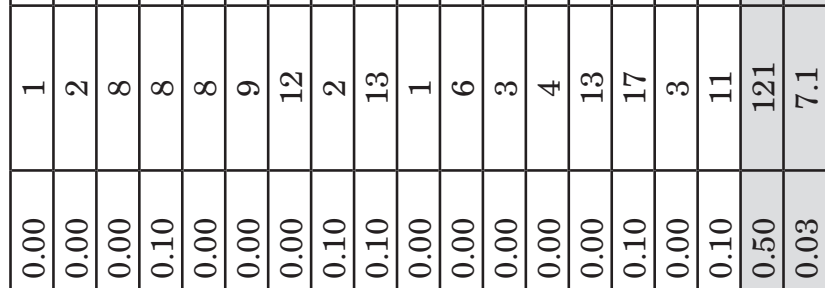

究

1

逭:

岁要

之ें

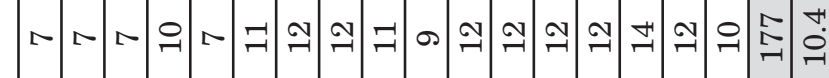

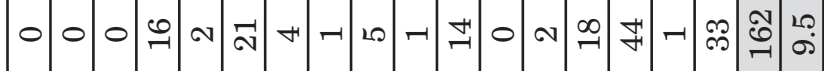

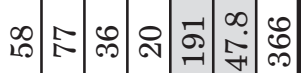

焉

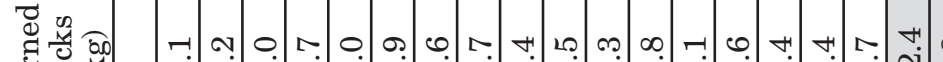
굴

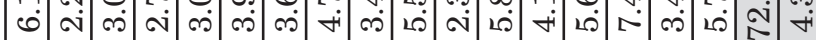

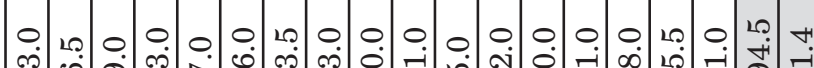

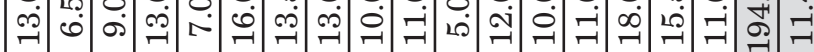

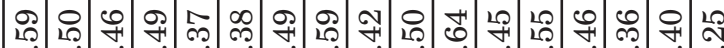

莡

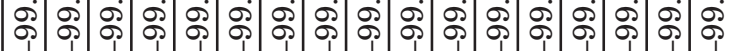

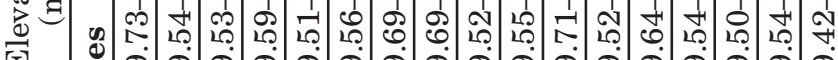

-

.

हूँ

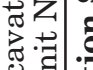

ฮั

国

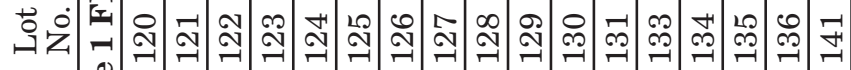

莹完

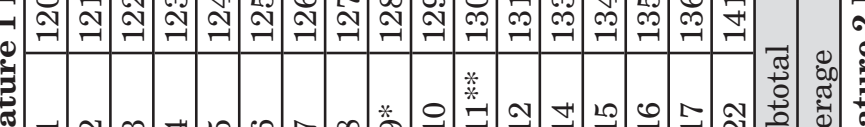

율

कृ

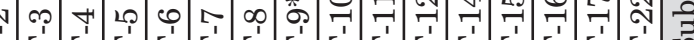

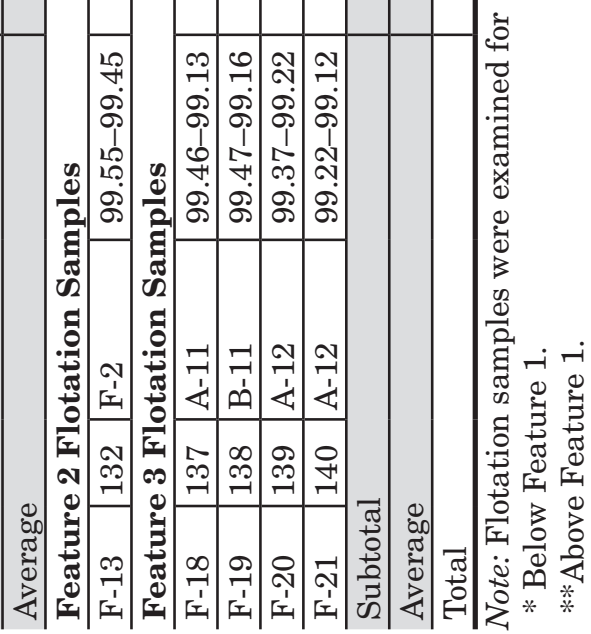


Data Recovery Investigations at the Tank Destroyer Site, Fort Hood

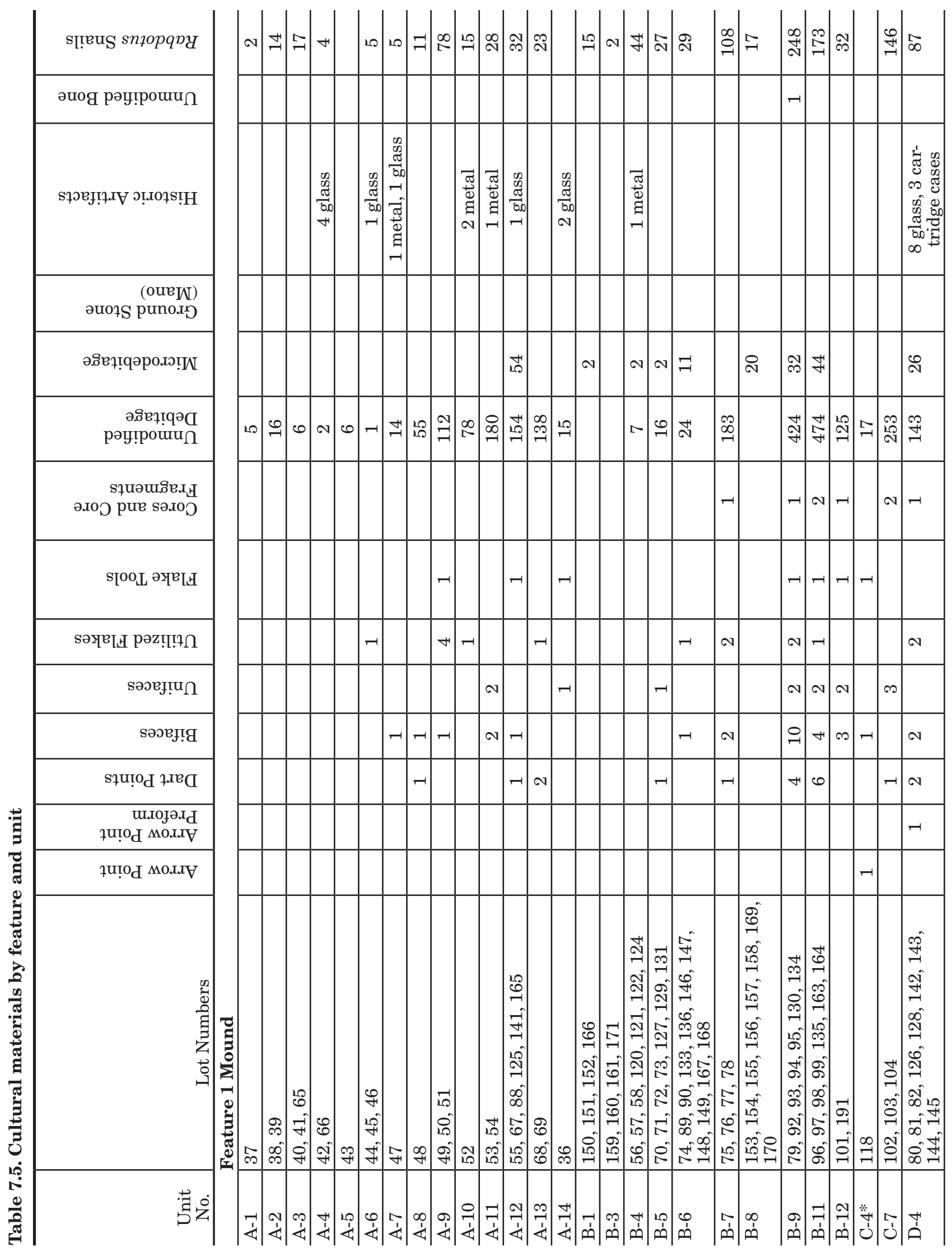




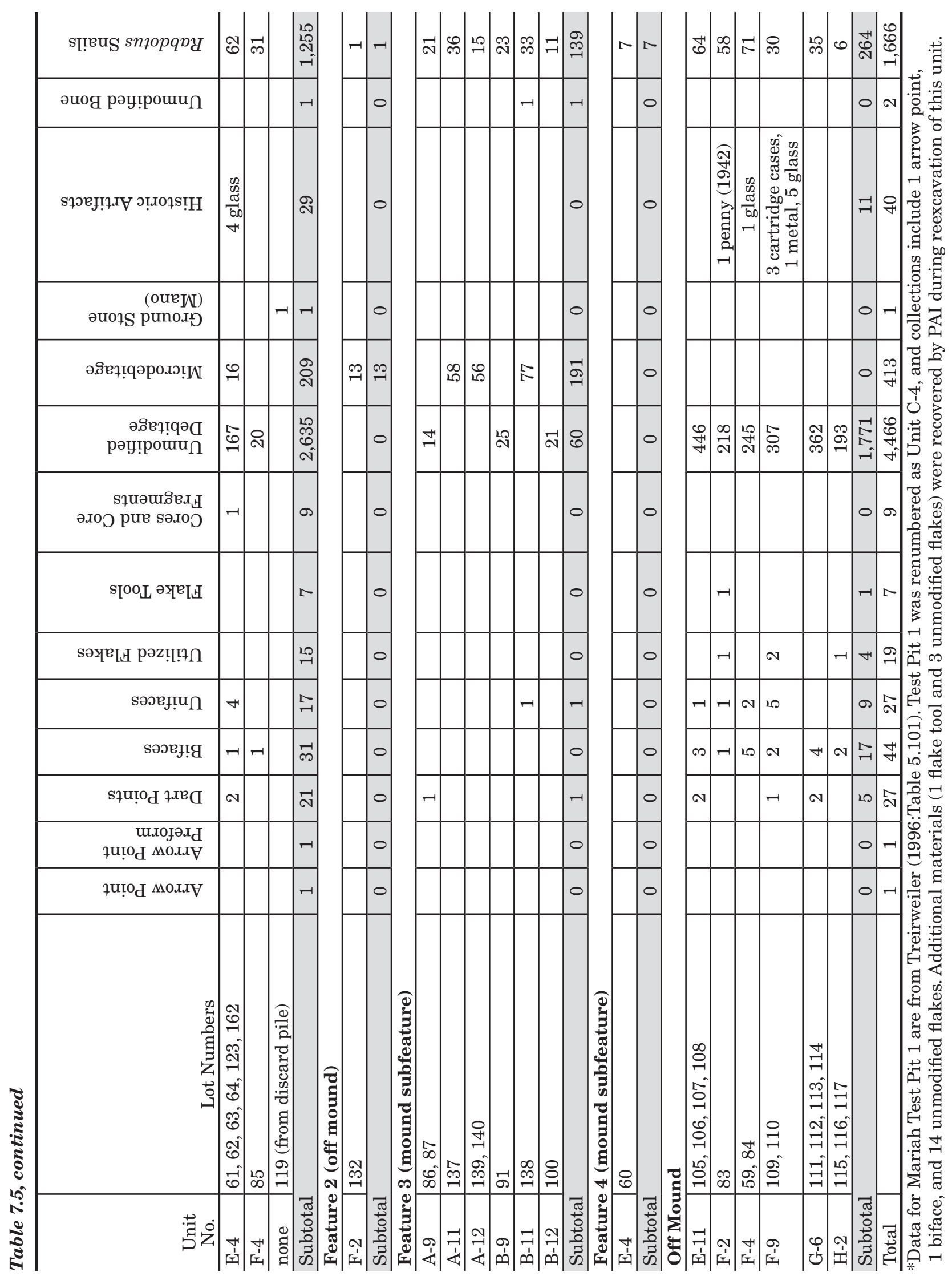




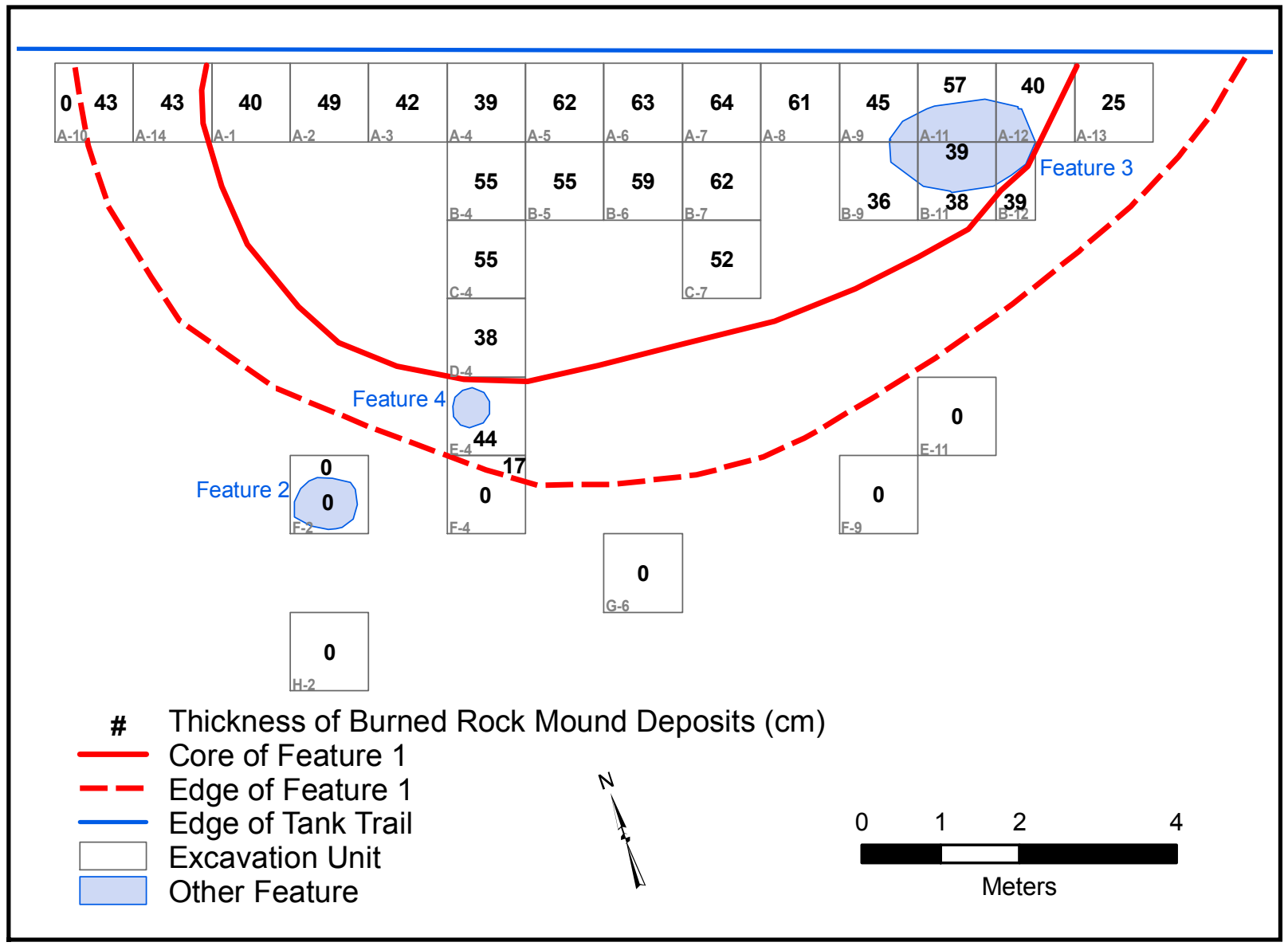

Figure 7.7. Map showing the thickness of the burned rock deposits across the Feature 1 mound. The thickness measurements in the partial units around Feature 3 are approximations because it was difficult to distinguish the top of the Feature 1 mound from the top of Feature 3.

edge of the tank trail had beveled the north edges of the Row A units, effectively reducing the volume of fill excavated from these units to about one-half of what would be in a complete $1 \times 1-m$ unit. Consequently, if the burned rock weights in the Row A units were multiplied by two, it would give a more realistic estimate of the true volume of burned rocks that would have been present before the modern disturbance. When this is taken into account, the extremely high burned rock weights seen in the Row B and Row $\mathrm{C}$ units in the core area do not look so unusual in comparison with Row A.

Looking at the quantities and densities of burned rocks allows for a quick comparison of the off-mound, mound, and earth oven areas (Table 7.6). The data show that the density of burned rocks is more than eight times higher in the burned rock mound than in the off-mound areas. The density of burned rocks in Feature 3 is about half that of the mound, but this figure would be too low if some portion of the heating element rocks were removed after the last cooking episode. It is interesting, however, that the $405 \mathrm{~kg}$ of burned rocks associated with Feature 3 is more limestone rock than is reported for experimental earth ovens (Mauldin et al. 2003:217). Dering (1999:664-666) reports that an average of $250 \mathrm{~kg}$ of rocks were used in experimental "Late Archaic" ovens $1.5 \mathrm{~m}$ in diameter and $50 \mathrm{~cm}$ deep. Leach et al. (2001:275-283) used $91 \mathrm{~kg}$ of rocks in an oven $1.3 \mathrm{~m}$ in diameter and $30 \mathrm{~cm}$ deep. And Thoms (1989:255-256) reports using $294 \mathrm{~kg}$ of rocks in a $2-\mathrm{m}$ diameter oven.

The burned rock size and weight data shown previously in Table 7.2 are more meaningful when converted to percentages as in Table 7.7. These data reveal one pattern that is interesting but certainly not unexpected. The general matrix in Feature 1 (the burned rock 


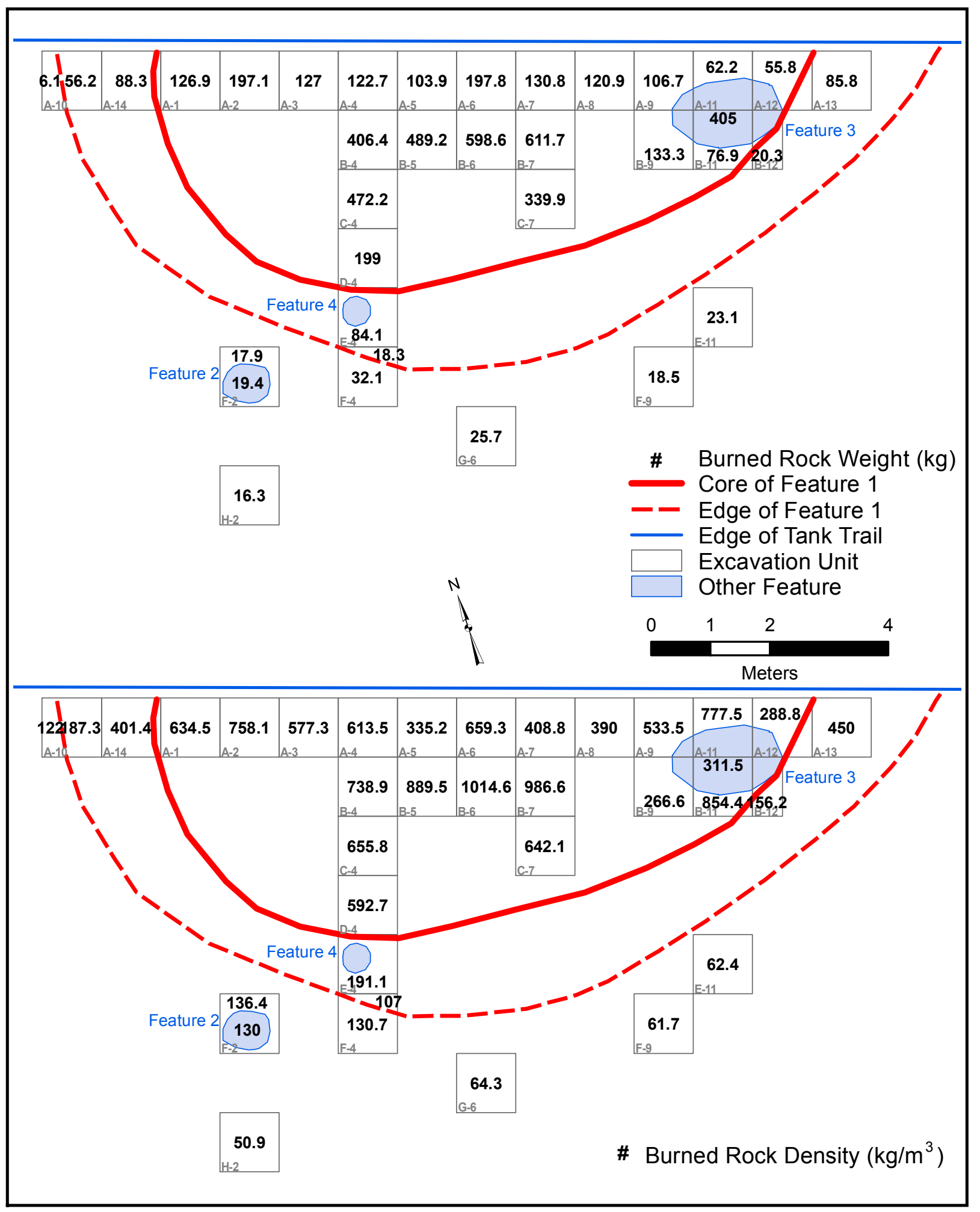

Figure 7.8. Maps showing the distribution of burned rocks by weight (top) and density (bottom) in and around the burned rock mound. The burned rock weights are by square meter, while the density numbers are converted to cubic meters based on the estimated excavation volumes. Note that the burned rocks from the three isolated matrix columns are excluded. 
Table 7.6. Burned rock weight data

\begin{tabular}{l|c|c|c}
\hline \multicolumn{1}{c|}{ Attribute } & $\begin{array}{c}\text { Feature 1 } \\
\text { Burned Rock Mound }\end{array}$ & $\begin{array}{c}\text { Feature 3 } \\
\text { Earth Oven }\end{array}$ & $\begin{array}{c}\text { Off-Mound } \\
\text { Excavations* }\end{array}$ \\
\hline Total weight of burned rocks $(\mathrm{kg})$ & 5165.50 & 405.0 & 139.70 \\
\hline Burned rock frequency data & \multicolumn{3}{|c}{} \\
\hline Hand-excavation area $\left(\mathrm{m}^{2}\right)$ & 23.25 & 2.0 & 6.40 \\
\hline Frequency of burned rocks $\left(\mathrm{kg} / \mathrm{m}^{2}\right)$ & 222.20 & 202.5 & 22.00 \\
\hline Burned rock density data & & & 1.9 \\
\hline Estimated excavation volume $\left(\mathrm{m}^{3}\right)$ & 8.57 & 311.5 & 72.40 \\
\hline Density of burned rocks $\left(\mathrm{kg} / \mathrm{m}^{3}\right)$ & 602.70 & & \\
\hline
\end{tabular}

*Feature 2 burned rocks are excluded from the off-mound excavations.

mound) is dominated by small burned rocks, while Feature 2 (the off-mound hearth) and Feature 3 (earth oven) contain abundant larger rocks. Approximately 92 percent of the Feature 1 rocks are under $15 \mathrm{~cm}$ in maximum size, and only 8 percent are burned rocks over $15 \mathrm{~cm}$. In contrast, 75 percent of the Feature 2 rocks and 43.5 percent of the Feature 3 rocks are larger than $15 \mathrm{~cm}$. Very large rocks (those over $25 \mathrm{~cm}$ ) were found only in the Feature 3 earth oven, and these were limited to a few large slabs that were used to line the cooking pit.

\section{Matrix Column Data}

The burned rock, sediment, and snail shell data derived from the five matrix column samples (see Table H.11) constitute a data set useful for defining the general matrix characteristics of the burned rock mound. Figure 7.9 shows the designations and elevations of the matrix sample columns from the Feature 1 burned rock mound. It indicates the weights of burned rocks and sediment from each matrix column sample, along with the total number of Rabdotus snail shells recovered in two different formats. Each matrix sample is from a $30 \times 30 \mathrm{~cm}$ square and is $10 \mathrm{~cm}$ thick. For the top and bottom levels that were less than $10 \mathrm{~cm}$ thick, the data are volume-adjusted data to account for thinner levels. Figure 7.10 compares the ratios of burned rocks to sediment, burned rocks to Rabdotus sp. shells, and sediment to Rabdotus sp shells. Figure 7.11 shows only the adjusted burned rock and sediment data for the matrix samples as a vertical bar graph. It graphically shows how much variation there is in the relative volume of burned rocks and sediments.

Table 7.7. Percentage of size classes of burned rocks by feature*

\begin{tabular}{|c|c|c|c|c|c|}
\hline Provenience & $<5 \mathrm{~cm}$ & $5-15 \mathrm{~cm}$ & $15-25 \mathrm{~cm}$ & $>25 \mathrm{~cm}$ & Total Percent \\
\hline \multicolumn{6}{|c|}{ General excavation burned rocks (discarded in the field) } \\
\hline Feature 1 & 17.6 & 74.3 & 8.1 & 0.0 & 100.0 \\
\hline Feature 2 & 0.0 & 34.8 & 65.2 & 0.0 & 100.0 \\
\hline Feature 3 & 1.4 & 53.8 & 42.3 & 2.5 & 100.0 \\
\hline Nonfeature & 38.2 & 55.0 & 6.8 & 0.0 & 100.0 \\
\hline \multicolumn{6}{|c|}{ Sample burned rocks (BR, F, SC, and TL samples) } \\
\hline Feature 1 & 29.0 & 60.9 & 9.4 & 0.7 & 100.0 \\
\hline Feature 2 & 0.0 & 7.5 & 92.5 & 0.0 & 100.0 \\
\hline Feature 3 & 57.3 & 7.3 & 35.5 & 0.0 & 100.0 \\
\hline Nonfeature & 100.0 & 0.0 & 0.0 & 0.0 & 100.0 \\
\hline \multicolumn{6}{|c|}{ All burned rocks (from general excavations and samples) } \\
\hline Feature 1 & 18.2 & 73.6 & 8.1 & 0.0 & 100.0 \\
\hline Feature 2 & 0.0 & 24.7 & 75.3 & 0.0 & 100.0 \\
\hline Feature 3 & 8.6 & 47.8 & 41.4 & 2.1 & 100.0 \\
\hline Nonfeature & 42.7 & 51.0 & 6.3 & 0.0 & 100.0 \\
\hline
\end{tabular}

* Percentage is based on recorded weights in kilograms 
产

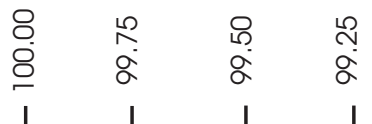
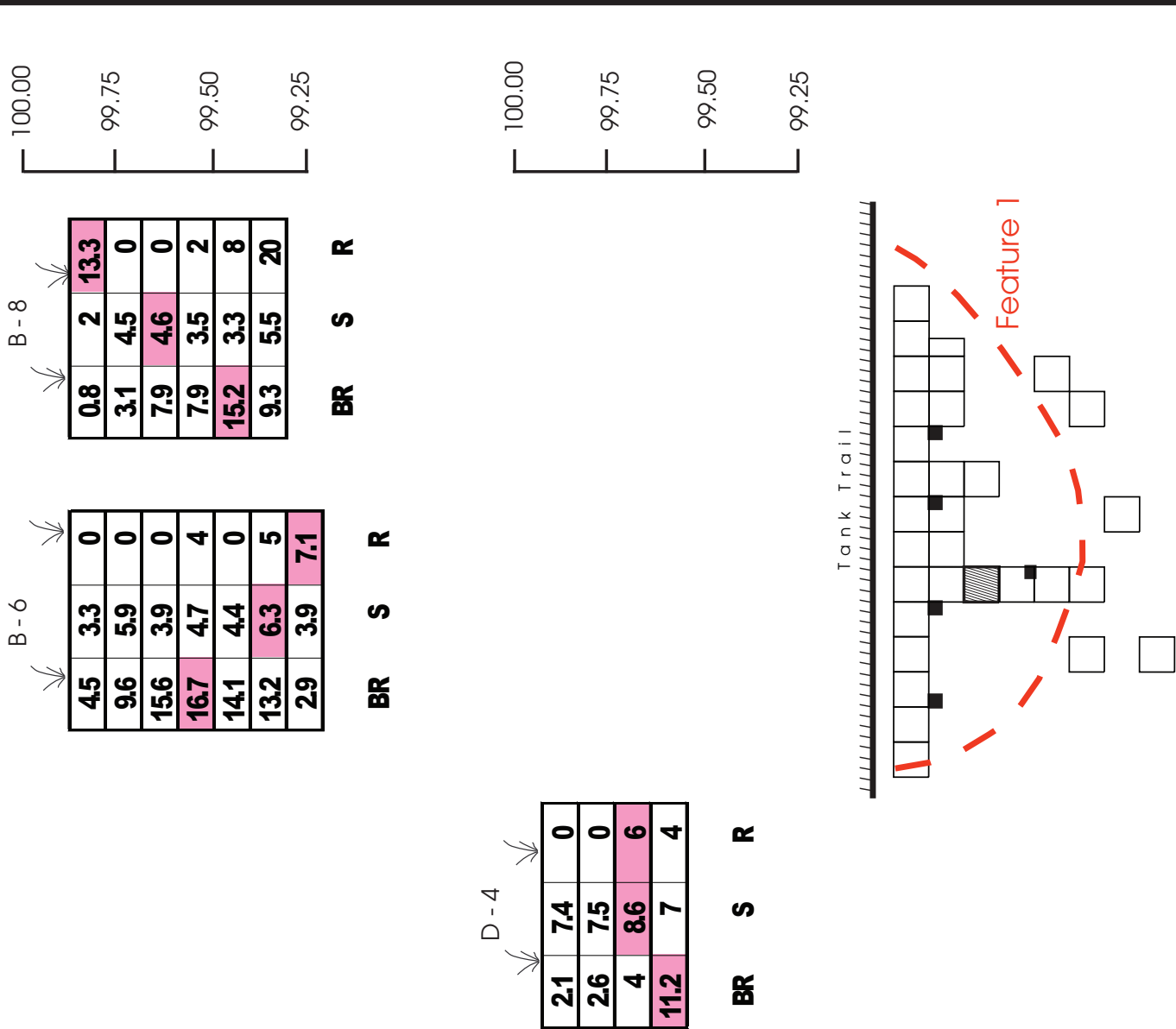

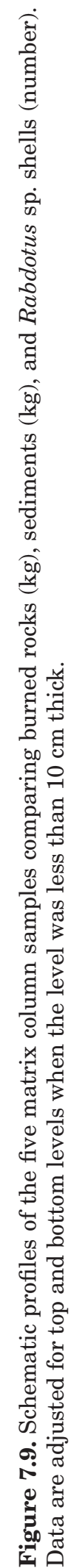
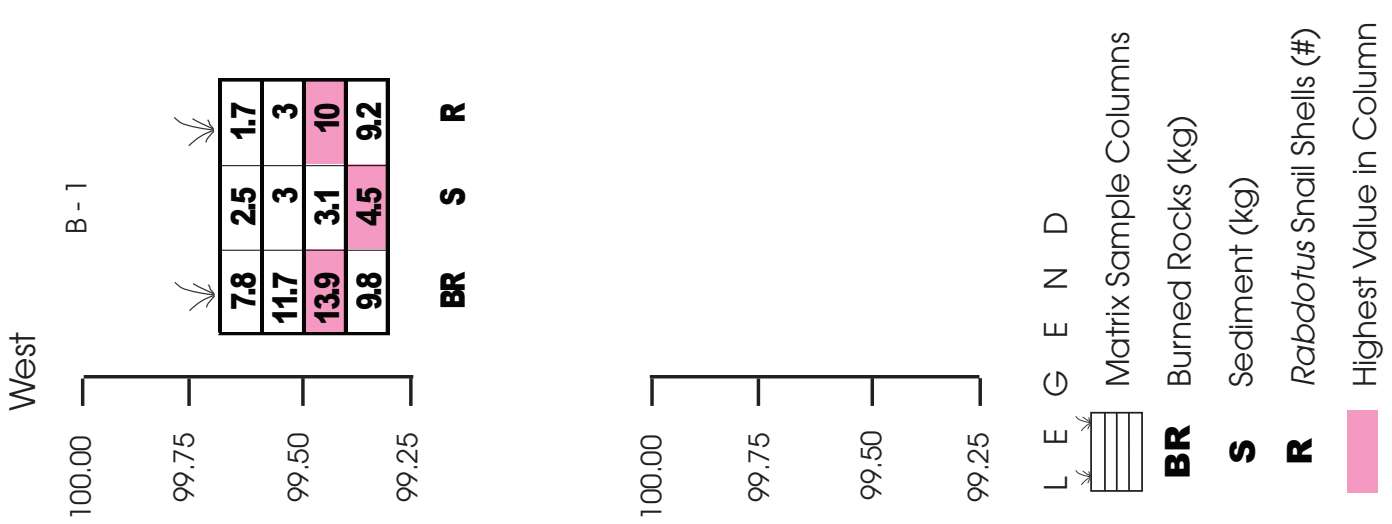


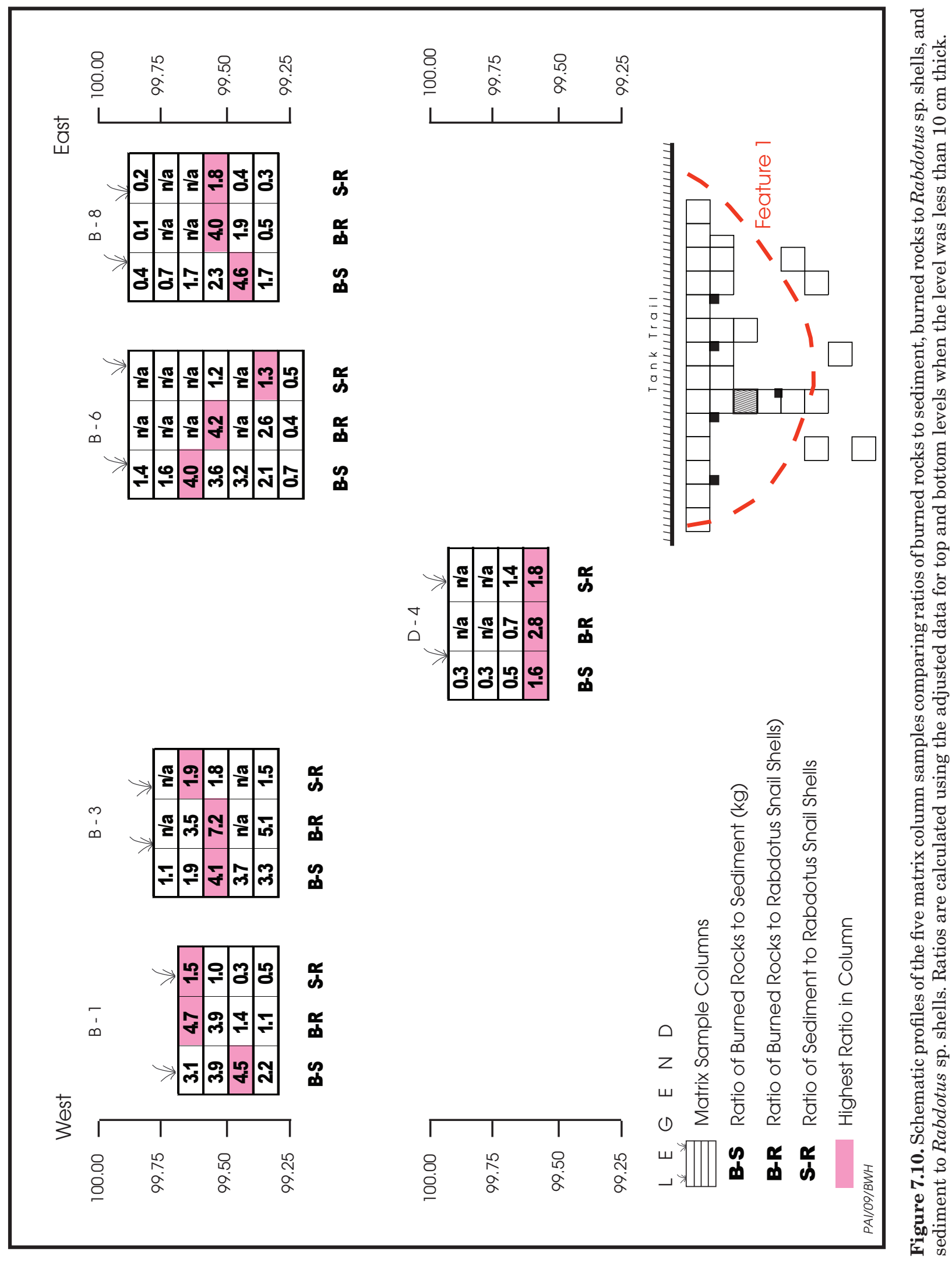


Chapter 7: Features and Spatial Analysis

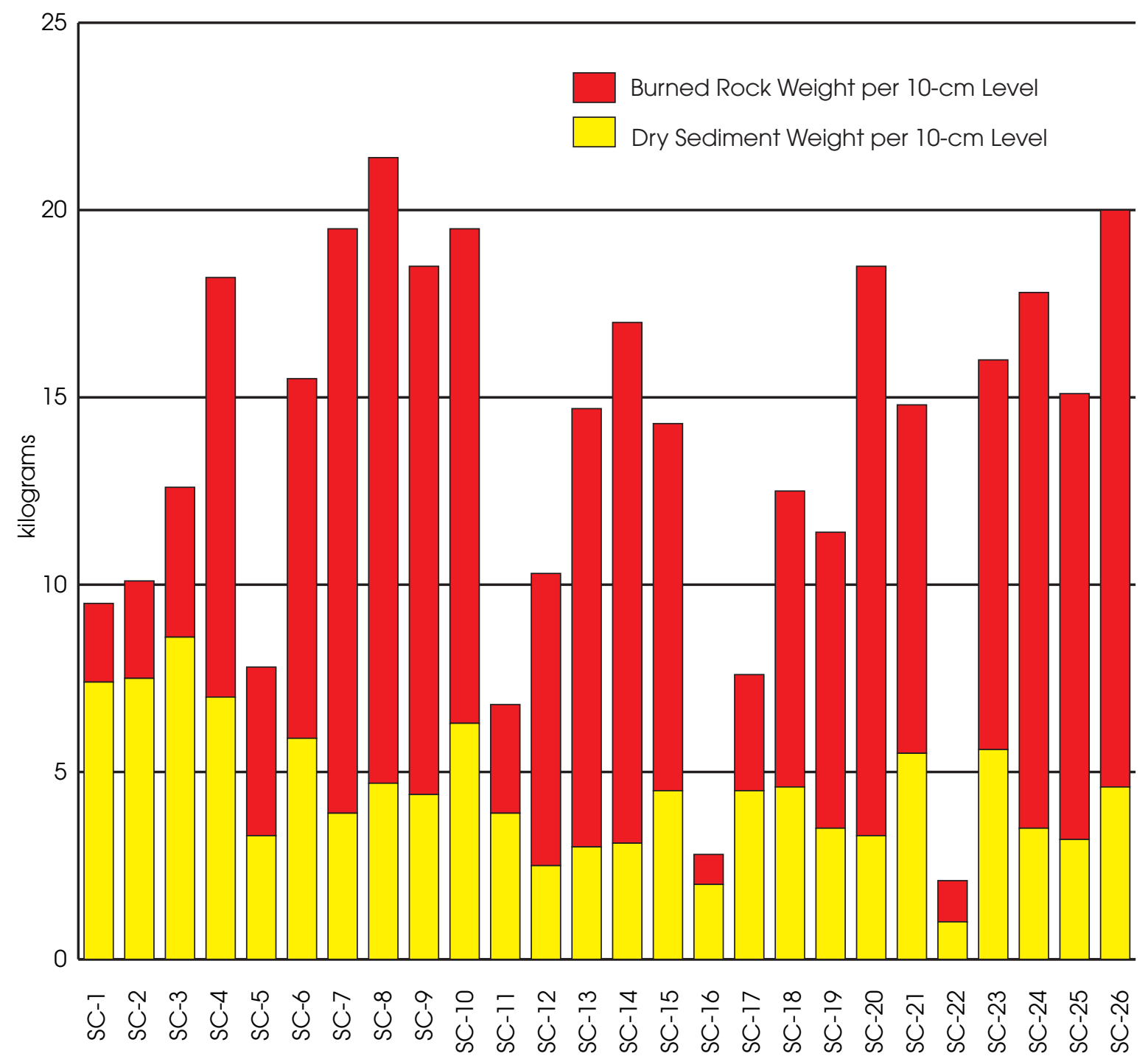

Figure 7.11. Bar graph comparing the total weight of burned rocks to sediment in all of the matrix column samples.

The patterns displayed in these graphic images appear to represent the jumbled deposits that characterize the matrix of a burned rock mound. Each matrix column is unique, and there are no clear relationships between the amount of burned rocks and sediment or the number of snail shells. Even when the volume-adjusted data for the matrix sample columns are converted to ratios, as in Figure 7.10 , there is no discernible patterning. In some ways, this data analysis "proves the obvious" in that we already know that the burned rock mound deposits are mixed by cultural activities and postdepositional processes, and upper deposits are disturbed to an unknown extent by modern activities.

\section{Artifact Data}

Only one ground stone artifact was recovered from the Tank Destroyer site: a limestone mano from the mound deposits, though it was found out of context in a backdirt pile. But grinding tools are probably grossly underrepresented relative to their true importance at this site, as is probably true at many burned rock midden sites in central Texas. Ground stone tools made of limestone are generally easy to identify if they are whole or large fragments that retain diagnostic morphological attributes, especially the distinctive angular shapes and smoothed surfaces characteristic of manos and metates. But fragments of grinding tools are notoriously 
hard to recognize when they are small, heatfractured pieces and their exteriors are coated with a thick carbonate buildup. In middens, such specimens are usually fragments of grinding tools that were worn out and recycled as hearthstones. The reuse of limestone grinding tools as hearthstones is a well-documented phenomenon in central Texas burned rock midden sites, and the ubiquity of grinding tool fragments speaks to the intensity of occupations and earth oven cooking episodes at any given site. Acknowledging the difficulties of identifying fragmented pieces of grinding tools and the fact that only one grinding tool was recovered from the Tank Destroyer site, the discussion that follows focuses solely on the chipped stone artifacts.

When one looks at the horizontal distributions of lithic debitage and chipped stone tools found within the burned rock mound (Figure 7.12), there are no discernible patterns. The locations of the chipped stone tools and debitage are seemingly random. In all likelihood, cultural trash and lithic debris was being regularly discarded onto the burned rock mound as the mound was accumulating, or these materials were inadvertently being added to the mound as sediment was borrowed from off-mound areas and piled on top of earth ovens as insulation. In both of these scenarios, the distributions of debitage and stone tools within the mound deposits are impossible to understand.

The limited off-mound sampling at the Tank Destroyer site was done to see if evidence of other domestic activities would be represented and interpretable. The horizontal distribution of chipped stone artifacts in the off-mound areas cannot be considered in any meaningful way because the area sample (just over 6 square meters) is simply too small. But a comparison of the artifact densities (per cubic meter) and frequencies (per square meter) between the mound and off-mound areas is interesting (Table 7.8). Overall, the burned rock mound yielded more than 1.5 times more artifacts than did the off-mound areas, but by frequency (per square meter) the off-mound areas yielded 2.5 times more artifacts (284.1 vs. 110.9 artifacts per $\mathrm{m}^{2}$ ). In terms of density, the $1.93 \mathrm{~m}^{3}$ volume of off-mound excavations yielded 1,807 artifacts, or 936 artifacts per $\mathrm{m}^{3}$-a density that is more than three times greater than in the mound itself, with only 284 artifacts per $\mathrm{m}^{3}$. It is somewhat surprising that the lithic artifact frequency by area would be much less in the burned rock mound because its secondary function was as a trash disposal area. The greater density by volume and frequency by area in the off-mound areas is interesting, and it seems logical to infer that the lithic materials around the mound were probably discarded or lost in close proximity to where they were being used. If so, it suggests that many domestic activities other than earth-oven cooking were occurring immediately around the burned rock mound. It is likely that some of the lithic artifacts found within the mound may have been intentionally discarded there to remove them from high-traffic paths or work areas around the midden. This is especially true of larger items that one would not want to step on, and it is notable that nine cores and core fragments were found in the mound but none were found in the off-mound areas. As suggested above, the other likely way in which lithic artifacts were introduced into the mound is when off-mound sediment was dug up and used as insulation to cover earth oven pits. This process would transport off-mound artifacts into the mound on a more or less regular basis. Unfortunately, as mound deposits accumulated and were churned up through time, these processes destroyed most of the contextual evidence that would be needed to differentiate between intentionally discarded debris (i.e., cleanup from off-mound activity areas) and fortuitously introduced debris.

The ratios of chipped stone tools to debitage are not significantly different between the mound and off-mound areas. The mound excavations produced 28.4 flakes per chipped stone tool, while the off-mound excavations produced a ratio only slightly higher at 49.2 flakes per chipped stone tool. Perhaps this similarity should be expected given the two likely processes for introducing chipped stone artifacts into the mound. In terms of assemblage diversity, the Tank Destroyer site has a fairly diverse set of lithic tools given the relatively small size of the tool sample (see Table 7.5). Bifacial tools include projectile points, knives, and an adze fragment, while the unifacial tools include knives, denticulates, various scrapers, a drill, and a notched flake. The number of tool types present in such a small tool assemblage (total number of tools $=$ 131 ) is rather notable and indicative of rich and diverse tool assemblage, which is suggestive of a great number of different activities taking place 


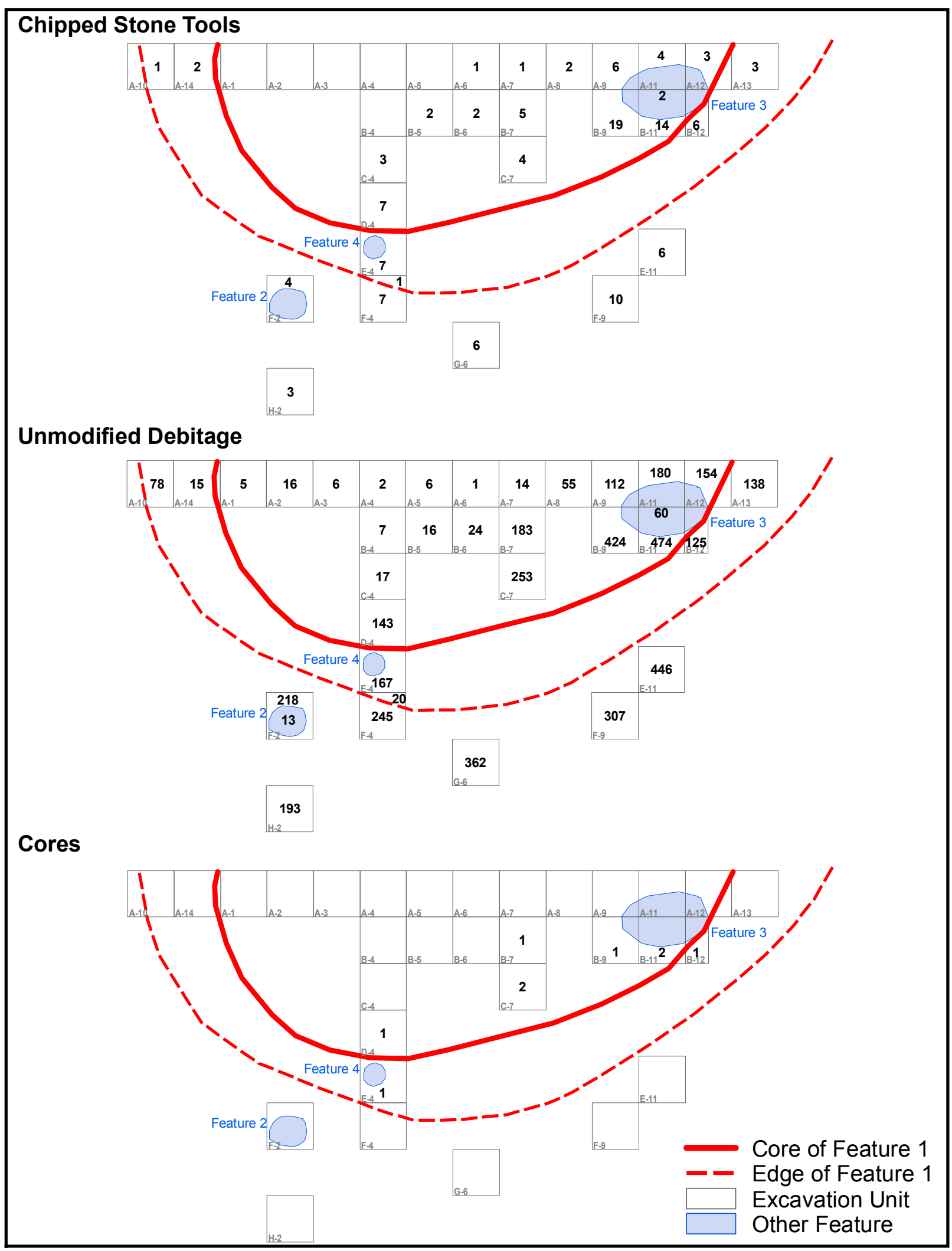

Figure 7.12. Distribution map of chipped stone tools, cores, and unmodified debitage (counts per square meter). 
Table 7.8. Density and frequency of lithic artifacts recovered from the burned rock mound, earth oven, and off-mound excavations

\begin{tabular}{l|c|c|c|c}
\hline \multicolumn{1}{c|}{ Attribute } & $\begin{array}{c}\text { Feature 1 } \\
\text { Burned Rock } \\
\text { Mound }\end{array}$ & $\begin{array}{c}\text { Feature 3 } \\
\text { Earth Oven }\end{array}$ & $\begin{array}{c}\text { Total Burned } \\
\text { Rock Mound } \\
\text { Excavations }\end{array}$ & $\begin{array}{c}\text { Total } \\
\text { Off-Mound } \\
\text { Excavations* }\end{array}$ \\
\hline Unmodified debitage & 2,635 & 60 & 2,695 & 1,771 \\
\hline Cores and core fragments & 9 & 0 & 9 & 0 \\
\hline Chipped stone tools & 93 & 2 & 95 & 36 \\
\hline Ground stone tools & 1 & 0 & 1 & 0 \\
\hline Total lithic artifacts & 2,738 & 62 & 2,088 & 1,807 \\
\hline Estimated excavation volume $\left(\mathrm{m}^{3}\right)$ & 8.57 & 1.30 & 9.87 & 1.93 \\
\hline Excavation area $\left(\mathrm{m}^{2}\right)$ & 23.25 & 2.00 & 25.25 & 6.36 \\
\hline Density of lithic artifacts $\left(\mathrm{no}\right.$ per $\left.\mathrm{m}^{3}\right)$ & 319.5 & 47.7 & 283.7 & 936.3 \\
\hline Frequency of lithic artifacts $\left(\right.$ no. per $\left.\mathrm{m}^{2}\right)$ & 117.8 & 31.0 & 110.9 & 284.1 \\
\hline
\end{tabular}

* Includes off-mound Feature 2, but no lithic artifacts were recovered.

at the site. The fact that some tool types, such as a drill and an adze, were found only in the off-mound areas suggests that these items could be indicative of primary activity areas located around the mound. But the size of the off-mound excavation sample is too small to draw any firm conclusions.

\section{Summary of the Tank Destroyer Burned Rock Mound}

Four conclusions may be drawn from the archeological data. First, we cannot determine the size and configuration of the large burned rock feature at the Tank Destroyer site with absolute certainty because a significant portion (as much as half) of this feature was destroyed before the archeological excavations occurred. ${ }^{3}$ However, a great deal of circumstantial evidence makes it highly likely that this feature was indeed an isolated burned rock mound that was generally circular to oval in plan view and had a flattened dome shape in profile. The shape and characteristics of this large feature are similar to many others on Fort Hood that are well documented as isolated burned rock mounds of that shape.

Second, this central feature is a burned rock mound with cultural debris that accumulated between 1500 B.C. and A.D. 1650 based on 16 radiocarbon dates and 17 diagnostic projectile points. However, because half of this feature was removed, we cannot be certain that it was

3 The absence of burned rocks in the tank trail suggests that a portion of the mound was actually bulldozed away during construction or maintenance of the tank trail. not in use longer than the available chronological evidence indicates. A 50 percent sample of a burned rock mound, however, is quite good, and the current chronological evidence may well represent the use life of the whole mound. If only the charcoal dates (excluding sample C-4, which dates to A.D. 1495-1651 [see Table 4.2]) and diagnostic dart points are considered, the mound feature was used primarily between 900 B.C. and A.D. 750 (see Figure 4.6).

The third conclusion is that we do not know if the burned rock mound had a central earth oven feature associated with it. While the Firebreak site (41CV595; Mehalchick et al. 2004:111-123, 141, 149) and 41CV594 indicate that multiple ovens within a mound do occur, the classic and more common configuration is a circular domed mound with one central cooking pit. a central oven feature within an isolated mound on Fort Hood would include the Feature 4 earth oven within the Area 3 mound at the Firebreak site (41CV595; Mehalchick et al. 2004:111-123, 141-149), and other earth ovens in mounds at 41CV984 and 41CV1553 (Thoms et al. 2014). The one earth oven that was discovered, Feature 3, was located on the eastern edge of the mound, 4 to $6 \mathrm{~m}$ from the hypothesized center of the mound. In all likelihood, the Tank Destroyer mound fit this classic mound model before half of it got destroyed.

The fourth conclusion is that we do not know what resources were being processed and cooked at the Tank Destroyer site, although circumstantial evidence suggests that geophytes-the underground root storage structures of bulbs, corms, rhizomes, taproots, and tubers-were 
used. Unfortunately, the poor preservation of organic remains at the Tank Destroyer site is a serious constraint for the current investigation, and there is no direct evidence that geophytes were cooked at this locality. But based on the recovery of charred plant remains from earth oven and midden contexts at many other sites on Fort Hood and elsewhere in central Texas, one may speculate that geophytes were the focus of the plant gathering and cooking activities at Tank Destroyer (see data summarized by Boyd et al. 2004:Table 8.18, Figures 9.4 and 9.5).

\section{THE STATUS OF BURNED ROCK MIDDEN STUDIES IN CENTRAL TEXAS}

It has been hypothesized for central Texas that large accumulations of burned rocks, generally known as burned rock middens, represent accumulations of residue generated by repeated cooking activities, and that the large amounts of burned rocks are related primarily to processing of plant foods (Black et al. 1997:294-301). In recent years, the plants that have come to the forefront as the leading candidates for what was being processed in middens are geophytes (Acuña 2006; Black and Thoms 2014; Dering 1999; Mehalchick et al. 2004:176-178; Raunkaiaer 1934:64; Thoms 1989:Table 2, 2008, 2009; Wandsnider 1997).

It has been suggested that large burned rock middens are associated with a broad range of cooking and other domestic activities, but that smaller burned rock mounds also exist and may represent specialized cooking features geared toward particular resources and subsistence strategies. This idea was developed using archeological evidence from Fort Hood (Bell and Coryell Counties of central Texas), and it is of considerable interest to archeological researchers (Abbott et al. 1996:583-585, 594-617; Black et al. 1997:288-289; Boyd and Mehalchick 2002; Kleinbach et al. 1995:771-775; Kleinbach et al. 1999:413-417; Mehalchick et al. 2000:212-215; Mehalchick et al. 2003:193-194). The distinction between large burned rock middens and isolated mounds may represent an important functional distinction in hunter-gatherer behavior relative to the differential use of resources, including the food plants, packing material, firewood, rocks, sediment, and water) across the landscape. Consequently, identifying the particular resources associated with burned rock middens and earth oven cooking features is critical, as is the need to distinguish the botanical materials used as fuel or packing material from the plants that were being cooked as food.

While flotation recovery of charred plant remains has been standard practice for many decades, recent advances made by the macrobotanical analysts now allow for more accurate identifications of charred botanical materials (e.g., Cortella and Pochettino 1994; Dering 2003; Oliveira et al. 2012; Piperno 2006). The use of microscopes (including scanning electron microscopes) often enables confident identifications to genus and species of very small fragments of charred plants (e.g., Smith 1984).

In addition, researchers have implemented experimental studies and specialized archeological field extraction methods and laboratory analyses to gather meaningful data on economic plants associated with burned rock features and on the burned rocks themselves. Special analyses that have been done on burned rocks include lipid (fatty acid) residue analysis, stable carbon and nitrogen isotopes, thermal demagnetization, petrography, diatoms, pollen, phytoliths, and starch grains. Much of this highly specialized research is in its infancy, and it is often very expensive, and the data interpretations are sometimes controversial (Kamiya 2011; Laurence et al. 2011; Quigg et al. 2000, 2002; Thoms and Laurence 2014; Thoms et al. 2014). But ongoing geophyte research at Fort Hood has been focusing on experimental work to understand the hydrothermal dynamics that occur within an earth oven, along with comparative modern and prehistoric phytolith and starch grain analyses (Laurence and Thoms 2014; Thoms et al. 2014). The continued application of such innovative analytical techniques will ultimately determine their utility and the importance of the archeobotanical data they produce. None of these specialized analytical techniques were attempted for the Tank Destroyer site.

After years of researching earth oven technologies all over North America, Thoms (2008) proposed a phenomenon he calls the "carbohydrate revolution." The carbohydrate revolution represents an intensification of the exploitation and use of plant foods and a broadening of foraging strategies within the North American savannahs between the southern Plains and 
southeast woodlands. Quintessential to this intensification and broadening of foraging strategies was the use of earth ovens-large rock-lined features capable of storing and releasing heat slowly over extended periods time, to render plant foods, particularly inulin-rich root foods, significantly more nutritious and palatable. The use of earth ovens and other large burned rock features had its genesis in the region nearly 9,000 years ago (Collins 1995:383; Collins 1998; Thoms 2008). The use of these features and their subsequent growth and development into burned rock middens and mounds clearly were an important aspect of a way of life that for the most part remained unchanged for thousands of years. While decades of research have noted that the use of these features was enduring and widespread, an understanding of the behavioral aspects associated with these features remains somewhat nebulous. This mystery, however, is slowly unraveling as more and more research focuses on various scales of study, from feature composition to feature distribution across the regional landscape (e.g., Black et al. 1997; Collins 1991), macrobotanical and plant microfossil remains (e.g., Dering 2003; Kamiya 2011; Thoms and Laurence 2014; Thoms et al. 2014), development of middle-range theory (e.g., Black et al. 1997; Stark 1997), and the ethnographic and ethnoarcheological records (e.g., Collins 2011; Stark 2002; Thoms 1989; Wandsnider 1997). With new technologies, methodologies, and theories, we may in fact soon be able to define and differentiate behavioral and social aspects of feature use, from simple family cookery to larger communal activities that may involve ritual and confirmation of social identity.

\section{THE TANK DESTROYER SITE AS A CENTRAL TEXAS BURNED ROCK MOUND}

One of the primary goals of the archeological data recovery at the Tank Destroyer site was to evaluate the structure, use life, and function of the large burned rock feature. The specific sets of archeological data described and analyzed earlier in this chapter are useful for characterizing the feature as a burned rock mound. We can never know for sure, but Feature 1 may have once had a single centrally located earth oven for most of its history, but the Feature 3 earth oven on its periphery indicates that the feature deviates slightly from the classic central Texas mound model. As we excavate more mound features across central Texas, it seems that the variations become the norm, making the classic model look less common. But we still want to know how the large burned rock feature at Tank Destroyer site fits into the bigger regional picture.

\section{Size and Shape of the Burned Rock Mound at the Tank Destroyer Site}

From a morphological standpoint, this discussion assumes that Feature 1 was an oval burned rock mound and that approximately half of the feature was destroyed and only the south half was investigated. ${ }^{4}$ Because a significant portion of the mound was destroyed, it is not known if this mound had a centralized cooking pit; the one earth oven pit that did survive was on the eastern periphery of the mound. It seems unlikely that all of the burned rocks were generated from the Feature 3 pit on the east edge of the mound, and there were probably other earth ovens that were destroyed. Unfortunately, it is unclear if this large feature was a classic central Texas burned rock mound with a single central cooking pit.

The size of the Feature 1 burned rock mound is calculated as follows:

- Dimensions of remaining mound $=14 \mathrm{~m}$ (east-west) by $5 \mathrm{~m}$ (north-south)

- $\quad$ Area of remaining mound $=56.5 \mathrm{~m} 2$

- Estimated area of destroyed portion of mound $=56.5 \mathrm{~m} 2$

- Estimated area of the total burned rock mound $=113 \mathrm{~m} 2$

Notably, $113 \mathrm{~m}^{2}$ is considered a minimal estimate of the area of the whole mound. If the feature was more circular than oval, a mound $14 \mathrm{~m}$ in diameter would cover an area of $153 \mathrm{~m}^{2}$.

\footnotetext{
4 It must be acknowledged that the estimate that 50 percent of the mound was destroyed could be inaccurate. It is possible that the complete feature was more circular and that the destroyed portion of the mound may have been much larger, perhaps accounting for 60-75 percent of the total mound. But the absence of burned rocks on the north side of the tank trail suggests that the 50 percent estimate is reasonable.
} 
The burned rock mounds and middens of central Texas are notorious for being jumbled masses of fire-cracked limestone, as opposed to smaller single-use burned rock features. The Tank Destroyer burned rock mound is no exception. But this does not mean that this feature is structureless. On the contrary, the Tank Destroyer mound has a characteristic domed shape and one intact remnant earth oven that attest to its structured nature. Given the chronological spread of radiocarbon dates and associated arrow and dart points, it is clear that this mound evolved over a long period, perhaps as much as 3,000 years (see Figures 4.3 and 4.6), through repetition of a single cultural behavior: earth oven cooking. Although we can never know for sure if the mound was a "center-focused midden" or which plant foods were processed in it, there is little doubt that the Tank Destroyer mound was a classic and highly structured hot rock cooking facility as described by Black and Creel (1997).

\section{Burned Rock Weights and Use-Episode Estimates}

One important way of examining burned rock mounds is to estimate the probable number of pit baking episodes that occurred. This calculation involves taking the weight of burned rocks that comprise an entire burned rock mound and dividing it by the weight of burned rocks associated with the central earth oven (or an average of multiple earth ovens). This gives the estimated number of earth oven baking episodes if one assumes that each limestone rock was used only once. But it is easy to adjust the calculations to allow for the possibility that the limestone rocks were used in multiple baking episodes before becoming fractured and being discarded. Then it is only a short step to estimate the volume of food that could be cooked in a single oven and derive a rough estimate of the overall volume of pit-baked foods represented by a single burned rock mound. The calculations of mound size and estimates of the number of cooking episodes and volume of processed foods is admittedly a theoretical exercise for the Tank Destroyer site, and its accuracy depends on the validity of many different assumptions.

To make the calculations and estimates mentioned above, the following assumptions are made (based mainly on data in Table 7.6):
- The average volume of rocks used in one earth oven cooking episode is $405 \mathrm{~kg}$.

- The limestone slabs in an earth oven could be used between one and four times each before thermal fracturing required they be discarded.

- The total weight of burned rocks in the mound (including the Feature 3 oven) is $5570.5 \mathrm{~kg}$, with an average of $220.6 \mathrm{~kg}$ per $\mathrm{m}^{2}$ across the mound.

- The area of the remaining one-half of the burned rock mound is $56.5 \mathrm{~m}^{2}$, and the estimated area of the total burned rock mound is $113 \mathrm{~m}^{2}$.

Using these assumptions, Table 7.9 presents the estimates of the number of earth oven cooking episodes. In this table, the estimates are for the hand-excavated portion of the mound only, the remaining half of the mound (includes the hand- and machine-excavated areas), and the complete burned rock mound assuming that half of it was destroyed. Assuming that the limestone rocks are used between one and four times each, the $113 \mathrm{~m}^{2}$ mound represents between 62 and 246 separate pit baking episodes. Since these cooking episodes appear to have occurred over a span of as much as 3,150 years, though concentrated within a 1,650 -year-long period between 900 B.C. and A.D. 750, it would appear that the overall use intensity of the Tank Destroyer site was relatively low.

It is likely that the use intensity of burned rock mounds in upland settings like the Tank Destroyer site would have been tied to the availability of geophyte food resources, firewood, and rocks in the immediate vicinity. Once an area was exploited intensively, people may have had to abandon the location for some time (probably many years) to allow the geophyte food resources and wood fuel to replenish. Previous research by Mauldin et al. (2003:220-231) suggests that the local availability of trees for wood fuel (especially oak in central Texas) was a critical factor determining the location and use intensity of burned rock mounds.

The next step in the calculations is to estimate the volume of food that could be cooked in an earth oven the size of Feature 3. The feature dimensions are $184 \times 132 \mathrm{~cm}$ and $39 \mathrm{~cm}$ deep. If it were a perfect elliptical cylinder, the total volume would be $185,988 \mathrm{~cm}^{3}$ or $1.85 \mathrm{~m}^{3}$. 
Table 7.9. Estimated number of cooking episodes represented by the burned rock mound at the Tank Destroyer site*

\begin{tabular}{|c|c|c|}
\hline & $\begin{array}{l}\text { Firing History of } \\
\text { Rocks (No. of Uses) }\end{array}$ & $\begin{array}{l}\text { Estimated No. of } \\
\text { Baking Episodes }\end{array}$ \\
\hline \multicolumn{3}{|l|}{ Hand-Excavated Mound Area } \\
\hline \multirow{4}{*}{$\begin{array}{l}\text { Assumes } 5,570.5 \mathrm{~kg} \text { of burned rock in the excavated mound } \\
\text { (including Feature } 3 \text { ) and } 405 \mathrm{~kg} \text { per earth oven, } \\
\text { calculated as: } 5,570.5 \mathrm{~kg} / 405 \mathrm{~kg}=13.75 \text { baking episodes. }\end{array}$} & 1 & 13.75 \\
\hline & 2 & 27.50 \\
\hline & 3 & 41.25 \\
\hline & 4 & 55.00 \\
\hline \multicolumn{3}{|l|}{$\begin{array}{c}\text { Remaining Half Mound } \\
\text { (includes hand- and machine-excavated portions) }\end{array}$} \\
\hline \multirow{4}{*}{$\begin{array}{l}\text { Assumes } 220.6 \mathrm{~kg} \text { burned rock per square meter x } 56.5 \text { square } \\
\text { meters }=12,463.9 \mathrm{~kg} \text { burned rock. } \\
\text { calculated as: } 12,463.9 \mathrm{~kg} / 405 \mathrm{~kg}=30.77 \text { baking episodes }\end{array}$} & 1 & 30.77 \\
\hline & 2 & 61.54 \\
\hline & 3 & 92.31 \\
\hline & 4 & 123.08 \\
\hline \multicolumn{3}{|l|}{ Total Mound Area } \\
\hline \multirow{4}{*}{$\begin{array}{l}\text { Assumes } 220.6 \mathrm{~kg} \text { burned rock per square meter x } 113 \text { square } \\
\text { meters }=24,927.80 \mathrm{~kg} \text { burned rock, } \\
\text { calculated as: } 24,927.8 \mathrm{~kg} / 405 \mathrm{~kg}=61.55 \text { baking episodes }\end{array}$} & 1 & 61.55 \\
\hline & 2 & 123.10 \\
\hline & 3 & 184.65 \\
\hline & 4 & 246.20 \\
\hline
\end{tabular}

* Estimates are for the excavated portion of the mound, the remaining portion of the mound, and the total mound area (estimated).

Estimating that just under half of this volume (let's say 46 percent) would be taken up by charred wood and hot rocks during a cooking episode, that leaves about $1.0 \mathrm{~m}^{3}$, or 1,000 liters (ca. 28.4 U.S. dry bushels) for the plant foods that were being cooked. Thus, the estimated volume of plants cooked in the whole burned rock mound in 62 to 246 cooking episodes would be $62,000-246,000$ liters.

It seems reasonable to assume that 1,000 liters of cooked geophytes would have been a substantial amount of food for a small hunter-gatherer group of perhaps 18 to 20 people in one to three families. It is impossible to derive precise calculations of the caloric food value of 1,000 liters of cooked geophytes because there are no caloric analyses available for the geophytes commonly recovered from prehistoric earth ovens (e.g., wild onion, eastern camas, false garlic, rain lily, dog's tooth) and we do not yet know all of the geophyte species (especially the unidentified tubers) that may have been used (Boyd et al. 2004:Figure 9.4).

The preceding discussion has admittedly focused on the purely practical aspects of earth oven cooking and the formation of burned rock mounds. This approach is an optimal foraging viewpoint that probably has some degree of validity, because people did often make decisions for practical reasons. But it also glosses over the complex social factors that probably came into play. The distinction between burned rock mounds and middens in central Texas was first recognized and described by archeologists working at Fort Hood (Abbott et al. 1996; Boyd et al. 2000; Boyd and Mehalchick 2002; and Kleinbach et al. 1995:767-775, 1999:411-417) but has been discussed by other researchers (Black et al. 1997:287-289). The broad anthropological question pertaining to these sites is: What do the differences between burned rock mounds and middens mean in terms of prehistoric social structure and use of the central Texas landscape through time? The Fort Hood evidence suggests that the differences between mounds and middens, and the variability within these feature classes, probably represents different hunter-gatherer behaviors occurring simultaneously. In Chapter 10, it is proposed that many of the hunter-gatherer behaviors relating to earth oven cooking were dictated not by the practical aspects of food production but by social factors. A great deal of the earth oven cooking that occurred throughout central Texas may have been related to various forms of communal activities, including feasting and other social rituals. 


\section{A LANDSCAPE ANALYSIS OF FORT HOOD ARCHEOLOGICAL AND ENVIRONMENTAL DATA}

Laura M. Short, Karl W. Kibler, and Douglas K. Boyd

\section{INTRODUCTION}

A large body of archeological data exists for prehistoric sites at Fort Hood, the largest single surveyed contiguous archeological landscape in central Texas. When used in conjunction with environmental data sets from Fort Hood, the archeological data offers the potential to address issues of hunter-gather adaptation and landscape use.

For this exploratory study, available archeological and environmental GIS databases were used to identify patterns in the distribution of burned rock mounds and middens at Fort Hood. Two types of landscape analyses were conducted. The first examines the environmental factors influencing the distribution of burned rock mounds and middens across the Fort Hood landscape using the existing GIS database and the ArcGIS program (Version 9.3.1) by ESRI. The second takes an independent look at the relationship between the locations of large burned rock cooking facilities on the Fort Hood lands and the presence of post oaks (Q. stellata) and Paluxy sands.

One previous study used archeological site data and GIS to conduct a landscape analysis of the Fort Hood lands. From 2002 to 2007, the Institute of Archaeology and Antiquity at the University of Birmingham, United Kingdom, conducted a "History Landscape Characterisation" project for the Cultural Resources Management Program at Fort Hood. The study, published by Barrett et al. (2007), looked at the distributions of all the prehistoric, historic, and military sites on Fort Hood in relation to the nine categories: Woodland-Related, Field Patterns, Open Land, Natural Landscape, Water-Related, Urban,
Defense, Communications, and Ritual. The resulting comparisons were made into a series of six maps (Barrett et al. 2007:Figures 45-50). But since most of the categories selected for that study relate to modern land use rather than environmental characteristics, the research shed little light on prehistoric behavior in the area that is now Fort Hood.

\section{LANDSCAPE STUDY 1: EXAMINING THE DISTRIBUTION OF BURNED ROCK MOUNDS AND MIDDENS ON FORT HOOD}

For many decades, the prehistoric cultural resources on the installation have been investigated through archeological surveys, extensive National Register eligibility testing, and limited data recovery investigations. The result is that almost the entire base has been surveyed (except for some portions of the live fire area and cantonment), creating an inventory that includes more than 1,100 prehistoric Native American sites (Barrett et al. 2007:Table 1). Of these, 60 contain burned rock mounds and 109 sites are burned rock middens. ${ }^{5}$ For as long as archeologists have been working at Fort Hood, they have wondered about the diversity of these large burned rock features and how they are distributed across the landscape. Multivariate classification, linear regression, and geographical weighted regression are statistical methods that can be used with different combinations of variables to explore the relationship between a

\footnotetext{
5 The Fort Hood CRM database provided for this analysis contains these sites: 107 middens, 2 possible middens, 58 mounds, and 2 possible mounds.
} 
diverse landscape, its ecological variations, and site locations.

This study looks at the sites with large burned rock features categorized as middens and mounds. The middens and mounds are excellent site types for a GIS study of the Fort Hood lands. As food processing centers closely linked to earth oven cooking of plant foods, it is expected that they should be closely tied to several ecological and resource variables. Unlike habitation sites, whose locations may be selected based on a complex set of cultural and ecological factors, mounds and middens are considered specialized plant processing localities, and their locations were more likely determined by environmental factors such as the availability of stone and firewood, as well as the characteristics of the sediment matrix into which earth ovens were dug. This does not imply that there were no social factors involved in the selection of locations for the earth oven cooking activities that generated the large mounds and middens (Chapter 7). However, mound and midden site locations were undoubtedly selected primarily for practical reasons related to the distribution of resources needed to accomplish specific food-processing tasks. Quite simply, you need a lot of rocks and wood to cook plant foods, particularly geophytes, in a below-ground pit, and the availability of these resources would have been an important, if not the first, consideration for prehistoric peoples were conducting these activities.

\section{The Fort Hood Study Area and GIS Database}

The Cultural Resources Management Program at Fort Hood provided the GIS database that comprises the bulk of the data used in this study. The existing Fort Hood data set represents the most complete archeological survey coverage of a large contiguous area in Texas (except Fort Bliss in West Texas). Because of the intensive survey coverage, the archeological site locations can be assumed to reflect most of the extant sites across the landscape. There are undoubtedly some archeological sites that have not been discovered (especially low-density sites and deeply buried alluvial sites along the major streams), but the Fort Hood site inventory is as good as can be expected for such a large area surveyed over many years by many different people.
The Fort Hood GIS data were used with minimal manipulation, with some exceptions, and was augmented with other data in some cases. Some data sets were obtained from other sources because the desired data or level of data did not exist in the Fort Hood GIS database (e.g., detailed soils data). Some information was obtained from other sources because a different data format was needed (e.g., Fort Hood's elevation data was provided in the form of contour lines, whereas creating slope coverage required a digital elevation model). And some additional data sets were created using ArcGIS calculations derived from variables within the Fort Hood database (e.g., distance to Paluxy sands).

The study area was defined by the boundaries of Fort Hood military reservation, a 218,458-acre property in northwestern Bell and southwestern Coryell Counties. The Fort Hood GIS database used here includes both archeological and environmental data covering all of Fort Hood. While environmental data were available for the entire base, archeological data were not because there are some unsurveyed areas (white areas in Figure 8.1). The unsurveyed tracts on Fort Hood are mostly in the central live fire area, with some in the cantonment, and they constitute approximately 17,375 acres. In addition, archeological data are not available for three types of extensively disturbed or inundated areas (grouped with the vegetation variable below), accounting for a total of 22,959 acres. These areas are excluded from most of the GIS analyses. Thus, the entire Fort Hood study area is the 218,458 -acre installation, called the "unmasked" area, while the restricted study area of 178,124 acres, representing 81.5 percent of Fort Hood, is called the "masked" area. The latter excludes the unsurveyed, extensively disturbed, and inundated areas.

While the GIS data represent relatively complete and thorough coverage of most of the base, it is not assumed that the environmental or archeological data are a perfect analog for the past landscapes on the Fort Hood lands. The archeological data are certainly not representative of all the prehistoric activities that occurred there over the past $12,000+$ years, and the modern environmental data sets reflect a landscape that has been significantly altered by many different groups who have occupied this land since the Native Americans created the burned rock mounds and middens. However, 


\section{Maps with site locations are not shown in report copies for public distribution.}

Figure 8.1. The Fort Hood landscape analysis study area. Map shows the locations of burned rock mounds, middens, scatters, and other features relative to selected vegetation groups and Paluxy sands. The white blocks indicate unsurveyed areas within Fort Hood.

while the modern vegetation is certainly not the same as it would have been in the past, it is assumed that the modern environmental variables may serve as proxy data that delineate past environmental zones. Certainly the modern analogs mean that we cannot fully understand the past ecological characteristics, but the differences between the modern environments are valid nonetheless, and there are likely to be some broad correlations between the modern environmental variables and site distributions on the prehistoric landscape.

The Fort Hood CRM program initially provided this database to the senior author to use in a GIS class taught at Texas A\&M in Spring $2010 .{ }^{6}$ The purpose of that original study was to determine if there was a relationship between the sandy Paluxy outcrops and the locations of

\footnotetext{
6 The class was Advanced Topics in GIS (ESSM/FRSC/ BAEN 652), Spatial Sciences Laboratory, Department
} of Ecosystem Science and Management. 
mounds and middens. It was concluded that the Fort Hood data were too coarse to determine if such a relationship exists. Building on this initial study, however, the senior author conducted a second round of GIS landscape analyses for Prewitt and Associates, but with a broader focus. The emphasis of this second analysis phase was still on the burned rock mounds and middens, but the objectives were expanded to look at their distributions across the entire base relative to many different environmental variables. The methods and results of both phases of the Fort Hood GIS landscape study are presented here.

All the data for the Fort Hood landscape study were analyzed using ArcGIS (version 9.3.1). Many different models were run in an exploratory fashion to look for correlations between different environmental and archeological variables. The discussions below describe the variables used and focus on those aspects of the GIS landscape analysis that proved to be the most informative. Many of the statistical terms used below are defined in the online ArcGIS Desktop 9.3 Help guide by ESRI. ${ }^{7}$

\section{Variables}

The 11 variables used in this study are described below. All rasters were set to roughly $28 \times 28 \mathrm{~m}$ pixels, as defined by the National Elevation Dataset (NED). The maps are projected in WGS 1984. The sources for these 11 variable data sets are as follows:

\begin{tabular}{ll} 
Variable & Data Source \\
\hline $\begin{array}{l}\text { Site Locations } \\
\text { Nonsite }\end{array}$ & $\begin{array}{l}\text { Provided by Fort Hood CRM } \\
\text { Racations }\end{array}$ \\
Geology & Provided by Fort Hood CRM \\
Distance to & Calculated from geology data \\
Paluxy Sands & provided by Fort Hood CRM \\
Vegetation & Provided by Fort Hood CRM \\
Distance to Post & $\begin{array}{l}\text { Calculated from post oak community } \\
\text { On Fort Hood data provided by the }\end{array}$ \\
& Nature Conservancy \\
Distance to & Calculated from USGS National \\
Streams & Hydrography Dataset \\
Soils & USGS-NRCS Soil Data Mart \\
Elevation & USGS-NAD digital elevation model \\
& from TNRIS
\end{tabular}

7 The ArcGIS Desktop 9.3 Help guide is available at http://webhelp.esri.com/arcgisdesktop/9.3/index. cfm?TopicName=welcome.

\begin{tabular}{ll} 
Variable & Data Source \\
\hline Slope & $\begin{array}{l}\text { Calculated from TNRIS elevation } \\
\text { data }\end{array}$ \\
Aspect & $\begin{array}{l}\text { Calculated from TNRIS elevation } \\
\text { data }\end{array}$
\end{tabular}

\section{Site Locations}

The locations of burned rock mound $(\mathrm{n}=55)$ and midden $(\mathrm{n}=94)$ sites were provided by the Fort Hood CRM program. No differentiation was made between types of burned rock mounds or middens. No data regarding site size or age were used in this analysis.

\section{Nonsite Locations}

Known site locations were removed from the study area, and then nonsite locations were randomly generated using the ArcGIS "Create Random Point" function. The randomly generated nonsite locations are used for statistical comparisons with known site locations.

\section{Geology}

The geology data set was provided by the Fort Hood CRM program. It includes the following 11 geological units:

Comanche Peak Limestone

Edwards Limestone

Georgetown Formation (Denton and Kiamichi clays and Fort Worth and Duck Creek limestones)

Georgetown Formation (Main Street Limestone)

Glen Rose Formation

Holocene Epoch

Paluxy Sand

Pleistocene Epoch

Walnut Clay (Gryphaeate Oyster Beds)

Walnut Clay (Lower)

Walnut Clay (Upper)

\section{Distance to Paluxy Sands}

Distance to Paluxy sands was determined by extracting the Paluxy sands polygons from the Fort Hood database geology layer, creating a separate layer, and then calculating the path distance accounting for slope. The path distance function in ArcGIS calculates the least difficult path based on horizontal and vertical factors (in this case slope), rather than a straight-line distance. This calculated distance from an archeo- 
logical site to the Paluxy sands more accurately reflects the way people would have moved across the landscape. The distance to Paluxy sands data vary from 0 to $29,550 \mathrm{~m}$ for the entire base and are displayed in $30-\mathrm{m}$ intervals.

\section{Vegetation}

The vegetation data set was provided by Fort Hood CRM program and includes the following 34 categories (names are verbatim from the database): 8

Acer grandidentatum-(Quercus muehlenbergii)/ Carex edwardsiana forest

*Bare ground

Buchloe dactyloides modified herbaceous vegetation Carya illinoinensis-Ulmus crassifolia/Elymus virginicus floodplain forest

Cephalanthus occidentalis/Ampelopsis arborea shrubland

*Developed

Disturbed herbaceous vegetation

Grassland with mulch

Juniperus ashei semi-natural forest

Juniperus ashei-Quercus buckleyi woodland

Juniperus ashei-Quercus sinuata var. breviloba woodland

Muhlenbergia reverchonii-Bouteloua curtipendulaDesmanthus velutinus herbaceous vegetation

Muhlenbergia reverchonii-Bouteloua hirsuta var. pectinata-Carex microdonta herbaceous vegetation

Platanus occidentalis-Salix nigra woodland

Prosopis glandulosa/Bouteloua curtipendulaNassella leucotricha woodland

Prosopis glandulosa-Ulmus crassifolia/Nassella leucotricha woodland

Quercus buckleyi-Fraxinus texensis-Juniperus ashei forest

Quercus fusiformis-(Celtis laevigata var. reticulata Ulmus crassifolia) woodland

Quercus fusiformis-(Quercus buckleyi)/Quercus sinuata var. breviloba-(Juniperus ashei) woodland

Quercus fusiformis/Schizachyrium scoparium woodland

Quercus fusiformis-Quercus buckleyi-Ulmus crassifolia/Schizachyrium scoparium woodland

Quercus macrocarpa-Carya illinoinensis/Cornus drummondii-Frangula caroliniana forest

Quercus muehlenbergii-Juglans major-(Ulmus rubra)/Verbesina virginica forest

Quercus sinuata var. breviloba shrubland

Quercus stellata-(Quercus marilandica-Ulmus crassifolia)/Schizachyrium scoparium woodland

Quercus stellata-(Ulmus crassifolia)/Callicarpa americana/Verbesina virginica woodland

Quercus stellata-(Ulmus crassifolia)/Sideroxylon lanuginosum/Nassella leucotricha Paluxy Sands woodland

8 Areas marked with an asterisk are devoid of natural vegetation because of extensive modern modifications or inundation.
Rhus lanceolata-Baccharis neglecta ruderal shrubland

Salix nigra/(Cephalanthus occidentalis) forest

Schizachyrium scoparium-(Sorghastrum nutans)Sporobolus compositus-Liatris mucronata herbaceous vegetation

Schizachyrium scoparium-Bouteloua curtipendulaNassella leucotricha herbaceous vegetation

Ulmus crassifolia-(Carya illinoinensis) small stream forest

Ulmus crassifolia-Celtis laevigata/Ilex decidua/ Elymus virginicus forest

*Water (i.e., permanent bodies of water where no land vegetation grows)

\section{Distance to Post Oaks}

Distance to post oaks was determined by extracting the polygons for all the Fort Hood database vegetation categories containing post oaks, creating a separate data layer, and then calculating the path distance accounting for slope. The path distance function in ArcGIS calculates the least difficult path based on horizontal and vertical factors (in this case slope), as opposed to a straight-line distance. A straightline distance and path distance may be the same in areas of low relief and gentle slopes. In rugged areas with high topographical relief, however, the path distance is always greater than the straight-line distance. Thus, the calculated path distance from burned rock mounds and middens to post oak environments more accurately reflects the way prehistoric peoples would have moved across the landscape. The distance to post oak data vary from 0 to $16,840 \mathrm{~m}$ and are displayed in 30-m intervals.

\section{Distance to Streams}

Stream data were obtained from the National Hydrography Dataset maintained by the United States Geological Survey (USGS). ${ }^{9}$ Distance to streams was determined by calculating the path distance to streams, accounting for slope. The path distance function in ArcGIS calculates the least difficult path based on horizontal and vertical factors (in this case slope) rather than a straight-line distance. This calculated distance from the burned rock mounds and middens to streams more accurately reflects the way prehistoric peoples would have moved across the landscape. The distance to stream

\footnotetext{
9 The National Hydrography Dataset is available at
} http://nhd.usgs.gov. 
data vary from 0 to $4,560 \mathrm{~m}$ and are displayed in $30-\mathrm{m}$ intervals.

\section{Soils}

Soils data were obtained from the USGS Soil Data Mart, maintained by the Natural Resources Conservation Services, part of the United States Department of Agriculture. ${ }^{10}$ The online Soil Data Mart provides access to the Soil Survey Geographic (SSURGO) database, and the available maps are vector-format digitized soil surveys linked to the National Soil Information System (NASIS) attribute database. The data set for Fort Hood contains 97 soil map units, consisting of 51 soil series and 5 nonsoil units (e.g., gravel pits, urban land, and water). The soils data for the Fort Hood lands includes the following units (map unit symbol: component name or local phase):

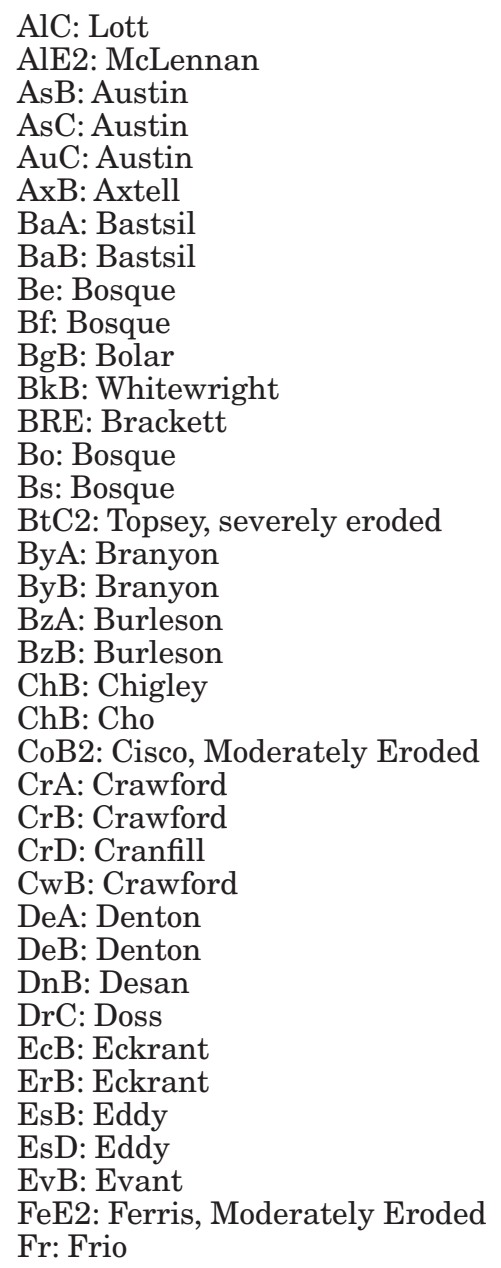

10 The Soil Data Mart databases may be downloaded from http://soildatamart.nrcs.usda.gov.
Fs: Frio

GeB: Georgetown

GP: Pits, gravel

HeB: Heiden

HeC: Heiden

HfD: Heiden

HgD2: Heiden, Moderately Eroded

HoA: Houston Black

HoB: Houston Black

HoC: Houston Black

HuC: Houston Black

KrA: Krum

KrB: Krum

LeB: Lewisville

LeC: Lewisville

LF: Dumps, sanitary landfill

LgC: Altoga

LgC: Lewisville

LuC: Lewisville

LyB: Georgetown

MeD2: Menard, Moderately Eroded

MnB: Minwells

NuC: Nuff

OgB: Oglesby

PaC: Patilo

PaD: Patrick

PcA: Payne

PcB: Payne

PdB: Minwells

PrB: Purves

PVD: Purves

QU: Pits, quarry

REF: Real

RgB: Riesel

SaA: San Saba

SaB: San Saba

SeC: Seawillow

SlB: Slidell

SnB: San Saba

SPD: Speck

SsB: Speck

StB: Stephen

StC: Stephen

SuC: Stephen

TpC: Topsey

TPF: Tarrant, PE >44

TPF: Purves

Tr: Tinn

TuC: Topsey

Ty: Tinn

Ub: Urban land

VeA: Venus

VeB: Venus

VeC: Venus

W: Water

WcA: Wilson

WcB: Wilson

WcC: Wilson

WsC2: Wise, moderately eroded

\section{Elevation}

Elevation data were obtained from Texas Natural Resource Information System, part 
of the Texas Water Development Board. ${ }^{11}$ The resolution of this data is $30 \mathrm{~m}$, with a vertical accuracy of $15 \mathrm{~m}$. The projection is UTM coordinate system, NAD 83, in meters above sea level. This elevation data are from the National Elevation Dataset (NED), a seamless data set with the best available raster elevation data maintained by the USGS. ${ }^{12}$ The sources for the NED are predominately USGS Digital Elevation Models ${ }^{13}$ which are raster representations of cartographic (i.e., elevation) data. These models are interpolated from known elevation points. The elevation data vary from approximately 180 to $373 \mathrm{~m}$ above mean sea level and are displayed in $10-\mathrm{m}$ intervals.

\section{Slope}

Slope data was extracted from the elevation data set mentioned above. Slope is the angle of the steepest downslope direction from a particular location, calculated for each burned rock mound or midden site. In this case, ArcGIS automatically calculated the slope for each unit of the raster using the elevation data. The slope data vary from 0 to 33 degrees and are displayed in 2-degree intervals.

\section{Aspect}

Aspect is the direction of the slope, defined as the steepest downslope direction from a particular location on the surface. ArcGIS automatically calculated the aspect data from the elevation data set for each unit of the raster. The measurements correspond with a compass, with 0 degrees being due north and continuing counterclockwise to 360 (also due north). Flat surfaces are assigned a value of -1 . ArcGIS was used to automatically calculate aspect from elevation data. Aspect data vary from -1 to 360 degrees and are displayed in 15-degree intervals.

\footnotetext{
11 Elevation data were downloaded from the Texas Natural Resource Information System website at http://www.tnris.org/datadownload.

12 For more information on the National Elevation Dataset, see http://ned.usgs.gov/Ned/faq.asp.

13 For more information on the USGS

Digital Elevation Models, see http://eros.usgs. gov/\#/Guides/dem.
}

\section{Layer Preparation}

The Fort Hood landscape analysis data were manipulated as described above to create an integrated set of variable layers, and all projections were transformed to match the elevation layer (WGS 1984, UTM Zone 14N). A mask for the full data set was created based on the boundaries of Fort Hood to define the study area, and all layers were clipped to conform to this boundary. All continuous data were reclassified to specific intervals as defined above for each variable. All vector layers were converted to raster. A second mask was created as a comparison layer to denote areas that have not undergone archeological survey, as well as the vegetation categories called "developed (13,770 acres)," "bare ground (8,489 acres)," and "water (700 acres)." The latter are artificial locations that do not reflect the natural environment, have no vegetation, and contain no sites due to modern alterations. This second mask was used to reject point locations. Attribute data were extracted by using the ArcGIS feature "Extract Values to Point" for point and nonpoint data, and the extracted data were then manually combined in Excel to create master attribute files.

\section{Analytical Methods and Results}

This section describes the analytical methods used to study the mound and midden sites relative to the environmental data across the Fort Hood landscape. One of the first steps in the analysis process was to create a supervised classification of the landscape using ArcGIS. A technique commonly used in range management and forestry, the basic idea is to identify known locations as "training areas" and then let the program assign classifications to the rest of the landscape (i.e., the unknown portion) based on selected variables. In this case, we hypothetically know where all the sites are located, so a sample of nonsite areas must be selected to serve as nonsite training areas. The data can then used to look for correlations between different types of sites and, if correlations are found, try to understand the variable affecting them.

For this first level of analysis, we attempted to use all the site type data for Fort Hood but quickly discovered that many individual sites 
consisted of multiple subareas that contained different site types (e.g., a lithic procurement area with a rockshelter, an open campsite with a midden, and sites with multiple rockshelters and mounds) and that there were problems with consistency in the site type classification names in the database. These issues complicated matters and could not be overcome without a careful site-by-site evaluation of all problematic sites. But the Fort Hood data set also has a site variable for prehistoric sites that contained certain types of features regardless of their official site type classification (Table 8.1).

\section{Comparison of Full Study Area and Masked Study Area: Maximum Likelihood Classification}

The next step in the analysis was to take the supervised classification of the landscape and conduct a "Maximum Likelihood Classification" (MLC) using ArcGIS, to compare the unmasked and masked study areas. The goal was to determine if removing the unsurveyed and disturbed areas would improve the statistical analyses and thus be a justification to exclude those areas

Table 8.1. Feature types in the Fort Hood CRM database and nonsite locations

\begin{tabular}{l|c|c}
\hline Prehistoric Feature Types in the Fort Hood Database & $\begin{array}{c}\text { Number of Sites in the } \\
\text { Unmasked Data Set }\end{array}$ & $\begin{array}{c}\text { Number of Sites in the } \\
\text { Masked Data Set }\end{array}$ \\
\hline Burned rock scatter & 23 & 23 \\
\hline Lithic scatter & 2 & 2 \\
\hline Midden & 107 & 2 \\
\hline Midden? & 2 & 54 \\
\hline Mound & 58 & 1 \\
\hline Mound? & 2 & 1 \\
\hline Quarry & 1 & 3 \\
\hline Rockshelter & 8 & 188 \\
\hline Unknown or other & 3 & 402 \\
\hline Total & 206 & 590 \\
\hline $\begin{array}{l}\text { Number of nonsite locations identified in the } \\
\text { supervised classification }\end{array}$ & 500 & \\
\hline Total locations & 706 & \\
\hline
\end{tabular}

Once it was verified that the mound and midden data in Table 8.1 accurately reflect all the Fort Hood sites with mounds and middens, this data set was used for all subsequent analyses along with the nonsite locations identified by the supervised classification. It was unclear whether the feature designation "burned rock scatters" is accurate, but no attempt was made to verify this. However, it is certain that the other feature designations are not representative and thus not useful for analyses. There are at least 150 sites on Fort Hood that are classified as rockshelters (Barrett et al. 2007:Table 1 ), but the eight in this data set are sites that have multiple subareas and include one or more rockshelters. Similarly, the lithic scatter, quarry, unknown, and other categories in this subset are not particularly meaningful. from continued analysis. In the series of figures that follow, these data sets are designated as "Unmasked Data" and "Masked Data."The identified "training sites" delineated the groups of independent variables or "class signatures" for the predefined classes. All sites and nonsites were used to define the class signatures. Analyzed variables are geology, distance to Paluxy sands, distance to streams, vegetation, distance to post oaks, soils, aspect, and slope. A visual inspection of the resulting maps shows a difference between the two models, but it is not readily apparent whether removing the unsurveyed areas actually improves the model (Figure 8.2). But the MLC also produces a confidence table with 14 confidence levels. Level 1 represents a 100 percent chance of being correct, and Level 14 represents 0.005 percent chance of being 
Maps with site locations are not shown in report copies for public distribution.

Figure 8.2. Maximum likelihood classification comparing the predicted locations of burned rock mounds, middens, and scatters for the unmasked and masked data. The unmasked data set includes all of Fort Hood, while the masked data set excludes the unsurveyed tracts and extensively disturbed and inundated areas. 
correct. As graphed in Figure 8.3, the confidence level data show a leftward shift between the masked and unmasked classifications, depicting a clear improvement in the confidence of the masked model over the unmasked one. Thus, all subsequent models were created using the masked data.

\section{Determining the Importance of Independent Variables: Ordinary Least Squares}

\section{SITES VERSUS NONSITES}

The goal of this analysis was to determine the important variables influencing the locations of prehistoric burned rock mound and midden sites. Ordinary least squares (OLS) is a basic type of regression analysis that looks for linear correlations and creates a global model for the variables in question. In addition to the regression, ArcGIS runs several other statistics to test for errors (Tables 8.2 and 8.3). The dependent variables were sites and nonsites, and the independent variables analyzed are geology, distance to Paluxy sands, distance to streams, vegetation, distance to post oaks, soils, aspect, and slope.

As shown in Table 8.2, this OLS model explains only 9 percent of the data variation (adjusted $\mathrm{R}^{2}=0.089971$ ). As shown in Table 8.3, the important variables in the OLS analysis are slope, distance to streams, and distance to Paluxy sands. When the logistic regression was rerun using only these variables, the adjusted $\mathrm{R}^{2}$ value increased slightly to 0.90911 . Thus, these three variables explain most of the variance in the first model. Diagnostic statistics automatically run by ArcGIS showed that the regression has problems, however. First, the Kroenker statistic was significant, which indicates that the data may be nonstationary. This simply means that the data are excessively volatile (varied and complex) and that different variables are important in different sections. Second, the Jarque-Bera statistic was significant, which indicates that there may be more explanatory variables, called misspecification, which is evident by the low $R^{2}$ value. In this case, part of the problem may be that the environmental variables that are important for mounds are different than the variables important for middens. Furthermore, running a Moran's I to test for spatial autocorrelation (things that are close tend to be more alike) showed a 5 to 10 percent chance of being autocorrelated, which is a signifier of misspecification (Z score: 1.780 , p-value: 0.075 ).

\section{MOUNDS VERSUS MIDDENS}

The goal of this study is to determine the important variables influencing whether a site is a mound or a midden. Again, ordinary least squares linear regression was used for this analysis (Tables 8.4 and 8.5). Dependent variables are mounds and middens. Analyzed independent variables are geology, distance to Paluxy sands, distance to streams, vegetation, distance to post oaks, soils, aspect, and slope.

As shown in Table 8.4, this model explains 19 percent (adjusted $R^{2}=0.190005$ ) of the variance in the mound and midden locations (see Table 8.3). As shown in Table 8.5, the important variables for this analysis were vegetation, distance to Paluxy sands, and geology. When the logistic regression was rerun using only these variables, the adjusted $R^{2}$ value actually increased to 20.02218 , confirming that these are key variables in the previous model. Again the Kroenker and Jarque-Bera statistics were significant, so this model is prone to the same problems as the previous one.

\section{Determining the Importance of Independent Variables: Geographically Weighted Regression}

\section{SITES VERSUS NONSITES}

Like the OLS regression analysis, the goal of this study is to determine the important variables influencing the location of mound and midden sites. While OLS builds one equation for the whole model, Geographically Weighted Regression (GWR) builds local equations for each feature in the data set. Because of this, GWR can overcome some of the problems associated with nonstationarity that were evident in the OLS. The dependent variables are sites and nonsites. The analyzed independent variables are geology, distance to Paluxy sands, distance to streams, vegetation, distance to post oaks, soils, aspect, and slope. 


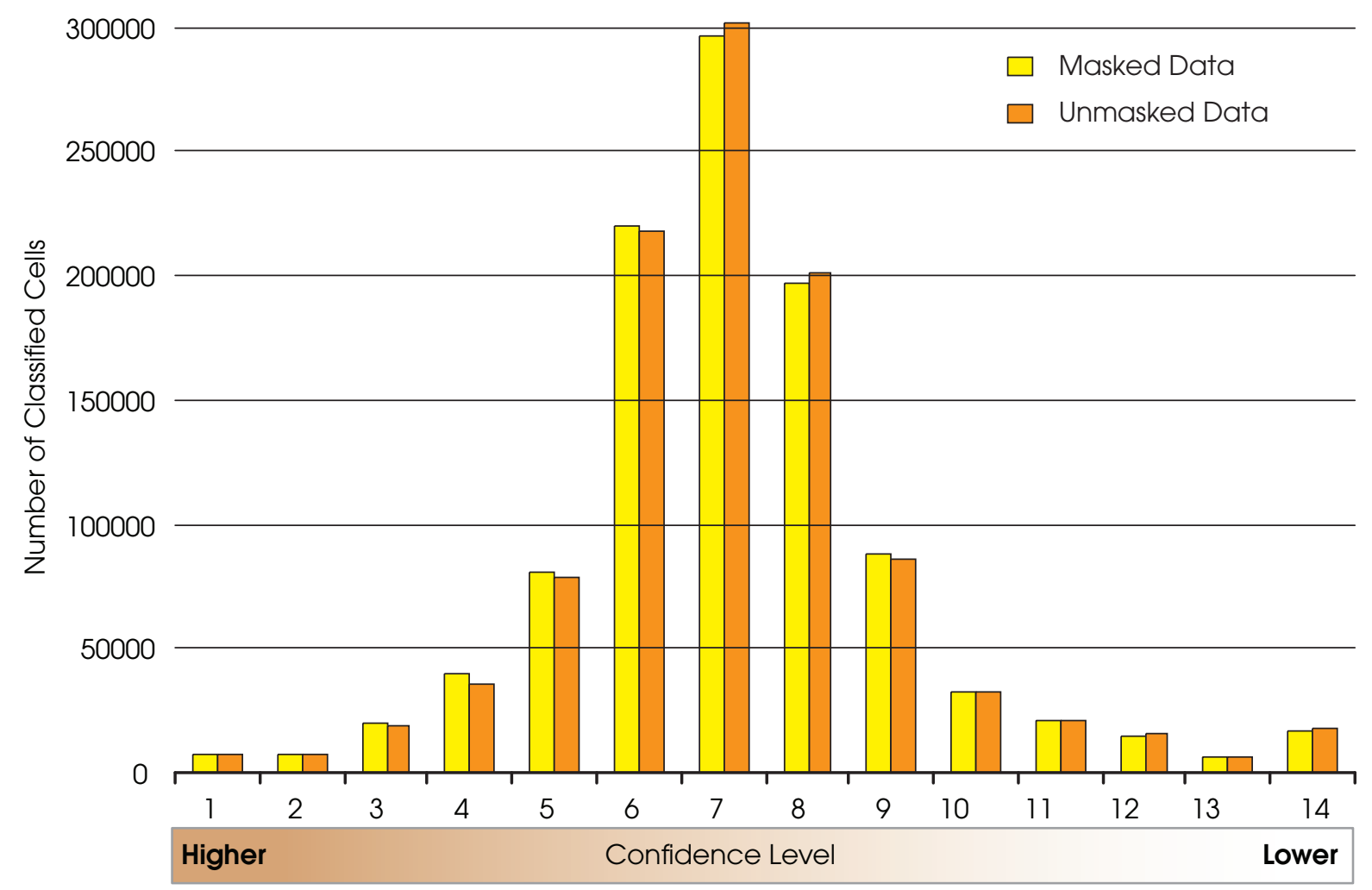

Figure 8.3. Graph of maximum likelihood classification confidence levels for the masked and unmasked data sets.

This model explained 23 percent of the variance (adjusted $\mathrm{R}^{2}=0.235534$ ). While this is still relatively low, there is little spatial autocorrelation (Moran's I: Z score $=1.08$, $\mathrm{p}$-value $=0.277)$ and clustering indicates misspecification or missing variables. Figure 8.4 is a map of the local $R^{2}$ values, and a visual inspection shows that there is low prediction value for the locations area along a north-south axis through the center of Fort Hood. This indicates that despite the low autocorrelation, there may be more factors for consideration in this corridor (range $=0.058-0.396$ ). When the logistic regression was rerun using only slope, distance to streams, and distance to Paluxy sands (the variables identified as important in the OLS model), the adjusted $\mathrm{R}^{2}$ value decreased to 0.204554 . Again there was little spatial autocorrelation (Moran's I: $\mathrm{Z}$ score $=1.11, \mathrm{p}$-value $=0.264)$. The $\mathrm{R}^{2}$ values are lower all around for this model; however, in addition to the central corridor, there is less predictive value for the south generally (range $=0.002-0.369)$.
The GWR analysis shows an improvement in predictive value for the restricted variables, but this data set probably has an issue with nonstationarity as well. There is still a large amount of unexplained variance, even though slope, distance to streams, and distance to Paluxy sands do seem to explain a large amount of this model. In addition, there appears to be something significant about the central north-south corridor that is missing in the model, but it is currently unclear what these factors might be.

\section{MOUNDS VERSUS MIDDENS}

Again, the goal of this GWR analysis was to determine the important environmental variables influencing whether a site is a burned rock mound or a midden. The dependent variables were mounds and middens, and the analyzed independent variables are geology, distance to Paluxy sands, distance to streams, vegetation, distance to post oaks, soils, aspect, and slope.

This model explains 26 percent (adjusted $\mathrm{R}^{2}=0.258227$ ) of the variance in the mounds 
Data Recovery Investigations at the Tank Destroyer Site, Fort Hood

Table 8.2. Ordinary least squares analysis diagnostics for sites and nonsites

\begin{tabular}{|c|c|c|c|c|}
\hline \multicolumn{5}{|c|}{$\begin{array}{c}\text { Diagnostics for Sites vs. Non-Sites, All Variables } \\
\text { (8 Environmental Variables) }\end{array}$} \\
\hline Statistic & Value & Statistic & Value & Explanation \\
\hline Multiple R-Squared & 0.103136 & Adjusted R-Squared & 0.089971 & $\begin{array}{l}\text { Measure of model } \\
\text { fit/performance. }\end{array}$ \\
\hline Joint F-Statistic & 7.834086 & $\begin{array}{l}\text { Prob }(>\mathrm{F}),(8,545) \\
\text { degrees of freedom }\end{array}$ & $0.000000^{*}$ & $\begin{array}{l}\text { Significant } \mathrm{p} \text {-value indicates } \\
\text { overall model significance. }\end{array}$ \\
\hline Joint Wald Statistic & 62.056646 & $\begin{array}{l}\text { Prob(>chi-squared), } \\
\text { (8) degrees of freedom }\end{array}$ & $0.000000^{*}$ & $\begin{array}{l}\text { Significant } p \text {-value indicates } \\
\text { robust overall model } \\
\text { significance. }\end{array}$ \\
\hline Koenker (BP) Statistic & 21.978724 & $\begin{array}{l}\text { Prob(>chi-squared), } \\
\text { (8) degrees of freedom }\end{array}$ & $0.004955^{*}$ & $\begin{array}{l}\text { Significant p-value indicates } \\
\text { biased standard errors; use } \\
\text { robust estimates. }\end{array}$ \\
\hline Jarque-Bera Statistic & 88.029816 & $\begin{array}{l}\text { Prob(>chi-squared), } \\
\text { (2) degrees of freedom }\end{array}$ & $0.000000^{*}$ & $\begin{array}{l}\text { Significant } \mathrm{p} \text {-value indicates } \\
\text { residuals deviate from a } \\
\text { normal distribution. }\end{array}$ \\
\hline \multicolumn{5}{|c|}{$\begin{array}{c}\text { Diagnostics for Sites vs. Non-Site, Restricted Variables } \\
\text { (3 Environmental Variables) }\end{array}$} \\
\hline Statistic & Value & Statistic & Value & Explanation \\
\hline Multiple R-Squared & 0.095843 & Adjusted R-Squared & 0.090911 & $\begin{array}{l}\text { Measure of model } \\
\text { fit/performance. }\end{array}$ \\
\hline Joint F-Statistic & 19.433801 & $\begin{array}{l}\operatorname{Prob}(>\mathrm{F}),(3,550) \\
\text { degrees of freedom }\end{array}$ & $0.000000^{*}$ & $\begin{array}{l}\text { Significant } p \text {-value indicates } \\
\text { overall model significance. }\end{array}$ \\
\hline Joint Wald Statistic & 55.471419 & $\begin{array}{l}\text { Prob(>chi-squared), } \\
\text { (3) degrees of freedom }\end{array}$ & $0.000000^{*}$ & $\begin{array}{l}\text { Significant p-value indicates } \\
\text { robust overall model } \\
\text { significance. }\end{array}$ \\
\hline Koenker (BP) Statistic & 18.753119 & $\begin{array}{l}\text { Prob(>chi-squared), } \\
\text { (3) degrees of freedom }\end{array}$ & $0.000307^{*}$ & $\begin{array}{l}\text { Significant p-value indicates } \\
\text { biased standard errors; use } \\
\text { robust estimates. }\end{array}$ \\
\hline Jarque-Bera Statistic & 90.120952 & $\begin{array}{l}\text { Prob(>chi-squared), } \\
\text { (2) degrees of freedom }\end{array}$ & $0.000000^{*}$ & $\begin{array}{l}\text { Significant } p \text {-value indicates } \\
\text { residuals deviate from a } \\
\text { normal distribution. }\end{array}$ \\
\hline
\end{tabular}

*Significant statistic

and middens, which is closer to what was predicted by OLS. This model, however, has a 5 to 10 percent chance of spatial autocorrelation (Moran's I: Z score $=1.73$, p-value $=0.083$, again indicating possible misspecification. Figure 8.5 is a map of the GWR for mounds and middens, and a visual inspection of the local $R^{2}$ values shows that the sites across the northern portion of Fort Hood have slightly lower values, but the range is much tighter than for the other models (range $=0.202-0.256$ ). Thus, even though explanatory value is still low, this model has more consistent explanatory value. When the logistic regression was rerun using only vegetation, distance to Paluxy sands, and geology (the variables identified as important in the OLS model), the adjusted $\mathrm{R}^{2}$ value decreased to 0.222399 . For this model, there was a high chance of spatial autocorrelation
(Moran's I: Z score $=3.76, \mathrm{p}$-value $=0.0001$, indicating a high probability of misspecification that would render this model unreliable. This model also has the same large range of $\mathrm{R}^{2}$ values (range $=0.126-0.326$ ), with the more significant values again favoring the southern portion of Fort Hood.

For the models including all variables, the GWR did not seem to show an improvement over the OLS. In terms of explanatory value, however, it showed that the GWR model has a better distribution than other models. Running the variables determined important in OLS showed a high probability of spatial autocorrelation, thus rejecting the notion that the GWR model for all variables is completely unreliable. Misspecification is still a problem, but it is unlikely that nonstationarity is an issue for the placement of mounds versus middens in this GWR. 
Chapter 8: A Landscape Analysis of Fort Hood Archeological and Environmental Data

Table 8.3. Statistics for ordinary least squares analysis comparing environmental variables with sites and nonsites

\begin{tabular}{l|c|c|c|c|c|c|c|c}
\hline \multicolumn{7}{c}{ Statistics for Model Running All Variables (8 Environmental Variables) } \\
\hline \multicolumn{1}{c|}{ Variable } & Coefficient & StdError & t-Statistic & Probability & Robust_SE & Robust_t & Robust_Pr & VIF \\
\hline Intercept & 0.274886 & 0.070986 & 3.87237 & $0.000130^{*}$ & 0.073673 & 3.731154 & $0.000222^{*}$ & - \\
\hline Vegetation & 0.003054 & 0.002376 & 1.285644 & 0.19912 & 0.002376 & 1.28561 & 0.199132 & 1.170364 \\
\hline Soil & -0.000739 & 0.002547 & -0.29017 & 0.771805 & 0.002722 & -0.271544 & 0.786081 & 1.226256 \\
\hline Slope & 0.036154 & 0.006177 & 5.852888 & $0.000000^{*}$ & 0.006399 & 5.649722 & $0.000000^{*}$ & 1.062859 \\
\hline $\begin{array}{l}\text { Distance to } \\
\text { Streams }\end{array}$ & -0.000455 & 0.000103 & -4.432758 & $0.000014^{*}$ & 0.000118 & -3.8676 & $0.000133^{*}$ & 1.036404 \\
\hline $\begin{array}{l}\text { Distance to } \\
\text { Post Oaks }\end{array}$ & 0.000004 & 0.000007 & 0.537779 & 0.590958 & 0.000007 & 0.552209 & 0.581039 & 1.139731 \\
\hline $\begin{array}{l}\text { Distance to } \\
\text { Paluxy Sands }\end{array}$ & -0.000006 & 0.000003 & -1.93847 & 0.053078 & 0.000003 & -2.01838 & $0.044033^{*}$ & 1.502337 \\
\hline Geology & -0.000619 & 0.007264 & -0.085223 & 0.9321 & 0.006963 & -0.088906 & 0.929174 & 1.354358 \\
\hline Aspect & -0.000267 & 0.000175 & -1.520637 & 0.128945 & 0.000186 & -1.431091 & 0.15299 & 1.028774 \\
\hline
\end{tabular}

\begin{tabular}{l|c|c|c|c|c|c|c|c}
\hline \multicolumn{7}{c}{ Statistics for Model Running Restricted Variables (3 Environmental Variables) } \\
\hline \multicolumn{1}{c|}{ Variable } & Coefficient & StdError & t-Statistic & Probability & Robust_SE & Robust_t & Robust_Pr & VIF \\
\hline Intercept & 0.243692 & 0.042875 & 5.683789 & $0.000000^{*}$ & 0.046513 & 5.239224 & $0.000000^{*}$ & - \\
\hline Slope & 0.036825 & 0.006014 & 6.123624 & $0.000000^{*}$ & 0.006288 & 5.856011 & $0.000000^{*}$ & 1.008385 \\
\hline $\begin{array}{l}\text { Distance to } \\
\text { Streams }\end{array}$ & -0.000441 & 0.000101 & -4.358695 & $0.000019^{*}$ & 0.000114 & -3.857054 & $0.000138^{*}$ & 1.006685 \\
\hline $\begin{array}{l}\text { Distance to } \\
\text { Paluxy Sands }\end{array}$ & -0.000005 & 0.000003 & -1.809374 & 0.070942 & 0.000003 & -1.798523 & 0.072647 & 1.014357 \\
\hline
\end{tabular}

* Significant variables.

\section{Summary}

The results show that there appears to be some relationship between the environmental variables and the mounds and middens, with less correlation between the environmental variables and the sites and nonsites.

For sites vs. nonsites, the OLS model explains less than 10 percent of the variance, and identifies slope, distance to streams, and distance to Paluxy sands as being significant variables. The statistics also identify the model as possibly being nonstationary and misspecified. The GWR analysis on the same data sets adjusts for the nonstationarity and improves the explanatory value to 23 percent, but there still appears to be some misspecification issues. An examination of the local $R^{2}$ values shows the central north-south corridor has a particularly low explanatory value, so an examination of other variables in that area could be worthwhile.

For mounds vs. middens, the OLS model explains roughly 20 percent of the variance, and identifies slope, distance to streams, and distance to Paluxy sands as being significant variables. The OLS statistics for this model also indicate that it may have issues with being nonstationary and misspecified. While the GWR does not particularly improve the explanatory value, it does show a relatively even distribution of local $\mathrm{R}^{2}$ values, indicating that the nonstationarity is probably accounted for. But there still appears to be some misspecification issues. GWR on the restricted variables results in an unreliable model, bringing into question the OLS identification of variable significance. Of particular interest here, however, is the fact that mounds and middens do appear to have statistically significant environmental variables influencing their locations.

Given the low explanatory values for both the OLS and GWR models, there is plenty of room for development and future research in Fort Hood landscape studies. This would, of course, include looking at a broader range of different variables and using different statistical methods to analyze the data. It is quite likely that there are some important environmental variables that were not considered in this study. Further manipulations of the raw data may reveal missing key environmental variables that could be meaningful. Examples would be creat- 
Table 8.4. Ordinary least squares analysis diagnostics for burned rock mounds and middens

\begin{tabular}{|c|c|c|c|c|}
\hline \multicolumn{5}{|c|}{$\begin{array}{c}\text { Diagnostics for Mounds vs. Middens, All Variables } \\
\text { (8 Environmental Variables) }\end{array}$} \\
\hline Statistic & Value & Statistic & Value & Explanation \\
\hline Multiple R-Squared & 0.233204 & Adjusted R-Squared & 0.190005 & $\begin{array}{l}\text { Measure of model fit/ } \\
\text { performance. }\end{array}$ \\
\hline Joint F-Statistic & 5.398283 & $\begin{array}{l}\text { Prob }(>\mathrm{F}),(8,142) \\
\text { degrees of freedom }\end{array}$ & $0.000006^{*}$ & $\begin{array}{l}\text { Significant p-value indicates } \\
\text { overall model significance. }\end{array}$ \\
\hline Joint Wald Statistic & 71.312369 & $\begin{array}{l}\text { Prob(>chi-squared), } \\
\text { (8) degrees of freedom }\end{array}$ & $0.000000^{*}$ & $\begin{array}{l}\text { Significant p-value indicates } \\
\text { robust overall model } \\
\text { significance. }\end{array}$ \\
\hline $\begin{array}{l}\text { Koenker (BP) } \\
\text { Statistic }\end{array}$ & 25.565572 & $\begin{array}{l}\text { Prob(>chi-squared), } \\
\text { (8) degrees of freedom }\end{array}$ & $0.001246^{*}$ & $\begin{array}{l}\text { Significant p-value indicates } \\
\text { biased standard errors; use } \\
\text { robust estimates. }\end{array}$ \\
\hline Jarque-Bera Statistic & 8.919987 & $\begin{array}{l}\text { Prob(>chi-squared), } \\
\text { (2) degrees of freedom }\end{array}$ & $0.011562^{*}$ & $\begin{array}{l}\text { Significant } p \text {-value indicates } \\
\text { residuals deviate from a normal } \\
\text { distribution. }\end{array}$ \\
\hline \multicolumn{5}{|c|}{$\begin{array}{c}\text { Diagnostics for Mounds vs. Middens, Restricted Variables } \\
\text { (3 Environmental Variables) }\end{array}$} \\
\hline Statistic & Value & Statistic & Value & Explanation \\
\hline Multiple R-Squared & 0.218174 & Adjusted R-Squared & 0.202218 & $\begin{array}{l}\text { Measure of model fit/ } \\
\text { performance. }\end{array}$ \\
\hline Joint F-Statistic & 13.673802 & $\begin{array}{l}\operatorname{Prob}(>\mathrm{F})(3147) \text { degrees } \\
\text { of freedom }\end{array}$ & $0.000000^{*}$ & $\begin{array}{l}\text { Significant p-value indicates } \\
\text { overall model significance. }\end{array}$ \\
\hline Joint Wald Statistic & 69.645855 & $\begin{array}{l}\text { Prob(>chi-squared), } \\
\text { (3) degrees of freedom }\end{array}$ & $0.000000^{*}$ & $\begin{array}{l}\text { Significant p-value indicates } \\
\text { robust overall model } \\
\text { significance. }\end{array}$ \\
\hline Koenker(BP)Statistic & 17.331049 & $\begin{array}{l}\text { Prob(>chi-squared), } \\
\text { (3) degrees of freedom }\end{array}$ & $0.000604^{*}$ & $\begin{array}{l}\text { Significant p-value indicates } \\
\text { biased standard errors; use } \\
\text { robust estimates. }\end{array}$ \\
\hline Jarque-Bera Statistic & 10.901515 & $\begin{array}{l}\text { Prob(>chi-squared), } \\
\text { (2) degrees of freedom }\end{array}$ & $0.004293^{*}$ & $\begin{array}{l}\text { Significant } p \text {-value indicates } \\
\text { residuals deviate from a normal } \\
\text { distribution. }\end{array}$ \\
\hline
\end{tabular}

* Significant statistic

ing variables to estimate land productivity for certain plant types or to rank streams by order instead of lumping them all together.

On a related note, the scale of data resolution may be a factor in the OLS and GWR models presented here. Determining a relationship between Paluxy sands and site locations, for example, most likely will require a finer resolution than is available with the existing data set (as discussed in the following section). Perhaps this could eventually be augmented using onground surveys to map Paluxy sediments at a finer scale.

Other statistical analyses could reveal patterns not yet apparent in these models and fine-tune those that are. Nonlinear regression techniques may also be appropriate for the data, but many of these require that the data be analyzed with high-end statistical programs such as S-plus or R. Additionally, tests of sev- eral inductively based models in a homogenous environment using a weighted regression and $\chi^{2}$ reveal techniques that may be applicable here (Whitley 2006). It also is possible that some types of simple analyses could be done to test problems proposed by this study (e.g., testing whether the data is nonstationary by dividing the study area up into several regions and seeing if logistic regression reveals the importance of different variables).

And finally, existing Fort Hood data includes absolute chronological evidence that could provide a measure of age control for some types of sites and features in the Fort Hood inventory (as of the end of 2011, there were over 480 radiocarbon dates obtained from hundreds of sites). Introducing the temporal element to the archeological site data would make the data analyses more complicated, but it is certainly a research direction worth exploring. 
Chapter 8: A Landscape Analysis of Fort Hood Archeological and Environmental Data

Table 8.5. Statistics for ordinary least squares analysis comparing environmental variables with burned rock mounds and middens

\begin{tabular}{|c|c|c|c|c|c|c|c|c|}
\hline \multicolumn{9}{|c|}{$\begin{array}{l}\text { Statistics for Model Running All Variables } \\
\text { (8 Environmental Variables) }\end{array}$} \\
\hline Variable & Coefficient & StdError & t-Statistic & Probability & Robust_SE & Robust_t & Robust_Pr & VIF \\
\hline Intercept & 1.217275 & 0.131268 & 9.273197 & $0.000000^{*}$ & 0.11681 & 10.42099 & $0.000000^{*}$ & - \\
\hline Vegetation & 0.017264 & 0.004504 & 3.832941 & $0.000197 *$ & 0.004525 & 3.815397 & $0.000210^{*}$ & 1.035907 \\
\hline Soil & -0.000619 & 0.004758 & -0.130015 & 0.89673 & 0.00399 & -0.15505 & 0.876997 & 1.312183 \\
\hline Slope & -0.005499 & 0.010225 & -0.537762 & 0.591589 & 0.009194 & -0.598057 & 0.550757 & 1.079136 \\
\hline $\begin{array}{l}\text { Distance to } \\
\text { streams }\end{array}$ & -0.000048 & 0.000223 & -0.213245 & 0.831442 & 0.000179 & -0.265351 & 0.791128 & 1.053057 \\
\hline $\begin{array}{l}\text { Distance to } \\
\text { post oaks }\end{array}$ & 0 & 0.000016 & 1662 & 0.988319 & 0.000015 & -0.0 & 538 & 1.271743 \\
\hline $\begin{array}{l}\text { Distance to } \\
\text { Paluxy Sands }\end{array}$ & -0.000013 & 0.000007 & -1.90507 & 0.058793 & 0.000006 & -2.126964 & $0.035144^{*}$ & 1.978745 \\
\hline Geology & 0.053392 & 0.016282 & 3.279255 & $0.001318^{*}$ & 0.013904 & 3.839935 & $0.000192^{*}$ & 1.5264 \\
\hline Aspect & -0.000501 & 0.000321 & -1.558691 & 0.121305 & 0.00028 & -1.791307 & 378 & 1.107442 \\
\hline \multicolumn{9}{|c|}{$\begin{array}{l}\text { Statistics for Model Running Restricted Variables } \\
\text { (3 Environmental Variables) }\end{array}$} \\
\hline Variable & Coefficient & StdError & t-Statistic & Probability & Robust_SE & Robust_t & Robust_Pr & VIF \\
\hline Intercept & 1.102889 & 0.101282 & 10.889241 & $0.000000^{*}$ & 0.091804 & 12.013465 & $0.000000^{*}$ & - \\
\hline Vegetation & 0.016755 & 0.004421 & 3.789777 & $0.000227^{*}$ & 0.004536 & 3.693999 & 0.000319* & 1.013399 \\
\hline $\begin{array}{l}\text { Distance to } \\
\text { Palxuy Sands }\end{array}$ & -0.000013 & 0.000005 & -2.355881 & $0.019789^{*}$ & 0.000005 & -2.489013 & $0.013916^{*}$ & 1.253475 \\
\hline Geology & 0.050357 & 0.014583 & 3.453115 & $0.000734^{*}$ & 0.012634 & 3.985947 & $0.000111^{*}$ & 1.243303 \\
\hline
\end{tabular}

*Significant variables

As a preliminary study, this landscape analysis of burned rock mounds and middens on Fort Hood was moderately successful. While far from perfect in providing explanatory frameworks for interpretation, the results do indicate some interesting directions to take Fort Hood landscape analyses in the future.

\section{LANDSCAPE STUDY 2: POST OAKS, PALUXY SANDS, AND BURNED ROCK FEATURES AT FORT HOOD}

The purpose of Landscape Study 1 was to determine if there were any relationships between environmental variables and the burned rock mounds and middens at Fort Hood, but it was concluded that the Fort Hood GIS data only hint at some possible meaningful relationships. Landscape Study 2 was designed to look for a possible correlation between the sandy Paluxy Formation outcrops and the locations of burned rock mounds and middens, but it concluded that the Fort Hood data were too coarse to determine if such a relationship exists. The coarse-grained nature of the data may have much to do with the fact that the Paluxy Formation is not accurately mapped in the existing geology data layer of the Fort Hood GIS database. The Paluxy outcrops on Fort Hood represent the distal margin of the lithological unit in this portion of central Texas, and the Paluxy is a relatively thin bed sandwiched between the Walnut clay and Glen Rose Formations (Figure 8.6). The Paluxy's limited and discontinuous exposure is not accurately mapped on large-scale maps like the 1:250,000 Geologic Atlas of Texas-Waco Sheet. ${ }^{14}$ In fact, the Waco Sheet does not map the Paluxy Formation within the boundaries of Fort Hood, except for a small area (< ca. 100 acres) along the northern upper valley margin of Cowhouse Creek, where it enters the base. Unfortunately, this mapped distribution is known to be quite

\footnotetext{
14 For discussions of the Paluxy Formation on Fort Hood, see Abbott (1994:329-331, 1995:814-816), and Kibler (1999:41-43).
} 


\section{Maps with site locations are not shown} in report copies for public distribution.

Figure 8.4. Local $R^{2}$ values for Geographically Weighted Regression (GWR) of sites vs. nonsites. 
Maps with site locations are not shown in report copies for public distribution.

Figure 8.5. Local $R^{2}$ values for Geographically Weighted Regression (GWR) of mounds vs. middens. 


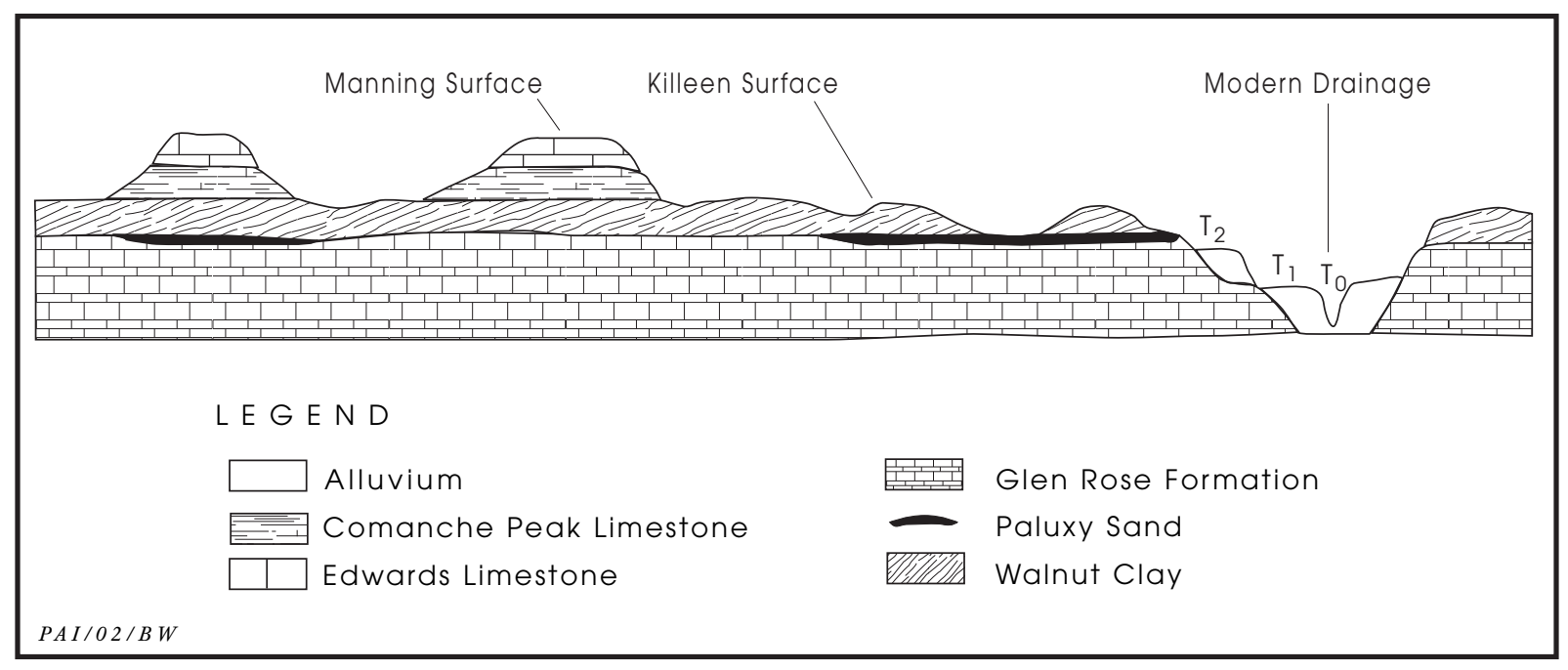

Figure 8.6. Generalized geologic cross section of the Lampasas Cut Plain in central Texas, showing the thin Paluxy Formation exposed at the lower margin of the Killeen surface (adapted from North 1992:Figure 3).

inaccurate. Field surveys over the years have encountered Paluxy outcrops in many other areas throughout the west-central portion of Fort Hood. Thus, the geologic data layer in the Fort Hood GIS database is of limited value for examining any relationship involving the Paluxy Formation. Abbott (1994:329) suggested that the mapped distributions of sandy Cisco and loamy Wise soils provide "a reasonable indication" of the areal extent of Paluxy outcrops, although he acknowledged the problems and probable inaccuracies inherent with using soil maps to define the extent of lithological units. Yet again, field surveys have encountered Paluxy outcrops and redeposited sands in areas not mapped as either of these soil series. These surveys have found that the Paluxy outcrops, though often limited in areal extent, are more common than any of the current geologic and soils data show. Finding a better or more accurate way to identify and map these outcrops would lessen the "coarse-grained" nature of the data and, consequently, provide more reliable data to examine the relationship between the Paluxy outcrops and the distributions of burned rock middens and mounds at Fort Hood.

With this in mind, another attempt to examine this relationship was made using an additional GIS data layer to augment the existing geologic and soils data and thus provide what is believed to be a more accurate picture of the spatial distribution of Paluxy outcrops at Fort Hood. The additional data set consists of a
GIS layer depicting three post oak communities on Fort Hood that was provided to Prewitt and Associates by the Nature Conservancy (Figure 8.7)..$^{15}$ The Fort Hood data set represents three distinct post oak communities: (1) on the Leon River in northern Fort Hood; (2) on the "redlands" area on the upland Manning surface; and (3) on the Paluxy sands in west-central Fort Hood. For the purposes of this study, only the latter post oak community is relevant. These communities were mapped initially using infrared aerial imagery and new technologies for differentiating between species, but they also were field checked by Nature Conservancy personnel (Charlotte Reemts, personal communication 2011).

The sandy substrate of the Paluxy Formation that occurs in western Fort Hood supports a post oak community much like the sandy Eocene formations of the inner Gulf Coastal Plain and the sandy Antlers Formation of the Western Cross Timbers support post oak communities. Field observations indicate that post oaks are almost always present and largely limited to where the Paluxy Formation and Paluxy-derived sand sheets are present in west-central Fort Hood. Post oaks do occur in some non-Paluxy environments at Fort Hood, but since the Paluxy Formation only crops out just below the Killeen surface scarp in the west-central portion of the base, the Leon River and Redlands post oak com-

\footnotetext{
15 The post oak community GIS data were provided courtesy of Charlotte Reemts, vegetation ecologist with the Nature Conservancy on Fort Hood.
} 


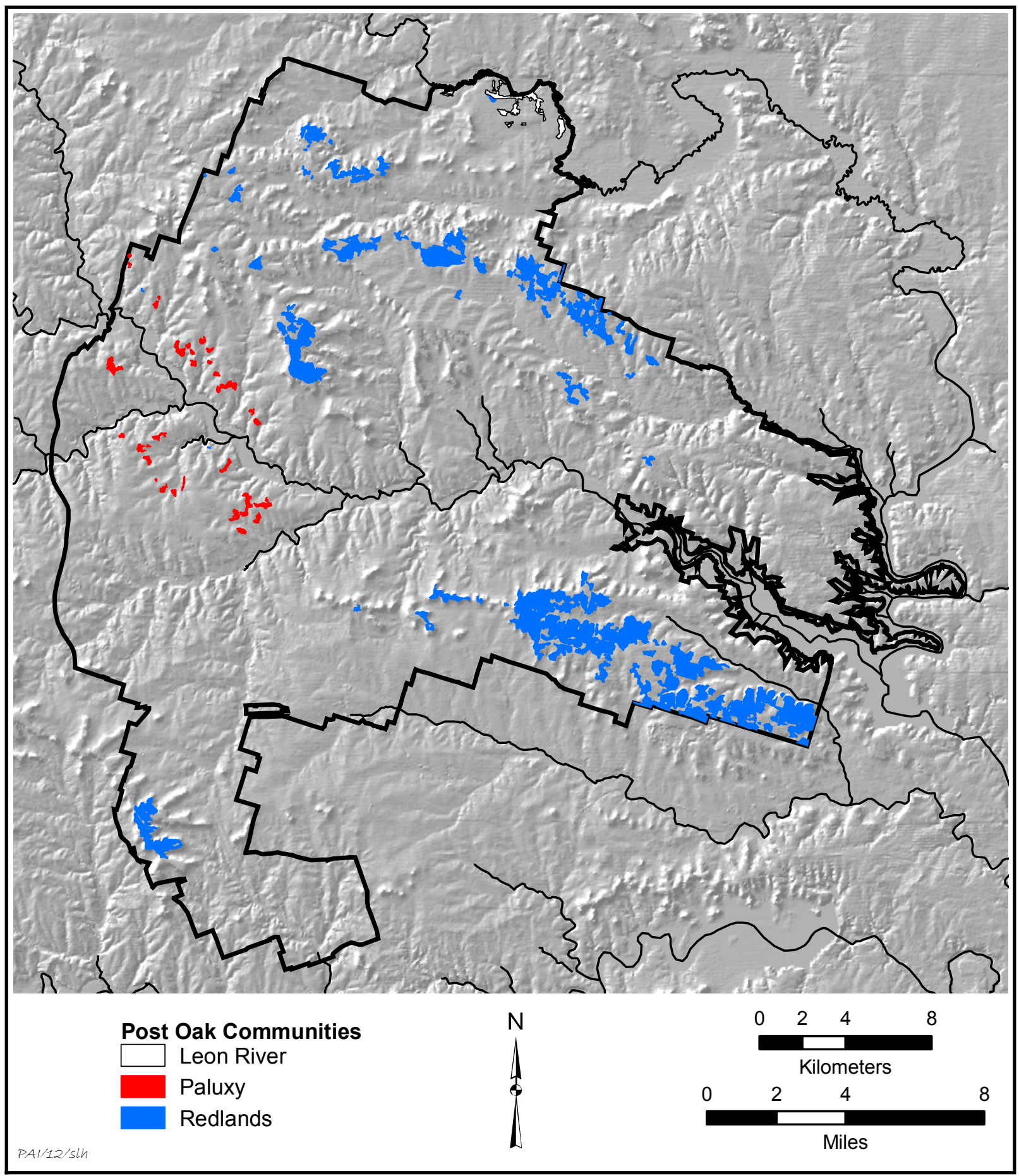

Figure 8.7. Distribution of three post oak communities on Fort Hood using data provided by the Nature Conservancy. The communities are geographically restricted to the Leon River in northern Fort Hood, the Paluxy environment (Paluxy Formation and redeposited sandy soils) in west-central Fort Hood, and the Redlands environment on the upland Manning surfaces north and south of the Cowhouse Creek drainage. 
munities are irrelevant when considering the past human use of the Paluxy environment.

Zooming in for a closer look at the westcentral portion of Fort Hood, Figure 8.8 shows the distribution of Paluxy outcrops using the geologic (Paluxy Formation) and soils (Cisco and Wise series) data. Figure 8.9 shows the distribution of Paluxy outcrops using the geologic and soils data along with the post oak data from Figure 8.7. This view is, in effect, an expanded distribution of the Paluxy environment on Fort Hood. Figure 8.10 shows the expanded Paluxy environment along with the locations of the 37 known Paluxy sites on Fort Hood. This figure distinguishes between Paluxy sites where burned rock mounds, middens, and/or earth ovens have been found and those where no such features are known.

A review of the 37 documented Paluxy sites on Fort Hood (Table 8.6) reveals that burned rock middens and mounds and earth ovens are present at 19 sites (51 percent) 41CV319, 41CV594, 41CV595 (Firebreak site), 41CV947, 41CV984, 41CV988, 41CV1027, 41CV1043, 41CV1049, 41CV1093, 41CV1141, 41CV1191, 41CV1194, 41CV1283, 41CV1296, and 41CV1391, 41CV1403, 41CV1415, and 41CV1553. Not all of the 37 Paluxy sites have witnessed the same level of work or excavation, so 19 sites with burned rock mounds, middens, and earth ovens should be viewed as a minimal number. There also is a correlation between the intensity of the archeological investigations and the number of burned rock features that are found. It is likely that the limited extent of the testing at many Paluxy sites is the only reason that more burned rock mounds, middens, and earth ovens have not been found.

Two sites-41CV595 and 41CV1553-provide examples that emphasize this point. Site 41CV595, the Firebreak site, has been examined by archeologists many times, including an intensive data recovery investigation. As summarized by Mehalchick et al. (2004:Chapter 7), the site was recorded in 1984 and examined again in 1985 . During the latter investigation, an isolated burned rock mound (Feature 1) was observed based on surface evidence. The site was shovel tested in 1992, and testing in 1993 include four backhoe trenches and four hand-excavated test units. The TRC Mariah Associates' archeologists reported two burned rock middens, one of which was the isolated mound seen in 1985. The second "midden" area, called Feature 2, was discovered in a backhoe trench and hand-dug units. Then in 1995, clearing of firebreaks during a large-range fire caused extensive damage to the site. This was followed by intensive archeological data recovery investigations by Prewitt and Associates in 2000. This work discovered and investigated a central earth oven in the Feature 1 mound and a series of three clustered earth ovens (Features 8,11 , and 15) associated with Feature 2, which appears to be an incipient burned rock mound or midden. The true extent of the use intensity of the site, especially in the Feature 2 area, did not become apparent until extensive work was completed. The Firebreak site has been investigated more intensively than any of the other 36 known Paluxy sites on Fort Hood.

The second case study is $41 \mathrm{CV} 1553$, which was investigated in 1992, 1993, 1999, and 2009. The site was discovered by Mariah Associates' archeologists in 1992, and they recorded the site in 1993. They observed several burned rock scatters and a "small burned rock midden" (Feature 1) based on surface evidence only. National Register testing in 1999 by Prewitt and Associates, Inc. revealed that Feature 1 was more accurately classified as a an "occupation zone" or perhaps an "incipient midden" (Mehalchick et al. 2003:209). But additional work by Prewitt and Associates in 2009 (Thoms et al. 2014) included a ground-penetrating radar survey that led to the discovery and excavation of a very discrete small burned rock mound (Feature 8) with a central earth oven. This feature was completely buried with no surface manifestation at all, and there were no surface artifacts in the vicinity to hint at its existence.

A few observations may be offered based on a review of the burned rock features at the 37 Paluxy sites (see Table 8.6) and the two case studies described above. (1) Burned rock mounds, middens, and earth ovens are more likely to have been discovered at Paluxy sites where intensive investigations have occurred. (2) Paluxy sites where no mounds, middens, or earth ovens are known may well contain such features. (3) Features that appear as surface as scatters or concentrations of burned rocks probably represent mounds or middens that are incipient (i.e., in early stages of formation), partially exposed, or extensively disturbed. (4) All the large burned rock features that have been 


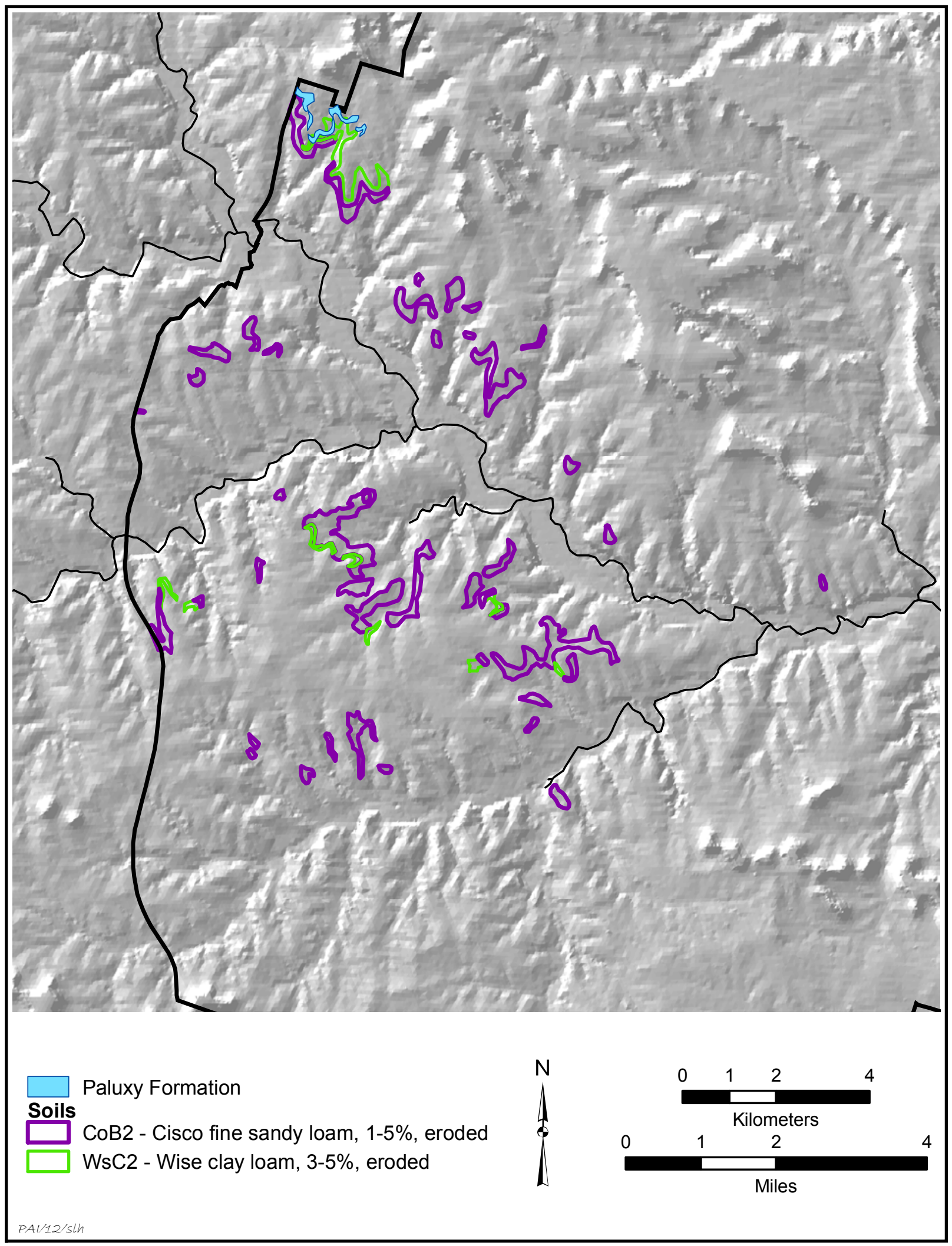

Figure 8.8. Distribution of Paluxy outcrops in west-central Fort Hood based on geologic and soils data. 


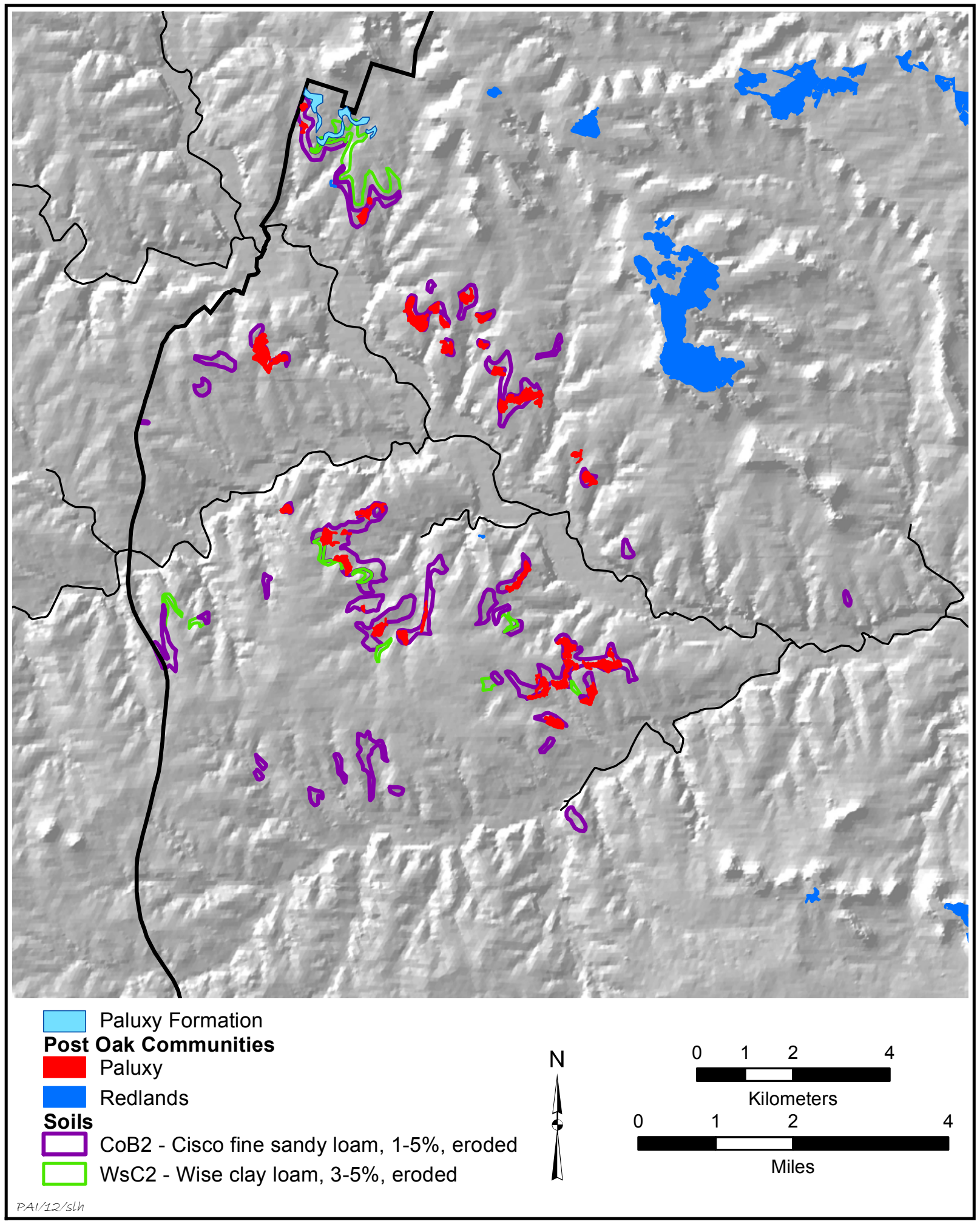

Figure 8.9. Distribution of expanded Paluxy outcrops in west-central Fort Hood based on geologic and soils data in conjunction with the distribution of post oak communities. Excluding the "redland" post oak community on the upland Manning surface in the northern portion of the map, much of the "Paluxy" post oak community corresponds with the known locations of Paluxy Formation and Paluxy soils. Areas where post oaks occur in isolation are probably unmapped outcrops of Paluxy Formation and redeposited Paluxy sands. 
Maps with site locations are not shown in report copies for public distribution.

Figure 8.10. Distribution of known Paluxy sites $(n=37)$ and expanded Paluxy outcrops from Figure 8.9. The image distinguishes between Paluxy sites with burned rock mounds, middens, and earth ovens $(n=19)$ and those without $(\mathrm{n}=18)$. 
Data Recovery Investigations at the Tank Destroyer Site, Fort Hood

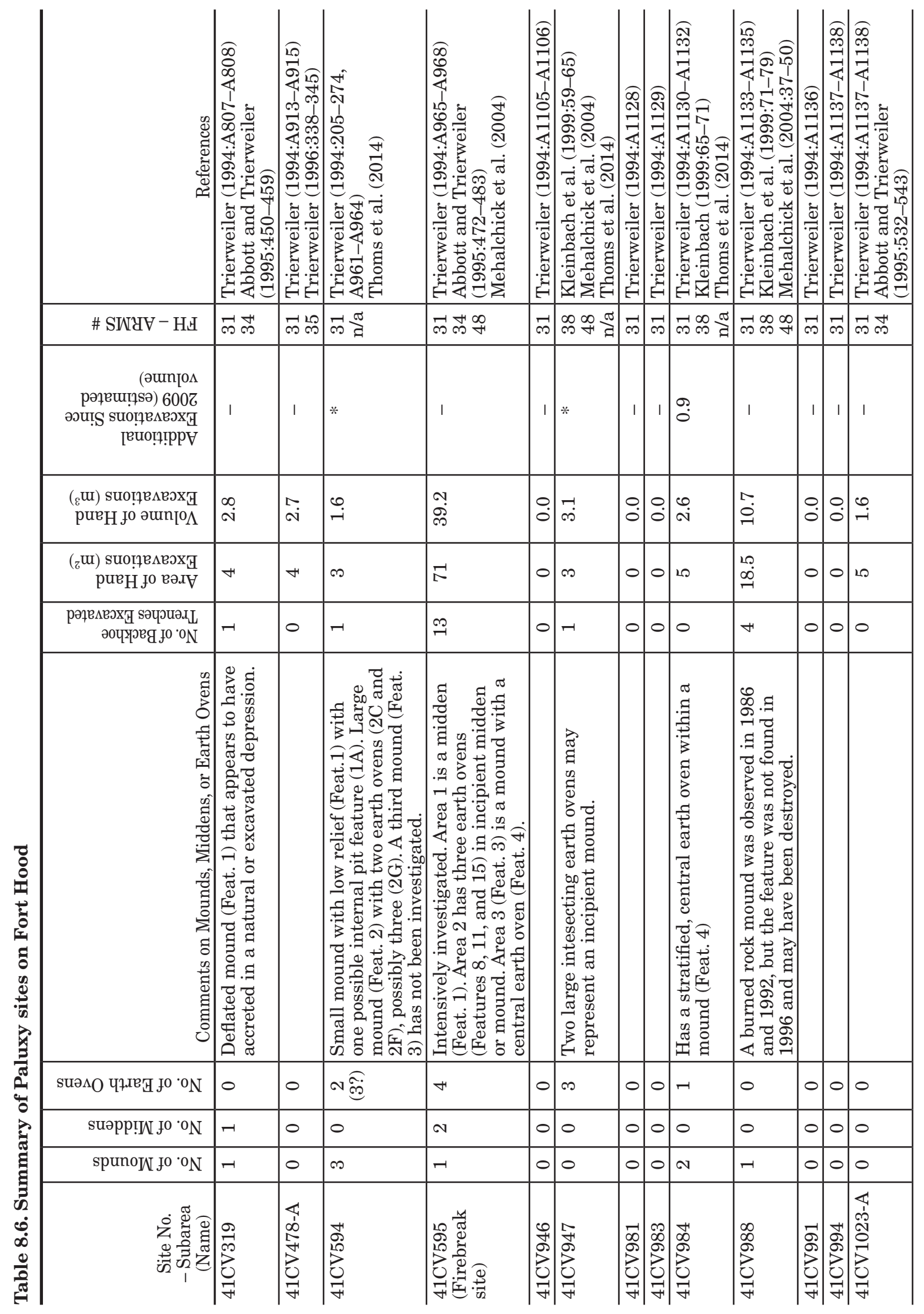




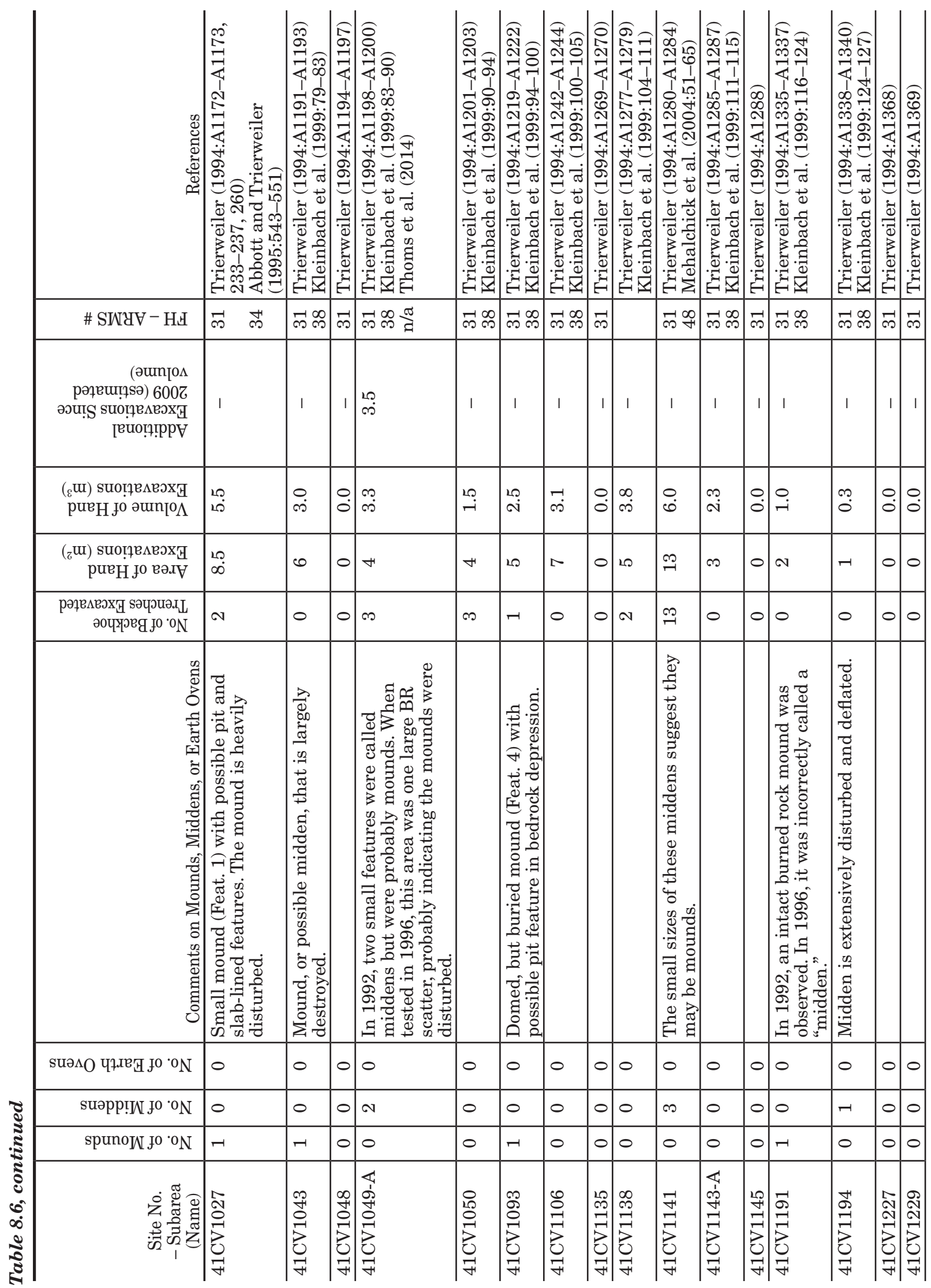




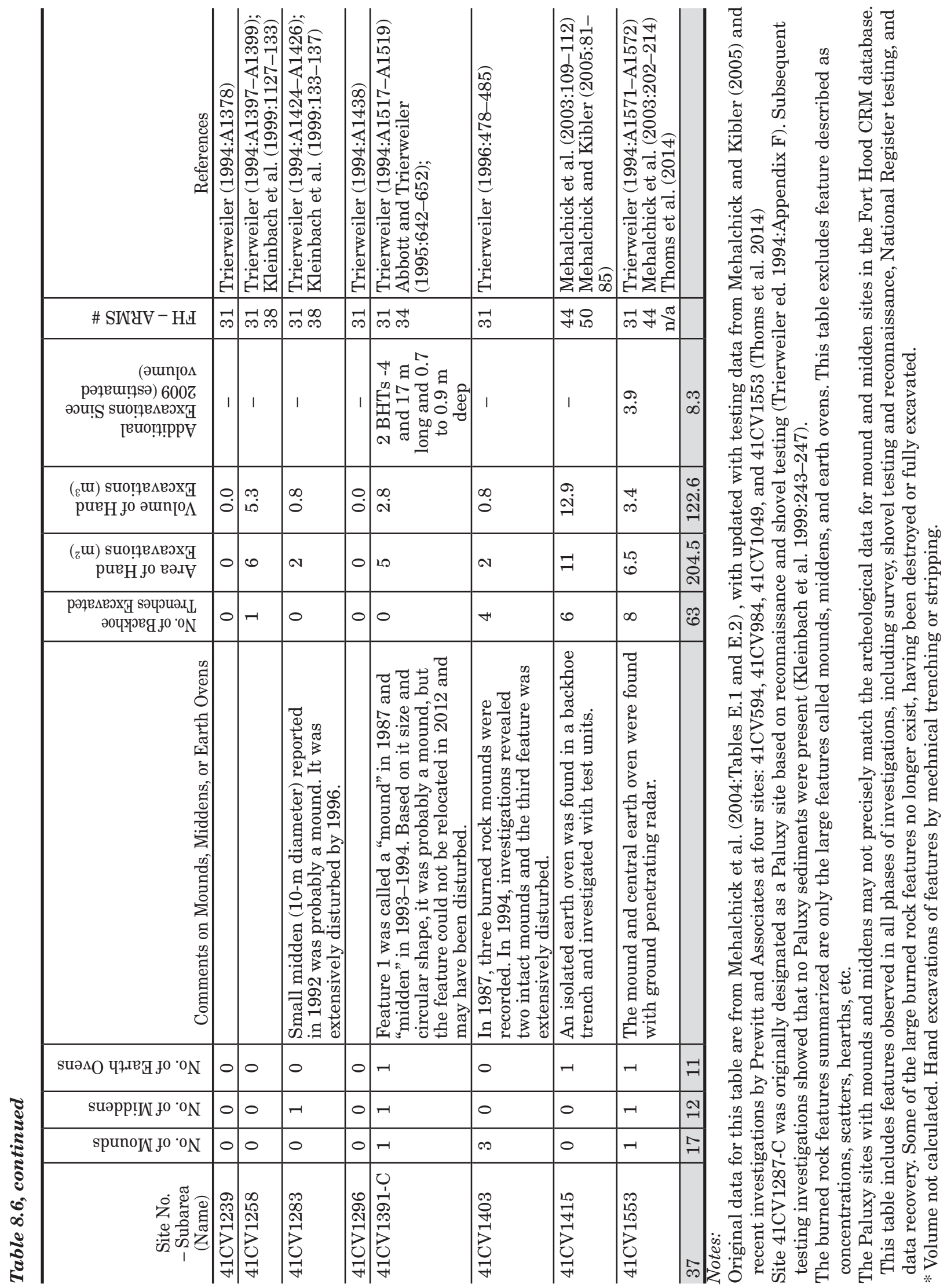


sufficiently investigated have demonstrated that they are associated with earth oven cooking features. And (5) all of the Paluxy site "middens" appear to share more attributes with burned rock mounds (i.e., they are isolated, relatively small, circular to oval in shape, and have domed relief in profile) than they do with the big classic middens found in riverine settings on Fort Hood (such as the intensively investigated midden at the Clear Creek Golf Course site, 41CV413 [Mehalchick et al. 2002]).

On Fort Hood, the burned rock middens and mounds are not just limited to the Paluxy environment. They occur in many other environments as well-in some cases perhaps for the same reasons that they occur within the Paluxy environment. When all sites with burned rock middens and mounds at Fort Hood are plotted, an interesting pattern emerges (Figure 8.11). ${ }^{16}$ Most of these sites are located within two environmental zones or niches. One of these is the Paluxy environment, and the second consists of the riparian zones along the high-order streams and their tributaries. While there may be several factors dictating or influencing this pattern, we know that the two resources that were unconditionally required for earth oven cooking are limestone rocks and firewood, and the importance of the latter is often overlooked. While limestone rocks are prevalent in many areas across Fort Hood, firewood is more abundant and more easily accessible in the Paluxy and riparian environments than in other environments on the base. Researchers have discussed the tremendous amounts of firewood necessary for heating rocks and cooking geophytes in earth ovens (e.g., Dering 1999; Mauldin and Nickels 2003; Thomas 1989). Like the rocks required in these endeavors, it is difficult to transport large amounts of firewood over long distances. Therefore, the accessibility of firewood undoubtedly played a significant role in determining the locations of large earth ovens that were used repeatedly and ultimately resulted in the formation of burned rock mounds and middens.

In a review of the ethnographic literature, Smart and Hoffman (1988:168-169) note that various factors affect firewood use decisions, including heat yield and quantity of smoke

$\overline{16}$ There are some minor discrepancies between the overall Fort Hood database of mound and midden sites and the Paluxy sites with mounds and middens summarized in Table 8.6. produced as well as availability and ease of collection. They also note that firewood collectors prefer fallen trees and dead or fallen limbs, which are lighter and burn easily because they are drier. Not all trees naturally shed limbs and branches throughout the year, so the strategy of using dead wood should affect the taxa that were collected and burned (Asch et al. 1972:6). Two of the more common taxa of natural pruning trees at Fort Hood are pecans (Carya illinoinensis) and various oak (Quercus sp.) species. These natural pruning trees are primarily limited to two areas on the Fort Hood landscape-riparian zones and the sandy substrate of the Paluxy Formation. Both support sinuous corridors of woodland and forest communities that traverse a landscape dominated by grasslands and savannas with limited firewood sources. For the Paluxy environment, the dominant self-pruning arboreal species is post oak, and in the riparian zones the species include pecan and various oaks, as well as an abundance non-self-pruning trees that would be available as firewood after windstorms. These factors, along with the environmental settings of many burned rock middens and mounds, suggest that the abundance and accessibility of firewood supplies were key factors determining where prehistoric earth oven cooking occurred on the Fort Hood landscape. From a social perspective, those in charge of collecting the firewood and rocks for earth oven cooking were probably the people who determined where on the landscape this activity took place. Many factors would have been considered in selecting areas for hot-rock cooking facilities, of course, but it would have been prudent to select suitable locations that required the least amount of effort for hauling the large amounts of firewood and rocks that were needed.

\section{Summary}

First recognized as a unique set of archeological resources on the Fort Hood landscape in the early 1990s (Abbott 1994:327-333, 1995:814-823), Paluxy sites have intrigued archeologists for several reasons. First, the Paluxy environment is geologically and floristically unique in that it primarily consists of an outcrop of fine quartz sands supporting an upland margin arboreal community in a limestone-dominated landscape of grasslands 
Maps with site locations are not shown in report copies for public distribution.

Figure 8.11. Distribution of all burned rock mounds and middens across Fort Hood. 
or grassland savannahs. Second, while the Paluxy environment comprises an estimated 2 to 3 percent of the Fort Hood landscape, it contains a relatively large number of sites, particularly for an upland setting. This suggests that the prehistoric inhabitants preferentially selected the environment. And third, Paluxy site tool and other artifact and feature assem- blages indicate that the use of this environment centered on the specific task of plant food processing in earth ovens (Kleinbach et al. 1999; Mehalchick et al. 2004). The results of this study suggest that one easily accessible and abundant resource-firewood-was a strong draw for prehistoric peoples to favor and use the Paluxy environment. 



\section{EXAMINING SOCIAL IDENTITY IN THE CENTRAL TEXAS LATE ARCHAIC}

9

John E. Dockall, Karl W. Kibler, and Douglas K. Boyd

\section{INTRODUCTION}

Recent work in central Texas has suggested that the Late Prehistoric and the initial phases of the historic periods were characterized by hunting and gathering groups with unique social identities that comprised regionally expansive social fields. Researchers have theorized that these groups are possibly comparable to recognized cultural phases (Prewitt 1981, 1985) or projectile point intervals (Collins 1995, 2004). Based on radiocarbon dates and projectile points recovered at the Tank Destroyer site, three Late Archaic cultural phases or projectile point intervals - the Uvalde, Twin Sisters, and Driftwood phases, roughly equivalent to the MarcosMontell-Castroville, Ensor-Frio-Fairland, and Darl intervals-represent the majority of the activities that occurred at the site. In this chapter we examine whether these phases are valid cultural concepts that represent distinct groups of people, and we consider what is required in the archeological record to enable researchers to recognize social identity, fields, and boundaries in the Late Archaic. However, before we can do this, a brief review of the work of Prewitt (1981, 1985), Collins (1995, 2004), and others is needed, as well as a brief discussion about social identity and what it means.

\section{ARCHEOLOGICAL PATTERNS OF THE CENTRAL TEXAS LATE ARCHAIC}

Ordering and synthesizing the archeological materials of central Texas into meaningful units of cultural materials and human behavior have been attempted several times since the middle of the twentieth century (e.g., Suhm 1960; Suhm et al. 1954; Weir 1976). In 1981, Prewitt $(1981,1985)$ provided a synopsis and discussion of the chronological and material culture evidence to support the existence of distinctive temporal phases of occupation for the broader region of central Texas. To develop this synopsis, he examined and grouped distinctive artifacts and features, mortuary practices, subsistence patterns, and evidence of extraregional trade into "phases" and estimated the age of each phase based on available radiocarbon dates. A series of 11 phases for the Archaic period and two phases for the Neo-Archaic (later referred to as the Late Prehistoric) were presented. Prewitt's research defined a number of cultural traits, some that were distinctive to certain phases and others that were shared by temporally adjacent phases. Radiocarbon assays from identified archeological components appeared to corroborate his assessments of each phase (Prewitt 1985). Aside from chronological differences between three of his phases-the Uvalde, Twin Sisters, and Driftwood-perhaps the most visible markers of each phase are the morphological differences in dart points. The Uvalde phase (ca. 300 B.C.-A.D. 200) is represented primarily by Castroville, Marcos, and Montell points. The Twin Sisters phase (ca. A.D. 200-A.D. 550) has the Ensor point as the dominant dart point form, and the Driftwood phase (ca. A.D. 550-A.D. 700) is associated with the Darl point.

Johnson and Goode (1994:18) have taken some issue with the chronological phases as defined by Prewitt and other researchers. Their problem with these various phases were that they have not proved to be particularly useful since the items and traits used to define them 
have not been shown to be consistently associated. Using climatic and archeological data, Johnson and Goode (1984) set out to redefine the subdivisions of the Early, Middle, and Late Archaic periods. For the Late Archaic period, Johnson and Goode (1994:29) employ subperiods I and II. One of the more intriguing aspects of their Late Archaic period discussion is the technological distinctiveness of the Late Archaic dart points. Subperiod I is associated with the Bulverde point, which Johnson viewed as possibly more related to such earlier dart point types such as Calf Creek, suggesting that Bulverde may be an intrusive type in the eastern part of the Edwards Plateau (Johnson and Goode 1994:29). Subperiod I, also with an abundance of Montell and Marshall type hafted bifacial projectile points, is seen by Johnson and Goode (1994:29-35) as having a material and behavioral link to the group(s) that made the earlier Pedernales points of subperiod I. Johnson discussed what he considered to be distinctive technological similarities between Montell, Marshall, and Pedernales points, such as the impressive technological blade thinning using billets that is common among all three dart point types. Also common to many archeological components that have produced these point types are broad, flat ovate knives made using similar techniques of thinning, indicating a probable technological relationship. Castroville points are seen as characteristic of the end of subperiod I and transitional into subperiod II (Johnson and Goode 1994:36-37). Other researchers have also speculated on the morphological and technological similarities among these point types (summarized in Johnson 1995:189-228). Point types characteristic of subperiod II are Marcos, Ensor, Frio, and Darl. Johnson and Goode (1994:37) compare the Marcos type to other corner-notched dart point forms from the Southern Plains, and Frio and Ensor are seen to have morphological similarities to point types in the southeast or eastern United States. Johnson's work is quite interesting in terms of suggesting that different point styles were associated with, or can be attributed to, different population groups with connections to adjacent geographic areas and incipient groups along the Edwards Plateau of central Texas. That technological aspects, such as billet thinning techniques, or dart point morphologies are used to speculate on groups and group movements is also intriguing and worth a closer examination in terms of social identity and social boundary theories (see below).

Collins (1995, 2004) employed archeological data from site deposits with high to moderate integrity to refine and expand Prewitt's work. The resulting cultural chronology dropped Prewitt's named phases and replaced it with archeological style intervals associated with distinctive projectile point types that are common in each interval. The last three dart point intervals for the Late Archaic period-Marcos-Montell-Castroville, Ensor-Frio-Fairland, and Darl—as defined by Collins reflect the same projectile point types employed by Prewitt in his earlier studies but follows the chronological suggestions proposed by Johnson and Goode (1994). As summarized by Collins (2004:122), the salient points made by Johnson and Goode (1994) are the increased diversity and complexity of archeological remains at the end of the Late Archaic that attest to certain types of human behavior not seen in earlier time periods. These would include the existence of Eastern Woodland influences in religious and mortuary practices.

Following Collins and others, Arnn (2007) argued that one of the key elements to identifying prehistoric social fields in central Texas is an archeological site with sufficient integrity, context, and evidence of repeated and redundant occupation by members of a distinct group over time. He proposed that the residential base camp or large open campsites represented the best opportunity to identify aspects of group social identity. Mortuary sites could also be added to that list for similar reasons. Both site types can contain evidence of "horizontal overprinting" of various activities and features from one period of use to the next, each associated with similar artifacts (Arnn 2007:21, 2012). Such patterning suggests a historical relationship among the several use periods. Arguably, both site types can be considered to represent "persistent places" on the social landscape (Littleton 2007; Littleton and Allen 2007; see also Spielmann 2008). Arnn (2007:22) proposes a scale of inquiry beginning with the residential base and expanding to the social boundary or border in any effort to define social fields. Certainly residential bases and mortuary locations would provide information that could be used to identify social fields and social boundaries. And it may be that established and repeatedly used mortuary locales in part 
represent social boundaries along buffer zones within certain resource areas.

It is necessary to couch any discussion of prehistoric social identity and social boundaries in terms of scale: local, regional, extra or macroregional. Following Arnn (2012:13-16), the three scales of interest are the band or community, the marriage group, and the long-distance network or social field. A scaled approach to studying social identity of forager groups must of course consider the landscape. Where people live on the landscape is basic to their own identity and the identities perceived by those with whom they interact. Each scale of identity is associated with archeological correlates of visibility. For band- or community-level identity, the correlate would be the residential base or campsite. The residential base also represents the basic unit for identity and social interaction at the family, band, and community level. Long-distance networks and social fields are the broadest level and are visible archeologically via the regional distributions of similar types of artifacts, decorative elements, assemblages, features, or even the technological ways of doing things. In the middle of the scale are marriage groups, which should link bands and communities together via a common language but should also have differences evident at the regional level. A scaled approach to social identity must also look at the distribution and use of artifacts manufactured from nonlocal materials and the manufacture of nonlocal styles of artifacts using local materials.

Arnn (2012:15-16) notes that the scalar approach to studying social identity is dynamic and includes both spatial and temporal dimensions of change and stasis. Prehistoric identities were not static but changed as the needs arose, and change could occur at any level of complexity, from band or community to larger regional levels.

\section{SOCIAL IDENTITY, SOCIAL FIELDS, AND SOCIAL BOUNDARIES}

To assess the utility of Late Archaic phases or projectile point intervals in determining the existence of prehistoric social fields and social identity, it is necessary to provide an acceptable definition of these phenomena and why they exist in the first place. A social identity is a "person's knowledge that he or she belongs to a social category or group" (Stets and Burke 2000:225). A social group is a "set of individuals who hold a common social identification or view themselves as members of the same social category" (Stets and Burke 2000:225). Social identity theory (following Tajfel [1972] and Tajfel and Turner [1986]) is an explanatory framework that proposes that an individual's behaviors reflects that individual's broader social units. Social structures such as groups, organizations, and cultures and an individual's association with these units are interrelated. Social identity theory predicts that individuals "think, feel, and act as members of collective groups, institutions and cultures" and that members of these collective groups share membership in several ways (Padilla and Perez 2003:43). Tajfel (1972:292) defined social identity as "the individual's knowledge that he belongs to certain social groups together with some emotional and value significance to him of his group membership." Social groups only exist in relationship to each other, and they have their descriptive value, properties, and social meaning in relation to other groups (Hogg 2001:186). Intergroup relations involve maintaining and protecting positive group distinctiveness and social identity for each group, which is reinforced by the group's understanding of group boundaries and how these boundaries are legitimized (Hogg 2001:186; Tajfel and Turner 1979).

Another important point for examining this issue is that social identity may be expressed at many different levels at the same time. On an individual level, one person within any society would have many identities simultaneously expressed in many different ways. Just as in modern societies, people in the past would have had multiple identities defined by such characteristics as sex (male, female, or other), age (e.g, a respected elder), general work (e.g., a farmer vs. a herder), particular job or craft specialization within the group (e.g., an arrow maker vs. a pottery maker), and membership in a particular subgroup (e.g., an elite warrior group). One's status might be measured in terms of leadership abilities in religion (e.g., a shaman or a priest), politics (e.g., a headman or a king), or economic pursuits (e.g., a local merchant or a traveling trader). Broadening the scope of research, Arnn (2012:13-16) suggests that there are three fundamental levels of social group identity that are most relevant to archeological research: (1) the band or community; (2) the 
endogamous marriage or linguistic group (composed of multiple bands or communities); and (3) the long-distance social network (composed of many larger marriage or linguistic groups). From an archeological perspective, then, it is important for researchers to be explicit about the particular level of identity that is being examined via material remains.

For hunter-gatherer groups during the Archaic period, "ethnicity," social identities, and social boundaries were used to create and maintain important contacts and relationships among widely dispersed population segments (Arnn 2012:153-163). This phenomenon has similarly been documented among the Hausa in Nigeria, where portions of the population are scattered in small groups over a large geographic area (Cohen 1969; Schortman 1989). Archeologists, however, should be careful in using the term "ethnicity" in reference to prehistoric, highly mobile foraging groups (Hegmon 1998:273-274). Boundaries that are defined on the basis of certain aspects of material culture may represent prehistoric social boundaries that cannot be equated with ethnicity without additional justification by the researcher.

An appropriate definition for social fields that emphasizes the role of objects can be taken from the work of Bourdieu (1985:723), who defines a social field as a multidimensional social space created by the actions and interactions of group members. That these social boundaries define ethnic groups is not implied. In his concept of social field, Bourdieu (1984) included the concept of "habitus," which represents the practices and accepted behaviors appropriate to an individual's status or role in a social group. Much like optimal foraging theory, which presents groups with a suite of options regarding the acquisition of resources based on their accessibility in terms of measurable variables such as time and energy, the concept of habitus presents the range of acceptable choices to respond to different situations. So Bourdieu did not consider it an inflexible set of options but a dynamic set of responses that change as the group dynamic changes. A group habitus is reflected in the group space and the use of objects considered appropriate by the group. Artifacts and artifact assemblages are key to defining prehistoric social fields because the items reflect the remaining physical manifestation of the former group and should reflect different group identities in the way that they did things and what was considered acceptable. The main thrust of Bourdieu's arguments is that objects (artifacts) materialize human behavior and certain symbolic aspects of that behavior.

At the outset of this discussion, it is important to remember that the symbolic features used by members of a group to identify themselves are often items that do not preserve well. As such, it may be impossible for archeologists to ever fully reconstruct the various group affiliations (Schortman 1989; Wobst 1977). The archeological visibility of prehistoric social groups with specific identities may be dependent on how discrete they are. For example, are personal and social identities discrete, or are they on a continuum of visibility? How strong are they? Huddy (2002) has argued that there are degrees of social identity and that these identities can range from the individual to the entire group. Huddy also argued that identity strength might be situational in some instances, especially when an individual or group is compelled by some reason to shift identities. Individuals maintain their identity with particular groups for various reasons, such as to reduce uncertainty or respond to resource scarcity (Padilla and Perez 2003:43). Social groups operate in dynamic contexts with group boundaries that can shift depending on changing conditions (e.g., economic, social, or ideological). The social contexts influence the nature of intergroup interaction and whether these interactions are peaceful or are associated with conflict. Group membership consists of two parts: first, the desire of the individual to belong to a specific group and, second, the desire of the individual to maintain his or her distinctiveness from individuals of other groups (Brewer 1991; Padilla and Perez 2003:43). One caveat is that an individual can have multiple and overlapping group memberships, and by extension, prehistoric social groups may also have overlapped accordingly.

One means of establishing, maintaining, and altering social boundaries and identities is through objects (Gal et al. 2004). Gal et al. refer to items that can be used to maintain social boundaries as "boundary objects." Boundary objects can be specific artifacts or perhaps attributes of artifacts that are recognizable to members of more than one social group. To understand the role of such items, it is necessary to consider the social structures of which 
they are a part (Gal et al. 2004:193). Boundary objects are used to develop and express social identities. Of potential significance for understanding prehistoric social boundaries, boundary objects can be abstract or physical artifacts that operate at the interfaces between different social communities. These items serve multiple purposes, from establishing and maintaining identities to bridging differences between groups sharing the same identity. Gal et al. (2004:194) indicate that boundary objects are dynamic and, if changed in one group, can in turn create the need for changes in adjacent groups. As the relationships between groups change, the meanings of boundary objects may change, or they may be replaced by entirely new boundary objects. Therefore, the interpreted meanings of these objects must be examined in the context of the specific relationships between groups at that time (Gal et al. 2004:197). For archeological research, this means that context is everything, and artifacts that may serve as boundary objects at the interface of two distinct social groups. Such objects may have different meaning(s) for each group. For example, an artifact type with a mundane function in one group may take on a different meaning as a trade item, and have a third meaning in the recipient group.

If certain lithic artifacts can function as boundary objects between groups and, by extension, as markers of specific group identities, how is this accomplished? Previous theoretical research on social identity involved assessing the presence of "style" in artifacts and whether certain attributes of stone artifacts were more "stylistic" or "functional," particularly in reference to Middle Paleolithic assemblages in Europe (Binford 1973; Binford and Binford 1966; Bordes 1973; Bordes and de Sonneville Bordes 1970). Projectile point morphology has been associated with similar debates in the literature. The crux of these debates has been the cause and meaning of formal variability in stone artifacts and whether or not it is indicative of any scale of "ethnicity." Barton (1997) provides a summary of the salient aspects of style and social identity in stone tools. Archeologists generally accept that artifact style can be used to define boundaries between social units. In effect, the artifacts appear to represent boundary objects discussed above. The critical debate has centered on how style can be defined in artifacts and what scale of social identity is defined (Conkey and Hastorf 1990; Hegmon 1992; Sackett 1985, 1990; Weissner 1983). Since archeologists have attempted to use various types of lithic artifacts to describe social boundaries and social groups, it only makes sense that the artifacts and attributes that are most likely to convey information between individuals and groups will be those that are most visible (Wobst 1977). Conversely, we can assume that lithic implements that have relatively short use lives may be rather deficient in their ability to transmit information regarding social group. According to Schortman (1989:57), technological items such as lithic tools are more frequently associated with adaptation and subsistence and may be extended more broadly among people belonging to different social groups. Hence, the spatial distribution of most technological items and features may not be primarily the result of their use as identity signals but more a result of their function in subsistence roles. The approaches to style by Sackett and others, however, are limiting in their focus on the artifact itself by suggesting that aspects of artifact design that cannot be explained in terms of function or technology can be explained as style. This approach suggests that artifact style is not necessarily intrinsic to its design and as such appears separate from technology; however, other researchers have suggested an intrinsic relationship between style and technology (e.g., Barton 1997; Dietler and Herbich 1998; Edens 1999; Hegmon 1998; Lechtman 1977). Technological style places an emphasis on the technical knowledge, expertise, and reasons why certain manufacture techniques exist within a group and how it is expressed in the artifact.

Barton (1997:144) makes the point that any social information regarding group membership that is intrinsic to lithic artifacts should be able to be recognizable to prehistoric people and archeologists. A particular problem with using lithic artifacts as markers of social identity is that they generally have short use lives and are continually resharpened and maintained before discard. This applies even to hafted implements such as scrapers, knives, and projectile points. So how do we determine the attributes or attribute suites of particular artifact types that are encoded with social information? By extension, how do we determine if particular assemblages of artifacts are indicative of prehistoric social 
groups? To assure that an artifact or attribute is conveying social information, it must be determined that they are not the result of other stochastic processes.

Archeologists have documented that stone artifacts do change in form through time and across regions, and the observed morphological variability in Texas projectile points within a given region and time period argues against a single group of people maintaining multiple styles through time. There are three possible explanations for this variability, according to Barton (1997:148-149). First is that observed variability among point types represent group selection through time and across space. Second, variability may represent arbitrary stages in the use life of a few types. Third, variability is the result of transport of discrete forms among different social groups and exchange of these forms between groups. Teltser (1995) and Barton (1997:149-150) note that in studies of lithic variability through time or among contemporaneous artifacts and assemblages, archeologists seek to establish the "social distance" between the groups that once used the tools.

One final cautionary note is warranted regarding stone artifacts as social identifiers. It is seldom true that archeologically recovered stone artifacts represent the complete tools that were used by prehistoric peoples. In almost all archeological sites in Texas (dry rockshelters being a notable expection), stone artifacts are simply the durable portions of the tools that survived in the archeological record, and the perishable components of the tool are generally absent in the archeological record. Stone projectile points survive in most archeological contexts, yet these small items are representative of very complex technological systems involving various types bows and arrows. From an identity standpoint, ethnographic evidence indicates that arrow shafts were laden with decorative and construction details that served as group social identifiers (Sinopoli 1991). Arrow point morphology can and did have some level of social meaning, but the nondurable parts of the bow and arrow weapon system (e.g., painted or engraved wooden shaft, proximal notching, fletching, and point hafting) encompassed most of the social identify symbolism that would have been meaningful to contemporary observers. Because of these realities, archeologists must exercise caution when interpreting identity using stone artifact morphology.

\section{SOCIAL GROUPS AND FIELDS IN PREHISTORIC TEXAS}

Across most of Texas and adjacent regions, much of the artifact data that archeologists deal with on a daily basis was produced by fluid groups of hunter-gatherers practicing a variable foraging and collecting subsistence strategy. Consequently, the boundaries between these groups (social and geographic, following Barton 1997:150) were often fluid, undefended, and open to intergroup exchange (e.g., of ideas, beliefs, artifacts, mates, and goods). In some cases, the artifacts of both groups may be discarded together. The likelihood that this would occur at the social boundary interface would depend on the social and geographic distances between the groups and the types of activities that would bring such groups together. Such interaction has been documented ethnographically by researchers among some African nomadic groups, and archeological evidence of this type of social relationship is also evident along the central Texas coast. At the Melon site (41RF21), Ricklis (1990:387-425) recovered convincing evidence of cooperative hunting of bison and deer by coastal and inland groups. The site sits on a low rise and yielded an assemblage that included lithics and ceramics. Ceramic sherds attributable to both Rockport ware and Toyah bone-tempered wares were recovered within spatially discrete areas of the site, with Rockport ware sherds in the southern half of the rise and Toyah wares in the northern half. Radiocarbon dates, stratigraphy, and faunal assemblage characteristics strongly suggest that the site reflects occupation of the site by distinct coastal and interior groups at the same time during the latter half of the thirteenth century A.D. The site inhabitants probably came together to hunt deer and bison, but the real reason for coalescing may have had as much to do with meeting a social need for group interaction. Other sites in the region demonstrate that both interior and coastal groups used the central Texas coastal area during Late Prehistoric times. A series of sites along the Aransas River suggest that there were seasonal differences in use of the coastal zone by inland and coastal groups, with inland groups moving into the area during the fall and winter, when Rockport groups had 
returned to campsites directly along the coastal margins (Ricklis 1990:422-423). The sites from Ricklis's Aransas River survey roughly overlap geographically along a zone about $40 \mathrm{~km}$ inland from the coast, which suggests the presence of a boundary possibly correlative with the inland boundary of Karankawa territory.

Perttula (2001) has presented additional evidence of the prehistoric presence of boundaries in a detailed summary of hunter-gatherer mortuary practices along the Rio Grande and central coastal plains of Texas. The amassed data suggests the existence of four or five distinct mortuary traditions across the region with the highest concentrations of burials and cemeteries along a line from Bexar to Nueces County. Another concentration of Archaic cemeteries is along the lower Colorado and Brazos Rivers (Perttula 2001:48-52). Interestingly, both of these areas have yielded the highest proportions of burials with evidence of interpersonal violence. The evidence suggests that the lower Brazos and Colorado Rivers and the middle reaches of the Nueces and San Antonio Rivers may have been zones of resource competition. Late Prehistoric cemeteries in central Texas, Corpus Christi Bay, and the Rio Salado/Rio Grande (Falcon Reservoir) area have yielded similarly compelling evidence of violence (Baker 2001; Perttula 2001:52). The Corpus Christi Bay and Falcon Reservoir areas had not yielded evidence of interpersonal violence during the preceding Archaic period, indicating that boundaries or group territories in these areas may have shifted for various reasons during the Late Prehistoric period. Evidence presented by Ricklis (1990) and Perttula (2001) indicate different approaches to social boundary maintenance (cooperation and cohabitation vs. boundary defense) that may reflect differing social or perhaps "ethnic" distance among groups involved and greater distinctions between "we" and "they."

One could imagine such scenarios along an interface between more western Caddo groups and eastern Edwards Plateau hunter-gatherer groups or between hunter-gatherer groups anywhere along the Edwards Plateau that come together seasonally in particular resource areas or differ in their seasonal use of a particular resource zone. We know that Caddo peoples were present on the Blackland Prairie east of the Edwards Plateau for bison hunts (Ricklis and Collins 1994:19). We also know that this area periodically hosted large encampments of diverse groups, including Caddo and others not from the immediate area (Campbell 1988; Ricklis and Collins 1994). Late Prehistoric period evidence of interpersonal violence (Baker 2001; Shafer 2006) indicates that a defended boundary zone may have been present at various times between more western Caddo groups and hunter-gatherer groups along the eastern Balcones Escarpment. Shafer (2006) presents a testable hypothesis that prairie Caddo groups were distinctly different from either the Austin or Toyah groups in central Texas and posits a western boundary for the prairie Caddo assemblages along the Balcones fault zone between the Brazos and Colorado river valleys. A distinctive assemblage that consists, among other items, of Caddoan-style ceramics, Gahagan bifaces, deer metapodial beaming tools, bone pins, and Bonham-Alba style arrow points marks this proposed boundary.

Other scenarios could also be developed. The distribution of artifacts such as projectile points (or perhaps artifacts with particular methods of manufacture like billet thinning) that exhibit spatial and temporal variability patterns should appear as overlapping concentrations that vary with the social distance between the groups. Analyzing and studying the spatial discard patterns among contemporaneous artifact types may be a more useful method of trying to define prehistoric social groups than ranking lithic assemblages based on the frequencies of different artifact types or projectile point styles (Barton 1997:153). Other distributions that do not fit the expectations of artifacts serving as boundary objects (Gal et al. 2004) may be explained in other ways. Similar types of technological and morphological data can be interpreted in a number of ways among different analysts, either as different technological and functional choices among the same social group (Schortman 1989; Shott and Weedman 2007) or as evidence of the interaction of two distinctly different social groups (Edens 1999).

It is certain that social identities existed during the Late Archaic and other time periods in the prehistory of central Texas. Wade (2003:222) notes that during the seventeenth century, at least 21 groups inhabited the Edwards Plateau. Although Wade presents her discussion in the context of native groups' relationships with Europeans in the early historic period, it seems 
highly unlikely that this degree of social group diversity developed in response to the arrival of Europeans and did not mimic prehistoric levels of group diversity in the region. This suggests that the broad Late Archaic archeological pattern we see across central Texas is not the product of a single group or monolithic culture; the key to unraveling this is identifying local or geographically restricted variants of artifact types that signal social identity (Mehalchick and Kibler 2008:371).

The picture presented for the Late Archaic is one of regional specialization, differentiation, and complexity for Texas and adjacent areas (Arnn 2012:153-165). Patterns that emerged during the Early and Middle Archaic culminated during the Late Archaic in a mosaic of cultural differences reflecting adaptation to specialized regional resources and environmental possibilities. Extraregional influences from northern Mexico, the Great Plains, and the southwestern and southeastern United States figure prominently in the local material culture. Significantly, central Texas is the only region that borders nearly all four of these cultural regions. Not only does this encompass a great deal of "ecotonal diversity," but it also should reflect a high degree of cultural diversity. Late Archaic peoples in Texas adapted their technology, subsistence, and settlement patterns to accommodate spatiotemporal variability in necessary resources, and thus became "intensive, localized, and specialized" (Arnn 2012:163). In part, the observed variability in material culture seen during the Late Archaic corresponds broadly to differences in regional climate and ecological characteristics across Texas. The second part of this variability picture includes the network of social interactions and relationships that developed between forager groups and evolved through time.

Hegmon (1998:273) suggests that although prehistoric material culture boundaries may exist and may indicate some type of social boundaries, these boundaries should not outright be equated with ethnicity until more is known regarding the social processes involved. Perhaps the closest that we have yet come to material culture boundaries are the projectile point intervals of Collins (1995, 2004). Previous data from Prewitt, Johnson, and Collins strongly suggest that with appropriate additional data on type distributions beyond our present nebulous understanding of the "ranges" of certain point types, we may be able to identify more clearly prehistoric social boundaries and groups. Barton (1997) cautions that researchers should first consider and eliminate other stochastic explanations, and Schortman (1989) reminds us that the spatial distribution of technological items most likely reflect their use in subsistence pursuits and not aspects of social identity. Collins (2004) has called for less effort pursuing the rather singular goal of chronology building and more concerted effort spent on analysis of high-integrity assemblages. Johnson and Goode's (1994) comments on discrete technological similarities like billet thinning present on Pedernales, Montell, and Marshall points is intriguing. The utility of certain technological aspects of stone tool manufacture as social identity markers has been little explored by Texas archeologists but has been more greatly explored by our colleagues in Europe and the greater Near East. Similar distinctive technological traits have also been identified among groups of Paleoindian assemblages. Such aspects of lithic technology (e.g., projectile points and large hafted and unhafted bifaces) have not been fully explored for later Archaic and Late Prehistoric period, but we do have a good understanding of particular assemblages of artifacts that appear to be diagnostic of broader groups with some type of social integrity such as Toyah and Austin phase (Johnson 1994; Prewitt 1981, 1985). Unfortunately, for the large region of the Edwards Plateau, with the exception of hafted projectile points and some other bifaces, much of the lithic technology remained the same. The exceptions to this may be some of the artifact types scattered across the area that hint at "interlopers" from the Eastern Woodlands and Southern Plains, or at the very least an influx of their technology via exchange (L. Johnson 1989; Johnson and Goode 1994).

\section{FUTURE RESEARCH ON SOCIAL IDENTITY IN THE LATE ARCHAIC OF CENTRAL TEXAS}

At present, we cannot confidently discuss the concept of social fields and social boundaries for central Texas and the Edwards Plateau, and we still only have the broadest understanding of what occurs at the interfaces of these loosely defined social boundaries. The literature that has provided our current understanding of Archaic and Late Prehistoric mortuary prac- 
tices in central Texas provides perhaps the best picture of such phenomena, but it is still at a broader scale than where we need to be in terms of our data use. Whether we are discussing Prewitt's Uvalde, Twin Sisters, and Driftwood phases or Collins's Marcos-Montell-Castroville, Ensor-Frio-Fairland, and Darl intervals, all are seemingly too geographically broad to represent a single social group based on the ethnographic and ethnohistorical literature and environmental diversity of central Texas. Certainly we do not want to think that the distributions of various styles of artifacts (e.g., projectile points or pottery) are equivalent to emically defined social units on a prehistoric landscape. Arnn (2012:165) noted that the Late Archaic appears to have been a time of many small communal groups that were adapting to life within specific ranges with associated local-scale cultural differences. His reminder that hunter-gatherer identity is intimately tied to the landscape is also important to remember. Given our current state of knowledge, can we infer that social boundaries at the local and regional scale were less permeable than during the Late Prehistoric? Does Arnn's present picture of the Toyah culture area indicate that by the Late Prehistoric these boundaries were less clearly defined, or were they defined differently during the Late Archaic? Given this, it is probably best to currently view these constructs as social fields composed of many smaller groups that share a nearly identical material culture.

To move beyond this, two things are needed before we can effectively begin to speak about social boundaries and social fields during the Late Archaic: (1) much more and better-quality data regarding the geographic distributions and metric/technical variations of artifact types; and (2) a better theoretical understanding of the modes and influences of artifact manufacture (Barton 1997; Dietler and Hebrich 1998). Certainly, knowledge regarding the use of various local and nonlocal raw materials would also provide greater information on prehistoric groups and group movements within a given region. Definition of social boundaries, identities, borders, buffer zones, territories, or other similarly termed spatial entities on the basis of geographic distributions of different styles of artifacts is fraught with problems if the concept of style is limited to only decorative elements and ignores the technological aspects of style (Dietler and Hebrich 1998; Edens 1999; Hegmon 1998; Lechtman 1977; Stark et al. 1998). Previous interpretations and continuing speculation regarding the suite of technological and typological characteristics/attributes that have been used to define "Toyah" for the Late Prehistoric serves as an example of the difficulties we face (e.g., Johnson 1994; Ricklis 1994) in attempting to define similar social phenomena during the Late Archaic in central Texas. Arnn (2012:142-143) argues that Toyah corresponds geographically with the Tejas Alliance, which is well documented in the historic period, and that it represents "a social field of considerable time depth." He goes on to present a good case for the existence of large social fields composed of smaller marriage groups and communities in the Late Archaic in many parts of Texas (Arnn 2012:153-167). And while Arnn (2012:219-233) summarizes some evidence for how to distinguish Classic Toyah marriage or linguistic groups in the archeological record, the reality is that this is much easier said than done. Even if future archeologists were more "methodologically and technologically sophisticated" in their efforts to distinguish social identities, "they will still be faced with a similarly limited material record" (Arnn 2012:45). And compared with Toyah, the archeological record of central Texas is comparatively sparse for any similar half-millenium time period within the Late Archaic period.

When reflecting on what it would require to begin to solidly address the concept of social identity during the Late Archaic of central Texas, one has to consider the quality and abundance of the existing data. Given Arnn's (2012) thoughtful and explorative treatise on defining classic Toyah social identity, a testable model is presented that can be applied to the Late Archaic data. Prewitt (1981, 1985), Johnson and Goode (1994), and Collins (2004) provide the important groundwork for such efforts, and Arnn (2012) provides an example of how it can be thoughtfully accomplished. Prewitt (1981:81-83) synthesized the pertinent literature of the day to identify site components, site types, tool kits and features, mortuary practices, subsistence, and social interactions for each archeological "phase." Following the lead of Johnson and Goode (1994), Collins (2004) refined the dating of selected "projectile point intervals" in the Late Archaic in central Texas by selecting only the very best components in terms of context 
and integrity. It is highly likely that none of the researchers would today consider their effort as the final word on the topic, but what they did is more consistent with the framework of Arnn's model of how prehistoric social identity can be addressed through archeological data.

For the Late Archaic, specifically central Texas, after almost a decade of additional research in the region, there has yet to be a synthesis that incorporates more recent archeological work that is needed in a wider study of prehistoric social identity. If we follow Arnn's model of what is necessary to begin such studies, at a minimum we would need a sufficiently large number of suitable sites (especially base camps and residential bases) that have excellent contextual integrity and good chronological control (Collins's gisement concept [Collins 1995]), and that are spatially located within a limited and well-defined geographic area (Arnn 2012:209-219).

Unfortunately, such sites are uncommon for the central Texas Late Archaic. Of Prewitt's (1981) list of sites for Uvalde, Twin Sisters, and Driftwood phases, Collins (1995) selected only three that had sufficient integrity and context for comparative analysis: Loeve Fox, Youngsport, and East Levee (41TG91). Some more recently excavated or reported sites that should be considered are Jonas Terrace (41ME29), Bessie Kruze (41WM13), and Lion Creek (41BT105). Table 9.1 lists sites that have moderate to highquality archeological data representative of the three projectile point intervals or cultural phases that comprise the latter part of the Late Archaic Period. Figures 9.1-9.3 show the locations of these sites, with the maps corresponding to Collins's Marcos-Montell-Castroville interval (Prewitt's Uvalde phase), Ensor-Frio-Fairland interval (Twin Sisters phase), and Darl interval (Driftwood phase) (Collins 1995, 2004; Prewitt 1981).

The geographic distribution of sites on these maps illustrates a major difficulty in examining social identity: there are too few high-quality sites, and those that do exist are too widely spread apart over a large geographic area. There are no reasonably distinct clusters of three or more sites that might approximate the territorial size of a band or community (stated by Arnn (2012:231-232, Figure 8.10 as being 1,000 to $1,600 \mathrm{~km}^{2}$ in size)—only small groups of sites that might approximate marriage/language groups. An in-depth literature review might identify a few more suitable Late Archaic sites in greater central Texas that could be added to the list, but meeting the rigorous requirements for occupation sites with good chronological control and contextual integrity is a significant limitation of the model.

Another possibility may be to consider looking at other sites and other site types around the periphery of the main cultural area as indicative of what occurs in the boundary zones. With regard to Toyah, Johnson (1994) identified the peripheral "shared area" as being around the classic Toyah area. Arnn (2012:213) considered the Toyah Shared Area as a zone in which "other point types and ceramic wares often overshadow Toyah assemblages." Arnn (2012:209) is quite correct in speaking of every Perdiz point as a "piece of regional social currency." This basic concept could be applied to any style of arrow or dart point, and it may be crucial in identifying Late Archaic sites along "shared areas" away from the central Texas homeland. The distribution of sites assigned to the Marcos-Montell-Castroville, Ensor-Frio-Fairland, and Darl intervals can be used to define core territories and identify similar peripheral "shared areas."

Mortuary sites and burned rock middens would provide two excellent avenues of research into defining such zones of interaction along social boundaries. Research by Hall (1981) and Ricklis (2011) demonstrate that cemeteries are a critical resource for understanding prehistoric social interactions and defining potential cultural boundaries and territories linked to specific geographic areas and ecological zones (such as riverine areas with pecan and oak tree habitats). Burned rock middens (including mounds) may also be underutilized for their potential social implications. Burned rock middens and mortuary areas reflect group behaviors resulting from repeated use of a single location over many decades, centuries, and even millennia. It may be necessary to start at the edges of greater central Texas to first define social boundaries, and then work inward toward the core area. Both site types would certainly have been the location of social group and communal activities, probably accompanied by feasting and rituals. Burned rock middens and mortuary areas both fit the definition of "persistent places" on the landscape (Littleton 2007; Littleton and Allen 2007; Spielmann 2008). It is not unrealistic to 
Table 9.1. Central Texas sites with significant components attributed to selected Late Archaic projectile point intervals and cultural phases

\begin{tabular}{|c|c|c|}
\hline Site No. & Site Name & References \\
\hline \multicolumn{3}{|c|}{ MARCOS-MONTELL-CASTROVILLE (Uvalde Phase) } \\
\hline 41BL104 & Evoe Terrace & Prewitt (1981) \\
\hline 41BL78 & Youngsport & Collins (1995), Prewitt (1981) \\
\hline 41BT105 & Lion Creek & Johnson (1997) \\
\hline 41CM1 & Oblate & Prewitt (1981) \\
\hline 41CM3 & Wunderlich & Prewitt (1981) \\
\hline 41CV1378 & Tank Destroyer & This report \\
\hline 41LM3 & McCann & Prewitt (1981) \\
\hline 41ME29 & Jonas Terrace & Johnson (1995) \\
\hline 41ME7 & Scorpion Cave & Prewitt (1981) \\
\hline 41TG91 & East Levee & Collins (1995), Prewitt (1981) \\
\hline $41 \mathrm{TV} 151$ & Jetta Court & Prewitt (1981) \\
\hline 41UV21 & La Jita & Prewitt (1981) \\
\hline 41UV60 & Anthon & Goode (2002) \\
\hline 41WM13 & Bessie Kruze & Johnson (2000) \\
\hline 41WM49 & John Ischey & Prewitt (1981) \\
\hline \multicolumn{3}{|c|}{ ENSOR-FRIO-FAIRLAND (Twin Sisters Phase) } \\
\hline 41BL104 & Evoe Terrace & Prewitt (1981) \\
\hline 41BL23 & Penny Winkle & Prewitt (1981) \\
\hline 41BL78 & Youngsport & Prewitt (1981) \\
\hline 41BT105 & Lion Creek & Johnson (1997) \\
\hline 41CM1 & Oblate & Prewitt (1981) \\
\hline 41CM3 & Wunderlich & Prewitt (1981) \\
\hline $41 \mathrm{CV} 1378$ & Tank Destroyer & This report \\
\hline 41LM3 & McCann & Prewitt (1981) \\
\hline 41ME7 & Scorpion Cave & Prewitt (1981) \\
\hline 41ML37 & Britton & Mehalchick and Kibler (2008) \\
\hline $41 \mathrm{TV} 75$ & Williams & Prewitt (1981) \\
\hline 41UV21 & La Jita & Prewitt (1981) \\
\hline 41UV60 & Anthon & Goode (2002) \\
\hline 41WM103 & Hoxie Bridge & Prewitt (1981) \\
\hline 41WM13 & Bessie Kruze & Johnson (2000) \\
\hline 41WM230 & Loeve Fox & Collins (1995), Prewitt (1981) \\
\hline \multicolumn{3}{|c|}{ DARL (Driftwood Phase) } \\
\hline 41BL104 & Evoe Terrace & Prewitt (1981) \\
\hline 41BT105 & Lion Creek & Johnson (1997) \\
\hline 41CV1378 & Tank Destroyer & This report \\
\hline $41 \mathrm{HI} 1$ & Kyle & Prewitt (1981) \\
\hline 41LM3 & McCann & Prewitt (1981) \\
\hline 41ML37 & Britton & Prewitt (1981) \\
\hline 41TV151 & Jetta Court & Prewitt (1981) \\
\hline $41 \mathrm{TV} 42$ & Smith Rockshelter & Prewitt (1981) \\
\hline $41 \mathrm{TV} 75$ & Williams & Prewitt (1981) \\
\hline $41 \mathrm{TV} 88$ & Pat Parker & Prewitt (1981) \\
\hline 41WM118 & Dobias-Vitek & Prewitt (1981) \\
\hline 41WM130 & Hoxie Bridge & Prewitt (1981) \\
\hline 41WM133 & Loeve & Prewitt (1981) \\
\hline 41WM230 & Loeve Fox & Collins (1995), Prewitt (1981) \\
\hline
\end{tabular}




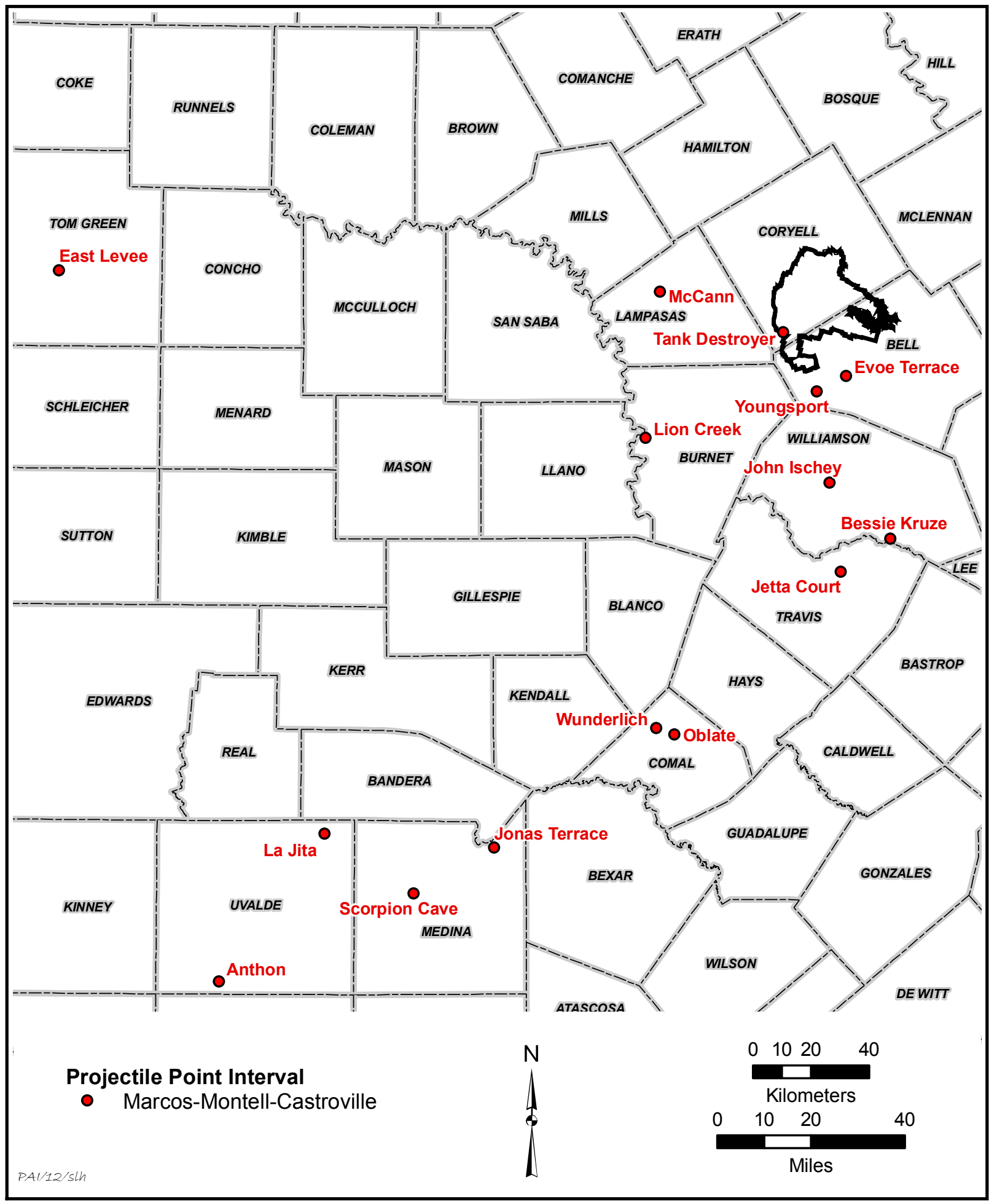

Figure 9.1. Map of sites containing significant components of the Marcos-Montell-Castroville projectile point interval, or Uvalde phase. 


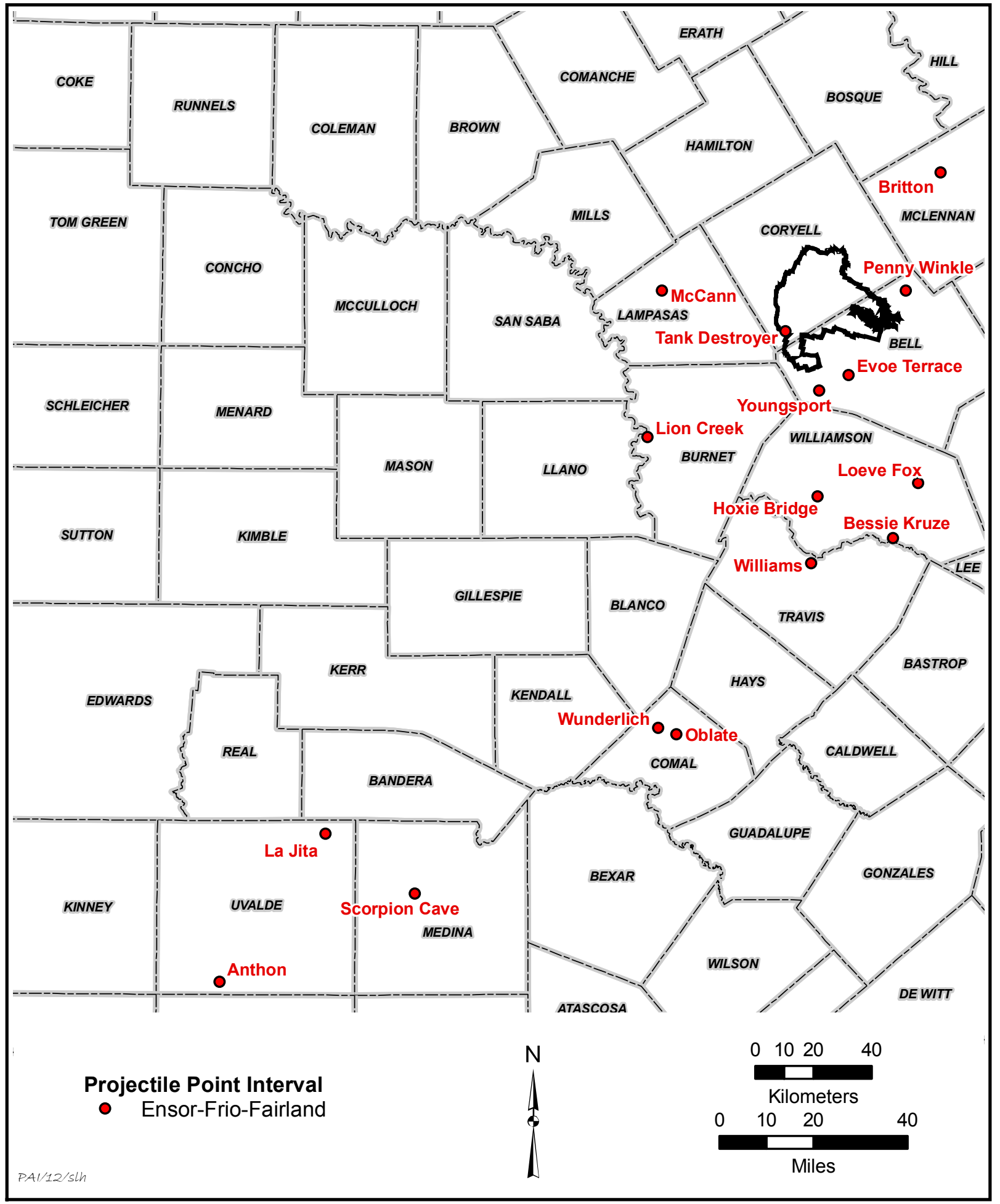

Figure 9.2. Map of sites containing significant components of the Ensor-Frio-Fairland projectile point interval, or Twin Sisters phase. 


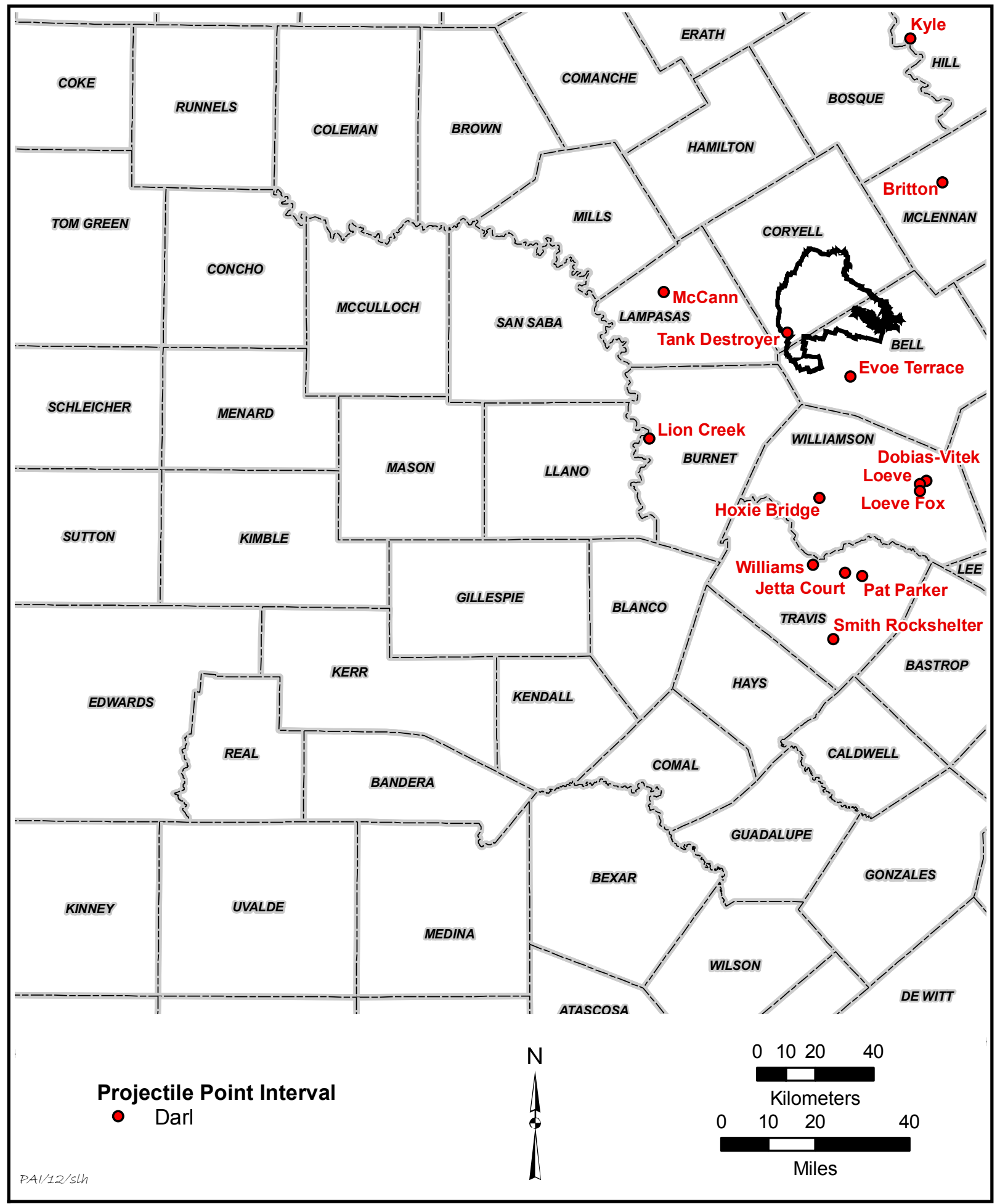

Figure 9.3. Map of sites containing significant components of the Darl projectile point interval, or Driftwood phase. 
predict that such site types significantly contributed to the maintenance of social boundaries and intergroup relationships over time and factored importantly in both settlement organization and distribution (following Parkinson 2006:36). Such locations were obviously important in demarcating specific resource exploitation zones and territories, but it also can be hypothesized that persistent places on the landscape functioned as visible evidence of a group's physical control of space as well (Kuusela et al. 2010). Sites such as cemeteries and burned rock middens, in effect, lay claim to ownership. A similar function may also be proffered for such prominent landscape and cultural features as the stone cairn burials in west-central Texas (Dial and Creel 2012).

Probably the most promising way to begin examining social identity in the Late Archaic would be to conduct DNA studies of the Late Archaic burial populations. Although the issue of conducting invasive and destructive tests on prehistoric and historic human skeletal remains is fraught with modern ethical and professional issues, the successful use of DNA in such research is well demonstrated (Kaestle and Hornsburgh 2002). DNA information has been effectively used to study identity at the level of the individual, family, local, and population levels (Kaestle and Hornsburgh 2002:96-101; Mills 2003; Ricaut et al. 2012). Similarly, stable carbon isotope analyses of human skeletal remains might also be useful to study social group identity, movements, and territorial boundaries (Hard and Katzenberg 2011). It is very easy to see how such data could be integrated into more traditional archeological studies of social identity to further refine levels of prehistoric social identity and social boundaries such as those proposed by Arnn (2012) and Stark (1998). 



\section{SUMMARY OF FINDINGS AT THE TANK DESTROYER SITE}

Karl W. Kibler and Douglas K. Boyd

\section{DATA RECOVERY INVESTIGATIONS}

Data recovery investigations at the Tank Destroyer site (41CV1378) were conducted in conjunction with a TxDOT road improvement project involving State Highway 9 and Tank Destroyer Blvd. The site is located near the planned intersection of the two roads. The investigations focused on a burned rock mound (Feature 1), one-half of which had been destroyed by an adjacent tank trail. The remaining portion of the mound measured approximately $14 \times 5 \mathrm{~m}$. Feature 1 contained two internal features: Feature 3, a large burned rock-filled pit or earth oven, and Feature 4, a small cluster of Rabdotus sp. shells (see Chapter 7). A small burned rock hearth (Feature 2) located just beyond the southwestern edge of the mound was also investigated. An area of $30.5 \mathrm{~m}^{2}$ and $11.8 \mathrm{~m}^{3}$ of cultural deposits were hand excavated, then an area of ca. $17.3 \mathrm{~m}^{2}$ was mechanically stripped.

Hand excavations covered ca. 45 percent of the remaining half of the mound, and mechanical scraping covered an additional estimated 30 percent of the extant mound to search for additional internal features, but none were found. A total of $5,570.5 \mathrm{~kg}$ of burned rocks was recovered from the mound (including Feature 3) excavations. Artifacts recovered from within the mound included a variety of chipped stone tools, including dart and arrow points, other bifaces, unifaces, flake tools, utilized flakes, cores, unmodified debitage, and a groundstone tool (see Chapter 6). The off-mound excavations yielded a similar diversity of artifacts, though fewer in number. There was virtually no preservation of faunal remains, save for snail shells, and poor preservation of botanical remains, which consisted of small charred wood pieces. No economic plants (i.e., processed food resources) were recovered despite the collection and processing of flotation samples.

Site occupation and midden use were dated by 16 radiocarbon assays on charred wood and Rabdotus sp. shells to 1500 B.C. through A.D. 1650. The date range for the diagnostic projectile points recovered from the site (200 B. C. to A.D. 1200) fits nicely within the range of radiocarbon dates. As a group, the radiocarbon dates and the projectile points suggest that the most intensive period of site use occurred between 1000 B.C. and A.D. 1200.

Unfortunately, the inability to recognize and define multiple components and the small assemblage, particularly the scarcity of certain artifacts and material types and categories, limits what can be said about the site. Given these limitations, a different analytical approach was taken. This approach was comprised of four tasks completed under work authorization issued by TxDOT after completion of the fieldwork, preliminary lab processing, and acquisition of radiocarbon assays. These four tasks consisted of: 1) analyses of the chipped stone and Rabotus sp. shell assemblages; 2) analysis of the burned rock data; 3) landscape analysis of burned rock mounds and middens at Fort Hood; and 4) examination of social identity in the Late Archaic period in central Texas.

\section{The Chipped Stone Assemblage and the TxDOT Lithic Analysis Protocol}

Including the earlier testing phase, the chipped stone assemblage recovered from the 
Tank Destroyer is relatively small, consisting of only 131 tools, 9 cores, and 4,466 pieces of unmodified debitage. Part of the analysis for the chipped stone assemblage included the implementation and evaluation of the TxDOT Chipped Stone Analytical Protocol. The full protocol (Version 2.3, dated July 16, 2010) is presented in Appendix C. We were asked to take the TxDOT protocol and developed it further by creating two blank data entry spreadsheets (Microsoft Excel) with instructions for the analysis of lithic tools (see Appendix D); and unmodified debitage and cores (see Appendix E). The 131 chipped stone tools recovered from 41CV1378 were then analyzed using the protocol (see Appendix F) as were the 9 cores and a ca. 10 percent sample of the unmodified debitage (see Appendix G). In the long term, the purpose of the protocol is to standardize replicability and decrease the variability in the analytical results between analysts with differing levels of experience so that interassemblage data are compatible.

The analysis of the chipped stone assemblage from $41 \mathrm{CV} 1378$ indicates that it is similar to those recovered from similar types of sites elsewhere on Fort Hood. It can be characterized as a low-density, generalized lithic assemblage with low tool diversity consisting of a mix of residential debris (e.g., broken and resharpened and discarded curated tools) and limited types of multifunctional tools (e.g., scrapers, denticulates, and utilized flakes). Such lithic assemblages reflect situations where a small number of tools must be used to complete many different tasks, part of a strategy employed by highly mobile groups whose members can only transport a limited amount of personal gear between residences.

Raw material procurement undoubtedly occurred within a few kilometers of the site, for the most part, based on the types of identified cherts in the assemblage. Despite this, there is a relative lack of both cores and cortex-bearing flakes. Core reduction, primarily represented by hard-hammer percussion flakes, only represents a small amount of the debitage assemblage and was associated with generalized core reduction and the production of flake blanks for a variety of edge-modified flake tools. Generally, small cortex-free flakes dominate the debitage assemblage.

Coinciding with the absence of cortex and small flake size predominance, the analyzed debris sample is dominated by flake types strongly indicative of lithic activities centered around the manufacture and maintenance of bifacial artifacts. It is probable that most of the biface manufacturing that occurred was conducted with the expressed purpose of replacing worn implements or to augment personal tool kits with bifacial tool blanks rather than to produce bifacial tools at the site. This strongly suggests that the need for bifacial tools was a continuous part of the groups' technology, and that the production of bifaces was done in conjunction with other resource procurement activities conducted at the site. In other words, tasks such as raw material procurement, tool manufacture, and repair were scheduled within the context of other activities.

The presence of denticulates, notched flakes, and burins/burin spall flakes indicate the need for a variety of multifunctional implements and perhaps certain function-specific implements at the site during its intermittent occupation. Use wear on these and utilized pieces of debitage demonstrates that a variety of tasks took place, including scraping and cutting. Burins were primarily used as specialized graving or scraping tools, probably for working wood or other similarly dense, hard materials.

The use of recycling is evidenced by the presence of bifaces, flakes, and other implements such as unifaces that are broken deliberately by smashing (radial fractures) and truncation (breakage into one or more pieces on an anvil of some sort). Burin spall retouch and deliberately broken artifacts yield edges that have durable facets that can be used for a variety of heavy scraping or planing tasks on hard materials. It is quite possible that these types of recycling activities could have made use of previously discarded tools and tool fragments from earlier occupations. In this case, lithic material (e.g., cores, tools, and flakes) discarded as clasts within the mound represents usable sources of raw material. Scavenging and recycling of lithic materials, and deliberate breakage of tools and tool fragments, places a different perspective on our understanding of lithic procurement and raw material reduction patterns that goes beyond the typical scenario for raw material procurement in chert-rich areas such as Fort Hood.

It was not uncommon for many historic Native American groups to scavenge lithic materials from archeological sites within 
their territories, especially bifacial tools and projectile points that could be easily reworked and reused (e.g., Amick 2007:226; Wandsnider 1989:430-436). Amick (2007:225) notes that this type of "secondary recycling," which involved procurement of stone tools and lithic debris from archeological sites, was very efficient because it could "provide high returns with relatively low investments of time and energy." Given the high density of archeological sites seen at Fort Hood and many other parts of the Edwards Plateau, it is reasonable to expect that many prehistoric groups in central Texas would have regularly engaged in secondary recycling. This behavior probably helps explain the large numbers of reused projectile points often found in central Texas sites.

There are two possible scenarios that could have produced the type of lithic assemblage seen at $41 \mathrm{CV} 1378$. First, the site could represent a residential base camp at which a range of technological activities occurred, accumulating through an unknown number of short-term intermittent occupations. Second, it may be a special-purpose site associated with the procurement and processing of specific resources. In this scenario, the site was reoccupied intermittently for periods of time, during which other activities not directly related to the function of the site were embedded and conducted. Overall, though, the lithic assemblage probably represents the congruence of strategies and activities that is the result of behaviors associated with both forager and collector mobility patterns and hence cannot be attributed exclusively to one strategy. Similar sites at Fort Hood have produced evidence supporting the idea of long-term continuity in repeated site use and patterns of resource exploitation from the Archaic into the Late Prehistoric period.

The implementation of the TxDOT Chipped Stone Analytical Protocol is discussed in Chapter 5 , which also includes an evaluation of the utility of this protocol. The protocol calls for developing spreadsheets with dropdown menus for the entry of observational and metric data on tools, cores, and unmodified debitage. For tools, the protocol consists of five parts: taxonomy, metric information, attributes, wear patterning, and raw material.

The protocol employs seven taxonomic levels to separate and define chipped stone tools. The highest taxonomic levels-technol- ogy and group-are straightforward and well defined. However, some of the lower taxonomic levels-subgroup, class, subclass, type, and subtype-are nebulous and not well defined. Given that, some of the less well-defined categories are believed to be of limited utility, particularly for tools with complex use lives (e.g., dart points recycled into burins). Particularly problematic is the goal of standardizing terminology, which even if categories are well defined and straightforward, would not prevent analysts with varying levels of experience from producing inconsistent results.

The collection of metric information on tools is fairly straightforward, and in most cases the analyst is only required to record maximum length, width, thickness, and weight. For most tool types this is clear; however, there is no standard for modified flake or blade tools unless the observable maximum dimension in question is recognizable. In addition, the protocol calls for measurements of edge angle. The discussion of this is rather short and would be more useful if expanded.

The protocol asks that observations on a series of attributes be recorded for each tool. Most of these attributes and their variables are straightforward and well defined, while others are limited and would greatly benefit from the addition of more variables (which in some cases we did). Wear patterning is another observation to be made. The discussion of this in the protocol is rather brief and tends to oversimplify the techniques and theory behind use-wear analysis, making its utility limited. The final category of observations that the protocol calls for is the identification of raw material. The raw material list provided in the protocol is useful and can be easily modified, adding or deleting certain materials to and from the list for specific projects.

Some aspects of the protocol are useful and easy to implement, while others are cumbersome and costly relative to the expected return in interpretive value. Providing better definitions for some of the categories might be helpful, though even with more thorough definitions, expecting analysts with different backgrounds and levels of experience to produce similar or standardized results in all categories is improbable. In the end, one analyst's bifacial core will almost always be other analyst's early-stage biface. Given this, simplifying the protocol might be a better option that would probably make it more useful. 
The protocol for the unmodified debitage is straightforward and fairly easy to implement, though use of the Minimum Number of Nodules (MNN) will be of limited value for assemblages from sites lacking tight temporal and spatial controls. The limited discussion of applying this technique to an assemblage is not very instructive for analysts who are unfamiliar with it. Also problematic are the cortex proportions for flakes, which seem to mimic those that have been used by other analysts but do nothing to eliminate subjectivity. Overall, though, the protocol for unmodified debitage is less cumbersome, far easier to implement, and much more likely to produce consistent results than the protocol for chipped stone tools.

\section{The Rabdotus sp. Shell Assemblage as a Food Source}

The results of the Rabdotus sp. shell assemblage analysis were not particularly robust, and in the end whether these snails served as a food source can not be confirmed with any level of confidence. The recovery of snail shells varied little between features and between feature and nonfeature contexts at the site. It appears that the density of snail shells was relatively consistent throughout most of the site, with an overall recovery of 140 snails per $\mathrm{m}^{3}$.

The age structure of the snail shells recovered from most of the features suggested that the population was natural and not the result of human selection of adult snails for food. Only Feature 3 yielded a large majority of adult snails, suggesting that those snails may represent a food source, albeit a very limited one. The context of these snail shells, however, makes this interpretation tenuous.

\section{Burned Rocks and Cooking Facilities}

A total of $5,792.6 \mathrm{~kg}$ of burned rocks were recovered from three burned rock features and nonfeature contexts at the Tank Destroyer site. The burned rock features consist of a classic domed, though slightly flattened by postoccupation disturbances, mound (Feature 1), a small off-mound hearth (Feature 2), and a burned rock-filled pit or earth oven (Feature 3 ) within the mound. Other than small pieces of charred wood, presumably representing fuel, no plant remains were recovered from these features, so it is not known what was being cooked. Some burned rocks are retained for curation for possible residue analysis in the future.

About half of the mound has been destroyed by an adjacent tank trail, but it is estimated to have been about $12 \mathrm{~m}$ in diameter. Feature 1 is fairly typical of burned rock mounds at Fort Hood, consisting of a jumbled mass of burned rocks surrounding an earth oven, though the only known earth oven (Feature 3) within Feature 1 is off center. The jumbled mass of burned rocks is dense at the core and less dense outside of the core. Radiocarbon dates, artifacts, and soil column data demonstrate that the mound accumulated through periodic use over at least a 3,150-year-long period, though discrete episodes of use and analysis units cannot be recognized or defined.

Based on the estimated size of the mound and burned rock weights from Feature 1 $(5,165.5 \mathrm{~kg})$ and Feature $3(405 \mathrm{~kg})$, it is estimated that the mound probably accumulated through 62 to 246 cooking events. This range of estimated cooking episodes depends on how many times a limestone rock can be heated in an earth oven before it fractures beyond the point of usefulness.

\section{Landscape Analysis of Burned Rock Mounds and Middens on Fort Hood}

Abbott et al.(1996:577-585) and Kleinbach et al.(1995:767-775) have suggested that burned rock mounds and middens are two distinct site types found on different parts of the landscape and characterized by different sets of activities. What these distinctions might mean in terms of prehistoric human behaviors is most clearly defined by Kleinbach et al. (1995:767-775), Abbott et al. (1996), Black et al. (1997:287-289), Boyd et al. (2000), Boyd and Mehalchick (2002), and Kleinbach et al. (1999:411-417). To better understand the differences between mounds and middens, the activities and behaviors that generated them, and how these things might have varied across the landscape through time, we conducted a comparative analysis of burned rock mounds and middens using the Fort Hood GIS database. The goal was to test the hypothesis that these feature types are indeed different from each other and are linked to particular 
landscape settings and other environmental variables.

We received permission from the Fort Hood CRM program to use the Fort Hood GIS database to conduct a baseline comparative analysis focusing on burned rock mounds and middens. We used the Fort Hood data as is, without manipulating the environmental and archeological variables. This analysis focused on whether there are any relationships between the archeological occurrences of burned rock mounds and middens and the environmental variables, and if so, how these relationships might relate to human behavior.

Working as a consultant for us, graduate student Laura Short of the Department of Anthropology, Texas A\&M University, conducted a study for use in our landscape analysis as part of a class project. She analyzed 11 variables within the Fort Hood GIS database and other data sets using a variety of statistical techniques to look for correlations. The results were not particularly robust, leading to the conclusion that the Fort Hood GIS data, especially the environmental variables, would have to be manipulated in some rather complex ways if we wanted to continue searching for links that might explain the distribution of burned rock mounds and middens across Fort Hood.

One aspect of Short's analysis was to examine the relationship between burned rock mounds and middens at Fort Hood to the outcrop of the Paluxy Formation. Archeological interest in this upland environment was first discussed by Abbott (1994:327-333; 1995:814-823), who noted that while the Paluxy environment made up no more than 2-3 percent of the Fort Hood landscape, it contained a relatively large number of sites and burned rock features. To Abbott, this suggested that the Paluxy environment was intentionally selected or favored by prehistoric hunters and gatherers in upland settings. In addition, he noted that Paluxy sites were qualitatively different than sites in other settings, yielding primarily burned rock features including earth ovens and burned rock mounds, low numbers of chipped stone artifacts, and tool assemblages of low diversity. These Paluxy site characteristics have also been noted by others (e.g., Kleinback et al. 1996; Mehalchick et al. 2004), suggesting that prehistoric hunters and gatherers conducted a different set of activities in the Paluxy environment than in other set- tings at Fort Hood. Given the nature of Paluxy site assemblages and the prevalence of burned rock mounds and earth ovens at Paluxy sites, Short's analysis, in part, attempted to look at the relationship between burned rock mounds and middens and the Paluxy environment. She concluded, however, that the data were "too coarse" to determine the nature of this relationship, if one existed. The coarse-grained nature of the data may have much to do with the fact that the Paluxy Formation is not accurately mapped or easily discernible in the data layers of the Fort Hood GIS database.

To get a better handle on the distribution of the Paluxy Formation outcrops at Fort Hood, PAI obtained another Fort Hood environmental data set-the distribution of post oaks-from the Nature Conservancy office at Fort Hood. Post oak communities are present wherever the Paluxy Formation crops out in west-central Fort Hood, and their distribution, along with that of Cisco and Wise soils, provide what is believed to be a more accurate representation of the Paluxy outcrop at Fort Hood. Once properly delineated, burned rock middens and mounds were then plotted and their relationship to the Paluxy examined. This exercise demonstrated that burned rock middens and mounds tend to occur in two environments: 1) the Paluxy, and 2) riparian zones along the high-order streams and their tributaries. While there may be several factors influencing this pattern, we know that there is one resource that is unconditionally required for earth oven cooking that is prevalent and easily accessible in both environments: firewood. Paluxy and riparian environments consist of relatively narrow, sinuous bands of arboreal communities that traverse a greater grassland or grassland savannah landscape.

A picture of earth oven use for plant food processing is becoming ever more clear, but the various plant foods recovered from these features are not limited to species from riparian and Paluxy environments. This suggests that earth oven processing in these environments is not necessarily linked only to the presence of plant food resource patches. The riparian zones are also arguably teeming with other vital resources, such as water and game, particularly deer, which are not available or are limited in the Paluxy environment. This fact is reflected in the greater number of artifacts and more diverse tool assemblages found in riparian 
zone archeological sites, as compared to Paluxy sites. All of this demonstrates a couple of fundamental aspects about burned rock middens and mounds at Fort Hood. First, earth oven cooking, and the resulting formation of burned rock middens and mounds, appears to be tied to firewood availability, as indicated by the fact that mounds and middens tend to cluster in two environments where firewood is abundant and easily accessible. Secondly, the use of the Paluxy environment and the activities that took place there were primarily related to earth oven cooking given the lack of other reliable resources (e.g, water), limited chipped stone tool and debris assemblages, and the ease of pit excavation in the sandy soils as noted by Abbott $(1994,1995)$ and other researchers.

\section{Social Identity in the Late Archaic of Central Texas}

The radiocarbon dates and diagnostic projectile points indicate that the human occupations and activities at the Tank Destroyer site primarily occurred during the Late Archaic, and more specifically during three time periods defined by Prewitt (1981) as the Uvalde, Twin Sisters, and Driftwood phases. The key markers of these phases, Marcos, Montell, Castroville, Ensor, Frio, Fairland, and Darl points, all (with the exception of Frio points) occur at the site. When initially defined by Prewitt, there appeared to be some meaningful differences among the three phases based on artifact and feature assemblages, mortuary practices, subsistence patterns, and extraregional exchange-differences that may represent different groups of people and changes through time in central Texas. Later research by Johnson and Goode (1994) organized the Late Archaic archeological record into units they called "patterns" that are based largely on subsistence pattern changes and technological differences among Late Archaic dart points. They viewed these patterns as evidence of different groups of people, some with ties to regions outside of central Texas. Collins (1995, 2004), expanding on Prewitt's earlier work and following that of Johnson and Goode (1994), referred to the various temporal units within the Late Archaic as archeological style intervals, which he based primarily on different dart point styles and types. Whether we use the term "phase," "pattern," or "interval," what Prewitt, Johnson and Goode, and Collins provide are syntheses that can serve as the basis for defining or identifying distinct groups of people within a geographically wide social field during the latter part of the Late Archaic in central Texas. We know these social phenomena existed, or at the very least it has been convincingly argued that they existed in central Texas during the later part of the Late Prehistoric and early Historic periods (ca. A.D. 1250 to 1750 ) by Arnn (2012) and Wade (2003). In particular, Wade $(2003: 228,231)$ notes that social groups and alliances did not arise in response to the presence of Europeans, that their complexities must be rooted in the past, prior to the arrival of Europeans. With that in mind, Arnn (2012) presents a testable model of how prehistoric social identity can be addressed through archeological data based on his research on classic Toyah social identity. This model that also be applied to the Late Archaic archeological data.

If we follow Arnn's model of what is necessary to begin such studies, at a minimum we would need a sufficiently large number of suitable sites (especially base camps and residential bases). Suitable sites should have excellent contextual integrity and good chronological control (c.f., Collin's [1995, 2004] gisement concept), and be located within a limited and welldefined geographic area (Arnn 2012:209-219). Unfortunately, such sites are few and far between for the central Texas Late Archaic. Of Prewitt's (1981) list of sites for Uvalde, Twin Sisters, and Driftwood phases, Collins (1995) selected only three that had sufficient integrity and context for comparative analysis. While there are some more recently excavated or reported sites that might be suitable and should be considered, there is still a relatively small number of sites within a very large region, and few locations within this region where sites are clustered in spatially limited areas. When the geographic distribution of sites pertaining to the model is considered, the difficulties in examining social identity become readily apparent. Quite simply, there are too few high-quality sites and they are too widely spread apart. There are no reasonably distinct clusters of three or more sites that might approximate the territorial size of a band or community (stated by Arnn [2012:231-232, Figure 8.10] as being 1,000 to 
$1,600 \mathrm{~km}^{2}$ in size), and only small groups of sites that might approximate marriage/language groups. An in-depth literature review might identify a few more suitable Late Archaic sites in greater central Texas that could be added to the list, but meeting the rigorous requirements for occupation sites with good chronological control and contextual integrity is a significant but necessary limitation of the model.

Although the data that are needed (as defined by Arnn) to address social identity in the Late Archaic is restrictive, other data sets that would augment the high-quality residential base camp data should be considered. Looking at sites around the periphery of the main cultural area can be used to help define core territories and identify peripheral "shared areas." Other site types, such as mortuary sites and burned rock middens, both of which fit the definition of "persistent places" on the landscape and are the result of behaviors that reflect repeated use of a single location over many decades, centuries, and even millennia, should be considered. These and other data of various scales ranging from an attribute of a single type of artifact to different site types may be the kinds of things needed to define and recognize social identity in the Late Archaic archeological record of central Texas.

\section{SOCIAL ASPECTS OF BURNED ROCK MIDDENS AND MOUNDS IN CENTRAL TEXAS}

Archeological research over the last decade or so has clearly demonstrated that earth ovens in central Texas are primarily related to the cooking of plant foods, particularly geophytes, and one of the results of repeated earth oven use at specific locations is the formation of various large burned rock features known throughout the literature by a plethora of names, including burned rock middens and burned rock mounds. Abbott et al. (1996:577-585) and Kleinbach et al. (1995:767-775; 1999:411-417) have argued that burned rock mounds and burned rock middens, though the product of similar actions, are in fact different types of features occupying different environments on the landscape and are associated with qualitatively and quantitatively different artifact assemblages. This distinction was first recognized and described at Fort Hood and has been observed by others working at Fort Hood (e.g., Boyd et al. 2000; Boyd and Mehalchick 2002) and other areas of central Texas (e.g., Black et al. 1997:287-289). A growing body of data seems to support the idea that burned rock mounds and middens have definable archeological differences that should reflect different human behaviors. These differences, particularly artifact content and association, suggest that these features are associated with different subsistence strategies. Following Binford's (1980) forager-collector model, burned rock middens would be part of a foraging strategy in which highly mobile groups move to the resources that are to be acquired and consumed, while burned rock mounds would be part of a collecting strategy in which specialized task groups acquire, process, and move the resources to consumers. While this illustrates how these features might have functioned within the subsistence strategies of prehistoric hunters and gatherers of central Texas, it ignores the social aspects of these features that surely existed but remain unclear.

Based on its morphology, composition, and artifact content and associations, the large burned rock feature (Feature 1) at the Tank Destroyer is a classic central Texas domed (though fairly flattened through postdepositional disturbance) burned rock mound, albeit with some variation from the norm regarding the location of its internal earth oven. Based on a range of estimated use lives of limestone rocks as heating elements, the burned rock content (weight), the size of the earth oven (Feature 3) within the burned rock mound, and the size of the mound itself, we estimated that Feature 1 may represent 62 to 246 cooking events, each producing approximately 1,000 liters (or ca. $28 \mathrm{U}$. S. dry bushels) of baked geophytes. For a group of 18 to 20 people, which based on the division of labor and labor schedules of ethnographically known hunters and gatherers is the minimal group size that could be maintained throughout most of the year (Binford 2001:233-234), this amount of food appears substantial, possibly providing enough calories from geophytes alone for the members of the group for several days. We know, however, that plant foods were not always processed in large volumes based on the recovery of charred geophytes from small features interpreted as family hearths (Mehalchick and Kibler 2008:146). Black and Thoms (2014:222) 
have recently argued that earth oven cooking in prehistory was much more widespread than we realize, and that large ovens were used "for purposes of storage or feasting and other forms of communal consumption." Obviously, larger earth ovens must have been used to produce food for groups larger than individual families. The range in the volume of food produced suggests that at times more than a few individual families were congregating for various reasons, but why?

The estimated 62 to 246 cooking episodes that occurred at the Tank Destroyer site took place over at least a 3,150-year period, which equates to an average of one cooking event every ca. 13 to 50 years. This suggests that the bulk processing of geophytes at any one burned rock mound in an upland setting was a fairly infrequent event. This could be due to the depletion of food and fuel resources near the site, or because the bulk processing of geophytes was linked to infrequent events that are not directly related to daily subsistence activities. Social group dynamics such as group aggregation, group fission, and dispersal are common elements of band-level societies and would occur infrequently throughout the year as compared to daily foraging and hunting for food. The processing of an estimated 1,000 liters of geophytes therefore might be tied to an aggregation of people far exceeding the minimum band size of 18 to 20 individuals.

Evidence that these cooking features, particularly large burned rock middens in riparian environments, were actually used by larger groups of people is admittedly circumstantial, but intriguing. Sites with burned rock middens, and the middens themselves, at Fort Hood and elsewhere in central Texas typically yield large and diverse numbers of tools, chipping debris, faunal remains, and other features. At Waco Lake, Mehalchick and Kibler (2008:367) noted that site components exhibiting high use intensity were also associated with burned rock midden formation. They attributed this greater site use intensity to larger aggregate groups and proposed a Late Archaic settlement-subsistence system characterized by group aggregation and group fission and dispersal that took place throughout the year, with the loci of aggregation marked by burned rock middens (Mehalchick and Kibler 2008:367-368). They further speculate that group aggregation probably took place in the spring when many geophytes are most nutritious, but they also suggest the spring scheduling could be rooted in a cosmology that viewed the seasonal change from winter to spring as a time of renewal and rebirth, hence group aggregation might also have been driven by the need for social and ritual activities important to the larger group's identity and viability. The timing of such an event is admittedly biased toward the idea of a long and enduring cold season followed by a warm season of plentiful resources. It ignores the fact that central Texas usually lacks a true long and enduring winter and that resource abundance and availability may be more aptly linked to moisture availability than temperature. Summer heat and drought in central Texas may be just as harsh on some resources as winter temperatures, if not more so. Given this, perhaps a more realistic schedule for the bulk processing of geophytes and associated group aggregation would be linked to the bimodal rainfall pattern of the region, which consists of peak late spring and early fall rains. Appreciable rainfall in the spring after cooler winter temperatures and in the early fall after typical hot and dry summer conditions would cause many plant resources to bloom and be more visible on the landscape. The rejuvenated landscape again could have played into that sense of renewal and rebirth, thus signaling to groups to schedule their social and ritual activities.

Obviously, sites with burned rock mounds tend not to display the same overall use intensity as sites with larger burned rock middens, so it is difficult to argue that mounds were the loci of large group aggregations. In a dynamic sense, though, sites and thus social group activities do not occur in isolation on the landscape but are part of a larger socioeconomic system. In this sense, we return to the idea of mounds being a part of a collecting strategy, where specialized task groups collect, process, and move resources to consumers. If our idea that the bulk processing of plant foods in earth ovens is a product of social group aggregation and ritual feasting is correct, then the larger aggregate groups that generated burned rock middens were also dispersing specialized task groups to collect and process geophytes away from the site of aggregation. This suggests that our larger aggregate group may have been processing the estimated 1,000 liters of geophytes in earth ovens at sev- 
eral scattered locations simultaneously. During a group aggregation for a social event, there might have been multiple ovens in use within a large midden at the base camp, as well as multiple ovens in mounds nearby. Unfortunately, we will probably never know the number of earth ovens operating simultaneously within the foraging radius of a large aggregate group base camp, and we cannot accurately estimate the total volume of geophytes processed in these features. Given these constraints, it would be difficult to estimate with any certainty what the total caloric and nutritional value of the all geophytes processed in a single feasting event for a large social group. If we are talking about the coalescence of several groups consisting of a few hundred individuals, it is probably safe to say that those values fall short of an individual's daily caloric and nutritional needs, which again suggests that the bulk processing of geophytes in earth ovens was not necessarily dictated by the practical aspects of food production but by social factors.

Returning to the Late Archaic settlementsubsistence system proposed by Mehalchick and Kibler (2008:368) for the Waco Lake area, this system can be viewed as a longstanding Late Archaic pattern of behavior that ultimately broke down at the beginning of the Late Prehistoric - a time that is often marked by evidence of violence and sharp population declines across central Texas (Prewitt 1981:83, 1985). There may be several factors behind this upheaval, including the end of more mesic conditions that prevailed across central Texas during the Late Archaic. Prior to the end of this mesic interval, it is possible that arboreal communities expanded beyond the riparian and Paluxy environments at Fort Hood, increasing the supplies of firewood across the landscape. In Chapter 8, we suggested that the location of burned rock middens and mounds was strongly linked to local firewood supplies, and previous research by Mauldin et al. (2003:220-231) also suggests that the local availability of firewood was a critical factor determining the location and use intensity of burned rock mounds. Increases in firewood supplies would have allowed larger groups to congregate more often and bulk process geophytes in more areas across the landscape. While the increased firewood supplies may have allowed for more frequent gatherings of larger groups of people using more of the landscape, it may have been spurred on by ceremonial rituals and religious ideological influences from more complex Woodland societies to the east (Johnson and Goode 1994), which may have required a more formal or organized ritual life. Again this suggests the possibility that earth oven cooking was not always tied to daily food production and that a significant amount of the procurement and processing of large quantities of root foods was tied to social and religious feasting events. It is acknowledged that possible religious influences from outside central Texas cannot account for earlier (ca. $>2000$ B.P.) middens and mounds, but it is notable that the proliferation of mounds and middens in the latter part of the Late Archaic coincides with a time when some (e.g., Prewitt 1981) suggest that central Texan cultures were participating in extensive exchange networks and thus were possibly exposed to ideas from outside of central Texas.

If these concepts have any merit, then it requires a substantial shift in thinking about the large burned rock features in central Texas. It challenges the idea that the earth ovens found at burned rock mounds and middens are only related to subsistence. It may be that many of earth ovens used to process bulk root foots are a product of communal feasting related to social gatherings and ritual events. Communal feasts were not random gatherings organized on the spur of the moment but planned events integrated into a group's cosmological beliefs and annual calendar. Spielmann (2002:197) argued that "The 'work' of communal feasting, thus, involves strategic planning over the course of a year or more and the intensification of a variety of subsistence activities whose products are targeted specifically for the feast." There is ample evidence for the use of earth ovens to cook large quantities of plant foods for communal feasting. Wills and Crown (2004:160) state that "a common ethnographic correlate of feasting is the earth oven or roasting pit, and while such features are often encountered archaeologically in the Southwest, they have not been the subject of detailed analyses of feasting." In their Comparative Studies of North American Indians, Driver and Massey (1957:233) present a discussion of earth ovens that mentions the communal aspects. Ethnographic records for camas roasting in the North Pacific Coast 
reveal that use of "large communal pits shared by a number of families." Carson (2002) presents one of the more in-depth studies of communal and ritual feasting involving earth ovens in the Hawaiian islands and Polynesia. The earth ovens there are called Ti ovens, so named for the plant that was most often being roasted. The ethnographic and archeological evidence show that very small earth ovens were used by single families, while medium and large ovens were used in multifamily to band-level social events involving communal politics and ritual. The ovens used in these communal contexts were called "uma ti," which distinguished them from smaller ovens used for subsistence. It is interesting that in almost all areas of the world where ethnography exists for earth ovens, they are connected to communal activities and feasting. But in central Texas where ethnographic accounts of Native people's daily lives before they were altered by European contact are virtually nonexistent, earth ovens are only discussed as subsistence-related features. Carson (2002:229, 345, 361) also notes ethnographic evidence of a connection between the root foods cooked in uma ti (communal earth ovens) and the production of intoxicating beverages used in communal events. This idea has not yet been given serious consideration in central Texas, but perhaps it should. 


\section{REFERENCES CITED}

Acuña, Laura I.

2006 The Economic Contribution of Root Foods and Other Geophytes in Prehistoric Texas. M.A. thesis. Department of Anthropology, Texas State University, San Marcos.

Amick, Daniel S.

2007 Investigating the Behavioral Causes and Archaeological Effects of Lithic Recycling. In Tools versus Cores:Alternative Approaches to Stone Tool Analysis, edited by Shannon P. McPherron, pp. 223-252. Cambridge Scholars Publishing, Newcastle, United Kingdom.

Andrefsky, William, Jr.

1998 Lithics: Macroscopic Approaches to Analysis. Cambridge University Press, Cambridge, United Kingdom.

Abbott, James T.

1994 Observations on Paluxy Sites. In Archeological Investigations on 571 Prehistoric Sites at Fort Hood, Bell and Coryell Counties, Texas, edited by W. Nicholas Trierweiler, pp. 327-333. Archeological Resource Management Series, Research Report No. 31. United States Army, Fort Hood.

1995 Observations on Paluxy Sand Sites. In NRHP Significance Testing of 57 Prehistoric Archeological Sites on Fort Hood, Texas, Vol. II, edited by James T. Abbott and W. Nicholas Trierweiler, pp. 814-823. Archeological Resource Management Series, Research Report No. 34. United States Army, Fort Hood.

Abbott, James T., and Charles D. Frederick

1990 Proton Magnetometer Investigations of Burned Rock Middens in West-Central Texas: Clues to Formation Processes. Journal of Archaeological Science 17:535-545.
Abbott, James T., Karl Kleinbach, and Gemma Mehalchick

1996 Features. In Archeological Testing at Fort Hood: 1994-1995, Vol. II, edited by W. Nicholas Trierweiler, pp. 577-617. Archeological Resource Management Series, Research Report No. 35. United States Army, Fort Hood.

Abbott, James T., and W. Nicholas Trierweiler (editors)

1995 NRHP Significance Testing of 57 Prehistoric Archeological Sites on Fort Hood, Texas, Vols. I and II. Archeological Resource Management Series, Research Report No. 34. United States Army, Fort Hood.

Ahler, Stanley A.

1989 Mass Analysis of Flaking Debris: Studying the Forest Rather Than The Tree. In Alternative Approaches to Lithic Analysis, edited by Donald O. Henry and George H. Odell, pp. 85-118. Archeological Papers of the American Anthropological Association No. 1.

1992 Use-Phase Classification and Manufacturing Technology in Plains Village Arrowpoints. In Piecing Together the Past: Applications of Refitting Studies in Archaeology, pp. 36-62. BAR International Series 578. BAR Oxford, England.

Amick, Daniel S.

2007 Investigating the Behavioral Causes and Archaeological Effects of Lithic Recycling. In Tools Versus Cores:Alternative Approaches to Stone Tool Analysis, edited by Shannon P. McPherron, pp. 223-252. Cambridge Scholars Publishing, Newcastle, UK.

Andrefsky, Jr., William

1998 Lithics: Macroscopic Approaches to Analysis. Cambridge University Press, Cambridge. 
2001 Lithic Debitage: Context, Form, Meaning. The University of Utah Press, Salt Lake City.

Arnn, John W., III

2007 Transformation and Persistence of Indigenous Cultural Identity During the Early Colonial and Late Prehistoric Periods in Texas. Ph.D. dissertation. Department of Anthropology, University of Kentucky.

2012 Land of the Tejas: Native American Identity and Interaction in Texas, A.D. 1300 to 1700. University of Texas Press, Austin.

Asch, Nancy B., Richard I. Ford, and David L. Asch 1972 Paleoethnobotany of the Koster Site: The Archaic Horizons. Reports of Investigations 24 and Illinois Valley Archeological Program Research Papers No. 6. Illinois State Museum, Springfield.

Baker, Joan

2001 No Golden Age of Peace:A Bioarchaeological Investigation of Interpersonal Violence on the West Gulf Coastal Plain. Unpublished Ph.D. dissertation. Department of Anthropology. Texas A\&M University.

Barrett, Glynn, Lucie Dingwall, Vince Gaffney, Simon Fitch, Cheryl Huckerby, and Tony Maguire

2007 Heritage Management at Fort Hood, Texas: Experiments in Historic Landscape Characterization. Archaeopress, Oxford, England. Archeological Resources Management Series, Research Report 57. United States Army, Fort Hood.

Barton, C. Michael

1997 Stone Tools, Style, and Social Identity: An Evolutionary Perspective on the Archaeological Record. American Anthropologist 7(1): 141-156.

Barton, C. Michael, Deborah I. Olszewski, and Nancy R. Coinman

1996 Beyond the Graver: Reconsidering Burin Function. Journal of Field Archaeology 23:111-125.

Beyries, Sylvie, and Veerle Rots

2008 The Contribution of Ethno-Archaeological Macro- and Microscopic Wear Traces to the Understanding of Archaeological Hide-Working Processes. In Prehistoric Technology 40 Years Later: Functional Studies and the Russian Legacy, edited by Laura Longo and Natalia Skakim, pp. 21-28. BAR British Series 1783, Oxford, United Kingdom.

Binford, Lewis R.

1973 Interassemblage Variability-The Mousterian and the "Functional" Argument. In The Explanation of Culture Change: Models in Prehistory, edited by Colin Renfrew, pp. 227-254. Duckworth, London.

1978 Nunamiut Ethnoarcheology. Academic Press, New York.

1980 Willow Smoke and Dogs' Tails: HunterGatherer Settlement Systems and Archaeological Site Formation. American Antiquity 45(1):4-20.

1982 The Archaeology of Place. Journal of Anthropological Archaeology 1: 5-31.

2001 Constructing Frames of Reference: An Analytical Method for Archaeological Theory Building Using Ethnographic and Environmental Data Sets. University of California Press, Berkeley.

Binford, Lewis R., and Sally R. Binford

1966 A Preliminary Analysis of Functional Variability in the Mousterian of Levallois Facies. American Anthropologist 68: 238-295.

Black, Stephen L.

1997 The Honey Creek Site, 41MS32. In Hot Rock Cooking on the Greater Edwards Plateau: Four Burned Rock Midden Sites in West Central Texas, Vol. 1, edited by Stephen L. Black, Linda W. Ellis, Darrell G. Creel, and Glenn T. Goode, pp. 99-167. Studies in Archeology 22, Texas Archeological Research Laboratory, the University of Texas at Austin.

Black, Stephen L., and Alston V. Thoms

2014 Hunter-Gatherer Earth Ovens in the Archaeological Record: Fundamental Concepts. American Antiquity 79(2):204-226.

Black, Stephen L., and Darrell G. Creel

1997 The Central Texas Burned Rock Midden Reconsidered. In Hot Rock Cooking on the Greater Edwards Plateau: Four Burned Rock Midden Sites in West Central Texas, Vol. 2, pp. 169-305. Studies in Archeology 22. Texas Archeological Research 
Laboratory, The University of Texas at Austin. Archeology Studies Program Report 2, Environmental Affairs Division, Texas Department of Transportation, Austin.

Black, Stephen L., and Linda W. Ellis

1997 Critically Observing and Recording Burned Rock Features. In Hot Rock Cooking on the Greater Edwards Plateau: Four Burned Rock Midden Sites in West Central Texas, Vol. 2, pp. 777-783. Studies in Archeology 22. Texas Archeological Research Laboratory, The University of Texas at Austin. Archeology Studies Program Report 2, Environmental Affairs Division, Texas Department of Transportation, Austin.

Black, Stephen L., Linda W. Ellis, Darrell G. Creel, and Glenn T. Goode

1997 Hot Rock Cooking on the Greater Edwards Plateau: Four Burned Rock Midden Sites in West Central Texas. 2 vols. Studies in Archeology 22. Texas Archeological Research Laboratory, The University of Texas at Austin. Archeology Studies Program Report 2, Environmental Affairs Division, Texas Department of Transportation, Austin.

Black, Stephen L., Kevin A. Miller, Glenn T. Goode, Linda W. Ellis, Susan Decker and Elton R. Prewitt

1997 Appendix C: Artifact Descriptions. In Hot Rock Cooking on the Greater Edwards Plateau: Four Burned Rock Middens in West Central Texas, Vol. 2, edited by S. Black, L.W. Ellis, D. Creel, and G. Goode, pp. 443-570. Studies in Archeology 22, Texas Archeological Research Laboratory, The University of Texas at Austin. Archeological Studies Program Report 2, Environmental Affairs Division, Texas Department of Transportation, Austin.

Blair, W. Frank

1950 The Biotic Provinces of Texas. The Texas Journal of Science 2(1):93-117.

Bleed, Peter

1986 The Optimal Design of Hunting Weapons: Maintainability or Reliability. American Antiquity 51:737-747.

Bordes, François

1973 On the Chronology and Contemporaneity of Different Paleolithic Cultures in France. In The Explanation of Culture Change: Models in Prehistory, edited by Colin Renfrew, pp. 217-226. Duckworth, London.

Bordes, François, and Denise de Sonneville Bordes

1970 The Significance of Variability in Paleolithic Assemblages. World Archaeology 2:61-73.

Bourdieu, Pierre

1984 Distinction: A Social Critique of the Judgement of Taste, translated by Richard Nice. Harvard University Press, Cambridge, Massachusetts.

1985 The Social Space and the Genesis of Groups. Theory and Society 14(6):723-744.

Bousman, C. Britt

1993 Hunter-Gatherer Adaptations, Economic Risk, and Tool Design. Lithic Technology 18:59-86.

Boyd, Douglas K., and Gemma Mehalchick

2002 The Clear Creek Golf Course Site: Understanding Central Texas Burned Rock Middens. In Geoarcheological Investigations at the Clear Creek Golf Course Site (41CV413), Fort Hood, Texas, by Gemma Mehalchick, Kyle Killian, Karl W. Kibler, and Douglas K. Boyd, pp. 71-85. Archeological Resource Management Series, Research Report No. 46. United States Army, Fort Hood.

Boyd, Douglas K., Gemma Mehalchick, and Karl W. Kibler

2004 Rethinking Paluxy Site Archeology. In Shifting Sands and Geophytes: Geoarcheological Investigations at Paluxy Sites on Fort Hood, Texas, by Gemma Mehalchick, Douglas K. Boyd, Karl W. Kibler, and Christopher W. Ringstaff, pp. 199-224. Archeological Research Management Series, Research Report No. 48. United States Army, Fort Hood.

Boyd, Douglas K., Gemma Mehalchick, and Ann M. Scott

2000 Burned Rock Mounds and Middens. In Planning Document for Treatment of National Register-Eligible Prehistoric Sites Under Section 106 of the National Historic Preservation Act, Fort Hood, Texas, pp. 3844. Unpublished document prepared by Prewitt and Associates, Inc., for the Cultural Resource Management Program, Environmental Division, Department of Public Works, United States Army, Fort Hood. 
Boyd, Douglas K., Christopher W. Ringstaff, and Gemma Mehalchick

2004 Analysis and Interpretations of Cultural Occupations at the FirebreakSite.InShifting Sands and Geophytes: Geoarcheological Investigations at Paluxy Sites on Fort Hood, Texas, by Gemma Mehalchick, Douglas K. Boyd, Karl W. Kibler, and Christopher W. Ringstaff, pp. 122-198. Research Report No. 48. United States Army Fort Hood, Archeological Resource Management Series. United States Army Fort Hood, Texas.

Bradbury, Andrew P., and Philip J. Carr

1995 Flake Typologies and Alternative Approaches: an Experimental Assessment. Lithic Technology 20:100-115.

Bradley, Bruce A.

1975 Lithic Reduction Sequences: A Glossary and Discussion. In Lithic Technology: Making and Using Stone Tools, edited by E. Swanson, pp. 5-14. Mouton Publishers, The Hague.

Brewer, M. B.

1991 The Social Self: On Being the Same and Different at the Same Time. Personality and Social Psychology Bulletin 17:475-482.

Brewington, Robbie L., John E. Dockall, and Harry J. Shafer

1995 Archaeology of 41MX5: A Late Prehistoric Caddoan Hamlet in Morris County, Texas. Reports of Investigations No. 1. Center for Environmental Archaeology, Texas A\&M University, College Station.

Brown, Kenneth M.

2002 Snails from the Quarter-Inch and EighthInch Screens. In The Smith Creek Bridge Site (41DW270): A Terrace Site in DeWitt County, Texas, by Dale Hudler, Keith Prilliman, and Thomas Gustavson, pp. 212-276. Studies in Archeology 35. Texas Archeological Research Laboratory, The University of Texas at Austin, and Archeology Studies Program, Report No. 17. Environmental Affairs Division, The Texas Department of Transportation, Austin.

Bureau of Economic Geology

1979 Waco Sheet, Geologic Atlas of Texas. Bureau of Economic Geology, The University of Texas at Austin.
Callahan, Errett

1979 The Basics of Biface Knapping in the Eastern Fluted Point Tradition: A Manual for Flintknappers and Lithic Analysts. Archaeology of Eastern North America 7:1-80.

Campbell, Thomas N.

1988 The Indians of Southern Texas and Northeastern Mexico: Selected Writings of Thomas Nolan Campbell. Texas Archeological Research Laboratory, The University of Texas at Austin.

Campbell, T. N., and T. J. Campbell

1981 Historic Indian Groups of the Choke Canyon Reservoir and Surrounding Area, Southern Texas. Choke Canyon Series, Vol. 1. Center for Archaeological Research, The University of Texas at San Antonio.

Carson, Mike T.

2001 Ti Ovens in Polynesia: Ethnological and Archaeological Perspective. The Journal of Polynesian Society 111(4):339-370.

Clark, John W., Jr.

1969 Implications of Land and Fresh-Water Gastropods in Archaeological Sites. Arkansas Academy of Science Proceedings $23: 38-52$

1976 Alvar Nunez and the Snail Rabdotus in Texas. The Nautilus 90(1):13-14.

Cohen, A.

1969 Custom and Politics in Urban Africa: A Study of Hausa Migrants in Yoruba Towns. University of California Press, Berkeley.

Collins, Michael B.

1974 A Functional Analysis of Lithic Technology among Prehistoric Hunter-Gatherers of Southwestern France and Western Texas. Ph.D. dissertation, University of Arizona, Tucson.

1975 Lithic Technology as a Means of Processual Inference. In Lithic Technology, Making and Using Stone Tools, edited by E. $\mathrm{H}$. Swanson, pp. 15-34. Aldine, Chicago, Illinois.

1991 Thoughts on Future Investigations of Burned Rock Middens. In The Burned Rock Middens of Texas: An Archeological Symposium, edited by Thomas R. Hester, pp. 1-24. Studies in Archeology 13. Texas 
Archeological Research Laboratory, The University of Texas at Austin.

1995 Forty Years of Archeology in Central Texas. Bulletin of the Texas Archeological Society 66:361-400.

2004 Archeology in Central Texas. In The Prehistory of Texas, edited by Timothy K. Perttula, pp. 101-126. Texas A\&M University Press, College Station.

2011 Heated Rocks as Evidence of Prehistoric Hunter-Gatherer Adaptations in Texas. Bulletin of the Texas Archeological Society 82:181-191.

Collins, Michael B. (editor)

1998 Wilson-Leonard: An 11,000-Year Archeological Record of Hunter-Gatherers in Central Texas, Vols. I-V. Studies in Archeology 31. Texas Archeological Research Laboratory, The University of Texas at Austin. Archeology Studies Program, Report 10. Environmental Affairs Division, Texas Department of Transportation, Austin.

Conkey, Margaret W., and Christine A. Hastorf (editors)

1990 The Uses of Style in Archaeology. Cambridge University Press, Cambridge.

Cortella, A. R. and M. L. Pochettino

1994 Starch Grain Analysis as a Microscopic Diagnostic Feature in the Identification of Plant Material. Economic Botany 48:171-181.

Cotterell, Brian, and Johann Kamminga

1979 The Mechanics of Flaking. In Lithic UseWear Analysis, edited by Brian Hayden, pp. 97-112. Academic Press, Inc., New York.

1987 The Formation of Flakes. American Antiquity 52:675-708.

Crabtree, Don E.

1966 AStoneworker's Approach to Understanding and Replicating the Lindenmeier Folsom. Tebiwa 9(1):3-39.

Dellar, D. Brian and Christopher J. Ellis

2001 Evidence for Late Paleoindian Ritual from the Caradoc Site (AfHj-104), Soutwestern Ontario, Canada. American Antiquity 66 (2):267-284).
Dering, J. Philip

1999 Earth Oven Processing in Archaic Period Economics: An Example from a Semi-Arid Savannah in South-Central North America. American Antiquity 64(4):659-674.

2003 Botanical Perspectives on Land Use in the Cross Timbers and Prairies: Plant Remains from Burned Rock Middens in Brown County, Texas. In Archaeological Testing to Determine the National Register Eligibility Status of 18 Prehistoric Sites on Camp Bowie, Brown County, Texas, Vol. 2 , by Raymond P. Mauldin, David L. Nickels, and Cory J. Broehm. Archaeological Survey Report No. 334. Center for Archaeological Research, The University of Texas at San Antonio.

Dial, Susan W., and Michael B. Collins

1998 Bifaces, Bifacial Tools, Perforators, Burins, and Spalls. In Wilson-Leonard, An 11,000-year Archeological Record of Hunter-Gatherers in Central Texas, Volume II: Chipped Stone Artifacts, edited by Michael B. Collins, pp. 537-596. Studies in Archeology 31, Texas Archeological Research Laboratory, The University of Texas at Austin. Archeology Studies Program, Report 10, Texas Department of Transportation, Environmental Affairs Division, Austin, Texas.

Dial, Susan, and Darrell Creel

2012 The Stone Cairns of West-Central Texas. Texas Beyond History. Electronic document, http://www.texasbeyondhistory. net/cairn/index.html, accessed September 12,2012 .

Dibble, Harold L., and Michel Lenoir (editors)

1995 The Middle Paleolithic Site of Combe Capelle Bas (France). The University Museum Press, Philadelphia.

Dickens, William A.

1993a Lithic Analysis. In Archaeological Investigations in Bull Branch: Results of the 1990 Summer Archaeological Field School, edited by David L. Carlson, pp. 79115. Archaeological Resource Management Series, Research Report No. 22. United States Army, Fort Hood.

1993b Lithic Artifact Analysis. In Archaeological Investigations in Spicewood Creek: Results of the 1991 Summer Archaeological Field School, edited by David L. Carlson, pp. 75111. Archeological Resource Management 
Series, Research Report No. 22. United States Army, Fort Hood.

Dietler, Michael, and Ingrid Herbich

1998 Habitus, Techniques, Style: An Integrated Approach to the Social Understanding of Material Culture and Boundaries. In The Archaeology of Social Boundaries, edited by Miriam T. Stark, pp. 232-263. Smithsonian Institution Press, Washington.

Diggs, George M. Jr., Barney L. Lipscomb, and Robert J. O'Kennon

1999 Shiners and Mahler's Illustrated Flora of North Central Texas. Botanical Research Institute of Texas (BRIT), Fort Worth.

Dockall, John

1991 Chipped Stone Technology at the NANRuin, Grant County, New Mexico. Unpublished M.A. thesis. Department of Anthropology, Texas A\&M University, College Station, Texas.

Dockall, John and Douglas K. Boyd

2006a Excavations at the Comal Power Plant Site, 41CM25. In Geoarcheological and Historical Investigations in the Comal Springs Area, LCRA Clear Springs Autotransformer Project, Comal County, Texas, by John E. Dockall, Douglas K. Boyd and Lannie Ethridge Kittrell, pp. 41-79. Reports of Investigations No. 149. Prewitt and Associates, Inc., Austin, Texas.

2006b Excavations at the Landa Park Golf Course Site, 41CM167. In Geoarcheological and Historical Investigations in the Comal Springs Area, LCRA Clear Springs Autotransformer Project, Comal County, Texas, by John E. Dockall, Douglas K. Boyd and Lannie Ethridge Kittrell, pp. 81-111. Reports of Investigations No. 149. Prewitt and Associates, Inc., Austin, Texas.

Dockall, John E., Stephanie L. Katauskas, and Ross C. Fields

2008 National Register Testing of Four Sites in the Sabine Mine's Area M, Harrison County, Texas. Reports of Investigations No. 157. Prewitt and Associates, Inc., Austin.

Dockall, John, and Charlotte D. Pevney

2007 Lithic Assemblages: Transitions of Holocene Technological Organization. In Archaeological and Paleoecological Investigations at the Richard Beene Site in South-Central Texas, 2007, edited by
Alston V. Thoms and Rolf D. Mandel, pp. 177-228. Reports of Investigations No. 8. Center for Ecological Archaeology, Texas A\&M University, College Station, Texas.

Driskell, Boyce N.

1986 The Chipped Stone Tool Production/Use Cycle. BAR International Series 305. BAR, Oxford, England.

Driver, Harold E., and William C. Massey

1957 Comparative Studies of North American Indians. Transactions of the American Philosophical Society, New Series 47(2):165-456.

Edens, Christopher

1999 The Chipped Stone Industry at Hacinebi: Technological Styles and Social Identity. Paleorient 25(1):23-33.

Frederick, Charles D., Michael D. Glascock, Hector Neff, and Christopher M. Stevenson

1994 Evaluation of Chert Patination as a Dating Technique: A Case Study from Fort Hood, Texas. Archeological Resource Management Series, Research Report No. 32. United States Army, Fort Hood.

Frederick, Charles D., and Christopher Ringstaff 1994 Lithic Resources at Fort Hood: Further Investigations on 571 Prehistoric Sties at Fort Hood, Bell and Coryell Counties, Texas, edited by W. Nicholas Trierweiler, pp. 125-181. Archaeological Resource Management Series, Research Report No. 31. United States Army, Fort Hood.

Frison, George C., and Bruce A. Bradley

1980 Folsom Tools and Technology at the Hanson Site, Wyoming. University of New Mexico Press, Albuquerque.

Fullington, Richard W.

1978 Analysis of Molluscan Remains Aquilla Lake, Texas. Appendix I in An Evaluation of Archaeological Resources at Aquilla Lake, S. A. Skinner, C. Shaw, K. Huckabay, and M. L. Bartsch, pp. 213-228. Report 117. Archaeology Research Program, Southern Methodist University, Dallas.

Gadus, E. Frances, Marie E. Blake, Martha Doty Freeman, and Karl W. Kibler

1999 National Register Testing of Prehistoric and Historic Sites and Survey of Placement Areas, Channel to Victoria, Calhoun and Victoria Counties, Texas. Reports 
of Investigations, No. 121. Prewitt and Associates, Inc., Austin, Texas.

Gadus, E. Frances, Ross C. Fields, and Karl W. Kibler

2006 Data Recovery Excavations at the J.B. White Site (41MM341), Milam County, Texas. Reports of Investigations No. 145. Prewitt and Associates, Inc., Austin.

Gal, Uri, Youngjin Yoo, and Richard J. Boland

2004 The Dynamics of Boundary Objects, Social Infrastructures, and Social Identities. Case Western Reserve University, USA. Sprouts: Working Papers on Information Systems, 4(11). Available at http://sprouts.aisnet. org/4-11.

Goode, Glenn T.

2002 The Anthon Site:A Prehistoric Encampment in Southern Uvalde County, Texas. Report No. 38. Texas Department of Transportation, Environmental Affairs Division, Archeological Studies Program, Austin, Texas.

Goodyear, Albert C.

1993 Toolkit Entropy and Bipolar Reduction: A Study of Interassemblage Lithic Variability Among Paleo-Indian Sites in the Northeastern United States. North American Archaeologist 14(1):1-23.

2005 Evidence of Pre-Clovis Sites in the Eastern United States. In Paleoamerican Origins: Beyond Clovis, edited by R. Bonnichsen, B. Lepper, D. Stanford, and M. Waters, pp. 103-12. Texas A\&M University Press, College Station.

Gramly, Richard M.

1980 Raw Materials Source Areas and "Curated" Tool Assemblages. American Antiquity 45(4):823-833.

Hall, Grant D.

1981 Allens Creek: A Study in the Cultural Prehistory of the Lower Brazos River Valley, Texas. Research Report 61. Texas Archeological Survey, The University of Texas at Austin.

Hard, Robert J., and M. Anne Katzenberg

2011 A Stable Isotope Study of Hunter-GathererFisher Diet, Mobility, and Intensification on the Texas Gulf Coastal Plain. American Antiquity 76(4):709-751.
Hayward, O. T., Peter M. Allen, and David L. Amsbury

1990 Lampasas Cut Plain-Evidence for the Cyclic Evolution of a Regional Landscape, Central Texas. In Geological Society of America Guidebook 2, p. 122. Dallas.

1996 Lampasas Cut Plain: Episodic Development of an Ancient and Complex Regional Landscape, Central Texas. In Guidebook to Upland, Lowland, and In BetweenLandscapes in the Lampasas Cut Plain, edited by David L. Carlson, pp. 1-1 through 1-97. Friends of Pleistocene South-Central Cell 1996 Field Trip. Department of Anthropology, Texas A\&M University, College Station, and Department of Geology, Baylor University, Waco.

Hegmon, Michelle

1992 Archaeological Research on Style. Annual Review of Anthropology 21:517-536.

1998 Technology, Style and Social Practices: Archaeological Approaches. In The Archaeology of Social Boundaries, edited by Miriam T. Stark, pp. 264-279. Smithsonian Institution Press, Washington.

Hester, Thomas R., and T. C. Hill, Jr.

1975 Eating and Land Snails in Prehistoric Southern Texas: Ethnohistoric and Experimental Data. The Nautilus 89(2):37-38.

Highley, Cheryl Lynn

1986 Archaeological Investigations at 41LK201, Choke Canyon Reservoir, Southern Texas. Choke Canyon Series, Vol. 11. Center for Archaeological Research, The University of Texas at San Antonio.

Hill, Robert T.

1901 Geography and Geology of the Black and Grand Prairies, Texas, with Detailed Descriptions of the Cretaceous Formations and Special Reference to Artesian Waters. In Twenty-first Annual Report to the United States Geological Survey 1899-100, Part 7-Texas.

Hogg, Michael A.

2001 A Social Identity Theory of Leadership. Personality and Social Psychology Review 5(3): 184-200. 
Holmes, William Henry

1894 Natural History of Flaked Stone Implements Memoirs of the International Congress of Anthropology, edited by C. S. Wake, pp. 120-139. Schulte, Chicago.

Huddy, Leonie

2002 Context and Meaning in Social Identity Theory: A Response to Oakes. International Journal of Political Psychology 23(4):825-838.

Jeske, Robert J., and Rochelle Lurie

1993 The Archaeological Visibility of Bipolar Technology: An Example from the Koster Site. Midcontinental Journal of Archaeology 18(2):131-160.

Johnson, Jay K.

1986 Amorphous Core Technologies in the Midsouth. Midcontinental Journal of Archaeology 11:135-151.

1989 The Utility of Production Trajectory Modeling as a Framework for Regional Analysis. In Alternative Approaches to Lithic Analysis, edited by Donald O. Henry and George H. Odell, pp. 119-138. Archeological Papers of the American Anthropological Association No. 1.

Johnson, LeRoy Jr.

1989 Great Plains Interlopers in the Eastern Woodlands in Late Paleoindian Times: The Evidence from Oklahoma, Texas, and Areas Close By. Office of the State Archeologist, Texas Historical Commission. Report Series 36 .

1994 The Life and Times of Toyah-Culture Folk as Seen from the Buckhollow Encampment, Site 41KM16 of Kimble County, Texas. Texas Antiquities Permit No. 35. Office of the State Archeologist, Record 38. Texas Historical Commission and Texas Department of Transportation, Austin.

1995 Past Cultures and Climates at Jonas Terrace 41ME29, Medina County, Texas. Office of the State Archaeologist Report 40. Texas Historical Commission, Austin, Texas.

1997 The Lion Creek Site (41BT105):Aboriginal Houses and Other Remains of a Prehistoric Ranchería in the Texas Hill Country (Burnet County). Archeological Studies Program Report 1, Environmental Affairs Division, Texas Department of Transportation,
Austin, and Office of the State Archeologist Report 41,

2000 Life and Death as Seen at the Bessie Kruze Site (41WM13) on the Blackland Prairie of Williamson County, Texas. Report No. 22. Texas Department of Transportation, Environmental Affairs Division, Archeological Studies Program, Austin, Texas.

Johnson, Leroy Jr., and Glenn T. Goode

1994 A New Try at Dating and Characterizing Holocene Climates, as Well as Archeological Periods, on the Eastern Edwards Plateau. Bulletin of the Texas Archeological Society 65:1-51.

Johnson, LeRoy, Jr., Dee Ann Suhm, and Curtis D. Tunnell

1962 Salvage Archeology of Canyon Reservoir: The Wunderlich, Footbridge, and Oblate Sites. Bulletin 5. Texas Memorial Museum, Austin.

Kaestle, Frederika A., and K. Ann Horsburgh

2002 Ancient DNA in Anthropology: Methods, Applications, and Ethics. Yearbook of Physical Anthropology 45:92-130.

Kamiya, Masahiro

2011 Use of Raman Spectroscopy on Wild Plants and Fire-Cracked Rocks to Analyze Organic Residues. Presentation to the Council of Texas Archeologists. Fall 2011 Meeting, October 28, 2011, Fort Worth, Texas.

Kehoe, Alice B.

2005 Expedient Angled-Tang Endscrapers: Glimpsing Women's Work in the Archaeological Record. In Gender and Hide Production, edited by Lisa Frink and Kathryn Weedman, pp. 133-142. AltaMira Press, New York.

Kelly, Robert L.

1983 Hunter-Gatherer Mobility Strategies. Journal of Anthropological Research 39(3):277-306.

1992 Mobility/Sedentism: Concepts, Archaeological Measures, and Effects. Annual Review of Anthropology 21:43-66.

1995 The Foraging Spectrum: Diversity in Hunter-Gatherer Lifeways. Smithsonian Institution Press, Washington, D.C. 
Kibler, Karl W.

1999 Paluxy Geomorphic Investigations: Site Stratigraphy, Sediments, and Formation Processes. In National Register Testing of 42 Prehistoric Archeological Sites on Fort Hood, Texas: The 1996 Season, by Karl Kleinbach, Gemma Mehalchick, Douglas K. Boyd, and Karl W. Kibler, pp. 39-58. Archeological Resource Management Series, Research Report No. 38. United States Army, Fort Hood.

Kibler, Karl W., and Ann M. Scott

2000 Archaic Hunters and Gatherers of the Balcones Canyonlands: Data Recovery at the Cibolo Crossing Site (41BX377), Camp Bullis Military Reservation, Bexar County, Texas. Reports of Investigations, No. 126. Prewitt and Associates, Inc., Austin, Texas.

Kleinbach, Karl, Gemma Mehalchick, James T. Abbott, and J. Michael Quigg

1995 Investigated Features. In NRHP Significance Testing of 57 Prehistoric Archeological Sites on Fort Hood, Texas, edited by James. T. Abbott and W. Nicholas Trierweiler, pp. 765-801. Archeological Resource Management Series, Research Report No. 34. United States Army, Fort Hood.

Kleinbach, Karl, Gemma Mehalchick, Douglas K. Boyd, and Karl W. Kibler

1999 National Register Testing of 42 Prehistoric Archeological Sites on Fort Hood, Texas: The 1996 Season. Archeological Resource Management Series, Research Report No. 38. United States Army, Fort Hood.

Knecht, Heidi

1988 Upper Paleolithic Burins. BAR International Series 434. British Archaeological Reports, Oxford.

Kuhn, Steven L.

1990 Diversity Within Uniformity: Tool Manufacture and Use in the Pontinian Mousterian of Latium (Italy). Ph.D. dissertation, Department of Anthropology, University of New Mexico.

1991 "Unpacking”Reduction:Lithic Raw Material Economy in the Mousterian of WestCentral Italy. Journal of Anthropological Archaeology 10:76-106.
1995 Mousterian Lithic Technology:An Ecological Perspective. Princeton University Press, Princeton, NJ.

Kuusela, Jari-Matti, Samuel Vaneeckhout and Jari Okkonen

2010 Places of Importance and Social Communication: Studying the PreRoman Cairn Field of Viirikallio in Laihia, Finland. Estonian Journal of Archaeology $14: 22-39$.

Larson, Mary Lou

1994 Toward a Holistic Analysis of Chipped Stone Assemblages. In The Organization of North American Prehistoric Chipped Stone Tool Technologies, edited by Philip J. Carr, pp. 57-69. Archaeological Series 7, International Monographs in Prehistory, Ann Arbor, Michigan.

Larson, Mary Lou, and Marcel Kornfeld

1997 Chipped Stone Nodules: Theory, Method, and Examples. Journal of Lithic Technology 22(1):4-18.

Laurence, Andrew R., Alston V. Thoms, Vaughn M. Bryant, and Cassandra McDonough

2011 Airborne Starch Granules as a Potential Contamination Source at Archaeological Sites. Journal of Ethnobiology 31(2):213-232.

Leach, Jeffrey D., D. L. Nichels, B. K. Moses, and R. Jones

2001 Estimating Rates of Burned Rock Discard: Results from an Experimental Earth Oven. In Test Excavations at the Culebra Creek site, 41BX126, San Antonio, Texas, by D. L. Nickels, C. B. Bousman, J. D. Leach, and D. A. Cargill, pp. 275-282. Archaeological Survey Report, No. 265. Center for Archaeological Research, University of Texas at San Antonio. And Archeological Studies, Report No. 3. Environmental Affairs Division, Texas Department of Transportation, Austin.

LeBlanc, Raymond

1992 Wedges, Pieces Esquillees, Bipolar Cores, and Other Things: An Alternative to Shott's View of Bipolar Industries. North American Archaeologist 13(1):1-14. 
Lechtman, Heather

1977 Style in Technology: Some Early Thoughts. In Material Culture: Style, Organization, and Dynamics of Technology, edited by Heather Lechtman and R. S. Merrill, pp. 3-20. West Publishing, New York and St. Paul, Minnesota.

Littleton, Judith

2007 From the Perspective of Time: HunterGatherer Burials in South-Eastern Australia. Antiquity 81:1013-1028.

Littleton, Judith, and Harry Allen

2007 Hunter-Gatherer Burials and the Creation of Persistent Places in Southeastern Australia. Journal of Anthropological Archaeology 26:283-298.

Lucas, Jason R., and Charles D. Frederick

1998 Burned Rock as Artifact. In Investigation and Experimentation at the Higgins Site (41BX184), Vol. I, by Stephen L. Black, Kevin Jolly, Charles D. Frederick, Jason R. Lucas, James W. Karbula, Paul R. Takak, and Daniel R. Potter, pp. 163-188. Module 3 of Archeology Along the Wurzback Parkway. Studies in Archeology 27, Texas Archeological Research Laboratory, University of Texas at Austin.

Masson, Marilyn

1998 Analysis of Debitage. In Wilson-Leonard:An 11,000-year Archeology Record of HunterGatherers in Central Texas, Vol. 3:Artifacts and Special Artifacts Studies, edited by Michael B. Collins, pp. 683-702. Studies in Archaeology 31. Texas Archaeological Research Laboratory, University of Texas at Austin.

McCaleb, Nathan L.

1985 Soil Survey of Coryell County, Texas. United States Department of Agriculture, Soil Conservation Service, in cooperation with Texas Agricultural Experiment Station and United States Department of the Army, Fort Hood.

Mauldin, Raymond P., and Daniel S. Amick

1989 Investigating Patterning in Debitage from Experimental Bifacial Core Reduction. In Experiments in Lithic Technology, edited by Daniel S. Amick and Raymond P. Mauldin, pp. 67-88. BAR International Series 528, Oxford, United Kingdom.
Mauldin, Raymond P., and David L. Nickels

2003 Burned Rock Middens in Texas. In Archaeological Testing to Determine the National Register Eligibility Status of 18 Prehistoric Sites on Camp Bowie, Brown County, Texas, Vol. 1, Raymond P. Mauldin, David L. Nickels, and Cory J. Broehm, pp. 217-231. Archaeological Survey Report No. 334. Center for Archaeological Research, The University of Texas at San Antonio.

Mauldin, Raymond P., David L. Nickels, and Cory J. Broehm

2003 Archaeological Testing to Determine the National Register Eligibility Status of 18 Prehistoric Sites on Camp Bowie, Brown County, Texas. 2 vols. Archaeological Survey Report, No. 334. Center for Archaeological Research, The University of Texas at San Antonio.

Mehalchick, Gemma, and Douglas K. Boyd

2007 Interim Report on Data Recovery of a Burned Rock Mound at 41CV1378 on Fort Hood, Coryell County, Texas. Report submitted to the Environmental Affairs Division, Texas Department of Transportation, Austin. Prewitt and Associates, Inc., Austin, Texas.

Mehalchick, Gemma, Douglas K. Boyd, Karl W. Kibler, and Christopher W. Ringstaff

2004 Shifting Sands and Geophytes: Geoarcheological Investigations at Paluxy Sites on Fort Hood, Texas. Archeological Research Management Series, Research Report No. 48. United States Army, Fort Hood.

Mehalchick, Gemma, and Karl W. Kibler

2005 National Register Testing of Nine Prehistoric Sites on Fort Hood, Texas: The 20012002 Season. Research Report No. 50, Archeological Resource Management Series, United States Army Fort Hood.

2008 Hunters and Gatherers of the North Bosque River Valley: Excavations at the Baylor, Britton, McMillan, and Higginbotham Sites, Waco Lake, McLennan County, Texas. Reports of Investigations, No. 156. Prewitt and Associates, Inc., Austin, Texas.

Mehalchick, Gemma, Karl W. Kibler, Amy M. Holmes, Christopher W. Ringstaff, and Douglas K. Boyd

2003 National Register Testing of 19 Prehistoric Archeological Sites at Fort Hood, Texas: The 
2000-2001 Season. Archeological Resource Management Series, Research Report No. 47. United States Army, Fort Hood.

Mehalchick, Gemma, Kyle Killian, Karl W. Kibler, and Douglas K. Boyd

2002 Geoarcheological Investigations at the Clear Creek Golf Course Site (41CV413), Fort Hood, Texas. Archeological Resource Management Series, Research Report No. 46. United States Army, Fort Hood.

Mehalchick, Gemma, Karl Kleinbach, Douglas K. Boyd, and Karl W. Kibler

2000 Geoarcheological Investigations and National Register Testing of 52 Prehistoric Archeological Sites at Fort Hood, Texas: The 1997 Season. Research Report No. 39. Archeological Resource Management Series, United States Army, Fort Hood.

Mehalchick, Gemma, Karl Kleinbach, Douglas K. Boyd, Steve A. Tomka, and Karl W. Kibler 1999 National Register Testing of 19 Prehistoric Archeological Sites at Fort Hood, Texas: The 1995 Season. Research Report No. 37. Archeological Resource Management Series, United States Army, Fort Hood.

Mills, Lisa Ann

2003 Mitochondrial DNA Analysis of the Ohio Hopewell of the Hopewell Mound Group. Unpublished Ph.D. dissertation, Department of Anthropology, Ohio State University, Columbus.

Morrow, Carol A.

1984 A Biface Production Model for GravelBased Chipped Stone Industries. Lithic Technology 13:20-28.

Mueller-Wille, Catherine S., and David L. Carlson 1990 Archaeological Survey at Fort Hood, Texas: Fiscal Year 1986, Other Training Areas. Archaeological Resource Management Series, Research Report No. 21. United States Army, Fort Hood.

Muto, Guy R.

1971 A Stage Analysis of the Manufacture of Stone Tools. In Great Basin Anthropological Conference 1970: Selected Papers, edited by M. Aiken, pp. 109-118. University of Oregon Anthropological Papers, Eugene.

Natural Fibers Information Center

1987 The Climates of Texas Counties. Bureau of Business Research, The University of Texas at Austin, in cooperation with the
Office of the State Climatologist, Texas A\&M University, The University of Texas at Austin.

Neck, Raymond W.

1994 Interpretations of Molluscan Remains from the Mustang Branch Site (41HY209). In Archaic and Late Prehistoric Human Ecology in the Middle Onion Creek Valley, Hays County, Texas, Vol. 2-Topical Studies, by Robert A. Ricklis and Michael B. Collins, pp. 491-497. Studies in Archeology 19. Texas Archeological Research Laboratory.

Nelson, Margaret C.

1991 The Study of Technological Organization. In Archaeological Method and Theory, Vol. 3, edited by Michael B. Schiffer, pp. 57-100. The University of Arizona Press, Tucson.

Nordt, Lee C.

1992 Archaeological Geology of the Fort Hood Military Reservation, Fort Hood, Texas. Archaeological Resource Management Series, Research Report 25. United States Army, Fort Hood.

1993 Additional Geoarchaeological Investigations at the Fort Hood Military Reservation, Fort Hood, Texas. Archaeological Resource Management Series, Research Report No. 25. United States Army, Fort Hood.

1995 Geoarchaeological Investigations of Henson Creek: A Low-Order Tributary in Central Texas. Geoarchaeology 10(3):205-221.

Odell, George H.

1979 A New and Improved System for the Retrieval of Functional Information from Microscopic Observations of Chipped Stone Tools. In Lithic Use-Wear Analysis, edited by Brian Hayden, pp. 329-345. Academic Press, New York.

1980 Toward a More Behavioral Approach to Archaeological Lithic Concentrations. American Antiquity 45(3):404-431.

1989 Experiments in Lithic Reduction. In Experiments in Lithic Technology, edited by Daniel S. Amick and Raymond P. Mauldin, pp. 163-198. BAR International Series 528, Oxford, United Kingdom.

2004 Lithic Analysis. Kluwer Academic/Plenum Publishers, New York. 
Odell, George H., and Frieda Odell-Vereecken 1980 Verifying the Reliability of Lithic Usewear Assessments by "Blind Tests": The Low Power Approach. Journal of Field Archaeology 7(1):87-120.

Oliveira, Hugo R., Peter Civán, Jacob Morales, Amelia Rodríguez-Rodríguez, Diane L. Lister and Martin K. Jones

2012 Ancient DNA in Archaeological Wheat Grains: Preservation Conditions and the Study of Pre-Hispanic Agriculture on the Island of Gran Canaria (Spain). Journal of Archaeological Science 39:828-835.

Padilla, Amado M., and William Perez

2003 Acculturation, Social Identity, and Social Cognition: A New Perspective. Hispanic Journal of Behavioral Sciences 25(1):35-55.

Parkinson, William A.

2006 Tribal Boundaries: Stylistic Variability and Social Boundary Maintenance during the Transition to the Copper Age on the Great Hungarian Plain. Journal of Anthropological Archaeology 25:33-58.

Parry, William J., and Robert L. Kelly

1987 Expedient Core Technology and Sedentism. In The Organization of Core Technology, edited by Jay K. Johnson and Carol A. Morrow, pp. 285-304. Westview Press, Boulder, CO.

Perttula, Timothy K.

2001 Hunter-Gatherer Mortuary Practices in the Rio Grande Plains and Central Coastal Plains Archeological Regions of Texas. $L a$ Tierra 28(3-4):2-83.

Piperno, Dolores R.

2006 Phytoliths: A Comprehensive Guide for Archaeologists and Paleoecologists. AltaMira Press. Lanham, Maryland.

Prewitt and Associates, Inc.

2007 Data Recovery Field Investigation Plan for 41CV1378 on Fort Hood, Coryell County, Texas. Unpublished manuscript submitted to the Environmental Affairs Division, Texas Department of Transportation, Austin, Texas.

Prewitt, Elton R.

1981 Cultural Chronology in Central Texas. Bulletin of the Texas Archeological Society 52:65-89.
1985 From Circleville to Toyah: Comments on Central Texas Chronology. Bulletin of the Texas Archeological Society 54:201-238.

Prilliman, Keith L., and C. Britt Bousman

1998 Unifacial Tools. In Wilson-Leonard, An 11,000-year Archeological Record of Hunter-Gatherers in Central Texas, Volume II: Chipped Stone Artifacts, edited by Michael B. Collins, pp. 597-632. Studies in Archeology 31. Texas Archeological Research Laboratory, The University of Texas at Austin. Archeology Studies Program, Report 10. Environmental Affairs Division, Texas Department of Transportation, Austin, Texas.

Proctor, C. V. Jr., J. H. McGowen, and W. T. Haenggi

1970 Geologic Atlas of Texas-Waco Sheet. Bureau of Economic Geology, The University of Texas at Austin.

Quigg, J. Michael, Chris Lintz, Grant Smith, and Scott Wilcox

2000 The Lino Site: A Stratified Late Archaic Campsite in a Terrace of the San Idelfonzo Creek, Webb County, Southern Texas. TRC Technical Reports 23756. Report 20. Archeological Studies Program, Environmental Affairs Division, Texas Department of Transportation, Austin.

Quigg, J. Michael, Shane Pritchard, and Grant Smith

2002 The Boiler Site (41WB557): Utilization of an Upland Setting over the last 4200 Years, Webb County, Texas. TRC Technical Reports 27277. Texas Department of Transportation, Environmental Affairs Division, Archeological Studies Program, Report 45.

Raunkaiaer, C.

1934 The Life Forms of Plants and Statistical Plant Geography. Clarendon Press, Oxford, England.

Ricaut, Francois-Xavier, Murray P. Cox, Marie Lacan, Christine Keyser, Francis Duranthon, Bertrand Ludes, JeanGuilaine, and Eric Crubezy

2012 A Time Series of Prehistoric Mitochondrial DNA Reveals Western European Genetic Diversity was Largely Established by the Bronze Age. Advances in Anthropology 2(1):14-23. 
Ricklis, Robert A.

1990 A Historical Cultural Ecology of the Karankawan Indians of the Central Texas Coast: A Case Study in the Roots of Adaptive Change. Ph.D. dissertation. Department of Geography, University of Texas at Austin.

1994 Limited Testing at the Mustang Lake Site, 41CL3, under Special Use Permit 73683 and ARPA Permit Aransas-94. Coastal Archaeological Studies, Corpus Christi, Texas.

2011 New Perspectives on the Archaic of the Texas Coastal Plain: The Buckeye Knoll Site (41VT98) on the Lower Guadalupe River near Victoria, Texas. Bulletin of the Texas Archeological Society 82:31-76.

Ricklis, Robert A., and Michael B. Collins

1994 Archaic and Late Prehistoric Human Ecology in the Middle Onion Creek Valley, Hays County, Texas. Studies in Archeology 19. Texas Archeological Research Laboratory, The University of Texas at Austin.

Root, M. J., J. D. William, M. Kay, and L. K. Shifrin 1999 Folsom Ultrathin Biface and Radial Break Tools in the Knife River Flint Quarry Area. In Folsom Lithic Technology, Explorations in Structure and Variation, edited by D. S. Amick, pp. 144-168. International Monographs in Prehistory, Archaeological Series 12, Ann Arbor, Michigan.

Rots, Veerle

2005 Wear Traces and the Interpretation of Stone Tools. Journal of Field Archaeology 30(1):61-73.

2008 Hafting and Raw Materials from Animals: Guide to the Identification of Hafting Traces on Stone Tools. Anthropozoologica 43(1):43-66.

Sackett, James R.

1985 Style and Ethnicity in the Kalahari: A Reply to Weissner. American Antiquity 50(1):154-159.

1990 Style and Ethnicity in Archaeology: The Case for Isochresticism. In The Uses of Style in Archaeology, edited by Margaret W. Conkey and Christine A. Hastorf, pp. 32-43. Cambridge University Press, Cambridge.
Schortman, Edward M.

1989 Interregional Interaction in Prehistory: The Need for a New Perspective.American Antiquity 54(1):52-65.

Sellards, E. H., W. S. Adkins, and F. B. Plummer

1932 The Geology of Texas, Volume I: Stratigraphy. University of Texas Bulletin No. 3232. Bureau of Economic Geology, The University of Texas at Austin.

Shafer, Harry J.

1970 Notes on Uniface Retouch Technology. American Antiquity 35(4):480-487.

1973 Lithic Technology at the George C. Davis Site, Cherokee County, Texas. Unpublished Ph.D. dissertation, Department of Anthropology, University of Texas at Austin.

2006 People of the Prairie:A Possible Connection to the Davis Site Caddo. Texas Department of Transportation, Environmental Affairs Division, Archeological Studies Program and Prewitt and Associates, Inc., Austin, Texas.

Shafer, Harry J., and Richard G. Holloway

1977 Organic Residue Analysis and Stone Tool Function from Hinds Cave, Val Verde County, Texas: a Progress Statement. In Archeological and Botanical Study of Hinds Cave, Val Verde County, Texas. Texas A\&M University Anthropology Laboratory Special Series No. 1, College Station.

1979 Organic Residue Analysis in Determining Stone Tool Function. In Lithic UseWear Analysis, edited by Brian Hayden, pp. 385-399. Studies in Archaeology Series, Academic Press, New York.

Shea, John J.

1991 The Behavioral Significance of Levantine Mousterian Industrial Variability. Vols. I and II. Ph.D. dissertation, Department of Anthropology, Harvard University, Cambridge, Massachusetts. University Microfilms, Ann Arbor, Michigan.

Shott, Michael J.

1986 Technological Organization and Settlement Mobility: An Ethnographic Examination. Journal of Anthropological Research 42(1):15-51.

1989 Bipolar Industries: Ethnographic Evidence and Archaeological Implications. North American Archaeologist 10(1):1-24. 
1999 On Bipolar Reduction and Splintered Pieces. North American Archaeologist 20(3):217-238.

Shott, Michael J., and Kathryn J. Weedman

2007 Measuring Reduction in Stone Tools: an Ethnoarchaeological Study of Gamo Hidescrapers from Ethiopia. Journal of Archaeological Science 34:1016-1035.

Sinopoli, Carla M.

1991 Style in Arrows:AStudy of An Ethnographic Collection from the Western United States. In Foragers in Context: Long-Term, Regional, and Historical Perspectives in Hunter-Gatherer Studies, edited by Preston T. Miracle, Lynn E. Fisher, and Jody Brown, pp. 63-87. Michigan Discussions in Anthropology 10. University of Michigan, Ann Arbor.

Smart, Tristine Lee, and Ellen S. Hoffman

1988 Environmental Interpretation of Archaeological Charcoal. In Current Paleobotany: Analytical Methods and Cultural Interpretations of Archaeological Plant Remains, edited by Christine A. Hastorf and Virginia S. Popper, pp. 167205. The University of Chicago Press, Chicago and London.

Smith, Bruce D.

1984 Chenopodium as a Prehistoric Domesticate in Eastern North America: Evidence from Russell Cave, Alabama. Science 226(4671):165-167.

Spielmann, Katherine A.

2002 Communal Feasting, Ceramics, and Exchange. In Identity, Feasting, and the Archaeology of the Greater Southwest, edited by Barbara J. Mills, pp. 210-232. University Press of Colorado, Boulder.

2008 Crafting the Sacred: Ritual Places and Paraphernalia in Small-Scale Societies. Research in Economic Anthropology 27:37-72

Stark , Miriam T. (editor)

1998 The Archaeology of Social Boundaries. Smithsonian Institution Press, Washington, D.C.

Stark, Miriam T., Mark D. Elson, and Jeffery J. Clark

1998 Social Boundaries and Technical Choices in Tonto Basin Prehistory. In The Archaeology of Social Boundaries, edited by M. T. Stark, pp. 308-231. Smithsonian Institution Press, Washington, D.C.

Stark, Richard

1997 Piedras Calientes: Ethnohistory, Experimental Archaeology, and Cooking with Hot Stones. Unpublished M.A. thesis, Department of Anthropology, The University of Texas at Austin.

2002 Comidas de la Tierra: An Ethnoarchaeology of Earth Ovens. Unpublished Ph.D. dissertation, Department of Anthropology, The University of Texas at Austin.

Stets, Jan E., and Peter J. Burke

2000 Identity Theory and Social Identity Theory. Social Psychology Quarterly 63(2):224-237.

Stuiver, M. and Paula J. Reimer

1993 Extended ${ }^{14} \mathrm{C}$ Data Base and Revised CALIB $3.0{ }^{14} \mathrm{C}$ Age Calibration Program. Radiocarbon 35(1):215-230.

Suhm, Dee Ann

1960 A Review of Central Texas Archeology. Bulletin of the Texas Archeological Society 29:63-107.

Suhm, Dee Ann, Alex D. Krieger, and Edward B. Jelks

1954 AnIntroductory HandbookTexas Archeology. Bulletin of the Texas Archeological Society 25

Sullivan, Alan P., III, and Kenneth C. Rozen

1985 Debitage Analysis and Archaeological Interpretation. American Antiquity 50:755-779.

Tajfel, G.

1972 La Catégorisation Sociale. In Introducion à la Psychologie Sociale, edited by $\mathrm{S}$. Moscovici, pp. 272-302. Larousse, Paris.

Tajfel, H., and J. C. Turner

1979 An Integrative Theory of Intergroup Conflict. In The Social Psychology of Intergroup Relations, edited by W.G. Austin and S. Worchel, pp. 33-47. Brooks and Cole, Monterrey, California.

1986 The Social Identity Theory of Intergroup Behavior. In The Social Psychology of Intergroup Behavior, edited by S. Worchel and W.G. Austin, pp. 7-24. Nelson-Hall, Chicago. 
Teltser, Patrice A.

1991 Generalized Core Technology and Tool Use: A Mississippian Example. Journal of Field Archaeology 18:363-375.

1995 Culture History, Evolutionary Theory, and Frequency Seriation. In Evolutionary Archaeology: Methodological Issues, edited by Patrice A. Teltser, pp. 51-68. University of Arizona Press, Tucson.

Thoms, Alston V.

1989 The Northern Roots of Hunter-Gatherer Intensification: Camas and the Pacific Northwest. Unpublished Ph.D. dissertation. Department of Anthropology, Washington State University.

2008 Ancient Savannah Roots of the Carbohydrate Revolution in South-Central North America. Plains Anthropologist 53(205):121-136.

2009 Rocks of Ages: Propagation of Hot-Rock Cookery in Western North America. Journal of Archaeological Science 31(2):213-232.

Thoms, Alston V., and Andrew R. Laurence

2014 Experimental Archaeology and PlantMicrofossil Taphonomy in Prolonged Baking of Geophytes. Journal of Archaeological Science. In Review.

Thoms, Alston, V., Karl W. Kibler, and Douglas K. Boyd (editors)

2014 Earth Ovens and Prehistoric Plant Use: Archeological and Botanical Investigations of Burned Rock Features on the Fort Hood Military Reservation in Central Texas. Archaeological Resource Management Series, Research Report No.65, United States Army, Fort Hood. In Preparation.

Titmus, Gene

1985 Some Aspects of Stone Tool Notching. In Stone Tool Analysis: Essays in Honor of Don E. Crabtree, edited by M.G. Plew, J.C. Woods, and M.G. Pavesic, pp. 243264. University of New Mexico Press, Albuquerque.

Tomaskova, Silvia

2005 What is a Burin? Typology, Technology, and Interregional Comparison. Journal of Archaeological Method and Theory 12(2):79-115.
Torrence, Robin

1983 Time Budgeting and Hunter-Gatherer Technology. In Hunter-Gatherer Economy in Prehistory, A European Perspective, edited by Geoff Bailey, pp. 11-22. Cambridge University Press, New York.

1989a Tools as Optimal Solutions. In Time, Energy and Stone Tools, edited by Robin Torrence, pp. 1-6. New Directions in Archaeology Series, Cambridge University Press, New York.

1989b Retooling: Towards a Behavioral Theory of Stone Tools. In Time, Energy and Stone Tools, edited by Robin Torrence, pp. 57-66. New Directions in Archaeology Series, Cambridge University Press, New York.

Trierweiler, W. Nicholas (editor)

1994 Archeological Investigations on 571 Sites at Fort Hood, Bell and Coryell Counties, Texas, Appendix A: Data Compendium. Archeological Resource Management Series, Report No. 31. United States Army, Fort Hood.

1996 Archeological Testing at Fort Hood, 1994-1995, Vol. I. Archeological Resource Management Series, Report No. 35. United States Army, Fort Hood.

Tringham, Ruth, Glenn Cooper, George Odell, Barbara Voytek, and Anne Whitman

1974 Experimentation in the Formation of Edge Damage: A New Approach to Lithic Analysis. Journal of Field Archaeology 1:171-196.

Tsirk, Are

1979 Regarding Fracture Initiations. In Lithic Use-Wear Analysis, edited by Brian Hayden, pp. 83-96. Academic Press, Inc., New York.

U.S. Army

1979 Ecological Baseline Report, Fort Hood, Texas. Environmental Impact Statement, Overall Mission, Fort Hood, Texas. Prepared by Espey Huston and Associates, Austin.

U.S. Department of Agriculture

1985 Soil Survey of Coryell County, Texas. U.S. Department of Agriculture, Washington, D.C., in cooperation with the Texas Agricultural Experiment Station and the United States Department of the Army, Fort Hood, Texas. 
Data Recovery Investigations at the Tank Destroyer Site, Fort Hood

Wade, Maria F.

2003 The Native Americans of the Texas Edwards Plateau-1582-1799. University of Texas Press, Austin.

Wandsnider, LuAnn

1989 Long-Term Land Use, Formation Processes, and the Structure of the Archaeological Landscape: A Case Study from Southwestern Wyoming. Ph.D. dissertation, Department of Anthropology, University of New Mexico, Albuquerque.

1997 The Roasted and the Boiled: Food Composition and Heat Treatment with Special Emphasis on Pit-Hearth Cooking. Journal of Anthropological Archaeology 16:1-48.

Waters, Michael R., Charlotte D. Pevny, and David L. Carlson

2011 Clovis Lithic Technology: Investigation of a Stratified Workshop at the Gault Site, Texas. Texas A\&M University Press, College Station.

Weber, Carey

1994 A Replication Technique for Andice/Bell Points. In Archaic and Late Prehistoric Human Ecology in the Middle Onion Creek Valley, Hays County, Texas, edited by Robert A. Ricklis and Michael B. Collins, pp. 629-651. Studies in Archeology 19, Texas Archeological Research Laboratory, The University of Texas at Austin.

Weir, Frank A.

1976 The Central Texas Archaic. Unpublished Ph.D. dissertation, Department of Anthropology, Washington State University, Pullman.

Weissner, Polly

1983 Style and Social Information in Kalahari San Projectile Points. American Antiquity 48:253-276.

Whitley, Thomas G.

2006 Predictive Modeling in a Homogenous Environment:An Example from Charleston Naval Weapons Station, South Carolina. In GIS and Archaeological Site Location Modeling, edited by Mark W. Mehrer and Konnie L. Wescott, pp. 326-361. CRC Press, Taylor \& Francis Group, Boca Rotan, Florida.

Whittaker, John C.

1994 Flintknapping: Making and Understanding Stone Tools. University of Texas Press, Austin.

Wills, W. H. and Patricia L. Crown

2004 Commensal Politics in the Prehispanic Southwest: An Introductory Review. In Identity, Feasting, and the Archaeology of the Greater Southwest, edited by Barbara J. Mills, pp. 153-172. University Press of Colorado, Boulder.

Wobst, H. M.

1977 Stylistic Behavior and Information Exchange. For the Director: Research Essays in Honor of James B. Griffin, edited by C. E. Cleland, pp. 317-344. Anthropological Papers of the Museum of Anthropology No. 61. Ann Arbor.

Woodruff, C. M. Jr., and P. L. Abbott (editors)

1986 The Balcones Escarpment: Geology, Hydrology, Ecology, and Social Development in Central Texas. Geological Society of America.

Young, David E., and Robson Bonnichsen 1984 Understanding Stone Tools: A Cognitive Approach. Peopling of the Americas Process Series, Vol. 1. Center for the Study of Early Man, University of Maine at Orono. 


\title{
APPENDIX A: Geophysical Surveys at 41CV1378
}

\author{
Chester P. Walker
}

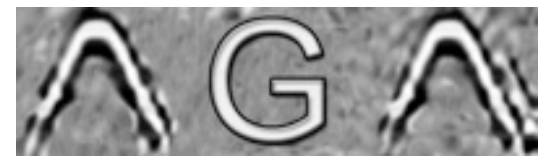

Archaeo-Geophysical Associates, LLC

AGA Report 2007-9 



\section{INTRODUCTION}

Prewitt and Associates, Inc. (PAI) contracted with Archaeo-Geophysical Associates, LLC, to conduct surveys using multiple geophysical methods to identify and delineate burned rock archeological features at 41CV1378. Magnetometer and ground-penetrating radar surveys were conducted on June 8, 2007.

\section{SUMMARY OF TECHNICAL INFORMATION}

Archeogeophysics employs a range of techniques for the nondestructive prospecting of archaeological deposits. These techniques have been developed for a range of applications, mostly geological in nature, but have been adapted for specific use in archaeology through rigorous field collection techniques and unique data-processing programs specifically developed for archaeo-geophysics.

The different geophysical techniques map, record, or sense different variables or properties of the soil and the objects within the soil. The instruments are differentially affected by variables such as moisture, metal trash or debris, and transmission of signals such as cell phones and transmission lines. Data collection is also impacted differently for each of the geophysical instruments by physical impediments such as trees, pavement, fences, and vegetation.

Archaeologists have found that the first line of defense against this complex matrix of variables is to come to the field prepared to collect data with several different instruments. This approach not only increases the margin of success, but can often enhance the visibility of the target (Kvamme 2006a:57-58; Kvamme et al. 2006:251). Archaeogeophysical investigations have a long history of success in helping to focus archaeological excavations to specific locations, and in the right conditions, they can be used as a primary source of archaeological data (Kvamme 2003).

\section{Magnetometer}

Magnetometer and gradiometer surveys measure slight variations in the magnetic properties of soil. Magnetometers are useful tools for archeogeophysicists in part due to the fact that data can be collected and processed quickly.
When conditions are right, magnetometers have proven useful in locating negative relief features such as pits and post molds as well as thermally altered features such as fire hearths and burned structures (Bruseth and Pierson 2004; Creel et al. 2003; Frederick and Abbott 1992; Schambach and Lockhart 2003; Walker and Perttula 2007; Walker and Schultz 2006; Walker et al. 2003).

Magnetometers record the minute fluctuations that sediments and objects have on the earth's magnetic field. This is known as "induced magnetism" because these objects do not maintain their own magnetic field. If the effects of this induced magnetism are strong enough compared to the surrounding soil matrix, pit features or post molds can be identified or resolved in the geophysical data. A second type of magnetism, called "remnant magnetism," is created when an object maintains its own magnetic field. This occurs when an object is thermally altered, thus creating a magnetic state called "thermoremanent magnetism" (Kvamme 2006b:207). The magnetometer used in the current study is detailed by Bartington and Chaman (2004).

\section{Ground-Penetrating Radar}

Ground-penetrating radar (GPR) is an active, noninvasive technique that uses a shielded surface antenna to transmit pulses of radar energy, generally high-frequency electromagnetic (EM) waves, that reflect off buried objects, features, or geological bedding contacts (Conyers 2004:23-28). Waves are detected using a receiving antenna and recorded in nanoseconds (ns). Travel time determines the approximate depth of recorded anomalies (Conyers and Lucius 1996).

The success of GPR is largely based on such site conditions as soil type, sediment mineralogy, and moisture content (Conyers 2004; Kvamme 2003). Ideal soil is dry and homogenous with minimal clay. Less ideal conditions include clay and poorly drained soils and mediums with high magnetic permeability (Conyers 2004).

\section{FIELD METHODS}

The specific settings used for the instruments differ greatly; however, there are a few general data collection concepts that apply to all 
three technologies. The density of the data set is controlled by two factors: (1) traverse interval-the distance between the passes the instrument makes as it is passed back and forth across the collection area; and (2) sample interval- the distance between readings the instrument records as it passes along each traverse. There are standard starting points for these settings, but ultimately this depends on many factors, including the size and depth of the target, the nature of the sediment matrix, land use of the collection area, duration of the survey, and the investigative scope of the research design.

Magnetic data was collected using a 0.5$\mathrm{m}$ traverse interval and a $0.125-\mathrm{m}$ ( 8 readings per meter) sample interval. Radar data was collected at a $0.5-\mathrm{m}$ traverse interval, and 32 samples per $\mathrm{m}$ were recorded. Both instruments were passed over the grids in a bidirectional pattern.

\section{DATA PROCESSING}

All data were processed and filtered to remove extraneous false readings (spikes and dropouts). Processing levels the data sets so adjacent grids are combined into a single image with no "grid lines." Data sets were processed to enhance the visibility of the target features through statistical manipulation of the recorded data as well as through image processing of the image file output. After each processing step, the results are closely compared to their previous state to assure that data manipulation is not in fact decreasing the clarity and quality of the data, and thus avoiding artifacts of data processing.

The general goal of data processing is to lessen the effects of background "noise" and to enhance the quality of the signal or target. In field geophysics in general, and archeogeophysics in particular, the term noise is used to discuss any return that is not a result of the object under investigation-referred to as the "target" or "signal." Hence, in some cases what is considered noise can in another case become the signal or target (Milsom 2005:13-14). For resolving targets, accuracy of the geophysical readings are not as important as the contrast between the target and its surrounding matrix.

Magnetometer data was processed using ArchaeoSurveyor 2.0. The data was first clipped to 3 standard deviations. Clipping replaces all values outside a specified minimum and maximum range. These minimum and maximum values are specified in either absolute values or \pm standard deviations (SD). This process is used to remove extreme data point values and aids in normalizing the histogram of the data. Archaeological details are subtle, and having a normal distribution of data allows the fine detail to show through with clarity.

Next the data was destriped using a zero median. Destriping is a process used to equalize the underlying differences between grids caused by instrument drift, inconsistencies during setup, delays between surveying adjacent grids, or heading error from magnetic instruments. The mean, mode, or median of each grid or traverse is subtracted from the grid or traverse, effectively zeroing the mean, mode, or median. When the mean is used, thresholds are set to exclude extreme data points.

Radar data was processed using GPR Slice. Amplitude slice maps were created, and a velocity analysis was conducted. Amplitude slice-maps are a three-dimensional tool for viewing differences in reflected amplitudes across a given surface at various depths. They are generated through comparison of reflected amplitudes between raw vertical profiles. Amplitude variations are analyzed at each location in a grid where there is a recorded reflection. The individual profiles are combined into a data cube, and the amplitudes of all traces are compared to the amplitudes of all nearby traces. This database can then be "sliced" horizontally and displayed to show the variation in reflection amplitudes at a sequence of depths in the ground.

\section{RESULTS}

Both the magnetometer and GPR data show the limits, and to some degree the structural detail, of the burned rock mound at 41CV1378. This mound was partially exposed by a road cut and is easily visible. Remote sensing was used to delineate the full dimensions and internal structure of the feature.

The magnetometer data (Figures A.1 and A.2) shows the burned rock feature the most clearly. The road cut removed a sizable portion of the feature, leaving a section exposed in the road cut and a semicircular arch extending southwest of the road cut. Figure A.1 shows the approximate edges of the exposed portions of the feature. 
A semicircular high (dark) magnetic anomaly extends northeast of the disturbed area-much of which is actually the sloped edge of the road cut. Figure A.3 displays the high magnetic returns associated with the burned rock feature. There appears to be a weak positive magnetic return in the center of the circular anomaly. This return, which is approximately $25 \mathrm{~cm}$ southeast of the top of the slope caused by the road cut, is possibly the center of the burned rock feature, and the stronger positive magnetic features surrounding it are being interpreted as the outer ring of burned rock.

Radar data shows a similar pattern (Figures A.4 and A.5). The 8-19 cm (2-6 ns) amplitude slice map shows a semicircular anomaly of high amplitude reflections. In the center of this is an anomaly also with a high amplitude. The pattern

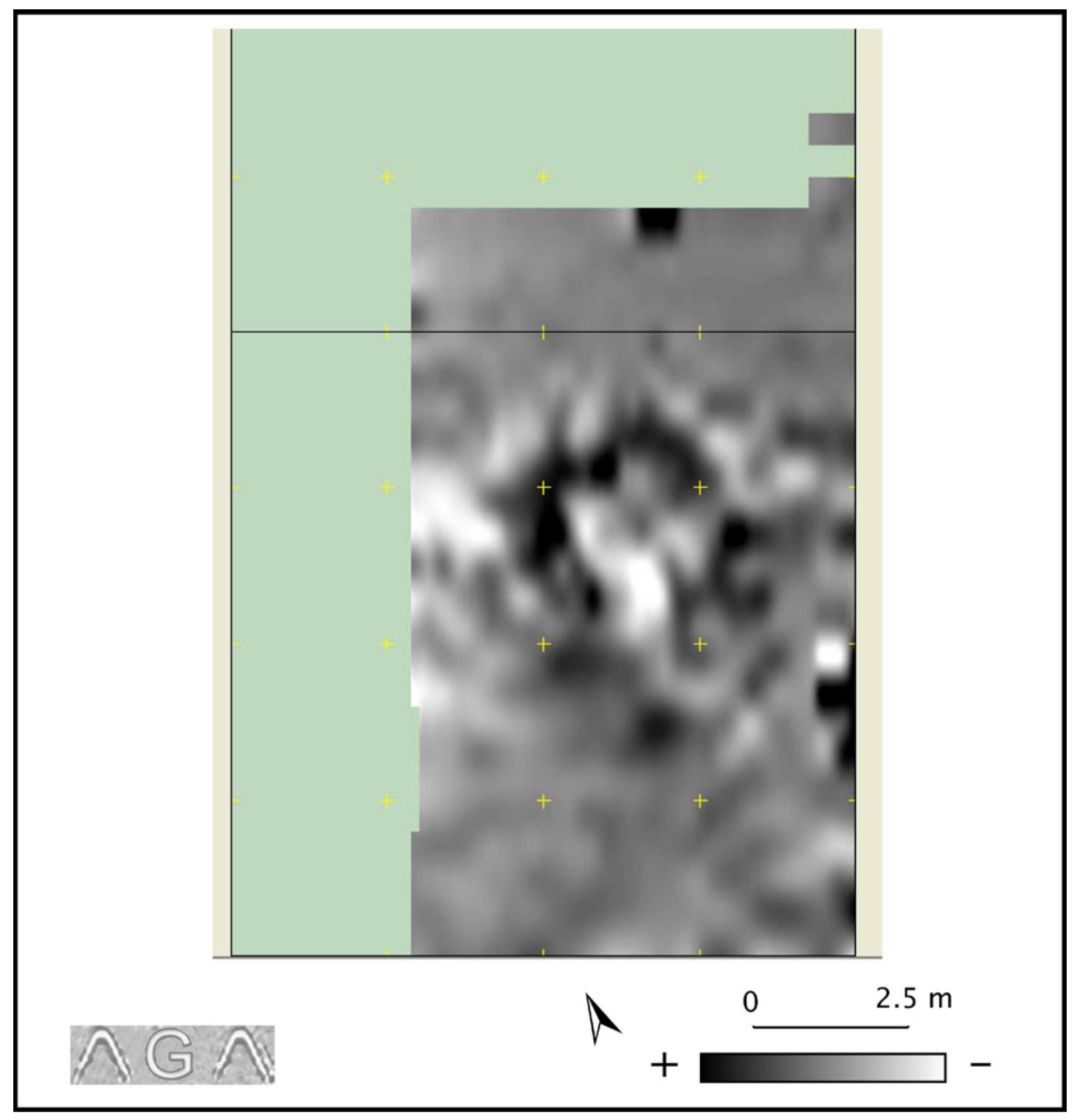

Figure A.1. Magnetometer data. 


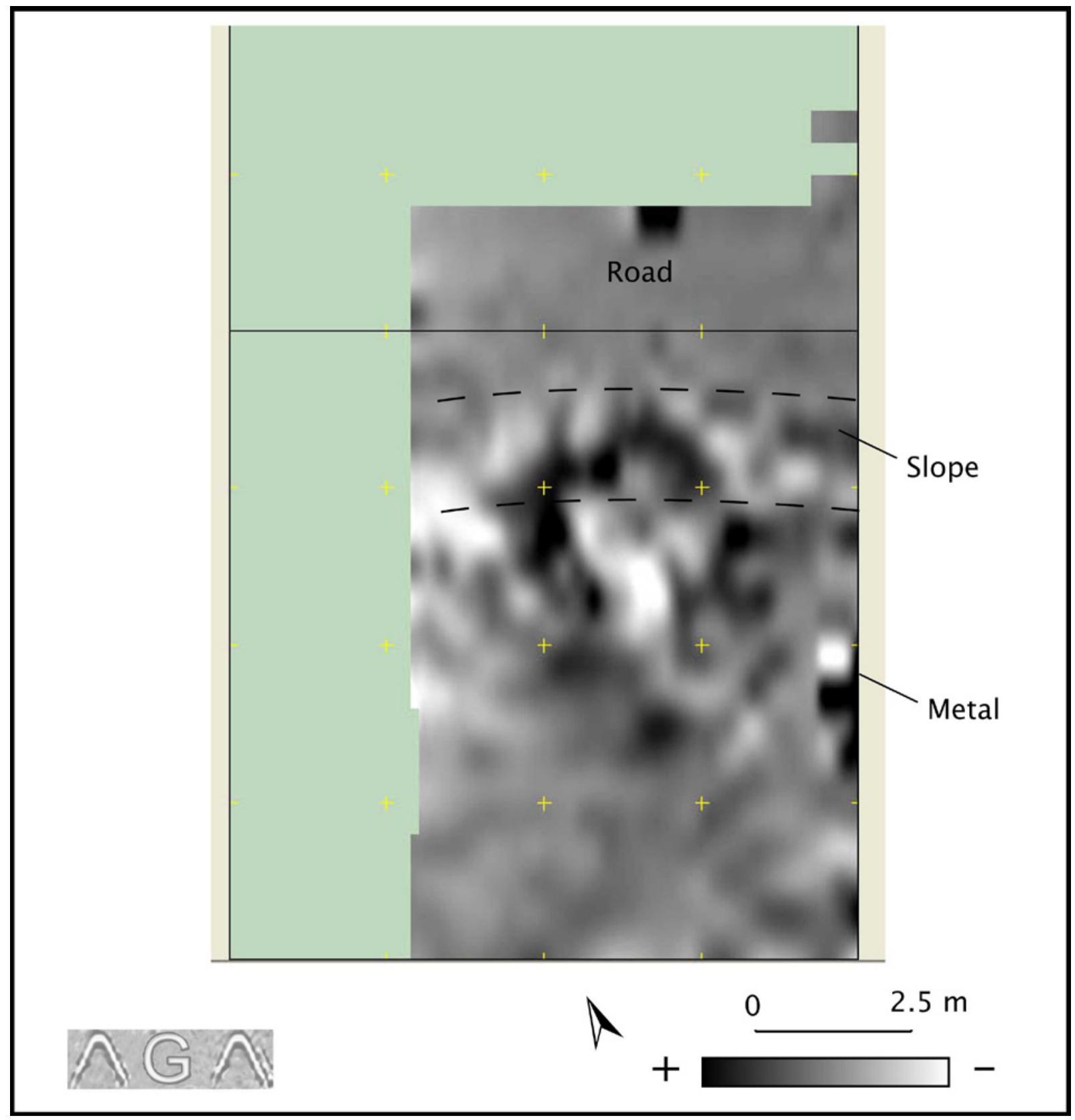

Figure A.2. Magnetometer data with interpretations.

pinches out in the 21-32 cm (6-9 ns) time slice. The central feature appears to pick back up in the $25-36 \mathrm{~cm}(7-10 \mathrm{~ns})$ time slice. It should be pointed out that due to the physical constraints of radar data collection, the last traverse collected was at the top of the slope. Thus the patterns observed in both data sets match up reasonably well. Velocity analysis was performed on a subtle hyperbola in the 12 radar traverse to calculate the depths presented in Figure A.4.

Both data sets suggest that there is a central pitlike feature containing burned rocks that possibly continues deeper than the surrounding burned rock deposits. According to the radar data, this central pit appears to terminate between 45 and $55 \mathrm{~cm}$ below surface. 


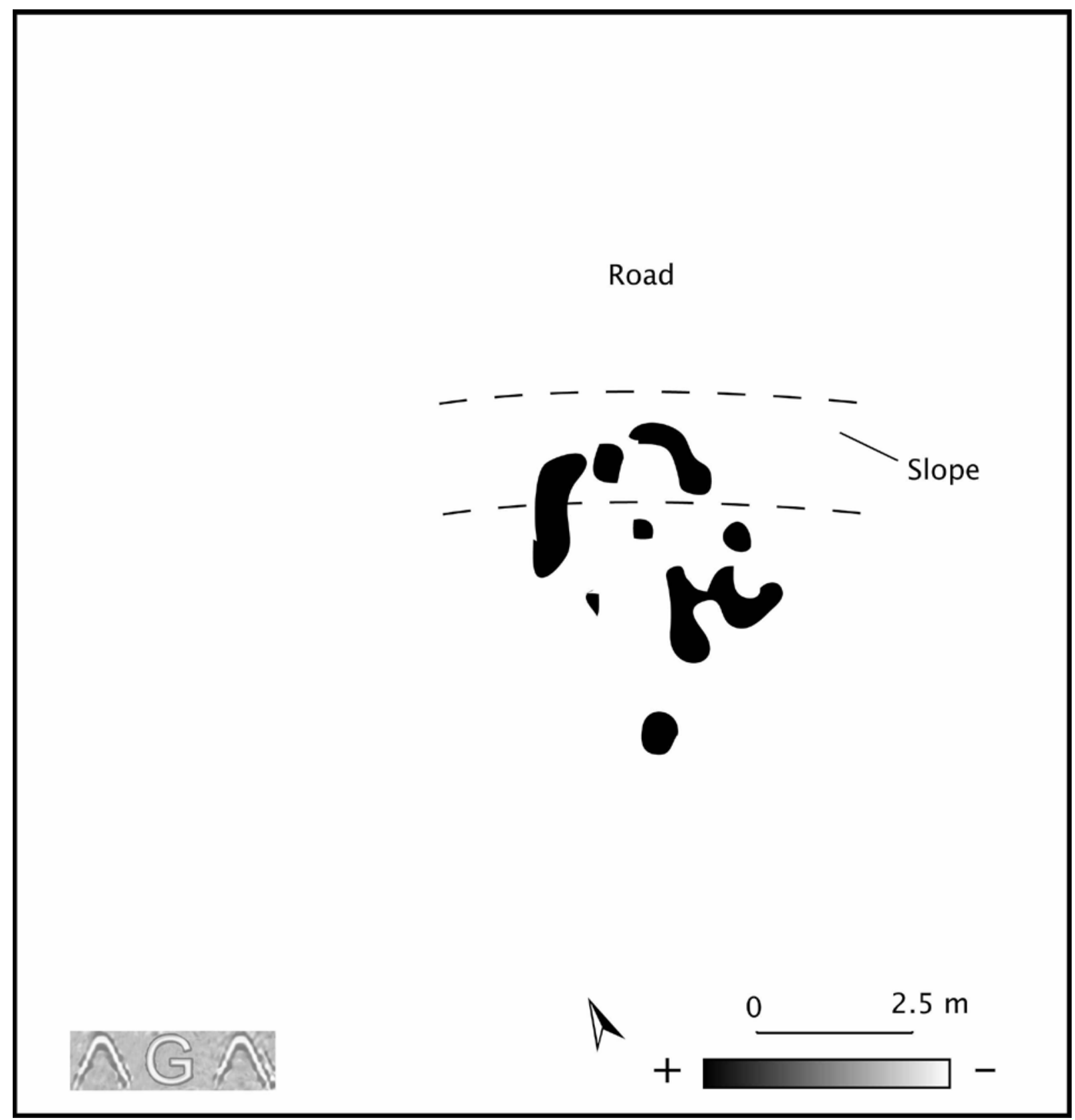

Figure A.3. Positive magnetic anomalies. 

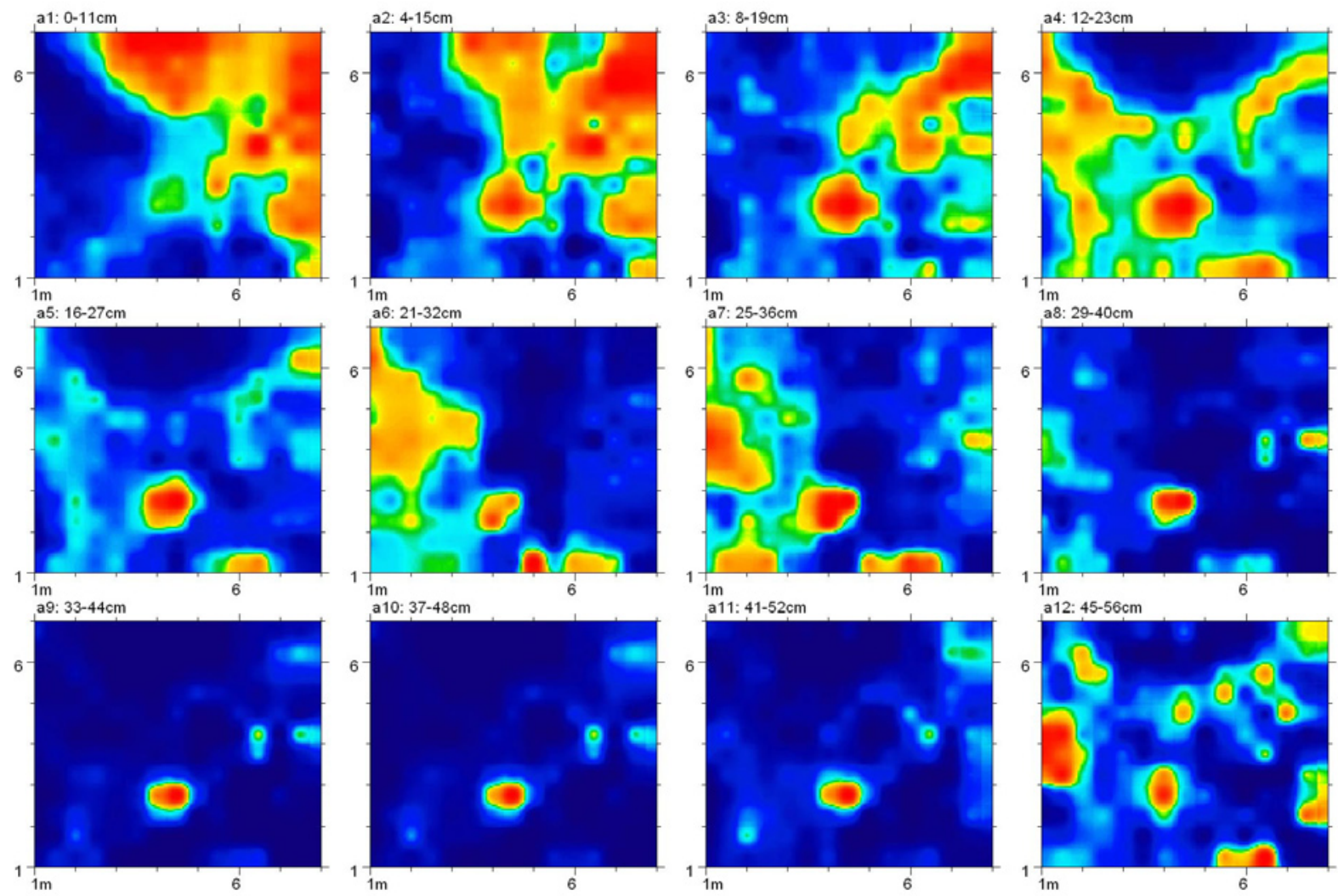

Figure A.4. GPR amplitude slice maps.

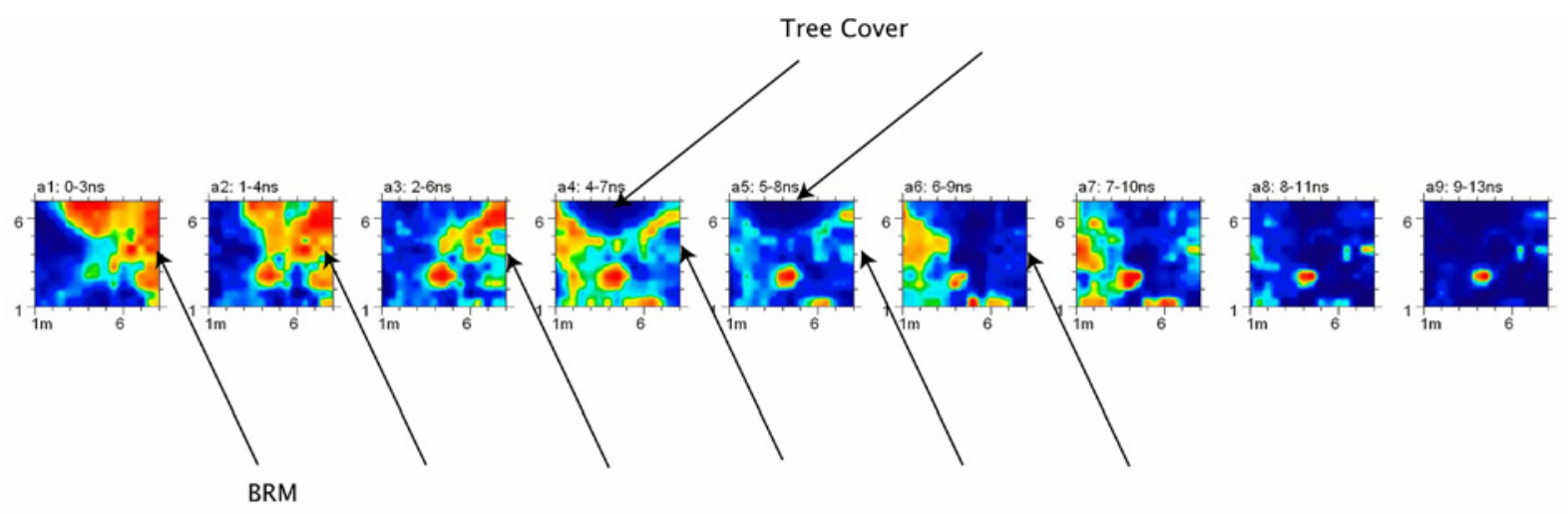

Figure A.5. GPR amplitude slice maps with interpretations. 


\section{REFERENCES CITED}

Bartington, Geoff, and C. E. Chapman

2004 A High-Stability Fluxgate Magnetic Gradiometer for Shallow Geophysical Survey Applications. Archaeological Prospection 11:19-34.

Bruseth, James E., and Bill Pierson

2004 Magnetometer Survey at the George C. Davis Site (41CE19). Current Archeology in Texas 6(1):7-9.

Conyers, Lawrence B.

2004 Ground-Penetrating Radar. AltaMira Press, Walnut Creek, California.

Conyers, Lawrence B., and Jeffrey Lucius

1996 Velocity Analysis in Archaeological GroundPenetrating Radar Studies. Archaeological Prospection 3:25-38.

Creel, Darrell G., Dale Hudler, Samuel M. Wilson, T. Clay Schultz, and Chester P. Walker

2003 A Magnetometer Survey of Caddoan Mounds State Historic Site. Technical Report 51. Texas Archeological Research Laboratory, The University of Texas at Austin.

Frederick, Charles, and James T. Abbott

1992 Magnetic Prospection of Prehistoric Sites in an Alluvial Environment: Examples from NW and West-Central Texas. Journal of Field Archaeology 19(2):139-153.

Kvamme, Kenneth

2003 Geophysical Surveys as Landscape Archaeology. American Antiquity 68:435-457.

2006a Integrating Multidimensional Geophysical Data. Archaeological Prospection 13:57-72.

2006b Magnetometry: Nature's Gift to Archaeology. In Remote Sensing in Archaeology: An Explicitly North American Perspective, edited by Jay K. Johnson, pp. 205-234. University of Alabama Press, Tuscaloosa.
Kvamme, Kenneth, Jay K. Johnson, and Bryan S. Haley

2006 Multiple Methods Surveys: Case Studies. In Remote Sensing in Archaeology: An Explicitly North American Perspective, edited by Jay K. Johnson, pp. 205-233. University of Alabama Press, Tuscaloosa.

Milsom, John

2005 Field Geophysics: The Geological Field Guide Series, Third edition. Wiley, West Sussex, United Kingdom.

Schambach, Frank F., and Jamie J. Lockhart

2003 The 2001-2002 Investigations by the Arkansas Archeological Survey and the Arkansas Archeological Society at the Tom Jones Site (3HE40), a Late 14thEarly 15th Century Caddo Mound Group in Southwest Arkansas. Paper presented at the 45th Annual Caddo Conference, Arkadelphia, Arkansas.

Walker, Chester P., and Timothy K. Perttula

2007 Remote Sensing at the Horace Cabe Site (41BW14). Caddo Archaeology Journal $16: 37-44$.

Walker, Chester P. and T. Clay Schultz

2006 Magnetometer Survey and Results. In An Intensive Cultural Resources Survey and Remote Sensing and Geomorphological Investigations for the Bowie County Levee Realignment Project, Bowie County, Texas and Little River County, Arkansas, by Scott A. Sundermeyer, John T. Penman, and Timothy K. Perttula pp. 158-168. Miscellaneous Reports, Report of Investigations No. 29. LopezGarcia Group, Dallas, Texas.

Walker, Chester P., T. Clay Schultz, Darrell G. Creel, Dale Hudler, and Samuel M. Wilson

2003 Magnetometer Survey and Intrasite Structure of the George C. Davis Site in Northeast Texas. Paper presented at the 11th East Texas Archeological Conference, Texarkana, Texas. 



\section{APPENDIX B: Inventory of Cultural}

Materials from 41CV1378 



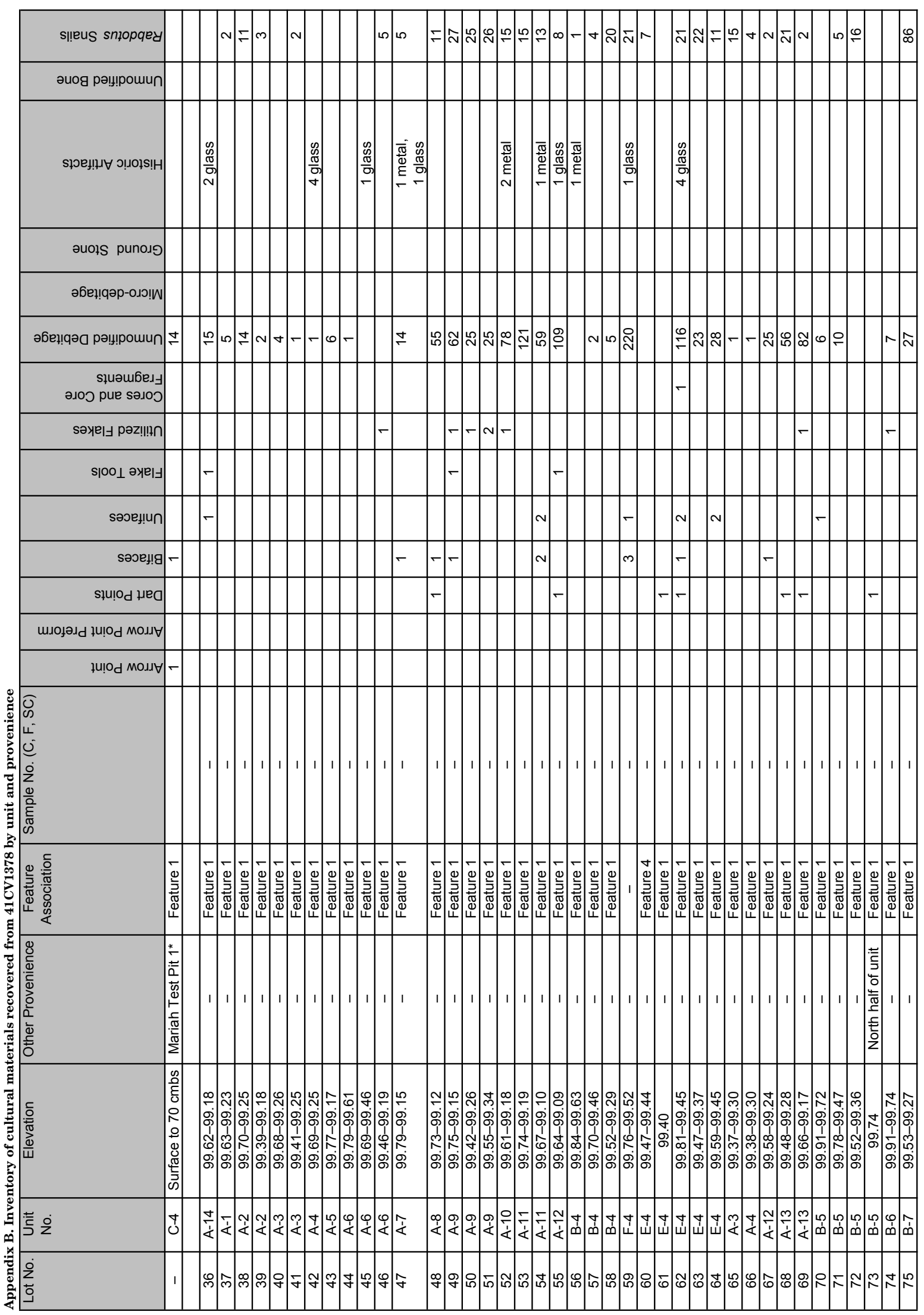


Data Recovery Investigations at the Tank Destroyer Site, Fort Hood

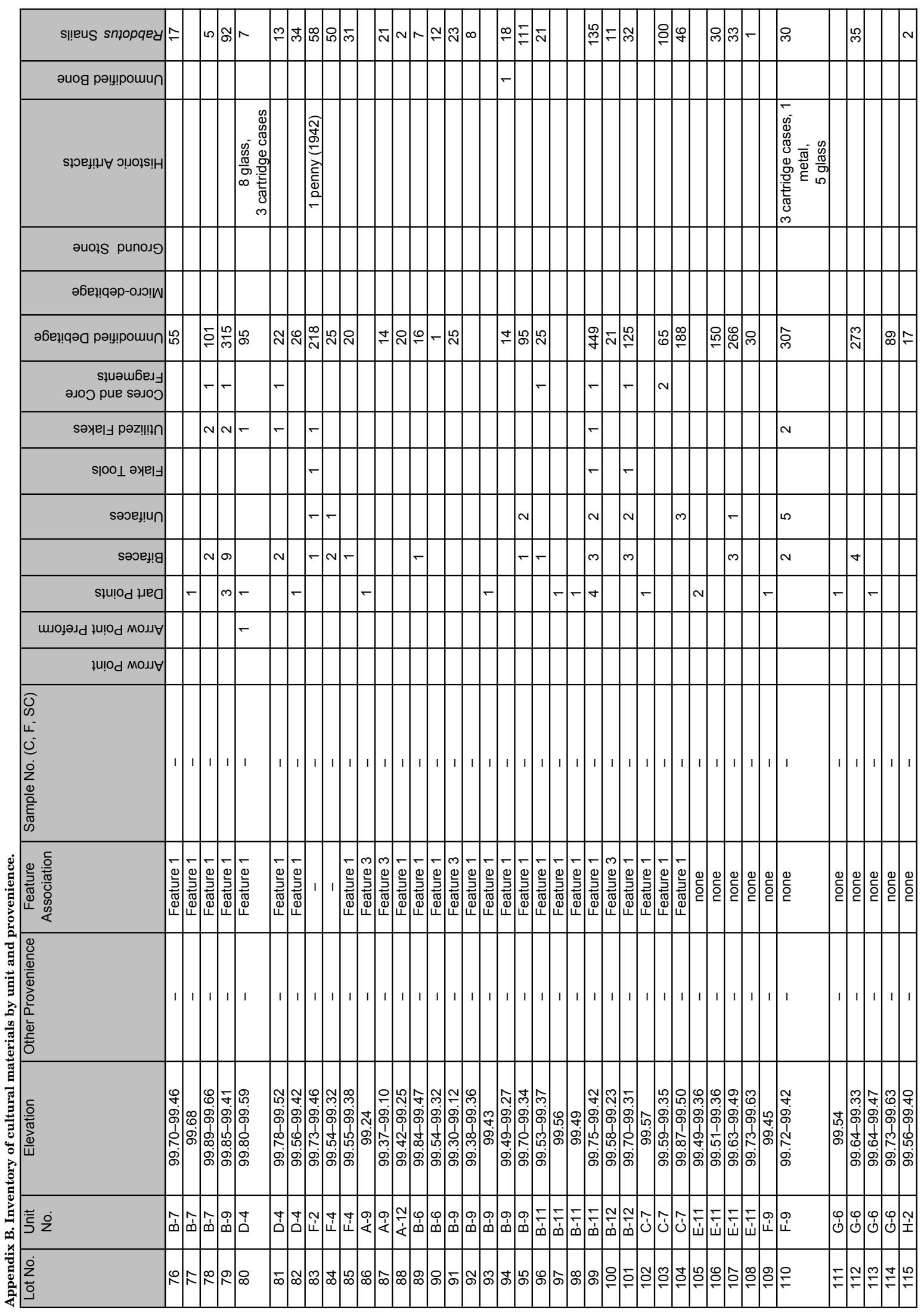




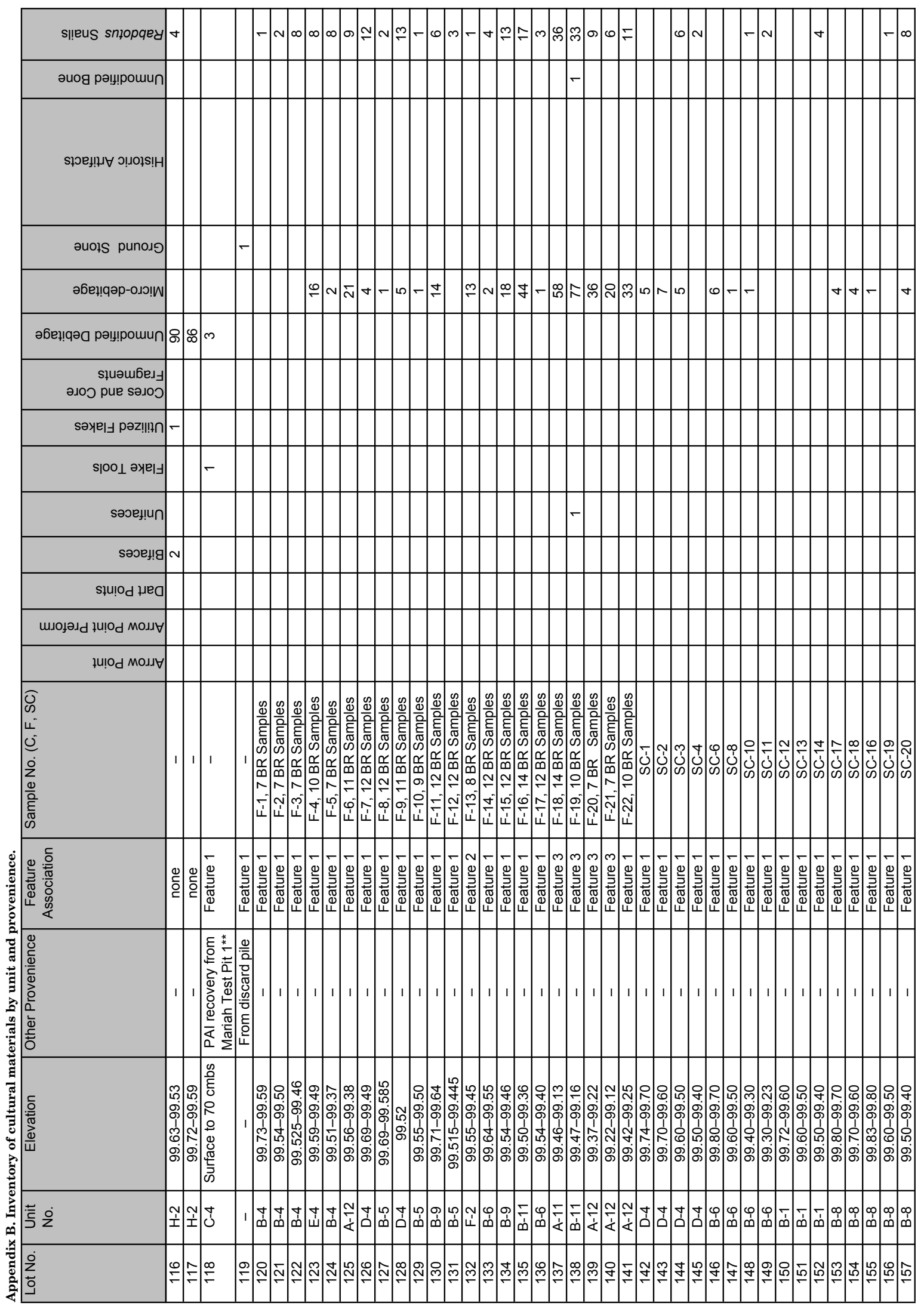


Data Recovery Investigations at the Tank Destroyer Site, Fort Hood

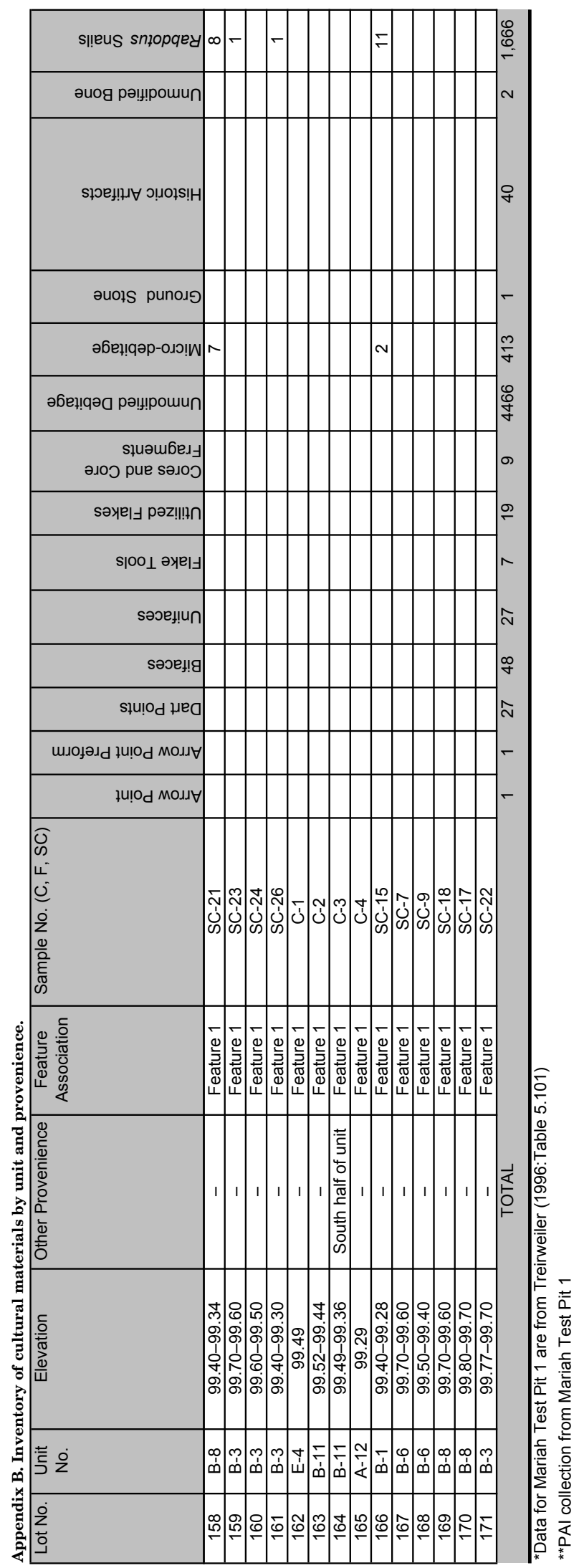




\section{APPENDIX C: TxDOT Chipped Stone}

Analytical Protocol 



\section{Chipped Stone Analytical Protocol TxDOT Archeological Studies Program}

This protocol specifies the observations to be made with respect to chipped-stone artifacts during fieldwork and analysis. It is TxDOT's position that data should be collected with problem-oriented research in mind, but that is not to say that it needs to be used in the context of the study that it is collected in - just that it needs to be systematically reported so that future researchers have access to the data for the purposes of developing innovative research designs. The specific observations included within this protocol have been selected because they have proven valuable for addressing important questions of prehistory, and because they can be feasibly accomplished in most laboratory settings within a reasonable time frame. The implementation of this protocol will not undermine the collection of additional data so long as the need for additional data can be justified with respect to specific research needs. We recognize that reasonable disagreement is possible with respect to those choices.

The following discussion of procedures is designed as a guide for using the data coding key that is part of the TxDOT chipped stone protocol. Data coding is important to the process of recording standardized observations within the proposed state-wide database that will facilitate inter-site comparisons and allow researchers to more readily address regional-scale research questions. It is TxDOT's intent that this protocol be used when analyzing any form of chipped stone tool or core. This portion of the protocol does not address the analysis of groundstone tools or chipped stone non-tools (e.g. symbolic forms).

\section{Taxonomy}

The artifact taxonomy presented here has been designed as a means to record various levels of analytical data for each specimen, and to move beyond a strict reliance on static artifact names and types. It is hoped that this taxonomy will help identify technological traditions and preferences of technique within and between groups, landscapes, regions, and periods. Taxonomic classification of stone tools will also provide the eventual database with greater analytical potential.

\section{Technology}

Technology, as used here, relates to the suite of techniques used to produce a lithic implement. The primary distinction at this level is between (1) chipped-stone, and (2) groundstone, although minor categories may be considered. This will be used to separate lithic artifacts at the broadest analytical level. TxDOT anticipates the development of a groundstone protocol in 2009.

\section{Group}

At the next lower taxonomic level, lithic objects classified as chipped stone (non-debitage) may be separated into two distinct groups. The first group is Tools, and includes objects that represent or were intended for (in the case of performs) direct functionality. The second group is Non-tools, representing objects of indirect functionality (ex. cores), or objects of an instructional, symbolic or artistic nature (ex. Early Archaic multi-notched lithics). For the purposes of this protocol, only those artifacts grouped as chipped-stone tools are considered.

Version 2.3

Dated July 16, 2010 


\section{Data Recovery Investigations at the Tank Destroyer Site, Fort Hood}

\section{Subgroup}

Tool subgroup identifies the primary technique of manufacture. Chipped-stone tools are classified into one of three subgroups: (1) simple detachment-based; (2) complex detachment-based; and (3) core-based. Detachment-based tools are derivative of larger cores. Simple detachment-based tools are classified as either blades or flakes, and are used with modest to no modification following detachment. Complex detachment-based tools undergo substantial modification prior to use. Such tools most commonly originate as macro-flakes or macro-blades detached from a sizable core. The form is then reduced through bifacial or unifacial percussion and, unlike simple detachment-based tools, proceeds through several identifiable reduction stages prior to use. Core-based tools are constructed from material cores (most often in the form of tabular or nodular cobbles) rather than detachments. Such tools are reduced through bifacial or unifacial percussion and proceed through several identifiable reduction stages prior to use. Differentiating between corebased and complex detachment-based tools may not be possible. Complex detachment-based tools can often only be distinguished from core-based tools when they retain characteristics of their origin. These may include a remnant bulb of percussion, striking platform, or (more typically) identifiable ventral surface.

\section{Class}

A tool class identifies the general form of the tool with implicit information relevant to understanding the techniques of manufacture. For simple detachment-based tools, classes include flakes and blades. For both complex detachmentbased tools and core-based tools, classes include bifaces and non-bifaces.

\section{Subclass}

The subclass of a tool provides additional information with respect to its class, often related to the degree to which the producer adhered to a predetermined manufacturing template. A subclass also encodes implicit information relevant to understanding the degree of expediency with which the tool was crafted. Tools classified as either flakes and blades are sub-classified as either modified or unmodified. Such tools are sub-classified as modified when additional stages of manufacture are required following their initial detachment prior to their use. Sequent flake unifaces, end scrapers, drills, and backed blades are a few examples of modified simple detachment-based tools.

Tools classified as either bifaces or non-bifaces are sub-classified as either formal or informal. If tools fit within a standardized, pervasive, recognizable morphology, they are considered formal as the producer is presumed to have been following a traditional manufacturing template. Unique tool forms that (typically) appear more expedient in design are considered informal.

\section{Type}

A tool's type identifies aspects of its use. Complex detachment-based and core-based tools should be typed according to their function. Function should be determined through use-wear analysis using the methods and observations outlined below. Some examples of biface tool types include projectiles, adzes, choppers, and knives. Examples of non-biface tool types include scrapers, adzes, and gouges.

Simple detachment-based tools sub-classified as modified flakes should also be typed according to their function (ex. burin, drill, graver, etc.). Simple detachment-based tools sub-classified as unmodified flakes should only be typed as expedient. Simple detachment-based tools sub-classified as unmodified blades should be typed according to their morphology. Common unmodified blade types include dihedral and polyhedral varieties. Simple detachment-based tools sub-classified as modified blades should be typed according to modification form (ex. backed, stemmed, etc.). 


\section{Subtype / Identity}

The identity of a tool form (its subtype) corresponds to how it is commonly identified within the classical typological system. Thus, a projectile may be identified as Angostura, Bell, Clovis, Dalton, Ensor, etc. For tools classified as flakes and blades, the appropriate identity will most often be "not applicable" (an exception would be a Clovis blade).

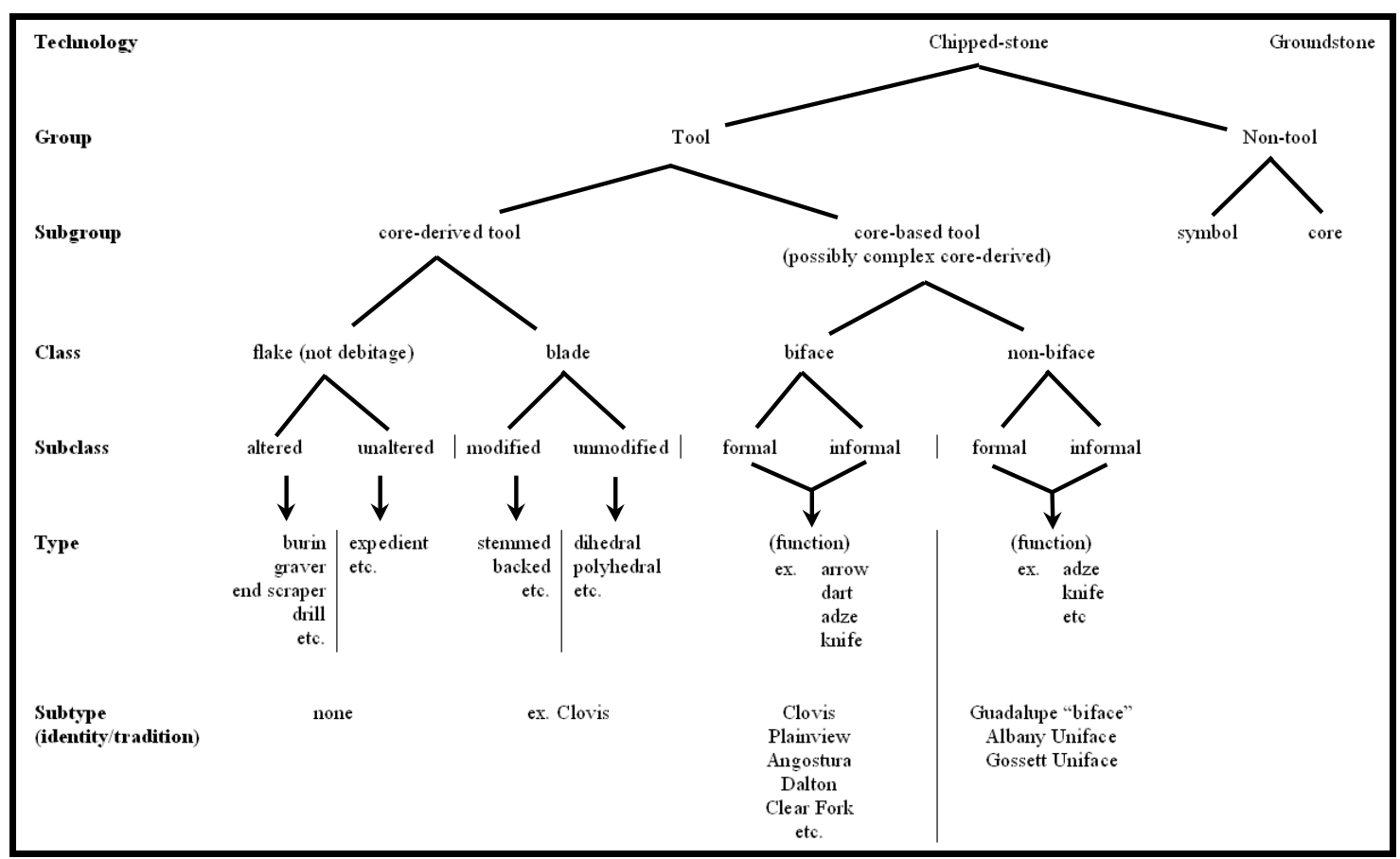

Figure 1: Artifact taxonomy for chipped stone tools based on technological attributes and reduction characteristics.

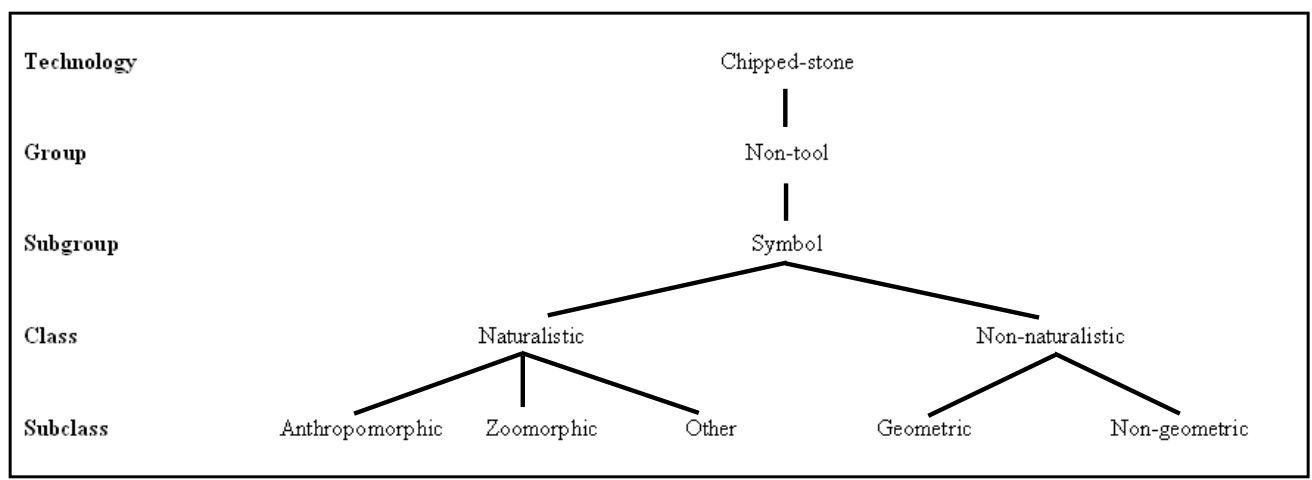

Figure 2: Artifact taxonomy for chipped stone objets with primarily non-utilitarian, symbolic purpose. 


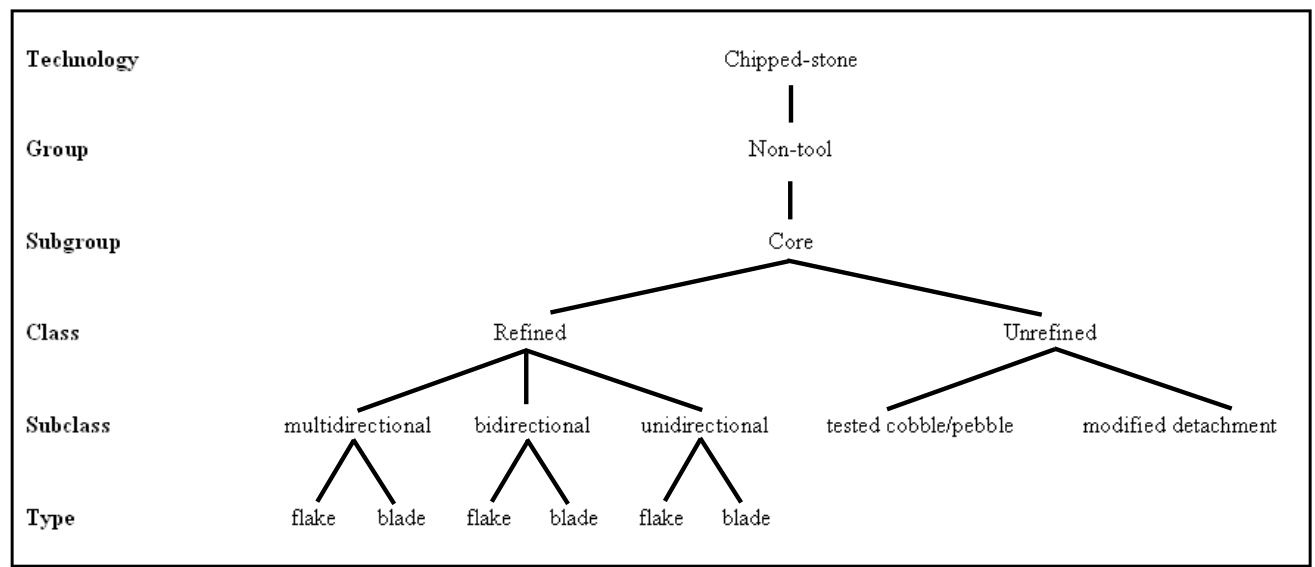

Figure 3: Taxonomy for chipped stone cores. These are not tools, but rather the objective piece from which tool forms are extracted.

\section{Metric Information}

\section{Max length}

Record the maximum observed length of the tool form to the nearest whole millimeter. Do not project or estimate unrepresented portions of the tool form. Using calipers, take this measurement directly from the tool.

\section{Max width}

Record the maximum observed width of the tool form to the nearest whole millimeter. Do not project or estimate unrepresented portions of the tool form. Using calipers, take this measurement directly from the tool.

\section{Figure 4: Metric measurements recorded directly from tool.}

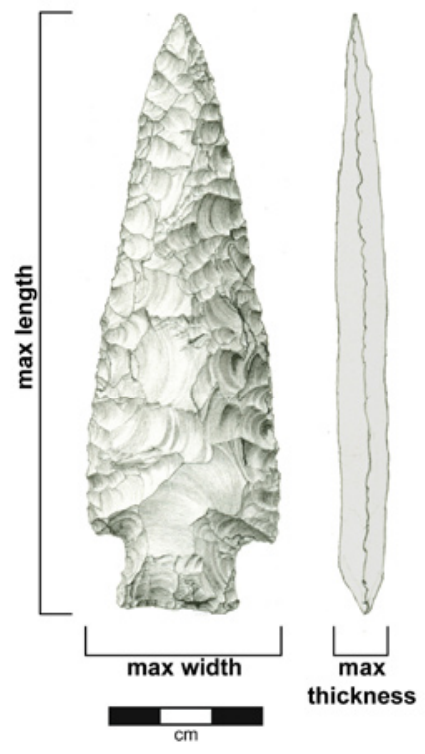

\section{Max thickness}

Record the maximum observed thickness of the tool form to the nearest whole millimeter. Do not project or estimate unrepresented portions of the tool form. Using calipers, take this measurement directly from the tool.

\section{Weight}

Record the weight of the tool to the nearest whole gram.

\section{Edge angle}

The edge angle of the tool should be recorded as an average measure along the used margin of the form. This should be recorded to the nearest $5^{\circ}$ interval. Measurements should be made using a goniometer and recorded directly from the tool. Some extrapolation is acceptable where the edge has been blunted from use and the original angle can be determined. 


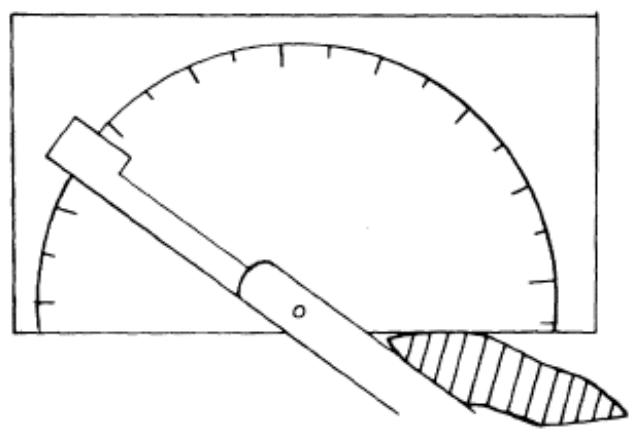

Figure 5: Edge angle can be recorded with the use of a goniometer. As the exact angle may vary across the length of the use edge, it is sufficient to record edge angle to the nearest $5^{\circ}$ increment.

\section{Attributes}

\section{Stage}

Linear reduction models assist in determining the stage of manufacture an artifact reached within an idealized trajectory. Linear reduction models provide a framework for understanding the functional and behavioral relationships among related sets of artifacts (Collins 1975; Goode 2002; Patterson 1977, Shafer 1983, 1985; Sollberger 1977; Tsirk 1979), and are typically based on theoretical abstractions or on experimental replication (Crabtree 1966). Classifying tools in accordance with a linear reduction scheme allows for a more precise study of manufacturing concerns, and it provides a conceptual model for determining the degree of morphologic variation that finished trajectories may be expected to exhibit. When assessing trait or design variability, it will be most productive to compare finished tool forms that have not been extensively remodeled through recycling efforts. The criteria for determining stage of manufacture used in this work closely follow that of Black et al. (1997).

Five stages in the life cycle trajectory of tools are recognized in this protocol: (1) initial package reduction, (2) blank preparation, (3) preform shaping and thinning, (4) final edge trimming and sharpening, and (5) rejuvenated forms. Assessing manufacturing stage is not a wholly objective enterprise (Goode 2002). Lithic reduction is a linear process, and its separation into discrete units of activity is necessarily subjective. Also, the fragmentary nature of some artifacts, the retention of trace amounts of surface cortex on finished forms, and variability in production patterns due to raw material variability and individual skill all contribute to the occasional difficulty in assigning production stage. However, observing this process in stepwise fashion provides a useful proxy measure for detecting potentially important variations in the organization of lithic resource exploitation.

The first stage of the linear reduction model, initial package reduction, reflects the beginning steps of tool manufacture and includes preliminary reduction efforts such as cortex removal, mass thinning, and initial shaping. At this stage, objective pieces typically retain some cortex on one or both faces and reduction is dominated by hard-hammer percussion. Tool forms in their initial production stage are generally irregular in outline, exhibit unrefined edges, and do not provide an indication of the intended manufacturing trajectory. However, tools may be employed as crude "choppers" even at this early stage (Goode 2002: 36). Most expedient tool forms will be assigned to this reduction stage.

The second category, blank preparation, is characterized by the production of a less generalized form with a limited set of possible final trajectories. Tool forms in this stage of manufacture, called "blanks" (Crabtree 1972), typically exhibit little if any cortex, although completed tools may exhibit traces of cortex on occasion. As blanks, tools receive further reduction of mass through thinning, which is accomplished with some hard-hammer, but primarily soft-

Version 2.3

Dated July 16, 2010 
hammer percussion. Blanks require refinement of lateral margins, which may appear sinuous on bifacial forms. Incipient stems may be initially observed at this stage.

The third category, preform shaping and thinning, is characterized by the artisan's full commitment toward a single or very limited number of morphological forms, producing what is commonly called a "preform" (Crabtree 1972b). Preforms exhibit a significant reduction in thickness when compared to blanks, and soft-hammers are used almost exclusively for purposes of reduction. Cortex is rare on these late stage forms. Artifacts categorized as performs approximate their final design and generally lack only refinement of lateral edges and minor facial thinning. Edges are nearly straight and exhibit minor sinuosity. This is the final stage of production to use direct percussion.

The fourth category, final edge trimming and sharpening, includes artifacts that are very near or have reached the end stage of their manufacture. Tools within their final production stage require minor reduction along their margins, which is accomplished exclusively through pressure flaking and indirect percussion. Notching, edge grinding, and final stem preparation are completed at this stage. Artifacts having reached their end stage presumably represent tools that were discarded (often due to breakage), cached, lost, or otherwise abandoned. Finished forms require no additional production efforts, and commonly exhibit use-related edge modification (use-wear). Edges have not been remodeled through refurbishing efforts.

The final category, rejuvenated forms, describes artifacts that exhibit pronounced edge retouch or remodeling, a marked reduction in size, or evidence of adaptation to a secondary production trajectory in response to failure or discontinuation of the initial tool form. Tool rejuvenation and other forms of recycling provide important information regarding the perceived value of the resource.
00. [Indeterminate] IND
03. [Preform] PRF
01. [Initial Reduction] INR
04. [Final Stage] FST
02. [Blank] BLK
05. [Rejuvinated] REJ
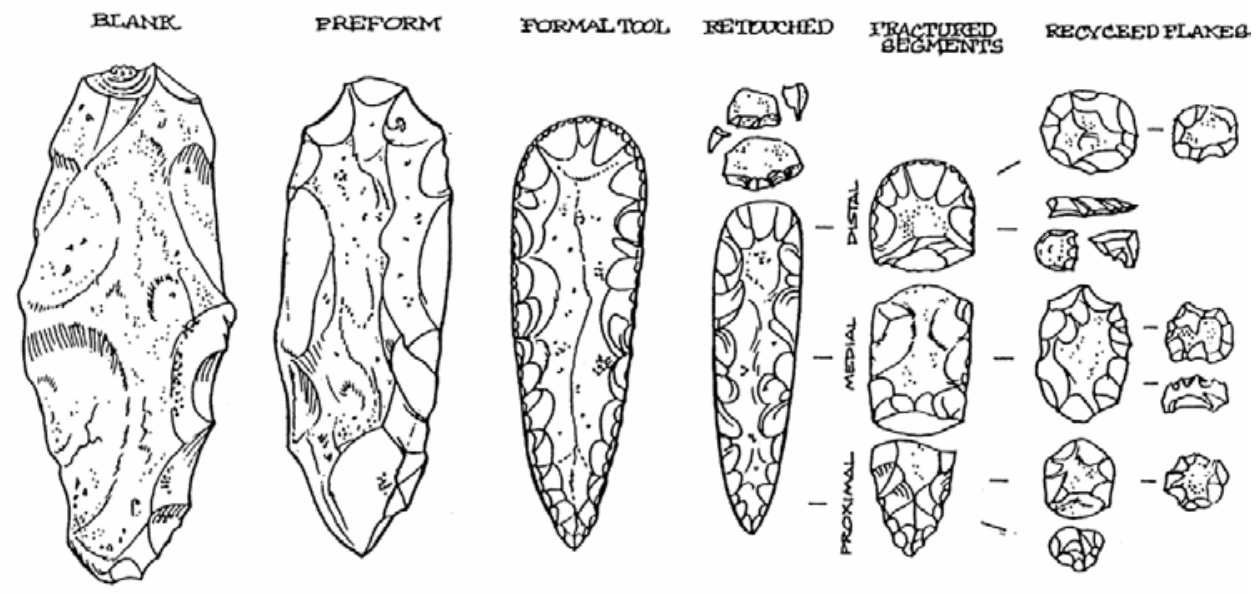

(2) wheresoo

Figure 6: In the illustration above, "retouched" and "fractured segments" are generally represented by Stage 5 (rejuvenated forms) in the TxDOT Chipped Stone Analytical protocol. However, it should be noted that "fractured segments" will often be identified as belonging to a perform of finished tool, and should be categorized appropriately. The final category, "recycled flakes," would be difficult to identify as deriving from an original formal tool in most instances, and many objects of this character would be included in the lithic assemblage as debitage. Such objects should only be identified as rejuvenated forms when the analyst is certain that a precursor form existed.

Version 2.3 
Figure 7: In the reduction sequence to the right, "stage one: blank" and "stage two: edged biface" each would be classified under Stage 2 (blank preparation) of the TxDOT Chipped Stone Analytical protocol. Similarly, "stage three: thinned biface" and "stage four: perform" would be classified as Stage 3 (perform shaping and thinning) under the TxDOT Chipped Stone Analytical protocol. The "stage five" shown here
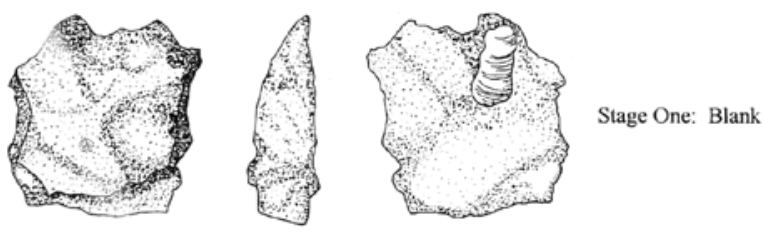
relates to Stage 4 of the TxDOT protocol.
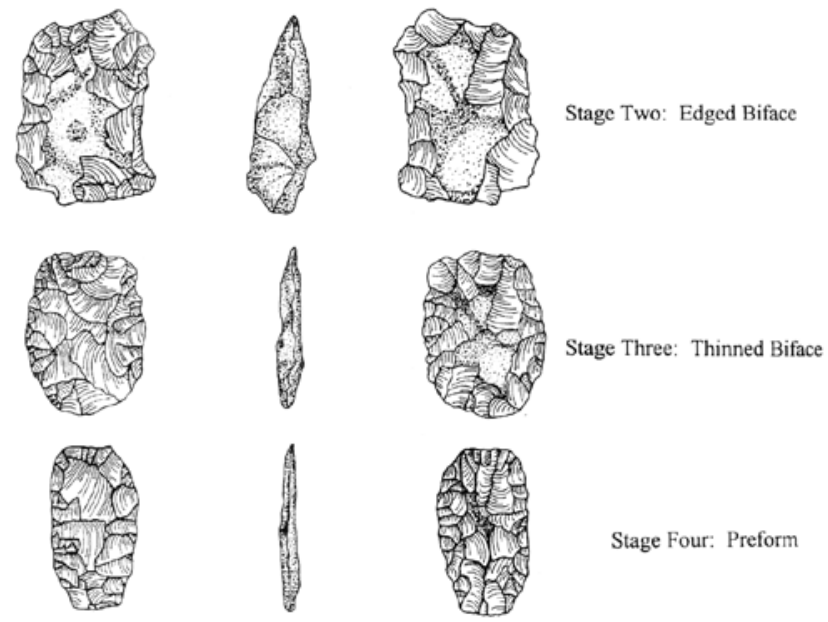

Stage Four: Preform
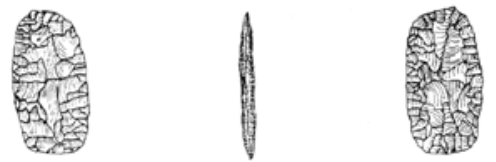

Stage Five: Finished Biface

14. Portion

A significant number of tools are recovered in a fragmentary state and it is important to record the portion represented. Identify partial forms as "fragments" when too little of the tool remains to determine what part of the tool is represented. As it is occasionally difficult to determine whether a piece corresponded to a proximal or distal segment, even when it was clear that one or the other is represented, an "indeterminate" category has been included.
00. [Indeterminate] IND
01. [Complete] CMP
02. [Distal] DIS
03. [Distal-medial] DME
04. [Medial] MED
05. [Proximal-medial] PME

06. [Proximal] PRX

07. [Lateral edges missing] LEM

08. [Fragment] FRG

09. [Barb/ shoulder] BSH

10. [Ear / tang] ETG

11. [Stem] STM 


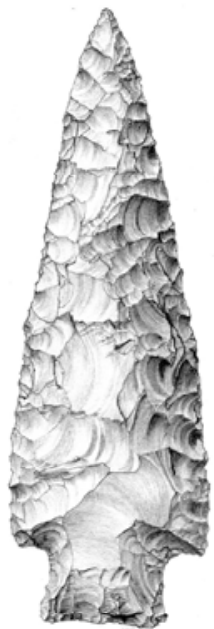

complete

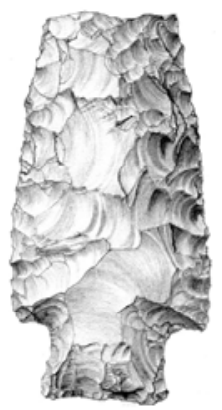

proximal-medial

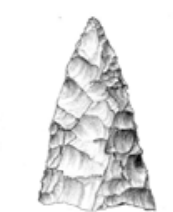

distal

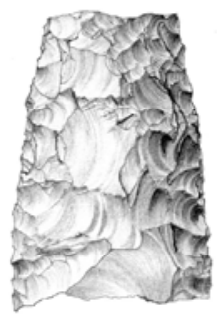

medial

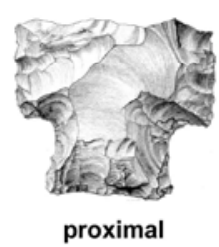

proximal

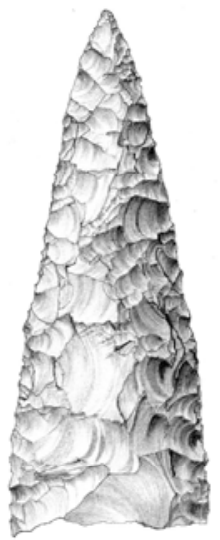

distal-medial

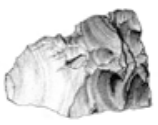

fragment

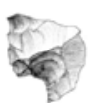

shoulder

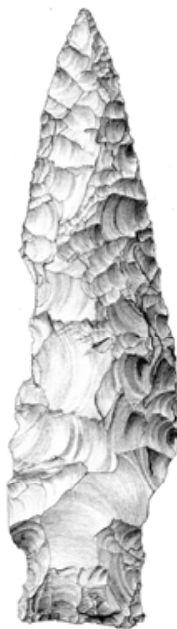

lateral edges missing

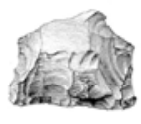

stem

Figure 8: Chipped stone tools are more often discovered in a broken state. Recording the portion of the tool that was recovered is necessary for adding context to metric measurements.

\section{Failure / Discard}

Determining the reason why a particular tool form was discarded is seldom a straightforward endeavor. Oftentimes such a determination cannot be made at all. However, where a cause of discard can be determined, valuable insights regarding production specialization and standardization, raw material conservation, use context, and cultural ideology may be gleaned.

The context of tool discard can be identified as production-related, use-related, and incidental. Productionrelated discard occurs when tools are discarded during manufacture as the result of technical mistakes or material deficiencies. Use-related discard can result from stress or impact fractures, excessive dulling, material exhaustion, useloss, or caching. Tool forms may also be lost unintentionally. Each mode of discard will have distinct implications for the likelihood of artifact recovery.

Several factors are also known to complicate determinations of discard cause. Secondary tool modification and material recycling may complicate determinations of failure, as can patina development. Excessive thermal alteration can also present an obstacle for assessing the probable cause of original discard as it is often difficult to determine the point at which the object was altered. Artifacts can be subjected to excessive heat following their discard, as when

Version 2.3

Dated July 16, 2010 


\section{Appendix C: TxDOT Chipped Stone Analytical Protocol}

affected by modern or ancient surface fires. The over-firing of raw material blanks or preforms may also have contributed to fire-damage.

\section{Production-related Discard}

Several authors have previously described snap or bending fractures (Crabtree 1972:60; Whittaker 1994:213; and Tsirk 1979:84). This fracture results when the lithic material is subjected to bending forces that exceed the material's elastic limits. Snap fractures often occur during tool production due to the knapper's failure to provide the objective piece with adequate support as it is reduced. In so doing, vibrations radiate throughout the tool form with each percussive strike, causing a fracture at the point where the elasticity of the material can no longer absorb the vibrations (Whittaker 1994: 213). Bending fractures can also occur quite commonly as the result of tool use. Use derived bending fractures manifest as lateral truncations that often display a rolled or lipped edge along one side of the termination (Shafer 1985: 283). When a rolled lip is observed, it often indicates that the tool was subjected to excessive torque during use. Snap fractures may also derive from material flaws, such as cavities or crystalline inclusions, which cause disharmony in the radiation of percussion waves through the material, or simply produce areas of weak structural integrity (see discussion of material flaws below). Step and hinge fractures present analogous difficulties for tool production or recycling, and while morphologically distinct, they are formed through similar circumstances. They are treated as a single category of failure in this protocol. A step fracture happens when the outward force is too great causing the flake to bend to the point of breaking. This is typically caused by hitting the core with a motion that is too fast which pulls the flake way faster than the propagation through the core; thus causing the snap to occur (Crabtree 1972: 92; Whittaker 1994: 109). Step fractures are similar to snap fractures with regard to the fracture mechanics of brittle solids in that they result in the truncation of material due to the unchanneled dispersion of percussive force. Hinge fractures occur when inadequate percussive force is applied to reduction efforts, preventing the flake from traveling the desired distance (Whittaker 1994: 109). However, rather than the flake being prematurely truncated as in step fractures, hinge fractures are characterized by the full termination of the flake. This termination occurs earlier than the intended point of egress, producing a rounded or blunt break and a disproportionate distribution of material mass that impedes further reduction efforts (Crabtree 1972: 68). Further reduction efforts often produce stacked step fractures or continued hinging, resulting in the inability to further reduce medial areas or to rejuvenate worn-out tool forms (Whittaker 1994: 109). Although they are morphologically dissimilar, the causes of hinge and step fractures, as well as the ensuing impediments for material reduction, are nearly equivalent (Whittaker 1994: 109). While step and hinge fractures often occur in the production of stone tools, they may also occur through tool use. Flakes may be inadvertently removed when tools come into contact with other materials as they are used in various tasks. Regardless of the trajectory stage, step and hinge fracture present a challenge to future reduction efforts, and may necessarily result in discard.

Failure and discard may also occur during reduction and rejuvenation efforts as the result of platform loss. The loss or collapse of a workable striking platform is often the consequence of improper reduction techniques or unanticipated fractures that leave no viable surface on which to strike and remove a desirable flake. Platform loss can occur during efforts to remove excessive mass from the medial areas of cores, preforms, and recycled tools, and may result in the inability to remove a desired mass without compromising the dimensional requirements of the desired trajectory. 
Material flaws generally manifest as mineral inclusions or cavities that differ compositionally from the package material. Irregular cleavage planes constitute another material obstacle that can impact the success of manufacturing efforts. When encountered, these flaws can produce anomalous fractures that complicate or preclude further reduction efforts. Common material flaws include macrocrystalline quartz, calcite, or fossil inclusions, as well as solution cavities and thermally-induced fractures. Production failures resulting from unanticipated thermally-induced fractures should be classified as "excessive heat" rather than "material flaw."

Cotterell and Kamminga (1979) describe overshot (outrepassé) failures as those that that result from the application of excessive percussion force, and which cause the fracture path to dive into the objective piece and remove more than the intended mass. Such fractures often occur during the bifacial thinning of blanks and preforms, or in the removal of blades from prepared cores. While failures of this type are most frequently observed during primary production, they may also occur during rejuvenation efforts. Discard will generally be motivated by excessive medial thinning or unrecoverable compromise of the objects design.

Perverse fractures, as defined by Crabtree (1972b: 82), are a spiral or twisting break that initiates at the point of percussion and follows through the object, causing its segmentation. In terms of causation, perverse fractures are the result of a hair-line fracture that resulted from a previous blow. The spiral perverse fracture picks up the old fracture thus resulting in failure. These differ from snap/bending fractures as they are not the result of excessive vibration, but result from a poor choice of striking angle and/or percussion force (as well as a bit of bad luck) that results in the plane of fracture traveling through rather than across the objective piece.

When more than one failure trait is expressed by an artifact, record the most significant cause for failure. For example, if a snap fracture resulted during production due to a fossil or crystalline quartz inclusion within the material, record material flaw as the cause of failure. In conjunction with other features of the assemblage, this information may potentially reveal preference patterns in raw material usage vis-à-vis specific tool classes, correlations between tool forms and discard patterning, and idiosyncratic differences in production skill.

\section{Use-related Discard}

Stone tools may be lost in their use-context in myriad ways. Points attached to an errant arrow may be lost or broken; as well they may be carried off embedded in game that was not subsequently subdued. Tools can also be continuously curated and used to the point of material exhaustion. Objects may also be cached in the process of ritual activity, such as when placed in burials. The motives for use-related discard may only be definitively discerned in a limited number of cases. Points with distal spalling, perhaps combined with a stress fracture above the hafting element, may be understood to have suffered an impact fracture. Tools recovered within a burial context may be identified as cached. Heavily recycled forms that cannot practically be further reduced through percussion or pressure flaking to yield an acute edge angle may be identified as exhausted tools. However, complete forms with light or no use-wear are commonly recovered at sites in contexts that do not explicitly indicate caching. When a discard motivation is ambiguous, "indeterminate" should be selected among the alternatives provided below. 
Incidental Discard

Incidental discard includes actions that removed objects from their systemic context by means other than manufacturing error, caching, or use (see Schiffer 1972), such as through dropping or misplacing them. However, this category of discard is a theoretical construct, the objective identification of which cannot be systematized. Thus, it is not included as an analytical option for assessing discard.

$\begin{array}{llllll}00 . & \text { [Indeterminate] IND } & 04 . & {[\text { Hinge / step] HST }} & 08 . & \text { [Excessive heating] HTF } \\ \text { 01. } & \text { [Snap / end shock] ESH } & 05 . & {[\text { Overshot (outrepasse)] OVR }} & 09 . & \text { [Exhausted] EXH } \\ \text { 02. } & \text { [Impact / bending] BND } & 06 . & {[\text { Material flaw] MFL }} & 10 . & \text { [Cached] CHD } \\ 03 . & \text { [Perverse] PRV } & 07 . & {[\text { Platform loss] PLL }} & & \end{array}$
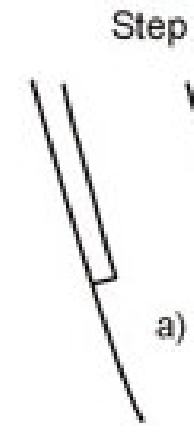

a)

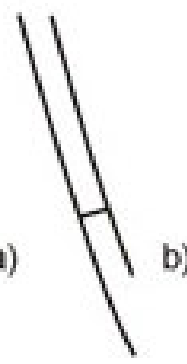

Hinge

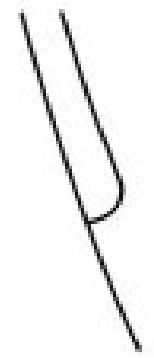

\section{Plunging (Outrépassé)}

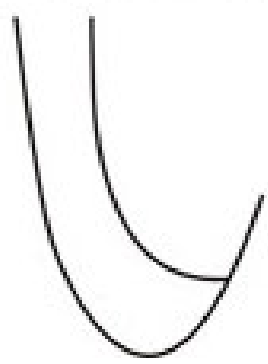

Figure 9: These terminations are often observed on bifacial blanks and preforms that were discarded in the process of manufacture. For the purposes of the protocols, step and hinge fractured are recorded as a single category of failure as the result in a very similar obstruction to the knapper.

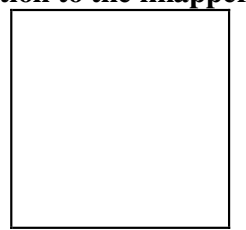

Figure 10: These terminations illustrate additional failures that may render the objective piece unusable or incapable of further reduction and recycling. 


\section{Alteration (choose dominant form)}

Material alteration addresses the transformation of structural and compositional properties that occurs as the result of natural and cultural processes. Natural processes include chemical and mechanical weathering, often resulting in patina or material decay. Thermal alteration is an example of material alteration through cultural processes.

An accurate assessment of thermal alteration is often inhibited by artifact size, patina formation, and unfamiliarity on the part of the researcher with some of the lithological variability expressed by select raw materials. Lithic raw materials typically undergo significant and detectable lithological changes with prolonged exposure to heat. Such changes are often desirable and may be deliberately generated by tool producers through controlled firing. Heattreated materials may be more easily worked by the artisan as the process renders low-quality materials more knappable (albeit while making them more brittle and decreasing their durability).

The identification of heat-treated materials brings culture process and the details of economic activity to the fore. Nonetheless, it is frequently difficult to distinguish purposefully treated materials from those that were incidentally burned. Incidental firing occurred in antiquity through controlled vegetation burns, as well as the occasional burning of middens or other cultural deposits. Historic-age and modern incidental firing may have resulted from burning off surface vegetation when preparing land for cultivation of pasture.

Lithic assemblages often exhibit other forms of material alteration that can obscure the study of raw material properties. The most common of these is the development of a weathering rind that is often identified as a white patina. The rind may be semi-translucent to opaque and is typically less than $3 \mathrm{~mm}$ in thickness. The development of a yellow to reddish brown "stain" may also develop on lithic artifact surfaces in iron-rich soils. The chemical processes that lead to the development of black (often dark blue) patinas is not completely understood. They most often occur in inundated deposits. Carbonate deposits and pigment staining occur rarely, the former being most common in coastal areas and the latter more common in ritual contexts.
00. [None observed] NOB
03. [White patina] WHP
06. [Pigment staining] PIG
01. [Indeterminate] IND
04. [Black patina] BKP
02. [Thermal] THR
05. [Oxide staining (yellowing)] OXS
07. [Carbonate build-up] CRB
99. [Other] ОТн

\section{Edge morphology (17a distal; 17b left lateral; 17c right lateral)}

Please indicate the shape of the working edge of the tool. Measuring from a line strung between edge termini, an edge is characterized as very convex if the distance from the cord to the maximum outward projection of the edge is greater than or equal to $5 \mathrm{~mm}$. Similarly, an edge is considered convex if the distance from the cord to the maximum outward projection of the edge is between $4.9 \mathrm{~mm}$ and $2 \mathrm{~mm}$. Edges are considered straight if the maximum inward or outward projection of the edge from the cord is no more than $1.9 \mathrm{~mm}$. An edge is considered concave if the distance from the cord to the maximum inward projection of the edge is between $4.9 \mathrm{~mm}$ and $2 \mathrm{~mm}$. An edge is characterized as very concave if the distance from the cord to the maximum inward projection of the edge is greater than or equal to $5 \mathrm{~mm}$. An edge is considered recurved if the maximum outward projection of the edge from the cord is greater than or equal to $2 \mathrm{~mm}$, and the maximum inward projection of the edge from the cord is also greater than or equal to $2 \mathrm{~mm}$.
00. [Indeterminate] IND
03. [Convex] CVX
06.. Very Convex VCX
01. [Straight] STR
04. [Recurved] RCV
07. Very Concave VCV
02. [Concave] CCV
05. [Serrated] SER
99. [Not applicable] NAP

Version 2.3

Dated July 16, 2010 


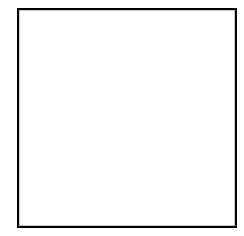

Figure 11: Edge morphology has its greatest utility in characterizing projectile points, darts, and knives.

\section{Flake scar pattern}

Flake scars are the impressions that remain on the face of a flaked stone artifact which are produced by the detachment of flakes during tool manufacture. The pattern of flake removal may offer important insights relating to the distribution of design templates and techniques of manufacture, as well as offer a means by which to observe variability in production design at different spatial scales.

00. [Indeterminate] a flaking pattern cannot be determined. IND

01. [Collateral] a flaking style that is characterized by parallel flakes emanating from opposing edges which meet in the center of the blade, forming a median ridge. COL

02. [Horizontal transverse] a flaking style that is characterized by horizontal, parallel flake scars emanating along one edge, traveling across the face of the blade, and terminating at the opposing edge. HTR

03. [Oblique transverse] a flaking style that is characterized by long, diagonal, parallel flake scars emanating along one edge, traveling across the face of the blade, and terminating at the opposing edge. OBT

04. [Random] flake removals do not reflect an aesthetic template in their distribution or alignment. RDM

99. [Not applicable] (expedient flake tools are one form of tool that will not exhibit a flake scar pattern). NAP

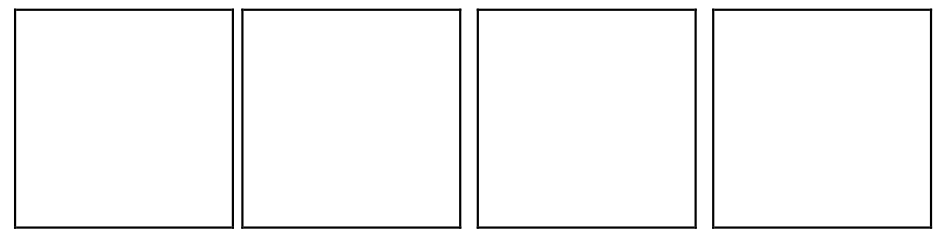

Figure 12: Patterns of flake removal in edge construction, potentially related to flaking technique, tool function, aesthetic display, and social identity.

\section{Edge construction type}

Edge construction type references the location and form of preparatory edge construction on the objective piece. There are a variety of ways in which an edge may be constructed on a chipped stone object. The most basic choice is between bifacial and unifacial constructions. Such choices carry implications for accurately assigning tools to a subgroup, distinguishing between techniques used during production, assessing the foci of use, and determining the angle of the resulting edge. Variability may also occur among subtypes, potentially alluding to differences in raw material access, tool function, or nuances of social identity. For example, while the lateral margins of some Perdiz points are bifacially constructed, others exhibit unifacially beveled edges. The constructed working edge(s) of a tool may be characterized using the following descriptions:

\section{0. [Indeterminate] IND \\ 01. [Bifacial-distal] BFD}

02. [Bifacial-bilateral] BFB

03. [Bifacial-unilateral] BFU

Version 2.3

Dated July 16, 2010 

04. [Bifacial-distal-bilateral] BDB
05. [Bifacial-distal-unilateral] BDU
06. [Bifacial-circumferential] BFC
07. [Unifacial-distal] UFD
08. [Unifacial-bilateral] UNB
09. [Unifacial-unilateral] UNU

10. [Unifacial-distal-bilateral] UDB

11. [Unifacial-distal-unilateral] UDU

12. [Unifacial-circumferential] UNC

13. [Other] OTH

99. [Not applicable] NAP

\section{Proximal edge grinding \\ Not Observed NOB \\ Observed OBS}

\section{Wear Patterning}

The following use-wear observations can be made macroscopically using an 18-20X jeweler's loop, and is considered low-power magnification. Low-power magnification is assumed to imply magnification between 18x-power and 100x-power. This portion of the protocol has not been designed for high-power magnification and Scanning Electron Microscopy. Use-wear characterization using low-power magnification has been shown to successfully identify the range of motion an object was used in, and, to a lesser degree, the hardness of the contact material. High-power magnification is generally needed to accurately identify contact material and detect finer details of object use. The lowpower use-wear characterization advocated here will find its greatest utility in quantifying the presence or absence of use, identifying the area of use on a specific piece, and in identifying variability in use among specific artifact types and subtypes. The use-wear categories described below are not mutually exclusive - tools may exhibit more than one form of wear.

Edge modification is not always the product of material use. Other natural and cultural processes, such as trampling and archeological excavation, have been shown to produce edge modification similar to that developed through actual use (McBrearty, et al. 1998; Shea and Klenck 1993; Tringham, et al. 1974). Such processes obviously affect the recognition of some patterns of wear more than others, and may be particularly relevant for detecting true usewear on simple detachment-based tools. Distinguishing use-derived flake terminations along the lateral margins of tools is perhaps the most equivocal functional assessment; although Odell and Odell-Vereecken (1980) state that the patternlessness of such incidental attrition is detectable and, thus, can be distinguished from actual use-wear with a high level of accuracy. Tools may exhibit a form of polish in deflationary zones derived from aeolian processes, and may exhibit battered edges within fluvial deposits. Given the possibility that edge modification derived from trampling or other processes, "attrition" use wear should only be record for artifacts that exhibit both a distinct, clustered pattern of edge alteration and worn or polished facets in the area of proposed use. While this undoubtedly underestimates the actual amount of use-wear exhibited throughout the assemblage, it substantially increases the accuracy with which positive determinations were made.

The degree of expedient tool use within an assemblage provides one means by which the level and importance of material conservation may be evaluated. Regions characterized by a scarcity of utilitarian lithic raw materials have been shown to exhibit higher levels of material recycling. Careful attention to and recording of use-wear may also provide important information related to spatial and temporal variability expressed within tool classes, types and subtypes. 


\section{Appendix C: TxDOT Chipped Stone Analytical Protocol}

\section{Flaking attrition}

Material mass is often removed from the working edge of a tool during the process of use. Much of this attrition is in the form of small flake removals that typically exhibit feathered or stepped terminations. Accurate recording of use-derived attrition requires an analyst to distinguishing these removals from trimming flakes that are detached along a tool's edge in the final preparation stage prior to use. Use-derived attrition can often be distinguished from preparatory trimming as it creates a more obtuse edge angle in the area of use than is expected based on observing edge characteristics elsewhere on prepared, but unused portions of the tool. Use-derived attrition may also remove areas of polish that have developed along tool margins, which may also produce sharper facets that contrast in the area of use with more polished and rounded facets.

Data is coded to record the presence and location of flaking attrition as its distribution on a tool form is a significant indication of tool function. Observations shall be recorded as follows:

00. NPR [Not present] Use if flaking attrition is not observed.

01. BFD [Bifacial-distal] Use if the working edge of a tool is located along the distal margin and attrition is observed on each face (dorsal and ventral)

02. BFB [Bifacial-bilateral] Use if both lateral margins exhibit use-derived attrition and the attrition has resulted in removals on both faces.

03. BFU [Bifacial-unilateral] Use if only one lateral margin (left or right) exhibits use-derived attrition and the attrition has resulted in removals on both faces.

04. BDB [Bifacial-distal-bilateral] Use if both lateral margins and the distal margin exhibit use-derived attrition and the attrition has resulted in removals on both faces. This option will be select if one of the lateral margins exhibits unifacial attrition.

05. BDU [Bifacial-distal-unilateral] Use if only one lateral margin (left or right) and the distal margin exhibit use-derived attrition and the attrition has resulted in removals on both faces.

06. BFC [Bifacial-circumferential] Use if the lateral margins along the entire circumference of the tool form exhibit use-derived attrition and the attrition has resulted in removals on both faces.

07. UFD [Unifacial-distal] Use if the distal margin exhibits use-derived attrition and the attrition is observed on only one face.

08. UFB [Unifacial-bilateral] Use if both lateral margins exhibit use-derived attrition and the attrition has resulted in removals on only one face.

09. UFU [Unifacial-unilateral] Use if only one lateral margin (left or right) exhibits use-derived attrition and the attrition has resulted in removals on only one face.

10. UDB [Unifacial-distal-bilateral] Use if both lateral margins and the distal margin exhibit use-derived attrition and the attrition has resulted in removals on only one face.

11. UDU [Unifacial-distal-unilateral] Use if only one lateral margin (left or right) and the distal margin exhibit use-derived attrition and the attrition has resulted in removals on only one face.

12. UFC [Unifacial-circumferential] Use if the lateral margins along the entire circumference of the tool form exhibit use-derived attrition and the attrition has resulted in removals on only one face.

Version 2.3 
Data Recovery Investigations at the Tank Destroyer Site, Fort Hood

13. UBO [Unifacial-bilateral-oppositional] This form of attrition is most typically found on tools used as drills or awls. Use if both lateral margins exhibit use-derived attrition and the attrition has resulted in removals along the opposing margins of each face.

14. OTH [Other] Use if none of the above apply and enter a description in the text box provided. 

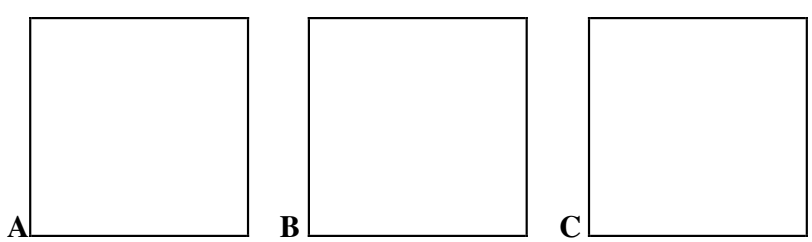

Figure 13: Examples of lateral edge flaking attrition. (A) bifacial-unilateral; (B) unifacial-unilateral; (C) platform abrasion and (likely) post-depositional removals.

\section{23. Smoothing}

Crushing and smoothing describe the form of wear attained through battering, grinding, or polishing. The tool is typically blunted through battering or abrasion against a hard contact material in the process of use. Crushed working surfaces may be a normally achieved trait with little effect of tool utility, such as with hammerstones. Alternatively, crushed surfaces may be an undesired consequence of use and material attrition that necessitates edge resharpening. Smoothing is typically the result of intensive abrasion and is commonly observed on tools used for grinding, polishing, or burnishing (uncommon among chipped-stone tools). Once identified, the distribution of this wear should be recorded using one of the following descriptions:

$\begin{array}{ll}\text { 00. } & \text { NPR [Not present] Use if attrition through } \\ & \text { crushing or smoothing is not observed. } \\ \text { 01. } & \text { DIS [Distal] } \\ \text { 02. } & \text { DLT [Distal-lateral] } \\ \text { 03. } & \text { UNL [Unilateral] } \\ 04 . & \text { BLT [Bilateral] }\end{array}$

$\begin{array}{ll}\text { 05. } & \text { FLS [Facial Smoothing] } \\ \text { 06. } & \text { FCS [Facet Smoothing] } \\ \text { 07. } & \text { CIR [Circumferential] } \\ \text { 08. } & \text { PPX [Primary Proximal] } \\ 09 . & \text { SPX [Secondary Proximal] }\end{array}$

24. Polish

The use-wear category "polish" describes lustrous areas on the tool, typically located at the distal or lateral margins, but occasionally noted on medial surfaces. Record polish as "shallow" when it is restricted to within $5 \mathrm{~mm}$ of an edge. Define polish as "deep" when it extends beyond $5 \mathrm{~mm}$ from the edge of its origin.

The origin of polish is not well understood despite having been the subject of generous scholarly attention (Odell 2001). Research into the nature of use-polish is generally focused either on the patterns of polish formed on stone tools as the result of a specific set of activities (cf. Aoyama 1999; Keeley 1977, 1980; Semenov 1964), or on the genesis and composition of polish itself (Fullagar 1991; Grace 1996; Odell 2001). In controlled studies where specific tool forms were utilized in a defined set of prescribed behaviors, researchers have had considerable success in correlating patterns of polish distribution and composition with the specific activities that generated its development. However, studies have also shown that a diverse set of activities may produce virtually identical patterns of use-polish (Lewenstein and Walker 1984). Researchers have also found that specific patterns of polish development do not correlate well with isolable tasks on multifunctional tools (Clark 1988). It is perhaps best to consider that the form of the tool, the raw material used in its manufacture, and the patterns of wear (in any form) observed will provide a range of functional possibilities and limitations for how the tool was used in a particular cultural and techno-environmental setting.

Three processes other than primary contact during use may cause the development of a lustrous sheen, and they should not be recorded as use-derived polish. The first, hafting polish, develops through secondary, use-associated

Version 2.3
Dated July 16, 2010 
contact. Hafting polish is formed through the tools contact with a hafting element or fastening material. Hafting polish, when present, will typically manifest on both lateral and medial surfaces nearer the proximal end of a tool. Hafting can also be associated with worn, ground, or otherwise blunted lateral margins. Evidence for hafting should be nominally recorded separately from use-wear (see \#26 below).

The second process that inhibits the detection of use-derived polish is thermal alteration. In extreme cases, lithic material will become vitrified through over-exposure to heat, producing a lustrous sheen that covers the surface of the artifact and resembles use-derived polish. Grinding, the third process, is a specialized manufacturing technique that results in the development of a luster across the ground surface. The luster forms through the extensive abrasion required in the production process rather than from use.

00. NPR [Not present] Use if use-derived polish is not observed.

01. SHD [Shallow distal $<5 \mathrm{~mm}$ ]

02. DED [Deep distal $>5 \mathrm{~mm}$ ]

03. SHL [Shallow lateral $<5 \mathrm{~mm}$ ]

04. DEL [Deep lateral $>5 \mathrm{~mm}$ ]

05. UFM [Unifacial-medial]

06. BFM [Bifacial-medial]

07. BPL [Bipolar]

08. PRX [Proximal]
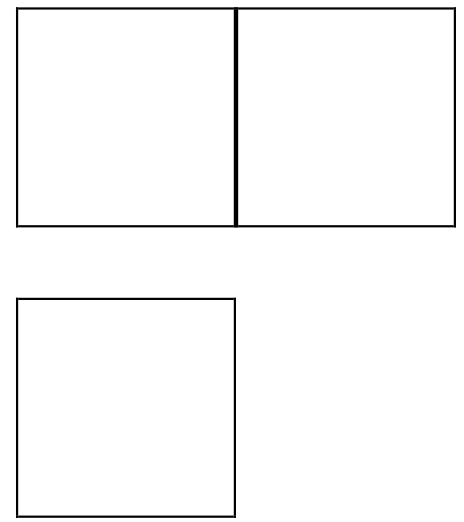

Figure 14: Patterns of polish formation and distribution related to use wear. The formation of polish is dependent on the nature of the tool construction material, nature of the contact material, and duration of use.

\section{Etching / pitting}

Etching and pitting refer to striations or small cavities produced through abrasive contact (Semenov 1964). As with polish, such markings may occasionally derive from production techniques, although this is generally only a concern for tool forms featuring ground or pecked and ground bits. Etching and pitting are better studied microscopically. The macroscopic techniques used in this study are useful for detecting moderate to deep scarring and abrasion that are characteristic of working soils with a significant sand content, but they may have less utility in detecting wear left from working in clayey soils. Striations (etching) may be located along either the distal or lateral margins of the tool. When located at the distal margin they most often run perpendicular to the edge. The extent to which they proceed from the distal margin across the face of a tool can provide some measure of how far the tool penetrated into a contact material.

\section{Version 2.3}

Dated July 16, 2010 
When located along the lateral margins striations more typically run parallel the edge. Striations may be created through quarrying, soil working, planing, polishing, grinding, or any extended lateral movement across a hard or abrasive surface.
00. $\quad$ NPR [Not present] Use if attrition through etching or pitting is not observed.
01. SHD [Shallow distal $<5 \mathrm{~mm}$ ]
02. DED [Deep distal $>5 \mathrm{~mm}$ ]
03. SHL [Shallow lateral $<5 \mathrm{~mm}$ ]
04. DEL [Deep lateral $>5 \mathrm{~mm}$ ]

05. UFM [Unifacial-medial]

06. DSM [Distal-medial]

07. CIR [Circumferential]

08. MBF [Medial-bifacial]

09. BPL [Bipolar]

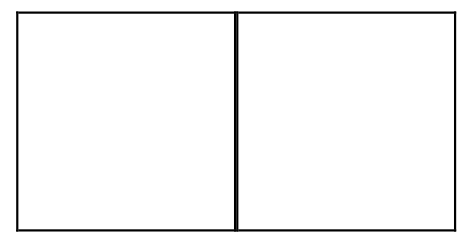

Figure 15: Development of lateral scars (or striations) developed on the working edge of the tool derived from abrasive and repeated contact between the tool form and contact material.

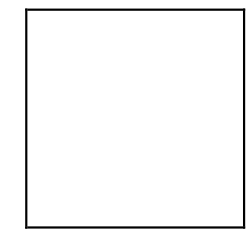

Figure 16: Scanning Electron Microscope (SEM) image of etch makes left on the surface of an artifact as the result of tool use. The marks reveal the motion of use relative to edge areas and the relative hardness of the contact material. 


\section{Data Recovery Investigations at the Tank Destroyer Site, Fort Hood}

\section{Hafting evidence}

Hafting may be identified through lateral edge dulling toward the proximal end of the tool form, polish along the proximal lateral margins and proximal facial facets, and the residual presence of a masticate such as asphaltum. Note the presence or absence of this evidence. $\quad \square$ Not Observed NOB $\quad \square$ Observed OBS

\section{Raw Material}

\section{Lithology}

The lithologic character of raw materials should be identified to the best, most accurate extent reasonable. The lithic analyst should specifically identify materials only to the extent that they are certain that the information provided is accurate. The most common raw materials have been coded for use. Materials not included in the list provided should be coded as "other" and specifically identified in the text field provided.

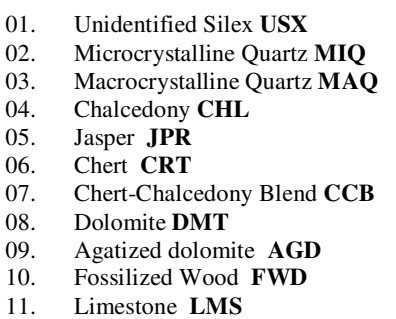

$\begin{array}{ll}\text { 12. } & \text { Silicified Limestone SLM } \\ \text { 13. } & \text { Quartzite QZT } \\ \text { 14. } & \text { Novaculite NVC } \\ \text { 15. } & \text { Rhyolite RHY } \\ \text { 16. } & \text { Basalt BSL } \\ \text { 17. } & \text { Serpentine / Greenstone SGR } \\ \text { 18. } & \text { Steatite (soap stone) STT } \\ \text { 19. } & \text { Granite GRT } \\ \text { 20. } & \text { Marble MAR } \\ 21 . & \text { Gneiss GNS } \\ 22 . & \text { Schist SCT }\end{array}$

$\begin{array}{ll}\text { 23. } & \text { Silt-stone SST } \\ \text { 24. } & \text { Obsidian OBS } \\ \text { 25. } & \text { Manning Fused Glass MFG } \\ \text { 26. } & \text { Ironized sandstone ISS } \\ \text { 27. } & \\ & \\ \text { 96. } & \text { Unidentified Sedimentary USM } \\ \text { 97. } & \text { Unidentified Igneous UIG } \\ \text { 98. } & \text { Unidentified Metamorphic UMM } \\ \text { 99. } & \text { Other OTH }\end{array}$

\section{Source identification (REMOVE FROM PROTOCOL)}

The source areas provided below represent those commonly identified in available literature, but the list is by no means exhaustive (see Banks 1990; Turner and Hester 1999). The identification of lithic raw material source is intended to provide a means to address issues of resource mobilization. However, many issues exist in accurately identifying source areas. For example, Uvalde Gravels contain a good amount of Edwards Chert. The raw material source area should be identified to the most accurate level possible without unsupportable speculation. It is expected that raw material source areas will not generally be identifiable.

In general, raw material sourcing is assessed using visual identification for chert, chalcedony, and quartzite artifacts as chemical characterization studies have not been reliable in determining source areas. Successful identification of specific resource outcrops is often impossible, but some confidence regarding the general can be gained by matching artifacts (formal tools and debitage) to geological samples taken from individual resource outcrops (ex. Edwards, Alibates, Maravillas, Ogallala). Relevant criteria to consider in matching archaeological materials to geological samples include lithology, material hardness, relative grain size, color, the presence or absence of banding and other irregularities, and the presence and composition of micro-fossils and other inclusions (cf. Morrow 1994). In most instances, determining the area of procurement depends on artifact mass as only large pieces will retain enough compositional character to distinguish between geographically discrete resource areas.

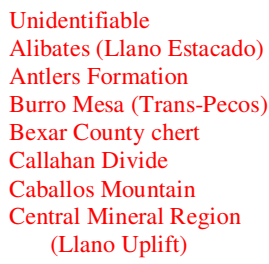

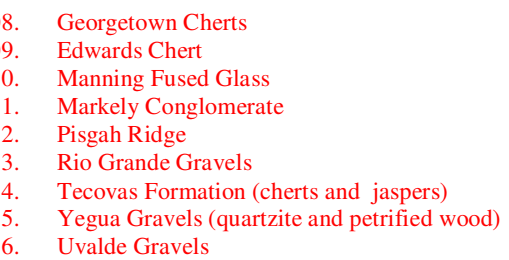

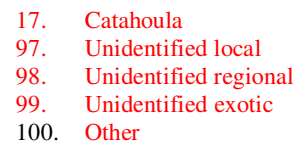

100. Other 


\section{Projectile point data}

28. Point Class
00. NAP Not Applicable
02. SDN Side Notched
04. TRI Triangular
01. CRN Corner Notched
03. STM Stemmed
05. LAN Lanceolate

\section{Point Data}

Table 1: Shaded rows have automatically populated data and should not be manually entered.

\begin{tabular}{|c|c|c|c|c|c|c|c|}
\hline & 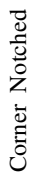 & 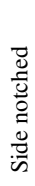 & 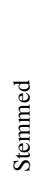 & 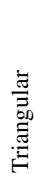 & 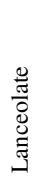 & Measurement & Description \\
\hline 8 & $\mathbf{X}$ & $\mathbf{x}$ & $\mathbf{X}$ & $\mathbf{x}$ & $\mathbf{X}$ & point length & same as question \#8 above \\
\hline 9 & $\mathbf{X}$ & $\mathbf{x}$ & $\mathbf{X}$ & $\mathbf{X}$ & $\mathbf{X}$ & point width & same as question \#9 above \\
\hline 29.1 & $\mathbf{X}$ & $\mathbf{X}$ & $\mathbf{X}$ & $\mathbf{x}$ & $\mathbf{X}$ & point ratio & Tool width divided by tool length \\
\hline 29.2 & $\mathbf{X}$ & $\mathbf{x}$ & $\mathbf{X}$ & $\mathbf{x}$ & $\mathbf{X}$ & blade length $(\mathrm{L})$ & \\
\hline 29.3 & $\mathbf{X}$ & $\mathbf{X}$ & $\mathbf{X}$ & $\mathbf{x}$ & $\mathbf{X}$ & blade length $(\mathrm{R})$ & \\
\hline 29.4 & $\mathbf{X}$ & $\mathbf{X}$ & $\mathbf{X}$ & $\mathbf{X}$ & $\mathbf{X}$ & base/stem length or basal inflection & \\
\hline 29.5 & $\mathbf{X}$ & $\mathbf{x}$ & $\mathbf{X}$ & & & base/stem width & \\
\hline 29.6 & $\mathbf{X}$ & $\mathbf{x}$ & $\mathbf{x}$ & & $\mathbf{x}$ & neck thickness & \\
\hline 29.7 & $\mathbf{X}$ & $\mathbf{X}$ & $\mathbf{x}$ & & $\mathbf{x}$ & neck width & $\begin{array}{l}\text { May be the same as \#30d in stemmed forms, and the same as \#9 in } \\
\text { Lanceolate forms. }\end{array}$ \\
\hline 29.8 & $\mathbf{X}$ & $\mathbf{x}$ & & & & notch depth (L) & \\
\hline 29.9 & $\mathbf{X}$ & $\mathbf{x}$ & & & & notch depth (R) & \\
\hline 29.10 & $\mathbf{X}$ & $\mathbf{x}$ & & & & notch ratio & Average notch depth divided by width of point \\
\hline 29.11 & $\mathbf{X}$ & $\mathbf{X}$ & $\mathbf{X}$ & $\mathbf{x}$ & $\mathbf{X}$ & base to blade ratio (length) & 29e divided by $30 \mathrm{a}$ \\
\hline 29.12 & $\mathbf{X}$ & $\mathbf{X}$ & $\mathbf{X}$ & & & base to blade ratio (width) & $29 \mathrm{f}$ divided by $30 \mathrm{~b}$ \\
\hline 29.13 & $\mathbf{x}$ & $\mathbf{x}$ & $\mathbf{x}$ & & & base/stem ratio & $\begin{array}{l}0 \text { (indeterminate), } 1 \text { (short; }<=0.7 \text { ), } \\
2 \text { (proportionate; }>0.7 \&<1.3 \text { ), } 3 \text { (long; }>=1.3 \text { ) } \\
\end{array}$ \\
\hline 29.14 & $\mathbf{x}$ & $\mathbf{x}$ & $\mathbf{x}$ & & & base form & $\begin{array}{l}0 \text { (indeterminate), } 1 \text { (convex), } 2 \text { (straight), } 3 \text { (concave), } \\
4 \text { (notched), } 5 \text { (pointed), } 6 \text { (bulbar) }\end{array}$ \\
\hline 29.15 & & & $\mathbf{x}$ & & & stem form & $\begin{array}{l}0 \text { (indeterminate), } 1 \text { (contracting), } 2 \text { (parallel), } 3 \text { (expanding), } \\
4 \text { (asymmetrical) }\end{array}$ \\
\hline 29.16 & & & & $\mathbf{X}$ & $\mathbf{x}$ & distal base form & $\begin{array}{l}0 \text { (indeterminate), } 1 \text { (convex; }>=1 \mathrm{~mm}) \\
2 \text { (straight; }<1 \mathrm{~mm} \&>-1 \mathrm{~mm}), 3 \text { (concave; }<-1 \mathrm{~mm} \text { ) }\end{array}$ \\
\hline 29.17 & & & $\mathbf{X}$ & & $\mathbf{x}$ & lateral base/stem form & $\begin{array}{l}0 \text { (indeterminate), } 1 \text { (contracting), } 2 \text { (parallel), } \\
3 \text { (expanding - exhibits tangs), } 4 \text { (asymmetrical) }\end{array}$ \\
\hline 29.16 & $\mathbf{x}$ & $\mathbf{x}$ & $\mathbf{x}$ & $\mathbf{x}$ & $\mathbf{x}$ & blade curvature (L) & $\begin{array}{l}0 \text { (indeterminate), } 1 \text { (very convex; }>=5 \mathrm{~mm}), \\
2 \text { (convex; }<5 \mathrm{~mm} \&>=2 \mathrm{~mm}), 3(\text { straight; }<2 \mathrm{~mm} \&>-2 \mathrm{~mm}), \\
4 \text { (concave; }<=-2 \mathrm{~mm}), 5(\text { recurved; }<-2 \mathrm{~mm} \&>2 \mathrm{~mm})\end{array}$ \\
\hline 29.19 & $\mathbf{x}$ & $\mathbf{x}$ & $\mathbf{X}$ & $\mathbf{x}$ & $\mathbf{X}$ & blade curvature $(\mathrm{R})$ & $\begin{array}{l}0 \text { (indeterminate), } 1 \text { (very convex; }>=5 \mathrm{~mm}), \\
2(\text { convex } ;<5 \mathrm{~mm} \&>=2 \mathrm{~mm}), 3(\text { straight; }<2 \mathrm{~mm} \&>-2 \mathrm{~mm}), \\
4(\text { concave } ;<=-2 \mathrm{~mm}), 5(\text { recurved; }<-2 \mathrm{~mm} \&>2 \mathrm{~mm})\end{array}$ \\
\hline 29.20 & $\mathbf{X}$ & $\mathbf{X}$ & $\mathbf{X}$ & & & shoulder angle $(\mathrm{L})$ & \\
\hline 29.21 & $\mathbf{X}$ & $\mathbf{X}$ & $\mathbf{X}$ & & & shoulder angle $(\mathrm{R})$ & \\
\hline 29.22 & & & $\mathbf{X}$ & & & shoulder junction & 0 (indeterminate), 1 (curved), 2 (angular), 3 (straight) \\
\hline 29.23 & & & & $\mathbf{X}$ & & base angle $(\mathrm{L})$ & \\
\hline 29.24 & & & & $\mathbf{X}$ & & base angle $(\mathrm{R})$ & \\
\hline 29.25 & $\mathbf{X}$ & $\bar{X}$ & $\mathbf{X}$ & $\mathbf{x}$ & $\mathbf{X}$ & index of symmetry & $? ? ? ? ? ?$ \\
\hline
\end{tabular}

Version 2.3 


\section{TXDOT PROTOCOL FOR DEBITAGE ANALYSIS}

\section{Research Methods: Debitage}

There is a great deal of information that may be gained from the study of debitage in archaeological assemblages, and researchers have debated the utility of various classes of information, as well as their situational applicability, accuracy, and level of efficiency (Ahler 1989; Andrefsky 1998; Baumler and Downum 1987; Johnson 1989; Magne 1989; Sullivan and Rossen 1985). The analytical process described here provides a useful synthesis of attribute analysis and mass analysis that captures the maximum amount of critical basic data for large collections within a workable time frame while allowing a wide range of research questions to be addressed.

Within this protocol, no linear measurements are recorded for individual artifacts (i.e. flake length, width, thickness, and curvature; platform angle, width, and thickness). Such measurements rarely lend themselves to addressing important or innovative research questions. They also require a large amount of time to collect when analyzing sizable collections and this time investment is invariably unwarranted when assessed against the amount of useful information returned.

\section{Metric Attributes (Number and Weight)}

In the interest of analytical efficiency, there is also no good reason to weigh individual flakes. Counts and weights will be assessed for artifact groupings (analytical assemblages) that are created through the analytical process.

\section{Minimum Number of Nodules (MNN)}

An assessment of MNN is designed to record the minimum number of individual packages of raw material (nodules) that contributed to a specific analytical assemblage. This may be relevant for determining the number/volume of tools produced, the number of individuals participating in the production activity, raw material preferences, or the degree of deflation, comingling, or disturbance reflected in the assemblage being analyzed. This assessment should be based on observations of raw material type and material properties, and may be augmented through the use of ultraviolet fluorescence. Analysts should consider the effects of differential patination and thermal alteration on observable raw material features when assessing MNN. Analysts should favor lumping over splitting in determining MNN (additional nodules should only be recorded when flakes within an analytical assemblage can not have been derived from the same source package).

\section{Form (Completeness - flake vs. frag vs. shatter)}

Sullivan and Rozen (1985:759) have advocated using the analytical categories "complete flake", "broken flake", "flake fragment", and "debris" for the study of flake assemblages, and have illustrated the tendency for each to be represented in different proportions at various stages of manufacture (see also Baumler and Downum 1987). There are many variables that undermine the utility of this approach. Landscapes used for pasture or cultivation, particularly in near-surface deposits, are highly susceptible to trampling and to disturbance by agricultural machinery. Either agent will distort the ratio of complete to broken flakes in such contexts. The movement of artifacts in vertic soils or within contexts characterized by erosion and re-deposition, root disturbances, and ancient cultural disturbances such as area maintenance (to name only a few) are equally likely to affect this ratio. Interpreting manufacturing stage through the percentage of whole vs. broken flakes requires preservation of integrity, in both individual specimens and the original composition of the assemblage, with little post-depositional alteration. Due to the rarity of such an occurrence, the interpretive worth of the categories advocated by Sullivan and Rozen find their greatest utility when used with experimental assemblages.

Version 2.3 
This protocol does not require that only those flakes within an assemblage that retain a striking platform (whole and proximal flakes) be included for data collection and analysis. While this would reduce spurious data produced through postdepositional processes, distal flake fragments may be excluded from analyses by most statistical packages.

Size-grade Analysis

Sort all debitage by size-grade using nested sieves with 1-inch, 3/4-inch, 1/2--inch, and 1/4-inch apertures. Size-grade analysis offers an alternative to taking standard metric measurements of maximum flake length, width, thickness (cf. Andrefsky 1998: 96-100) that substantially increases the efficiency with which large samples may be studied (Ahler 1989). When combined with supplementary data, such as the percentage of dorsal cortex present and platform type, size-grade analysis provides researchers with valuable information regarding production trajectory, the method and organization of raw material procurement, technology of production, production efficiency, and the level of material curation (Ahler 1989; Baumler and Downum 1987; Behm 1983; Bradbury and Franklin 2000).
01. [1-inch sieve]
03. [1/2-inch sieve]
02. [3/4-inch sieve]
04. [1/4-inch sieve]

\section{Cortex Percent}

Perhaps the most common use of cortex observations in debitage analysis is for assessing the stage of manufacture represented by the flake assemblage. Researchers commonly use one of two models of assigning meaning to the percentage of cortex present. The first uses the relative amount of cortex present on each flake to place the individual piece within a linear reduction model, under the assumption that only flakes produced during the initial phases of tool manufacture will exhibit a high percentage of dorsal cortex. Andrefsky (1998:111) refers to this as the "triple cortex" approach, and it can be recognized by the identification of primary, secondary, and tertiary (or interior) flakes. As researchers Sullivan and Rozen (1985:756-757) have pointed out, however, there is little standardization among those employing the triple cortex approach, such that the flakes designated as primary may be required to have as much as 100 percent dorsal cortex or be permitted to exhibit as little as 50 percent. Similarly, the percentage of dorsal cortex required to identify a secondary flake ranges between 100 and 0 percent depending on the researcher, while the percentage of dorsal cortex required for the identification of a tertiary flake ranges from between 0 and 25 percent (Sullivan and Rossen 1985:757). As a significant number of tertiary flakes -which are often regarded as evidence of final stage manufacture- are produced in the initial phases of core reduction, the traditional classification of debitage into primary, secondary, and tertiary flakes has very little analytical worth.

Ahler (1989:90) has pointed out that the presence of cortex in a lithic waste assemblage, as well as the utility of information gleaned from its study, will vary according to the nature of the raw material, how it was quarried, the reduction technology employed, and the stage of manufacture represented by the assemblage. Also, the presence of cortex at any reduction stage is dependent on the initial presence of cortex prior to reduction (Andrefsky 1998:113-114). The nature of raw material outcrops, the method of quarrying employed, and the technology of production affects the viability of using cortex percent as an indicator of production stage. Even under the best of circumstances, cortex percent may only provide data relevant to broadly distinguish early reduction stages from later stages (Mauldin and Amick 1989:71). Debitage is able to more accurately inform reduction stage and artifact class when classified according to size, percentage of cortex represented, and platform type. The following size categories should be used for classifying debitage.

Version 2.3

Dated July 16, 2010 


\section{Cortex Percentages}
00. [0\%]
02. [26-50\%]
04. [76-100\%]
01. [1-25\%]
03. [51-75\%]

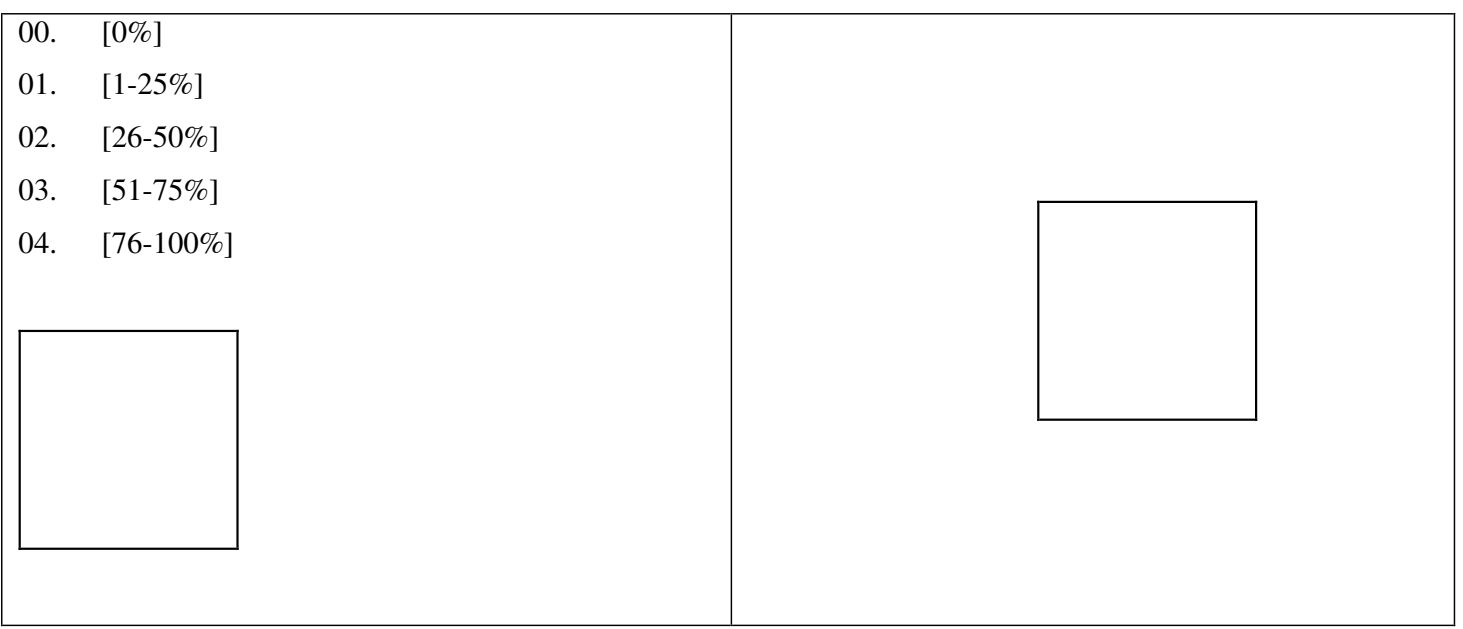

Figure 17: The graphic above illustrates both a quantifiable and a qualitative means by which to measure or estimate the amount of remnant dorsal cortex on a lithic flake.

Platform Type

The striking platform of a flake is the point of contact where the percussor initiated the flake detachment. The morphology of the platform can yield valuable information pertaining to the stage of manufacture represented by the flake, which in turn reflects the presence, character, and organization of activity areas. Platform morphology can also inform production technology (Andrefsky 1998).

Platform types should be recorded as indeterminate, cortical, flat, complex, abraded, faceted, multifaceted, and rejuvenated (cf. Andrefsky 1998:93-96). Cortical platforms are those that retain some amount of unmodified cortex, and are generally attributable of early production stages. Cortical flakes also generally, but do not necessarily, exhibit dorsal cortex beyond the platform. Flat striking platforms exhibit a smooth, un-faceted striking surface. Flakes detached from unidirectional cores generally exhibit flat platforms (Andrefsky 1998:94), although flakes with flat striking platforms may also be produced in the early stages of bifacial core reduction. Faceted striking platforms exhibit one or more facets, reflecting the removal of previous flakes from the same general area. Although researchers have had some success in determining manufacturing stage using facet counts (Mauldin and Amick 1989; McAnany 1988), time constraints and unresolved ambiguity in directly correlating facet count with manufacturing stage in an uncontrolled archeological sample undermine the desirability of including this finer resolution. A simple distinction between single-faceted platforms and multifaceted platforms is advocated in this protocol. Flakes that exhibit bifacial mass removal, often referred to as bifacial thinning flakes, are categorized as having complex platforms. Abraded platforms are those that exhibit attrition caused by purposeful edge preparation procedures. Such platforms are generally rounded or ground in appearance, and often exhibit multiple tiny step fractures. Marginal abrasion is a common practice for preparing a striking platform, and serves as a method of altering the direction of percussor force, which produces a more predictable flake removal (Andrefsky 1998:96; Whittaker 1994). Abraded platforms are produced in all phases of tool manufacture, but are more common in later stages of production. Finally, rejuvenated platforms reflect tool resharpening and

Version 2.3 
often display remnant use wear along a focal margin. Assemblages dominated by rejuvenated platforms indicate tool maintenance rather than tool production.

Indeterminate identifications generally result from poorly represented (fractured) or wholly absent platforms, or from poor resolution caused by heavy patina. To be clear, it is not desirable to record platform width and thickness or the number of facets present on the dorsal surface of flakes. These attributes are not efficiently recorded through mass analysis procedures, and the information they provide may be ascertained through other means, such as multivariate analysis incorporating the percentage of dorsal cortex present with flake size and platform type.

00. IND [indeterminate]

01. CRT [cortical] flakes with cortex observed on striking platforms are produced in the initial stage of package reduction.

02. FLA [flat] a single facet, caused by characterizes the striking platform.

03. FCT [faceted] two facets are observed on the platform. Assemblages dominated by flakes with double faceted platforms are generally produced in early stage blank production.

04. MLT [multifaceted] multiple facets are observed on the platform. Assemblages dominated by flakes with multifaceted platforms are generally produced through work on later stage preforms.

05. ABR [abraded] the platform exhibits ground margins

06. CMP [complex] complex platforms are bifacial.

07. REJ [rejuvenated] rejuvenated platforms are indicative of recycling and will typically exhibit worn edges and remnant polish.

08. MSG Missing 


\begin{tabular}{|c|c|c|}
\hline Platform Type & Platform Description & Illustration \\
\hline missing & $\begin{array}{l}\text { Flakes are defined as having missing striking platforms if they } \\
\text { are flake fragments or shatter that are missing the proximal } \\
\text { segment of the flake that includes the point of fracture initiation. }\end{array}$ & \\
\hline cortical & $\begin{array}{l}\text { Flakes with cortex observed on striking platforms are generally } \\
\text { produced in the initial stage of package reduction. }\end{array}$ & \\
\hline flat & $\begin{array}{l}\text { A single facet, caused by a single previous flake removal, } \\
\text { characterizes this type of striking platform. Flat (single faceted) } \\
\text { platforms are common in early stages of decortification, and are } \\
\text { common to blade manufacture when combined with notably } \\
\text { abraded edges. }\end{array}$ & \\
\hline dihedral-faceted & $\begin{array}{l}\text { A surface having or formed by two intersecting faces. } \\
\text { Assemblages dominated by flakes with double faceted } \\
\text { platforms are generally produced in early stage blank } \\
\text { production. }\end{array}$ & \\
\hline multifaceted & $\begin{array}{l}\text { Multiple facets are observed on the platform but only along one } \\
\text { face of the object. Light abrasion may or not be present. } \\
\text { Assemblages dominated by flakes with multifaceted platforms } \\
\text { are generally associated with later stages of biface reduction but } \\
\text { can also be present in early stage biface reduction. }\end{array}$ & \\
\hline abraded & $\begin{array}{l}\text { Abraded platform exhibit grinding that may obliterate facet } \\
\text { ridges and/or exhibit platform edge smoothing/rounding visible } \\
\text { to the naked eye or under low power magnification. Heavily } \\
\text { abraded platforms are often "dull" or smooth to the touch. } \\
\text { Abraded platforms serve to strengthen a platform edge } \\
\text { allowing for the application of greater force loads thus } \\
\text { increasing successful flake propagation and decreasing } \\
\text { platform failure by crushing or collapse. }\end{array}$ & \\
\hline complex & $\begin{array}{l}\text { Complex platforms exhibit pressure or light percussion scars on } \\
\text { the proximal-dorsal flake surface originating from the platform } \\
\text { edge. These scars are typically associated with precision } \\
\text { platform preparation (i.e., isolation and orientation) of late } \\
\text { stage bifaces where manufacturing failure rates increase } \\
\text { proportional to width to thickness ratios. }\end{array}$ & \\
\hline rejuvenated & $\begin{array}{l}\text { Rejuvenated platforms are indicative of recycling and will } \\
\text { typically exhibit worn edges and remnant polish. }\end{array}$ & \\
\hline indeterminate & $\begin{array}{l}\text { In some instances, the platform type will not be determinable } \\
\text { even when the striking area is present. This is typically caused } \\
\text { by crushing of the platform at the instant of production, or by }\end{array}$ & \\
\hline
\end{tabular}

Version 2.3 
post-depositional weathering.

Figure 18: 10-20x magnification (hand lens or loop) is recommended for viewing platforms on debitage in the $1 / 4$ $1 / 2$ inch size grades.

\section{Thermal Alteration}

Thermal alteration is used here to describe the process of purposefully subjecting lithic materials to a heat source as a means to affect raw material properties. Lithic raw materials typically undergo significant and detectable lithological changes with prolonged exposure to heat. Such changes are often desirable and may be deliberately generated by tool producers through controlled firing. Heat-treated materials may be more easily worked by the artisan, thus rendering low-quality materials more useful (albeit while making them more brittle and decreasing their durability).

An accurate assessment of thermal alteration is often inhibited by artifact size, patina formation, the production of comparable attributes through incidental fire exposure, and unfamiliarity on the part of the researcher with the lithological variability expressed by select raw materials in their natural state. The identification of heat-treated materials can bring culture process and the details of economic activity to the fore. For example, the presence of thermal alteration in combination with an assessment of platform type and cortex representation can indicated the trajectory stage at which the objective piece was heattreated. Nonetheless, it is frequently difficult to distinguish purposefully treated materials from those that were incidentally burned. Given the inherent difficulty with distinguishing between materials were purposefully heat-treated (cultural process) as opposed to fire-affected (incidental alteration resulting from both natural and cultural processes), debitage should be recorded as thermally altered, not altered, or indeterminate with regard to alteration conservatively and through incorporation of ancillary data. Identifying alteration on pieces with insufficient mass is unreliable, and so all small-sized debitage that is not minimally captured by a $1 / 2$-inch mesh sieve should be recorded as indeterminate. If a piece has been determined to be altered its context and association should be considered (e.g. if other artifact classes for the same context similarly burned the piece is more likely to have been incidentally fire affected).
00. IND [indeterminate]
01. AOB [thermal alteration observed]
02. ANO [thermal alteration not observed]

\section{Analytical Process}

By combining the above attributes into criteria lists and then recording the number and aggregate weight of flakes that fit a given set of criteria, this system allows for numerous unique attribute combinations for all debitage within a given provenience. This system works efficiently for large volumes of material and produces an easily queried database.

First, flakes from a given provenience should be sorted by raw material or individual package where it is obvious that the assemblage represents the reduction of separate material packages and such packages are distinctly identifiable. Uniquely identifiable raw material groupings will represent distinct analytical assemblages within each provenience. Next, for each separate package group, sort whole and proximal flakes by size within a given spatial context. Following this, inspect flakes within the 1-inch and 3/4-inch sieve size groups for evidence of use-wear (use-derived edge modification is unlikely to be reliably reflected on smaller flakes and they should not be evaluated for possible use). Remove utilized flakes for analysis under the chipped-stone tool protocol. Next, within each size group, sort flakes according to whether or not they are thermally altered (this step will not be performed for the two smallest size groups). Sort flakes within each alteration group (or size group if not

Version 2.3
Dated July 16, 2010 
separated by alteration) according to the amount of dorsal cortex that is present. From each of the cortex groups, sort flakes by platform type. Finally, count and record the total number of flakes in each of these final groupings and collectively weigh them in grams (round to the nearest gram) and record the MNN for the grouping.

Data derived from formal tool and debitage analyses are complementary. Each data set provides a more informed perspective on the other. Individually, however, each data set may make a distinct contribution with respect to illuminating a particular set of cultural processes and behaviors.

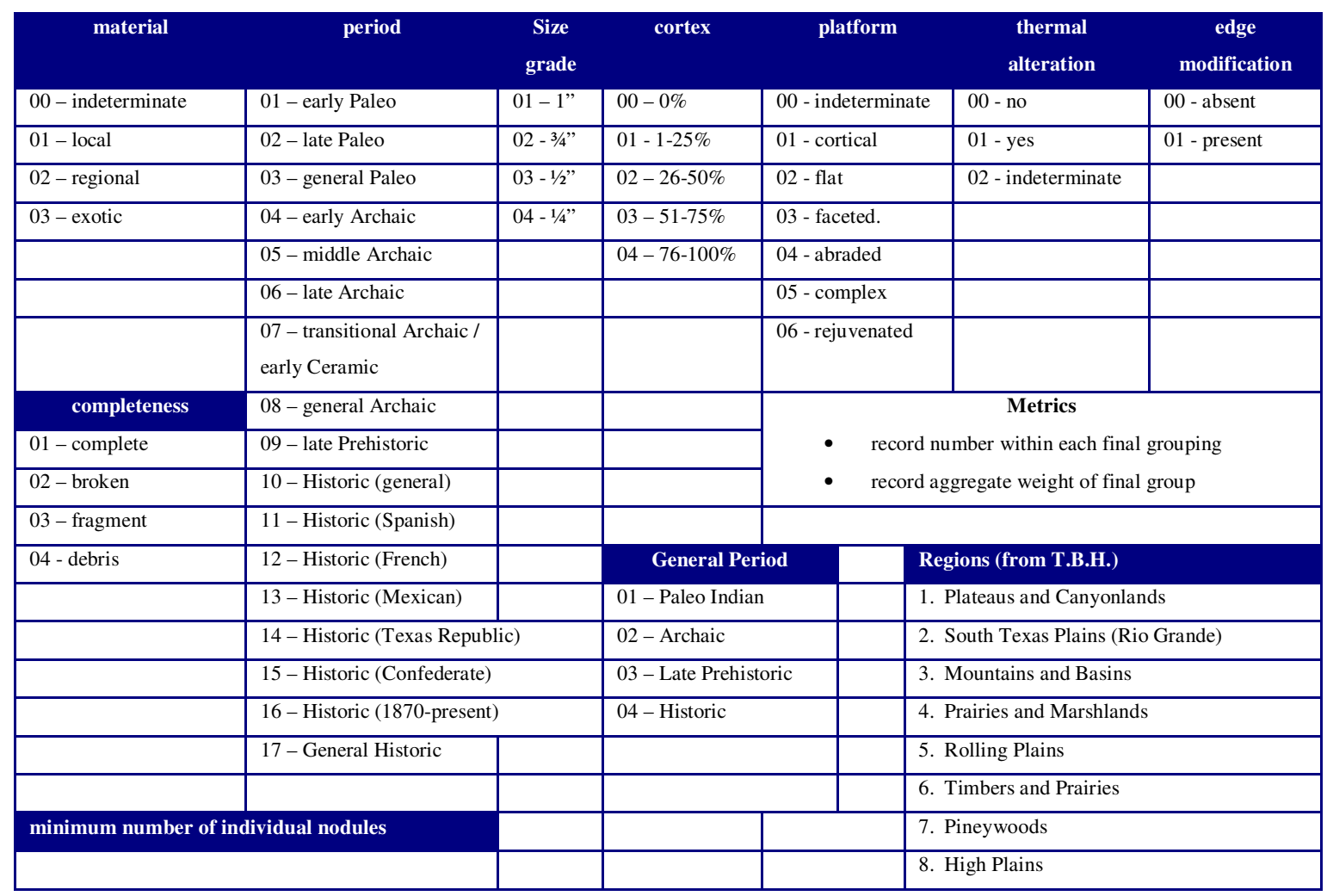

Version 2.3 
TAXONOMY

1. Technology:

2. Group:

3. Subgroup:

4. Class:

5. Subclass:

6. Type:

7. Identity:

\section{METRIC INFO / Measurements}

8. Max length (mm):

9. Max width (mm):

10. Max thickness (mm)

11. Weight (g):

12. Edge angle (working edge averaged to nearest $\left.5^{\circ}\right)$

\section{ATTRIBUTES \\ 13. Stage \\ 00 . Indeterminate \\ 01 . Initial reduction \\ 02 . Early stage forming \\ 03. Late stage perform \\ 04. Finished product \\ 05. Recycled}

14. Portion

00. Indeterminate
01. Complete
02. Distal
03. Distal-medial
04. Medial
05. Proximal-medial
06. Proximal
07. Lateral edges missing
08. Fragment
09. barb / shoulder
10. ear / tang
11. stem

15. Failure

00. Indeterminate

01. Snap / end shock

02. Impact/ bending

03. Perverse

04. Hinge / Step

05. Overshot (outrepasse)

06. Material flaw

07. Platform loss

08. Excessive heating

09. Exhausted

16. Alteration

00 . None observed

01. Thermal

02. White patina

03. Black patina

04. Oxide staining (yellowing)

05. Pigment staining

06. Carbonate build-up

17. Edge Morphology $[17 \mathrm{a}=\mathrm{L} ; 17 \mathrm{~b}=\mathrm{R}]$ 00 . Indeterminate

01. Concave

02. Convex

03. Recurved

04. Serrated

18. Flake Scar Pattern

00. Indeterminate

01. Collateral

02. Horizontal transverse

03. Oblique transverse

04. Random

99. Not Applicable

Version 2.3
19. Edge Construction Type

00 . Indeterminate

01. Bifacial - distal

02. Bifacial - bilateral

03. Bifacial - unilateral

04. Bifacial - distal - bilateral

05. Bifacial - distal - unilatera

06. Bifacial - circumferential

07. Unifacial - distal

08. Unif - bilat - conforming

09. Unif - bilateral - opposing

10. Unifacial - unilateral

11. Unif - distal - bilateral-conform

12. Unif - distal - bilateral-opp

13. Unifacial - distal - unilateral

14. Unifacial - circumferential

15. Other

99. Not applicable

20. Proximal edge grinding

$\square$ Not observed

$\square$ Observed

WEAR PATTERNING (macroscopic)

21. Flaking

00. Not present

01. Bifacial - distal

02. Bifacial - bilateral

03. Bifacial - unilateral

04. Bifacial - distal - bilateral

05. Bifacial - distal - unilatera

06. Bifacial - circumferential

07. Unifacial - distal

08. Unifacial - bilateral - conform

09. Unifacial - bilateral - opposing

10. Unifacial - unilateral

11. Unif - distal - bilateral-conform

12. Unif - distal - bilateral-opposing

13. Unifacial - distal - bilateral

14. Unifacial - distal - unilateral

15. Unifacial - circumferential

16. Other

22. Crushing 24. Smoothing

00. Not Present

01. Distal

02. Distal - lateral

03. Unilateral

04. Bilateral

05. Facial smoothing

06. Facet smoothing

07. Circumferential

08. Primary proximal

09. Secondary proximal

25. Polish

00. Not present

01. Shallow distal $(<5 \mathrm{~mm})$

02. Deep distal $(>5 \mathrm{~mm})$

03. Shallow lateral $(<5 \mathrm{~mm})$

4. Deep lateral $(>5 \mathrm{~mm})$

05 . Unifacial medial

06. Bifacial medial

07. Bipolar

08. Proximal
26. Etching / Pitting

00. Not present

01 . Shallow distal $(<5 \mathrm{~mm})$

02. Deep distal $(>5 \mathrm{~mm})$

03. Shallow lateral $(<5 \mathrm{~mm})$

04 . Deep lateral $(>5 \mathrm{~mm})$

05 . Unifacial medial

06. Distal medial

07. Circumferential

08. Medial bifacial

09. Bipolar

27. Hafting evidence

$$
\square \text { Not observed }
$$

$\square$ Observed

$\square$ Not Applicable

\section{RAW MATERIAL}

28. Lithology

01. Unidentified Silex

02. Microcrystalline Quartz

03. Macrocrystalline Quartz

04. Chalcedony

05. Jasper

06. Chert

07. Chert-Chalcedony Blend

08. Dolomite

09. Agatized dolomite

10. Fossilized Wood

11. Limestone

12. Silicified Limestone

13. Ortho-Quartzite

14. Meta-Quartzite

15. Novaculite

16. Rhyolite

17. Basalt

18. Serpentine / Greenstone

19. Steatite (soap stone)

20. Granite

21. Marble

22. Gneiss

23. Schist

24. Silt-stone

25. Obsidian

26. Manning Fused Glas

27. Ironized sandstone

96. Unidentified Sedimentary

97. Unidentified Igneous

98. Unidentified Metamorphic

99. Other

29. Major Sources

00. Unidentifiable

01. Alibates (Llano Estacado)

02. Antlers Formation

03. Burro Mesa (Trans-Pecos)

04. Bexar County chert

05. Callahan Divide

06. Caballos Mountain

07. Central Mineral Region (Llano Uplift)

08. Georgetown Cherts

09. Edwards Chert

10. Manning Fused Glass

11. Markely Conglomerate

12. Pisgah Ridge

13. Rio Grande Gravels

14. Tecovas Formation (cherts and jaspers)

15. Yegua Gravels (quartzite and petrified wood)

16. Uvalde Gravels

17. Catahoula

97. Unidentified local

98. Unidentified exotic

99. Other 


\section{Questions for Middle-Level and High-Level Theory Using Debitage Data}

* Many of these questions can be best or only answered with respect to complementary data from other material classes.

\section{QUESTIONS}

1. What is being produced? (biface, blades, expedient flakes, points, etc... may be identified by class or type).

2. What techniques were used in its production? (bifacial reduction, prismatic core, bipolar reduction, channel flaking, etc...).

3. What part of the production process is represented by the available assemblage? (stage in trajectory).

4. What function was the objective piece meant to serve? (this deduction is generally only possible when waste can be related to finished products with observable use-wear, or production failures of known function)

5. How many were being made? (flake-to-tool ratios are inherently spurious without diagnostic flakes and researchers should be cautious when addressing this issue; raw material type differences may be valuable in establishing a minimum number objects produced)

6. Who was making it? (age, gender, and social status are typically central to this issue, and the question may be best addressed -if it is indeed possible to do so- with respect to the context and composition of deposit, and its association with identified activity areas)

7. How many people were involved in creating the assemblage and what was their relationship? (this assumes that the waste actually has the meaning that we assign to it and that variation in flaking is not the result of an ancillary feature in the manufacturing process such as raw material type and quality)

8. Is this where the constituent components of the assemblage originally entered the archeological record? (the integrity of deposit should be considered with respect to natural and cultural transformation processes, including disturbances and patterns of refuse disposal)

9. Was the product for immediate use? (consider degree of material curation, production stage, environmental setting, and degree of expediency in tool design)

10. Was the material easy to come by? (consider the local availability of the raw material, as well as the degree of material curation and conservation observed in the relative percentage of use-wear observed on flakes, and the degree to which tools are recycled)

11. Was the raw material easy to use? (this assessment of raw material quality may be addressed through error rates and thermal alteration, but also in consideration of the amount of material mass that remains when expended tools are discarded) 


\section{References Cited}

Ahler, S. A.

1988 Mass Analysis of Flaking Debris: Studying the Forest Rather than the Tree. In Alternative Approaches to Lithic Analysis, edited by D. O. Henry and G. H. Odell, pp. 85-118. Archaeological Papers, Vol. 1. American Anthropological

Association, Washington, DC.

1989 Experimental Knapping with KRF and Midcontinent Cherts: Overview and Applications. In Experiments in Lithic Technology, edited by D. S. Amick and R. P. Mauldin, pp. 199-234. Vol. 528. British Archaeological Reports International

Series 528. Oxford.

Andrefsky, W.

1998 Lithics: Macroscopic Approaches to Analysis. Cambridge Manuals in Archeology. Cambridge University Press, Cambridge, England.

Aoyama, K.

1999 Ancient Maya State, Urbanism, Exchange, and Craft Specialization: Chipped Stone Evidence from the Copán Valley and the La Entrada Region, Honduras. University of Pittsburgh Memoirs in Latin American Archaeology, No. 12. University of Pittsburgh Dept. of Anthropology, Pittsburgh, PA.

Banks, L. D.

1990 From Mountain Peaks to Alligator Stomachs: A Review of Lithic Sources in the Trans-Mississippi South, the Southern Plains, and Adjacent Southwest. Oklahoma Anthropological Society, Memoir No. 4.

Baumler, M. F. and C. E. Downum

1987 Between Micro and Macro: A Study in the Interpretation of Small-sized Lithic Debitage. In Experiments in Lithic Technology, edited by D. S. Amick and R. P. Mauldin, pp. 101-116. British Archaeological Reports International Series 528. Oxford.

Behm, J. A.

1983 Flake Concentrations: Distinguishing Between Flintworking Areas and Secondary Deposits. Lithic Technology 12(1):9-16.

Black, S., K. Miller, G. Goode, L. Ellis, S. Decker, and E. Prewitt

1997 Appendix C: Artifact Descriptions. In Hot Rock Cooking on the Greater Edwards Plateau: Four Burned Rock Midden Sites in West Central Texas, Volume 2, edited by S. Black, L. Ellis, D. Creel, and G. Goode, pp. 443-570. Studies in Archeology 22, Texas Archeological Research Laboratory, The University of Texas at Austin. Report 2, TxDOT ENV Archeological Studies Program.

S. L. Black, L. W. Ellis, D. G. Creel, and G. T. Goode

1997 Hot Rock Cooking on the Greater Edwards Plateau: Four Burned Rock Midden Sites in West Central Texas. 2 vols. Studies in Archeology 22, Texas Archeological Research Laboratory, The University of Texas at Austin, and Archeology Studies Program, Report 2, Environmental Affairs Division, Texas Department of Transportation, Austin.

Bradbury, A. P. and J. D. Franklin

2000 Raw Material Variability, Package Size, and Mass Analysis. Lithic Technology 25(1):42-58.

Clark, J. E.

1988 The Lithic Artifacts of La Libertad, Chiapas, Mexico: An Economic Perspective. Papers of the New World Archaeological Foundation, 52. Brigham Young University, Provo, UT.

Collins, M. B.

1975 Lithic Technology as a Means of Processual Inference. In Lithic Technology: Making and Using Stone Tools, edited by E. H. Swanson, pp. 15-34. Mouton Publishers, The Hague, The Netherlands.

Cotterell, B., and J. Kamminga

1979 The Mechanics of Flaking. In Lithic Usewear Analysis, edited by B. Hayden, pp. 97-112. Academic Press, New York.

Crabtree, D. E.

1966 A Stoneworker's Approach to Analyzing and Replicating the Lindenmeire Folsome. Tebiwa 9(1):3-39.

1972a The Cone Fracture Principle and the Manufacture of Lithic Materials. Tebiwa 15(2):29-42.

Version 2.3

Dated July 16, 2010 
1972b An Introduction to Flintworking. Occasional Papers of the Idaho State University Museum, Pocatello, ID. Fullagar, R. L. K.

1991 The Role of Silica in Polish Formation. Journal of Archaeological Science 18(1):1-24.

Goode, G.

2002 The Anthon Site: A Prehistoric Encampment in Southern Uvalde County, Texas. Report 8, TxDOT ENV Archeological Studies Program.

Grace, R.

1996 Use-wear Analysis: The State of the Art. Archaeometry 38(2):209-229.

Johnson, J. K.

1989 The Utility of Production Trajectory Modeling as a Framework for Regional Analysis. In Alternative Approaches to Lithic

Analysis, edited by D. O. Henry and G. H. Odell, pp. 119-138. Archaeological Papers, Vol. 1. American Anthropological Association, Washington, DC.

Keeley, L. H.

1977 An Experimental Study of Microwear Traces on Selected British Paleolithic Implements. Unpublished Ph.D. dissertation, School of Archaeology, Oxford University.

1980 Experimental Determination of Stone Tool Uses: A Microwear Analysis. Prehistoric Archeology and Ecology. University of Chicago Press, Chicago.

Lewenstein, S. M. and J. Walker

1984 Obsidian Chip / Manioc Grating Hypothesis. Journal of New World Archaeology 6(2):25-38.

Magne, M. P. R.

1989 Lithic Reduction Stages and Assemblage Formation Processes. In Experiments in Lithic Technology, edited by D. S. Amick and R. P. Mauldin, pp. 15-32. British Archaeological Reports International Series 528. Oxford.

Mauldin, R. P. and D. S. Amick

1989 Investigating Patterning in Debitage from Experimental Bifacial Core Reduction. In Experiments in Lithic Technology, edited by D. S. Amick and R. P. Mauldin, pp. 67-88. British Archaeological Reports International Series 528. Oxford.

McAnany, P. A.

1988 The Effects of Lithic Procurement Strategies on Tool Curation and Recycling. Lithic Technology 17(1):3-11.

McBrearty, S., L. Bishop, T. Plummer, R. Dewar and N. Conard

1998 Tools Underfoot: Human Trampling as an Agent of Lithic Artifact Edge Modification. American Antiquity 63(1):108-129.

Morrow, T.

1994 A Key to the Identification of Chipped-Stone Raw Materials Found on Archaeological Sites in Iowa. Journal of the Iowa Archeological Society 41: 108-129.

Odell, G. H.

2001 Stone Tool Research at the End of the Millenium: Classification, Function, and Behavior. Journal of Archaeological Research 9(1):45-100.

Odell, G. H. and F. Odell-Vereecken 1980 Verifying the Reliability of Lithic Use-Wear Assessments by 'Blind Tests': The Low-Power Approach. Journal of Field Archaeology 7(1):87-120.

Patterson, P.

1977 A Lithic Reduction Sequence: A Test Case in the North Fork Reservoir Area, Williamson County, Texas. Bulletin of the Texas Archeological Society 48: 44-54.

Schiffer, M.

1972 Archaeological Context and Systemic Context. American Antiquity 37: 156-165.

Version 2.3 
Semenov, S.

1964 Prehistoric Technology trans. M. Thompson. Cory, Adams, and MacKay, London.

Shafer, H. J.

1983 The Lithic Artifacts of the Pulltrouser Area: Settlements and Fields. In Pulltrouser Swamp: Ancient Maya Habitat, Agriculture, and Settlement in Northern Belize, edited by P. D. Harrison and B. L. Turner, pp. 212-245. University of Texas Press, Austin.

1985 A Technological Study of Two Maya Workshops at Colhá, Belize. In Stone Tool Analysis: Essays in Honor of Don E. Crabtree, edited by M. G. Plew, J. C. Woods and M. G. Pavesic, pp. 277-315. University of New Mexico Press, Albuquerque.

Shea, J. J., and Klenck, J. D.

1993 An Experimental Investigation of the Effects of Trampling on the Results of Lithic Microwear Analysis. Journal of Archaeological Science 20(2):175-194.

Sollberger, J. B.

1977 Fracture Theory, Applied to Flaking Forces and Lithic Analysis. Paper presented at the Conference for Use-Wear, Simon Fraser University, Burnaby, British Columbia, Canada.

Sullivan, A. P. and K. C. Rozen

1985 Debitage Analysis and Archaeological Interpretation. American Antiquity 50(4):755-779.

Tringham, R., G. Cooper, G. H. Odell, B. Voytek and A. Witman

1974 Experimentation in the Formation of Edge Damage: A New Approach to Lithic Analysis. Journal of Field Archaeology 1(1/2):171-196.

Tsirk, A.

1979 Regarding Fracture Initiations. In Lithic Use-wear Analysis, edited by B. Hayden, pp. 83-96. Academic Press, New York.

Turner, E and T. Hester

1999 A Field Guide to Stone Artifacts of the Texas Indians. Gulf Publishing, New York.

Whittaker, J. C.

1994 Flintknapping: Making and Understanding Stone Tools. 1st ed., University of Texas Press, Austin. 



\title{
APPENDIX D: Lithic Analysis Protocol Blank Data Entry Form for Chipped Stone Tools
}

\author{
Digital Only
}

For the Texas Department of Transportation, Archeological Studies Program's

Chipped Stone Analytical Protocol, Version 2.3,

July 16, 2010

This appendix contains an electronic copy of the data entry form for chipped stone tools that follows the Chipped Stone Analytical Protocol, Version 2.3 (July 16,2010), developed by the Archeological Studies Program, Texas Department of Transportation. The form follows the original protocol design closely, although it does incorporate some minor changes that were needed for clarity. These changes (added categories) are highlighted in red at the top of the spreadsheet.

The data entry form is a spreadsheet in Microsoft Excel format. In order to standardize entries for data compatibility, all TEXT variables used in the data entry form must be chosen from the dropdown lists that are linked to the "Drop Down List" worksheet. If any of these links are broken, the data entry form will not work properly.

While some of the data entry variables, such as "site number," are self- explanatory, others need some type of instructional comments. Consequently, the blank data entry form also includes one row that has user instructions for each variable. 



\section{APPENDIX E: Lithic Analysis Protocol Blank Data Entry Form for Unmodified Debitage and Cores}

\section{Digital Only}

For the Texas Department of Transportation, Archeological Studies Program's

Chipped Stone Analytical Protocol, Version 2.3,

July 16, 2010

This appendix contains an electronic copy of the data entry form for unmodified debitage and cores that follows the Chipped Stone Analytical Protocol, Version 2.3 (July 16, 2010), developed by the Archeological Studies Program, Texas Department of Transportation. The form follows the original protocol design closely, although it does incorporate some minor changes that were needed for clarity.

The data entry form is a spreadsheet in Microsoft Excel format. In order to standardize entries for data compatibility, all TEXT variables used in the data entry form must be chosen from the dropdown lists that are linked to the "Drop Down List" worksheet. If any of these links are broken, the data entry form will not work properly.

While some of the data entry variables, such as "site number," are self- explanatory, some type of instructional comments are need for many of the variables. Consequently, the blank data entry form also includes one row that has user instructions for each variable. 



\section{APPENDIX F: Lithic Analysis Protocol Form for Chipped Stone Tools from 41CV1378}

\section{Digital Only}

For the Texas Department of Transportation, Archeological Studies Program's

Chipped Stone Analytical Protocol, Version 2.3,

July 16, 2010

This appendix contains an electronic copy of the completed data entry form for the analysis of the chipped stone tools recovered from 41CV1378. It follows the Chipped Stone Analytical Protocol, Version 2.3 (July 16, 2010), developed by the Archeological Studies Program, Texas Department of Transportation, and modified by Prewitt and Associates, Inc. 



\section{APPENDIX G: Lithic Analysis Protocol Form for Unmodified Debitage and Cores from 41CV1378}

\section{Digital Only}

For the Texas Department of Transportation, Archeological Studies Program's

Chipped Stone Analytical Protocol, Version 2.3,

July 16, 2010

This appendix contains an electronic copy of the completed data entry form for the analysis of the unmodified debitage and cores recovered at 41CV1378. It follows the Chipped Stone Analytical Protocol, Version 2.3 (July 16, 2010), developed by the Archeological Studies Program, Texas Department of Transportation, and modified by Prewitt and Associates, Inc. 



\section{APPENDIX H: Burned Rock Analysis Data}

for $41 \mathrm{CV} 1378$ 

The primary focus of the Prewitt and Associates, Inc. (PAI) archeological investigations at 41CV1378 was on the large burned rock mound and the smaller burned rock features within and around it. Not surprisingly, the most common cultural materials encountered were burned limestone rocks. The burned rocks are important objects for understanding the hot rock cooking involving earth ovens and formation of the large waste piles described as burned rock mounds and middens. This appendix summarizes the data acquisition and analytical processes for examining the burned rocks, and presents both raw and calculated data in tabulated formats. Interpretations of these data are presented elsewhere in this report (see Chapter 7).

\section{COLLECTION OF BURNED ROCKS AND BURNED ROCK DATA}

Five types of burned rock data were obtained during the data recovery investigations. The bulk of the burned rocks were encountered in the general excavations, and for each unit the burned rocks were examined, quantified, and discarded in the field. For each provenience, the burned rocks were sorted into four size classes, and the burned rocks for each group were weighed. In addition, burned rocks were recovered from four types of samples collected in the field. Burned rocks were recovered in Flotation samples (sediment samples taken for flotation and recovery of macrobotanical remains) and as Matrix Column samples (called Soil Columns in the field). The latter consisted of five $30 \times 30$ $\mathrm{cm}$ columns where everything was collected to provide data on the fine-grained (sediment) and course-grained (inclusions and burned rocks) components of the burned rock mound matrix following recommendations by Black and Ellis (1997). Finally, individual burned rocks were mapped and collected in the field as Burned Rock samples (for possible extraction of organic remains and radiocarbon dating or microfossil analysis) and as Thermoluminesence samples (for possible TL dating). Table H.1 summarizes of all the burned rocks and sediment associated with the general collections and four types of samples. The raw analytical data for the burned rocks are presented in Tables H.2 to H.4. Table H.2 presents the burned rocks weights for the general excavations, with the data separated into the four size classes and linked to unit numbers and associated features. Table H.3 presents the burned rock weights for the collected samples, with the data separated into the four size classes and linked to unit numbers and associated features. Table H.4 presents the burned rock weights for all the sample contexts, with the data separated into the four size classes and linked to unit numbers and cultural features.

Table H.1. Summary of burned rock and sediment sample data for $41 \mathrm{CV} 1378$

\begin{tabular}{|l|c|c|c|c|}
\hline Provenience & $\begin{array}{c}\text { No. of } \\
\text { Samples } \\
\text { Collected }\end{array}$ & $\begin{array}{c}\text { Total Burned } \\
\text { Rock Weight (kg) }\end{array}$ & $\begin{array}{c}\text { Total Sediment } \\
\text { Volume } \\
\text { (liters) }\end{array}$ & $\begin{array}{c}\text { Total Sediment } \\
\text { Weight } \\
(\mathrm{kg})\end{array}$ \\
\hline $\begin{array}{l}\text { General Excavation } \\
\text { (burned rocks documented in the field and } \\
\text { discarded) }\end{array}$ & -- & $5,353.8$ & -- & -- \\
\hline $\begin{array}{l}\text { Matrix Column Samples } \\
\text { (designated as SC samples; called soil column } \\
\text { in the field) }\end{array}$ & 26 & 226.6 & 121.0 & 107.4 \\
\hline $\begin{array}{l}\text { Flotation Samples } \\
\text { (designated as F samples) }\end{array}$ & 22 & 103.6 & 296.5 & -- \\
\hline $\begin{array}{l}\text { Burned Rock Samples } \\
\text { (designated as BR samples) }\end{array}$ & 36 & 26.7 & & -- \\
\hline $\begin{array}{l}\text { Thermoluminescence Samples } \\
\text { (designated as TL samples) }\end{array}$ & 14 & 18.9 & -- & - \\
\hline
\end{tabular}


Data Recovery Investigations at the Tank Destroyer Site, Fort Hood

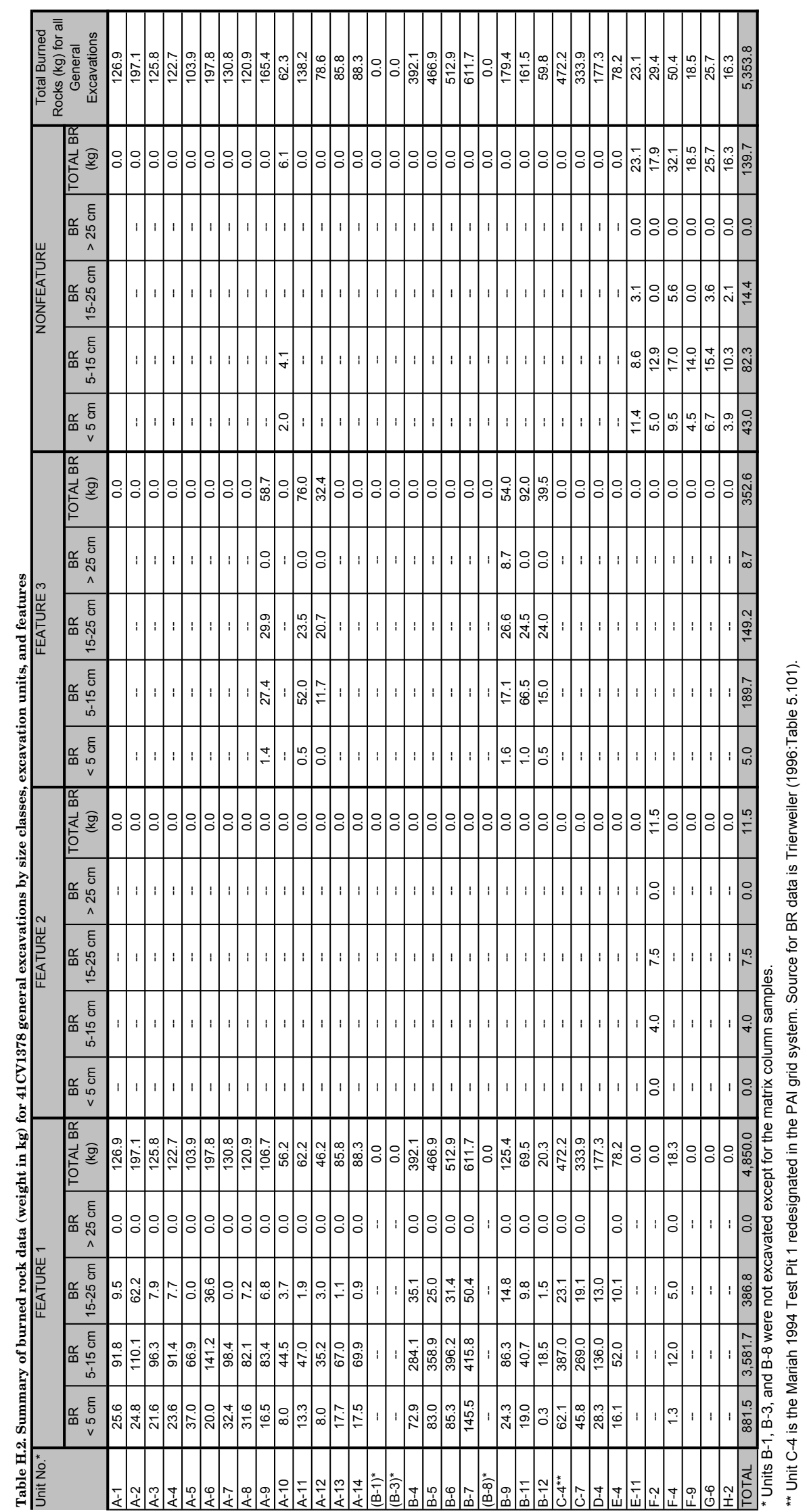




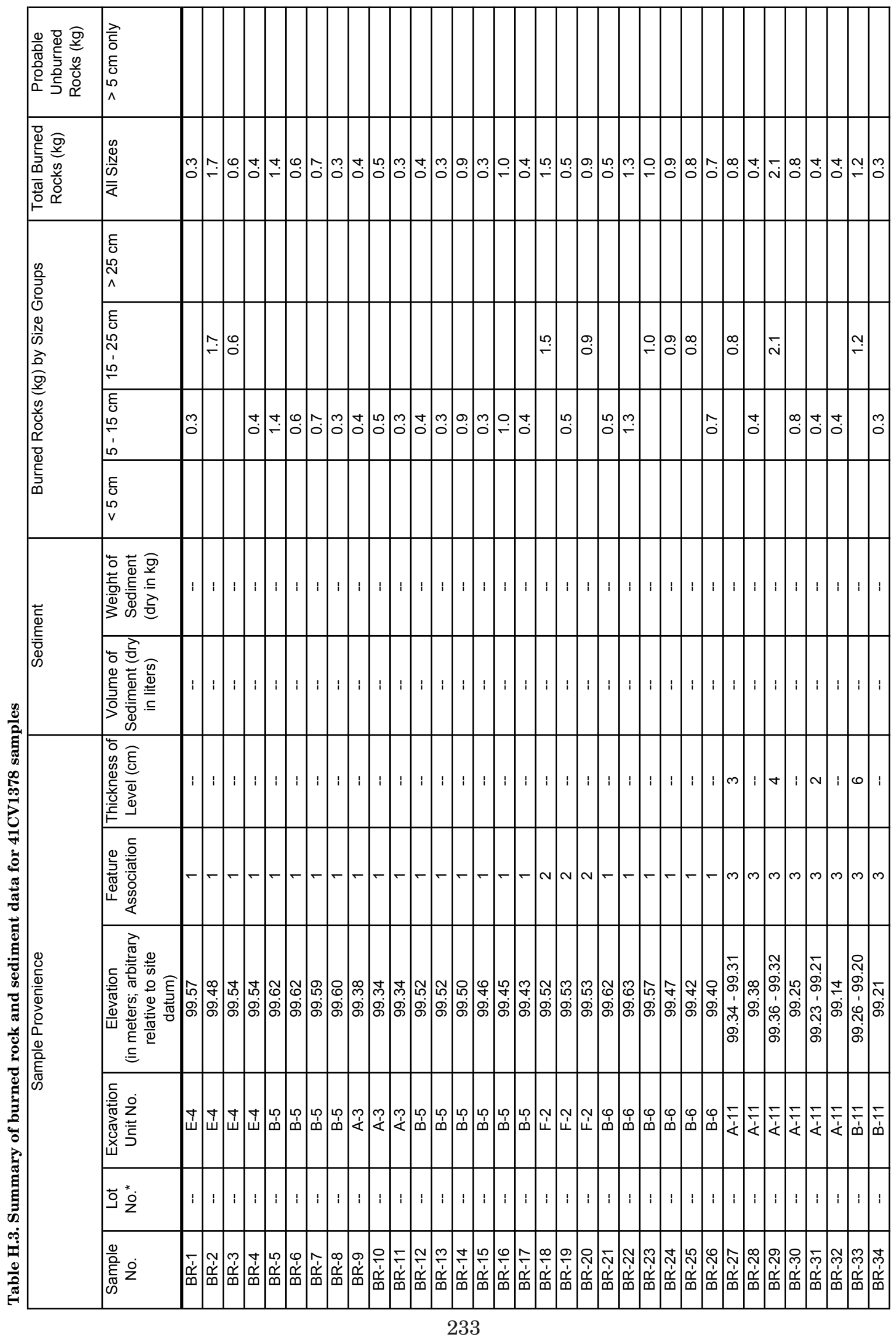




\begin{tabular}{|c|c|c|c|c|c|c|c|c|c|c|c|c|c|c|c|c|c|c|c|c|c|c|c|c|c|c|c|c|}
\hline 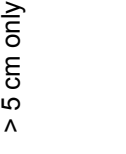 & & 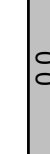 & & & & & & & & & & & & & & & & & & & $\begin{array}{l}m \\
0 \\
0\end{array}$ & $\stackrel{n}{\square}$ & & & & & & \\
\hline 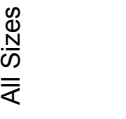 & 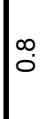 & & & 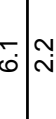 & & $\hat{N}$ & \begin{tabular}{l|l} 
\\
ल)
\end{tabular} & $\stackrel{\circ}{\infty}$ & $\widehat{\vec{f}}$ & & Sُ| & $\begin{array}{l}\infty \\
\infty \\
\infty\end{array}$ & $\stackrel{\sim}{\stackrel{\sim}{\leftarrow}}$ & 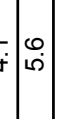 & $\stackrel{\sim}{\sim}$ & & 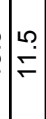 & $\stackrel{\leftrightarrow}{\hookrightarrow}$ & & 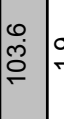 & 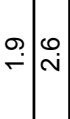 & $\begin{array}{lll}0 & 0 \\
\dot{\sigma} & 0 & \\
& & \\
\end{array}$ & & 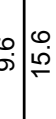 & \begin{tabular}{|l|}
$\hat{\circ}$ \\
$\stackrel{\circ}{*}$
\end{tabular} & 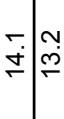 & & $\stackrel{m}{m}$ \\
\hline 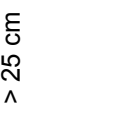 & & c & & & & & & & & & & & & & & & & & & ${ }_{0}^{\circ}$ & & & & & & & & \\
\hline 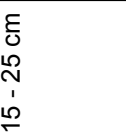 & 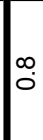 & : & & & & & & & & & & & & & & & & & & ${ }_{0}^{0}$ & & $\mid$\begin{tabular}{c|c}
$\sim$ \\
\hdashline \\
\end{tabular} & & $\stackrel{\circ}{\circ}$ & & 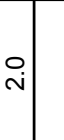 & & | \\
\hline 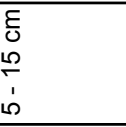 & . & 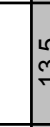 & 巳 & & & & & & & & & & & & & & & & & & 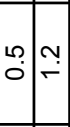 & 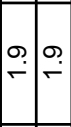 & $\because \underset{0}{\circ}$ & 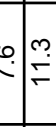 & & 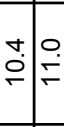 & & O \\
\hline $\begin{array}{l}E \\
\begin{array}{c}E \\
0 \\
D \\
v\end{array}\end{array}$ & & c & s. & $\dot{i} \underset{\sim}{ }$ & & $\hat{N}$ & 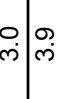 & $\stackrel{\leftrightarrow}{\infty}$ & $\widehat{\vec{f}}$ & & ڤ̊: & $\mid \begin{array}{l}\infty \\
\infty \\
i\end{array}$ & 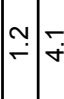 & 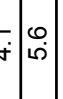 & $\stackrel{\sim}{\wedge}$ & • & 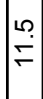 & $\stackrel{m}{m}$ & & . & $\stackrel{\square}{-} \because$ & $\circ: 0 ?$ & 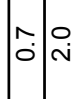 & 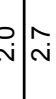 & $\stackrel{\sim}{\circ}$ & 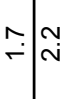 & & 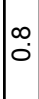 \\
\hline 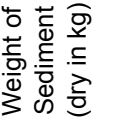 & 1 & 1 & & & 1 & 1 & $1: 1$ & 1 & $:$ & : & $1: 1$ & 1 & $\begin{array}{llll} & 1 & :\end{array}$ & : $: 1$ & 1 & $1:$ & 1 & $: 1$ & & $\begin{array}{lll}0 & 1 \\
\dot{0} & 0\end{array}$ & 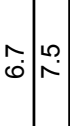 & $\mid$\begin{tabular}{l|l}
0 & 0 \\
$\infty$ & $\infty$ \\
\end{tabular} & $\stackrel{m}{\stackrel{m}{\infty}} \underset{\infty}{\infty}$ & $\begin{array}{c}\sigma \\
\text { S. }\end{array}$ & $\hat{\dot{r}}$ & 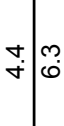 & $\hat{\mathrm{N}}$ & 官. \\
\hline 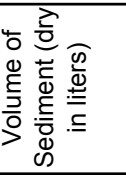 & i & $:$ & & 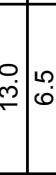 & Oे & : & 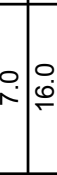 & : & $\mid \begin{array}{l}0 \\
\stackrel{\rho}{\vec{j}}\end{array}$ & & \begin{tabular}{l|l} 
\\
\end{tabular} & $\stackrel{\mathrm{O}}{\mathrm{i}}$ & 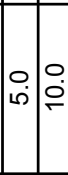 & 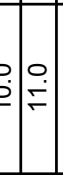 & $\left|\begin{array}{l}0 \\
\infty \\
0\end{array}\right|$ & 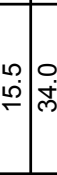 & $\left|\begin{array}{l}\mid \\
0 \\
0 \\
\sigma\end{array}\right|$ & 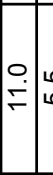 & & 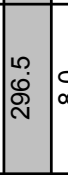 & \begin{tabular}{l|l}
0 & $\infty$ \\
$\infty$ & $\infty$ \\
\end{tabular} & 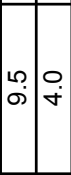 & 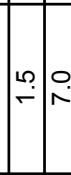 & 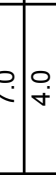 & $\stackrel{m}{\stackrel{\rho}{\dot{f}}}$ & $\stackrel{m}{\rightarrow} \underset{\sim}{\stackrel{\rho}{r}}$ & : & $\stackrel{\circ}{\circ}$ \\
\hline 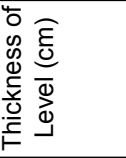 & 0 & & & $\forall \nabla$ & & $\div$ & $\forall \stackrel{\infty}{\square}$ & $2 \stackrel{2}{2}$ & $\circ$ & $?$ & & $\wedge$ & $\circ 0$ & $\$ \infty$ & 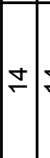 & 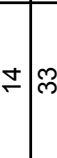 & $\bar{m}$ & 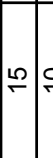 & $=F$ & & $\infty \div$ & $\div 0$ is & $\checkmark P$ & 이은 & 우 & 웅으 & & $\cong$ \\
\hline 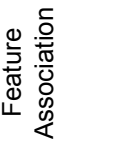 & $m$ & & & $-r$ & & -1 & r & - & - & & & - & & -1 & & $m$ & & $m$ & $0 \mid-$ & & & & & & $1-1$ & & & - \\
\hline 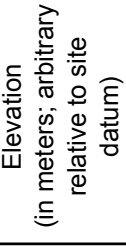 & 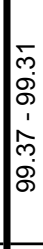 & 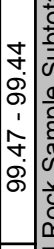 & है & 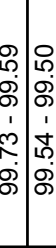 & 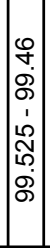 & 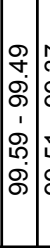 & 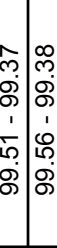 & 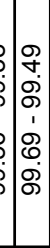 & 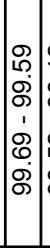 & 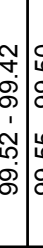 & 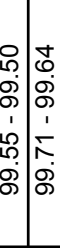 & 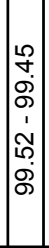 & 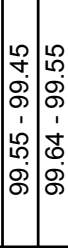 & 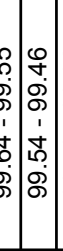 & 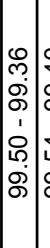 & 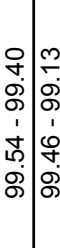 & 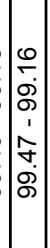 & 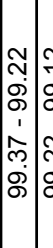 & 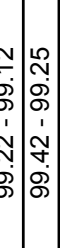 & 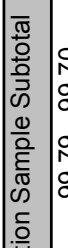 & 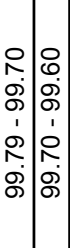 & 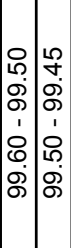 & 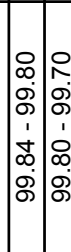 & 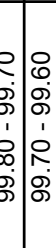 & $\begin{array}{l}0 \\
1 \\
0 \\
8 \\
1 \\
\vdots \\
0 \\
\dot{8} \\
8\end{array}$ & 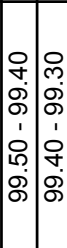 & 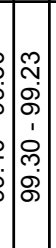 & 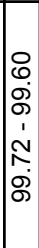 \\
\hline 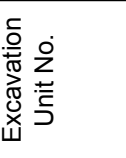 & $\bar{c}_{\bar{c}}$ & 1 & 总 & $\dot{\infty}$ & $\begin{array}{l}\checkmark \\
\oplus \\
\oplus\end{array}$ & 崫 & 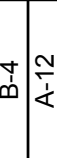 & Dे & $\left|\begin{array}{l}0 \\
\infty \\
\infty\end{array}\right|$ & & فُ & $\mid \begin{array}{l}\infty \\
\infty \\
\infty\end{array}$ & 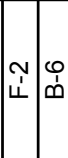 & 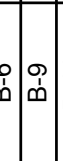 & $\overline{\dot{\omega}}$ & 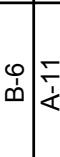 & $\overline{\dot{\omega}}$ & $\frac{1}{\dot{T}}$ & $\frac{1}{\frac{1}{d}} \frac{\dot{x}}{4}$ & $\frac{\pi}{4}$ & 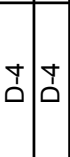 & ดे & 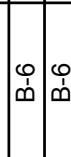 & 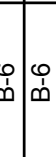 & $\left|\begin{array}{l}0 \\
\dot{\omega}\end{array}\right|$ & \begin{tabular}{|lll}
0 & 0 \\
$\dot{\omega}$ & $\dot{1}$
\end{tabular} & & $\bar{\omega}$ \\
\hline${ }^{\circ} \stackrel{*}{\circ}$ & & : & & 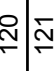 & $\stackrel{\mathbb{N}}{\simeq}$ & 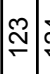 & 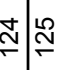 & 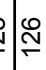 & $\stackrel{\mathbb{N}}{\stackrel{N}{*}}$ & $\stackrel{\infty}{\text { N }}$ & 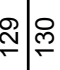 & $\bar{m}$ & $\stackrel{m}{=} \underset{\sim}{\stackrel{m}{m}}$ & $\begin{array}{lll}n \\
?\end{array}$ & 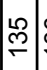 & 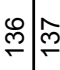 & 㐫 & $\stackrel{m}{=}$ & $\underset{f}{\dot{f}}$ & & 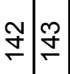 & $\underset{\forall}{I}$ & 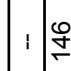 & 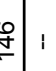 & $\hat{f}$ & $1: \underset{f}{\infty}$ & & 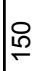 \\
\hline 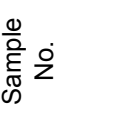 & 1 & ஜ & & 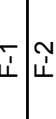 & $m$ & +1 & 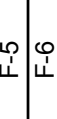 & C & $\infty$ & & $\begin{array}{l}0 \\
\dot{L} \\
\dot{L}\end{array}$ & $\cong$ & \begin{tabular}{|l|l}
$\dot{m}$ & $\overrightarrow{\dot{L}}$ \\
$\dot{\vec{L}}$
\end{tabular} & 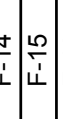 & $\mid$ & 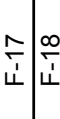 & $\stackrel{0}{-}$ & ని & $\underset{\substack{i\\
}}{\mathbb{N}}$ & & 足 & 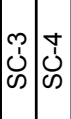 & \begin{tabular}{l|l} 
& \\
& 0 \\
0 & 0 \\
0 & 0 \\
$\infty$ & 0 \\
$\infty$
\end{tabular} & 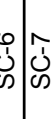 & $\left|\begin{array}{l}\infty \\
0 \\
0 \\
\infty\end{array}\right|$ & 焉 & 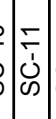 & ; \\
\hline
\end{tabular}


Appendix H: Burned Rock Analysis Data

\begin{tabular}{|c|c|c|c|c|c|c|c|c|c|c|c|c|c|c|c|c|c|c|c|c|c|c|c|c|c|}
\hline 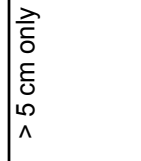 & & 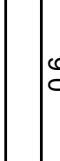 & : & & & & & & & & $\stackrel{L}{\sim}$ & & & & & & & & & & & & & $\stackrel{\sim}{\sim}$ & \\
\hline 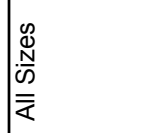 & $\stackrel{\hat{\varepsilon}}{F}$ & 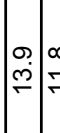 & & ल) & & & & $\begin{array}{l}\infty \\
0 \\
0\end{array}$ & & $\stackrel{?}{\stackrel{\leftrightarrow}{\rightleftarrows}}$ & 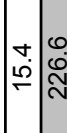 & $\stackrel{\square}{\stackrel{\square}{*}}$ & & $\stackrel{0}{-}$ & 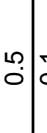 & & $\stackrel{+}{\rightarrow}$ & $\stackrel{\sim}{-}$ & & & & : & $\stackrel{\substack{\infty \\
\infty}}{\infty}$ & {$\left[\begin{array}{l}\infty \\
\stackrel{\rho}{\rho} \\
m\end{array}\right.$} & \\
\hline $\begin{array}{l}\text { E } \\
\text { N } \\
\text { N } \\
\wedge\end{array}$ & & $\overline{\mathrm{N}}$ & & & & & & & & & $\overline{\mathrm{N}}$ & & & & & & & & & & & & & $\mid \bar{N}$ & \\
\hline 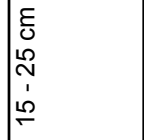 & m. & $\stackrel{\circ}{\circ} \mid 7$ & & & & 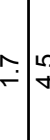 & & & & $\dot{j} \mid \stackrel{i}{i}$ & 恼 & $\stackrel{+}{-}$ & $\stackrel{\leftrightarrow}{\longrightarrow}$ & $\stackrel{0}{-}$ & & & $\stackrel{+}{\leftarrow}$ & 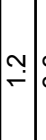 & 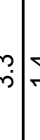 & 7 & & & 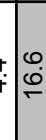 & $\mid \begin{array}{l}\infty \\
\tilde{i}\end{array}$ & \\
\hline 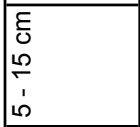 & बें & \begin{tabular}{lll} 
& \\
\hdashline & 0 \\
\hdashline & 0
\end{tabular} & & م & $\begin{array}{c}\infty \\
\dot{0} \\
\end{array}$ & مُ مُ & 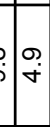 & 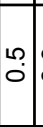 & $\begin{array}{lll}\infty \\
\infty \\
\infty\end{array}$ & $\stackrel{\infty}{\infty}$ & 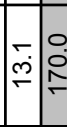 & & & & בְ: & $\dot{\vdots}$ & & & & & & . & $\stackrel{m}{\mathrm{~N}}$ & 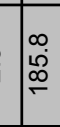 & \\
\hline $\begin{array}{l}\varepsilon \\
0 \\
0 \\
v \\
v\end{array}$ & مُ & $\hat{0}$ & 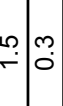 & 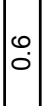 & 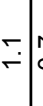 & 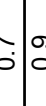 & $\hat{0}$ & $\stackrel{m}{\infty}$ & خ & $\stackrel{0}{\circ}$ & 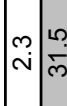 & & & & & & & & & & & & $\because$ & $\mid \begin{array}{c}\Gamma \\
\stackrel{\rho}{\sigma}\end{array}$ & \\
\hline 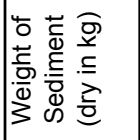 & $\stackrel{\circ}{\circ}$ & $\underset{m}{-i s}$ & 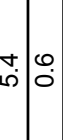 & 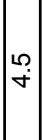 & & Pُ & $\stackrel{m}{\infty}$ & $\hat{o}$ & مُ & 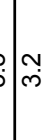 & $\mid \begin{array}{l}0 \\
\dot{\tau} \\
\dot{\sim}\end{array}$ & i & 1 & 1 & 1 & & 1 & 1 & $\begin{array}{lll}1 & 1\end{array}$ & i & 1 & 1 & & $\mid \begin{array}{c}0 \\
\hat{0} \\
1.0\end{array}$ & \\
\hline 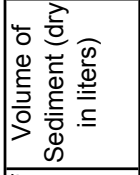 & $\stackrel{\circ}{\circ}$ & & & $\left|\begin{array}{l}0 \\
i \\
\end{array}\right|$ & & $\stackrel{f}{\dot{f}}$ & 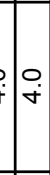 & $\stackrel{m}{\rightarrow}$ & & $\stackrel{\circ}{\circ}$ & $\mid$ & i & 1 & i & 1 & & : & 1 & : & $1:$ & i & 1 & : & | & 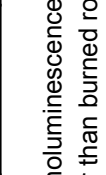 \\
\hline 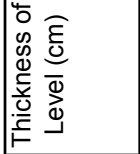 & 으 & 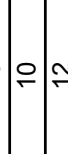 & & $1 ㅇ$ & & & 0 & $\wedge$ & & 20 & 으 & & $N$ & N & $\diamond$ & & $m$ & $\infty$ & & 0 & & $\nabla$ & & & 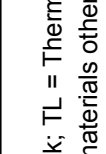 \\
\hline 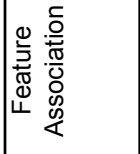 & - & & & & & & - & - & & & - & & & $N$ & - & & & $m$ & & $m$ & & & & & 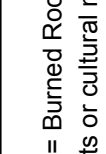 \\
\hline 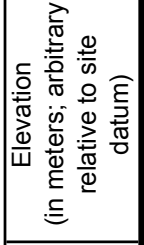 & 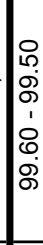 & 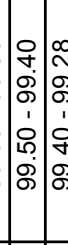 & 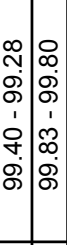 & 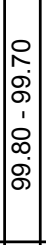 & 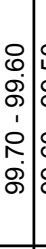 & 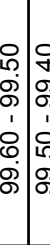 & 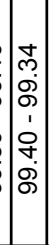 & 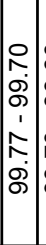 & 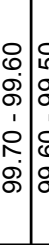 & 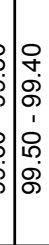 & 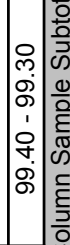 & 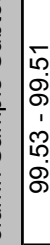 & 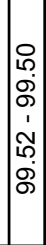 & 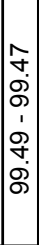 & 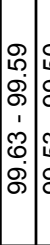 & 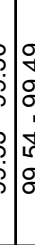 & 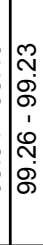 & 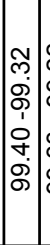 & 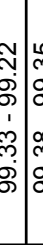 & 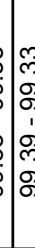 & 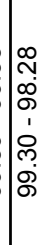 & 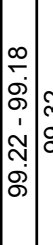 & 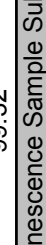 & 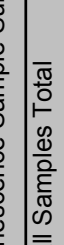 & 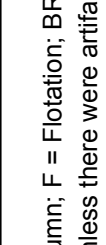 \\
\hline 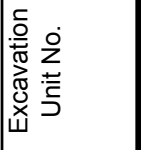 & $\bar{\omega}$ & $\bar{\infty}$ & $\bar{\omega}: \bar{\infty}$ & 足 & $\begin{array}{l}\infty \\
\infty \\
\dot{\omega}\end{array}$ & 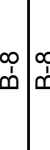 & 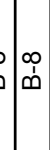 & 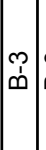 & $m$ & $\stackrel{m}{\infty}$ & 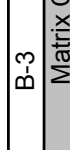 & ָั & $\mid \begin{array}{c}\mathcal{L} \\
\stackrel{\sim}{*}\end{array}$ & $\left|\begin{array}{|}\multirow{1}{*}{} \\
\dot{L}\end{array}\right|$ & ఫُ & & $\overbrace{\infty}$ & $\underset{\dot{\omega}}{\bar{\phi}}$ & $=$ & 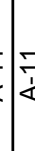 & & $\underset{\perp}{\mp}$ & 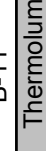 & & 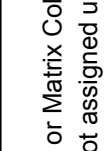 \\
\hline${ }^{*}{ }^{*}$ & 5. & $\underset{1}{\infty}$ & 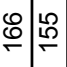 & 梁 & 苟 & \begin{tabular}{l|l}
0 & 1 \\
$\stackrel{1}{2}$ & 15
\end{tabular} & 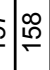 & $:$ & 赵 & $?$ & $\bar{\sigma}$ & ; & 1 & 1 & 1 & 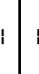 & 1 & 1 & ; & 1 & & i & & & o. \\
\hline $\mid \frac{0}{0}$ & 's & 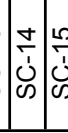 & 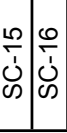 & $\begin{array}{l}\hat{1} \\
\dot{0} \\
0\end{array}$ & 竞 & 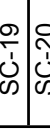 & $\begin{array}{l}\dot{v} \\
\dot{v}\end{array}$ & 勇 & 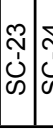 & \begin{tabular}{|l|l}
\multirow{v}{*}{} \\
$\vdots$ \\
$\vdots$
\end{tabular} & $\mid$\begin{tabular}{l}
0 \\
\multirow{2}{0}{} \\
0 \\
0
\end{tabular} & ت. & $\stackrel{\sim}{\stackrel{P}{F}}$ & $m$ & 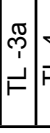 & & نִ & . & & & & $\cong$ & & & 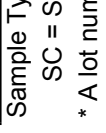 \\
\hline
\end{tabular}


Data Recovery Investigations at the Tank Destroyer Site, Fort Hood

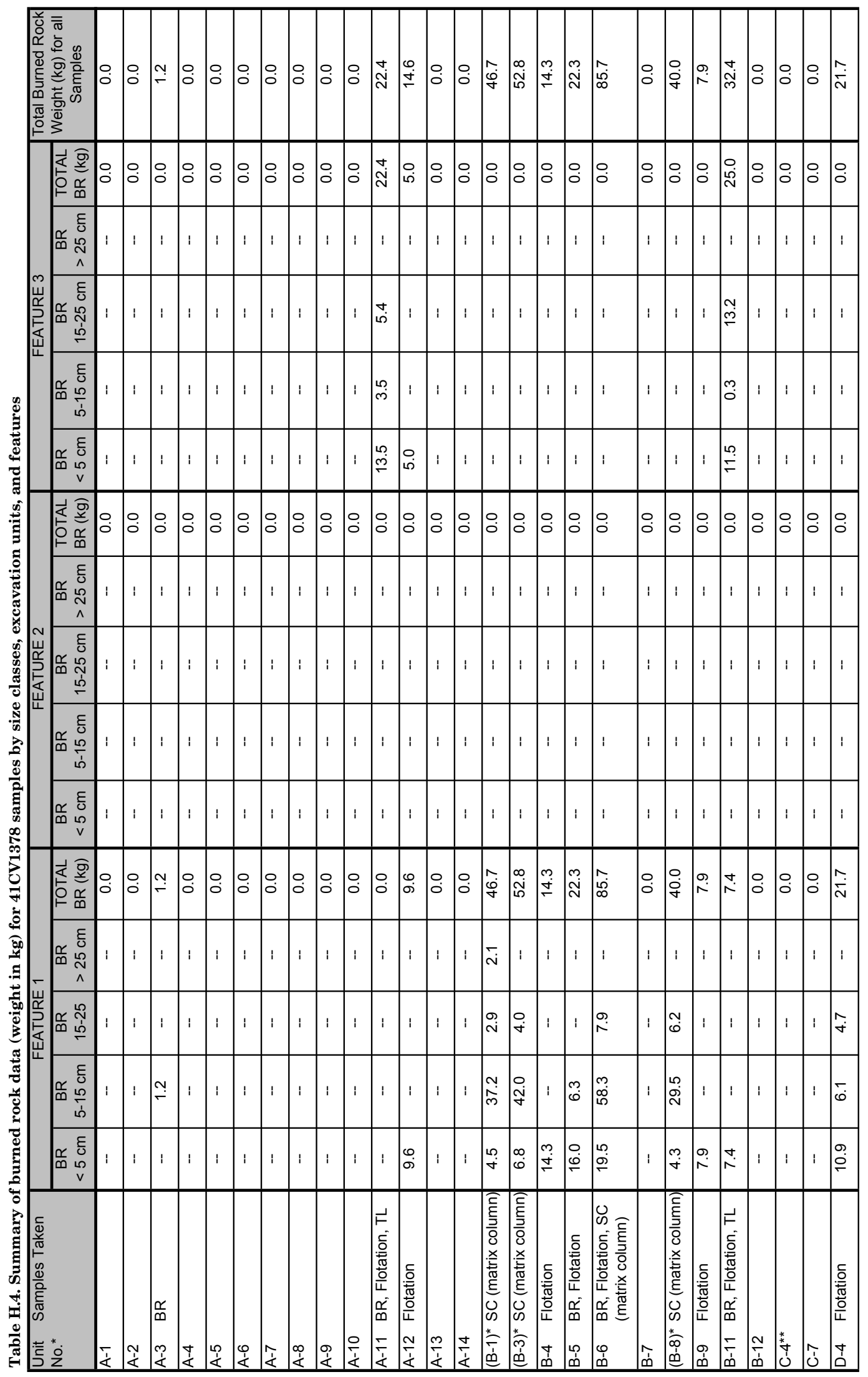




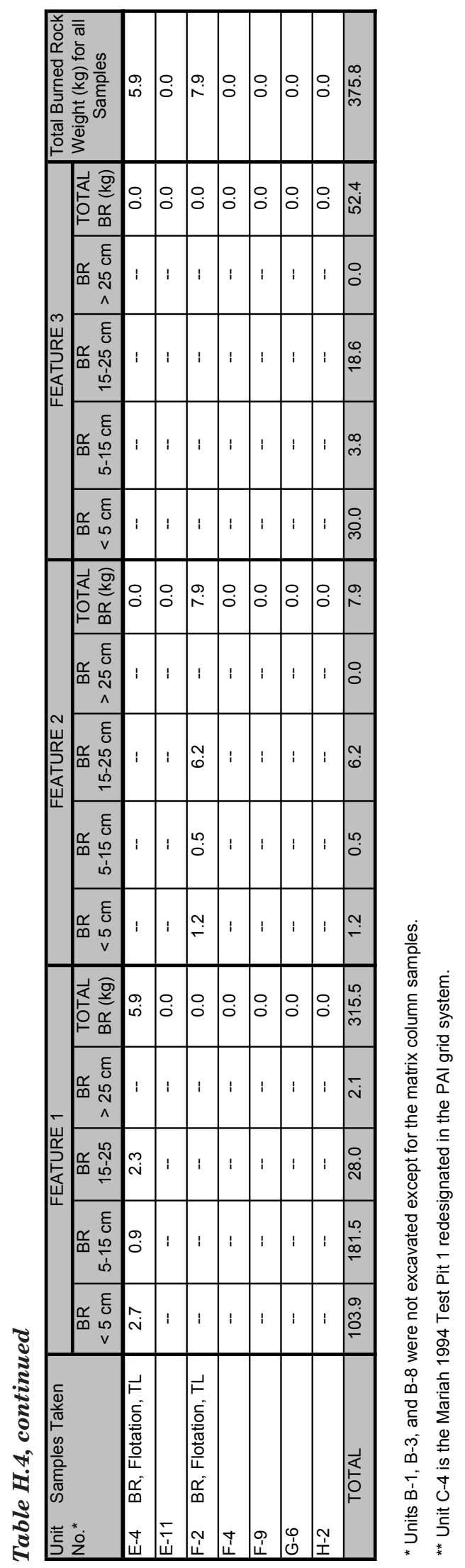


For this analysis, the original Test Pit 1 excavated by TRC Mariah Associates, Inc. archeologists in 1994 was redesignated as Excavation Unit C-4 in the PAI grid system. The original data for Test Pit 1 were presented by Trierweiler (1996:477, Table 5.101), and this included weights of burned rocks by levels. However, the burned rocks were not sorted into size classes, so the Mariah data was not directly comparable with the PAI data. But when PAI archeologists reexcavated Mariah's old Test Pit 1 , they screened all the backfill and sorted the burned rocks into the four size categories to obtain weights. In the reexcavation, the burned rock weights added up to $465.6 \mathrm{~kg}$, which was only $6.6 \mathrm{~kg}$ short of Mariah's original calculated burned rock weight of $472.2 \mathrm{~kg}$. It was then relatively easy to adjust these numbers using a weighted average to bring the PAI data up to the original calculated weight of $472.2 \mathrm{~kg}$, as shown in Table H.5.

The next step in the analytical process was to combine the burned rock data from all the general excavations and the all the sample proveniences. Table H.6 combines the data from the general excavations (see Table H.2) and sample contexts (see Table H.3) to show the total burned rocks weights by excavation units and features. Then, the burned rock size data from the general excavations (see Table H.2) were combined with the burned rock size data from the sample proveniences (see Table H.4) to create Table H.7 that shows the size class data for all burned rocks at $41 \mathrm{CV} 1378$ by excavation units and cultural features.

\section{BURNED ROCK DENSITY DATA}

The next step in the burned rock data analysis involved quantifying the volume of the hand excavated fill from each excavation units and feature provenience so that the burned rock data could be converted from raw weight to weight per square meter. Table H.8 presents the volumetric calculations for all of the testing and data recovery excavations at 41CV1378. Once the volumetric data were compiled, the data were used to create Table H.9, a comprehensive inventory of burned rock weights and excavation volumes that are converted to burned rock weight per square meter for each provenience (excavation unit and feature context). This table provides the most meaningful data that were used to examine the horizontal distribution of burned rocks in and around the burned rock mound at 41CV1378 (see Chapter 7).

Table H. 5. Adjusted burned rock data for Mariah's 1994 Test Pit 1.

\begin{tabular}{|l|c|c|c|}
\hline \multicolumn{1}{|c|}{ Size Class } & $\begin{array}{c}\text { "Burned Rock Weight } \\
(\mathrm{kg}) \\
\text { by PAI" }\end{array}$ & $\begin{array}{c}\text { Weighted Adjustment } \\
(\mathrm{kg})\end{array}$ & $\begin{array}{c}\text { "Adjusted } \\
\text { Weight (kg)" }\end{array}$ \\
\hline$<5 \mathrm{~cm}$ & 61.2 & +0.9 & 62.1 \\
\hline $5-15 \mathrm{~cm}$ & 381.6 & +5.4 & 387.0 \\
\hline $15-25 \mathrm{~cm}$ & 22.8 & +0.3 & 23.1 \\
\hline$>25 \mathrm{~cm}$ & 0.0 & +0.0 & 0.0 \\
\hline Total Weight & 465.6 & +6.6 & 472.2 \\
\hline
\end{tabular}

* Mariah's Test Pit 1 is renumbered as Excavation Unit C-4 in the data recovery site grid used by Prewitt and Associates, Inc 


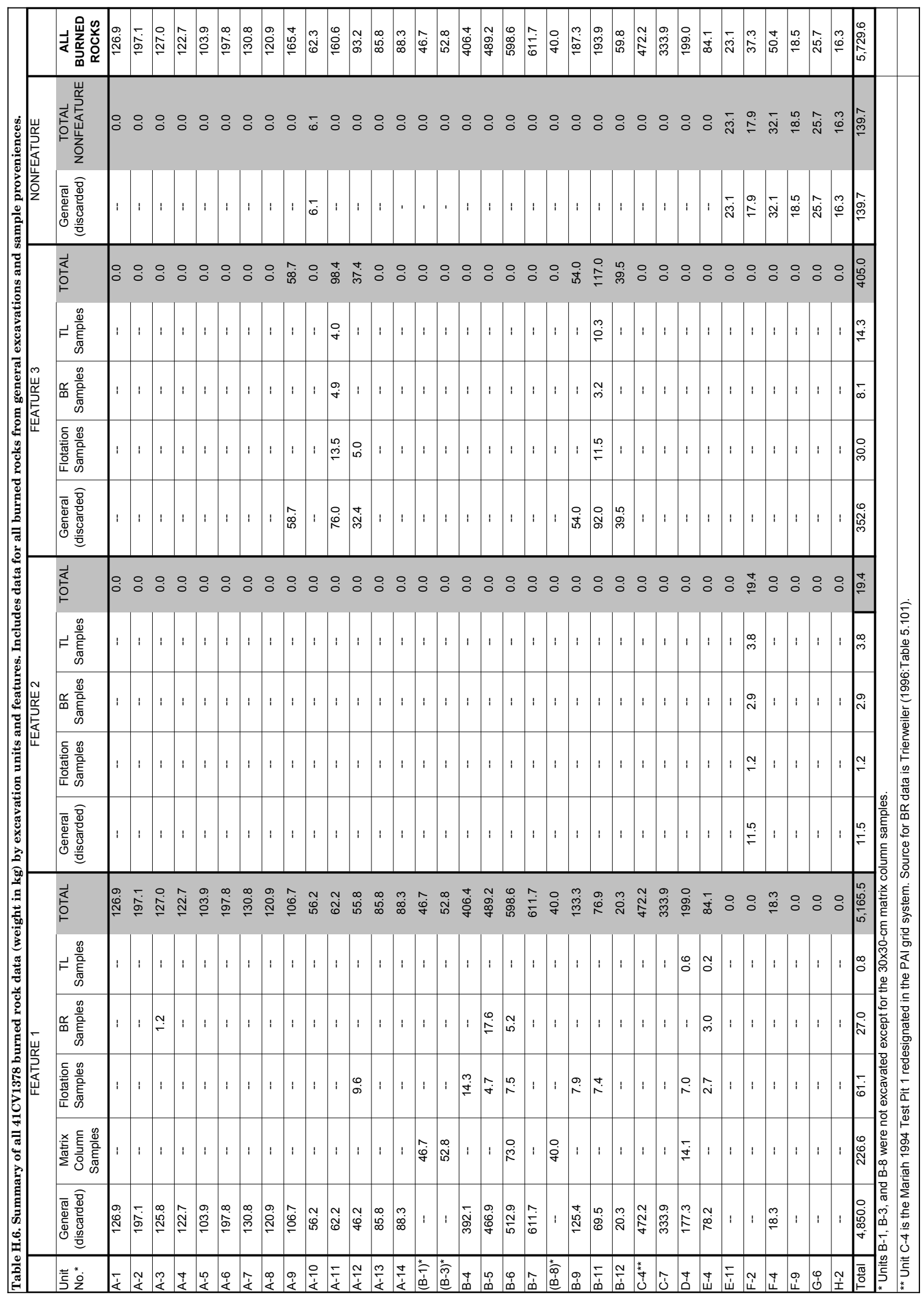




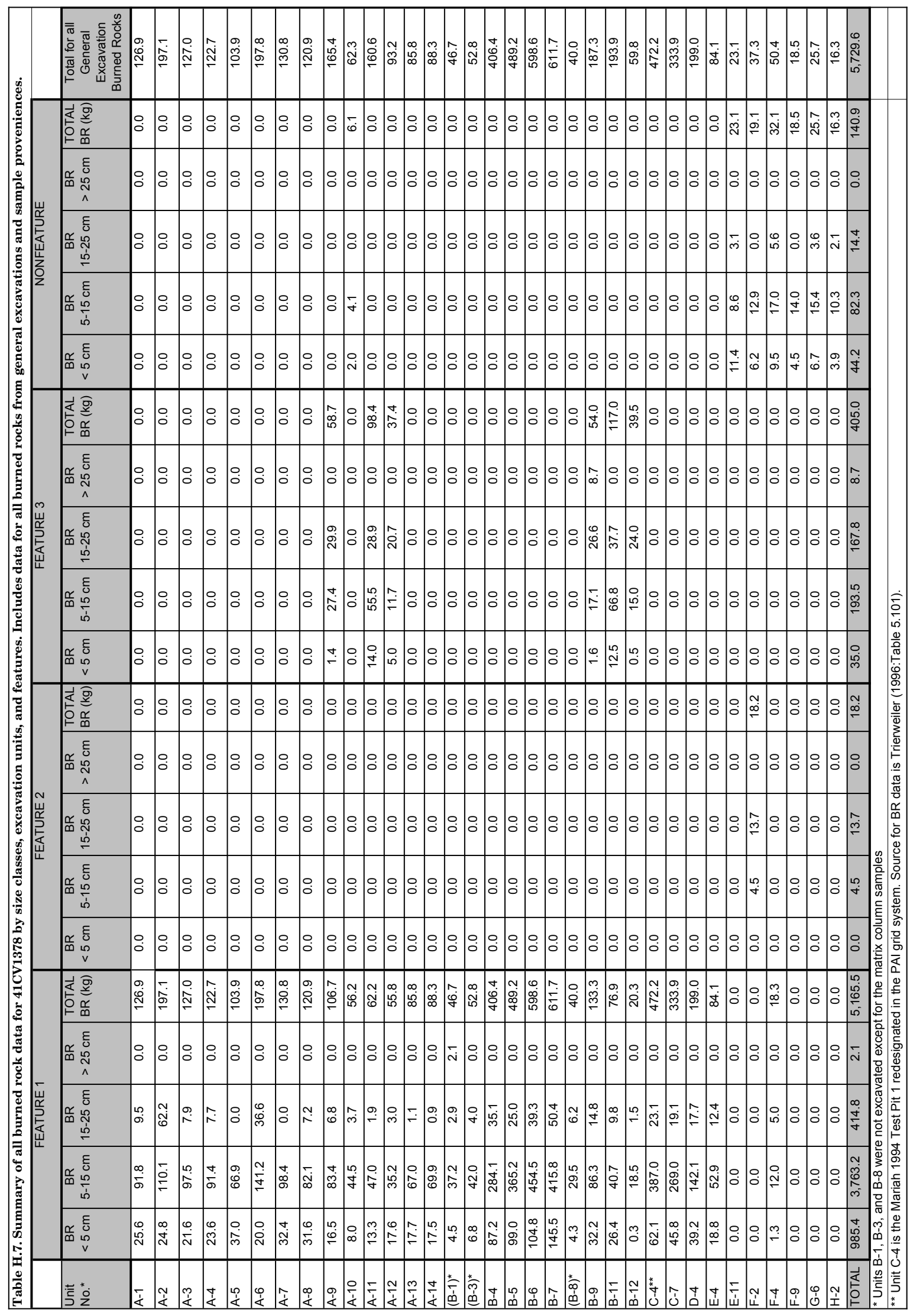




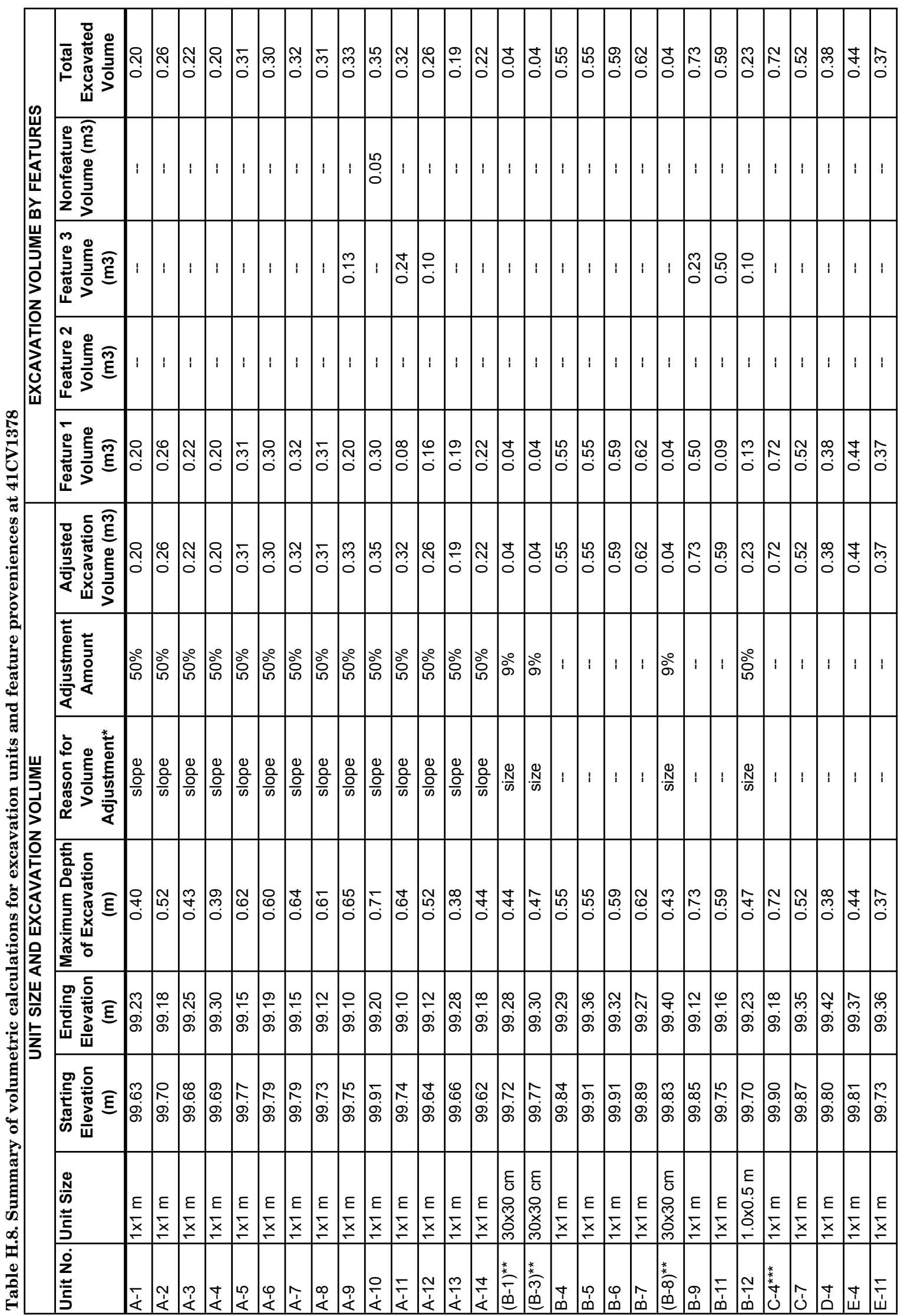




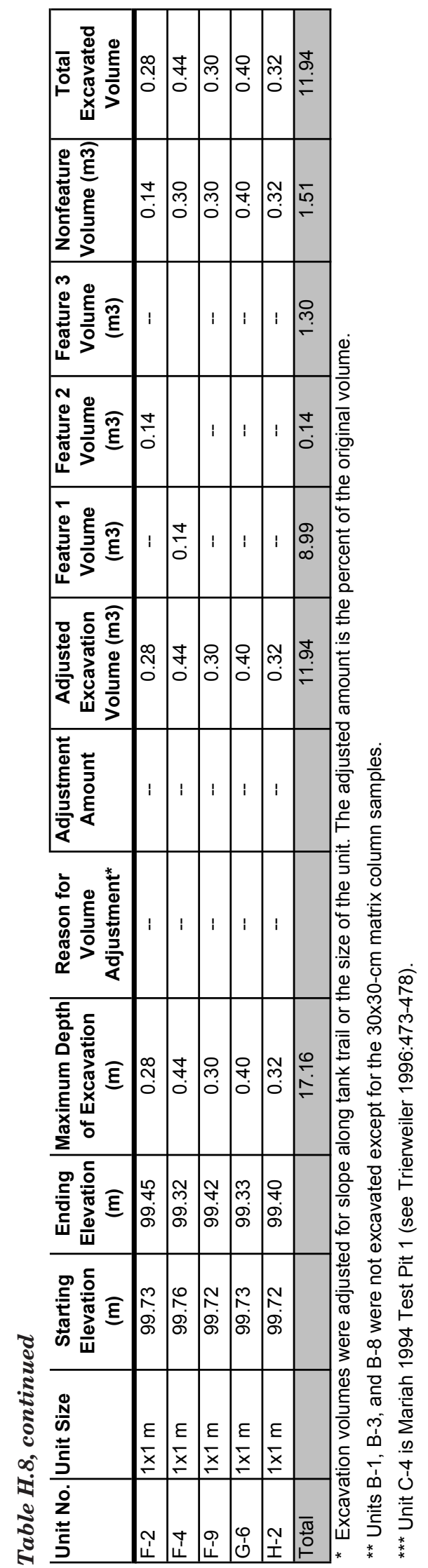




\begin{tabular}{|c|c|c|c|c|c|c|c|c|c|c|c|c|c|c|c|c|c|c|c|c|c|c|c|c|c|c|c|c|c|}
\hline \multirow{3}{*}{ 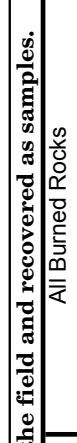 } & 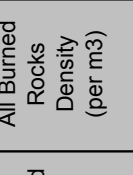 & 党 & \multicolumn{2}{|c|}{ 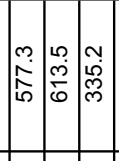 } & \multicolumn{2}{|c|}{ 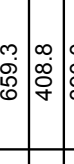 } & 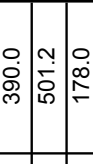 & \multicolumn{2}{|c|}{ 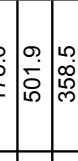 } & 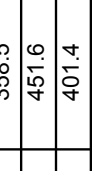 & \multicolumn{2}{|c|}{ 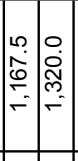 } & 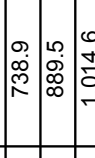 & \multicolumn{2}{|c|}{ 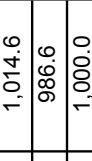 } & 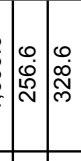 & \multicolumn{2}{|c|}{ 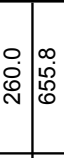 } & 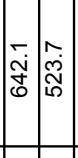 & \multicolumn{2}{|c|}{ : } & 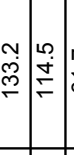 & 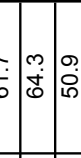 & \multicolumn{3}{|c|}{ 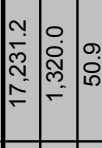 } & \multicolumn{3}{|c|}{$\mid \begin{array}{l}\infty \\
\dot{0} \\
0 \\
0\end{array}$} \\
\hline & 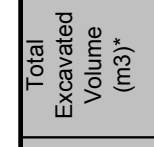 & 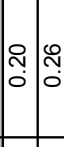 & דָָ & $\begin{array}{c}\substack{0 \\
0} \\
\end{array}$ & & N్m & $\int_{\substack{\infty \\
0 \\
0}}$ & & 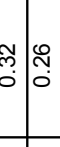 & 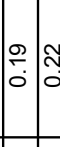 & 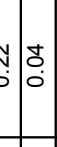 & & مُ & 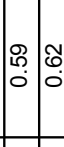 & $\left|\begin{array}{l}0 \\
0 \\
0\end{array}\right|$ & & ָָּ & \begin{tabular}{|c|c|c}
$\tilde{N}$ \\
\hdashline \\
0
\end{tabular} & 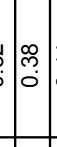 & & 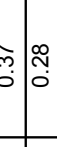 & $\begin{array}{lll}4 & 0 \\
0 & 0 \\
0 & 0 & 0\end{array}$ & 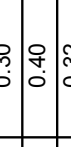 & ্ָল & \begin{tabular}{l|l}
$N$ \\
0
\end{tabular} & & & 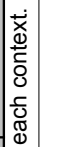 & \\
\hline & 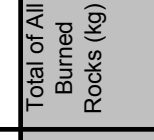 & 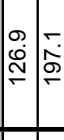 & 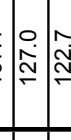 & & & 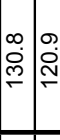 & 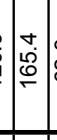 & & 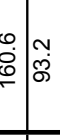 & 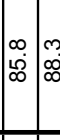 & & & 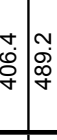 & 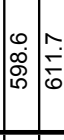 & & & $\begin{array}{l}\infty \\
8 \\
0 \\
0\end{array}$ & 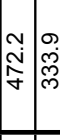 & 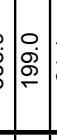 & & 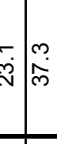 & 界 & & & $\frac{\vec{\sigma}}{\dot{\sigma}}$ & & & & \\
\hline \multirow{3}{*}{ 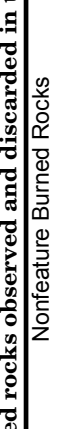 } & 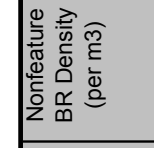 & $\because: 0$ & 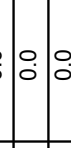 & $\begin{array}{l}0 \\
0\end{array}$ & $: 0$ & 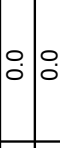 & 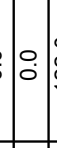 & & $: 0$. & $\begin{array}{lll} & 0 \\
0 & 0\end{array}$ & $\begin{array}{lll}0 \\
0\end{array}$ & & $\circ: 00$ & 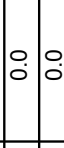 & 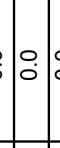 & & 0 & 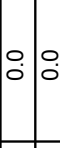 & $\dot{3}: \dot{0}$ & 웅 & 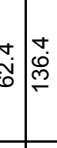 & $\begin{array}{l}0 \\
\dot{i} \\
0 \\
0 \\
0\end{array}$ & 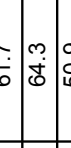 & & $\left|\begin{array}{l}0 \\
\ddot{0} \\
\end{array}\right|$ & & & 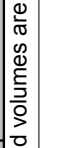 & \\
\hline & 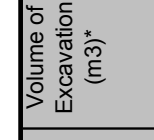 & 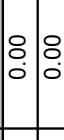 & $\int_{0}^{\infty}$ & & & 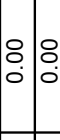 & $\left|\begin{array}{l}8 \\
0 \\
0\end{array}\right|$ & & $: \begin{array}{l}8 \\
:\end{array}$ & $\mid$\begin{tabular}{l}
0 \\
\hdashline \\
0
\end{tabular} & & & : & $\because 8$ & 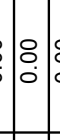 & & $\mid$ & $\underset{0}{0}$ & 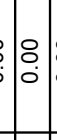 & & $\overbrace{0}^{\infty}$ & @্ & $\begin{array}{l}b \\
b \\
b\end{array}$ & & $\mid \begin{array}{l}o \\
f \\
0\end{array}$ & & & & \\
\hline & 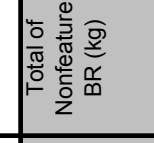 & $\because: 0$. & $:$ & $: 0: 0$ & ${ }_{0}^{\circ}$ & $\stackrel{0}{\circ}: 0$. & $\stackrel{0}{\circ}$ & & $: 0$. & $\begin{array}{lll}0 & 0 \\
& 0\end{array}$ & 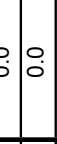 & & 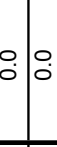 & $\because: 0$. & 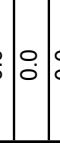 & & $:$ & 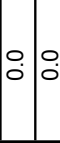 & $\dot{0}$ & : & 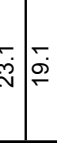 & 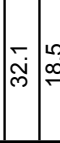 & & & $\overline{\mathrm{j}}$ & & & 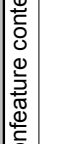 & \\
\hline \multirow{3}{*}{ 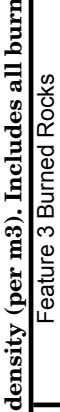 } & 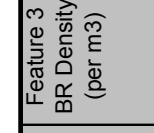 & $\because: 0$. & $\because 0$ & $: 0_{0}^{\circ}: 0$. & $: 0$ & $: 0: 0$ & $\mid$ & & 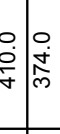 & $\because: 0$ & $: 0$ & & $:{ }_{0}^{\circ}$ & $\because: 0$ & 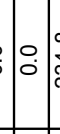 & & $\mid \begin{array}{l}0 \\
0 \\
0 \\
0\end{array}$ & 8. & $\dot{0}$ & & : & 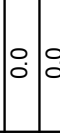 & 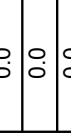 & $: \begin{array}{l}m \\
0 \\
0 \\
0 \\
0 \\
0\end{array}$ & 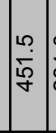 & & & & \\
\hline & 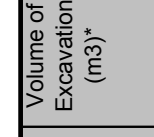 & ن. & $\mid$\begin{tabular}{l}
0 \\
\hdashline \\
0
\end{tabular} & \begin{tabular}{l|l|l|l|} 
\\
0 & 0 & 0 \\
0
\end{tabular} & & $::_{0}^{0}$ & $\mid$\begin{tabular}{l}
$m$ \\
\hdashline
\end{tabular} & & \begin{tabular}{l}
\multirow{2}{*}{} \\
\hdashline
\end{tabular} & : & $\begin{array}{l}0 \\
:\end{array}$ & & : & 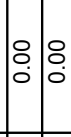 & $\dot{0}$ & & $\frac{0}{0}$ & 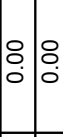 & $\begin{array}{l}8 \\
0 \\
0\end{array}$ & : & 8 & 80 & s: & & $\begin{array}{l}0 \\
0 \\
0 \\
0\end{array}$ & & & 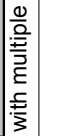 & \\
\hline & 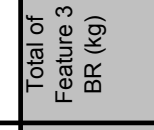 & $\because 0$. & $\because 0$ & 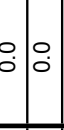 & $: 0$ & 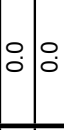 & $\hat{a}$ & & \begin{tabular}{l|l}
\multirow{2}{\infty}{} \\
\hdashline
\end{tabular} & $\because: 0$ & $\begin{array}{lll}0 & 0 \\
0\end{array}$ & & 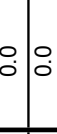 & $: 0$. & $: 0$ & & $\mid$ & $: 0$. & $\dot{0}$ & ㅇ. & $0^{\circ}: 0$. & $: 0$ & $: 0$ & & $\left|\begin{array}{l}0 \\
\stackrel{P}{F} \\
\end{array}\right|$ & & & & \\
\hline \multirow{3}{*}{ 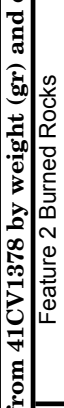 } & 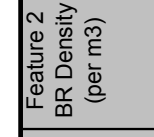 & $: 0:$ & $: 0$ & $: 0: 0$ & $\because$ & $\because: 0$ & $:$ & & 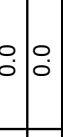 & $: 0$ & 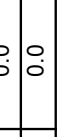 & & $::^{\circ}$ & $\therefore: 0$. & $: 0$ & $:\left.\right|_{0} ^{\circ}$ & $: 0$ & $\because: 0$. & $\dot{0} 0$ & : & 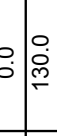 & $\therefore: 0$ & $\dot{b}: \circ:$ & 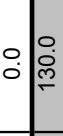 & $\frac{\pi}{\Sigma}$ & & & & \\
\hline & 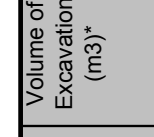 & $\begin{array}{l}0 \\
0 \\
0\end{array}$ & $: 0$ & 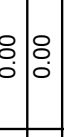 & & 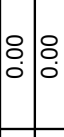 & 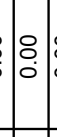 & & $\begin{array}{lll}8 & 0 \\
0 & 0 & 0 \\
0\end{array}$ & $: 0$ & 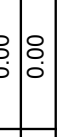 & & : & \begin{tabular}{l}
0 \\
\hdashline \\
\hdashline
\end{tabular} & 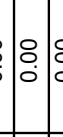 & & 8 & $: O_{0}^{\circ}$ & 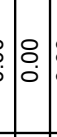 & & : & $: 0$ & 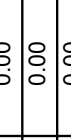 & $\begin{array}{l}8 \\
0 \\
0\end{array}$ & $\frac{\pi}{\pi}$ & & & & \\
\hline & |이 & $\because: 0$. & $: 0$ & $\begin{array}{l}0 \\
0\end{array}$ & 0 & $\because: 0$ & 0 & & $\begin{array}{l}0 \\
:\end{array}$ & $\because: 0$ & 0 & ○. & 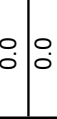 & $\because: 0$. & 0 & $:$ & 0. & $: 0_{0}^{\circ}$ & $b_{0}$ & : & סִ & $\therefore: 0$ & : & $: \begin{array}{c}\text { D. } \\
:\end{array}$ & $\frac{\pi}{\Sigma}$ & & & & \\
\hline \multirow{4}{*}{ 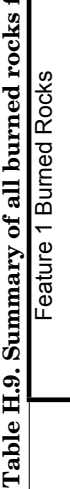 } & 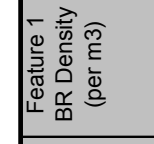 & 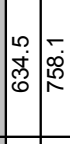 & 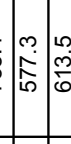 & 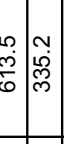 & 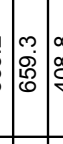 & \begin{tabular}{|l|l} 
& \\
$\infty$
\end{tabular} & 痌 & & 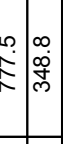 & 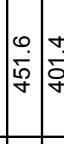 & 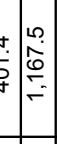 & & 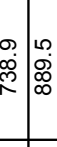 & 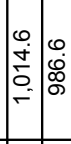 & 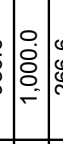 & & & 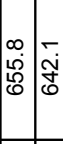 & & & $: 0$. & 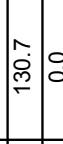 & $:$ & 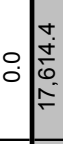 & $\begin{array}{l}0 \\
0 \\
0 \\
0 \\
- \\
-\end{array}$ & & & & \\
\hline & 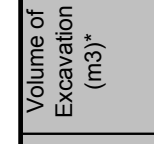 & 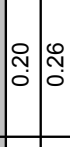 & ִָ & $\begin{array}{l}c \\
0\end{array}$ & $\left.\begin{array}{lll}0 \\
0 \\
0\end{array}\right)$ & 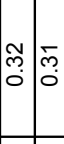 & : & & \begin{tabular}{l|l}
$\infty$ \\
0 \\
0
\end{tabular} & $\mid \begin{array}{c}0 \\
0 \\
0 \\
0\end{array}$ & 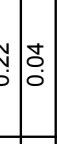 & مُ & مُ & : & $\begin{array}{l}0 \\
0 \\
0\end{array}$ & & $\frac{m}{0}$ & 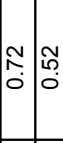 & $\mid \begin{array}{c}\infty \\
\infty \\
0 \\
0\end{array}$ & f & $\underbrace{8}_{0}$ & $\stackrel{\square}{\square}$ & 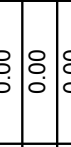 & \begin{tabular}{l}
8 \\
\hdashline \\
\hdashline
\end{tabular} & $\left|\begin{array}{l}N \\
0 \\
0\end{array}\right|$ & & & & \\
\hline & 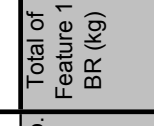 & 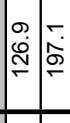 & $\underset{\substack{0 \\
\stackrel{N}{N}}}{\stackrel{N}{N}}$ & 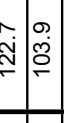 & 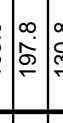 & 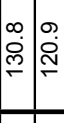 & 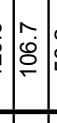 & 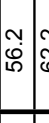 & 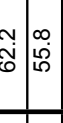 & 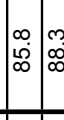 & \begin{tabular}{ll|l}
$:$ \\
$:$
\end{tabular} & & 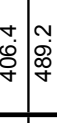 & 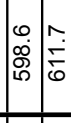 & $\vdots$\begin{tabular}{l}
0 \\
\hdashline \\
$\dot{q}$
\end{tabular} & 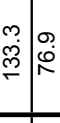 & & 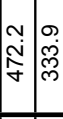 & 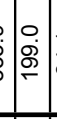 & 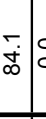 & 0. & $\begin{array}{l}\infty \\
\infty \\
\infty \\
\infty\end{array}$ & $\dot{0}: \circ$ & $: \underbrace{\infty}_{0}$ & $\begin{array}{l}\hat{\vdots} \\
\overline{\mathbf{c}} \\
\end{array}$ & & & $\mid$ & \\
\hline & $\sum_{x}^{0}$ & $\begin{array}{l}N_{2}^{2} \\
\end{array}$ & 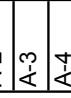 & $\begin{array}{l}* \\
\dot{y} \\
\end{array}$ & & {$\left[\begin{array}{ll}2 \\
\end{array}\right.$} & & & & & 主啇 & & & & 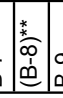 & & & & $\mid$ & & & 童隊 & $\begin{array}{cccc}0 & 0 \\
1 & 0 & 3 \\
1\end{array}$ & & . & & & & \\
\hline
\end{tabular}




\section{MATRIX COLUMN SAMPLES}

The matrix sample columns were processed in the PAI laboratory in February 2009. The laboratory procedures used were as follows: Step 1. Screening:

Each samples was run through a 1/4-inch screen to separate the fine fraction from course fraction

Step 2. Processing of Course Fraction:

a. All artifacts were picked from the course fraction, and bagged and tagged by sample provenience

b. All snail shells were picked from the course fraction, and bagged and tagged by sample provenience

c. Each sample with artifacts or snails was assigned a new lot number linked to the sample column and level.

d. The rocks were sorted unburned $(>5 \mathrm{~cm})$ and burned, and the burned rocks were sorted into four size categories:

$<5 \mathrm{~cm}$

$5-15 \mathrm{~cm}$

$15-25 \mathrm{~cm}$

$>25 \mathrm{~cm}$

e. The burned rocks for each size category were weighed $(\mathrm{kg})$ and the data recorded.

f. All the burned rocks were saved temporarily, but ultimately were discarded.

Step 3. Processing of Fine Fraction:

a. The volume of sediment was measured and recorded (dry in liters)

b. Measure and record weight of sediment (dry in kg)

c. Save small bag of sediment for now
The burned rock and sediment data for all of the matrix column samples is presented in Table H.10. The raw data consists of counts of snails shells and weights of the sediment (fine-grained matrix) and burned rocks (course-grained matrix) for each matrix sample level. Each level was $10-\mathrm{cm}$ thick except for the first and last levels that varied in thickness. To account for these differences in some levels, the raw data were mathematically converted to the standardized 10-cm level. As shown in Table H.11, these volume-adjusted data were then used to calculate the following ratios of interest: (1) the ratio of burned rocks to sediment (BR kg/Sediment $\mathrm{kg}$ ); (2) the ratio of burned rocks to snails (BR $\mathrm{kg} / \#$ of Snails); and (3) the ratio of sediment to snails (Sediment kg/\# of Snails). Selected cells in Table H.11 are highlighted to emphasize the matrix column levels with the highest density of snails, burned rocks, and sediment, as well as those levels with the highest calculated ratios. In all cases, the cells with the highest number are highlighted in descending order until the cumulative total of the highlighted cells reaches one-third of the total. Interpretations of these data are presented in Chapter 7.

\section{REFERENCES CITED}

Black, Stephen L., and Linda W. Ellis

1997 Critically Observing and Recording Burned Rock Features. In Hot Rock Cooking on the Greater Edwards Plateau: Four Burned Rock Midden Sites in West Central Texas, Vol. 2, pp. 777-783. Studies in Archeology 22. Texas Archeological Research Laboratory, The University of Texas at Austin. Archeology Studies Program Report 2, Environmental Affairs Department, Texas Department of Transportation, Austin.

Trierweiler, W. Nicholas (editor)

1996 Archeological Testing at Fort Hood, 1994-1995, Vol. I. Archeological Resource Management Series, Report No. 35. United States Army, Fort Hood. 


\begin{tabular}{|c|c|c|c|c|c|c|c|c|c|c|c|c|c|c|c|c|c|c|c|c|c|c|c|c|c|c|}
\hline 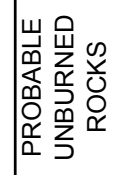 & 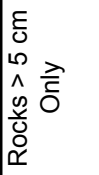 & $\overbrace{0}^{\infty}$ & 1 & $\stackrel{\sim}{-}$ & $1 \stackrel{1}{1} \stackrel{1}{\stackrel{1}{r}}$ & $\begin{array}{l}?: 11 \\
:=1\end{array}$ & 1 & 1 & 1 & & \begin{tabular}{l|l}
$\ddot{O}_{0}$ & 1
\end{tabular} & : & $\begin{array}{lll}1 & 1 \\
\end{array}$ & $\begin{array}{lll}1 & 1\end{array}$ & $\begin{array}{l}1 \\
1 \\
0 \\
0\end{array}$ & $\begin{array}{l}0 \\
0 \\
0\end{array}$ & \begin{tabular}{|l|l|l} 
& 1
\end{tabular} & \begin{tabular}{l|l}
1 & 1
\end{tabular} & 1 & 1 & $1 \mid \begin{array}{l}0 \\
0\end{array}$ & \begin{tabular}{l|l}
1 & 1
\end{tabular} & 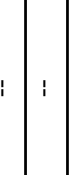 & 1 & : $:{ }^{\circ}$ & $\stackrel{\sim}{\sim}$ \\
\hline 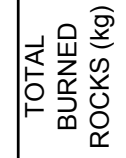 & 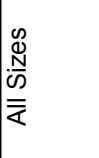 & $\stackrel{\leftrightarrow}{\longrightarrow}$ & 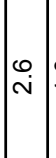 & 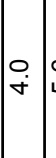 & 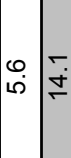 & $\stackrel{\infty}{\circ}$ & $\ddot{\circ}$ & 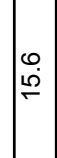 & $\left(\begin{array}{l}\hat{\theta} \\
\stackrel{\leftrightarrow}{\circ}\end{array}\right.$ & 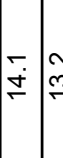 & 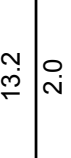 & 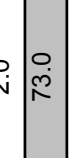 & $\stackrel{m}{\sigma}=$ & 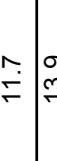 & \begin{tabular}{r|l}
$\qquad$ \\
\hdashline
\end{tabular} & $\left|\begin{array}{r}\hat{0} \\
\dot{g}\end{array}\right|$ & 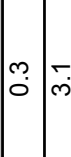 & $\dot{r} \stackrel{9}{r}$ & 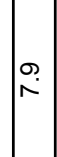 & 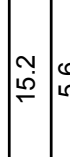 & 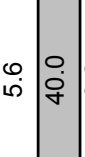 & \begin{tabular}{l|l}
$\infty$ \\
\hdashline \\
\hdashline & 0
\end{tabular} & 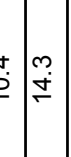 & 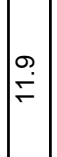 & 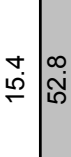 & 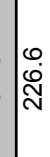 \\
\hline & $\begin{array}{l}E \\
\text { U } \\
\stackrel{N}{N} \\
\wedge\end{array}$ & 1 & 1 & 1 & 10 & \begin{tabular}{l|l}
$j^{3}$ & 1
\end{tabular} & 1 & 1 & 1 & \begin{tabular}{l|l}
1 & 1
\end{tabular} & \begin{tabular}{l|l}
1 & 1
\end{tabular} & $\because$ & $\begin{array}{lll}1 & 1\end{array}$ & 1 & $\bar{v} \mid 1$ & $\overline{\mathrm{N}}$ & \begin{tabular}{|l|l|l}
1 & 1
\end{tabular} & \begin{tabular}{l|l}
1 & 1
\end{tabular} & 1 & 1 & $1 \mid \begin{array}{l}0 \\
0\end{array}$ & $\begin{array}{lll}1 & 1\end{array}$ & $\begin{array}{l:l}1 & 1\end{array}$ & 1 & $1:{ }_{0}^{\circ}$ & ì \\
\hline 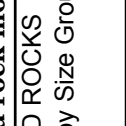 & 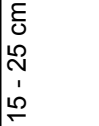 & : & 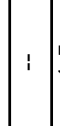 & $\mid$ & \begin{tabular}{l|l} 
& \multirow{r}{*}{}
\end{tabular} & $\begin{array}{ll} & 1\end{array}$ & i & $\stackrel{0}{\leftarrow}$ & $\stackrel{\leftrightarrow}{\stackrel{\circ}{-}}$ & 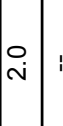 & \begin{tabular}{l|l}
1 & 1
\end{tabular} & 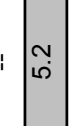 & בְ: & $\stackrel{3}{\overbrace{0}^{\prime}}:$ & S. & 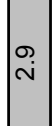 & \begin{tabular}{|l|l|l}
1 & 1
\end{tabular} & $: 1$ & $\stackrel{\therefore}{=}$ & $\mid \begin{array}{l}\infty \\
\stackrel{\sigma}{*}\end{array}$ & $: \mid \begin{array}{c}\tilde{\omega} \\
\tilde{\omega}\end{array}$ & $\begin{array}{lll}1 & 1\end{array}$ & 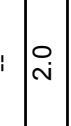 & ì & $1: \stackrel{\circ}{\circ}$ & 㐫 \\
\hline 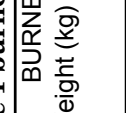 & 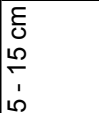 & | & 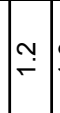 & $\stackrel{\leftrightarrow}{\longrightarrow}$ & \begin{tabular}{l|l} 
\\
\end{tabular} & $F$ & 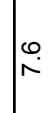 & $\stackrel{m}{\rightleftarrows}$ & $\mid \stackrel{0}{\stackrel{0}{\grave{M}}}$ & 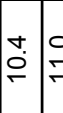 & 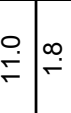 & 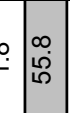 & \begin{tabular}{|l|l}
0 & 0 \\
$\infty$ & 0 \\
$\infty$
\end{tabular} & $\stackrel{\leftrightarrow}{\circ}$ & 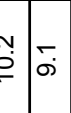 & $\begin{array}{l}\mathrm{N} \\
\tilde{m} \\
\bar{m}\end{array}$ & $\stackrel{n}{N}$ & \begin{tabular}{l|l}
$\mathfrak{v}^{\circ}$ & $\infty$ \\
0
\end{tabular} & $\begin{array}{ll}10 \\
10 \\
0\end{array}$ & $\begin{array}{ll}\infty & \circ \\
\infty & \varnothing\end{array}$ & 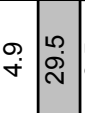 & \begin{tabular}{|l|l}
0 \\
\hdashline \\
\hdashline
\end{tabular} & 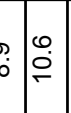 & $\mid \begin{array}{l}\infty \\
\infty \\
\infty\end{array}$ & 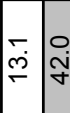 & 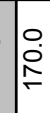 \\
\hline & $\mid \begin{array}{l}E \\
\text { D } \\
b \\
\mathrm{v}\end{array}$ & $\stackrel{+}{\rightleftarrows}$ & $\stackrel{.}{\square}$ & 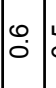 & "ִ & $\dot{s}$ & $\stackrel{\circ}{\stackrel{i}{ }}$ & $\hat{\mathrm{N}}$ & $\mid$ & $\because \approx$ & \begin{tabular}{l|l}
\multirow{N}{*}{} & \multirow{O}{*}{}
\end{tabular} & 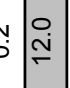 & \begin{tabular}{l|l} 
& \multicolumn{1}{c}{} \\
0 & \\
0 &
\end{tabular} & $\stackrel{0}{\circ}$ & مُ & $\begin{array}{l}\infty \\
\dot{*} \\
\end{array}$ & $\begin{array}{lll}m & 0 \\
0 & 0 & 0 \\
0\end{array}$ & $\stackrel{5}{\circ}$ & $\hat{\circ}$ & $\ddot{0}$ & \begin{tabular}{c|c|c|}
$\hat{o}$ & $m$ \\
$\dot{\sigma}$
\end{tabular} & 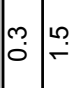 & $\stackrel{\overbrace{}}{=}$ & $\stackrel{\circ}{-}$ & $\begin{array}{lll}m & \infty \\
\stackrel{N}{*} & \infty \\
0\end{array}$ & مُ \\
\hline$\sum_{u}$ & 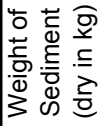 & $\hat{0}$ & 足 & 0 & & $\stackrel{m}{\longrightarrow}$ & is & 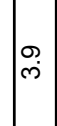 & $\stackrel{\sim}{f}$ & \begin{tabular}{|l|l}
$\forall$ \\
$\forall$ \\
$\forall$ \\
0
\end{tabular} & \begin{tabular}{c|c}
$\stackrel{m}{0}$ & $\hat{N}$
\end{tabular} & 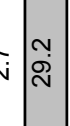 & लि & 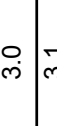 & $\begin{array}{c}\sigma \\
\dot{m}\end{array}$ & $\mid$ & \begin{tabular}{l|l} 
\\
\hdashline
\end{tabular} & \begin{tabular}{l|l}
$\stackrel{f}{f}$ & $\stackrel{\leftrightarrow}{f}$
\end{tabular} & مי & 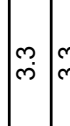 & \begin{tabular}{l|l|}
$\stackrel{\infty}{m}$ & $\infty$ \\
$\stackrel{m}{\rightleftharpoons}$ & $\infty$
\end{tabular} & & \begin{tabular}{l|l} 
\\
\hdashline
\end{tabular} & 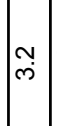 & 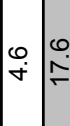 & 莗 \\
\hline प्र & 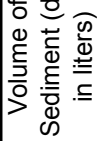 & : & $\mid \begin{array}{l}m \\
\infty \\
\infty\end{array}$ & & \begin{tabular}{l|l}
$\circ$ & $\infty$ \\
$\dot{+}$ & $\stackrel{0}{N}$
\end{tabular} & ִִ & $\stackrel{\circ}{r}$ & $\underset{\dot{f}}{\stackrel{0}{*}}$ & $\stackrel{m}{+}$ & \begin{tabular}{|l|l}
$m$ \\
$\dot{\sigma}$
\end{tabular} & $\begin{array}{l}\stackrel{\circ}{r} \\
\stackrel{\rho}{r}\end{array}$ & 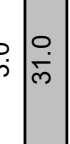 & $\underset{\forall}{\circ}$ & 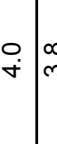 & 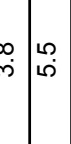 & $\stackrel{m}{\stackrel{m}{\sim}}$ & 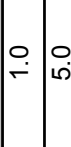 & \begin{tabular}{l|l}
0 \\
\end{tabular} & $\underset{\dot{\sigma}}{\stackrel{O}{*}}$ & $\underset{\dot{\sigma}}{\circ}$ & \begin{tabular}{l|l|}
$\circ$ & $\infty$ \\
$\dot{\sigma}$ & $\stackrel{N}{N}$
\end{tabular} & $\begin{array}{lll}m & \stackrel{m}{-} \\
\end{array}$ & \begin{tabular}{l|l|}
$\stackrel{p}{\circ}$ & $\stackrel{p}{+}$ \\
\end{tabular} & $\stackrel{\circ}{\dot{r}}$ & 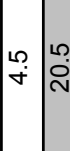 & 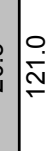 \\
\hline 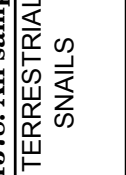 & 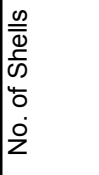 & 0 & 0 & 0 & & 0 & 0 & 0 & + & o & ما مـ & $\neq$ & $\sim$ & $m:$ & $P F$ & 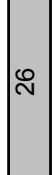 & +0 & 0 & N & $\infty ?$ & $\stackrel{\oplus}{\stackrel{\oplus}{\sim} \mid}$ & \begin{tabular}{l|l}
0 & $\mathrm{~m}$
\end{tabular} & $\cap N$ & 0 & $m \infty$ & $\infty \mid \propto$ \\
\hline \multirow{6}{*}{$\sum_{\overline{1}}^{\overline{1}}$} & 恖 & 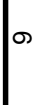 & $\circ$ & 음 & ما & r & 우 & & 은 & & $\therefore \wedge$ & & $\cong$ & $\div \%$ & $\stackrel{2}{2} \cong$ & & $m ?$ & $? \div$ & 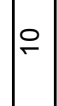 & 은 & 0 & 우 & $2 \div$ & 으 & 으 & \\
\hline & 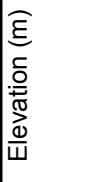 & 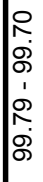 & $\begin{array}{l}8 \\
0 \\
\dot{8} \\
1 \\
2 \\
? \\
8 \\
8\end{array}$ & 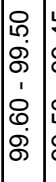 & 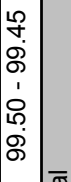 & $\begin{array}{l}\infty \\
\infty \\
0 \\
8 \\
8 \\
1 \\
\vdots \\
0 \\
8 \\
8\end{array}$ & 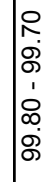 & $\begin{array}{l}0 \\
0 \\
8 \\
8 \\
1 \\
1 \\
R \\
8 \\
8 \\
8\end{array}$ & 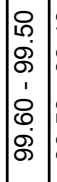 & 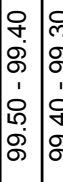 & 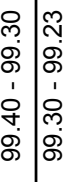 & 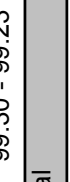 & 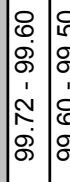 & 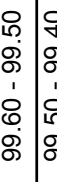 & 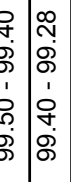 & & 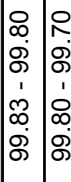 & 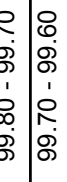 & $\mid \begin{array}{l}0 \\
0 \\
8 \\
8 \\
1 \\
8 \\
0 \\
8 \\
8\end{array}$ & 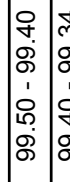 & 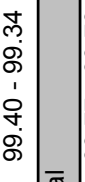 & 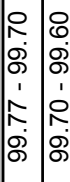 & 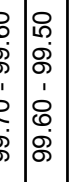 & $\mid \begin{array}{l}0 \\
0 \\
8 \\
8 \\
1 \\
0 \\
b \\
0 \\
8 \\
0\end{array}$ & $\mid \begin{array}{l}0 \\
0 \\
0 \\
\sigma \\
1 \\
0 \\
0 \\
0 \\
\sigma \\
\sigma\end{array}$ & \\
\hline & 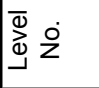 & - & N & $m$. & $+\sqrt{\circ}$ & - & N & $m$ & + & 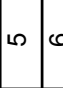 & $0 \wedge$ & $-\left|\begin{array}{l}0 \\
\stackrel{0}{0} \\
0 \\
\infty\end{array}\right|$ & - & $\sim$ & $m+$ & $\mid \begin{array}{l}\stackrel{0}{\circ} \\
\bar{\emptyset} \\
\dot{\omega}\end{array}$ & $-\alpha$ & 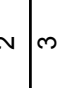 & $\sigma$ & $\infty$ & $0\left|\begin{array}{l}\overrightarrow{0} \\
\circ \\
\infty \\
\dot{\omega} \\
\dot{0}\end{array}\right|$ & $-\alpha$ & $v m$ & $\nabla$ & & $\left\{\begin{array}{l}0 \\
0 \\
0 \\
0 \\
0\end{array}\right.$ \\
\hline & 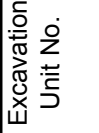 & a & 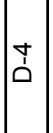 & ذ. & ๑5 & o & $\mid \begin{array}{l}\varrho \\
\omega\end{array}$ & $\begin{array}{l}0 \\
0 \\
\infty\end{array}$ & \begin{tabular}{|l|}
0 \\
$\infty$ \\
$\omega$
\end{tabular} & & 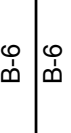 & $\mathfrak{l}^{5}$ & $\bar{\infty}$ & $\overline{\dot{\omega}} \overline{\dot{\alpha}}$ & 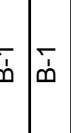 & & 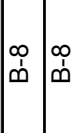 & \begin{tabular}{l|l}
$\infty$ & $\infty$ \\
$\dot{D}$ &
\end{tabular} & $\begin{array}{l}\infty \\
\infty \\
\infty\end{array}$ & $\mid \begin{array}{ll}\infty & \infty \\
\infty & \infty \\
\infty & \alpha \\
\alpha\end{array}$ & 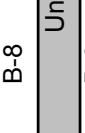 & 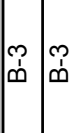 & $\begin{array}{l}\dot{p} \\
\dot{p} \\
\dot{\omega}\end{array}$ & $\begin{array}{l}m \\
\tilde{\infty} \\
\infty\end{array}$ & & $\bar{\sigma}$ \\
\hline & $\dot{s}^{*} \dot{0}$ & $\underset{2}{2}$ & $\mid \frac{\mathfrak{g}}{\square}$ & 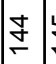 & $\stackrel{\wp}{f}$ & : & $\stackrel{\mathscr{P}}{\sigma}$ & 1 & F & $: 1 \underset{f}{\infty}$ & \begin{tabular}{l|l}
$\stackrel{\infty}{+}$ & $\stackrel{g}{\square}$ \\
\end{tabular} & & in & हू & 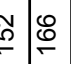 & & 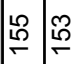 & 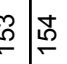 & 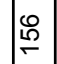 & th & $\stackrel{\infty}{\stackrel{\infty}{\rho}}$ & 1 量 & 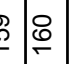 & 1 & $\underline{\underline{E}}$ & \\
\hline & 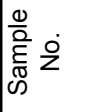 & & $\left|\begin{array}{l}\approx \\
\tilde{\omega} \\
\infty\end{array}\right|$ & $\mid \begin{array}{l}m \\
\dot{d} \\
\infty\end{array}$ & & 电 & 品 & $\hat{u}$ & 舟 & $\mid \begin{array}{l}8 \\
\dot{d} \\
\dot{u}\end{array}$ & 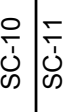 & & 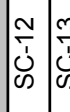 & 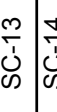 & 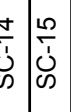 & & 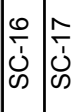 & 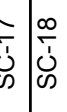 & 号 & 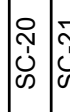 & ঠ্ত্ & 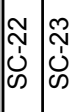 & 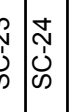 & $\mid$ & 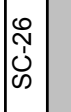 & \\
\hline
\end{tabular}




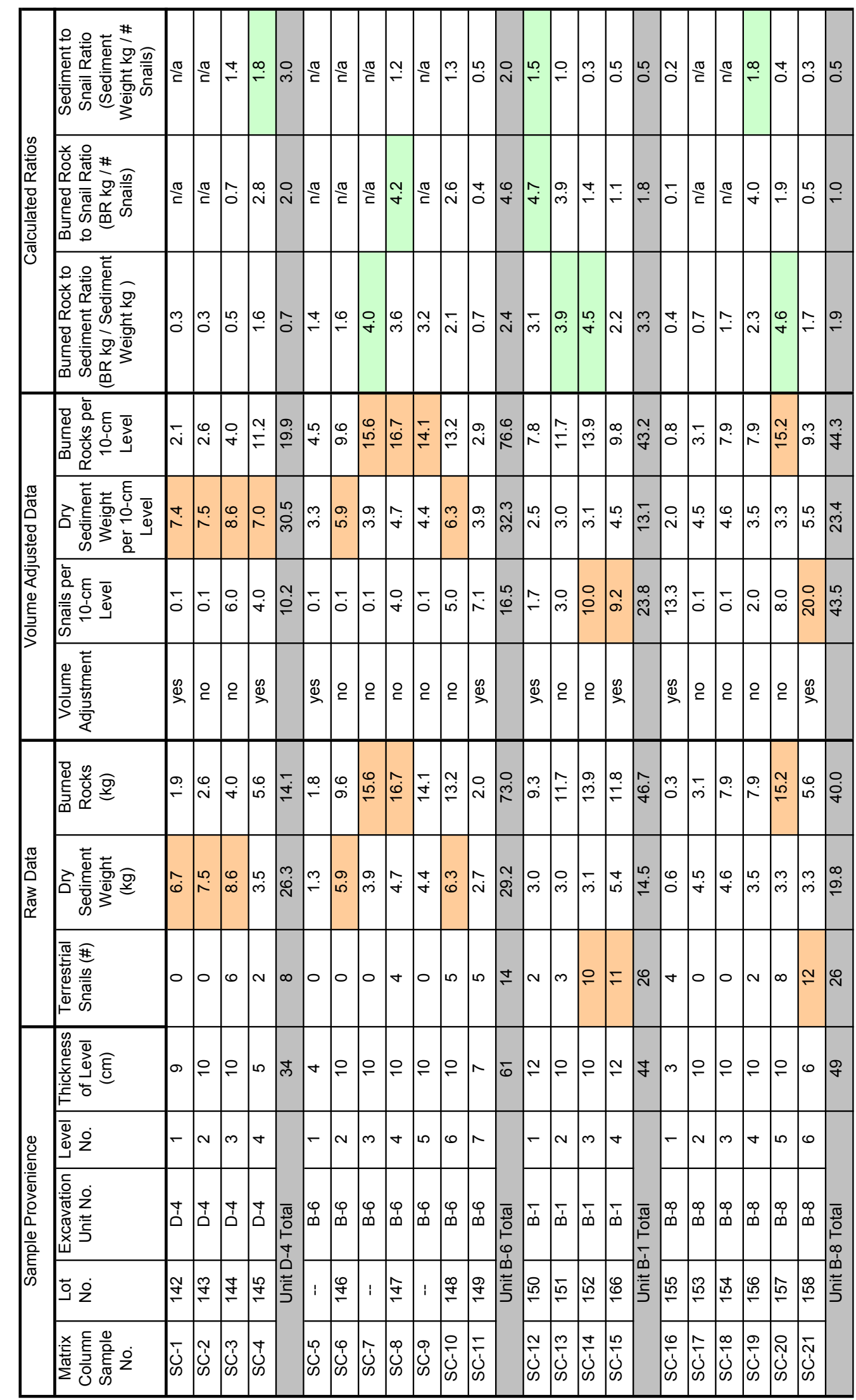




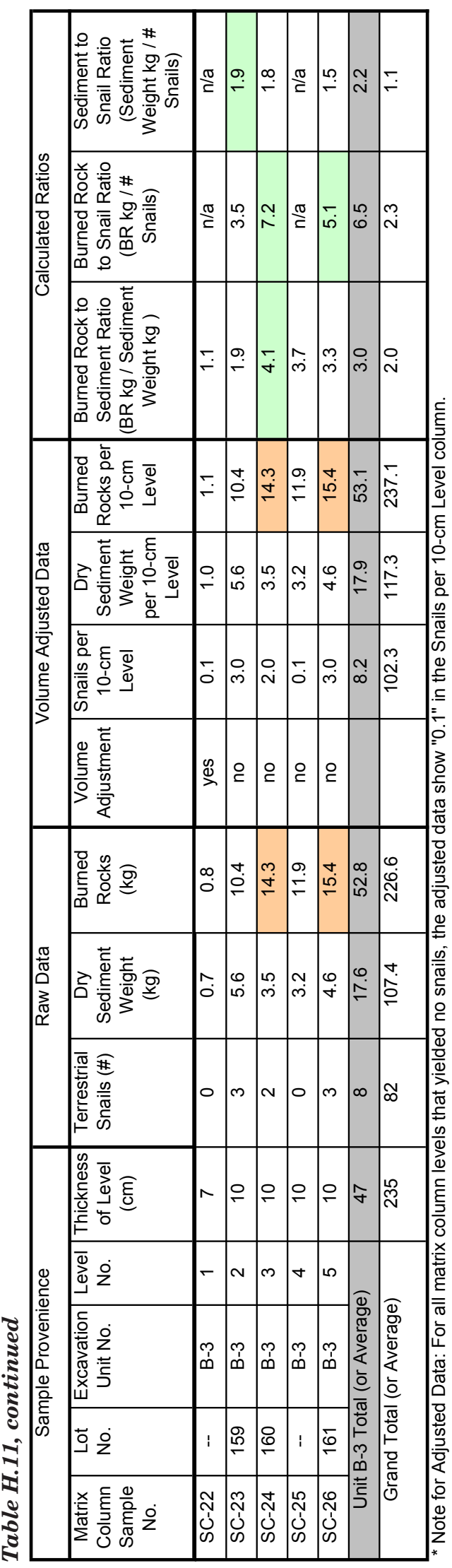

\begin{tabular}{|c|c|c|c|}
\hline 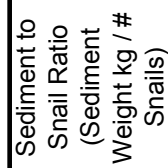 & & $\sigma^{\circ}$ & o. \\
\hline 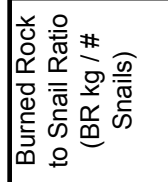 & $\stackrel{N}{\sim}$ & $\stackrel{m}{\sim}$ & $\because$ \\
\hline 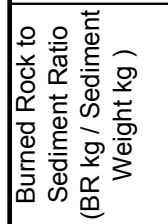 & $\stackrel{\circ}{\circ}$ & $\stackrel{\circ}{i}$ & m. \\
\hline 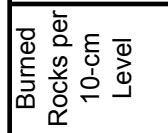 & $\stackrel{\Lambda}{\underline{o}}$ & के & $\infty^{\infty}$ \\
\hline 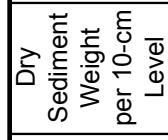 & $\left.\right|_{\infty} ^{0}$ & קִน & $\stackrel{0}{-}$ \\
\hline 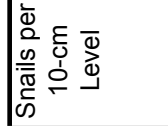 & & O্ & 0 \\
\hline
\end{tabular}

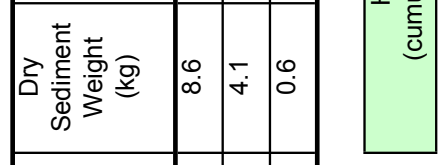

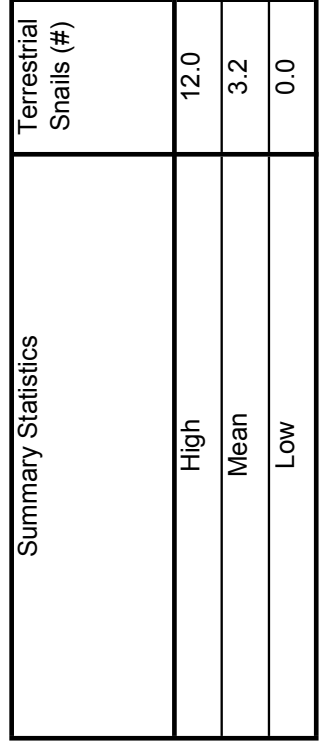

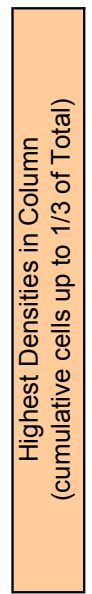


